\title{
Using natural adjuvants to \\ stimulate anti-tumour immune \\ responses
}

\author{
By \\ Sabine Kuhn \\ Malaghan Institute of Medical Research
}

\begin{abstract}
A thesis
submitted to the Victoria University of Wellington in fulfilment of the requirements for the degree of Doctor of Philosophy in Biomedical Science
\end{abstract}

Victoria University of Wellington

2012 



\section{Abstract}

The anti-tumour immune response is often not potent enough to prevent or eradicate disease. Dendritic cells (DCs) are professional antigen-presenting cells that are critical for the initiation of immune responses. While DCs frequently infiltrate tumours, lack of activation together with immuno-suppressive factors from the tumour can hamper an effective anti-tumour immune response.

In this thesis, the ability of microbial stimuli and danger signals to overcome suppression and re-programme DCs and macrophages to an immuno-stimulatory phenotype was investigated. Whole live Mycobacterium smegmatis and BCG were used to provide multiple pathogen-associated molecular patterns. The intracellularly-recognised toll-like-receptor (TLR) ligands CpG and Poly IC, as well as the extracelullarly recognised TLR ligand LPS, and the danger signal monosodium-urate crystals (MSU) were also included.

Bone-marrow derived DCs were found to respond to all adjuvants in vitro and DCs in tumour cell suspensions could be activated ex vivo. To assess the ability of adjuvants to enhance anti-tumour responses in vivo, immune-competent mice bearing established subcutaneous B16F1 melanomas were injected peri-tumorally with the different adjuvants. In line with previous reports, $\mathrm{CpG}$ treatment was effective in delaying tumour growth and increasing survival. A similar effect was found with Poly IC, but not with LPS, M. smegmatis, BCG or MSU alone. Combination of M. smegmatis + MSU, however, significantly delayed tumour growth and prolonged survival, while combinations of MSU + BCG or LPS were ineffective. Similar results were obtained using the B16.OVA melanoma and E.G7-OVA thymoma subcutaneous tumour models. In addition, Poly IC and MSU + M. smegmatis reduced primary tumour growth as well as lung metastases in the orthotopic 4T1 breast carcinoma model. 

Both Poly IC and MSU + M. smegmatis elicited an anti-tumour immune response that required CD8 T cells as well as NK cells. These treatments also resulted in increased proliferation of CD8 T cells and NK cells in tumour-draining lymph nodes, augmented infiltration of effector cells into the tumour, as well as enhanced production of inflammatory cytokines by effector cells and DCs in tumours. In addition, MSU + M. smegmatis also stimulated CD4 T cell proliferation, tumour-infiltration and activation, while at the same time decreasing the frequency of regulatory $\mathrm{T}$ cells in tumours.

Activation of a successful immune response to tumours was associated with early induction of IL-12 and IFN $\gamma$, as well as moderate levels of pro-inflammatory cytokines at the tumour site and systemically. Furthermore, anti-tumour activity correlated with the induction of inflammatory monocyte-derived DCs in tumour-draining lymph nodes. These DCs were also observed in adjuvant treated tumours and their appearance was preceded by accumulation of inflammatory monocytes at the tumour site.

These findings suggest that specific natural adjuvants can successfully modify the tumour environment and enhance the innate and adaptive anti-tumour immune response to delay tumour progression and increase survival. 


\section{Acknowledgments}

I would like to express my heartfelt gratitude to my supervisor Prof. Franca Ronchese for giving me this opportunity to do my Ph.D. and for her patience, guidance and her willingness to let me pursue new ideas. Her insight and wealth of immunological knowledge will continue to inspire me. I also wish to thank my co-supervisor Dr Joanna Kirman, for sharing her expert knowledge about mycobacteria in immunology, and for her support and encouragement throughout this thesis project. I am grateful to Dr Jacquie Harper, who provided helpful insights into monocyte biology and valuable advice. I would also like to thank Dr Darren Day for taking on the role of administrative supervisor and facilitating my Ph.D. at Victoria University.

I am grateful to Mark (Jianping) Yang and Evelyn Hyde for teaching me how to handle mice and many other techniques when I first started. Special thanks go to Evelyn Hyde, Fenella Rich and Lisa Shaw for their assistance with ever expanding experiments. I would also like to thank all other members of the Immune Cell Biology group, and my summer student Courtney O'Sullivan, for their willingness to help and for being great company in the lab. Thank you to the support staff, especially of the Cell Technology Suite and the Biological Research Unit, who do an amazing job at the Malaghan Institute and enabled my experiments to run smoothly.

I would like to acknowledge my fellow Ph.D. students for their support and friendship and many great discussions, scientific and otherwise. Throughout my thesis project, I have had the opportunity to get to know great colleagues in "The yellow submarine", "Siberia" and "The Crow's nest" offices. I really enjoyed working in the supportive and intellectually challenging environment of the Malaghan Institute and would like to thank the director, Prof. Graham LeGros, for constantly driving critical thinking and research excellence.

I greatly appreciated the financial support from the Wellington Medical Research Foundation, Lottery Health Research Grants, the Cancer Society Wellington Branch 
and the Australasian Society for Immunology, who provided funding for my project and travel awards that enabled me to present my work at international conferences. I would also like to thank the Deutsche Akademische Austauschdienst and Victoria University for my Ph.D. scholarships.

Finally, I want to express my gratitude to all my friends and family, for their ongoing support and encouragement. In particular, I want to thank Stefanie Steiger, Sonai Lim, and Mimi and Flaviu Hodis, who have become great friends since I arrived in New Zealand. In memory of my parents, who have always encouraged me and helped me become the person I am today: I would have loved to share the experience with you. I am very lucky to have my wonderful partner Sebastian Riedle come to New Zealand with me. His constant encouragement, cheer and love have kept me motivated throughout my Ph.D - I couldn't have done it without him. 
viii

\section{Table of Contents}

$\begin{array}{lr}\text { Abstract } & \text { iii } \\ \text { Acknowledgements } & \text { vi } \\ \text { Table of Contents } & \text { viii } \\ \text { List of Figures } & \text { xiii } \\ \text { List of Tables } & \text { xvi } \\ \text { List of Abbreviations } & \text { xviii }\end{array}$

1 Introduction $\quad 1$

1.1 Tumour immune surveillance . . . . . . . . . . . . . . . . . 3

1.1.1 Evidence from animal models . . . . . . . . . . . . . . . . . 4

1.1.2 Clinical evidence . . . . . . . . . . . . . . . . 5

1.1.3 Recognition of tumour cells . . . . . . . . . . . . . 6

1.1.4 Elimination of tumour cells by cytotoxic immune cells . . . . . . 9

1.1.5 Mechanisms of direct tumour cell killing . . . . . . . . . . . . . 10

1.1.6 Cytokines in the anti-tumour immune response . . . . . . . . . 11

1.2 Tumour immune escape . . . . . . . . . . . . . . . . 15

1.2.1 Escape through antigen loss . . . . . . . . . . . . . 15

1.2.2 Escape through resistance to cell death . . . . . . . . . . . . . 16

1.2.3 Direct suppression of effector T cells by tumour-derived factors . 17

1.2.4 Immune-suppression via tumour-associated macrophages . . . . 18

1.2.5 Immune-suppression via regulatory $\mathrm{T}$ cells . . . . . . . . . . . . 19

1.2.6 Immune-suppression via myeloid-derived suppressor cells . . . . 21

1.3 Dendritic cells . . . . . . . . . . . . . . . . . 23

1.3.1 Initiation of adaptive immune responses . . . . . . . . . . . . 23

1.3.2 Activation of innate immune responses . . . . . . . . . . . . . . 24

1.3.3 Activation states of DCs . . . . . . . . . . . . . . 24

1.3.4 Antigen presentation to T cells . . . . . . . . . . 26

1.3.5 Subtypes of DCs . . . . . . . . . . . . . . . . . . . . . . 29

1.3.6 Role of DCs in anti-tumour immune responses . . . . . . . . . . 32

1.4 Natural adjuvants . . . . . . . . . . . . . . . . . . . 33

1.4.1 TLR ligands . . . . . . . . . . . . . . . . . 34

1.4 .2 NLR ligands . . . . . . . . . . . . . . . . . . . . . 36

1.4.3 RLR ligands . . . . . . . . . . . . . . . . . . . 37

1.4.4 Control of adaptive immunity . . . . . . . . . . . . . 38

1.5 Tumour immunotherapy . . . . . . . . . . . . . . . . . . . . . 40

1.5.1 Cell based immunotherapy . . . . . . . . . . . . . . . . . . . 41

1.5.2 Cell free immunotherapy . . . . . . . . . . . . . . . . 43 
1.6 Aims of this thesis . . . . . . . . . . . . . . . . . . 47

2 Material and methods $\quad 49$

2.1 Material . . . . . . . . . . . . . . . . . . 50

2.1.1 Labware . . . . . . . . . . . . . . . . . . 50

2.1.2 Reagents and buffers . . . . . . . . . . . . . . . . 52

2.1.3 Cytokines and growth factors . . . . . . . . . . . . 61

2.1.4 Antibodies and fluorophores . . . . . . . . . . . . 62

2.1.5 Mycobacteria . . . . . . . . . . . . . . . 65

2.1.6 Tumour cell lines . . . . . . . . . . . . . . . . . . . . . . 65

2.1 .7 Mice . . . . . . . . . . . . . . . 66 66

2.2 Methods . . . . . . . . . . . . . . . . . . . . 68

2.2.1 Cell culture . . . . . . . . . . . . . . . . . . . . 68

2.2.2 Tissue processing . . . . . . . . . . . . . . 70

2.2.3 Flow cytometry . . . . . . . . . . . . . . 73

2.2.4 Gene expression analysis with real-time PCR . . . . . . . 76

2.2.5 Cell purification . . . . . . . . . . . . . . . . . . 78

2.2.6 Tumour models . . . . . . . . . . . . . . . . . . . . 79

2.2.7 Assays of cell function . . . . . . . . . . . . . . . . . . . . . . . . . . . . . . . 81

2.2 .8 Data analysis . . . . . . . . . . . . . . . . . . 82

3 Identification of adjuvants for the activation of tumour-infiltrating $\begin{array}{ll}\text { DCs } & 85\end{array}$

3.1 Introduction . . . . . . . . . . . . . . . . . . . 86

3.2 Aims . . . . . . . . . . . . . . . . . . . . 88

3.3 Results . . . . . . . . . . . . . . . . . . . . 89

3.3.1 Different adjuvants induce varying degrees of activation of GMCSF/IL-4 BMDCs . . . . . . . . . . . . . . . . . . . . . 89

3.3.2 Different adjuvants induce varying degrees of activation of Flt3L BMDCs . . . . . . . . . . . . . . . . 95

3.3.3 DCs infiltrate tumours and show a poorly activated phenotype .100

3.3.4 The immune infiltrate of murine melanomas consists mainly of DCs and monocytes . . . . . . . . . . . . . 103

3.3.5 Validation of flow cytometry to analyse immune infiltrate in tumours . . . . . . . . . . . . . . . 105

3.3.6 DCs from tumours can be activated by adjuvants ex vivo . . . . 108

3.4 Discussion . . . . . . . . . . . . . . . . . . . . . . . 112 
4 Treatment with specific adjuvants delays tumour growth and activates DCs in vivo

4.1 Introduction . . . . . . . . . . . . . . . . . . . . . . . . 124

4.2 Aims . . . . . . . . . . . . . . . . . . . 126

4.3 Results . . . . . . . . . . . . . . . . . . . . 127

4.3.1 Selected adjuvants have anti-tumour activity in vivo . . . . . . 127

4.3.2 Effect of one treatment with adjuvants on tumourinfiltrating APCs . . . . . . . . . . . . . . . . 131

4.3.3 A single adjuvant administration affects DCs in tumour-draining LNs . . . . . . . . . . . . . . . . 139

4.3.4 A single peri-tumoral adjuvant treatment induces systemic cytokine release . . . . . . . . . . . . . . . . . . . . 144

4.3.5 Local induction of cytokines by one peri-tumoral adjuvant administration . . . . . . . . . . . . . . . . 147

4.3.6 Changes in DCs in tumour-draining LNs are sustained after repeated adjuvant administration . . . . . . . . . . 150

4.3.7 Impact of repeated adjuvant administration on monocytes and DCs in tumours . . . . . . . . . . . . . . . . . . . . . 154

4.4 Discussion . . . . . . . . . . . . . . . . . . . . 160

5 Effective adjuvants activate both innate and adaptive effector cells 171

5.1 Introduction . . . . . . . . . . . . . . . . . . . . . . . . 172

5.2 Aims . . . . . . . . . . . . . . . . . . . . 174

5.3 Results . . . . . . . . . . . . . . . . . . . . . . 175

5.3.1 Effect of adjuvant treatment on in vivo $\mathrm{T}$ cell proliferation . . . . . . . . . . . . . . . . 175

5.3.2 A single adjuvant treatment has a minor impact on effector cell numbers and phenotype in tumours . . . . . . . . . . . . . . 180

5.3.3 Repeated adjuvant treatment increases infiltration and activation of effector cells in tumours . . . . . . . . . . . . . . . 182

5.3.4 Repeated adjuvant treatment increases the capacity of effector cells in tumours to produce pro-inflammatory cytokines . . . . . 184

5.3.5 T cells from adjuvant-treated mice delay onset of tumour growth in naïve recipients . . . . . . . . . . . . . . . . . . . 188

5.3.6 Adaptive immune cells are required for the anti-tumour effect of adjuvants . . . . . . . . . . . . . . . . . . . 193

5.3.7 Both adaptive and innate immune cells are necessary for the anti-tumour effect of adjuvants . . . . . . . . . . . . . . 195 
5.3.8 MSU + M. smegmatis reduces Tregs and induces CD4 T cell activation in tumours . . . . . . . . . . . . . . . . . . . 199

5.4 Discussion . . . . . . . . . . . . . . . . . . . . 202

6 Optimisation of adjuvant administration to delay tumour growth and reduce metastases $\quad 215$

6.1 Introduction . . . . . . . . . . . . . . . . . . 216

6.2 Aims . . . . . . . . . . . . . . . . . . . . 218

6.3 Results . . . . . . . . . . . . . . . . . . . . 219

6.3.1 Optimisation of the number and frequency of treatments with Poly IC and MSU + M. smegmatis . . . . . . . . . . . . . . . 219

6.3.2 Heat-killed and live M. smegmatis + MSU both activate anti-tumour immunity . . . . . . . . . . . . . 222

6.3.3 Effect of Poly IC and MSU + M. smegmatis treatment contralateral to the tumour site . . . . . . . . . . . . . . 225

6.3.4 Contralateral MSU + M. smegmatis treatment requires adaptive immmunity . . . . . . . . . . . . . . . . . 227

6.3.5 Differential immune response to contralateral and peri-tumoral $\mathrm{MSU}+$ M. smegmatis treatment . . . . . . . . . . . . 228

6.3.6 The capacity of adjuvants delay primary tumour growth is similar in melanoma, thymoma and breast carcinoma models . . . . 233

6.3.7 Peri-tumoral Poly IC and MSU + M. smegmatis treatments reduce metastases . . . . . . . . . . . . . . . . . . 237

6.4 Discussion . . . . . . . . . . . . . . . . . . . . . . 239

7 General discussion $\quad 249$

7.1 Cytokines induced by adjuvant treatment . . . . . . . . . . . . . . . . 250

7.2 Accumulation of monocytes and monocyte-derived DCs in response to adjuvants . . . . . . . . . . . . . . . . . . 253

7.3 Potential of tumour-infiltrating monocytes to function as MDSCs . . . 255

7.4 Activation of adaptive and innate effector cells by adjuvant treatment . 256

7.5 Implications of the findings of this thesis . . . . . . . . . . . . . . 257

7.5.1 Tumour-infiltrating monocytes may exert suppressive or stimulating functions . . . . . . . . . . . . . . . . 257

7.5.2 Monocyte-derived DCs in tumour immunotherapy . . . . . . . . 258

7.5.3 Potential for the combination of adjuvant treatment with blockade of immuno-suppression . . . . . . . . . . . . . . . . 259

7.6 Summary and conclusions . . . . . . . . . . . . . . . . . . . 260

7.7 Future directions . . . . . . . . . . . . . . . . . . . 262 
xii

References

Appendix

A LSRII specifications . . . . . . . . . . . . . . . . . . 304

B Distribution of cell frequencies and tumour sizes . . . . . . . . . . . . 305

C 96-well-plate quantitative PCR of B16F1 gene expression . . . . . . . . 307

D Spleen monocytes frequency, numbers and suppressive function . . . . . 311

E Publications . . . . . . . . . . . . . . . . . . . . . 314 


\section{List of Figures}

Figure 3.1 Time course of GM-CSF/IL-4 BMDC activation by adjuvants in vitro . . . . . . . . . . . . . . . . . . . . . . . . 90

Figure 3.2 GM-CSF/IL-4 BMDC activation by adjuvants in vitro . . . . . 92

Figure 3.3 Cytokine production by GM-CSF/IL-4 BMDC activated by adjuvants in vitro . . . . . . . . . . . . . . . . . . . . . . . 93

Figure 3.4 Time course and MSU titration for Flt3L BMDC activation by adjuvants in vitro . . . . . . . . . . . . . . . . . . . . . 96

Figure $3.5 \quad$ Activation of Flt3L pDCs by adjuvants in vitro . . . . . . . . . 98

Figure 3.6 Activation of Flt3L cDC subpopulations by adjuvants in vitro . 99

Figure 3.7 Cytokine production by Flt3L BMDCs activated by adjuvants in vitro . . . . . . . . . . . . . . . . . . . . . . . . 100

Figure 3.8 Identification of DCs in murine melanomas . . . . . . . . . . . 101

Figure 3.9 DCs in tumours are mainly CD11b+ . . . . . . . . . . . . . 102

Figure 3.10 Representative gating strategy for flow cytometric analysis of the immune cell infiltrate in B16 tumours. . . . . . . . . . . . 104

Figure 3.11 Idenfitication of immune cell types in B16F1 tumours . . . . . 105

Figure 3.12 Blood cells reperesent a small proportion of the tumour immune infiltrate . . . . . . . . . . . . . . . . 106

Figure 3.13 Blood cells do not skew cell type analysis of the tumour immune infiltrate by flow cytometry . . . . . . . . . . . . . 107

Figure 3.14 DCs from B16F1 tumours can be activated by exposure to adjuvants ex vivo . . . . . . . . . . . . . . . . . . . . . . 110

Figure 3.15 Adjuvant treatment induces secretion of pro-inflammatory cytokines in B16F1 tumours ex vivo . . . . . . . . . . . . . . . 111

Figure 4.1 Effect of different MSU doses on B16F1 tumour growth. . . . 128

Figure 4.2 Some adjuvants delay tumour growth . . . . . . . . . . . . . . 130

Figure 4.3 Representative gating of lymphoid and myeloid immune cells in tumours . . . . . . . . . . . . . . . . . . . . 132

Figure 4.4 Some adjuvants affect immune infiltrate in tumours after a single adjuvant treatment . . . . . . . . . . . . . . . 133

Figure 4.5 Some adjuvants increase monocyte infiltrate in tumours after a single treatment . . . . . . . . . . . . . . . . 135

Figure 4.6 Macrophages in tumours remain largely unaffected by one adjuvant treatment . . . . . . . . . . . . . . . . 136

Figure 4.7 DCs in tumours are not activated after a single adjuvant treatment . . . . . . . . . . . . . . . . 138 
Figure 4.8 DC numbers and subsets in dLN change after a single adjuvant treatment . . . . . . . . . . . . . . . 140

Figure 4.9 Some adjuvants activate DCs in dLN after a single administration141

Figure 4.10 DC in dLN increase their capacity to produce cytokines after a single adjuvant treatment . . . . . . . . . . . . . . . . . 143

Figure 4.11 Peri-tumoral administration of some adjuvants increases serum cytokine concentrations . . . . . . . . . . . . . . . 145

Figure 4.12 Changes in the expression of immune-related molecules in tumours after one adjuvant treatment . . . . . . . . . . . . . . . 149

Figure 4.13 MSU + M. smegmatis and Poly IC continue to induce inflammatory DCs in dLNs after 4 treatments . . . . . . . . . . . . 151

Figure 4.14 Inflammatory DCs in dLN are activated by some adjuvants after 4 treatments . . . . . . . . . . . . . . . . 153

Figure 4.15 Number of immune cells in tumours. . . . . . . . . . . . . . . . 154

Figure 4.16 Inflammatory monocytes remain increased after $4 \mathrm{MSU}+$ M. smegmatis treatments . . . . . . . . . . . . . 156

Figure 4.17 Inflammatory DCs are increased in tumours after 4 adjuvant treatments . . . . . . . . . . . . . . . 158

Figure 4.18 Effective adjuvants increase the capacity of tumour-DCs to produce pro-inflammatory cytokines . . . . . . . . . . . . . 159

Figure 5.1 Adjuvant treatment enhances tumour-antigen specific CD8 T cell proliferation . . . . . . . . . . . . . . . 176

Figure 5.2 Successful adjuvants result in increased numbers of tumour-specific CD8 T cells in dLN . . . . . . . . . . . . . . . . . . 177

Figure 5.3 Only MSU + M. smegmatis treatment stimulates proliferation of tumour-antigen specific CD4 T cells in dLN . . . . . . . . . 178

Figure 5.4 Only MSU + M. smegmatis treatment increases tumour-specific CD4 T cell proliferation and numbers in dLN . . . . . . . . . . 179

Figure 5.5 A single adjuvant treatment leads to minor changes in intratumoral effector cells . . . . . . . . . . . . . . . . . . 181

Figure 5.6 Repeated treatment with effective adjuvants substantially increases immune infiltrate in tumours and alters its composition 183

Figure 5.7 Repeated treatment with effective adjuvants substantially increases the percentage of intra-tumoral effector cells and changes their phenotype . . . . . . . . . . . . . . . . . 185

Figure 5.8 Repeated treatment with effective adjuvants increases the capacity of intra-tumoral CD8 $\mathrm{T}$ cells to produce pro-inflammatory cytokines . . . . . . . . . . . . . . . 186 
Figure 5.9 Repeated treatment with effective adjuvants enhances the capacity of intra-tumoral NK cells to produce pro-inflammatory cytokines . . . . . . . . . . . . . . . . . 187

Figure 5.10 Adoptive transfer of splenic T cells from adjuvant treated tumourbearing mice into naïve recipients . . . . . . . . . . . . . . . . 189

Figure 5.11 Adoptive transfer of splenic T cells from adjuvant treated tumourbearing mice delays onset of tumour growth . . . . . . . . . . . 191

Figure 5.12 Increasing the numbers of adoptively transferred splenic T cells from adjuvant treated tumour-bearing mice does not improve tumour protection in recipients . . . . . . . . . . . . . . 192

Figure 5.13 The anti-tumour effect of Poly IC and MSU + M. smegmatis treatment requires adaptive immunity . . . . . . . . . . . . 194

Figure 5.14 Depletion of CD4 T cells, CD8 T cells, Tregs and NK cells in tumour-bearing adjuvant-treated mice . . . . . . . . . . . . . 196

Figure 5.15 Both CD8 T cells and NK cells are required for the anti-tumour effect of Poly IC and MSU + M. smegmatis treatment . . . . . 197

Figure 5.16 MSU + M. smegmatis but not Poly IC treatment reduces the frequency of Tregs in tumours . . . . . . . . . . . . . 200

Figure 5.17 MSU + M. smegmatis but not Poly IC treatment induces CD25+FoxP3- CD4 T cells in tumours . . . . . . . . . . . . . 201

Figure 6.1 Repeated administration increases the anti-tumour effect of Poly IC and MSU + M. smegmatis treatments . . . . . . . . . 220

Figure 6.2 More frequent or earlier start of adjuvant administration does not significantly improve anti-tumour activity . . . . . . . . . . 221

Figure 6.3 Live and dead M. smegmatis in combination with MSU are equally effective at delaying tumour progression . . . . . . . . 223

Figure 6.4 A single treatment with dead M. smegmatis + MSU has the same effects on intratumoral immune cells as live M. smegmatis + MSU . . . . . . . . . . . . . . . . . . . 224

Figure 6.5 Dead M. smegmatis + MSU increases the number of inflammatory DCs in dLN similar to live M. smegmatis + MSU . . . 225

Figure 6.6 Poly IC must be administered at the tumour site while MSU + M. smegmatis is effective even if injected into the contralateral flank . . . . . . . . . . . . . . . . 226

Figure 6.7 MSU + M. smegmatis contralateral treatment requires adaptive immunity . . . . . . . . . . . . . . . . . 2227

Figure 6.8 A single MSU + M. smegmatis contralateral treatment does not affect the immune infiltrate in tumours . . . . . . . . . . . 229 
Figure 6.9 A single MSU $+M$. smegmatis contralateral treatment does not affect DCs in tumour-draining LN . . . . . . . . . . . . 230

Figure 6.10 MSU + M. smegmatis contralateral treatment induces proliferation of tumour specific CD4 and CD8 T cells . . . . . . . 231

Figure 6.11 Repeated MSU + M. smegmatis contralateral treatment has similar effects on the composition of the immune infiltrate in tumours as peri-tumoral administration . . . . . . . . . . 232

Figure 6.12 Adjuvant treatment in a thymoma model is effective at delaying tumour growth . . . . . . . . . . . . . . . . . . 234

Figure 6.13 Treatments with selected adjuvants delay tumour growth in an orthotopic breast carcinoma model similar to their effect against melanoma . . . . . . . . . . . . . . . . 236

Figure 6.14 Treatment with Poly IC or MSU + M. smegmatisreduces metas-

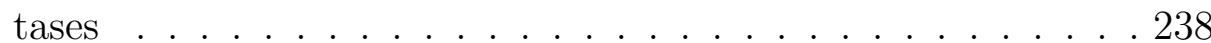

Figure 7.1 Working model of the effects of adjuvant treatments on the anti-tumour immune response. . . . . . . . . . . . . . . 251

Figure B.1 B16F1 tumour sizes are consistent with Gaussian distribution. 305

Figure B.2 Infiltration of immune cells into tumours is consistent with Gaussian distribution. . . . . . . . . . . . . . . . 305

Figure B.3 Accumulation of DCs in dLNs is consistent with Gaussian distribution. . . . . . . . . . . . . . . 306

Figure D.1 Frequency and number of monocytes in spleens of adjuvant treated tumour bearing mice. . . . . . . . . . . . . . . . . 311

Figure D.2 Suppression of T cell proliferation by Ly6Chi monocytes. . . . 312

Figure D.3 Suppression of T cell proliferation by Ly6G+ cells. . . . . . . . 313 


\section{List of Tables}

Table 2.1 Labware . . . . . . . . . . . . . . . . . . . . 50

Table 2.2 Antibodies . . . . . . . . . . . . . . . . . . 63

Table 2.3 Isotype Controls . . . . . . . . . . . . . . . . . . . . . . . . . . 64

Table 2.4 Real-time PCR assays . . . . . . . . . . . . . . . . . . . . . 77

Table 4.1 Number of mice per treatment group and experiment for B16F1 tumour growth . . . . . . . . . . . . . . . . . . . . 129

Table A.1 LSR II Lasers . . . . . . . . . . . . . . . . . . . . . . . . . . . . 304

Table A.2 LSR II Detectors . . . . . . . . . . . . . . . . . . . . . . . . . 304

Table C.1 96 -well-plate quantitative PCR CT values . . . . . . . . . . . 307 
xviii

\section{List of Abbreviations}

\begin{tabular}{|c|c|}
\hline $2-\mathrm{ME}$ & 2-Mercaptoethanol \\
\hline $\mathrm{ACT}$ & Ammonium Chloride Tris \\
\hline AIDS & Acquired immuno-deficieny syndrome \\
\hline ANOVA & Analysis of Variance \\
\hline AP-1 & activator protein 1 \\
\hline $\mathrm{APC}$ & Antigen presenting cell \\
\hline $\mathrm{APC}$ & Allophycocyanin \\
\hline ATP & Adenosine triphosphate \\
\hline B6 mice & B6.SJL-Ptprc ${ }^{\mathrm{a}} \mathrm{Pep} 3^{\mathrm{b}} /$ BoyJArc mice \\
\hline $\mathrm{BCG}$ & Mycobacterium bovis Bacillus Calmette-Guérin \\
\hline $\mathrm{BM}$ & Bone marrow \\
\hline BMDC & Bone marrow-derived dendritic cell \\
\hline $\mathrm{CCR}$ & Chemokine (C-C motif) receptor \\
\hline CCL & CC chemokine ligand \\
\hline $\mathrm{CD}$ & Cluster of differentiation \\
\hline $\mathrm{cDC}$ & Conventional dendritic cell \\
\hline cDNA & Complementary deoxyribonucleic acid \\
\hline CFA & Complete Freund's adjuvant \\
\hline CFDA-SE & Carboxyfluorescein diacetate succinimidyl ester \\
\hline CFSE & Carboxyfluorescein succinimidyl ester \\
\hline $\mathrm{CFU}$ & Colony forming units \\
\hline cIMDM & Complete Iscove's modified Dulbecco's medium \\
\hline $\mathrm{CpG}$ & Oligodeoxynucleotides with adjoining cytosine and guanine bases \\
\hline $\mathrm{CT}$ & Cycle threshold \\
\hline CTL & Cytotoxic T lymphocyte \\
\hline CTLA-4 & Cytotoxic T lymphocyte associated antigen 4 \\
\hline CXCR & Chemokine (C-X-C motif) receptor \\
\hline $\mathrm{Cy}$ & Cyanine \\
\hline DAMP & Danger associated molecular pattern \\
\hline DAPI & 4',6-Diamidino-2-phenylindole \\
\hline $\mathrm{DC}$ & Dendritic cell \\
\hline $\mathrm{dH}_{2} \mathrm{O}$ & Distilled water \\
\hline $\mathrm{dLN}$ & Draining lymph node \\
\hline DMSO & Dimethyl sulfoxide \\
\hline DNA & Deoxyribonucleic acid \\
\hline dsRNA & Double-stranded RNA \\
\hline
\end{tabular}


DT

DTR

EDTA

FACS

FBS

FITC

Flt3L

FMO

Foxp3

FSC

G-CSF

GFP

GM-CSF

HBSS

HIF

i.p.

i.v

IDO

IFN

Ig

IL

IMDM

iNOS

IRF

$-\mathrm{L}$

LB broth

LC

LN

LPS

MACS

MAGE

MAPK

MCP-1

M-CSF

MDA-5

MDP

MDSC

MFI
Diphteria toxin

Diphteria toxin receptor

Ethylenediaminetetraacetic acid

Fluorescence activated cell sorting

Foetal bovine serum

Fluorescein isothiocyanate

Fms-like tyrosine kinase 3 ligand

Fluorescence minus one - staining control

Forkhead box P3

Forward scatter

Granulocyte colony-stimulating factor

Green fluorescent protein

Granulocyte-macrophage colony-stimulating factor

Hank's Buffered Salt Solution

Hypoxia inducible factor

Intraperitoneal

Intravenous

Indoleamine 2,3-dioxygenase

Interferon

Immunoglobulin

Interleukin

Iscove's modified Dulbecco's medium

Inducible nitric oxide synthase

Interferon regulatory factor

Ligand

Luria-Bertani broth

Langerhans cell

Lymph node

Lipopolysaccharide

Magnetic cell sorting

Melanoma associated antigen

Mitogen-activated protein kinase

Macrophage chemoattractant protein 1

Macrophage colony-stimulating factor

Melanoma differentiation associated factor 5

Muramyl-dipeptide

Myeloid derived suppressor cell

Median fluorescence intensity 


\begin{tabular}{|c|c|}
\hline $\mathrm{MHC}$ & Major histocompatibility complex \\
\hline MIC & MHC class I polypeptide-related sequence \\
\hline MPL & Monophosphoryl lipid A \\
\hline mRNA & Messenger ribonucleic acid \\
\hline MSU & Monosodium urate crystals \\
\hline MyD88 & Myeloid differentiation primary response protein 88 \\
\hline $\mathrm{NF} \kappa \mathrm{B}$ & Nuclear factor $\kappa$-light-chain-enhancer of activated B cells \\
\hline NK & Natural killer \\
\hline NKT & Natural killer T \\
\hline NOD & Nucleotide-binding oligomerization domain containing protein \\
\hline NLR & Nucleotide-binding, leucine-rich-repeat containing receptor \\
\hline OADC & Oleic albumin dextrose catalase (growth supplement) \\
\hline OTI & Ovalbumin transgenic-I \\
\hline OTII & Ovalbumin transgenic-II \\
\hline OVA & Ovalbumin \\
\hline PAMP & Pathogen-associated molecular pattern \\
\hline PBS & Phosphate buffered saline \\
\hline PCR & Polymerase chain reaction \\
\hline PD-1 & Programmed death receptor 1 \\
\hline $\mathrm{PE}$ & Phycoerythrin \\
\hline PerCP & Peridinin chlorophyll protein \\
\hline $\mathrm{pDC}$ & plasmacytoid dendritic cell \\
\hline PI & Propidium iodide \\
\hline Poly IC & Polyinosinic-polycytidylic acid \\
\hline PRR & Pattern recognition receptor \\
\hline $\mathrm{RAG}$ & Recombination activating gene \\
\hline RIG-I & Retinoic acid incucible gene I protein \\
\hline RLR & RIG-I-like receptor \\
\hline ROS & Reactive oxygen species \\
\hline SA & Streptavidin \\
\hline s.c. & Subcutaneous \\
\hline SD & Standard deviation \\
\hline $\mathrm{SE}$ & Standard error \\
\hline SCID & Severe combined immunodeficieny \\
\hline $\mathrm{SSC}$ & Side scatter \\
\hline TAA & Tumour associated antigen \\
\hline TAE & Tris-acetate-ethylenediaminetetraacetic acid \\
\hline TAP & Transporter associated with antigen processing \\
\hline
\end{tabular}


TCR

TGF

Th

TLR

TNF

TRAIL

Treg

TRIF

$\mathrm{V} \alpha$

$\mathrm{V} \beta$

VEGF
$\mathrm{T}$ cell receptor

Transforming growth factor

$\mathrm{T}$ helper

Toll-like receptor

Tumour necrosis factor

TNF-related apoptosis-inducing ligand

Regulatory $\mathrm{T}$ cell

TIR-domain-containing adaptor-inducing interferon $\beta$

Variable $\alpha$ region of the $\mathrm{T}$ cell receptor

Variable $\beta$ region of the $\mathrm{T}$ cell receptor

Vascular endothelial growth factor 

Chapter 1

\section{Introduction}


Cancer remains a major cause of death world-wide [1]. Depending on location and grade, cancers can be treated by surgery, chemotherapy or radiation therapy [2]. Surgery that involves removal of the entire tumour mass can be curative. However, if the tumour is not accessible, has infiltrated the tissue locally or has metastasised throughout the body, radiation or chemotherapy are commonly used. These treatments rely on damaging the DNA of the quickly proliferating tumour cells to eliminate them. However, all of these treatments are also carcinogenic Travis, 2002, p48958. In addition, a small population of tumour cells may be resistant to chemotherapeutic drugs. Therefore, the therapy can select for the resistant cells, which then grow out and lead to disease recurrence. Targeted therapies against specific tumour mutants have now been developed, which use monoclonal antibodies that bind to a tumour-specific antigen [3].

Immunotherapy has the potential to complement existing therapies. Immune cells are highly specific, can directly eliminate their targets and seek out even individual tumour cells throughout the body [2]. While spontaneous activation of an effective anti-tumour immune response is rare, it can lead to complete tumour regression [4]. Immunotherapy aims to boost or elicit the anti-cancer immune response. Cell-based immunotherapy approaches have induced some complete and partial responses in cancer patients and the dendritic cell (DC) vaccine provenge has now been approved for use in prostate cancer by the US Food \& Drug Administration [5]. However, clinical response rates are still low and these treatments are labour intensive and expensive.

Other strategies aim to block tumour-suppressive molecules or activate stimulatory receptors to improve the anti-tumour response. Ipilimumab and BMS-936558, antibodies against inhibitory molecules on cytotoxic $\mathrm{T}$ cells, have shown promising results in clinical trials [6-8]. The mycobacterium Mycobacterium bovis Bacillus Calmette-Guérin (BCG) is currently used to activate the immune response in patients with superficial bladder cancer $[9,10]$. 
As research on the receptors that the immune system employs to detect pathogens has progressed, a range of natural and synthetic ligands has been developed. These structures all activate immune cells and can potentially be used as adjuvants to stimulate the immune response both in vaccination and in a cancer therapy setting. Some adjuvants, namely oligodeoxynucleotides containing CpG motifs ( $\mathrm{CpG}$ ), Polyinosinicpolycytidylic acid (Poly IC) and Imiquimod, have shown promising results in animal models of cancer [11-13]. In clinical trials, the results with $\mathrm{CpG}$ have been disappointing [14]. The studies of Poly IC in humans indicate that it has similar effects to viral vaccines and can increase the efficacy of chemotherapy and radiation therapy in brain cancer $[15,16]$. A topical cream of Imiquimod is currently used to treat warts and basal cell carcinoma [17]. These studies all show that adjuvants can be used to activate the anti-tumour immune response. However, the understanding of their mechanism of action and the immune parameters required to induce a successful anti-tumour response remain limited.

The aim of this introductory chapter is to review the literature on the current understanding of the anti-tumour immune response, the activation of immune cells by adjuvants and their potential for immunotherapy.

\subsection{Tumour immune surveillance}

The role of the immune system to prevent and eliminate infection with pathogens such as viruses, bacteria and parasites is well recognised. However, the immune response is also critical for the prevention of tumours. First, it protects the host from virus-induced tumours by clearing viral infections. Secondly, the timely elimination of pathogens limits inflammation, which, especially in a chronic setting, can be conducive to tumour formation. Thirdly, there is now ample evidence that the immune system can specifically identify and eliminate tumour cells, thus preventing the outgrowth of tumours. This third process is termed cancer immune surveillance. The idea that the immune system can protect the body from malignant disease was already suggested 
by Paul Ehrlich in 1909 [18]. In the 1950s, Burnet and Thomas further advanced this concept, proposing that immune cells could directly eliminate cancer cells [19], but conclusive evidence in support has only emerged in the last 15 years [20-22].

\subsubsection{Evidence from animal models}

One of the first observations of a tumour-protective effect of the immune response was that administration of interferon (IFN) could increase the survival of tumour-bearing mice [23]. Later studies found that endogenous $\operatorname{IFN} \gamma$ was required to protect mice against the growth of transplanted tumours [24]. The importance of IFN $\gamma$ for tumour control was further substantiated by studies in $\operatorname{IFN} \gamma$ receptor deficient mice that showed an increased incidence of both chemically induced and spontaneous tumours [20], and was also confirmed by independent work on IFN $\gamma$ knock out mice [25].

Subsequently to the identification of cytokines as important mediators to prevent tumours, the cells required to mediate the tumour-protective function of the immune system were characterised. Recombination activating gene (Rag) 2-/- mice that lack T, B and Natural Killer T (NKT) cells [26] were found to be more susceptible to both chemically induced and spontaneous tumours [21]. Similar results were obtained in Rag1-/- and severe combined immuno-deficient (SCID) mice, which are also deficient in T, B and NKT cells [27], thus demonstrating the tumour-protective function of host lymphocytes. Furthermore, gene targeted mice lacking NKT cells or depleted of either T cells or Natural Killer (NK) cells revealed differential roles of both cell types in tumour immune surveillance [28]. In addition to conventional $\mathrm{T}$ cells, $\gamma \delta \mathrm{T}$ cells were also shown to play a role in tumour protection [29]. These studies collectively demonstrate a prominent role of both adaptive and innate immune cells in preventing tumour growth.

The mechanisms through which immune cells controlled tumour growth, were found to include both cytokine production and direct elimination of tumour cells. Lymphocyte- 
mediated cytotoxicity was first described as a mechanism of immune control of lymphoma by Smyth et al. [22]. Further experiments revealed independent roles of IFN $\gamma$ and the cytotoxic molecule perforin in the control of chemically induced sarcomas and transplantable carcinomas [25]. Thus, there is strong evidence for tumour immune surveillance in rodent models.

\subsubsection{Clinical evidence}

In humans, indirect evidence for the importance of the immune system in the control of cancer comes both from tumour-suppressed patients, as well as from observations of spontaneous anti-tumour immune responses that correlate with better survival.

Acquired immuno-deficiency syndrome (AIDS) patients have higher rates of some cancers $[30,31]$. As many of the malignancies that show increased frequencies in AIDS patients are associated with viral infections, such as lymphomas (Epstein-Barr virus), Kaposi's sarcoma (herpes viruses) and cervical cancer (human papilloma viruses), this has been argued to reflect a reduction in anti-viral immunity rather than impeded tumour immune surveillance. However, patients treated with immuno-suppressive drugs following organ transplants also have a higher risk of cancer, including non-infectious colon, lung, renal and pancreatic cancers $[32,33]$. In rare cases [34], these tumours are donor-derived and may arise from the outgrowth of undiagnosed micro-metastases already present in the organ transplant that develop in the tumour-suppressed recipient. The majority of tumours in transplant recipients develop de novo [34]. The higher incidence of cancers in immuno-suppressed patients indicates that in healthy individuals the immune system plays an important role in preventing the formation or outgrowth of tumours.

Spontaneous regression of tumours, which is sometimes observed in cancer patients, is probably due to an anti-tumour immune response. William Coley noted that especially patients that contracted a severe bacterial infection associated with fever had a 
higher rate of spontaneous cancer regression. Between 1880 to 1910 he was the first to develop an immunotherapy approach based on the injection of a mixture of pyrogenic bacteria, known as Coley's toxin, which resulted in complete and permanent regression of inoperable sarcomas in about $10 \%$ of patients [35]. Subsequently, spontaneous anti-tumour immune responses were characterised in more detail. Antibody responses to autologous cancers were first identified in a subset of patients in the 1970s [36]. To date, antibodies to more than 100 tumour-associated antigens (TAAs) have been described [37]. These studies indicate that the human immune system can recognise tumour cells and react to them. T cells that are specific for tumour antigens and infiltrate malignant lesions have also been identified in many patients [38]. The strongest evidence for anti-tumour $\mathrm{T}$ cell responses in humans is probably provided by studies that report $\mathrm{T}$ cell expansion accompanying the spontaneous regression of melanoma lesions $[39,40]$.

Even when the immune system cannot fully control the tumour growth, tumour-infiltration by T cells, Natural Killer (NK) cells, or NKT cells has been shown to be a positive prognostic factor, predicting better survival in a range of cancers [41-43]. Both the quality and quantity of infiltrating lymphocytes was correlated with patient survival in colon and lung cancer and was a more powerful independent prognostic factor than pathological staging [42-44]. A high ratio of cytotoxic T cells (CTLs) to regulatory $\mathrm{T}$ cells (Tregs) appears correlate better with increased survival [44, 45] than infiltration by Tregs alone, which has variably been associated with poor [46] or better prognosis [47].

\subsubsection{Recognition of tumour cells}

To enable immune cells to eliminate cancer cells, they need to both recognise the tumour cells and become sufficiently activated to initiate a response. While tumour cells arise from normal body cells, they can express new or altered antigens that make them susceptible to recognition and elimination by T cells or NK cells. 
During the transformation process, tumour cells acquire several mutations and often show genetic instability and altered chromosomes. This can lead to the expression of neo-antigens, such as the fusion protein BCR-ABL in human chronic myelogenous leukaemia, which can be recognised by $\mathrm{T}$ cells [48]. In addition, some tumours that are associated with infection by oncogenic viruses express viral proteins, which are a foreign target for T cells [49]. Examples include the E6 and E7 proteins of human papillomavirus in cervical cancer [50] and K1 epitopes of Kaposi's sarcoma-associated herpesvirus [51].

In addition, tumour cells can aberrantly express antigens that are normally only found in immune-privileged sites, such as cancer-testis antigens [52]. Cancer testis antigens are not normally expressed throughout the body and therefore $\mathrm{T}$ cells are not tolerised against them and can react to their presence on tumour cells. Examples include the melanoma antigen (MAGE) family proteins, as well as NY-ESO-1 family proteins, which are expressed by melanomas as well as squamous cell carcinomas and a proportion of breast cancers [38, 53].

Cancer cells can also over-express differentiation antigens, such as gp100 and Melan-a in melanoma cells $[38,54]$, or growth factor receptors such as Her2 in breast and ovarian carcinomas [55]. As these antigens are also present in normal tissue cells, they are called tumour associated antigens (TAAs). The abnormally high levels of TAAs on tumour cells may induce them to be immunogenic, as $\mathrm{T}$ cells specific for TAAs have been described $[54,55]$.

In addition, tumour cells often display stress-antigens, such as MICA/B (Major Histocompatibility Complex (MHC) class I polypeptide-related sequence A/B), which are ligands for activating NK cell receptors [56]. At the same time, malignant cells often lose or down-regulate the expression of classical MHC I molecules that would otherwise inhibit NK cells, thus becoming sensitive to NK cell mediated killing [57].

While these studies demonstrate that there are tumour-specific and associated antigens that can be recognised by $\mathrm{T}$ cells, the initiation of anti-tumour $\mathrm{T}$ cell responses 
is less well understood. Janeway proposed that the immune system only becomes activated after sensing of pathogen associated molecular patterns (PAMPs). However, this could not explain the rejection of tissue transplants and the occasional spontaneous anti-tumour responses. Matzinger then proposed that 'danger signals' released from dying cells can also activate the immune response [58]. Classical 'danger signals' are induced early during tumour development. These include type I IFNs, which activate DCs and promote the induction of adaptive anti-tumour immune responses [59-61]. In addition, tumour cells often proliferate more rapidly than their blood supply develops and therefore the centre of solid tumours becomes necrotic. During necrotic cell death, cellular components are released. Some of these are recognised by the immune system as damage-associated molecular patterns (DAMPs). DAMPs include high mobility group box 1 proteins, adenosine triphosphate (ATP) and uric acid, which have all been shown to activate immune cells in response to cell death [62-64]. This can lead to local inflammation, recruitment of immune cells and may enable the initiation of the anti-tumour immune response [62, 65]. Tumour cell lysis by innate immune cells such as NK cells and macrophages, may also contribute to the early release of DAMPs and danger signals that alert the immune system to the tumour.

Cell death that is accompanied by the release of danger signals and DAMPs has been termed "immunogenic" cell death to differentiate it from apoptosis which normally occurs during physiological cell turnover and does not activate an immune response, thereby avoiding auto-immunity [66]. It has now become clear that radiation therapy as well as many chemotherapeutic agents induce immunogenic death of tumour cells. There is mounting evidence that the subsequent activation of DCs and the ensuing immune response contribute substantially to the success of these therapies in patients [66]. 


\subsubsection{Elimination of tumour cells by cytotoxic immune cells}

NK cells, T cells and macrophages can all directly kill target cells and several studies have shown that they can all contribute to anti-tumour immunity. However, the process of tumour elimination before the onset of apparent disease has not been directly visualised in vivo. Rather, it has been inferred from the earlier onset or greater penetrance of cancer in animals genetically deficient in or depleted of certain immune cell subsets, recognition molecules, cytotoxic molecules and cytokines. A deeper understanding of the roles of different immune cell types in the anti-tumour response may enable the design of better strategies to enhance their function.

As described in the previous section, tumours can express ligands that make them sensitive to lysis by NK cells. A distinct role for NK cells in controlling tumour growth was demonstrated in NK cell deficient mice by Smyth et al. [28].

T cells have been implicated in tumour control by the increased incidence of chemically induced tumours in mice lacking B, T and NKT cells [21, 27]. Subsequently, separate roles for $\alpha \beta$ and $\gamma \delta$ T cells in the protection from tumours were shown [29]. It has been demonstrated that CTLs can directly kill tumour cells [67]. The anti-tumour activity of CD4 T helper (Th) cells was initially mainly attributed to providing help for the priming of cytotoxic CD8 T cells [68] (see also Chapter 1.3.4). However, subsequent studies also demonstrated a 'post-licensing' role for CD4 T cells that were required for the maintenance and tumour infiltration of CD8 T cells $[69,70]$. In addition, in the specific situation of lymphopenia, CD4 T cells may even develop cytotoxic activity and directly eliminate tumour cells in vivo [71].

Macrophages often infiltrate both murine and human tumours, but their role is controversial. They can be either tumoricidal or promote tumour growth, depending on their activation state [72]. Classically activated macrophages that play a role in the clearance of infections are generally referred to as M1, while alternatively activated macrophages involved in wound-healing are designated M2. While 
M1-like macrophages are associated with tumour elimination, M2-like macrophages were linked with tumour progression [73]. Thus, a high density of M1-like compared to M2-like macrophages infiltrating non-small cell lung cancers was associated with extended survival of patients [74]. Macrophages can recognise tumour cells through their altered membrane composition as well as surface carbohydrates [75, 76], and in some experimental murine tumours macrophages were required for tumour rejection [77]. Their anti-tumour activity comprises both the direct elimination of cancer cells via phagocytosis and secretion of cytotoxic factors $[76,78]$ and the induction of adaptive immunity and enhancement of the cytotoxic activity of $\mathrm{T}$ cells [79]. Their tumour-promoting functions will be discussed in more detail below.

\subsubsection{Mechanisms of direct tumour cell killing}

The mechanisms for the elimination of tumour cells depend both on the immune cell type and on sensitivity of the tumour targets to different death pathways. Target cell killing can be mediated through the release of cytotoxic molecules such as perforin and granzymes, or through the interaction of death receptors ligands on effector cells with death receptors on the targets [80].

Perforin deficient mice have a higher incidence of chemically induced tumours and show more metastases [25]. In addition, perforin is required by both CTLs and NK cells to reject injected, chemically induced and virally induced cancers [81]. Although in vivo perforin mediated killing was found to be the dominant mechanism of tumour cell elimination [82], NK cells and CTLs have also been shown to express death receptor ligands such as FAS ligand (FASL) and tumour necrosis factor-related apoptosis-inducing ligand (TRAIL) [83, 84]. Tumours that express the death receptor FAS can thus be directly killed via FAS-FASL interactions $[83,85]$. In addition, a study using perforin and FASL single and double knock-out T cells showed impaired killing of B16 melanomas in vivo when the double deficient CTLs were used, compared to just perforin-/- CTLs [84]. This demonstrates a contribution of FASL to tumour 
cell elimination. TRAIL is also important for the control of tumours, as TRAIL-/mice had a higher rate of chemically induced tumours and showed defects in the NK cell mediated control of metastasis $[86,87]$. The relative importance of these different killing mechanisms may depend on the expression of death receptors on tumour targets, as well as on their level of anti-apoptotic molecules that can impede death receptor induced apoptosis.

Macrophages can contribute to tumour elimination via secretion of toxic nitric oxide (NO). A study by Miguel et al. demonstrated that macrophages in progressing murine tumours produced low levels of NO, while macrophages from regressing tumours secreted much higher levels of NO ex vivo. In addition, the inducible nitric oxide synthase (iNOS) was required for tumour rejection in vivo [79]. Macrophages in the brain (microglia) were also found to specifically eliminate brain tumour cells via NO, while sparing normal brain cells [78]. In vitro experiments further suggest that the balance of iNOS and arginase activity in macrophages determines their tumoricidal versus tumour-promoting function [88].

\subsubsection{Cytokines in the anti-tumour immune response}

Many cytokines have been studied in the context of tumour immunology. While some exclusively affect immune cells, the majority can either be produced by tumour cells themselves or be sensed by and directly affect cancer cells. This may be part of the reason why some cytokines have an ambiguous role in tumour immunology and can both suppress and promote tumour growth, often depending on the exact type of cancer or even individual tumours.

IFN $\gamma$ is required for protection against the growth of transplanted, chemically induced and spontaneous tumours $[20,24,25]$. It has both direct effects on tumour cells and on the immune response. In some tumours, IFN $\gamma$ has an anti-proliferative role [89], and in others it shows direct pro-apoptotic activity [90]. IFN $\gamma$ is a signature cytokine 
of Th1 CD4 T cells, which promote the induction and licensing of CTLs. At the same time, IFN $\gamma$ enhances the immunogenicity of tumour cells by up-regulating expression of MHC I molecules [21, 24], thereby increasing the sensitivity of tumour cells to CTL killing [21].

Type I IFN is also tumour protective, as its neutralisation increases the growth of transplanted tumours [91]. Furthermore, IFN $\alpha$ receptor deficient mice have a higher incidence of chemically induced sarcomas than $\operatorname{IFN} \alpha$ sufficient animals [61]. The roles of IFN $\gamma$ and type I IFN partially overlap, as they both have anti-proliferative activity [89]. However, while IFN $\gamma$ mainly works through a direct effect on the tumour cells, the anti-tumour activity of type I IFN is largely mediated by immune cells [61]. Type I IFN can activate DCs [59, 60], increase the cytotoxicity of NK cells by up-regulating TRAIL expression [92], and promote the generation and survival of CTLs by preventing activation induced cell death [93].

Type III IFN (IFN $\lambda$ ) has also been shown to have anti-tumour effects, as its expression in several murine tumours leads to tumour rejection or growth delay [94, 95]. IFN $\lambda$ directly increased tumour cell immunogenicity by up-regulating MHC I expression [94]. However, the mechanism by which $\operatorname{IFN} \lambda$ enhanced rejection in $\operatorname{IFN} \lambda$-resistant tumours remains to be elucidated [94].

The key role of interleukin-12 (IL-12) is the polarisation of Th1 responses, which are crucial for tumour protection [96]. Mice that lack IL-12 or IL-12 signalling components have severely reduced levels of IFN $\gamma$ and a higher incidence of chemically induced tumours $[97,98]$. In contrast, the closely related cytokine IL-23 appears to promote tumour growth, as IL-23 knock out mice exhibit reduced tumour growth [98].

IL-10 is generally regarded as a regulatory or suppressive cytokine. It is implicated in the down-regulation of immune responses, induction of regulatory $\mathrm{T}$ cells. It also promotes tolerance via the inhibition of DC activation [99]. In humans, high IL-10 levels correlate with tumour progression [100]. In vitro IL-10 increased the proliferation of human melanoma cells and led to down-regulation of MHC and adhesion 
molecules [101]. Conversely, mice that lack IL-10 are less prone to the development of chemically induced sarcomas [102]. Moreover, in the murine B16 melanoma model, IL-10 favours tumour growth by inhibiting macrophage activation, inducing tumour cell proliferation and promoting angiogenesis [103].

Transforming growth factor $\beta$ (TGF $\beta$ ) has been named for its ability to transform cell lines and was therefore thought to be a tumour-promoting factor. Indeed, it can be secreted by tumour cells and is associated with poor prognosis and metastasis in many human cancers $[104,105]$. Similarly, TGF $\beta$ was required for metastasis in a murine model of colon cancer [106]. However, tumour suppressor activity of TGF $\beta$ has also been described [107] and several human tumours harbour TGF $\beta$ inactivating mutations [108]. This apparent contradiction may be due to the overlap of signalling pathways downstream of TGF $\beta$ with many known pro-oncogenes. In in vitro and murine models of metastasis, TGF $\beta$ was found to collaborate with Ras [109] and the mutational state of these oncogenic factors may influence the outcome of TGF $\beta$ signalling.

The role of IL-17 in cancer development and progression is also controversial. IL-17-/mice have been shown to be more susceptible to lung melanoma [110]. In this model, IL-17 was required to provide sufficient inflammation to activate CTLs. However, IL-17 deficient animals have a lower incidence of chemically induced skin cancer [111]. Furthermore, IL-17 was found to promote melanoma and bladder cancer growth in mice via induction of IL-6 [112]. Moreover, in patients with ulcerative colitis, CD4 T cells that expressed both IL-17 and the Treg marker Forkhead box P3 (FoxP3) were identified [113]. These IL-17+ Tregs both suppressed T cell activation and induced secretion of pro-inflammatory cytokines [113], suggesting that they may dampen the immune response while favouring disease-promoting inflammation. These "cross-over" T cells are in line with previous studies that reported plasticity between Tregs and Th17 cells $[114,115]$. Therefore it appears that IL-17 induces inflammation that can either be associated with immune activation or suppression, possibly 
depending on other factors in any given microenvironment, and can thereby either promote or hinder the anti-tumour immune response.

The pro-inflammatory cytokines tumour necrosis factor $\alpha$ (TNF $\alpha)$, IL-6 and IL-1 $\beta$ likewise have been both implicated in enhancing tumour development and growth, as well as being required for tumour eradication. Thus, $\mathrm{TNF} \alpha$ deficient mice are more susceptible to chemically induced sarcomas [102], but display increased resistance to chemically induced skin carcinogenesis [116]. Pro- and anti-tumour activity of TNF $\alpha$ has even been documented in the same tumour model, depending on the expression of oncogenic Ras [117]. TNF $\alpha$ is a pleiotropic cytokine with many down-stream effects. Among its tumour-promoting activities, it can enhance the production of pro-angiogenic factors from tumour cells [118]. In its function as tumour suppressor, it has been shown to promote differentiation of immature myeloid cells into M1-like macrophages and their tumoricidal activity [119].

Elevated levels of IL-6 are reported in cancer patients [100]. Furthermore, IL-6 contributes to progression in ovarian and colorectal cancer and is associated with a higher risk for liver cancer [120-122]. Its ability to skew CD4 T cells from an anti-tumour Th1 phenotype towards a tumour-promoting Th2 profile may favour tumour development and progression [123]. On the other hand, IL-6 as well as IL- $1 \beta$ and TNF $\alpha$ levels were consistently found to be elevated in tumours that were successfully controlled by the immune system [124]. In addition, IL-6 has been found to contribute to up-regulation of MHC expression on tumour cells, inhibit TGF $\beta$ and restore the cytotoxic function of NK cells [125, 126].

A study on the role of IL-1 in tumour development found that IL- $1 \beta$ deficient mice develop chemically induced sarcomas more slowly, while IL-1 $\beta$ receptor antagonist deficient mice grow them faster [127]. These experiments clearly demonstrated that IL-1 favoured tumour initiation. However, IL-1 receptor and the Nucleotide-binding, leucine-rich-repeat containing receptor P3 (NLRP3), which is required for IL-1 secretion, were necessary for CTL priming in response to dying tumour cells and for the 
effectiveness of chemotherapy [128].

The tumour-promoting as well as suppressing roles of a range of pro-inflammatory cytokines including TNF $\alpha$, IL-6 and IL- $1 \beta$ are probably all related to the role of inflammation in cancer development, progression and eradication. Acute inflammation generally precedes the induction of adaptive immunity. However, chronic inflammation promotes tumorigenesis at all stages by inducing genotoxic stress, cellular proliferation, angiogenesis and invasion [129]. Chemically induced tumour models often require an inflammatory event for the initiation of tumour growth [102, 122, 127]. However, the same inflammatory factors contribute to tumour protection at later stages as they facilitate the induction of immunity in response to tumour cell death $[128,129]$.

\subsection{Tumour immune escape}

As discussed in the previous section, there is ample evidence that the immune system can recognise and eradicate tumours. However, the pressure of the immune response on the proliferating, genetically unstable tumour cells can also select for tumour variants that escape the immune response through loss of antigen or resistance to cell death. Furthermore, tumours can also create and environment that is immune suppressive and can even subvert infiltrating immune cells to promote tumour progression.

\subsubsection{Escape through antigen loss}

Correlative evidence from melanoma patients suggests that tumours lose antigens to escape the immune response as metastatic lesions or tumours progressing after immunotherapy express lower levels of several tumour antigens than primary tumours $[130,131]$. In addition, in a patient that had generated CTLs against various autologous tumour antigens, recurring lesions were found to have lost these antigens 
[132].

Genetic instability of tumours and elimination of more immunogenic variants by the immune response likely result in progressive selection of less immunogenic antigen loss variants. Antigen loss may result directly from mutations of the epitopes recognised by CTLs. Studies in mice have found spontaneous point mutations in antigens from immune escape variants of tumours [133]. In addition, immunotherapy targeted to different domains of a model antigen induced mutations preventing immune recognition precisely in the targeted portion [134].

Another mechanism of antigen loss is the alteration of MHC molecules presenting peptide antigens to $\mathrm{T}$ cells. After immunotherapy, tumours from several melanoma patients were found to have lost the $\beta$-microglobulin subunit of MHC I, resulting in the absence of surface antigen [135]. Other human cancers have also been reported to lack MHC expression $[136,137]$. In addition, antigen presentation on tumour cells can also be impaired by loss of components required for antigen processing and loading onto MHC I, including the transporter associated with antigen processing (TAP), tapasin and the immuno-proteasome subunit LMP2 [138]. Failure to respond to IFN $\gamma$ can also contribute to low expression of MHC I and thus evasion of immune recognition [139].

In addition to loss of $\mathrm{T}$ cell antigens or antigen presentation, shedding of NK cell ligands has also been implicated in tumour immune escape. Patients with gastrointestinal cancers have elevated levels of the stress molecule MICA in the serum and proteolytic shedding of MICA from tumour cells appears to lead to internalisation and inactivation of its receptor on NK cells [140, 141].

\subsubsection{Escape through resistance to cell death}

A further mechanism of tumour immune escape is the resistance to cell death. This can be conferred by mutations in death receptors such as TRAIL receptors and FAS 
that render them dysfunctional $[142,143]$. Inhibition of cell death in tumours can also occur through mutations in the signalling cascade following death receptor engagement [144]. Furthermore, over-expression of anti-apoptotic molecules can counteract death signals and impair tumour cell killing [145].

\subsubsection{Direct suppression of effector $\mathrm{T}$ cells by tumour-derived factors}

Tumours frequently express and secrete molecules that directly suppress $\mathrm{T}$ cell responses. Human pulmonary, ovarian and colon carcinomas have been shown to express high levels of programmed death ligand 1 (PD-L1) [146]. Expression of PD-L1 in murine tumours increases apoptosis of activated, tumour-specific T cells and promotes tumour growth in vivo [146].

In addition, the majority of human tumours express the tryptophan catabolising en-

zyme indoleamine 2,3-dioxygenase (IDO) [147]. When immunogenic murine tumours were manipulated to express IDO, they failed to be rejected due to a lack of $\mathrm{T}$ cell accumulation [147]. IDO impairs the function of effector cells via causing tryptophan starvation together with the accumulation of the toxic metabolite L-kynurenine, which inhibits T cell and NK cell proliferation in vitro [148]. Furthermore, tumour derived IDO has been shown to mediate conversion of CD4 T cells into Tregs [149].

TGF $\beta$ expressed by tumors [105] also has direct inhibitory effect on $\mathrm{T}$ cells. It can limit CD8 T cell expansion [150] and inhibit Th1 differentiation of CD4 T cells [151]. Moreover, the selective blockade of TGF $\beta$ signalling in $\mathrm{T}$ cells restored their anti-tumour activity in a murine cancer model [152].

In addition to directly impeding eradication by $\mathrm{T}$ cells, tumour-derived factors also recruit Tregs and subvert the function of myeloid cells and macrophages to become immuno-suppressives and tumour-promoting. This indirect inhibition of anti-tumour immunity is discussed below. 


\subsubsection{Immune-suppression via tumour-associated macrophages}

Macrophages are generally identified by high expression of CD68 in humans and F4/80 in mice, as well as their distinct morphology in tissue sections [153-155]. In human cancer, the prevailing role of macrophages is in the promotion of tumour progression, angiogenesis and metastasis and frequently a high infiltration of macrophages is linked to poor prognosis $[156,157]$.

Many tumours actively recruit macrophages to the tumour site via secretion of chemoattractant factors. Thus, macrophage chemoattractant protein-1 (MCP-1) is associated with a high level of macrophage infiltration in human breast cancer [158] and has been shown to directly recruit macrophages to the tumour site in a murine tumour model [159]. Furthermore, macrophages are recruited by tumour-derived vascular endothelial growth factor (VEGF), which also promotes angiogenesis [160].

While classically activated M1-like macrophages can eliminate tumour cells as discussed above, M2-like alternatively activated macrophages normally have a role in wound-healing and can promote cancer by favouring tissue remodelling and angiogenesis, and suppressing immunity [73]. Tumours can skew macrophages towards a tumour-promoting M2-like phenotype $[154,161]$. This is partly due to the hypoxic environment in solid tumours, which induces hypoxia inducible factor- $\alpha$ (HIF-1 $\alpha)$ in macrophages [162]. HIF-1 $\alpha$ can directly bind to the promotor region of VEGF and enhance its production [162]. Macrophage expression of HIF-1 $\alpha$ is also involved in $\mathrm{T}$ cells suppression and promotion of tumour progression [153]. In addition, Tregs can also induce M2-like activation of macrophages [155].

Tumour-associated macrophages show up-regulated production of the regulatory cytokines IL-10 and TGF $\beta$, as well as VEGF and several CC chemokine ligand (CCL) chemokines $[154,161]$. In addition, they fail to increase pro-inflammatory cytokines like IL-1 $\beta$, IL-6 and TNF $\alpha$ in response to stimulation with bacterial lipopolysaccaride (LPS) [154]. They also express low levels of MHC and IL-12, and are thus im- 
paired in their ability to activate T cells. Moreover, tumour infiltrating macrophages up-regulate arginase [154]. Arginase and iNOS share arginine as a common substrate and both have been shown to directly inhibit $\mathrm{T}$ cells [163]. This may be partly due to depletion of the amino-acid arginine. In addition, arginase was found to inhibit T cell proliferation by irreversibly blocking IL-2 production [163]. Lastly, tumour-associated macrophages can also recruit Tregs to the tumour site via secretion of CCL22 [46].

Thus, M2-like tumour-infiltrating macrophages can suppress the immune response via secretion of regulatory cytokines, direct inhibition of $\mathrm{T}$ cells combined with a lack of $\mathrm{T}$ cell stimulation and recruitment of Tregs.

\subsubsection{Immune-suppression via regulatory $\mathrm{T}$ cells}

Murine Tregs are characterised by the expression of the transcription factor FoxP3 [164], which is necessary for their suppressive function [165]. In humans, Tregs are also FoxP3+, but effector $\mathrm{T}$ cells can transiently express low levels of FoxP3 upon activation [166]. Prior to the discovery of FoxP3, CD25 was widely used as a marker of Tregs, but as CD25 is also expressed by activated $\mathrm{T}$ cells, it cannot be viewed as a reliable marker of Treg [167]. Tregs are normally induced in the thymus [168], but TGF $\beta$ can convert FoxP3-CD4+ T cells into FoxP3+ Tregs in the periphery [169].

Tumours frequently show profound infiltration by Tregs $[46,170,171]$ and high Treg infiltration has been associated with poor prognosis in several human cancers [46, 172]. However, Treg infiltration does not always correlate with bad prognosis [47]. Instead the ratio of Tregs to effector $\mathrm{T}$ cells was found to be a more reliable prognostic factor $[44,45]$. Furthermore, depletion of Tregs induces regression of a range of syngeneic experimental tumours in mice [173]. Tumours can actively recruit Tregs by inducing macrophages to produce the chemokine CCL22 [46]. In addition, tumour-derived 
TGF $\beta$ has been shown to directly convert CD4 T cells into Tregs independent of proliferation $[174,175]$.

Tregs can suppress the anti-tumour immune response at several stages. Tregs can inhibit the activation and proliferation of effector $\mathrm{T}$ cells, impair their cytotoxic function and even directly kill effector cells at the tumour site [176-181]. Interaction with activated DCs is critical for the initiation of $\mathrm{T}$ cell responses, as will be described in more detail in the next section. Tregs have been shown to inhibit the expression of activation markers on DCs and to reduce the interaction of effector T cells with DCs $[176,182]$. In tumour-bearing mice, but not in tumour-free animals, Tregs could also directly kill DCs in tumour-draining lymph nodes (LNs), thus preventing the initiation of an anti-tumour immune response [183]. Tregs can also secrete adenosine, TGF $\beta$ and IL-10, all of which can inhibit T cell proliferation [184-186]. Furthermore, Tregs can prevent the transcription of IL-2 by activated CD4 T cells, thus reducing the levels of this crucial cytokine for T cell proliferation $[177,178]$.

T cell effector functions can also be blocked by Tregs. They have been shown to inhibit the release of $\operatorname{IFN} \gamma[178]$ and cytotoxic granules $[179,180]$. One study also showed that the function of CTLs at the tumour site was impaired via Treg suppression of IFN $\gamma$ production from CD4 Th cells [187].

Treg induced inhibition of IL-2 may cause effector cell death indirectly by cytokine deprivation induced apoptosis [188]. It has also been proposed that Tregs can directly eliminate target cells using GzmB and perforin [189, 190]. This is supported by the findings of Cao et al., who showed that wild type but not perforin deficient Tregs correlated with increased apoptosis of CD8 T cells and NK cells in the tumour and thus promoted tumour growth [181].

An inverse correlation between Treg and NK cell infiltration has also been reported in human tumours [191]. In mouse models of cancer, Tregs suppressed cytoxicity of NK cells in a TGF $\beta$ dependent manner and depletion of Tregs increased NK cell dependent tumour growth suppression [191, 192]. 
Tregs can therefore potently inhibit the anti-tumour immune response both at the initiation and the effector stages. In addition, they can impair the cytotoxic anti-tumour function of innate NK cells as well as adaptive CTLs.

\subsubsection{Immune-suppression via myeloid-derived suppressor cells}

Myeloid-derived suppressor cell (MDSC) is a term used to describe a heterogenous population of myeloid cells that accumulate in cancer patients [193, 194] and tumour-bearing mice $[195,196]$ and can suppress anti-tumour immunity.

The first report describing an inhibitory function of Gr-1+ myeloid cells on tumour growth came from Schreiber's group [197]. Initially, CD11b and Gr-1 were widely used to identify MDSCs in mice [198]. However, Gr-1 recognises both Ly6C and Ly6G [199]. When antibodies to distinguish these two molecules became available, Ly6C $^{\text {hi }}$ Ly6G- monocytic and Ly6C lo $^{\text {Ly }} 6 \mathrm{G}+$ granulocytic MDSC subtypes were characterised $[195,196]$. However, the expression of CD11b, Gr-1 and Ly6C or Ly6G is not unique to MDSCs and not all CD11b+ Gr-1+ cells are tumour-suppressive [200, 201]. Therefore, to conclusively identify MDSCs functional studies of suppressive activity are required. The ability to distinguish the Ly6C ${ }^{\text {hi }}$ Ly6G- and Ly6C ${ }^{\text {lo }}$ Ly6G+ subsets has also revealed that the monocytic MDSCs have a higher suppressive capacity compared to the granulocytic subset and are the MDSCs mainly responsible for immune dysfunction in cancer $[195,196]$.

It has now become clear that in the absence of tumours CD11b + Gr- $1+$ cells reside mainly in the bone marrow and represent less than $5 \%$ of splenocytes [196, 202]. In addition, these steady-state CD11b + Gr-1+ cells do not exhibit significant $\mathrm{T}$ cell suppression [198].

Tumours induce the accumulation of MDSCs in the blood and spleen [196, 198, 202, 203]. A multitude of factors have been implicated in the expansion and activation of MDSCs. Among these, granulocyte-macrophage colony-stimulating factor (GM-CSF) 
[203, 204], VEGF [205, 206], macrophage colony-stimulating factor (M-CSF) [207] and CCL2 [195, 208] have been identified to mediate expansion and recruitment of MDSCs to tumour sites in several studies both in mouse models and human cancer. In addition, it is becoming clear that MDSCs also require activation to exert their suppressive function. IFN $\gamma[154,209,210]$, IL-4 [163, 210] and IL-13 [210, 211] have all been described to induce MDSC activation.

MDSCs express the enzymes arginase 1 and iNOS, both of which use arginine as a substrate. Depletion of arginine can lead to T cell suppression via down-regulation of the CD3 $\zeta$-chain of the T cell receptor (TCR) [212] and inhibition of the up-regulation of the cell-cycle regulators cyclin D3 and cyclin-dependent kinase 4 [213]. NO produced by iNOS can inhibit IL-2 signalling components in T cells, thereby preventing $\mathrm{T}$ cell proliferation [214]. Furthermore, NO can inhibit MHC II expression in DCs [215], thus limiting antigen-presentation to T cells. In addition, NO production by MDSCs can induce apoptosis of T cells $[216,217]$. However, as described above, NO can also induce tumour cell death and thus act as a tumour suppressor. The pro- versus anti-tumour effect of NO may depend on local interaction with other factors in each tumour and also during the progression of tumour growth. In addition, the level of NO may be critical, with high concentrations observed to be tumoricidal, while lower amounts were tumour-suppressive [79]. Thus, similar to inflammatory cytokines, an acute burst of high amounts of NO appears to be tumour-suppressive, while chronic release favours tumour progression through immune-suppressive mechanisms.

Reactive oxygen species (ROS) also play a role in the suppressive activity of MDSCs. Increased production of ROS by MDSCs has been observed both in patients with cancer and tumour mouse models [194, 198]. Furthermore, inhibition of ROS in MDSCs fully restored T cell responses against murine fibrosarcomas [198].

Peroxynitrite, which results from the chemical reaction of NO with the superoxide anion, has also been implicated as a mediator of MDSC immune suppression. Increased levels of amino-acid nitrosylation linked to peroxynitrites, are associated with poor 
prognosis in a range of cancers $[218,219]$. Bronte et al. found that nitrosylation via peroxynitrites inhibited prostate cancer-infiltrating $\mathrm{T}$ cells and that their responsiveness could be restored by blocking iNOS and arginase [220]. Moreover, nitrosylation of tyrosine residues in the TCR complex by MDSC-derived peroxynitrites was shown to directly inhibit antigen-recognition by tumour-specific CD8 T cells [221].

In addition to directly suppressing $\mathrm{T}$ cell activation and function, MDSCs can also produce tumour-suppressive cytokines such as IL-10 and recruit Tregs to the tumour site via production of TGF $\beta[222,223]$.

\subsection{Dendritic cells}

\subsubsection{Initiation of adaptive immune responses}

DCs are the sentinels of the immune system and play a key role in the induction and regulation of immune responses [224]. They reside in peripheral tissues in an immature state and continuously sample the antigenic environment. In the absence of microbial stimuli or danger signals, DCs spontaneously mature and migrate to the draining LNs (dLNs) to present the captured antigen to circulating $\mathrm{T}$ cells. This results in the induction of peripheral tolerance in antigen specific T cells [225]. In contrast, DCs that take up antigen in the context of inflammatory or danger signals become activated, migrate to the draining LN and present antigen to $\mathrm{T}$ cells with additional signals, leading to $\mathrm{T}$ cell activation and differentiation into effector cells [226]. The activation status of DCs, its impact on the generation of $\mathrm{T}$ cell tolerance and activation and the molecular pathways and cytokines involved in antigen presentation will be discussed in more detail in section 1.3.3 and 1.34. In addition, DCs can be divided into several subsets that differ in their ability to respond to activation stimuli and subsequently to activate immune responses as described in section 1.3.5 and 1.4. 


\subsubsection{Activation of innate immune responses}

While DCs are generally regarded to be critical for the initiation of adaptive immune responses, they can also activate innate immune cells. Fernandez et al. demonstrated that activated DCs can enhance NK cell cytotoxicity and IFN $\gamma$ production, resulting in the control of tumour growth in mice [227]. The DC-NK cell interaction also has reciprocal effects on the DCs, which were shown to be more mature and secrete more IL-12 when they were exposed to NK cells [228]. Furthermore, activated DCs can recruit NK cells to LNs during inflammation to favour DC-NK cell interactions [229]. Similar reciprocal DC-NK cell activation has been suggested to play a role in the immune response against human tumours [230].

\subsubsection{Activation states of DCs}

The nomenclature used to described DCs in different activation states is not consistent throughout the literature. In this thesis, DCs that are sampling antigen but not presenting it yet are referred to as immature. DCs that have up-regulated co-stimulatory molecules and acquired the capacity to migrate to dLNs and present antigen to T cells are termed mature. Mature DCs are further divided into tolerogenic DCs and activated DCs depending on their function in inducing tolerance or activation in naïve T cells.

Immature DCs reside in all tissues throughout the body and function to sample the antigenic environment. They are characterised by low surface expression of MHC II molecules and co-stimulatory molecules, together with a high capacity to take up antigen [224]. Antigen capture can occur via receptor-mediated endocytosis, for example through CD205 (DEC205) [231], CD206 (mannose receptor) [232], or Fc $\gamma$ receptors (for immune complexes) [233]. DCs also show high rates of phagocytosis [234] and pinocytosis [232]. This variety of antigen uptake mechanisms contributes to the high efficiency of DCs in antigen presentation and initiation of immune responses. 
Under steady-state conditions, a small number of tissue DCs that have taken up self-antigens mature spontaneously. Maturation is accompanied by up-regulation of surface expression of MHC II and co-stimulatory molecules, as well as LN homing receptors such as the chemokine (C-C motif) receptor 7 (CCR7), which enables the DCs to migrate to the dLNs [235]. Naïve T cells that encounter their cognate antigen presented by these DCs are either deleted, rendered anergic or induced to become Tregs [236-238]. This process is known as peripheral tolerance and is thought to ensure the elimination of auto-reactive $\mathrm{T}$ lymphocytes that have escaped thymic deletion [239].

Maturation and activation of DCs occurs in response to danger signals, which are released from dying cells, conserved pathogen patterns or inflammatory stimuli [64, 240]. Upon activation, DCs express very high levels of surface MHC II and co-stimulatory molecules [224, 241]. Simultaneously, DCs down-regulate the expression of receptors involved in antigen capture. Switching in the expression of chemokine receptors from chemokine (C-X-C motif) receptor 1 (CXCR1) to CXCR4 and CCR7 enables activated DCs to migrate from the peripheral tissues to the T cell areas of the LNs and spleen [242]. Thus, phenotypically activated and tolerogenic DCs are very similar, although the level of MHC II and co-stimulatory molecules differs: activated DCs display very high surface levels, whereas tolerogenic DCs show intermediate expression [243]. In addition, activated DCs secrete considerably more IL-12 and other cytokines that are necessary for the differentiation of CD8 T cells into fully licensed cytotoxic effector cells [244, 245]. Tolerogenic DCs, in contrast, produce large quantities of the immune regulatory cytokine IL-10 [246].

Thus, the activation status of DCs determines whether antigen presentation to T cells results in tolerance or immunity. The degree of stimulation, the pattern of co-stimulatory molecules expressed and the cytokines secreted by DCs also regulate the type of effector and memory cells that is induced [247]. Mounting the right type of immune response in turn is crucial to clear pathogens and control disease [248]. 


\subsubsection{Antigen presentation to $\mathrm{T}$ cells}

In lymphoid tissues, DCs are scanned by circulating T cells for their cognate antigens. The two major classes of $\mathrm{T}$ cells are distinguished by their surface expression of either CD4 or CD8. CD4 and CD8 T cells both recognise antigens that have been processed by antigen-presenting cells (APCs) into peptides and loaded onto MHC I and MHC II. CD4 T cells recognise peptide-MHC II complexes and once activated can differentiate into either Th1, Th2, Th17, T follicular helper or Treg cells [249, 250], whereas CD8 T cells specifically bind to peptide-MHC I complexes and become CTLs [251].

\subsubsection{MHC I and II presentation pathways}

Distinct pathways lead to the generation of peptide loaded MHC I and MHC II complexes and not all cell types are equally able to use them. Peptides formed by degradation of endogenous proteins in the cytosol are translocated into the endoplasmic reticulum, where they are loaded onto new MHC I molecules. The associated peptideMHC I complexes are then transported to the cell surface via the Golgi apparatus, where they can be recognised by CD8 T cells [226, 251]. This pathway operates in all cell types, so that any virus infected cell can present viral antigens on their surface MHC I complexes.

In contrast, exogenous antigen is taken up and degraded in endomsomes. Fusion of antigen-rich endosomes and MHC II containing endosomes enables loading of the peptides onto the MHC II molecules, which are then also transported to the cell surface for recognition by CD4 T cells $[252,253]$. Only professional APCs, namely B cells, macrophages and DCs, are capable of presenting antigen on MHC II, with DCs being most effective [254]. As a result of this compartmentalisation of antigen presentation pathways, endogenous antigen is presented on MHC I and exogenous antigen is presented on MHC II. 
In a situation, in which DCs are not themselves infected with a virus, this strict separation would impede the efficient initiation of an anti-viral immune response by DCs [255]. Thus, it is not surprising that a third mechanism has been identified to enable presentation of exogenous antigen on MHC I. This phenomenon has been termed cross-presentation and was first described by Michael Bevan in 1976 [256]. To date, several mechanisms for cross-presentation have been found: Some involve retrograde transport of exogenous protein into the cytosol, where it can then enter the endogenous MHC I presentation pathway, while others involve only endosomal compartments and MHC I recycling from the cell surface. Which pathway is most important in vivo, or if they are used simultaneously or depending on the infection encountered by the immune system, remains to be elucidated [257]. Also, only few cell types are capable of cross-presentation. Macrophages can cross-present antigen only at a very low rate, whereas some DC subsets are extremely effective cross-presenters. In particular, the CD8+ population of LN resident DCs and the CD103+ population of the migratory DCs have been shown to specialise in cross-presentation of exogenously acquired antigen on MHC I (for DC subsets see chapter 1.3.5) [258-260].

\subsubsection{Co-stimulatory molecules and cytokines}

Recognition of peptide-MHC complexes alone is not sufficient to generate a $\mathrm{T}$ cell response. On the contrary, it normally induces $\mathrm{T}$ cell tolerance. Additional signals in the form of co-stimulatory molecules and cytokines are required to fully activate naïve T cells.

CD80 and CD86 are co-stimulatory molecules, which are up-regulated on DCs upon activation [261]. Binding of CD80 and CD86 to CD28 on T cells promotes T cell proliferation, survival and cytokine secretion [262, 263]. Intriguingly, both CD80 and CD86 also bind to the inhibitory receptor cytotoxic T lymphocyte associated antigen 4 (CTLA-4) on T cells [264]. This interaction results in blockage of $\mathrm{T}$ cell proliferation 
and cytokine secretion and serves an important role in regulating immune responses and maintaining T cell homeostasis [264, 265]. Programmed death (PD) is another inhibitory molecule expressed by $\mathrm{T}$ cells and interacts with PD-L1 and PD-L2 on DCs [266, 267]. The balance of inhibitory PD-1 and co-stimulatory CD80/86-CD28 signalling may determine the threshold between $\mathrm{T}$ cell tolerance and $\mathrm{T}$ cell activation [266]. PD-L1 expression is not limited to DCs, and interaction of tissue PD-L1 with PD-1 on T cells has also been implicated in peripheral tolerance [268]. Furthermore, PD-1 expression is up-regulated on exhausted $\mathrm{T}$ cells and has also been linked to T cell dysfunction in cancer [269, 270].

CD40 is another co-stimulatory molecule expressed on DCs [271]. Binding of CD40 to its ligand CD40L on activated T cells leads to further activation of DCs. They up-regulate co-stimulatory molecules and produce the cytokine IL-12, which is important for T cell activation and Th1 differentiation [271]. CD40 also enables CD4 T cells to provide help for activation and licensing of CD8 T cells to become CTLs [272, 273]. Other co-stimulatory molecules have been identified, but will not be a focus of this thesis.

The types and levels of cytokines produced during $\mathrm{T}$ cell activation are important factors in determining the type of $\mathrm{T}$ helper cell that is generated. While a milieu rich in IL-4 favours a Th2 response, IL-6 and TGF $\beta$ induce Th17 cells to develop, and IL-10 has been implicated in the generation of Tregs [235]. For the generation of fully licensed CTLs, IL-12 is thought to be indispensable [274]. This is often called the third signal, in addition to antigen-MHC I complex recognition (signal 1) and binding of co-stimulatory molecules (signal 2). The main source of IL-12 in vivo are DCs that have interacted with T helper cells [245, 275]. 


\subsubsection{DC survival}

The efficiency of antigen presentation to CD4 and CD8 T cells is also affected by DC survival. In general, mouse DCs have been shown to have a rapid turnover (half-life 1.5-3 days), with slightly different life spans for different DC subtypes. Exposure of DCs to some microbial activation signals promotes survival, whereas others enhance cell death [276]. In addition, immature DCs are sensitive to lysis by NK cells [277] and antigen-loaded DCs can be killed by CTLs [278]. Studies on the effect of DC survival via manipulation of the expression of pro- or anti-apopototic proteins have found that prolonged DC survival was associated with increased CD4 and CD8 T cell responses and beneficial anti-tumour effects [276, 279, 280].

\subsubsection{Subtypes of DCs}

DCs form a heterogeneous cell population with many distinct subsets, which differ both in localisation and function in the immune system [281]. Generally, monocytederived inflammatory DCs, plasmacytoid DCs (pDCs) and conventional DCs (cDCs) are distinguished. In addition, cDCs can be divided into lymphoid tissue resident and migratory DCs and further subtypes are defined based on surface markers.

\subsubsection{Conventional lymphoid tissue resident DCs}

Three types of lymphoid tissue resident DCs have been described in mouse spleens. $\mathrm{CD} 4-\mathrm{CD} 8 \alpha+(\mathrm{CD} 8 \alpha+\mathrm{DCs}), \mathrm{CD} 4+\mathrm{CD} 8 \alpha-(\mathrm{CD} 8 \alpha-\mathrm{DCs})$ and CD4-CD8 $\alpha-($ double negative (DN) DCs) [282]. To date, no functional role has been attributed to CD4 or CD8 molecules on DCs [281]. Additional markers used to discriminate between lymphoid tissue resident DCs are CD11b and CD205. CD11b is present both on CD8 $\alpha$ and DN DCs, whereas CD205 is only present on CD8 $\alpha+$ DCs [283]. CD8 $\alpha+$ DCs are functionally different from CD $8 \alpha$ - DCs, as CD $8 \alpha+$ DCs produce more IL-12 and 
are superior at cross-presenting antigen [245, 284, 285]. However, a later study suggests that not all CD $8 \alpha+$ DCs cross-present efficiently but that a subset of langerin+ DCs within the CD8 $\alpha+$ DCs is critical for cross-priming and IL-12 production in response to systemic antigens [286]. In humans, the CD141+ (BDCA3+) CLEC9A+ (DNGR-1+) DCs have recently been identified independently by several groups to be the equivalent of mouse CD8 $\alpha+$ both developmentally and for their superior ability to produce IL-12 and cross-present antigen [287, 288]. Moreover, DC subtypes express different receptors to recognise microbial products. Toll-like receptor (TLR) 3, for example, which binds to double-stranded RNA and is thus implicated in recognition of viruses, is only expressed on CD8 $\alpha+$ DCs $[289,290]$. On the other hand, CD8 $\alpha+$ DCs lack TLR5 (binding to bacterial flagellin) and TLR7 (receptor for single stranded RNA) [291] (The different pathogen recognition receptors (PRRs) are discussed in more detail in Section 1.4). Distinct profiles of PRRs on DC subsets suggest that they are specialised to respond to certain types of pathogens and are functionally diverse.

\subsubsection{Conventional migratory DCs}

Migratory DCs differ according to their tissue of origin. The skin has widely been used to study DCs in the mouse and has led to the identification of at least three cDC subsets. Langerhans cells (LC) of the epidermis were the first DCs identified [292]. They sample antigen and upon activation migrate to the dLNs. They are langerin+ CD11b+ CD205hi CD103-. Initially, langerin was used as a marker to distinguish LCs from dermal DCs, until it became clear that a group of migratory dermal DCs also expresses langerin [293-295]. However, LC express considerably higher levels of CD205 compared to dermal DCs, making it possible to identify them in this way. Two functionally distinct groups of dermal DCs can be characterised based on their

expression of CD11b and CD103 [292]. CD103+CD11b- DCs have been shown to cross-present viral antigen on MHC I to CD8 T cells, whereas CD103-CD11b+ dermal 
DCs seem to be mainly responsible for antigen presentation on MHC II to CD4 T cells [260]. In addition to directly presenting antigen to T cells in dLNs, migratory DCs have also been shown to transfer antigen to LN-resident DC populations for presentation [296].

\subsubsection{Plasmacytoid DCs}

Human pDCs are identified as CD4+ CD45RA+ IL-3R $\alpha+$ ILT3+ ILT1- CD11clineage- cells, whereas mouse pDCs are CD45RA + CD11c ${ }^{\text {lo }}[297,298]$. Their main function is the production of large amounts of type-I interferons (IFNs) in response to viral RNA and DNA, recognised via the receptors TLR7 and TLR9 [299]. Activated pDCs can also present antigen to $\mathrm{T}$ cells, but they do not induce $\mathrm{T}$ cell proliferation and effector differentiation as efficiently as cDCs, probably due to lower surface levels of MHC II and co-stimulatory molecules [300]. Type I IFNs inhibit virus replication and activate NK cells, macrophages and cDCs. Thus, pDCs play an important role in anti-viral responses and provide a link between innate and adaptive immunity $[297,298]$.

\subsubsection{Monocyte-derived inflammatory DCs}

It has long been noted that bone marrow (BM) cells can be differentiated into DCs in the presence of GM-CSF in vitro [301] and many clinical trials have used GM-CSF generated DCs for the vaccination of cancer patients [302-305]. Such GM-CSF BMDCs resemble monocyte-derived DCs that arise during inflammation in vivo [306]. In mice, inflammatory monocyte-derived DCs are characterised by expression of CD11b, Ly6C and intermediate levels of CD11c [307]. They have been shown to accumulate at infection sites and to be able to induce functional Th1 responses in vivo [307, 308]. Under inflammatory conditions, they can become the major DC subset to produce IL-12 and monocyte-derived DCs have been shown to be required for the clearance of 
some infections [307, 309].

\subsubsection{Role of DCs in anti-tumour immune responses}

DCs are the most potent APCs and are crucial for the initiation of immune responses [224]. The activation of anti-tumour immune responses also depends on BMderived APCs and cannot be achieved by antigen-presentation on tumour cells alone [310].

DCs have been found to infiltrate both murine and human tumours [311-313]. In mice, they are mainly comprised of CD11b + CD8 $\alpha$ - B200- cDCs [311], but a small population of B220+ pDCs has also been observed [311, 314]. Both cDCs and pDCs have been reported to infiltrate human tumours [312, 313, 315, 316]. As DC subsets differ in their ability to induce cytotoxic $\mathrm{T}$ cell responses as described above, different DC types can show varying roles in cancer development. In patients with ovarian or breast cancer, infiltration of pDCs was found to correlate with poor prognosis, whereas other DC subsets did not affect clinical outcome [312, 316].

The activation state of DCs is critical for the induction of immune-responses versus tolerance, as described above. In patients with melanoma, increased infiltration by mature DCs correlated with better prognosis [317]. In addition, a high number of DCs infiltrating murine melanomas alone was not sufficient to induce tumour regression, but tumour rejection could be achieved by addition of an adjuvant to activate DCs [318]. Further evidence for a link between DC activation state and their role in the anti-tumour immune response comes from a study by Movassagh et al. that identified activated DCs co-localising with CTLs in regressing melanoma lesions [4], suggesting that activated DCs can promote anti-tumour immunity.

While tumour-infiltrating DCs have been shown to readily induce proliferation of antigen-experienced T cells [319], they were unable to prime naïve tumour-specific T cells ex vivo [314] or to activate CTLs in vivo [320]. In tumour-draining LNs, 
they induce anergy leading to tolerance instead of anti-tumour immunity [321]. This failure of DCs to induce anti-tumour immunity has been suggested to be due to insufficient activation [314] and could be overcome by addition of DC activating factors in combination with blocking tumour-suppressive IL-10 [322].

Tumours can actively recruit DCs and even skew DC development to enhance pDC formation [323]. In the tumour micro-environment, factors like IL-10, TGF $\beta$ and VEGF secreted by the tumour or tumour-infiltrating MDSCs and macrophages can inhibit DCs [324, 325], rendering them tolerogenic. As described above, tolerogenic DCs cause T cell deletion, anergy and induction of Tregs [236-238]. Tumours have been shown to directly stimulate DCs to produce TGF $\beta$ and thus favour the expansion of Tregs [222]. Furthermore, prostaglandin E2 abundant in a range of tumours, can induce secretion of CD25 as a decoy-IL-2 receptor and production of IDO in DCs, leading to inhibition of $\mathrm{T}$ cell proliferation and cytokine production [326]. Moreover, tumours can induce DCs to secrete the suppressive factors IL-10, NO and VEGF and to suppress $\mathrm{T}$ cells proliferation via expression of arginase I [325].

Thus, DCs infiltrating tumours can promote either tumour rejection or tumour progression, depending on their activation status.

\subsection{Natural adjuvants}

It is crucial for the immune system to only react to pathogens but not to innocuous substances. Therefore, cells of the innate immune system, such as macrophages and DCs, express PRRs that can recognise conserved PAMPs [299]. Upon binding of the corresponding ligands to their receptors, the immune cells become activated and an immune response appropriate to the recognised type of pathogen is mounted. In addition, danger signals and DAMPs released from dying cells can also be sensed by immune cells and lead to activation [58-64]. A range of receptors for PAMPs and DAMPs, including TLRs, NLRs and RLRs (Rig-I-like receptors) and their ligands 
have been identified and will be discussed in more detail below. Such ligands can be described as natural adjuvants, as they are natural activators of the immune response. In contrast, adjuvants used clinically for vaccination, such as aluminium salts and oilin-water emulsions, can be considered artificial immune-stimulators.

Adjuvants have long been used in vaccination to stimulate the immune response. Alum, a mixture of aluminium salts, and complete Freund's adjuvant (CFA), consisting of a oil-in-water emulsion with heat-killed mycobacteria, were among the first adjuvants described [327]. It has now become clear that the mycobacteria contained in CFA stimulate the immune response by activating DCs, as they express multiple TLR ligands as well as a NLR ligand [328, 329].

\subsubsection{TLR ligands}

TLRs can be subdivided in receptors expressed on the cell surface with specificity for extracellular bacterial or protozoan compounds (TLR 1, 2, 4, 5, 6 and 11) and receptors expressed in intracellular vesicles that function to recognise viral pathogens (TLR 3, 7, 8 and 9). Together, they are capable of recognising a wide range of pathogens: TLR1/TLR2 and TLR2/TLR6 bind to lipoteichoic acid on Gram positive bacteria [330, 331], TLR4 recognises LPS on Gram negative bacteria [332], TLR5 detects flagellin of motile bacteria [333] and TLR11 binds to profilin of Toxoplasma gondii and Escherichia coli [334]. Typically, viral replication intermediates are recognised by intracellular receptors. TLR3 senses double-stranded RNA [335], TLR7 and TLR8 bind to single-stranded RNA [336-338] and TLR9 is a receptor for unmethylated CpG motifs in DNA [339]. Unmethylated CpG sites are rare in vertebrate DNA, where the cytosine of the $\mathrm{CpG}$ motif is generally methylated, but are common in bacterial and viral genomes.

All of these TLRs are expressed by macrophages and DCs, and some can also be expressed by mast cells and B cells [299]. Expression among different DC subsets, 
however, varies. CD8 $\alpha+$ DCs, for example, highly express TLR3, but lack TLR5 and TLR7 [289-291]. Conversely, pDCs have high levels of TLR7 and TLR9 [291].

Binding of a TLR to its respective ligand initiates signalling cascades that result in the activation of transcription factors, which are involved in production of inflammatory cytokines and up-regulation of co-stimulatory molecules. With the exception of TLR3, all other TLRs recruit the adaptor molecule myeloid differentiation primary response protein 88 (MyD88) [340]. TLR3 signals exclusively via TIRdomain-containing adapter-inducing interferon- $\beta$ (TRIF), while TLR4 can engage both MyD88 and TRIF pathways [340]. TRIF signalling results in the activation of the transcription factor interferon regulatory factor (IRF) 3, which induces IFNs and IFN inducible genes. In contrast, MyD88 can activate Nuclear factor $\kappa$-light-chainenhancer of activated B cells (NF $\kappa \mathrm{B})$, activator protein (AP)-1 and IRF7, depending on its interaction with other signalling components [340].

Following the identification of natural ligands for the different receptors, commercially manufactured TLR ligands became available. These include the TLR2 ligand Pam3Cys [341], the TLR3 ligand Poly IC [335], the TLR4 ligand monophospholyl lipid A (MPL) [342], the TLR7 ligand Imiquimod [343] and several oligodeoxynucleotides containing CpG motifs (CpG) that stimulate TLR9 [344]. A clinical study of CpG reported that it was well tolerated, but showed little anti-tumour activity [14]. In contrast, Poly ICLC (a stabilised form of Poly IC) appeared to induce immune responses as potent as a viral vaccine in humans [15] and showed promising anti-tumour activity in mice and a small pilot study in glioma patients $[16,345,346]$. MPL and Imiquimod are already in clinical use, as MPL is included in the human papillomavirus vaccine [342], while Imiquimod is applied in topical creams for the eradication of warts and skin cancer [17]. TLR ligands are therefore promising adjuvants for the use in cancer immunotherapy. 


\subsubsection{NLR ligands}

NLRs represent a large family of intracellular receptors that contain a C-terminal leucine-rich repeat, a central nucleotide-binding oligomerisation domain (NOD) and one of four possible N-terminal domains [347]. These N-terminal domains can be used to divide NLRs into sub-families using A for the acidic transactivation domain, B for the baculoviral inhibitory repeat domain, $\mathrm{P}$ for the pyrin domain or $\mathrm{C}$ for the caspase recruitment domain, followed by a number (e.g. NLRP3).

Upon activation, a number of NLRs, including NLRP3, interact with different adaptor molecules to form multi-protein complexes called inflammasomes. Ultimately, inflammasomes form a platform for cleavage of pro-caspase 1 into its active form, caspase 1, which in turn cleaves pro-IL-1 $\beta$, pro-IL-18 and pro IL-33 into biologically active IL-1 $\beta$, IL-18 and IL-33 [348, 349]. These cytokines are then secreted and exert important pro-inflammatory roles. Inflammasomes have been implicated in detection of various pathogens and products of pathogens, as well as stress and danger signals. The NLRC4 inflammasome, for example, is activated in macrophages following infection with intracellular pathogens such as Salmonella enterica serovar Typhimurium or Pseudomonas aeruginosa [350, 351]. The most-studied inflammasome, however, is the NLRP3 inflammasome. It can recognise bacterial pore-forming toxins, ATP, DNA, bacterial RNA, and crystals such as silica, asbestos, monosodium urate crystals (MSU) and alum [347, 352-354]. The mechanism of NLRP3 activation has only partly been clarified: NLRP3 inflammasome agonists may either act through lysosomal damage or via the generation of mitochondrial-derived ROS, which then in some way activate NLRP3 [355]. Recently, thioredoxin-interacting protein has been indicated as the direct ligand for NLRP3 in this signalling pathway [356].

The NLRs NOD1 and NOD2 (NLRC1 \& NLRC2, respectively) differ from many other NLRs, as they do not form inflammasomes, but instead signal via mitogen-activated protein kinase (MAPK) and $\mathrm{NF} \kappa \mathrm{B}$ pathways. The transcription factor $\mathrm{NF} \kappa \mathrm{B}$ in turn 
activates a range of pro-inflammatory genes. One of the best characterised activators for NOD1 and NOD2 is mycobacterial peptidoglycan, and for NOD2 in particular its muramyl-dipetide (MDP) component [329, 357]. NOD2 mediated recognition of bacteria has been shown to be non-redundant with TLR signals [329] and NOD1/2 ligands can activate DCs and enhance cross-priming [358].

While most studies on inflammasome-forming NLRs so far have been performed in macrophages, they are also expressed in cDCs and monocytes [359]. One NLRP3 ligand, MSU, has recently been suggested for use as a vaccine platform and was found to be safe for application in humans [360]. One study also showed that uric acid released from tumour cells activated anti-tumour immunity and that MSU administration could promote tumour rejection [361]. However, the possibility to use DAMPs such as MSU for anti-tumour immunotherapy has not been investigated yet.

\subsubsection{RLR ligands}

RIG-I -(Retinoic acid incucible gene I protein)-like receptors (RLRs) include RIG-I and melanoma differentiation associated factor 5 (MDA-5). RIG-I is specific for 5' triphosphate single stranded (ss) RNA with a double stranded (ds) RNA component, a form of nucleic acid associated with many negative ssRNA viruses and some dsDNA viruses $[362,363]$. MDA-5 interacts preferentially with long dsRNA, a PAMP occuring in infections with positive ssRNA viruses [364].

Intracellular virus recognition through RLRs leads to engagement of the shared adaptor molecule mitochondrial antiviral signalling protein [365]. In contrast, the other RNA sensors engage different signalling molecules. TLR3 signals through TRIF and TLRs 7, 8 and 9 employ MyD88 [340]. All of these signalling cascades ultimately result in activation of the transcription factors $\mathrm{NF} \kappa \mathrm{B}$ and IRF, subsequent production of type I IFNs and other pro-inflammatory and anti-viral cytokines, and induction of an adaptive immune response [366]. 
In addition to being a TLR3 ligand, Poly IC can be recognised by either RIG-I or MDA-5, depending on its length [367]. Poly IC of $>2 \mathrm{kbp}$ length is preferentially recognised by MDA-5, while Poly IC molecules shorter than 300bp are bound by RIG-I [367]. Furthermore, activation of monocyte-derived DCs but not steady-state DCs was found to be partially dependent on MDA-5 [368] and optimal induction of CTL responses in vivo required both TLR3 and MDA-5 [369]. In addition, MDA-5 is not limited to immune cells and expression in non-haematopoietic cells can contribute to successful CTL responses after Poly IC vaccination [370].

\subsubsection{Control of adaptive immunity}

\subsubsection{Direction of the type of immune response}

Recognition of PAMPs and danger signals by PRRs is important in the initiation and regulation of the correct type of adaptive immune response. Thus, recognition of fungal cell wall components such as $\beta$-glucan and mannan by PRRs drives Th17 responses, which are required to clear fungal infections of the mucosa [371, 372]. Similarly, different TLRs can trigger Th1, Th2, Th17 and CTL T cell responses via activation of distinct cytokine profiles in DCs [373, 374]. This capacity is not limited to surface receptors, as cytosolic RLRs and some NLRs have also been demonstrated to activate adaptive immune responses $[369,375]$. In one study, a cytosolic DNA sensor pathway was even found to be sufficient to activate Th1 CD4 T cells, cytotoxic CD8 T cells and antibody responses [376]. For many PRRs the relation between the type of ligand recognised and the height and type of ensuing immune response is not yet fully understood and still needs further studies. 


\subsubsection{Requirement for innate signals}

For DCs, direct recognition of pathogens through PRRs seems to be a prerequisite for the successful activation of $\mathrm{T}$ cell responses. As mentioned before, DCs are the most important cell type to distinguish self from foreign molecules to ensure maintenance of tolerance to innocuous agents and induction of immunity to harmful pathogens. To become sufficiently activated to initiate adaptive immune responses, DCs need to sense PAMPs or DAMPs as they acquire antigen.

In addition, it has been suggested that full activation of DCs may require recognition of more than one danger signal or PAMP [377]. A combination of ligands that signal through the different adaptor molecules MyD88 and TRIF was suggested to provide optimal synergistic stimulation [378]. On the other hand, exposure of DCs to inflammatory cytokines alone without the delivery of PAMPs or DAMPs leads to up-regulation of co-stimulatory molecules, but cannot induce cytokine production [379]. Therefore, DCs matured with cytokine cocktails alone are not as effective at stimulating immune responses as DCs that encounter pathogens in vivo. One TLR ligand frequently used to achieve DC maturation is the TLR4 ligand LPS. LPS can induce very fast and efficient short-term DC activation, but can result in exhaustion, DC cell death and down-regulation of cytokine production over longer periods of time, especially at hight doses [380]. Simultaneous recognition of multiple TLRs that trigger synergistic signalling pathways seems to be needed to induce optimal production of cytokines from DCs. Especially the combination of intrinsically recognised ligands like the TLR8 ligand imidazoquinoline resiquimod and extrinsic ligands such as LPS has been found to increase the amounts of IL-12 secreted from DCs [377].

While migratory DCs may readily encounter PAMPs as they take up antigen, the sensing of PAMPs by lymphoid-tissue resident DCs is much less clear. Nevertheless, the non-migratory CD8 $\alpha+$ DCs specialise in the induction of effective CTL responses [258]. Migratory DCs may deliver both antigens and PAMPs to the LN resident DCs 
for recognition and processing [381]. A different theory is that migratory DCs activate CD4 Th cells which then interact with the LN resident DCs that in turn activate and license CTLs. According to this model, direct PAMP recognition is replaced by activation of the appropriate type of CD4 T cells which then deliver information about the type of pathogen to the lymphoid tissue resident DCs [382].

\subsubsection{Implication of innate regulation of adaptive responses}

Together, studies in recent years have found that the induction of adaptive immune responses depends largely on innate signals [299]. The detection of PAMPs and danger signals by DCs and other cell types serves to ensure that immune responses are only mounted to pathogens, while tolerance is maintained towards host cells. The recognition of conserved features of groups of similar pathogens through PRRs then results in signalling cascades which activate production of intracellular and extracellular mediators. Changes in cytokine milieu and expression of surface markers in turn confer information about the type of immune response that is needed for protection and interact with other cells of the immune system [374]. A considerable amount of signals and interplay between pathogens and various cell types of the immune system have been discovered and the emerging insights may help in the design of effective adjuvants for vaccines and tumour immunotherapies. Addition of appropriate PAMPs as adjuvants to the delivered antigens may drive the desired type of immune response, resulting in long-lasting memory and protection.

\subsection{Tumour immunotherapy}

Immunotherapy approaches aim at using the power of the host's immune system to eradicate disease. Generating anti-tumour responses de novo or enhancing existing responses holds a huge potential to complement current cancer therapies.

Immune cells are capable of distinguishing healthy from tumour cells, have power- 
ful and highly specific effector functions, can migrate to distant sites and develop long-lasting memory [383]. Thus, the generation of an effective anti-tumour immune response should result in the specific and total eradication of all cancerous cells and protection from recurrence of disease with minimal side effects to the body.

The type of immune response that is desirable for successful immunotherapy may be inferred from the large amount of studies on tumour immune surveillance and the occasional reports of spontaneous anti-tumour immune responses in patients. As described in Chapter 1.1, tumours are variable and the most effective anti-tumour immune response will depend on the susceptibility of the target cells to different cytotoxic pathways. In general, the activation and recruitment of NK cells and CTLs appears desirable. Furthermore, a Th1 cytokine environment providing substantial amounts of IFN $\gamma$ and inducing IL-12 secretion for the induction of CTLs is likely to be beneficial. Induction of broad, polyclonal responses may limit the possibility of tumour immune escape via loss of specific antigens. A reduction of the infiltration of inhibitory immune cells such as Tregs or the dampening of inhibitory factors, including IL-10 and IDO may synergise with immune activation to achieve an optimal anti-tumour effect. The ideal strategy to initiate a beneficial anti-tumour immune response remains to be determined, but several approaches are outlined below.

\subsubsection{Cell based immunotherapy}

\subsubsection{Adoptive $\mathrm{T}$ cell transfer}

A direct method to increase the amount of tumour-specific CTLs is the adoptive transfer of tumour-antigen specific $\mathrm{T}$ cells, which can lead to tumour regression in murine cancer models [69, 384, 385].

In the 1980s, Rosenberg first pioneered the adoptive transfer of tumour-infiltrating lymphocytes as a cancer therapy in melanoma patients [386]. To increase the survival 
of transferred T cells, non-myeloablative conditioning prior to cell transfer or injection of high-dose IL-2 is often used [386, 387]. It was shown that conditioning dramatically improved the anti-tumour efficacy of transferred T cells by removing endogenous cells that act as cytokine sinks [388]. In addition, T cells can also be engineered genetically to express a tumour-specific T cell receptor [389]. Several studies demonstrated a clinical benefit of $\mathrm{T}$ cell transfer especially in melanoma patients, thus illustrating the potential of the immune response for cancer therapy [386, 387, 389].

\subsubsection{Dendritic cell vaccination}

Activated and antigen loaded DCs have been used as vaccines against cancer. As DCs are critical for the initiation of immune responses, this is thought to result in activation of all appropriate effector cells. Haematopoietic precursors or monocytes can be isolated from patients' blood and differentiated into DCs, frequently with the use of GM-CSF and IL-4 [390]. These GM-CSF generated DCs are thought to correspond to inflammatory monocyte-derived DCs in vivo [306]. As they differ from steady-state migratory and LN resident DCs [306], they may not represent the best DC type for the induction of anti-tumour immune responses. The choice of antigen for DC vaccination can also be problematic. The use of synthetic peptides is highly specific, but requires exact knowledge of the expressed tumour antigens and is prone to lead to tumour evasion through antigen loss [302]. Other methods like the loading of DCs with tumour lysates [303], fusion of DCs with tumour cells [304], or electroporation of DCs with tumour RNA [305] have the potential to induce an immune response against a large spectrum of tumour antigens, but are limited by the availability of tumour sample.

Furthermore, DCs will induce tolerance instead of immunity if not sufficiently activated. Most studies so far have used monocyte conditioned medium and several cytokines, which leads to up-regulation of co-stimulatory molecules and migration 
in vivo [379], but is probably not suited to induce cytokine production by DCs. In contrast, DCs exposed to microbial stimuli secrete cytokines, but data about the duration of this cytokine production after transfer in vivo is not yet available. The frequently used stimulus LPS is a strong short-term DC activator, but seems to cause exhaustion and down-regulation of cytokine production over longer periods of time [380]. Moreover, it has been suggested that optimal cytokine production by DCs requires activation of multiple receptors for microbial products, triggering synergistic signalling pathways [377].

Clinical trials involving DC vaccination have shown improved survival in metastatic melanoma, prolonged time in remission in patients with follicular lymphoma and increased overall survival in prostate cancer patients [391-393]. Following these results, the prostate cancer DC vaccine provenge was the first DC vaccine for cancer to gain US Food \& Drug Administration approval [5].

While both T cell transfer and DC vaccination protocols highlight the potential of immunotherapy of cancer, they still show only limited anti-tumour effects in a small percentage of patients. In addition, they are labour intensive, require good manufacturing practice approved laboratories and specially trained staff. This renders cell-based immunotherapies expensive and limits their applicability.

\subsubsection{Cell free immunotherapy}

Monoclonal antibodies, cytokines and adjuvants can also be used to modulate the anti-tumour immune response. These cell-free immunotherapy approaches for cancer are generally less expensive than cell-based protocols and easily applicable in patients. 


\subsubsection{Monoclonal antibodies}

Antibodies can be used to block molecules involved in tumour progression, such as Bevacizumab that blocks VEGF, Cetuximab, which blocks the epidermal growth factor receptor and Trastuzumab which targets Her2/neu [3]. All of these antibodies are used clinically to treat colorectal cancer and Trastuzumab is also used in patients with other Her2+ malignancies. In addition, Rituximab targeting CD20 on lymphoma and Alemtuzumab specific for CD52 on chronic lymphocytic leukaemia may exert their anti-tumour functions by inducing antibody-dependent cytotoxicity [3]. However, most studies on the effect of these antibodies in cancer therapy have focused on blocking tumour-signalling pathways and much less is known about the involvement of immune pathways in their effects.

Other antibodies have been developed to block tumour-suppressive molecules and thereby boost anti-tumour immunity. These include Ipilimumab, which targets CTLA-4 and the anti-PD1 antibody BMS-936558 [6-8]. CTLA-4 is an inhibitory receptor expressed on T cells which binds to CD80 and CD86 [264]. Anti-CTLA-4 antibody enhances anti-tumour immunity in mice $[394,395]$ and patients with melanoma $[6,7]$. However, its use in patients is limited by its severe toxicity $[6,7]$. PD-1 is also an inhibitory molecule on $\mathrm{T}$ cells and has been linked to exhaustion and dysfunction in cancer $[266,269,270]$. The results of a phase I clinical study of antibodies against PD-1 and PD-L1 in patients with a range of different tumours have been promising, showing $>20 \%$ or $6-17 \%$ objective, durable responses, respectively [8, 396].

\subsubsection{Cytokines that activate the immune response}

High-dose IL-2 therapy has long been used to activate anti-tumour immunity in patients suffering from metastatic melanoma and renal cell carcinoma. High-dose IL-2 is associated with durable long-term responses in $13-15 \%$ of patients [397, 398]. The 
high dose is required to achieve anti-tumour responses, however, the majority of patients do not benefit from high-dose IL-2 therapy and the treatment is associated with severe toxicity [399].

Recently, the use of IL-12 injections at the tumour site to boost anti-tumour immunity has been evaluated in a phase I trial. In combination with Paclitaxel and Trastuzumab, IL-12 treatment was safe and showed promising activity in patients with Her2+ tumours [400]. Further studies will be needed to determine the potential of IL-12 administration to enhance anti-tumour immunity.

\subsubsection{Adjuvants}

Adjuvants that stimulate the immune response via PRRs are also used to activate anti-tumour immunity. The attenuated mycobacterium BCG has been used for the treatment of bladder cancer in patients since 1976 [9]. When repeated instillations are administered, BCG was even found to be superior to chemotherapy [10]. However, despite its long use in the clinic, little is known about the mechanism by which BCG activates the anti-tumour response. Severe local inflammation appears to occur in the bladder, but its effects on DCs are largely unknown [401]. A very recent study showed that a single instillation was sufficient to disseminate BCG to the dLNs and prime IFN $\gamma$ producing $\mathrm{T}$ cells [402]. However, multiple treatments were required to induce $\mathrm{T}$ cell infiltration in the bladder. In addition, this study showed that preexisting BCG-specific T cells could accelerate and enhance the response, and such T cells could be generated by subcutaneous vaccination prior to BCG instillation in the bladder [402].

The other clinically used adjuvant in the immunotherapy of cancer is Imiquimod. This TLR7 agonist induces local production of IFN $\alpha$, IL-12 and TNF $\alpha$. It has been shown to recruit pDCs and result in a Th1 response and activation of CTLs [17, 343]. A topical cream containing Imiquimod is used in the treatment of warts, basal cell 
carcinoma and other pre-cancerous and early malignant skin lesions [17].

Other adjuvants, such as Poly IC and CpG have shown very promising results in murine models of immunotherapy, but yielded varying results in clinical trials for cancer immunotherapy and are not currently used as standard treatments for cancer patients $[12,14,16,403]$. 


\subsection{Aims of this thesis}

DCs have been found to infiltrate most human and murine tumours. However, they appear to lack sufficient activation to induce potent anti-tumour immunity. The hypothesis of this thesis was that microbial stimuli and danger signals may be able to overcome immune-suppression in tumours and re-programme DCs and macrophages to an immuno-stimulatory phenotype. Adjuvants that induce Th1 responses and CTLs are likely to be the most beneficial stimuli for anti-tumour immunity. Therefore, adjuvants associated with Th1 induction were chosen for this study. Whole live Mycobacterium smegmatis and BCG were selected to provide multiple PAMPs. The intracellularly-recognised TLR ligands CpG and Poly IC, as well as the extracelullarly recognised TLR ligand LPS, and the danger signal MSU were also included. Both individual adjuvants and adjuvant combinations were assessed in this study.

The specific aims of this thesis were:

- To investigate which adjuvants or adjuvant combinations could activate DCs in vitro and in vivo.

- To identify adjuvants that could induce tumour regression or delay of tumour growth in vivo.

- To elucidate which cytokines, growth factors and DC activation parameters or subsets were associated with successful adjuvants compared to ineffective adjuvants.

- To determine, which effector cells mediated a successful adjuvant induced immune response.

- To evaluate whether adjuvants that were effective against primary murine melanomas could also delay tumour growth in other cancer models or reduce metastases. 

Chapter 2

Material and methods 


\section{$2.1 \quad$ Material}

\subsubsection{Labware}

Table 2.1: Labware

\begin{tabular}{|c|c|}
\hline Product & Supplier/Distributor \\
\hline $\begin{array}{l}\text { Acrodisc }^{\mathrm{TM}} 13 \mathrm{~mm} \& 32 \mathrm{~mm} \text { syringe fil- } \\
\text { ters with } 0.2 \mu \mathrm{m} \text { Supor }^{\circledR} \text { membrane }\end{array}$ & PALL Life Sciences, Cornwall, U.K. \\
\hline Axygen Micro Tubes $0.6 \& 1.7 \mathrm{~mL}$ & $\begin{array}{l}\text { Axygen Scientific Inc., Union City, CA, } \\
\text { USA }\end{array}$ \\
\hline $\begin{array}{l}\text { Axygen sterile pipet tips 1000, } 200 \& \\
10 \mu \mathrm{L}\end{array}$ & USA \\
\hline $\begin{array}{l}\text { BD } 1 \mathrm{~mL} \text { Tuberculin syringes \& BD } \\
10 \mathrm{~mL} \text { syringes }\end{array}$ & BD BioSciences, Bedford, MA, USA \\
\hline $\begin{array}{l}\text { BD Falcon }{ }^{\mathrm{TM}} \text { polystyrene sterile conical } \\
\text { tubes: Blue Max } 50 \mathrm{~mL} \& \text { Blue Max Jr. } \\
15 \mathrm{~mL}\end{array}$ & \\
\hline $\begin{array}{l}\text { BD Falcon }{ }^{\mathrm{TM}} \text { polystyrene sterile } 5 \mathrm{~mL} \\
\text { round bottom tubes }\end{array}$ & \\
\hline $\begin{array}{l}\text { BD Falcon }{ }^{\mathrm{TM}} \text { polystyrene sterile tissue } \\
\text { culture dishes } 100 \times 20 \mathrm{~mm}\end{array}$ & \\
\hline $\begin{array}{l}\text { BD Falcon }{ }^{\mathrm{TM}} \text { polystyrene sterile multi- } \\
\text { well tissue culture plates: } 6 \text { well, } 24 \text { well } \\
\& \text { Microtest }^{\mathrm{TM}} \text { U-bottom } 96 \text { well plates }\end{array}$ & \\
\hline $\begin{array}{l}\text { BD Falcon }{ }^{\mathrm{TM}} \text { polystyrene sterile tissue } \\
\text { culture flasks: } 200 \mathrm{~mL} \& 600 \mathrm{~mL}\end{array}$ & \\
\hline $\begin{array}{l}\text { BD Falcon }{ }^{\mathrm{TM}} \text { polystyrene sterile serolog- } \\
\text { ical pipettes }(5,10 \& 25 \mathrm{~mL})\end{array}$ & \\
\hline
\end{tabular}


Table 1: Labware (continued)

\begin{tabular}{|c|c|}
\hline Product & Supplier/Distributor \\
\hline $\begin{array}{l}\text { BD Falcon }{ }^{\mathrm{TM}} \text { nylon cell strainers }(40 \& \\
70 \mu \mathrm{m})\end{array}$ & \multirow[t]{3}{*}{ BD BioSciences, Bedford, MA, USA } \\
\hline $\begin{array}{l}\text { PrecisionGlide }^{\mathrm{TM}} \text { needles: } 18,20,25 \& \\
27.5 \text { gauge }(\mathrm{G})\end{array}$ & \\
\hline $\begin{array}{l}\text { Ultra-Fine }^{\mathrm{TM}} \text { needle insulin syringes } \\
(29 \mathrm{G}): 0.3,0.5 \& 1 \mathrm{~mL}\end{array}$ & \\
\hline $\begin{array}{l}\text { CellLine CL } 1000 \text { two-compartment } \\
\text { bioreactor flasks }\end{array}$ & $\begin{array}{l}\text { INTEGRA Biosciences Corp., Hudson, } \\
\text { NH, USA }\end{array}$ \\
\hline Cover Slips 22x22 mm, No. 1 thickness & Biolab Ltd., Auckland, NZ \\
\hline Cryo's $^{\mathrm{TM}}$ sterile cryotubes $2 \mathrm{~mL}$ & $\begin{array}{l}\text { Greiner Bio-One, Frickenhausen, Ger- } \\
\text { many }\end{array}$ \\
\hline $30 \mu \mathrm{m}$ MACS ${ }^{\circledR}$ pre-separation filters & \multirow[t]{2}{*}{ Miltenyi Biotech GmbH, Germany } \\
\hline $\begin{array}{l}\text { MACS }^{\circledR} \quad \text { autoMACS } \\
\text { Columns }\end{array}$ & \\
\hline Millipore $^{\mathrm{TM}}$ MX-Plates & Millipore Corp., Billercia, MA, USA \\
\hline$\mu$ ltra Amp 96-well PCR plates & $\begin{array}{l}\text { Sorensen }^{\mathrm{TM}} \text { Bioscience, Inc., Salt Lake } \\
\text { City, UT, USA }\end{array}$ \\
\hline 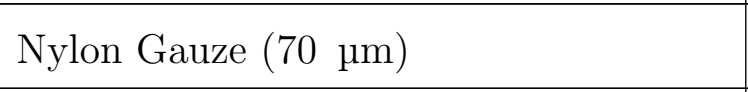 & NZ Filter Specialists Ltd., Auckland, NZ \\
\hline $\begin{array}{l}\text { Optical Adhesive Covers for Real-time } \\
\text { PCR }\end{array}$ & $\begin{array}{l}\text { Applied Biosystems, Foster City, CA, } \\
\text { USA }\end{array}$ \\
\hline $\begin{array}{l}\text { PCR } 0.2 \mathrm{~mL} \text { thin wall tubes; } 8 \text { strips \& } \\
\text { caps }\end{array}$ & $\begin{array}{l}\text { Axygen Scientific Inc., Union City, CA, } \\
\text { USA }\end{array}$ \\
\hline Petri dishes, $90 \mathrm{~mm}$ diameter & Labserv, Auckland, New Zealand \\
\hline $\begin{array}{l}\text { Slide-A-Lyzer Dialysis Cassettes } 10,000 \\
\text { MWCO }\end{array}$ & $\begin{array}{l}\text { Thermo Scientific, Portsmouth, NH, } \\
\text { USA }\end{array}$ \\
\hline
\end{tabular}


Table 1: Labware (continued)

\begin{tabular}{|l|l|}
\hline Product & Supplier/Distributor \\
\hline Sterile Carbon Steel Surgical Blades & Swann-Morton ${ }^{\circledR}$ Limited, Sheffield, \\
number $10 \& 22$ & England \\
\hline Superfrost $^{\circledR}$ Plus microscope slides & Biolab Ltd., Auckland, NZ \\
\hline Titertube $^{\mathrm{TM}}$ Microtubes $0.5 \mathrm{~mL}$ & Bio-Rad Laboratories, Hercules, CA, \\
\hline Transfer pipettes $(1 \mathrm{~mL})$ & USA \\
\hline
\end{tabular}

\subsubsection{Reagents and buffers}

\section{Mercaptoethanol (2-ME)}

$2 \mathrm{ME}$ was purchased from Sigma (St. Louis, MO, USA) as a $55 \mathrm{mM}$ solution in PBS and stored at $4{ }^{\circ} \mathrm{C}$.

\section{6-thioguanine}

6-thioguanine powder was purchased from Sigma (St. Louis, MO, USA) and stored at room temperature.

\section{Acetone}

Acetone was purchased from Merck (Darmstadt, Germany) and stored at room temperature.

\section{Agar}

Granulated Difco ${ }^{\text {TM }}$ agar was purchased from BD BioSciences, Bedford, MA, USA.

\section{Agarose}

Ultra-Pure $^{\mathrm{TM}}$ Agarose was purchased from Invitrogen, Auckland, NZ. 


\section{Alsever's Solution}

Dextrose, $\mathrm{NaCl}$ and sodium citrate (all from BDH Laboratory Supplies, Poole, England) were dissolved in distilled $\mathrm{H}_{2} \mathrm{O}$ (MilliQ) to give final concentrations of $20.5 \mathrm{mg} / \mathrm{mL}$ dextrose, $4.2 \mathrm{mg} / \mathrm{mL} \mathrm{NaCl}$ and $8.0 \mathrm{mg} / \mathrm{mL}$ sodium citrate. The $\mathrm{pH}$ was adjusted to 6.1 with $1 \mathrm{M}$ citric acid (BDH Laboratory Supplies, Poole, England) and the solution stored at room temperature until used.

Ammonium Chloride Tris (ACT) Lysis Buffer

ACT buffer containing $0.144 \mathrm{M} \mathrm{NH}_{4} \mathrm{Cl}$ and $0.017 \mathrm{M} \mathrm{HCl}$ was prepared by mixing 9 parts of $0.16 \mathrm{M} \mathrm{NH}_{4} \mathrm{Cl}$, pH 7.4 (Sigma, St. Louis, Missouri, USA) and 1 part of $0.17 \mathrm{M}$ Tris-HCl, pH 7.65 (Merck, Darmstadt, Germany) directly before use.

\section{Assay Buffer}

Assay Buffer for Multiplex detection of cytokines was prepared by adding $0.1 \%$ BSA, $0.05 \%$ Tween20, $0.005 \% \mathrm{NaN}_{3}$ and $2.5 \mathrm{mM}$ EDTA to $\mathrm{CaCl}_{2}$ and $\mathrm{MgCl}_{2}$ free PBS. Assay buffer was stored at $4^{\circ} \mathrm{C}$.

Bovine Serum Albumin (BSA)

BSA with low endotoxin levels was purchased from ICPbio Ltd. (Henderson, Auckland, NZ) in powder form and stored at $4{ }^{\circ} \mathrm{C}$.

\section{Brefeldin A}

Brefeldin A was purchased from eBioscience (San Diego, CA, USA) at 1000x concentration and stored at $4 \mathrm{C}$. To block cytokine secretion in cell culture, Brefeldin A was diluted to a $1 \mathrm{x}$ concentration in cIMDM.

\section{Carboxyfluorescein Diacetate Succinimidyl Ester (CFDA-SE)}

CFDA-SE was purchased as powder from Molecular Probes (San Diego, CA, USA) and suspended at $10 \mathrm{mM}$ in dimethyl sulfoxide (DMSO; Sigma, St. Louis, Missouri, USA). Single use aliquots for were stored at $-20^{\circ} \mathrm{C}$. 


\section{Chromatography Elution Buffer}

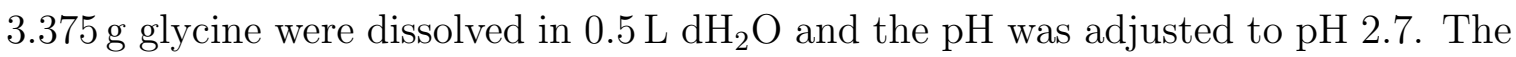
buffer was degassed and stored at room temperature.

\section{Chromatography Neutralising Buffer}

$12.114 \mathrm{~g}$ Tris- $\mathrm{HCl}$ were dissolved in $100 \mathrm{~mL} \mathrm{dH_{2 }} \mathrm{O}$. The solution was adjusted to $\mathrm{pH} 9.0$, degassed and stored at room temperature.

\section{Chromatography Wash Buffer}

$2.84 \mathrm{~g}$ Di-sodium-orthophosphate were dissolved in $1 \mathrm{~L} \mathrm{dH_{2 }} \mathrm{O}$ and $\mathrm{pH}$ was adjusted to $\mathrm{pH}$ 8.0. The buffer was degassed and stored at room temperature.

\section{Collagenase IV}

Collagenase IV (260 u/mg) was purchased as lyphilised powder from Worthington Biochemical Corporation (Lakewood, NJ, USA). The powder was stored at $-20^{\circ} \mathrm{C}$ and the desired amount of enzyme was diluted in HBSS directly before use.

\section{$C p G$ Oligodeoxynucleotides 1668 ( $C p G)$}

Transfection grade CpG ODN 1668 (ODN 1668 (sequence: 5-tccatgacgttcctgatgct-3; phosphorothionate linkages)) was purchased as lyophilised powder from GeneWorks (Thebarton, SA, Australia) and stored at $4{ }^{\circ} \mathrm{C}$. CpG was dissolved in PBS to give a stock concentration of $3.2 \mathrm{mg} / \mathrm{mL}$ and aliquots were stored at $-20^{\circ} \mathrm{C}$. In use aliquots were stored at $4{ }^{\circ} \mathrm{C}$ for a maximum of 4 weeks.

\section{DEPC-Treated Water}

RNase-free, DNase-free, pyrogen-free filtered DEPC treated water was purchased from Ambion Inc, (Austin, TX, USA).

\section{DNase I}

DNase I was purchased as a lyophilised powder from Roche (Mannheim, Germany), dissolved at $10 \mathrm{mg} / \mathrm{mL}$ in IMDM and stored at $-20^{\circ} \mathrm{C}$ until used. 


\section{Dubos Broth}

Difco $^{\mathrm{TM}}$ Dubos Broth Base was purchased from BD BioSciences (Bedford, MA, USA). The broth powder was dissolved in $\mathrm{dH}_{2} \mathrm{O}$ and autoclaved to sterilise. $10 \%$ OADC (oleic albumin dextrose catalase growth) supplement was filter sterilised and added to the warm medium. Medium was stored at $4{ }^{\circ} \mathrm{C}$ and used within one month. Sterility was tested by incubating $10 \mathrm{~mL}$ medium at $37^{\circ} \mathrm{C}$ for $3-5$ days.

\section{Elastase}

Elastase $(4.4 \mathrm{u} / \mathrm{mg})$ was purchased as a lyophilised powder from Worthington Biochemical Corporation (Lakewood, NJ, USA). The powder was stored at $-20^{\circ} \mathrm{C}$ and the desired amount of enzyme was diluted in HBSS directly before use.

\section{Ethanol}

Molecular grade $100 \%$ ethanol was purchased from Carlo Erba Reagents (Milan, Italy) and stored at room temperature until used.

\section{Ethylendiaminetetraacetic Acid (EDTA)}

EDTA was purchased in powder form from Sigma (St. Louis, MO, USA), dissolved in $\mathrm{dH}_{2} \mathrm{O}$ at a concentration of $0.5 \mathrm{M}$ and stored at room temperature.

Flow Cytometry (FC) Buffer

EDTA, $\mathrm{NaN}_{3}$ (both from Sigma, St. Louis, MO, USA) and FBS (GIBCO, Invitrogen, Auckland, NZ) were added to PBS at final concentrations of $10 \mathrm{mM}$ EDTA, $0.01 \%$ $\mathrm{NaN}_{3}$ and $2 \%$ FBS. FC buffer was stored at $4{ }^{\circ} \mathrm{C}$.

\section{Foetal Bovine Serum (FBS)}

Mycoplasma and virus screened and performance tested FBS containing $27 \mathrm{EU} / \mathrm{mL}$ was purchased from GIBCO (Invitrogen, Auckland, NZ) and stored in $25 \mathrm{~mL}$ aliquots at $-20^{\circ} \mathrm{C}$. After thawing, aliquots were stored at $4{ }^{\circ} \mathrm{C}$ for a maximum of 3 weeks. 
Geneticin $^{\circledR}($ G418)

The antibiotic Geneticin ${ }^{\circledR}$ was purchased from GIBCO (Invitrogen, Auckland, NZ) and stored in aliquots at $-20^{\circ} \mathrm{C}$.

\section{Glycerol}

$100 \%$ Glycerol was purchased from Sigma (St. Louis, MO, USA) and stored at room temperature.

Hank's Buffered Salt Solution (HBSS)

HBSS was purchased from GIBCO (Invitrogen, Auckland, NZ) and stored at $4{ }^{\circ} \mathrm{C}$.

\section{Intracellular cytokine detection kit}

The BD Cytofix/Cytoperm kit was purchased from BD Pharmingen (San Diego, CA, USA). The kit buffers 1x Cytofix/Cytoperm buffer and 10x Perm/Wash buffer were stored at $4{ }^{\circ} \mathrm{C}$. $10 \mathrm{x}$ Perm/Wash buffer was diluted to $1 \mathrm{x}$ Perm/Wash buffer with $\mathrm{dH}_{2} \mathrm{O}$ (MilliQ) directly prior to use.

Iscove's Modified Dulbecco's Medium (IMDM)

IMDM supplemented with GlutaMAX, $25 \mathrm{mM}$ HEPES buffer and $3.024 \mathrm{mg} / \mathrm{L} \mathrm{NaHCO} 3$ was purchased from GIBCO (Invitrogen, Auckland, NZ) and stored at $4{ }^{\circ} \mathrm{C}$ until used.

Complete Iscove's Modified Dulbecco's Medium (cIMDM)

IMDM was supplemented with $1 \%$ Penicillin-Streptomycin (GIBCO, Invitrogen, Auckland, NZ), $55 \mu \mathrm{M} 2 \mathrm{ME}$ and $5 \% \mathrm{FBS}$. Medium was stored at $4{ }^{\circ} \mathrm{C}$ for a maximum of 3 weeks.

\section{Isopropanol}

Analytical grade Isopropanol was purchased from Scharlau Chemie (Barcelona, Spain) and stored at room temperature until used. 


\section{Liberase TL}

Liberase TL was purchased as a lyophilised powder from Roche (Mannheim, Germany), dissolved in injection grade $\mathrm{dH}_{2} \mathrm{O}$ (2 parts) and IMDM (3 parts) at a concentration of $1 \mathrm{mg} / \mathrm{mL}$ and stored in single use aliquots at $-20^{\circ} \mathrm{C}$.

\section{Lipopolysaccharides (LPS)}

LPS from Escherichia coli, serotype 0111:B4, was purchased as a lyophilised powder from Sigma (St. Louis, MO, USA), dissolved in IMDM at a stock concentration of $1 \mathrm{mg} / \mathrm{mL}$ and stored at $4^{\circ} \mathrm{C}$.

\section{Luria-Bertani Medium (LB)}

Luria-Bertani broth powder (Miller) was purchased from Sigma (St. Louis, MO, USA), dissolved in $\mathrm{dH}_{2} \mathrm{O}$ according to the manufacturer's instructions and autoclaved. LB medium was stored at $4{ }^{\circ} \mathrm{C}$. For LB agar plates, $15 \mathrm{~g} / \mathrm{L}$ agar (Difco ${ }^{\mathrm{TM}}$, BD BioSciences, Bedford, MA, USA) were added to LB medium. LB agar medium was autoclaved and dispensed into sterile petri dishes while still warm. Plates were stored at $4^{\circ} \mathrm{C}$.

\section{Magnetic Separation (MACS) Beads}

Anti-CD8 $\alpha$, anti-CD4, anti-CD11c, anti-CD45 and anti-biotin MACS Microbeads were purchased from Miltenyi Biotec GmbH (Bergisch Gladbach, Germany) and stored at $4{ }^{\circ} \mathrm{C}$ until used.

\section{Methanol}

Analytical grade methanol was purchased from Scharlau Chemie (Barcelona, Spain) and stored at room temperature until used.

Polymerase Chain Reaction (PCR) reagents 2x Taqman expression master mix and taqman primers were all from Applied Biosystems, Foster City, CA, USA. The master mix was stored at $4{ }^{\circ} \mathrm{C}$ and primer aliquots were stored at $-20^{\circ} \mathrm{C}$. 


\section{Middlebrook $7 H 11$ Agar}

Difco $^{\text {TM }}$ Middlebrook 7H11 agar powder was purchased from BD BioSciences (Bedford, MA, USA). The 7H11 powder and $0.55 \%$ glycerol were dissolved in $\mathrm{dH}_{2} \mathrm{O}$ and autoclaved. When medium had cooled down, 10\% OADC supplement were added and the 7H11 medium was dispensed into sterile petri dishes. 7H11 agar plates were stored at $4{ }^{\circ} \mathrm{C}$.

\section{Monosodium Urate Crystals (MSU)}

MSU was prepared from uric acid as previously described [404]. Briefly, $250 \mathrm{mg}$ uric acid were dissolved in $50 \mathrm{~mL}$ boiling $\mathrm{dH}_{2} \mathrm{O}$ (MilliQ) and subsequently left to cool down to $50{ }^{\circ} \mathrm{C}$. The solution was filtered through a $0.2 \mu \mathrm{m}$ filter and reboiled. Crystal formation was induced by addition of $1 \mathrm{~mL} 5 \mathrm{M} \mathrm{NaCl}$. 7 days later, MSU crystals were washed with ethanol and acetone and dried. MSU crystals were $12 \mu \mathrm{m}$ to $25 \mu \mathrm{m}$ in size with needle-like appearance, as determined by light microscopy. Endotoxin content was assessed by LAL test and found to be $0.288 \mathrm{EU} / \mathrm{mg} \mathrm{MSU}$.

\section{Oleic Albumin Dextrose Catalase Growth Supplement, Middlebrook (OADC)}

Difco $^{\mathrm{TM}}$ OADC supplement was purchased from BD BioSciences (Bedford, MA, USA) and stored at $4{ }^{\circ} \mathrm{C}$. For addition to Dubos broth or Middlebrook 7H11 agar, a OADC was filter sterilised.

\section{Penicillin-Streptomycin}

Penicillin-Streptomycin (10 $000 \mathrm{U} / \mathrm{mL}$ and $10000 \mathrm{\mu g} / \mathrm{mL}$, respectively) was purchased in liquid form from GIBCO (Invitrogen, Auckland, NZ) and stored as single use aliquots at $-20^{\circ} \mathrm{C}$ until used.

\section{Phosphate Buffered Saline (PBS)}

$\mathrm{CaCl}_{2}$ and $\mathrm{MgCl}_{2}$ free PBS was purchased from GIBCO (Invitrogen, Auckland, NZ) and stored at room temperature. In use PBS was stored at $4{ }^{\circ} \mathrm{C}$. 


\section{Polyinosinic-Polycytidylic Acid (Poly IC)}

Low molecular weight Poly IC $(0.2-1 \mathrm{kbp})$ was purchased as lyohilised powder from InvivoGen (San Diego, CA, USA) and dissolved in endotoxin-free physiological water (containing $1.5 \% \mathrm{NaCl}$ ) provided by the supplier at a stock concentration of $20 \mathrm{mg} / \mathrm{mL}$. Stock aliquots were stored at $-20^{\circ} \mathrm{C}$ and in use aliquots were stored at $4{ }^{\circ} \mathrm{C}$ for a maximum of 4 weeks.

\section{Proteinase inhibition buffer}

A 25x stock solution was prepared by dissolving one Complete Protease Inhibitor Tablet (Roche, Mannheim, Germany) in $2 \mathrm{~mL}$ PBS. Aliquots were stored at $-20^{\circ} \mathrm{C}$ for up to three months. Prior to protein extraction, the stock solution was diluted to a 1x working solution in PBS.

\section{Reverse Transcription Kit}

The reverse transcription SuperScript Vilo ${ }^{\mathrm{TM}}$ kit was purchased from Invitrogen, Auckland, NZ, and stored at $-20^{\circ} \mathrm{C}$.

\section{RNA Extraction Kit}

The NucleoSpin ${ }^{\circledR}$ RNA L kit for extraction of RNA from medium-sized tissue samples was purchased from Macherey-Nagel GmbH \& Co. KG, Düren, Germany. The DNase powder was stored at $4{ }^{\circ} \mathrm{C}$, aliquots of DNase solution were stored at $-20^{\circ} \mathrm{C}$ and the remaining components were stored at room temperature as specified by the manufacturer.

\section{RPMI Medium 1640 (RPMI)}

RPMI supplemented with $4.5 \mathrm{~g} / \mathrm{L}$ D-glucose, $1.5 \mathrm{~g} / \mathrm{L}$ Sodium Bicarbonate, 1 mM Sodium Pyruvate, $10 \mathrm{mM}$ HEPES and $300 \mathrm{mg} / \mathrm{L}$ L-Glutamine was purchased from GIBCO (Invitrogen, Auckland, NZ) and stored at $4^{\circ} \mathrm{C}$ until used. 
Sodium Azide $\left(\mathrm{NaN}_{3}\right)$

$\mathrm{NaN}_{3}$ (Sigma, St. Louis, MO, USA) was purchased in powder form and dissolved in $\mathrm{dH}_{2} \mathrm{O}$ to a stock concentration of $5 \%$. The solution was stored at room temperature until used.

\section{Sodium Bicarbonate Buffer}

$\mathrm{NaHCO}_{3}(0.42 \mathrm{~g})$ was dissolved in $50 \mathrm{~mL} \mathrm{dH} \mathrm{H}_{2} \mathrm{O}$ to yield a $0.1 \mathrm{M}$ solution. Sodium bicarbonate buffer was prepared immediately prior to use and not stored.

\section{Sodium Chloride ( $\mathrm{NaCl})$}

$\mathrm{NaCl}$ was purchased in powder form from Sigma (St. Louis, MO, USA), dissolved in $\mathrm{dH}_{2} \mathrm{O}$ to a concentration of $1.8 \%$ and stored at room temperature.

\section{Sodium Hydroxide}

Sodium hydroxide was purchased from BDH Laboratory Supplies (Poole, England) in powder form. Different solutions were used to adjust the $\mathrm{pH}$ of buffers.

\section{$S Y B R^{\circledR}$ Safe}

SYBR ${ }^{\circledR}$ Safe DNA Gel Stain was purchased from Invitrogen, Auckland, NZ. The dye was added directly to the agarose-solution before the gels set to visualize nucleic acids.

Tris-acetate-EDTA (TAE) buffer

A 50x stock solution of TAE buffer containing $2 \mathrm{M}$ Tris Acetate and $50 \mathrm{mM}$ EDTA was purchased from GIBCO (Invitrogen, Auckland, NZ) and diluted to a 1x working solution using $\mathrm{dH}_{2} \mathrm{O}$.

\section{Tris Buffer}

Tris buffer was made by diluting $0.5902 \mathrm{~g}$ Tris in $50 \mathrm{~mL} \mathrm{dH}_{2} \mathrm{O}$. The $\mathrm{pH}$ was adjusted to 8.5 and the buffer was stored at room temperature until used. 
Trypsin/EDTA

Trypsin/EDTA solution containing $0.25 \%$ Trypsin and 1 mM EDTA in Hanks' Balanced Salt Solution was purchased from GIBCO (Invitrogen, Auckland, NZ), and aliquots were stored at $-20^{\circ} \mathrm{C}$. In use aliquots were stored at $4{ }^{\circ} \mathrm{C}$.

Tween $^{\circledR} 20$ and Tween ${ }^{\circledR} 80$

Tween 20 and Tween 80 were purchased from Sigma (St. Louis, Missouri, USA) and stored at room temperature until used.

Uric Acid

Uric acid was purchased in powder form from Sigma (St. Louis, MO, USA) and stored at room temperature.

\section{Wuerzburger Buffer}

Sterile PBS was supplemented with $0.5 \mathrm{M}$ EDTA, $10 \mathrm{mg} / \mathrm{mL}$ DNAse I and FBS to give final concentrations of $5 \mathrm{mM}$ EDTA, $20 \mu \mathrm{g} / \mathrm{mL}$ DNase and $1 \%$ FBS. The buffer was stored at $4{ }^{\circ} \mathrm{C}$ until used.

\subsubsection{Cytokines and growth factors}

Fms-like tyrosine kinase 3 ligand (Flt3L)

Recombinant murine Flt3L was produced using stationary phase cultures of the Chinese Hamster Ovary cell line CHO flagFlk2.cone5, kindly provided by Prof. Nicos Nicola (WEHI, Melbourne, Australia).

\section{Granulocyte-Macrophage Colony Stimulating Factor (GM-CSF)}

Recombinant murine GM-CSF was produced using stationary phase cultures of the murine X63 cell line, modified to secrete the full-length murine GM-CSF protein. The modified murine X63 cell line was kindly provided by Dr Antonius Rolink (Basel Institute for Immunology, Basel, Switzerland). 
Interleukin $4(I L-4)$

Recombinant murine IL-4 was produced using stationary phase cultures of a Chinese Hamster Ovary cell line (CHO), modified to secrete the full length murine IL-4 protein. The IL-4 producing CHO cell line was kindly provided by Dr Antonius Rolink (Basel Institute for Immunology, Basel, Switzerland).

Cytokines were collected by growing adherent cells in cIMDM, harvesting the culture supernatants and filtering through a $0.2 \mu \mathrm{m}$ serum filter. The cytokines were titrated using bone-marrow derived DC (BMDC) cultures (GM-CSF and IL-4 or FLt3L, respectively) to determine the optimal amount for good cell recovery and clear separation of DC subsets (Flt3L). The cytokines were stored as aliquots at $-80^{\circ} \mathrm{C}$. In use aliquots were stored at $4{ }^{\circ} \mathrm{C}$ for up to 2 weeks.

\subsubsection{Antibodies and fluorophores}

\section{Antibodies}

Antibodies specific to murine antigens were either purchased from BD Pharmingen (San Diego, CA, USA), eBioscience (San Diego, CA, USA), Biolegend (San Diego, CA, USA), AbD Serotec (Morphosys, Kidlington, U.K.) or purified in-house and labelled with biotin, FITC or Alexa Fluor 647 (see 2.2.1.2 \& 2.2.3.1). 
Table 2.2: Antibodies

\begin{tabular}{|c|c|c|c|}
\hline Specificity & Clone & Label & Source \\
\hline B220 & $6 \mathrm{~B} 2$ & Biotin, FITC & purified in-house \\
\hline B220 & $6 \mathrm{~B} 2$ & Pacific Blue, PerCP & BD Pharmingen \\
\hline CD3e & $2 \mathrm{C} 11$ & $\mathrm{APC}$ & BD Pharmingen \\
\hline CD4 & GK1.5 & Alexa Fluor 647, FITC & purified in-house \\
\hline CD4 & GK1.5 & Pacific Blue & BD Pharmingen \\
\hline $\operatorname{CD} 8 \alpha$ & 2.43 & Alexa Fluor 647, Biotin, FITC & purified in-house \\
\hline $\operatorname{CD} 8 \alpha$ & $53-6.7$ & $\begin{array}{l}\text { Alexa Fluor 700, APC-H7, PE, } \\
\text { PerCP-Cy5.5 }\end{array}$ & BD Pharmingen \\
\hline CD11b & $\mathrm{M} 1 / 70$ & $\begin{array}{l}\text { PE, PE-Cy5, PerCP-Cy5.5, } \\
\text { APC-Cy7 }\end{array}$ & BD Pharmingen \\
\hline CD11c & N418 & Alexa Fluor 647, Biotin, FITC & purified in-house \\
\hline CD11c & HL3 & $\mathrm{PE}, \mathrm{PE}-\mathrm{Cy} 7$ & BD Pharmingen \\
\hline CD19 & 1D3 & Biotin & BD Pharmingen \\
\hline $\mathrm{CD} 24$ & $\mathrm{M} 1 / 69$ & FITC & Biolegend \\
\hline CD24 & $\mathrm{M} 1 / 69$ & $\mathrm{PE}$ & eBioscience \\
\hline CD40 & $3 / 23$ & $\mathrm{PE}$ & BD Pharmingen \\
\hline CD45 & $30-\mathrm{F} 11$ & FITC, Pacific Blue, PE, Biotin & BD Pharmingen \\
\hline CD45.1 & A 20 & APC, Biotin, PE, PerCP-Cy5.5 & BD Pharmingen \\
\hline CD 45.2 & 104 & $\begin{array}{l}\text { APC, Biotin, FITC, PE, PE- } \\
\text { Cy5.5 }\end{array}$ & eBioscience \\
\hline CD80 & 16-10A1 & Biotin, PE & BD Pharmingen \\
\hline CD86 (B7.2) & GL1 & FITC & purified in-house \\
\hline CD86 (B7.2) & GL1 & V450 & BD Pharmingen \\
\hline DX5 & DX5 & Biotin & eBioscience \\
\hline $\begin{array}{l}\text { Fc } \gamma \text { RII/III } \\
(\mathrm{CD} 32 / \mathrm{CD} 16)\end{array}$ & $2.4 \mathrm{G} 2$ & none & purified in-house \\
\hline $\mathrm{F} 4 / 80$ & BM8 & FITC, PE, PerCP & eBioscience \\
\hline
\end{tabular}


Table 2: Antibodies (continued)

\begin{tabular}{|l|l|l|l|}
\hline Specificity & Clone & Label & Source \\
\hline Gr-1 & RB6-8C5 & Biotin & Biolegned \\
\hline IFN $\gamma$ & XMG1.2 & APC, PE & BD Pharmingen \\
\hline IL-12p40/70 & C15.6 & PE & BD Pharmingen \\
\hline Ly-6B.2 & $7 / 4$ & FITC & AbD Serotec \\
\hline Ly6C & HK1.4 & PE-Cy7 & Biolegend \\
\hline Ly6G & 1A8 & Biotin & BD Pharmingen \\
\hline $\begin{array}{l}\text { MHC class } \\
\left(\text { II }{ }^{\text {b }}\right)\end{array}$ & $3 J P$ & Alexa Fluor 647, FITC & purified in-house \\
\hline $\begin{array}{l}\text { MHC class } \\
(\text { II-A } / \text { I-E })\end{array}$ & M5/114 & FITC, PE & BD Pharmingen \\
\hline NK1.1 & PK136 & Biotin, FITC, PE & eBioscience \\
\hline Ter119 & Ter119 & Biotin & eBioscience \\
\hline TNF- $\alpha$ & MP6-XT22 & FITC, Pacific Blue & eBioscience \\
\hline V $\alpha 2$ & B20.1 & Biotin, FITC, PE & BD Pharmingen \\
\hline V $\beta 5.1 / 5.2$ & MR9-4 & Biotin, PE & BD Pharmingen \\
\hline
\end{tabular}

To identify positive staining, isotype control antibodies were used for some staining antibodies:

Table 2.3: Isotype Controls

\begin{tabular}{|l|l|l|l|}
\hline Specificity & Isotype & Isotype control clone & Source \\
\hline CD11b & Rat IgG2b $\kappa$ & A95-1 & BD Pharmingen \\
\hline CD11c & $\begin{array}{l}\text { Armenian Ham- } \\
\text { ster IgG1 } \lambda\end{array}$ & G235-2356 & BD Pharmingen \\
\hline IL-12p40/70 & Rat IgG1 $\kappa$ & R3-34 & BD Pharmingen \\
\hline IFN- $\gamma$ & Rat IgG1 $\kappa$ & R3-34 & BD Pharmingen \\
\hline F4/80 & Rat IgG2a $\kappa$ & R35-95 & eBioscience \\
\hline TNF- $\alpha$ & Rat IgG1 $\kappa$ & EBRG1 & eBioscience \\
\hline
\end{tabular}




\section{Cell viability dyes}

4,6-Diamidino-2-Phenylindole Dihydrochloride (DAPI) was purchased as a lyophilised powder from Invitrogen (Auckland, NZ) and dissolved in $\mathrm{dH}_{2} \mathrm{O}$ to a concentration of $5 \mathrm{mg} / \mathrm{mL}$. This solution was then further diluted to a stock solution of $200 \mu \mathrm{g} / \mathrm{mL}$ in FC buffer and stored in aliquots at $4{ }^{\circ} \mathrm{C}$ until used.

Live/Dead ${ }^{\circledR}$ fixable dead cell staining kit blue was purchased from Invitrogen (Auckland, NZ) and stored at $-20^{\circ} \mathrm{C}$. The lyohilised dye powder was dissolved in $50 \mu \mathrm{L}$ DMSO per vial as per manufacturer's instructions and stored at $4{ }^{\circ} \mathrm{C}$ for a maximum of 6 weeks.

\section{Streptavidin (SA)-Fluorophore conjugates}

SA-Alexa Fluor 555 was purchased from Invitrogen (Auckland, NZ) and stored in aliquots at $-20^{\circ} \mathrm{C}$. In use aliquots were stored at $4{ }^{\circ} \mathrm{C}$. SA-FITC, SA-PE, SA-PerCP, SA-PETR and SA-APC were purchased from BD Pharmingen and stored at $4{ }^{\circ} \mathrm{C}$.

\subsubsection{Mycobacteria}

The mycobacterial strains Mycobacterium smegmatis mc2155 and Mycobacterium bovis Bacillus Calmette-Guérin (BCG) Pasteur strain $1173 \mathrm{P}_{2}$ were kindly provided by Ronan O’Toole (School of Biological Sciences, Victoria University of Wellington, NZ) and AgResearch Wallaceville Animal Research Centre (Upper Hutt, NZ).

\subsubsection{Tumour cell lines}

The B16.OVA melanoma tumour cell line was generated by Drs. Edith Lord and John G. Frelinger, University of Rochester, Rochester, NY [405] and kindly gifted by Drs. Roslyn Kemp and Dick Dutton, Trudeau Institute, NY, USA. The parental melanoma tumour cell line B16.F1 was purchased from American Type Culture Collection (ATCC, Manassas, VA, USA). 
The E.G7-OVA tumour cell line was derived from the C57BL/6 mouse lymphoma cell line EL4 [406] and purchased from American Type Culture Collection (access number: CRL2113, ATCC, Manassas, VA, USA). E.G7-OVA cells contain one copy of the pAC-neo-OVA plasmid and constitutively synthesise and secrete OVA.

The breast carcinoma line $4 \mathrm{~T} 1$ is a 6 -thioguanine resistant cell line selected from the 410.4 tumor without mutagen treatment [407]. The 4T1 tumour cells were obtained from ATCC (access number CRL-2539, ATCC, Manassas, VA, USA).

Tumour cell lines were stored in $90 \% \mathrm{FBS}, 10 \% \mathrm{DMSO}$ at $-80^{\circ} \mathrm{C}$. Before use in experiments, tumour cells were cultured in cIMDM to obtain cells in exponential growth. OVA expressing cell lines were cultured in the presence of $0.5 \mathrm{mg} / \mathrm{mL}$ Geneticin ${ }^{\circledR}$ selective antibiotic. Adherent cells were detached by $1 \mathrm{~min}$ trypsinisation at $37^{\circ} \mathrm{C}$. Proteolysis was stopped by addition of an equal volume of FBS and cells were washed 3 times in PBS before use.

\subsubsection{Mice}

\subsubsection{Maintenance and ethical approval}

All mice were bred and maintained on standard laboratory food and water ad libitum in the Biomedical Research Unit of the Malaghan Institute of Medical Research. All experimental protocols were approved by the Victoria University Animal Ethics Committee and all procedures were carried out in accordance with the guidelines of Victoria University of Wellington.

\subsubsection{Mouse strains}

C57BL/6 and BALB/cJ (BALB/c) breeding pairs were obtained from the Jackson Laboratories (Bar Harbour, ME, USA). 
B6.SJL-Ptprc ${ }^{\mathrm{a}} \mathrm{Pep} 3^{\mathrm{b}} /$ BoyJArc (B6) mice were created by backcrossing the inbred strain SJL, expressing the Ptprca gene (CD45.1), onto the C57BL/6 (CD45.2) background [408]. Therefore, cells from C57BL/6 and B6 congenic mice can be on the basis of CD45.1 and CD45.2 expression. Breeding pairs were obtained from the Animal Resources Centre (Canning Vale, WA, Australia).

OTI and OTII mice [409, 410] were obtained from Dr. Sarah Hook, School of Pharmacy, Dunedin, NZ, with the permission of Prof. Frank Carbone, Melbourne University, Australia. $\mathrm{T}$ cells from OTI and OTII mice express V $\alpha 2 \mathrm{~V} \beta 5.1 / 5.2$ TCRs specific for $\mathrm{OVA}_{257-264}$ presented on $\mathrm{H}_{2}-\mathrm{K}^{\mathrm{b}}$ and $\mathrm{OVA}_{323-339}$ presented on $\mathrm{I}^{\mathrm{A}} \mathrm{A}^{\mathrm{b}}$, respectively. OTII mice were tested for expression of $\mathrm{V} \alpha 2 \mathrm{~V} \beta 5.1 / 5.2$ TCRs on peripheral blood CD4 T cells by flow cytometry.

OT-I x B6.SJL-Ptprca mice and OT-II x B6.SJL-Ptprca (OTI congenic and OTII congenic mice) were bred by crossing OTI or OTII mice (CD45.2) with B6 congenic mice (CD45.1) at the Malaghan Institute of Medical Research, Wellington, New Zealand.

Foxp3GFP mice carry an in-frame insertion of the enhanced green fluorescent protein (eGFP) gene into the first coding exon of the Foxp3 gene [411]. These mice produce a fully functional Foxp3 protein fused to the eGFP protein. Breeding pairs were obtained from Prof. Alexander Y Rudensky, University of Washington (Seattle, Washington, USA).

V(D)J Recombination Activation Gene RAG-1 deficient mice (RAG1-/- mice) were purchased from the Walter and Eliza Hall Institute (Melbourne, Australia). Rag1-/- mice are defective in their ability to recombine the $\mathrm{T}$ cell receptor (TCR) and $\mathrm{B}$ cell receptor (BCR) and as a consequence have no mature T or B cells [412].

Mouse strains were maintained by mating between siblings. For all experiments mice were sex matched and 6-8 weeks old at the beginning of the experiments. 


\subsection{Methods}

\subsubsection{Cell culture}

All cells were cultured at $37{ }^{\circ} \mathrm{C}$ with $5 \% \mathrm{CO}_{2}$ and $95 \%$ humidity.

\subsubsection{Culture of tumour cell lines}

E.G7-OVA and B16.OVA cell lines were grown in cIMDM containing $0.5 \mathrm{mg} / \mathrm{mL}$ G418. B16F1 tumour cells were grown in cIMDM without G418. Non-adherent E.G7-OVA tumour cells were harvested by spinning down cultures. Adherent B16 melanoma cells were detached from the flask by incubation for $1 \mathrm{~min}$ at $37^{\circ} \mathrm{C}$ in $3 \mathrm{~mL}$ Trypsin/EDTA. Proteolysis was stopped by 3 -fold dilution in cIMDM and cells were, washed, counted and replated.

4T1 breast carcinoma cells were cultured in RPMI containing $10 \%$ FBS. Adherent cells were dissociated by incubation with $1 \mathrm{mM}$ EDTA for $10 \mathrm{~min}$ at $37^{\circ} \mathrm{C}$ and collected by centrifugation. Cells were then washed in pre-warmed medium and counted before reseeding.

All tumour cells were washed 3 times in PBS before injection into mice.

\subsubsection{Propagation of hybridomas for antibody production}

B cell hybridomas stored in liquid $\mathrm{N}_{2}$ were thawed, washed and pre-cultured in IMDM containing $20 \%$ FBS in $25 \mathrm{~mL}$ flasks. When cells had grown up to $30 \mathrm{x} 10^{6}$, they were transferred into the cell compartment of CellLine flasks and IMDM containing 1\% FBS was added to the nutrient compartment. Cells were harvested twice a week, pelleted by centrifugation and re-seeded. The supernatant was kept for antibody purification. Nutrient medium was exchanged once per week. 


\subsubsection{Generation of dendritic cells from bone marrow pre- cursors}

C57BL/6 mice were euthanised and hind legs were detached at the hips and collected in IMDM. The muscle and connective tissue was removed, knee joints were separated and the ends of femurs and tibias cut off to gain access to the BM. The BM was flushed out with IMDM into a $50 \mathrm{~mL}$ tube using a 25 -gauge needle attached to a $10 \mathrm{~mL}$ syringe. Cell clumps were disrupted by vigorous pipetting and cells were filtered through $70 \mu \mathrm{m}$ nylon gauze. Cells were pelleted by centrifugation at $320 \mathrm{~g}$ for $5 \mathrm{~min}$. Red blood cells were lysed by incubation in $10 \mathrm{~mL} \mathrm{ACT}$ buffer per mouse for $5 \mathrm{~min}$ at $37^{\circ} \mathrm{C}$; lysis was stopped by addition of an equal volume of IMDM. Cells were pelleted again and live cells were identified by trypan blue (GIBCO, Invitrogen, Auckland, NZ) exclusion and counted using a haemocytometer. Cells were pelleted, resuspended in cIMDM and plated in 6-well plates.

For GM-CSF/IL-4 derived BMDC, BM cells were plated at $2 \times 10^{6}$ cells/well containing $10 \mathrm{ng} / \mathrm{mL}$ GM-CSF and $20 \mathrm{ng} / \mathrm{mL}$ IL-4. Cells were cultured at $37^{\circ} \mathrm{C}$ for 7 days. Cells were supplemented with nutrients on day 3 and 5 by replacing $2 \mathrm{~mL}$ of medium containing GM-CSF and IL-4 to again give a final concentration of $10 \mathrm{ng} / \mathrm{mL}$ GM-CSF and $20 \mathrm{ng} / \mathrm{mL}$ IL-4.

To generate DCs from BM using the Flt3L culture system, BM cells were plated at $5 \times 10^{6}$ cells/well containing a pre-determined amount of Flt3L (see 2.1.3). Cells were supplemented with Flt3L growth factor and nutrients on day 3,6 and 9 by replacing $2 \mathrm{~mL}$ of medium containing Flt3L to give the same total concentration as on day 1.

To activate BMDCs, different adjuvants were added at the indicated concentrations in the medium replacement on day 6 (for GM-CSF/IL-4 derived BMDCs) or day 9 (for Flt3L derived BMDCs) and analysis was performed 20 hours later, unless otherwise stated. 


\subsubsection{Culture of mycobacteria}

M. smegmatis

M. smegmatis was cultured in sterile LB medium containing $0.1 \%$ Tween 80 . One frozen aliquot was thawed, inoculated into $30 \mathrm{~mL}$ medium and cultured over night at $37^{\circ} \mathrm{C}$ and $200 \mathrm{rpm}$. The culture was then split into two $400 \mathrm{~mL}$ cultures and again grown over night. M. smegmatis was pelleted by centrifugation at $3,115 \mathrm{~g}$ for $30 \mathrm{~min}$ at $4{ }^{\circ} \mathrm{C}$ and washed 3 times in PBS containing $0.05 \%$ Tween 80 . Single use aliquots were stored at $-80^{\circ} \mathrm{C}$.

$\mathrm{BCG}$

BCG was cultured in sterile Dubos Broth supplemented with $10 \%$ OADC. One frozen aliquot of BCG was inoculated into $10 \mathrm{~mL}$ medium. After one week bacteria reached the logarithmic growth phase and were split 1:10 into fresh medium. BCG was grown up again and harvested after 5-7 days by centrifugation at $3,115 \mathrm{~g}$ for $30 \mathrm{~min}$ at $4{ }^{\circ} \mathrm{C}$. BCG was washed 3 times in PBS containing $0.05 \%$ Tween 80 before aliquots were stored at $-80{ }^{\circ} \mathrm{C}$.

Enumeration of bacterial titer (CFU)

Sterility of samples was tested by culturing one aliquot on blood agar plates for 5 days. To enumerate colony forming units (CFU), bacteria were thawed, sonicated twice for $20 \mathrm{~s}$ and serial 10-fold dilutions were plated out. M. smegmatis was grown on LB agar plates for 1-2 days, whereas BCG was cultured on Middlebrook 7H11 agar plates for 21 days.

\subsubsection{Tissue processing}

\subsubsection{Blood}

Blood was collected either from the tail vein (live mice) or the heart (sacrificed mice). For tail vein bleeding, mice were warmed up with a lamp, put into restrainers and tail veins were nicked with a sterile scalpel blade. For cardiac puncture, a $1 \mathrm{~mL}$ syringe with a 25 gauge needle was inserted through the skin and ribcage to puncture the heart. 
Blood for flow cytometry analysis was collected in $1.7 \mathrm{~mL}$ micro tubes containing at least an equal volume of Alsever's Solution. The blood samples were centrifuged at $420 \mathrm{~g}$ for $2 \mathrm{~min}$ and the supernatant was discarded. Cell pellets were resuspended in $1 \mathrm{~mL}$ ACT buffer and incubated at $37^{\circ} \mathrm{C}$ for $10 \mathrm{~min}$ to lyse red blood cells. Cells were centrifuged again for $2 \mathrm{~min}$ at $420 \mathrm{~g}$ and resuspended in $1 \mathrm{~mL} \mathrm{FC} \mathrm{buffer.} \mathrm{If} \mathrm{large} \mathrm{amounts} \mathrm{of} \mathrm{red} \mathrm{blood} \mathrm{cells} \mathrm{were}$ still visible, a second lysis step with $\mathrm{dH}_{2} \mathrm{O}$ was performed. Cell pellets were resuspended in

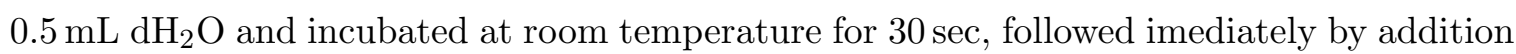
of $0.5 \mathrm{~mL} 1.8 \% \mathrm{NaCl}$. Cells were washed twice in $\mathrm{FC}$ buffer and stored on ice until fluorescent labelling.

Blood for serum analysis was collected into empty $1.7 \mathrm{~mL}$ micro tubes. Blood samples were left at $4{ }^{\circ} \mathrm{C}$ over night to clot. Cells were pelleted by centrifugation at $420 \mathrm{~g}$ for $2 \mathrm{~min}$. The supernatant was transferred to fresh $1.7 \mathrm{~mL}$ micro tubes and clarified by centrifugation at $14,000 \mathrm{~g}$ for $20 \mathrm{~min}$. Serum samples were stored in $50 \mu \mathrm{L}$ aliquots in $0.6 \mathrm{~mL}$ micro tubes at $-20^{\circ} \mathrm{C}$ until further analysis.

\subsubsection{Lymph nodes}

LNs were collected from sacrificed mice into 24-well plates containing IMDM. To release DCs, LNs were broken up using 18 gauge needles and then digested enzymatically in IMDM containing $0.1 \mathrm{mg} / \mathrm{mL}$ Liberase TL and $100 \mathrm{\mu g} / \mathrm{mL}$ DNase for $25 \mathrm{~min}$ at $37^{\circ} \mathrm{C}$. Digestion was stopped by addition of EDTA to a final concentration of $10 \mathrm{mM}$. Subsequently, LNs were pressed through $70 \mu \mathrm{m}$ cell strainers using the plunger of a $1 \mathrm{~mL}$ syringe. Cells were washed with either IMDM, FC buffer or Wuerzburger buffer, depending on their further use. If the release of DCs was not required, LNs were directly processed into single-cell suspensions by pressing through cell strainers without digestion. Cells were stored on ice until further use. 


\subsubsection{Spleens}

Spleens were collected into 24-well plates containing IMDM. To release DCs, spleens were injected with $2 \mathrm{~mL}$ of digestion solution containing $0.1 \mathrm{mg} / \mathrm{mL}$ Liberase TL and $100 \mu \mathrm{g} / \mathrm{mL}$ DNase in IMDM. Spleens were digested for $25 \mathrm{~min}$ at $37^{\circ} \mathrm{C}$. Digestion was stopped by addition of EDTA to a final concentration of $10 \mathrm{mM}$. Spleens were then processed into single-cell suspensions by pressing through a $70 \mu \mathrm{m}$ cell strainer using the plunger of a $1 \mathrm{~mL}$ syringe. Cells were pelleted by centrifugation at $400 \mathrm{~g}$ for $5 \mathrm{~min}$ at $4{ }^{\circ} \mathrm{C}$. The cell pellet was resuspended in $10 \mathrm{~mL} \mathrm{ACT} \mathrm{buffer} \mathrm{and} \mathrm{incubated} \mathrm{at} 37^{\circ} \mathrm{C}$ for $10 \mathrm{~min}$ to lyse red blood cells. $5 \mathrm{~mL}$ of IMDM were added and spleen cells centrifuged again. Subsequently, spleen cells were washed in the appropriate buffer of medium for each experiment and stored on ice until further use.

\subsubsection{Lungs}

Lungs were taken out onto paper towels, kept moist with HBSS and photographed for general appearance. After weighing, lungs were placed into 6-well plates containing $5 \mathrm{~mL}$ HBSS, washed in fresh HBSS and minced with a sterile scalpel blade. Lung tissue was digested in HBSS containing $1 \mathrm{mg} / \mathrm{mL}$ Collagenase IV and $1.32 \mathrm{mg} / \mathrm{mL}$ Elastase for $75 \mathrm{~min}$ at $37^{\circ} \mathrm{C}$ shaking at $150 \mathrm{rpm}$. Digested lungs were filtered through $70 \mu \mathrm{m}$ cell strainers using the tip of a $10 \mathrm{~mL}$ pipette to strain through remaining tissue. Cells were washed 3-times in HBSS, pelleted at $400 \mathrm{~g}$ for $5 \mathrm{~min}$ at $4^{\circ} \mathrm{C}$ and stored on ice.

\subsubsection{Tumours}

For flow cytometry analysis and cell culture, tumours were collected into 6-well plates containing IMDM and broken up with tweezers. Tumours were digested in IMDM containing $0.1 \mathrm{mg} / \mathrm{mL}$ Liberase TL and $100 \mathrm{\mu g} / \mathrm{mL}$ DNase for $25 \mathrm{~min}$ at $37^{\circ} \mathrm{C}$. Digestion was stopped by addition of EDTA to a final concentration of $10 \mathrm{mM}$. Tumours were then processed into single-cell suspensions by pressing through $70 \mu \mathrm{m}$ cell strainers using the plunger of a $1 \mathrm{~mL}$ 
syringe. Cells were pelleted by centrifugation at $320 \mathrm{~g}$ for $10 \mathrm{~min}$ at $4{ }^{\circ} \mathrm{C}$. The cells were washed once in FC buffer or IMDM, depending on the experiment. Cells were stored on ice.

For extraction of RNA the NucleoSpin RNA L kit (Macherey-Nagel, Düren, Germany) was used according to the manufacturer's instructions, with the exception that $15 \mathrm{~mL}$ tubes were centrifuged at $800 \mathrm{~g}$ instead of $4,500 \mathrm{~g}$ due to the properties of the centrifuge. Briefly, tumours were placed into lysis buffer containing 2-ME, weighed and processed using a tissue homogeniser. Tumour lysate was filtered and transferred to $1.7 \mathrm{~mL}$ tubes to allow clarification by spinning at 4,500 $\mathrm{g}$ for $10 \mathrm{~min}$ at room temperature. Subsequently, $70 \%$ ethanol was added and lysates were loaded onto silica columns. Columns were washed, DNA was digested and columns were washed again. RNA was eluted in water and stored at $-80^{\circ} \mathrm{C}$.

For analysis of the total tumour protein, tumours were snap frozen in liquid $\mathrm{N}_{2}$ and ground with a mortar and pestle on dry ice. The powder was transferred into $1.7 \mathrm{~mL}$ tubes containing proteinase inhibition buffer. The samples were vortexed for $2 \mathrm{~min}$ and clarified by centrifugation at $14,000 \mathrm{~g}$ for $10 \mathrm{~min}$ at $4{ }^{\circ} \mathrm{C}$. The protein protein content was determined using the BCA kit (Thermo Scientific, Portsmouth, NH, USA) and samples were stored in aliquots at $-20^{\circ} \mathrm{C}$.

\subsubsection{Flow cytometry}

\subsubsection{Purification and labelling of antibodies}

Supernatants from B-cell hybridomas were spun down at $800 \mathrm{~g}$ and filtered to $0.2 \mu \mathrm{m}$ low protein binding filters. Antibodies were purified over a protein G-Sepharose column (Pharmacia Biotech, Uppsala, Sweden) using low pressure chromatography. Briefly, the system was cleaned with ethanol, water and wash buffer before the sample was loaded. The column was then washed with wash buffer. Elution buffer was run over the column to elute bound antibody into tubes prepared with 1/40 volume of neutralising buffer. Antibody concentration was assessed by spectophotometry as absorption at $280 \mathrm{~nm}$. All fractions containing $>0.3 \mathrm{mg} / \mathrm{mL}$ antibody were combined and dialysed over night at $4{ }^{\circ} \mathrm{C}$ in PBS using dialysis 
cassettes. The final protein concentration was determined and antibody samples were stored at $-80^{\circ} \mathrm{C}$ until use.

For labelling with biotin, FITC or Alexa dyes, $0.5 \mathrm{mg}$ antibody was centrifuged at 10,000 $\mathrm{g}$ for $30 \mathrm{~min}$ at $4{ }^{\circ} \mathrm{C}$ to remove particles. Subsequently, the antibody was transferred to a vivaspin (10,000 MWCO) column and washed twice in sodium bicarbonate buffer. Then the antibody transferred into a $1.7 \mathrm{~mL}$ tube at $2.5 \mathrm{mg} / \mathrm{mL}$ bicarbonate buffer. Per $100 \mu \mathrm{g}$ antibody $10 \mu \mathrm{g}$ N -Hydroxysulfosuccinimide-biotin (Thermo Scientific, Portsmouth, NH, USA), FITC or Alexa dye (Molecular Probes, Invitrogen, Auckland, NZ) were added while vortexing and the mixture was incubated for $8 \mathrm{~min}$ at room temperature. Following the labelling, the antibody was transferred back to the same vivaspin column and washed once with Tris buffer and twice with PBS. Azide was added at $0.1 \%$ and the labelled antibody was stored at $4{ }^{\circ} \mathrm{C}$.

\subsubsection{Staining of surface markers}

Single cell suspensions were counted and washed once in FC buffer. For staining, 1-3 x 10 cells were dispensed into a 96-well plate. Cells were pelleted by centrifugation at $320 \mathrm{~g}$ for 2 min and supernatants were removed by flicking the plate once. Pellets were resuspended by vortexing the plate at low speed. If fixation of the cells was required before flow cytometry acquisition, staining with live/dead fixable blue was performed by incubating the cells for $15 \mathrm{~min}$ in a 1:500 dilution of the stock solution on ice. Cells were then pelleted and resuspended again. To block unspecific binding by Fc $\gamma$ Receptors II and III, cells were incubated in FC buffer containing $2.4 \mathrm{G} 2$ antibody at a predetermined saturating concentration for $10 \mathrm{~min}$ on ice. Fluorescently or biotin-labelled antibodies against the surface markers of interest were then added at the appropriate dilutions and the cells were incubated a further 10 min to 15 min on ice. Cells were washed once in FC buffer and then incubated with a fluorochrome-conjugated streptavidin at the appropriate concentration for $10 \mathrm{~min}$ on ice, where required. After labelling, cells were washed twice in FC buffer. Cells were resuspended in $200 \mu \mathrm{L}$ FC buffer and kept on ice. Directly before analysis by flow cytometry, the cell viability dye DAPI was added at final concentration of $0.003 \mu \mathrm{g} / \mathrm{mL}$. 


\subsubsection{Intracellular staining}

Cells were labelled with antibodies against surface markers as described in 2.2.3.2. After washing in FC buffer, cells were fixed in BD Cytofix/Cytoperm solution for 20 min at $4{ }^{\circ} \mathrm{C}$. Cells were then washed twice in BD Perm/Wash buffer and incubated in this buffer for $15 \mathrm{~min}$. Cells were pelleted, resuspended and stained for $30 \mathrm{~min}$ on ice with the appropriate dilutions of antibodies for intracellular markers or isotype controls. Cells were washed twice in Perm/Wash buffer and incubated another $30 \mathrm{~min}$ in Perm/Wash buffer to reduce background staining. Cells were washed once in FC buffer, resuspended in FC buffer and stored on ice for up to $2 \mathrm{~h}$ or at $4{ }^{\circ} \mathrm{C}$ over night before flow cytometry analysis.

\subsubsection{Acquisition and analysis}

Data was acquired using a FACScalibur or a LSRII SORP flow cytometer (Beckton-Dickinson, San Jose, CA, USA) and analysed with FlowJo version 9.3.1 software (Treestar Inc., CA, USA). For LSRII SORP instrument details, please see Appendix A. Live cells were identified based on their forward scatter (FSC) and side scatter (SSC) properties as well as on their ability to exclude the viability dyes live/dead fixable blue or DAPI. Unlabelled cells were used to adjust channel voltage settings. Cells labelled with a single antibody or dye and antibody-labelled CompBeads were used to compensate for spectral overlap between fluorophores. CompBeads were labelled for $10 \mathrm{~min}$ at room temperature in FC buffer containing the same antibody used in the experiment at a 1:10 dilution respective to the concentration used for cell labelling. Unbound antibody was diluted by excess buffer and labelled CompBeads were stored up to two weeks at $4{ }^{\circ} \mathrm{C}$. In some experiments, matched isotype control antibodies were used to control for background fluorescence due to nonspecific antibody binding. Where combination of many different fluorophores caused a rise in background staining or positively stained populations were hard to identify, fluorescence minus one (FMO) controls were used to determine gate positions. 


\subsubsection{Gene expression analysis with real-time PCR}

\subsubsection{Assessment of RNA quality}

RNA concentration and quality was determined on a ND-100 spectrophotometer by absorption at $230,260,280$ and $340 \mathrm{~nm}$. The 260:280 ratio was generally $>2$ and the 260:230 ratio was $>2.2$, indicating that there was little contamination with DNA or protein, respectively. Absorption at 340 was zero.

A sample of $2 \mu \mathrm{g}$ of RNA, as determined spectrophotometrically, were also run on a $2 \%$ agarose gel. Agarose was dissolved in 1x TAE buffer by heating in a microwave and SYBRSafe was added to a $1 \mathrm{x}$ concentration to label nucleic acids. RNA samples were mixed with BlueJuice $^{\text {TM }}$ loading dye (Invitrogen, Auckland, NZ) and loaded onto the gel. A 1 kbp DNA ladder (Invitrogen, Auckland, NZ) was included as a size marker. Gels were submerged in $1 \mathrm{x}$ TAE and electrophoresis was performed at $100 \mathrm{~V}$ for $45 \mathrm{~min}$. RNA was imaged in UVlight using a Biorad Geldoc unit.

\subsubsection{DNase digestion}

Samples that had a 260:280 ratio $<2$ as measured spectrophotometrically or showed bands additional to the expected $28 \mathrm{~S}$ and $18 \mathrm{~S}$ ribosomal RNA on the agarose gel were purified by DNase digestion using the Ambion ${ }^{\circledR}$ DNA-free ${ }^{\text {TM }}$ kit (Invitrogen, Auckland, NZ). Briefly, buffer and DNase were added to the samples and DNA was digested at $37^{\circ} \mathrm{C}$ for $30 \mathrm{~min}$. Subsequently, DNase inactivation agent was added, mixed and spun down. The RNAcontaining supernatant was transferred to fresh tubes and analysed again for RNA quality and quantity using spectophotometry and agarose gels.

\subsubsection{Reverse-Transcription}

The Superscript Vilo kit (Invitrogen, Auckland, NZ) was used according to the manufacturer's instructions to synthesise cDNA. Appropriate amounts of reaction mix and enzyme 
were combined with $10 \mu \mathrm{g}$ of RNA in PCR 8-strips. Controls with DEPC water instead of enzyme were also included. Reverse transcription was performed for $60 \mathrm{~min}$ at $42^{\circ} \mathrm{C}$, followed by enzyme inactivation at $85^{\circ} \mathrm{C}$. cDNA samples were stored at $-80^{\circ} \mathrm{C}$.

\subsubsection{Real-time PCR}

The Taqman 96-well plate inflammatory mouse array, as well as individual taqman assays were used in conjunction with the Taqman Expression Master Mix for real-time PCR (all from Applied Biosystems, Foster City, CA, USA). For the plate-based array, samples corresponding to $20 \mathrm{ng}$ to $40 \mathrm{ng}$ RNA were placed into the wells that already contained the lyophilised primers and probes. DEPC water and master mix were added and the plates were sealed with optical adhesive covers. For individual Taqman assays, stock solutions of the lyophilised primers and probes were prepared and stored at $-20^{\circ} \mathrm{C}$. Primers/probes were combined with the master mix and distributed in 96-well PCR plates. $20 \mathrm{ng}$ to $40 \mathrm{ng}$ RNA and DEPC water were added to a final volume of $25 \mu \mathrm{L}$. Non-reverse-transcribed and water controls were also included. Real-time PCR was run on an Applied Biosystems 7500 system. Samples were incubated for $2 \mathrm{~min}$ at $50^{\circ} \mathrm{C}$, followed by $15 \mathrm{~min}$ at $95^{\circ} \mathrm{C}$ and 40 cycles of $15 \mathrm{~s}$ at $95^{\circ} \mathrm{C}$ and $1 \mathrm{~min}$ at $60^{\circ} \mathrm{C}$. Cycle threshold (CT) values were determined automatically and $\Delta \Delta \mathrm{CT}$ values (normalised to $18 \mathrm{~S}$, compared to saline treated controls) were calculated for all samples.

Table 2.4: Real-time PCR assays

\begin{tabular}{|l|l|}
\hline Target & Assay \\
\hline multiple (96) & mouse immune response array 4414079 \\
\hline 18S ribosomal RNA & Mm03928990_g1 \\
\hline CSF2 (GM-CSF) & Mm00438328_m1 \\
\hline CSF3 (G-CSF) & Mm00438334_m1 \\
\hline IL-6 & Mm00446190_m1 \\
\hline IL-10 & Mm00439616_m1 \\
\hline NOS2 (iNOS) & Mm00440485_m1 \\
\hline CXCL-10 & Mm00445235_m1 \\
\hline
\end{tabular}




\subsubsection{Cell purification}

\subsubsection{Magnetic cell separation (MACS)}

Magnetic cell separation was performed using MACS Microbeads (Miltenyi Biotec, Germany) according to the manufacturer's instructions. Single cell suspensions were resuspended in $9 \mu \mathrm{L}$ Wuerzburger buffer per $10^{6}$ cells and filtered through $70 \mu \mathrm{m}$ cell strainers to remove cell clumps. Unspecific binding to Fc $\gamma$ Receptors II and III was blocked by incubation with 2.4G2 for 10 min on ice. For direct labelling, $1 \mu \mathrm{L}$ MACS Microbeads per $10^{6}$ cells was added and cells were incubated for 15 min at $4{ }^{\circ} \mathrm{C}$ with slow rotation. For indirect labelling, cells were first stained with the appropriate dilutions of biotin-labelled antibodies, washed once in FC buffer and then resuspended in $10 \mu \mathrm{L}$ Wuerzburger buffer containing $1 \mu \mathrm{L}$ Streptavidin MACS Microbeads per $10^{6}$ cells. Labelling was also performed for $15 \mathrm{~min}$ at $4{ }^{\circ} \mathrm{C}$ with slow rotation. Unbound Microbeads were removed by adding an excess of Wuerburger buffer and pelleting the cells at $320 \mathrm{~g}$ for $4 \mathrm{~min}$. Cells were resuspended at $10^{8}$ cells/mL for magnetic separation on the AutoMACS instrument (Miltenyi Biotec).

\subsubsection{OTI and OTII T cell purification from lymph nodes and spleens}

Single cell suspensions were prepared from LNs and spleens without enzymatic digestion (see 2.2.2.2 \& 2.2.2.3). T cells were isolated by depletion of other cell types. The cell suspension was incubated with a cocktail of biotinylated antibodies for Ter119, CD19, B220, NK1.1, CD11b, CD11c, GR-1, CD49b and MHC II, followed by Streptavidin-MACS Microbeads (Miltenyi Biotec, see 2.2.5.1). T cells were collected from the negative fraction after separation on the deplete programme on the AutoMACS. Cell purity was assessed by flow cytometry for CD4, CD 8 and appropriate TCR $\alpha \beta$ chains. Purity of combined CD4 T and CD8 T cells was greater than $90 \%$. 


\subsubsection{T cell purification from spleens of adjuvant treated mice}

Single cell suspensions were prepared from spleens without enzymatic digestion (see 2.2.2.3). T cells were isolated by direct labelling with anti-CD8 $\alpha$ (OTI) or anti-CD4 (OTII) MACS Microbeads (Miltenyi Biotec, see 2.2.5.1). T cells were collected from the positive fraction after separation on the possel programme on the AutoMACS. Cell purity was assessed by flow cytometry for CD4, CD8 and appropriate $\mathrm{TCR} \alpha \beta$ chains. $\mathrm{CD} 4 \mathrm{~T}$ cell purity was generally greater than $85 \%$ and CD8 T cell purity was greater than $80 \%$.

\subsubsection{Tumour models}

\subsubsection{Tumour challenge and growth monitoring}

Tumour cells grown in vitro were harvested during their exponential growth phase and washed thrice in PBS. B16F1, B16F10 or B16.OVA tumour cells were inoculated into the flank of C57BL/6 mice by subcutaneous (s.c.) injection of $10^{5}$ cells. EL4 and E.G7-OVA cells were inoculated by s.c. injection of $5 \times 10^{5}$ cells into the flank of C57BL/6 mice. 4T1 breast cancer cells were injected into the mammary fat pad of female BALB/c mice at $10^{4}$ cells/mouse. Tumour growth was monitored at least three times a week by palpation for very small tumours and measuring the bisecting diameters using Mitutoyo callipers for tumours $>3 \mathrm{~mm} .1 \mathrm{~mm}$ was substracted from each measurement to account for skin thickness. Mice were euthanised when tumour size exceeded $150 \mathrm{~mm}^{2}$.

\subsubsection{Adjuvant treatment}

Adjuvant treatment was administered by peritumoral injection in a maximal volume of $100 \mu \mathrm{L}$ PBS for tumours on the flank and $50 \mu \mathrm{L}$ PBS for tumours in the mammary fat pad. For tumours $>25 \mathrm{~mm}^{2}$, adjuvants were injected on 3 sites around the tumour. Doses were $2 \times 10^{6}$ CFU live BCG, $20 \mu g$ CpG, $20 \mu g$ LPS, $4 \times 10^{6}$ CFU live M. smegmatis, $500 \mu g$ MSU 
crystals, $2 \times 10^{6} \mathrm{CFU}$ live M. smegmatis $+250 \mu \mathrm{g}$ MSU crystals or $50 \mu \mathrm{g}$ Poly IC, unless otherwise stated. All adjuvants were diluted freshly in PBS before injection. Bacteria and MSU crystals were sonicated thrice for $10 \mathrm{sec}$ before usage. All combination treatments were given in separate injections of the two adjuvants, mainly to avoid clumping of MSU crystals with mycobacteria. Adjuvant treatment was generally administered after the appearance of palpable tumours every second day for 4 times total. In some experiments, different treatment schedules or dosing was used as stated in the figure legends.

\subsubsection{Adoptive cell transfer}

$\mathrm{T}$ cells were purified from either TCR transgenic mice or adjuvant treated tumour bearing mice as described in 2.2.5.2 \& 2.2.5.3. In some instances, cells were labelled with CFSE to assess in vivo proliferation (see 2.2.7.2). Cells were washed thrice in IMDM and injected intra-venously (i.v.) into the lateral tail vein of the recipient mice.

\subsubsection{Antibody Depletion of Cells}

CD4 T cells, CD8 T cells, NK/NKT cells and Tregs were depleted by intra-peritoneal (i.p.) injection of purified GK1.5, 2.43, PK136 or PC61 antibody, respectively. On day -1 and 0 with respect to the tumour inoculation, $200 \mu \mathrm{g}$ GK1.5, 2.43 or PK136 or $100 \mu \mathrm{g}$ PC61 were injected. Tumours were inoculated at day 0 and adjuvant treatment was performed from day 8 to day 14. Cell depletion was assessed by flow cytometry staining of the blood on days 0,7 and 15. CD4 T cells were $98 \%$ depleted, CD8 T cells were $99 \%$ depleted, NK1.1+ cells were $97 \%$ depleted and Tregs were $80 \%$ depleted on each of these time points.

\subsubsection{Metastasis assay}

Lungs from 4T1 tumour bearing mice were harvested and processed into single cell suspensions as described in 2.2.2.4. Cells were resuspended in RPMI containing (100 U/mLPenicillin and $100 \mu \mathrm{g} / \mathrm{mL}$ Streptomycin (GIBCO, Invitrogen, Auckland, NZ), 55 MM 2-ME, $10 \%$ FBS 
and $10 \mu \mathrm{g} / \mathrm{mL}$ 6-thioguanine. Lung cells were then seeded at 10-fold, 100-fold and 1000-fold dilutions into $10 \mathrm{~cm}$ tissue culture plates and incubated at $37{ }^{\circ} \mathrm{C} 5 \% \mathrm{CO}_{2}$ for 14 days. Following incubation, culture medium was discarded and $4 \mathrm{~T} 1$ colonies were fixed for $5 \mathrm{~min}$ in methanol. Cells were washed in $\mathrm{ddH}_{2} \mathrm{O}$, stained with $0.03 \%$ methylene blue solution for 5 min and washed again in $\mathrm{ddH}_{2} \mathrm{O}$. Plates were allowed to air dry before photographs were taken and blue $4 \mathrm{~T} 1$ colonies were counted.

\subsubsection{Assays of cell function}

\subsubsection{Cytokine production}

Cytokine production was either analysed by intracellular staining or multiplex bead assay of culture supernatant or serum. For intracellular staining, cells were isolated, plated in cIMDM containing $1 \mathrm{\mu g} / \mathrm{mL}$ GolgiStop (BD, San Diego, CA, USA) and/or Brefeldin A (eBioscience, San Diego, CA, USA) and incubated for $3-6$ hours at $37^{\circ} \mathrm{C}, 5 \% \mathrm{CO}_{2}$. Subsequently, cells were harvested by pipetting and stained for intracellular cytokines as described in 2.2.3.3.

For cytokine detection in culture supernatant or serum, multiplex polystyrene-bead kits from Invitrogen (Auckland, NZ), Biorad (Hercules, CA, USA) and Millipore (Merck Millipore Corporation, Billerica, MA, USA) were used as per the manufacturer's instructions. Briefly, supernatant was clarified by centrifugation at 1,000 $\mathrm{g}$ for $10 \mathrm{~min}$. Samples and reconstituted standards were added to pre-wet 96-well filter plates (Merck Millipore Corporation, Billerica, MA, USA) and incubated with a mixture of cytokine detection beads under agitation. Plates were washed and flow-through removed using a vacuum manifold (Biorad, Hercules, CA, USA). Appropriate biotinylated detection antibodies for the assayed cytokines were added and the incubated with the samples on a plate shaker. Subsequently, samples were stained with Streptavidin-PE, washed thrice and resuspended by vigorous shaking. Samples were then transferred into flat-bottom 96 -well plates and read on a Bio-Plex ${ }^{\text {TM }}$ system (Biorad, Hercules, CA, USA). Cytokine concentrations were calculated against the commercial standards using the provided software (Bio-plex ${ }^{\mathrm{TM}}$ manager software, Biorad, 
Hercules, CA, USA).

\subsubsection{T cell proliferation in vivo}

OTI or OTII T cells were enriched from LNs and spleens of OTI and OTII congenic mice by MACS as described in 2.2.5.2. T cells were resuspended in PBS at $5 \times 10^{6}$ cells $/ \mathrm{mL}$. CFSE was added at a final concentration of $1 \mu \mathrm{M}$; cells were immediately vortexed and incubated for $10 \mathrm{~min}$ at $37^{\circ} \mathrm{C}$. Equal volumes of FBS and IMDM were added to stop the CFSE staining reaction.

T cells were washed thrice in IMDM, counted and resuspended in IMDM at 5 to $10 \times 10^{6}$ cells $/ \mathrm{mL} .1-2 \times 10^{6}$ cells were injected i.v. into the lateral tail vein of C57BL/6 mice in a total volume of $200 \mu \mathrm{L}$. 3-5 days later, tumour-draining LNs were harvested, processed into single-cell suspensions (see 2.2.2.2) and analysed for $\mathrm{T}$ cell proliferation by flow cytometry (see 2.2.3).

\subsubsection{Data analysis}

\subsubsection{Software used for data analysis}

FlowJo version 9.3.1 software (Treestar Inc., CA, USA) was used to analyse flow cytometry data and to create flow cytometry plots and tables with percentages of gated cells. Microsoft Excel 2008 for Mac OS (Microsoft Corporation, Washington, USA) and GraphPad Prism version 5.00 for Mac OS X (GraphPad Software, San Diego California, USA) were used to create tables and graphs and for statistical analysis.

\subsubsection{Calculation of cell numbers}

Viable cells were identified in single cell suspensions from processed organs by trypanblue (GIBCO, Invitrogen, Auckland, NZ) exclusion and counted using a haemocytometer. Numbers of specific cell populations were calculated using the percentage of the cell type 
of interest among live cells obtained by flow cytometry analysis and the live cell counts obtained by trypan-blue exclusion.

\subsubsection{Statistical calculations}

Data was tested for normality using D'Agostino and Pearson's omnibus test. The results showed that tumour sizes in control and adjuvant treated groups as well as percentages of tumour-infiltrating immune cells and percentages of different cell types in LNs were consistent with a Gaussian distribution (see Appendix B). If experimental data was from similar experiments to those found to be consistent with Gaussian distribution, multiple groups were compared using one-way Analysis of Variance (ANOVA) with Tukey's post test. To compare two parameters across multiple groups, two-way ANOVA with Bonferroni's post test was used. If data was not consistent with Gaussian distribution or normality could not be tested due to small samples sizes or few repeats, multiple groups were compared using the Kruskal-Wallis test with Dunn's post test that does not assume normal distribution of the data. Tukey's post test, Bonferroni's post test and Dunn's post test all correct for errors introduced by comparing multiple groups. Two samples were compared using the nonparametric two-tailed Mann-Whitney test. Survival analysis was done using the log-rank (Mantel-Cox) test with Bonferroni's correction for multiple comparisons, where applicable. Mean and standard deviation (SD) or standard error (SE) is reported in graphs. 



\title{
Chapter 3
}

\section{Identification of adjuvants for the activation of tumour-infiltrating}

\author{
DCs
}




\subsection{Introduction}

DCs are crucial for the initiation of immune responses. Activated DCs are the most potent inducers of $\mathrm{T}$ cell priming and are thought to be vital in shaping the immune response. Insufficiently activated DCs, however, present antigen without the necessary co-stimulatory signals and cytokines, thereby promoting $\mathrm{T}$ cell tolerance. Tumours frequently secrete factors that inhibit DC activation, thus inducing them to be tolerogenic and leading to inhibition of the anti-tumour immune response $[222,324,326]$.

When DCs are fully activated, they increase their surface levels of MHC II molecules with bound peptide antigen for presentation. In addition, they up-regulate surface expression of co-stimulatory molecules, such as CD80, CD86 and CD40 [261, 271]. DCs can then be licensed to activate CD8 $\mathrm{T}$ cells by classic CD4 $\mathrm{T}$ cell help or alternatively by NK and NKT cell help, via CD40-CD40Ligand interaction [273, 413, 414]. This induces DCs to produce IL-12, a cytokine thought to be required as a third signal (besides antigen recognition and co-stimulation) to prime CD8 T cells [274, 275]. Furthermore, activated DCs also produce a range of pro-inflammatory cytokines, including IL-6 and TNF $\alpha$, as well as chemokines, such as CCL3, CCL4 and CCL17 to attract naïve T cells [414, 415].

During the initiation of an immune response to an infectious agent, DCs become activated as they recognise conserved structures on pathogens (PAMPs) via a range of specialised receptors as discussed in detail in Chapter 1.4. In addition to activating DCs, PAMPs also confer information to the DCs with respect to which type of immune response (Th1, Th2, Th17) is needed [341, 371, 373, 374]. Furthermore, DCs also integrate signals of multiple PAMPs and combination of intracellulary and extracellularly recognised TLRs has been shown to synergistically enhance IL-12 production from DCs [377]. In addition to microbial signals, DCs also sense tissue damage through endogenous danger signals such as ATP and MSU, which also results in DC activation [63, 64].

In vaccination, adjuvants that either contain or mimic PAMPs have long been used to confer activation signals to DCs. Traditionally, mycobacteria that express PAMPs recognised by multiple DC receptors have been included in complete Freund's adjuvant [327, 328]. More recently, several ligands for TLRs and other receptors, as well as endogenous dan- 
ger signals have been identified, some of which are currently in clinical trials as adjuvants $[64,416]$.

Different types of DCs vary in their expression of pathogen-recognition receptors and therefore in their response to specific pathogens [291]. To study the effects of TLRs and other adjuvants on DCs in detail, culture systems to develop DCs from bone-marrow precursors (BMDCs) have been established. The cytokines GM-CSF and IL-4 are frequently used to induce DC differentiation [417] and the resulting GM-CSF/IL-4 BMDCs display phenotypic and functional characteristics similar to DCs arising during GM-CSF secretion in inflammation in vivo [306]. However, GM-CSF is not necessary for generation of DCs in the steady-state in vivo [418]. Rather, Fms-like tyrosine kinase-3 ligand (Flt3L) is required for the formation of DCs from bone-marrow precursors in the absence of inflammation [419]. An in vitro culture system to generate BMDC using Flt3L, developed by Brasel et al., yields three DC subsets equivalent to plasmacytoid DCs (pDCs), CD8- and CD8+ cDCs in vivo $[420-422]$.

In a successful anti-tumour immune response, NK cells and cytotoxic T cells (CTLs) can eradicate tumour cells. IL-12 and a strong Th1 bias are required for the induction of CTLs [274]. In addition, a Th1 environment favours production of IFN- $\gamma$ by NK cells and T cells, which is directly toxic for some tumours and also increases the sensitivity of tumour cells to $\mathrm{T}$ cell killing $[21,24,89,90]$. Therefore, adjuvants that induce strong Th1 responses may be useful in activating strong anti-tumour immunity. Combination of intracellulary and extracellularly recognised adjuvants or microbial and endogenous danger signals may be superior to individual adjuvants. 


\subsection{Aims}

The purpose of this chapter was to identify adjuvants for the activation of DCs in tumours. The hypothesis was that adjuvants that strongly activated DCs in vitro to up-regulate antigen presentation, express co-stimulatory molecules and secrete Th1-promoting cytokines may also activate DCs in tumours and thereby induce potent anti-tumour immunity. In addition to analysing the activation of DCs by adjuvants in vitro, it was necessary to analyse DC infiltration and activation status in tumours to evaluate whether these DCs may be activated by adjuvant administration to induce anti-tumour immunity.

The specific aims were:

- To identify adjuvants that could activate BMDCs resembling inflammatory and steadystate DCs in vitro and induce them to produce Th1-promoting cytokines

- To assess whether combinations of adjuvants were superior to individual adjuvants in activating DCs

- To investigate whether murine tumours were infiltrated by DCs that may be targeted by adjuvant administration to induce anti-tumour immunity

- To assess whether DCs from murine tumours could be activated by exposure to adjuvants ex vivo 


\subsection{Results}

\subsubsection{Different adjuvants induce varying degrees of activation of GM-CSF/IL-4 BMDCs}

To study the activation of DCs by adjuvants and assess their production of Th1-promoting cytokines, initial experiments were done in vitro. For the first set of experiments, DCs were generated from murine bone marrow by culture with GM-CSF and IL-4 as described in Chapter 2.2.1. This well established culture system leads to differentiation of GM-CSF/IL-4 BMDCs that have similar characteristics to DCs arising during inflammation in vivo [306, 417].

To determine the best time point to assess activation of GM-CSF/IL-4 BMDCs, a time course was done. DCs were incubated with adjuvants and $6 \mathrm{~h}, 20 \mathrm{~h}$ and $30 \mathrm{~h}$ later, surface levels of the antigen-presentation molecule MHC II and the co-stimulatory molecules CD40 and CD86 was analysed by flow cytometry. The majority of cells was identified as live by DAPI exclusion and expressed the typical GM-CSF/IL-4 BMDCs markers CD11c and CD11b (Figure 3.1A). LPS activation of DCs for $20 \mathrm{~h}$ to $24 \mathrm{~h}$ is known to result in substantial up-regulation of surface markers and higher levels of cytokine production compared to shorter stimulation and therefore the effect of LPS was only analysed at this time point [423].

MHC II expression was up-regulated in M. smegmatis and MSU + M. smegmatis DCs already after $6 \mathrm{~h}$ (Figure $3.1 \mathrm{~B}$, right panel). However, CD40 and CD86 reached their highest expression levels only after $20 \mathrm{~h}$ and were not further enhanced at $30 \mathrm{~h}$ (Figure $3.1 \mathrm{~B}$, left $\&$ middle panels). At $20 \mathrm{~h}$, the extent of activation marker up-regulation was similar for M. smegmatis, MSU + M. smegmatis and LPS stimulated DCs (Figure $3.1 \mathrm{~B}$ ). Although MSU has been reported to activate murine BMDCs [64], only a very slight increase in activation marker expression was observed with the tested concentrations of this MSU preparation (Figure $3.1 \mathrm{~B}$ ). Overall, stimulation of GM-CSF/IL-4 BMDCs for $20 \mathrm{~h}$ appeared to result in the highest levels of activation marker expression and therefore this time point was chosen for further experiments. 
Next, the ability of a range of adjuvants to activate GM-CSF/IL-4 BMDCs was compared. In addition to the well-known DC stimulator LPS, the endogenous danger signal MSU, M. smegmatis as a live Th1-inducing bacterium with multiple PAMPs and the viral RNAmimic Poly IC were analysed. Furthermore, several combinations of these adjuvants were also tested.

In all adjuvant-treated DC cultures, the number of viable cells recovered was slightly decreased compared to unstimulated controls (Figure 3.2 A). Analysis of the side scatter (SSC) profiles of the GM-CSF/IL-4 BMDC revealed an increase in SSC in DCs that had been exposed to $250 \mu \mathrm{g} / \mathrm{mL}$ or $500 \mu \mathrm{g} / \mathrm{mL}$ MSU (Figure $3.2 \mathrm{~B}$ ). This suggests that these DCs were taking up MSU crystals.

A.
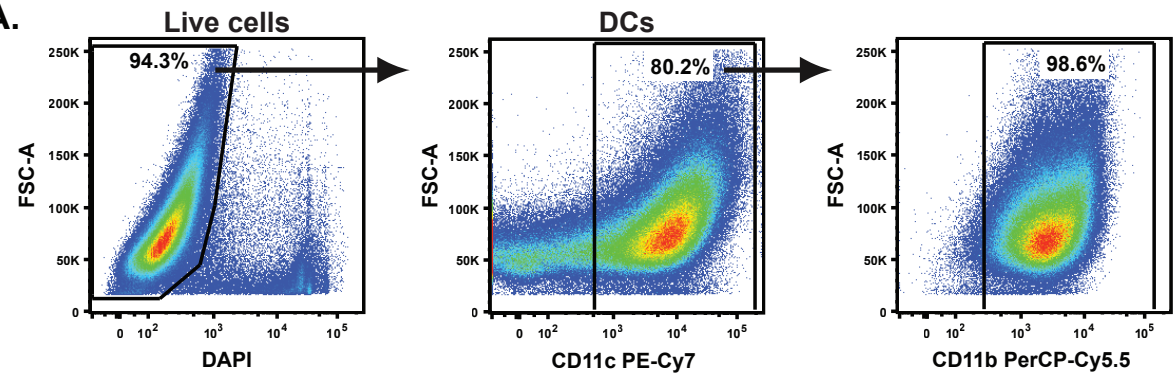

B.
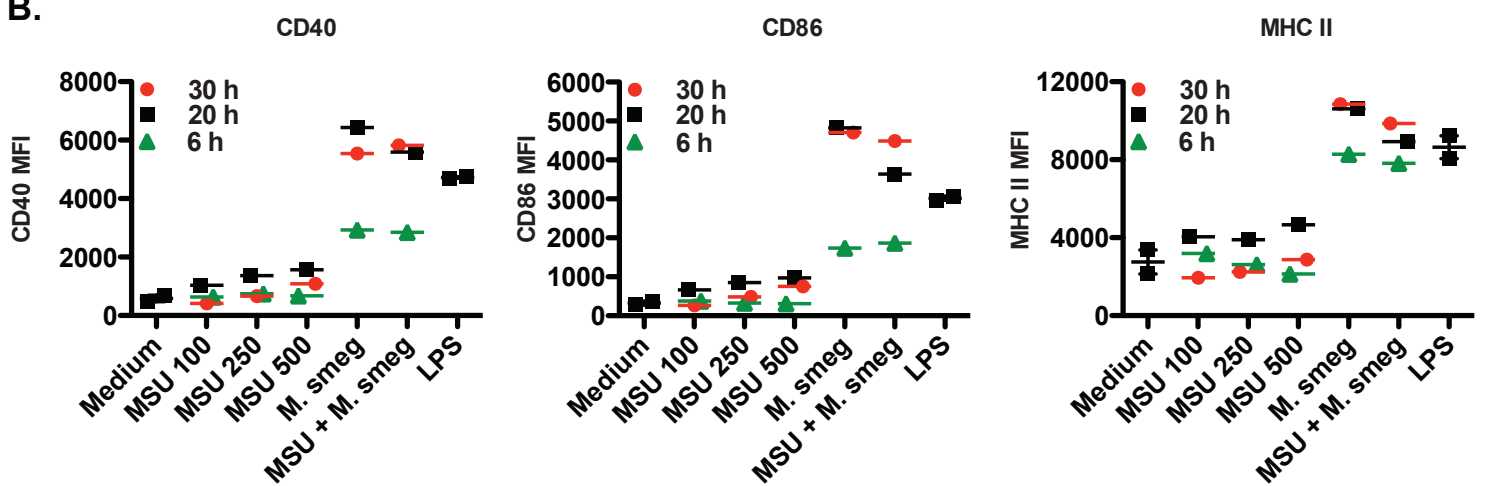

Figure 3.1: Time course of GM-CSF/IL-4 BMDC activation by adjuvants in vitro. DCs were generated from bone marrow of C57BL/6 mice by culture with GM-CSF and IL-4. On day 7 , the indicated adjuvants were added and DC activation was analysed at $6 \mathrm{~h}, 20 \mathrm{~h}$ and $30 \mathrm{~h}$ by flow cytometry. (A) Live cells were identified by DAPI exclusion and examined for the expression of CD11c and CD11b. Representative flow plots are shown. (B) The expression of CD40, CD86 and MHC II on CD11c+ DCs is shown as Median Fluorescence Intensity (MFI). MSU $100-500=100 \mu \mathrm{g} / \mathrm{mL}$ to $500 \mathrm{\mu g} / \mathrm{mL}$ MSU crystals; M. smeg $=2.0 \times 10^{6} \mathrm{CFU}$ live M. smegmatis per well; MSU + M. smeg = combination of $250 \mathrm{\mu g} / \mathrm{mL}$ MSU crystals and $1.0 \times 10^{6}$ CFU M. smegmatis per well; LPS $100 \mathrm{ng} / \mathrm{mL}$. Values of individual samples from one experiment are shown. 
As expected from the literature [341] the TLR4 ligand LPS induced up-regulation of CD40, CD80, CD86 and MHC II on BMDC, whereas the TLR3 ligand Poly IC did not activate GM-CSF/IL-4 BMDC (Fig. 3.2 C-F). The endogenous danger signal MSU also failed to induce a significant up-regulation of activation markers. However, M. smegmatis stimulation increased the surface levels of all activation markers in a dose-dependent manner (Fig. 3.2 CF). The effects of LPS, MSU and $M$. smegmatis on the activation of GM-CSF/IL-4 DCs confirm the initial results obtained during the activation time course (Figure 3.1).

The combination of M. smegmatis and MSU significantly increased expression of CD40, CD80, CD86 and MHC II compared to the levels observed with the same dose of M. smegmatis alone (Fig. 3.2 C-F). The extent of activation induced by MSU + M. smegmatis was actually similar to the level induced by a 2 -fold higher dose of $M$. smegmatis. In contrast, combination of LPS and Poly IC did not enhance the expression of CD40, CD80 and MHC II more than LPS alone and only slightly increased CD86 expression (Fig. 3.2 C-F).

These data indicate that LPS and M. smegmatis can activate GM-CSF/IL-4 BMDCs. Poly IC stimulation had little effect on expression of co-stimulatory molecules and MHC II on GM-CSF/IL-4 BMDCs. Exposure of DCs to MSU also did not increase activation marker expression, but addition of MSU significantly enhanced DC activation in response to M. smegmatis.

The supernatant from GM-CSF/IL-4 BMDC cultures was also analysed for the production of cytokines using a multiplex assay. MSU and Poly IC stimulation caused little cytokine secretion by GM-CSF/IL-4 BMDCs (Figure 3.3). LPS, LPS + Poly IC, M. smegmatis and MSU + M. smegmatis, on the other hand, all induced the production of IL- $1 \alpha$, IL- $1 \beta$, IL-12p70, IL-6 and TNF $\alpha$ (Figure 3.3). This result is consistent with the effect of these adjuvants on activation marker expression of GM-CSF/IL-4 BMDCs (Figure 3.2).

The highest levels of all of these cytokines were detected in response to LPS and LPS + Poly IC. Interestingly, the amount of IL- $1 \alpha$, IL- $1 \beta$, IL- 6 and TNF $\alpha$ secreted by GM-CSF/IL-4 BMDCs after LPS stimulation was not further enhanced by addition of Poly IC, but IL-12p70 levels were more than 5-fold higher in LPS + Poly IC compared to LPS treated cultures (Figure 3.3). In contrast, the combination of $250 \mu \mathrm{g} / \mathrm{mL} \mathrm{MSU}$ and $1.0 \times 10^{6} \mathrm{CFU}$ 
A.

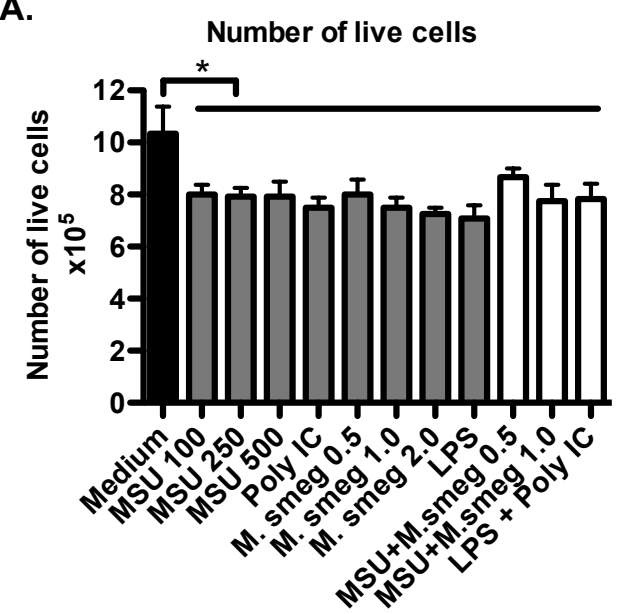

C.

CD40

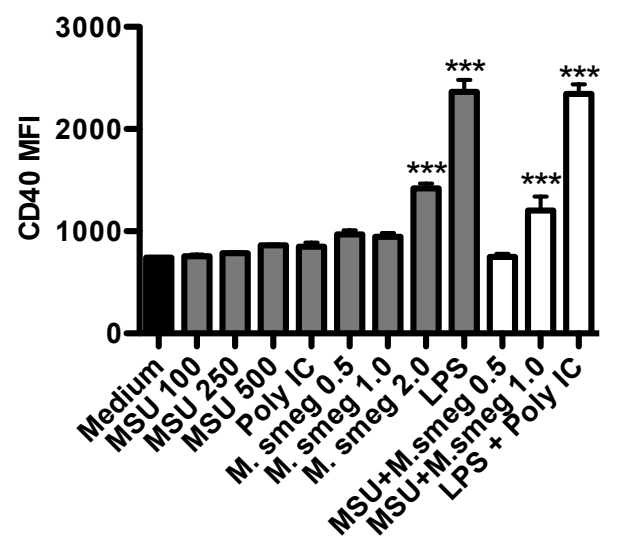

E.

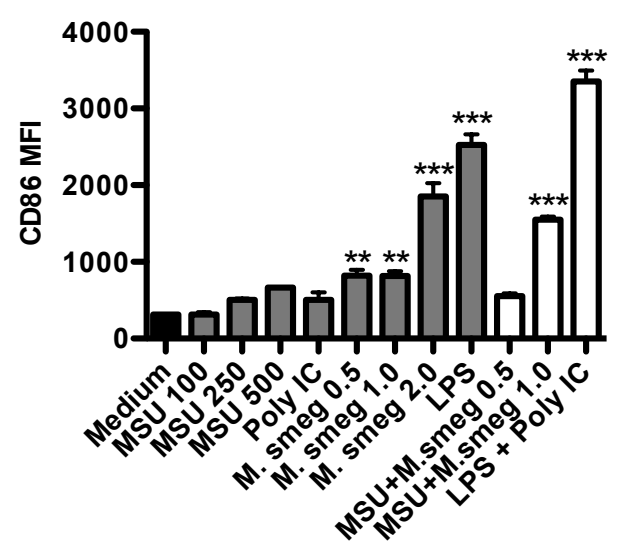

B.

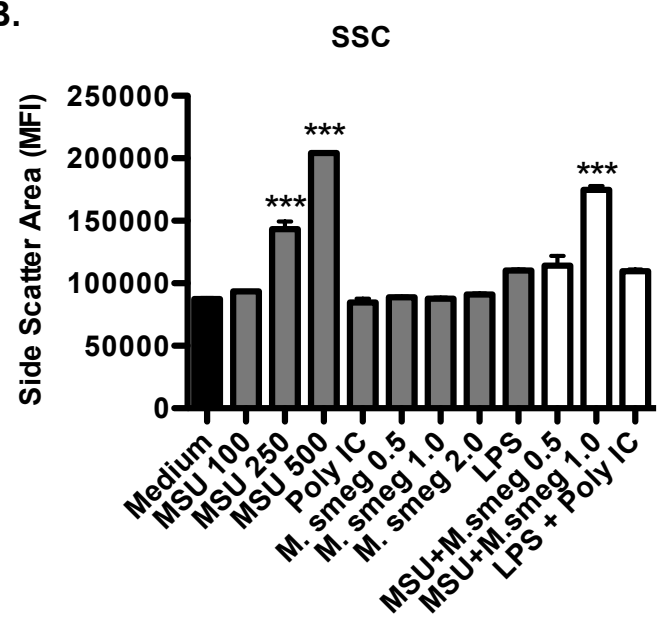

D.

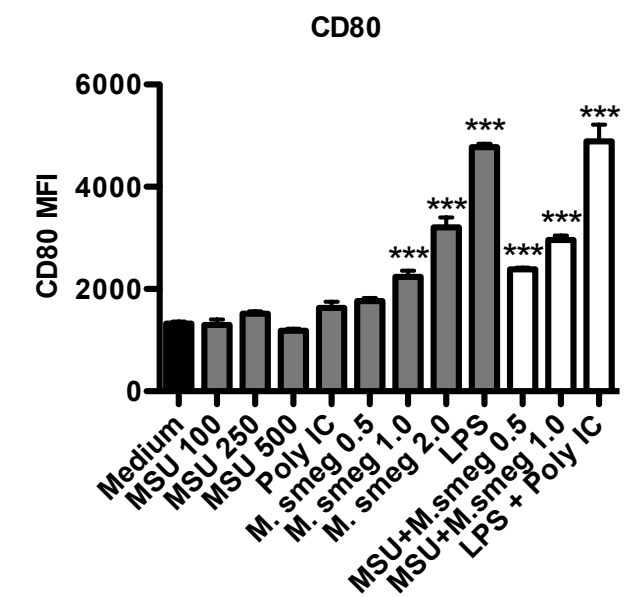

F.

MHC II

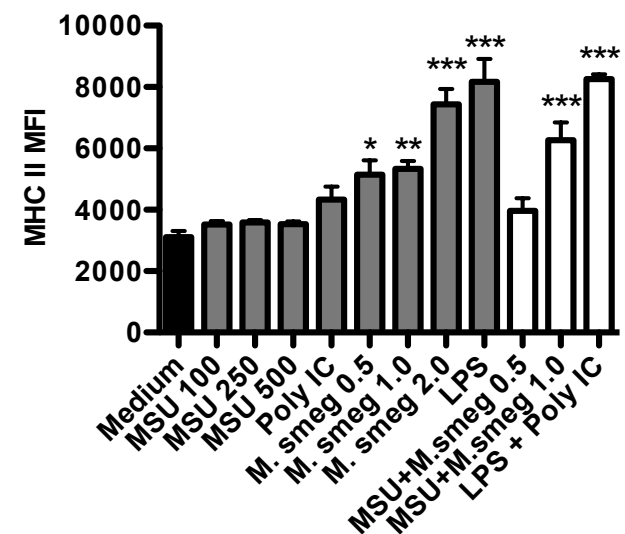

Figure 3.2: GM-CSF/IL-4 BMDC activation by adjuvants in vitro. GM-CSF/IL-4 BMDCs were generated, stimulated with adjuvants for $20 \mathrm{~h}$ and analysed by flow cytometry as described in Figure 3.1 A. (A) The number of live cells in each culture condition is shown. (B) The side scatter level is graphed as Median Fluorescence Intensity (MFI). (C-D) The expression of CD40, CD80, CD86 and MHC II on CD11c+ DCs is shown as MFI. Adjuvant doses were: MSU $100-500=100 \mathrm{\mu g} / \mathrm{mL}$ to $500 \mathrm{\mu g} / \mathrm{mL}$ MSU; Poly IC: $50 \mathrm{\mu g} / \mathrm{mL}$; M. smeg $0.5-2.0=0.5-2.0 \times 10^{6} \mathrm{CFU}$ live M. smegmatis per well; MSU+M.smeg $0.5=100 \mathrm{\mu g} / \mathrm{mL}$ MSU and $0.5 \times 10^{6}$ CFU M. smegmatis per well; MSU+M.smeg $1.0=250 \mathrm{\mu g} / \mathrm{mL}$ MSU and $1.0 \times 10^{6}$ CFU M. smegmatis per well; LPS $100 \mathrm{ng} / \mathrm{mL} ;$ LPS + Poly IC: $100 \mathrm{ng} / \mathrm{mL}$ LPS and $50 \mu \mathrm{g} / \mathrm{mL}$ Poly IC. ${ }^{*} p<0.05,{ }^{* *} p<0.01$ and ${ }^{* * *} p<0.001$ compared to medium controls as assessed by one-way ANOVA with Tukey's post test. The graphs show the mean value $+\mathrm{SE}$ from 3 samples per condition. Results are from one of two independent experiments with similar results. 
IL-1a

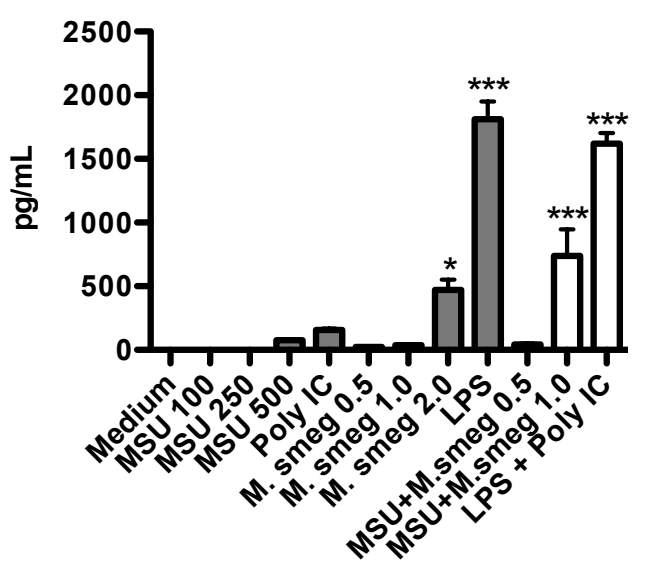

IL-12p70

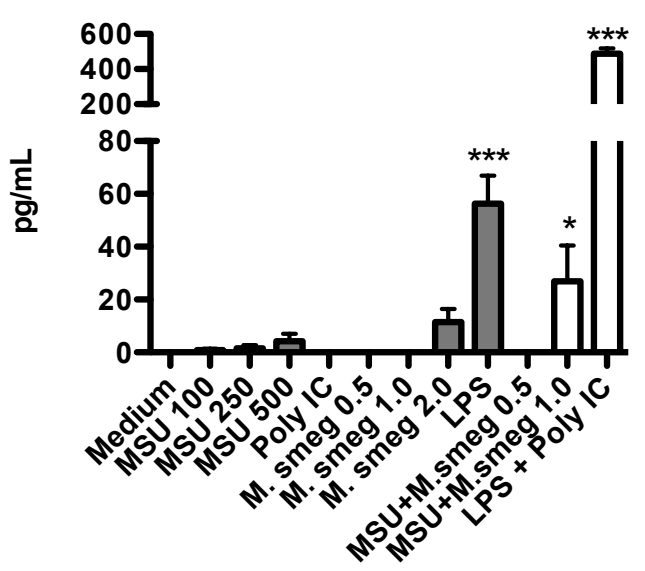

IL-6

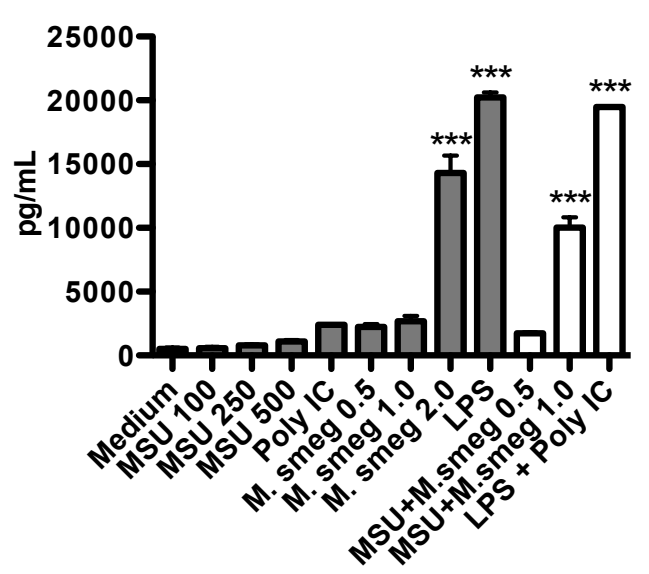

IL-1 $\beta$

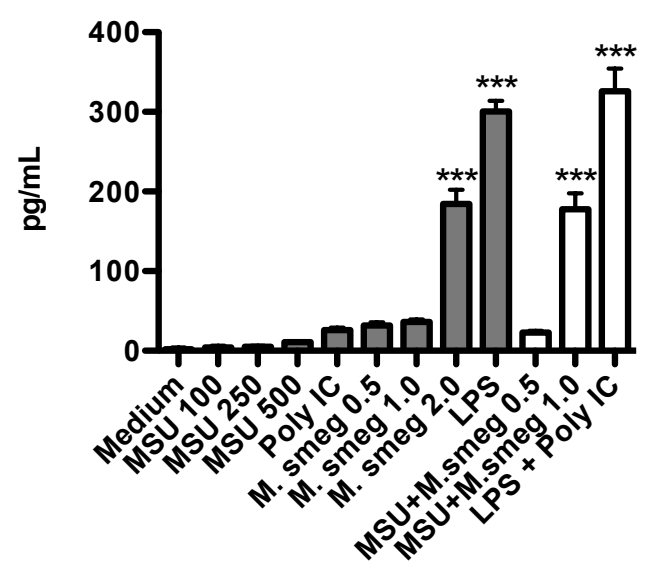

IL-10

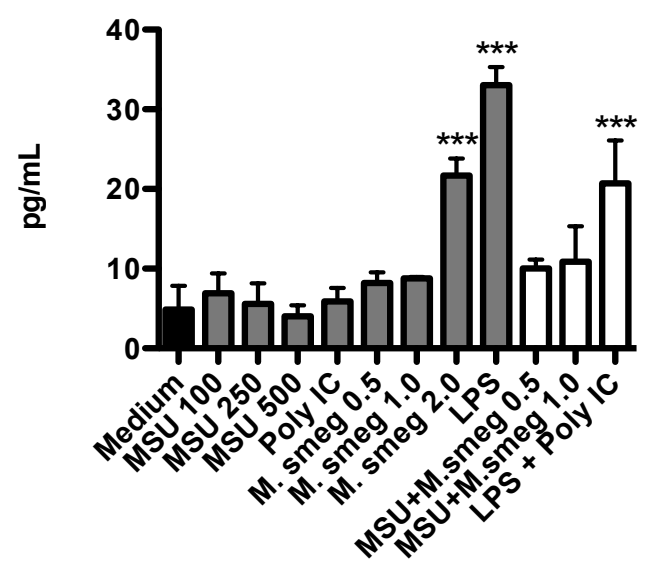

TNFa

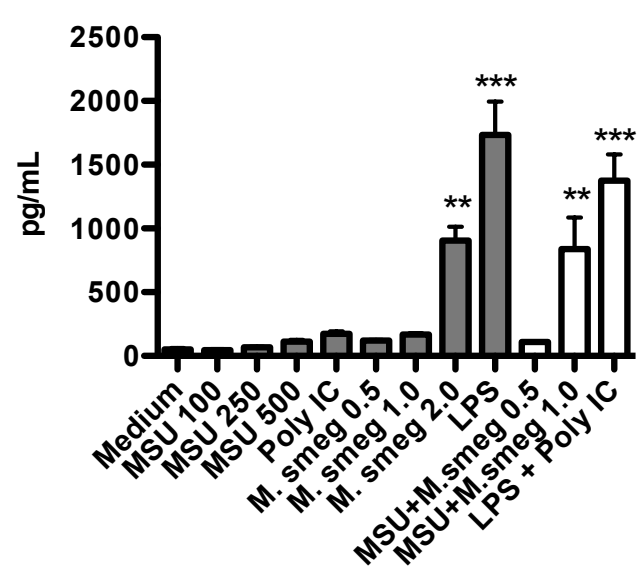

Figure 3.3: Cytokine production by GM-CSF/IL-4 BMDC activated by adjuvants in vitro. The supernatant of GM-CSF/IL-4 BMDCs activated with adjuvants for $20 \mathrm{~h}$ as described in Figure 3.2 was analysed for the production of cytokines. IL- $1 \alpha$, IL-1 $\beta$, IL-12p70, IL-10, IL-6 and $\mathrm{TNF} \alpha$ were detected in a multiplex bead assay. ${ }^{*} p<0.05,{ }^{* *} p<0.01$ and ${ }^{* * *} p<0.001$ compared to medium controls as assessed by one-way ANOVA with Tukey's post test. Bar graphs depict the mean value from 3 samples per condition + SE. Data are from one out of two similar independent experiments with comparable results. 
M. smegmatis resulted in considerable production of IL- $1 \alpha$, IL- $1 \beta$, IL-12p70, IL-6 and TNF $\alpha$, while neither $250 \mu \mathrm{g} / \mathrm{mL}$ MSU nor 1.0 x $10^{6}$ CFU M. smegmatis induced any significant cytokine secretion (Figure 3.3).

The highest levels of all of these cytokines were detected in response to LPS and LPS + Poly IC. Interestingly, the amount of IL- $1 \alpha$, IL- $1 \beta$, IL- 6 and TNF $\alpha$ secreted by GM-CSF/IL-4 BMDCs after LPS stimulation was not further enhanced by addition of Poly IC, but IL-12p70 levels were more than 5-fold higher in LPS + Poly IC compared to LPS treated cultures (Figure 3.3). In contrast, the combination of $250 \mu \mathrm{g} / \mathrm{mL}$ MSU and $1.0 \times 10^{6} \mathrm{CFU}$ M. smegmatis resulted in considerable production of IL- $1 \alpha$, IL-1 $\beta$, IL-12p70, IL-6 and TNF $\alpha$, while neither $250 \mathrm{\mu g} / \mathrm{mL}$ MSU nor $1.0 \times 10^{6}$ CFU M. smegmatis induced any significant cytokine secretion (Figure 3.3).

In addition to the induction of pro-inflammatory and Th1 cytokines, M. smegmatis, LPS and LPS + Poly IC also stimulated the production of the anti-inflammatory cytokine IL-10 (Figure 3.3). In contrast, $250 \mathrm{\mu g} / \mathrm{mL} \mathrm{MSU}+1.0 \times 10^{6} \mathrm{CFU}$ M. smegmatis induced proinflammatory cytokines and IL-12p70, but did not increase the secretion of IL-10.

In summary, GM-CSF/IL-4 BMDCs showed increased activation marker expression and cytokine secretion in response to M. smegmatis, LPS, LPS + Poly IC and MSU + M. smegmatis, while MSU or Poly IC alone had little effect. The highest levels of pro-inflammatory cytokines and IL-12p70 was induced by LPS + Poly IC stimulation. However, this adjuvant combination also increased the production of IL-10. MSU + M. smegmatis also enhanced the production of pro-inflammatory cytokines without inducing IL-10 secretion. 


\subsubsection{Different adjuvants induce varying degrees of activation of Flt3L BMDCs}

It is unclear, whether DCs in tumours resemble inflammatory DCs as modelled in the GM-CSF/IL-4 culture system or whether they are more similar to any of the steady-state DC types. Therefore, the effect of different adjuvants on steady-state DCs was investigated using Flt3L BMDCs. Flt3L cultures generate three DC subsets thought to correspond to steady-state $\mathrm{pDC}, \mathrm{CD} 8 \alpha+$ and $\mathrm{CD} 8 \alpha-\mathrm{cDC}$ in vivo [420-422].

As was done for the GM-CSF/IL-4 BMDCS, first a time course was performed to determine the best time point for DC activation analysis. Up-regulation of CD80 and MHC II expression on Flt3L DCs occurred already after $6 \mathrm{~h}$ of stimulation with M. smegmatis and MSU + M. smegmatis (Figure 3.4). Maximal CD40 and CD86 expression, however, was observed at $20 \mathrm{~h}$ and levels decreased slightly at $30 \mathrm{~h}$. Therefore, the $20 \mathrm{~h}$ time point was selected for analysis of DC activation in subsequent experiments.

The second goal of the experiment shown in Figure 3.4 was to evaluate whether MSU could activate Flt3L BMDCs. Up-regulation of CD40, CD86 and MHC II expression was observed in a dose-dependent manner at the $20 \mathrm{~h}$ time point (Figure 3.4). However, overall levels of CD40 and CD86 were considerably lower in MSU stimulated compared to LPS or M. smegmatis treated Flt3L BMDCs (Figure 3.4). For further experiments, the intermediate concentration of $250 \mathrm{\mu g} / \mathrm{mL} \mathrm{MSU}$ and a lower dose of $0.5 \times 10^{6} \mathrm{CFU}$ live M. smegmatis per well were chosen to avoid saturating the level of activation marker expression with either MSU or M. smegmatis alone and to enable any additive or synergistic effect of the combination of the two adjuvants to be assessed.

To study the effect of the different adjuvants on the activation of Flt3L DCs in more detail, the three DC populations in these cultures were analysed individually. Figure $3.5 \mathrm{~A}$ illustrates the gating strategy used to identify B220+ pDCs, as well as CD24+ cDCs (corresponding to $\mathrm{CD} 8 \alpha+\mathrm{cDCs}$ in vivo) and $\mathrm{CD} 11 \mathrm{~b}+\mathrm{cDCs}$ (corresponding to $\mathrm{CD} 8 \alpha-\mathrm{cDCs}$ in vivo).

pDCs were strongly activated by stimulation with a combination of mycobacteria (either 

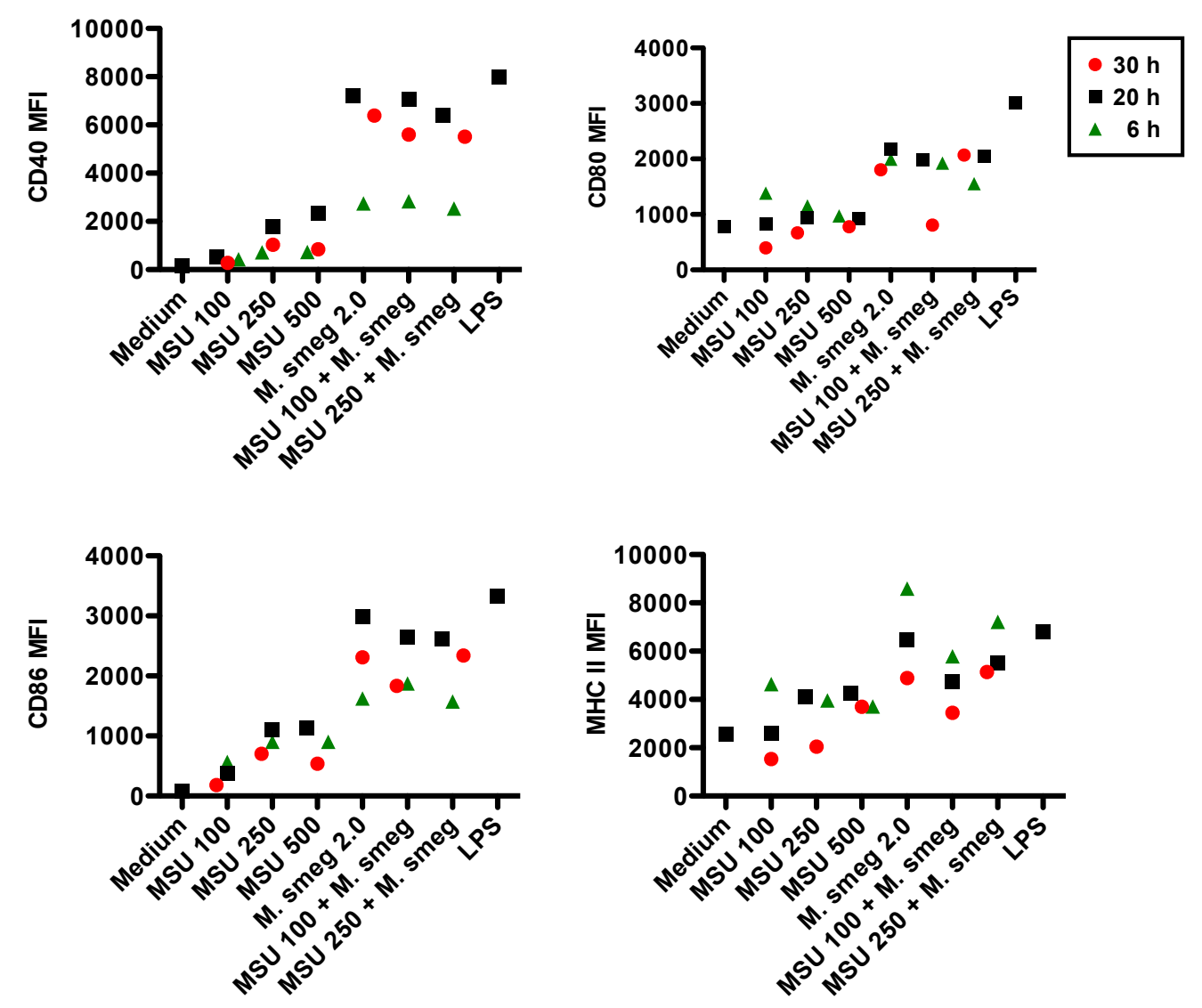

Figure 3.4: Time course and MSU titration for Flt3L BMDC activation by adjuvants in vitro. DCs were generated from bone marrow of C57BL/6 mice by culture with Flt3L. On day 10, indicated adjuvants were added and DC activation was analysed at $6 \mathrm{~h}, 20 \mathrm{~h}$ and $30 \mathrm{~h}$ by flow cytometry. DCs were gated on CD11c+ live cells as shown in Figure 3.1. The expression of CD40, CD86 and MHC II on the whole CD11c+ DC population is shown as Median Fluorescence Intensity (MFI). Adjuvants were used at the following concentrations: MSU $100-500=100 \mu \mathrm{g} / \mathrm{mL}$ to $500 \mu \mathrm{g} / \mathrm{mL}$ MSU crystals; M. smeg $2.0=2.0 \times 10^{6} \mathrm{CFU}$ live M. smegmatis per well; MSU $+\mathrm{M} . \mathrm{smeg}=$ combination of indicated doses of MSU crystals and $2.0 \times 10^{6}$ CFU live M. smegmatis; LPS $100 \mathrm{ng} / \mathrm{mL}$. Data from individual samples of one experiment are shown.

BCG or M. smegmatis) and MSU crystals, as judged by surface marker up-regulation (Fig. $3.5 \mathrm{~B}$ ). To a lesser extent, pDCs also up-regulated activation markers in response to CpG, while the other tested adjuvants had no effect pDCs (Fig. 3.5 B). In contrast, both CD24+ and CD11b + cDCs were activated by all adjuvants (Fig. 3.6). However, the degree of activation marker up-regulation varied for the different markers and among adjuvants. The effect of MSU was most variable, showing little up-regulation of CD40 and CD86, medium 
levels of CD80 and high levels of MHC II. CpG strongly up-regulated CD40, CD80 and MHC II, but induced only a limited increase in CD86 levels. Stimulation of DCs with a combination of two adjuvants did not further increase in expression of MHC II, while CD40, CD80 and CD86 levels were higher in DC activated with two adjuvants compared to the single stimuli.

Overall, the activation of CD24+ cDCs compared to CD11b + cDCs induced by each adjuvant was very similar. All adjuvants induced some degree of cDC activation and most resulted in comparable levels of activation marker up-regulation. In addition to responding to a broader range of adjuvants, cDCs also showed higher activation marker levels compared to pDCs.

To further assess the ability of adjuvants to activate Flt3L BMDCs and to polarize them towards a Th1 inducing phenotype, cytokine secretion in Flt3L BMDC cultures was analysed. IL-10 levels were below detection threshold in all of the tested samples. Nearly all adjuvants or combinations induced secretion of IL-12, TNF $\alpha$ and IL-6 (Figure 3.7). No significant cytokine production was detected in samples exposed to MSU. While Poly IC stimulation induced secretion of substantial amounts of IL-12, little TNF $\alpha$ or IL-6 and no IL-1 $\beta$ was detected in these cultures. Combination of adjuvants did not seem to enhance DC activation substantially. Only Poly IC + LPS stimulation had a marked effect on the secretion of IL-12 and IL-6, but did not increase the levels of TNF $\alpha$ over those seen with LPS alone. Interestingly, only stimulation with mycobacteria or a combination of mycobacteria and MSU crystals resulted in secretion of IL- $1 \beta$ from Flt3L BMDCs.

These data indicate that all adjuvants are capable of activating cDCs and with the exception of MSU also induce secretion of pro-inflammatory cytokines. However, only mycobacteria seem to induce activation of $\mathrm{pDCs}$ and production of IL-1 $\beta$. 


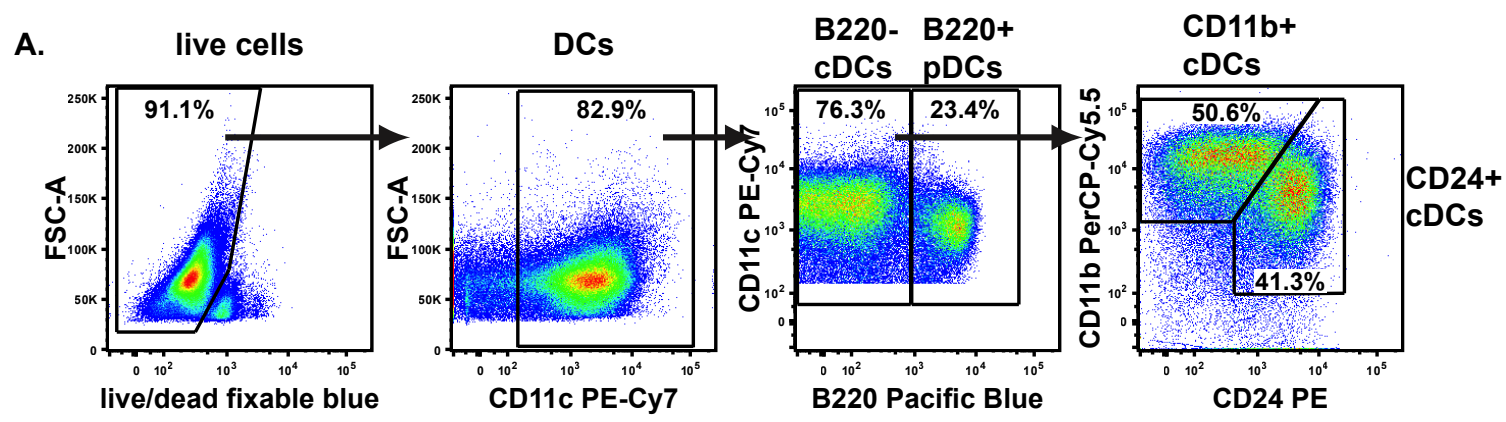

B. $\quad B 220+p D C s$
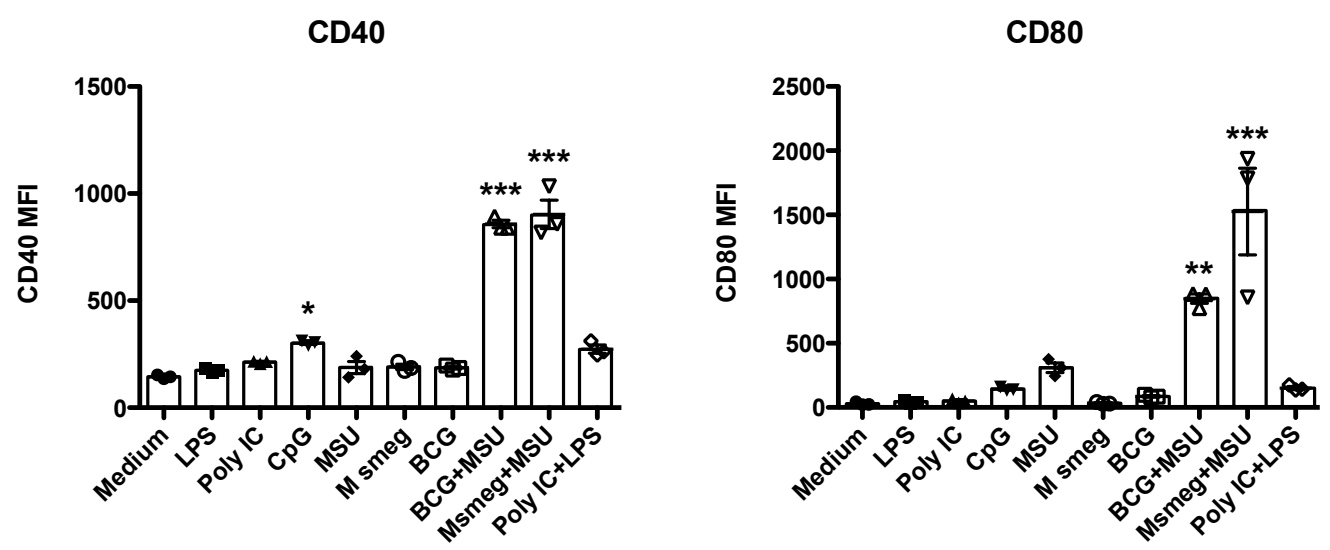

CD86
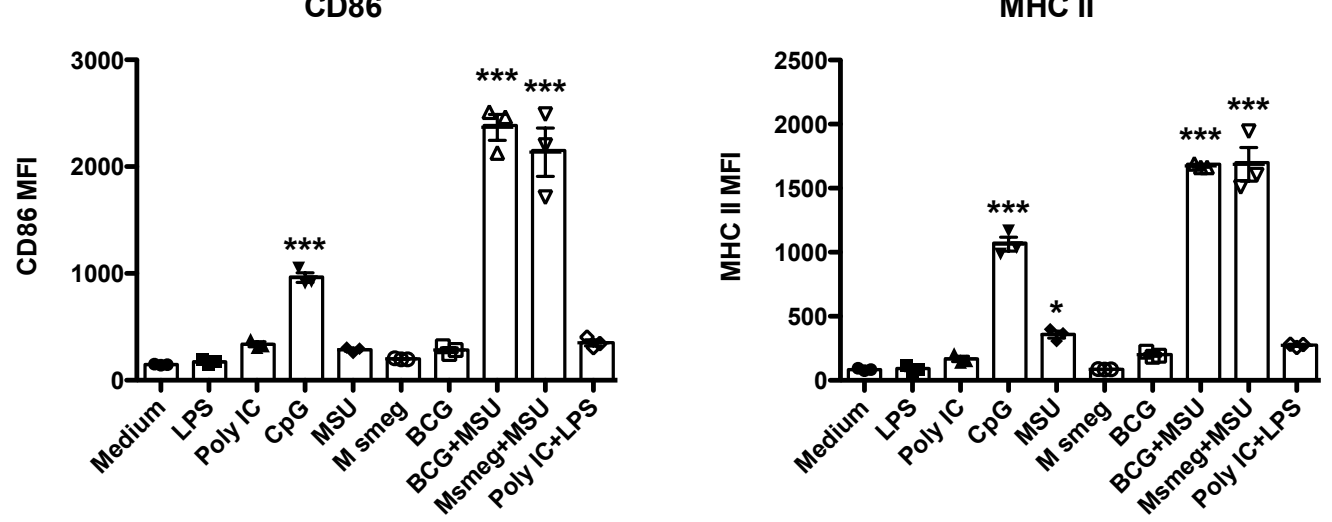

Figure 3.5: Activation of Flt3L pDCs by adjuvants in vitro. DCs were generated from bone marrow of C57BL/6 mice by culture with Flt3L. On day 10, indicated adjuvants were added and DC activation was analysed $20 \mathrm{~h}$ later by flow cytometry. (A) Representative gating of live cells, CD11c+ DCs, B220+ pDCs and CD11b+ or CD24+ cDCs. (B) The expression of CD40, CD86, CD80 and MHC II on B220+ pDCs is shown as Median Fluorescence Intensity (MFI). Adjuvants were used at the following concentrations: LPS $100 \mathrm{ng} / \mathrm{mL}$; Poly IC: $50 \mu \mathrm{g} / \mathrm{mL}$; CpG: $20 \mu \mathrm{g} / \mathrm{mL}$; MSU $250 \mu \mathrm{g} / \mathrm{mL}$; M. smeg $=0.5 \times 10^{6}$ CFU M. smegmatis per well; BCG $=0.5 \times 10^{6} \mathrm{CFU} B C G$ per well; $\mathrm{BCG}+\mathrm{MSU}$ combination of BCG and MSU; M. smeg+ MSU = combination of M. smegmatis and MSU; Poly IC + LPS $=$ combination of Poly IC and LPS. ${ }^{*} p<0.05,{ }^{* *} p<0.01$ and ${ }^{* * *} p<0.001$ compared to medium controls as assessed by one-way ANOVA with Tukey's post test. Data are shown as individual samples and mean $\pm \mathrm{SE}$ and are from one out of two similar experiments that gave comparable results. 
3 Identification of adjuvants for the activation of tumour-infiltrating DC

99

A.

CD24+ cDCs
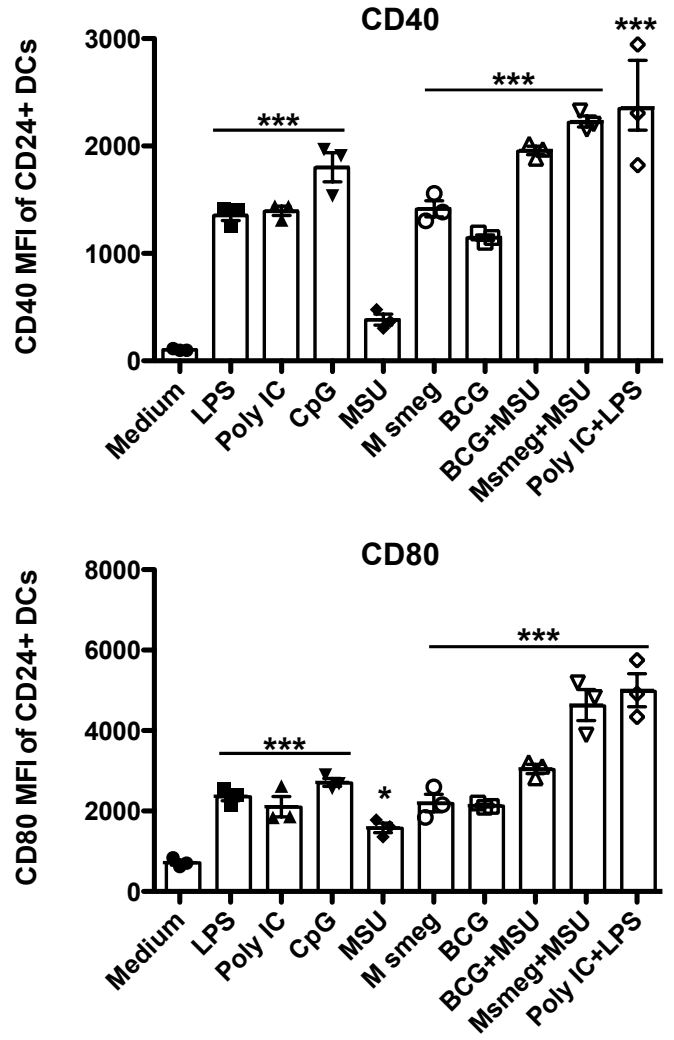

CD86
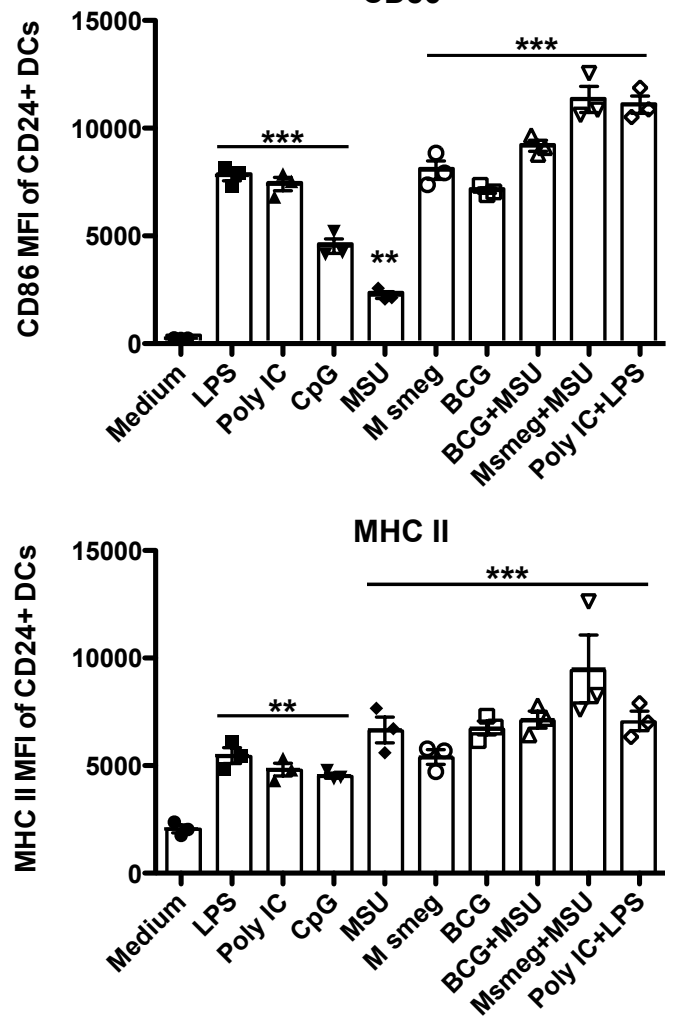

B.
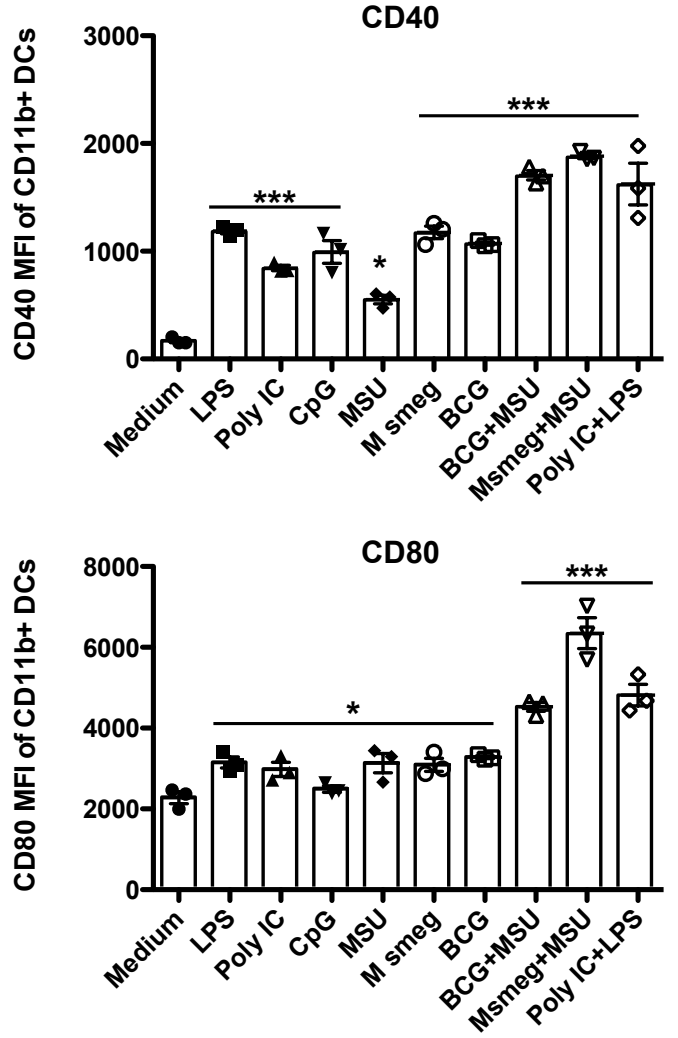

CD86
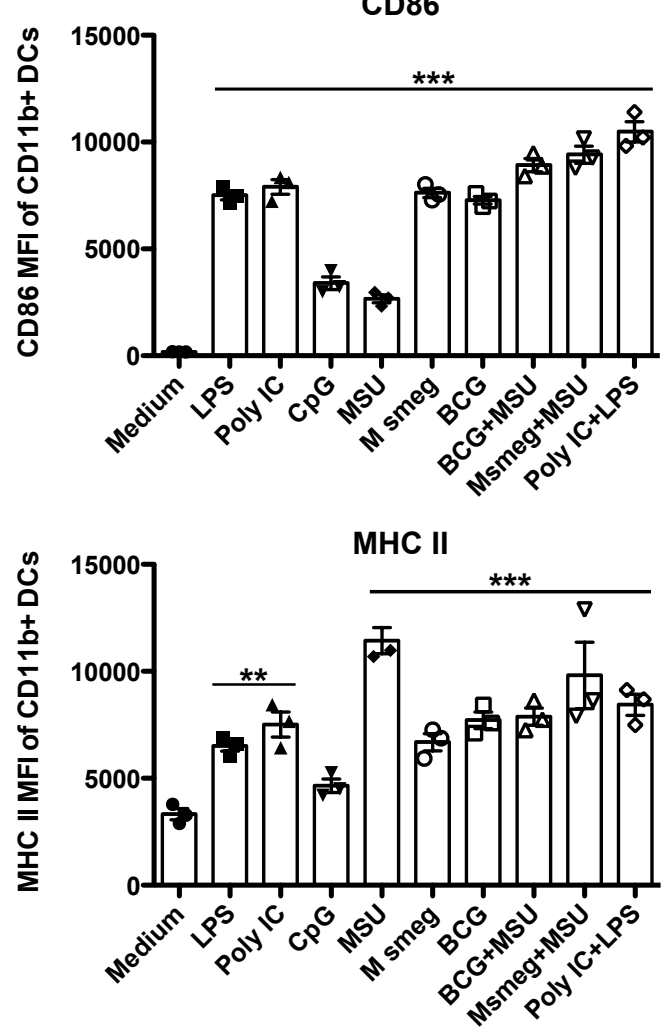

Figure 3.6: Activation of Flt3L cDC subpopulations by adjuvants in vito. Astivation marker expression is shown for the $\mathrm{CD} 24+\mathrm{cDC}(\mathbf{A})$ and the $\mathrm{CD} 11 \mathrm{~b}+\mathrm{cDC}(\mathbf{B})$ from the same experiment as in Figure 3.5. ${ }^{*} p<0.05,{ }^{* *} p<0.01$ and ${ }^{* * *} p<0.001$ compared to medium controls as assessed by one-way ANOVA with Tukey's post test. Data are shown as individual samples and mean $\pm \mathrm{SE}$ and are from one out of two similar experiments that gave comparable results. 
IL-12p70

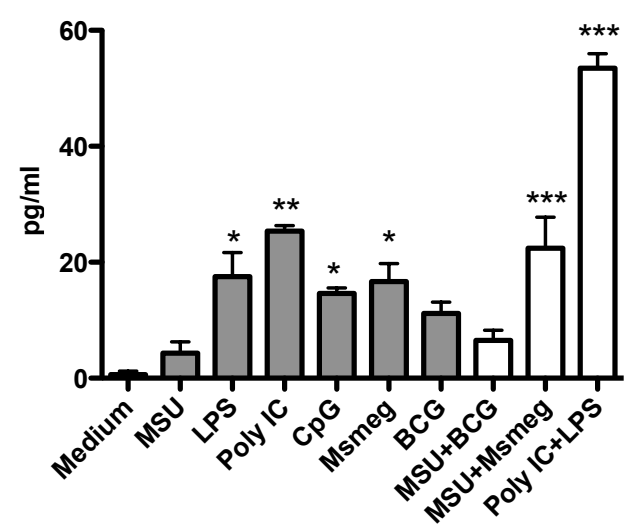

IL-6

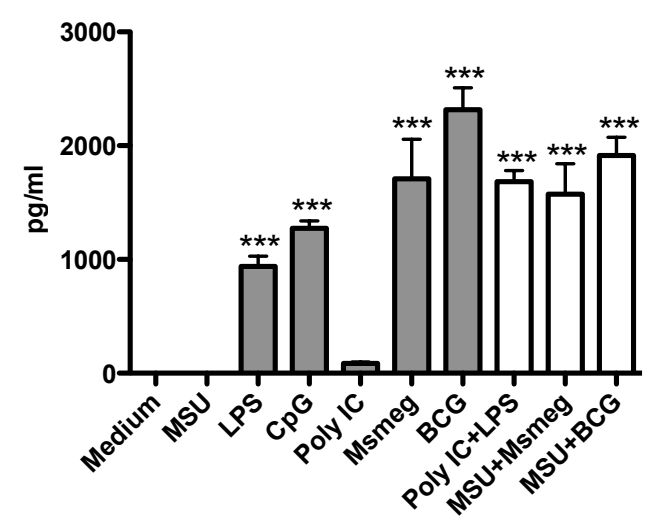

TNFa

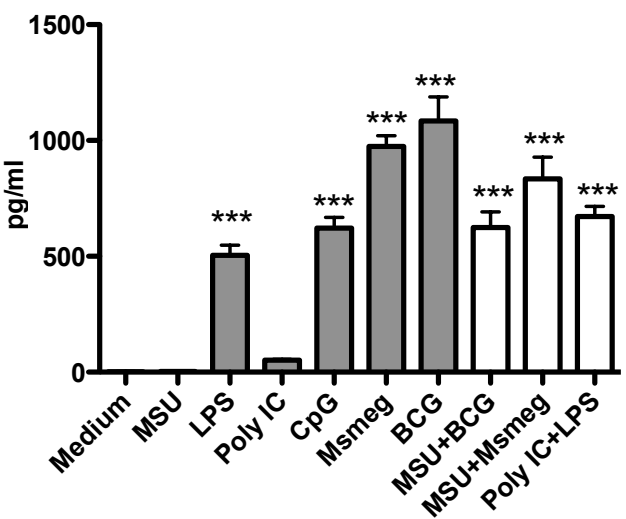

IL-1 $\beta$

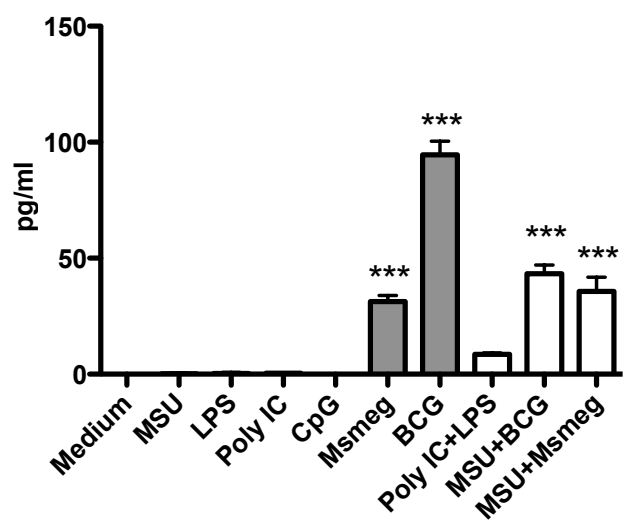

Figure 3.7: Cytokine production by Flt3L BMDCs activated by adjuvants in vitro. Supernatants of the same Flt3L DCs shown in Figure 3.5 were analysed for the production of cytokines. Il-12p70, TNF $\alpha$ and IL- 6 were detected in a multiplex bead assay. IL- $1 \beta$ was detected by sandwich ELISA. Data are depicted as mean + SE from 2 independent experiments with 3 samples per condition each. ${ }^{*} p<0.05,{ }^{* *} p<0.01$ and ${ }^{* * *} p<0.001$ for adjuvant compared to medium control as determined by using a Kruskal-Wallis test with Dunn's post test.

\subsubsection{DCs infiltrate tumours and show a poorly activated phenotype}

In order to evaluate whether tumour-infiltrating DCs could be a valuable target for activation by adjuvants, the presence of DCs in tumours and their activation status needed to be assessed. The transplantable B16F1 murine melanoma was chosen for this purpose, as these tumours grow relatively uniformly and are poorly immunogenic, therefore providing a good model to develop immunotherapies with potential clinical application. Furthermore, a B16 
A.

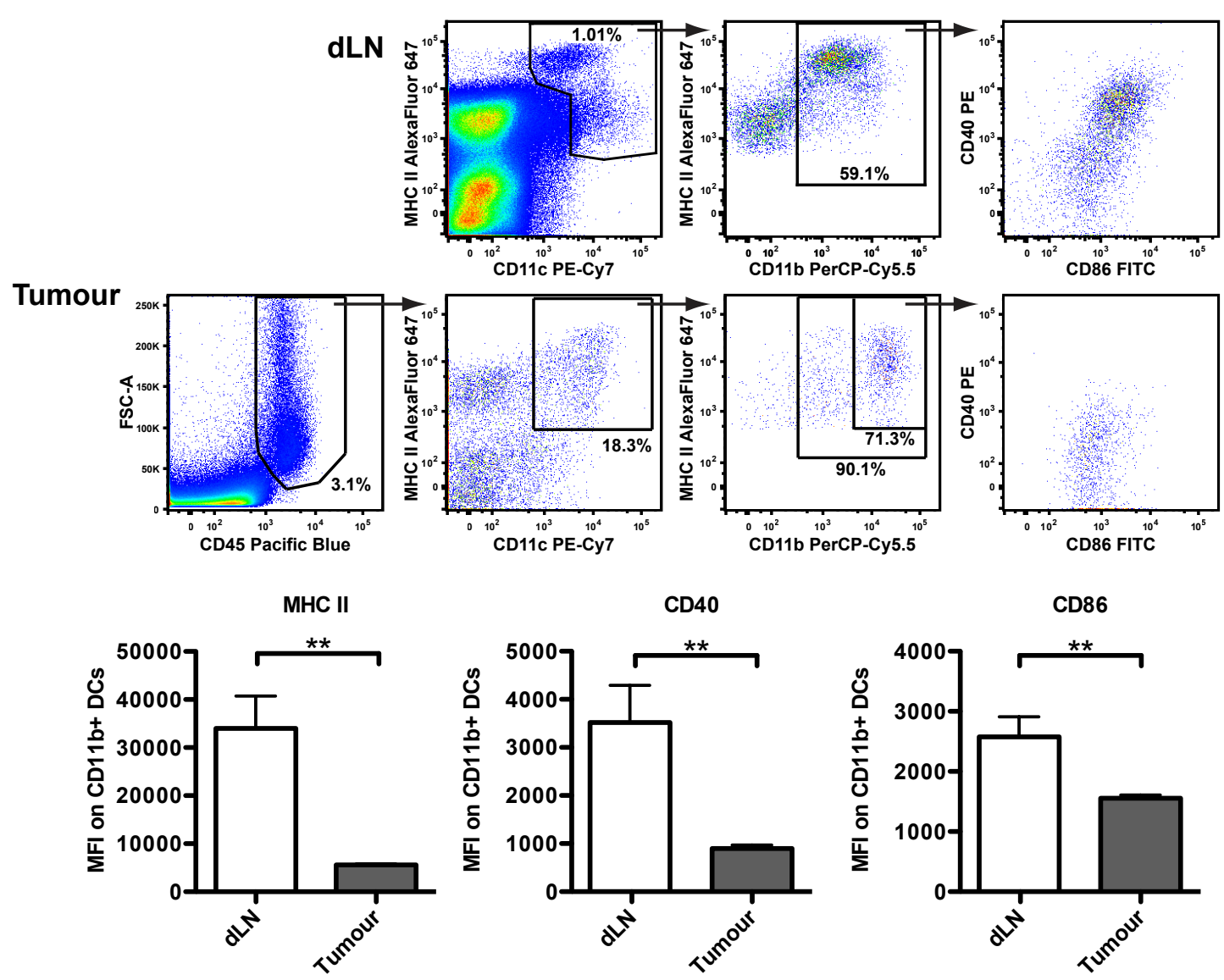

Figure 3.8: Identification of DCs in murine melanomas. DCs in B16 tumours of $4 \mathrm{~mm}$ to $6 \mathrm{~mm}$ diameter and tumour-draining LNs were analysed by flow cytometry. (A) Representative gating of DCs in dLN and tumours is shown. Cells were pre-gated on singlets by FSC and SSC and live cells (DAPI-). (B) Expression of the activation markers MHC II, CD40 and CD86 is shown as median fluorescence intensity (MFI) on CD11b+ DCs in dLN and tumours. Data are depicted as mean $+\mathrm{SE}$ from one experiment with 5 samples. ${ }^{* *} p<$ 0.01 as assed by a two-tailed Mann-Whitney test.

line expressing the model antigen OVA is available to enable the study of $\mathrm{T}$ cell responses with transgenic CD4 and CD8 T cells specific for this antigen.

Tumour cells were injected subcutaneously (s.c.) into syngeneic C57BL/6 mice. When tumours reached $4 \mathrm{~mm}$ to $6 \mathrm{~mm}$ in diameter, they were analysed for infiltration of DCs by flow cytometry. The activation status of DCs in the tumour was also compared to DCs in tumour-draining LN. First, live cells were identified by DAPI exclusion and were then gated using the common leukocyte marker CD45 to exclude auto-fluorescent tumour cells. Subsequently, DCs were identified as CD11c+ MHC II+ cells (Figure 3.8 A). Most tumour-infiltrating DCs expressed CD11b and about $70 \%$ showed very high CD11b levels. 
In dLNs, DCs were identified as live CD11c+MHC II+ cells, without the use of CD45 (Figure $3.8 \mathrm{~A}$ ). Approximately $60 \%$ of the DCs in dLNs were found to be CD11b+. Compared to tumour-infiltrating CD11b+ DCs, CD11b+ DCs in LNs expressed substantially higher levels of MHCII, CD40 and CD86 (Figure 3.8 A \& B).

To further characterise the remaining tumour-infiltrating DC population that did not express high levels of CD11b, other DC markers were included into the staining panel. Tumours of $10 \mathrm{~mm}$ to $15 \mathrm{~mm}$ diameter were analysed by flow cytometry, gating on live cells and CD45+ immune cells, followed by identification of DCs as CD11c+ MHC II+ cells (Figure 3.9 A). Similar to what was observed in the previous experiment, $80 \%$ of the tumour-infiltrating DCs expressed high levels of CD11b (Figure 3.9 A \& B). Only $5 \%$ of tumour-infiltrating DCs were B220+, indicating that they are pDCs (Figure 3.9 A \& B). CD11b+ DCs showed a more activated phenotype compared to pDCs, as assessed by the levels of MHC II expression (Figure $3.9 \mathrm{C}$ ).

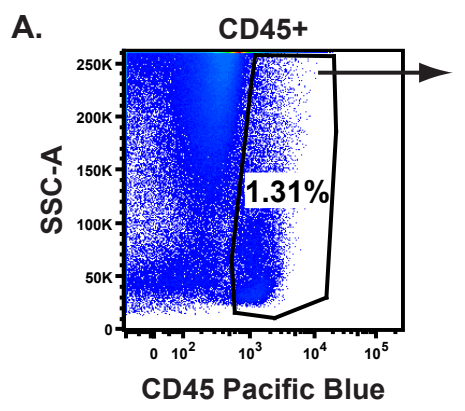

B.

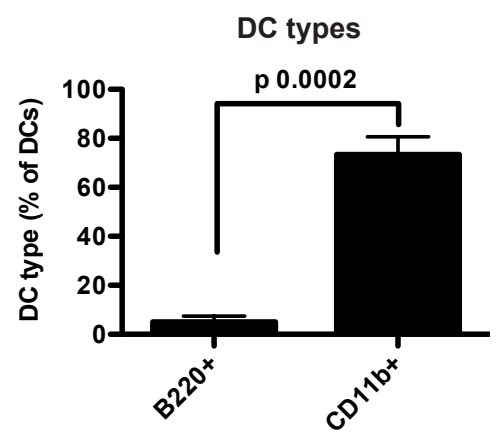

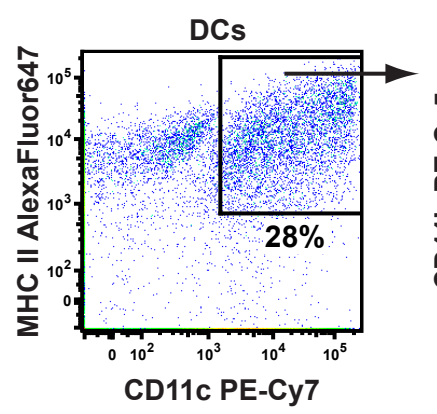

C.

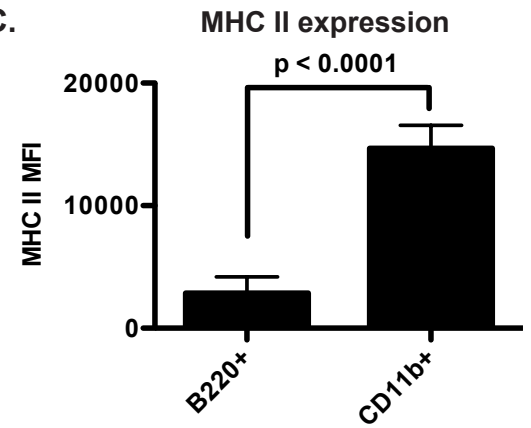

Figure 3.9: DCs in tumours are mainly CD11b+. CD11b+ and B220+ DCs were identified in B16 tumours of $10 \mathrm{~mm}$ to $15 \mathrm{~mm}$ diameter by flow cytometry. (A) Representative gating of DCs subtypes in tumours. Cells were pre-gated on singlets by FSC and SSC and live cells (DAPI-). (B) The percentage of the two DC subtypes among the total tumour-infiltrating DCs is depicted. (C) Expression of the activation marker MHC II on $\mathrm{CD} 11 \mathrm{~b}+$ and B220+ DCs in tumours is shown as median fluorescence intensity (MFI). Data are combined from two experiments with 8 and 10 mice, respectively, and are expressed as mean $+\mathrm{SD}$. The p-values were calculated using a two-tailed Mann-Whitney test. 
These data show that DCs infiltrate murine B16 tumours. The majority of tumour-infiltrating DCs expressed CD11b and low levels of the activation markers CD40, CD86 and MHC II compared to LN DCs.

\subsubsection{The immune infiltrate of murine melanomas consists mainly of DCs and monocytes}

To investigate which other immune cell types infiltrate murine B16 tumours, a more comprehensive analysis was undertaken. B16F1 tumour cells were injected s.c. into C57BL/6 mice and left to develop for 15 days. Tumours were then excised, processed into single cell suspensions and analysed by flow cytometry.

Tumour-infiltrating immune cells were gated as singlet, DAPI-, CD45+ events (Figure 3.10 A). Subsequently, CD4+ and CD8+ T cells and NK1.1+ NK cells were identified (Figure 3.10 B). In a separate staining panel, DCs were again gated as CD11c and MHC II expressing immune cells (Figure 3.10 C, left panel). Monocytes newly recruited to an inflammatory site have previously shown to express low levels of F4/80, whereas tissue-resident macrophages were F4/80 high [424]. Thus, monocytes and macrophages were identified as F4/80 intermediate FSC $^{\text {low }}$ and F4/80 high FSC $^{\text {high }}$ cells, respectively (Figure $3.10 \mathrm{C}$, middle panel). Both of these populations were also CD11b+ (not shown). B220+ MHC II + B cells were also observed.

In four separate experiments, the total percentage of infiltrating immune cells among all live cells (including tumour cells) was found to be 2-7\% (Figure $3.11 \mathrm{~A}$ ). Immune infiltration varied both between individual mice and between experiments. This may be due to variation in tumour size, as smaller tumours were generally found to have a higher percentage of infiltrating immune cells.

DCs were found to constitute a large proportion of the immune cells in tumours, accounting for about $30 \%$ of the total infiltrate (Figure $3.11 \mathrm{~B}$ ). Monocytes were present at a similar percentage. In contrast, macrophages, B cells and T cells only constituted a small fraction of the total immune infiltrate. Notably, CD8+ T cells and NK cells, which can potentially kill tumour cells through direct lysis, each constituted less than $5 \%$ of the immune cells in tumours. 
The analysis of the immune infiltrate in tumours shows that there are few effector cells present at the tumour site, while the majority of immune cells are DCs and monocytes. These myeloid cells might favour tumour progression but could possibly be harnessed to exert anti-tumour functions if appropriately activated by adjuvant treatment.

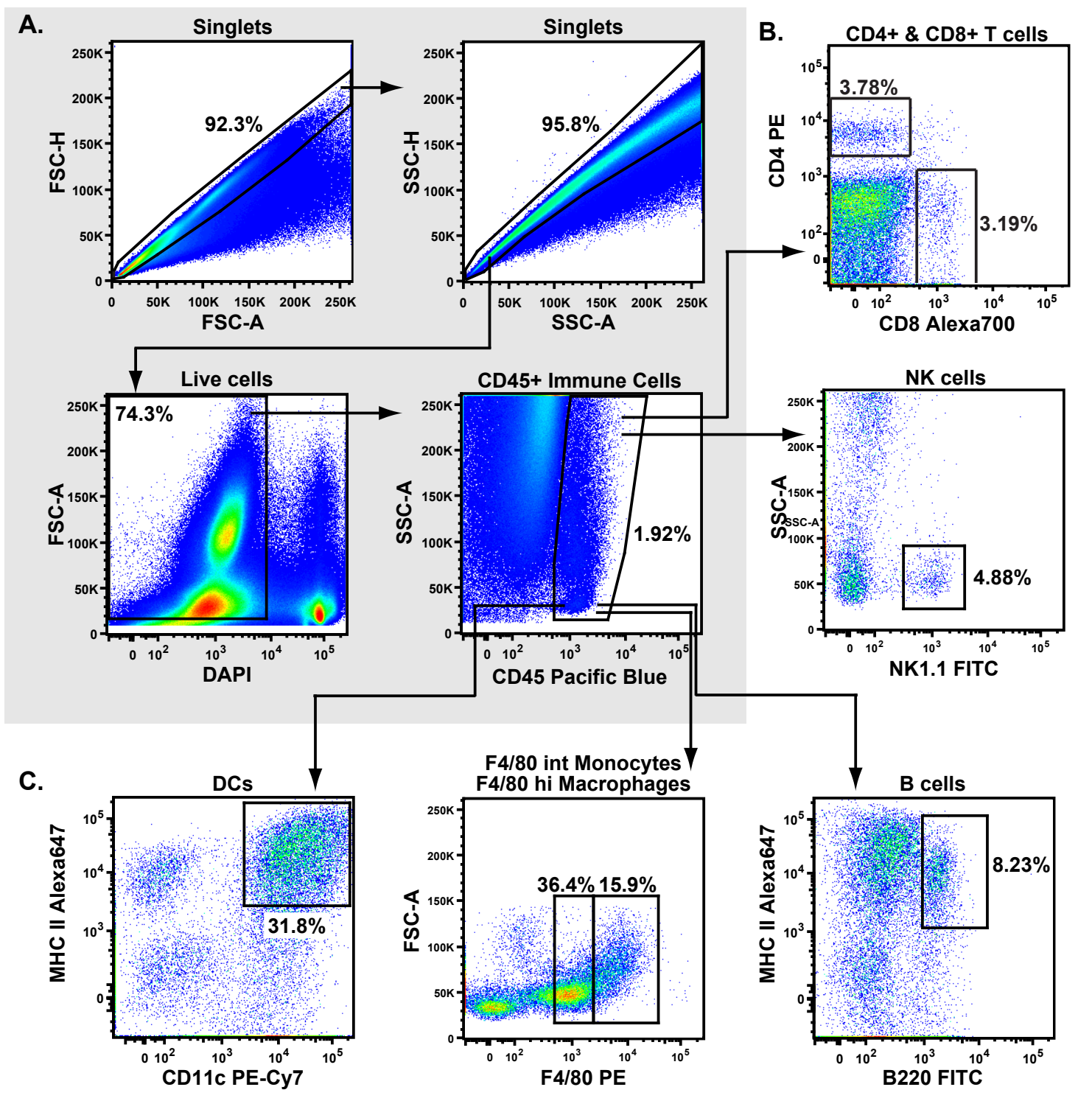

Figure 3.10: Representative gating strategy for flow cytometric analysis of the immune cell infiltrate in B16 tumours. Day 15 B16F1 tumours from C57BL/6 mice were processed into single cell suspensions, stained with antibodies and analysed by flow cytometry. Gating strategy is indicated by arrows. (A) Gating for singlets on FSC and SSC, live cells by DAPI exclusion and CD45+ immune cells. (B) CD45+ immune cells were subsequently gated on CD4 and CD8 to identify T cells and NK1.1 for NK cells. (C) In a separate staining panel, CD45+ immune cells were gated on MHCII+CD11c+ DCs, $\mathrm{F} 4 / 80^{\text {high }}$ FSC ${ }^{\text {high }}$ macrophages, F4/80 ${ }^{\text {int }} \mathrm{F} 4 / 80^{\text {int }}$ monocytes and B220+MHCII+ B cells. 
A.

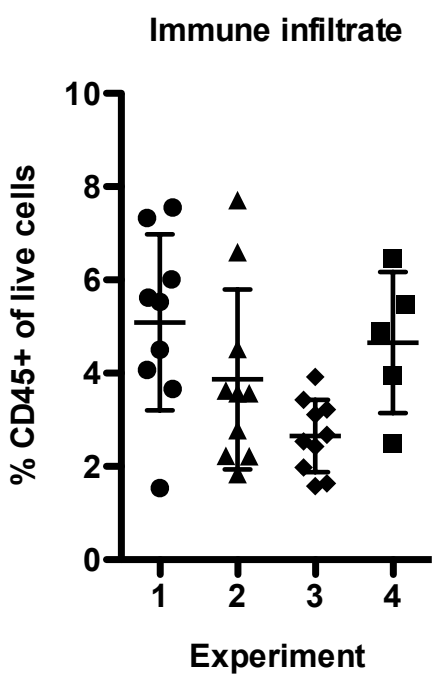

B. Immune cell types

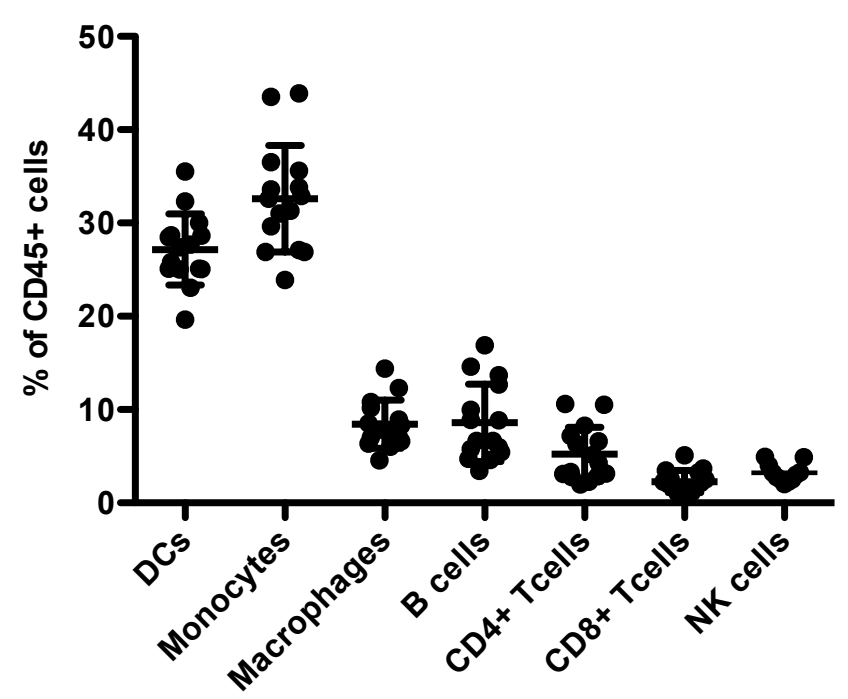

Figure 3.11: Identification of immune cell types in B16F1 tumours. Day 15 B16F1 tumours from C57BL/6 mice were processed into single cell suspensions, stained with antibodies and analysed by flow cytometry as shown in Figure 3.10. (A) The percentage of CD45+ immune cells among live cells is shown for four independent experiments. (B) The frequency of different immune cell types among the CD45+ immune infiltrate is graphed. Data are shown as mean \pm SD. Each symbol represents 1 mouse. Data in (B) are pooled from 2 independent experiments with 5 and 10 mice, respectively.

\subsubsection{Validation of flow cytometry to analyse immune infil- trate in tumours}

In previous experiments flow cytometry was used to analyse total immune cells and immune cell subtypes infiltrating murine melanomas. This method allows the concomitant examination of several cell surface markers to identify immune cell populations as well expression of activation markers for cell function on a per cell basis and is therefore widely used to study immune cells. As tumours display a low and variable level of infiltration by immune cells, it was important to verify that flow cytometric analysis accurately represented the immune cells in the tumour. In particular, the poor vasculature and occasional bleeding around the tumour site as tumours were excised from mice led to concerns that flow cytometric analysis might be skewed by immune cells from the blood contaminating the tumour tissue.

A method for labelling all blood cells by CFDA-SE injection in vivo, developed by Becker 
et al. to study immune cell recruitment to lymphoid tissues or sites of inflammation [425], was employed to address this concern. Briefly, a stock solution of CFDA-SE was diluted in ethanol and PBS and injected into the tail vein of tumour bearing mice. Subsequently organs were removed and analysed by flow cytometry.

Using this method, $>96 \%$ of blood cells were labelled with CFSE (Figure 3.12 A \& B left panels). In accordance with the results obtained by Becker et al., approximately $60 \%$ of spleen cells and nearly $15 \%$ of LN cells were labelled with CFSE (Figure 3.12 A \& B centre panels). Becker et al. attributed the stronger labelling of immune cells in the spleen both to labelling of intravascular immune cells in this organ and to leakage of the dye through the blood vessels.

Tumour samples from CFDA-SE injected mice displayed low levels of CFSE labelling, comparable to LNs (Figure 3.12 A \& B right panels). This indicates that the majority of CD45+

A.
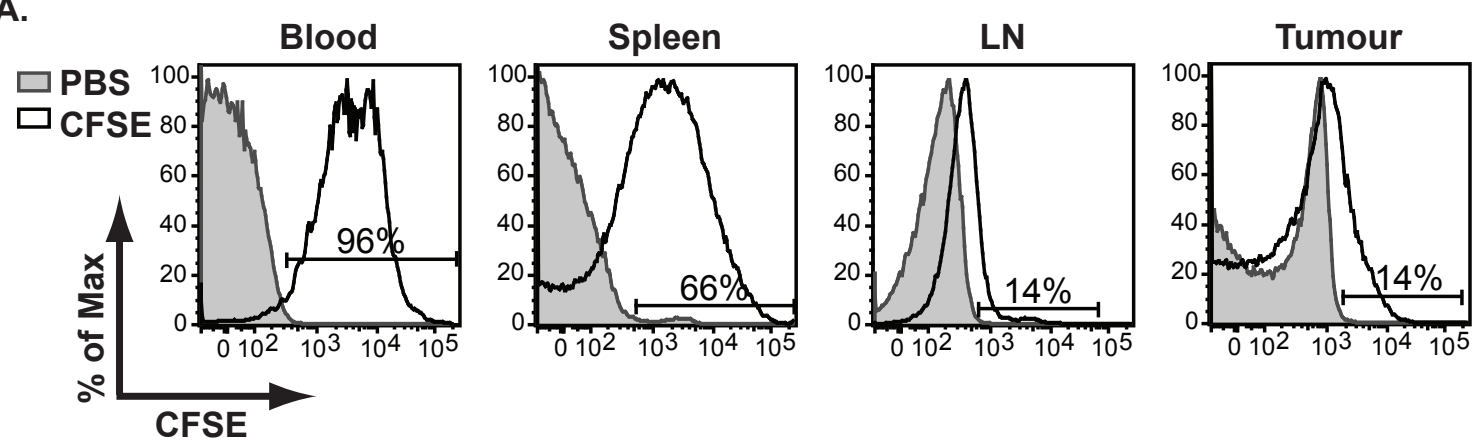

B.

Blood

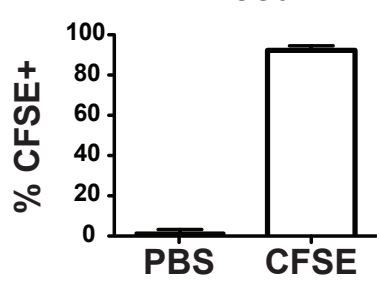

Spleen

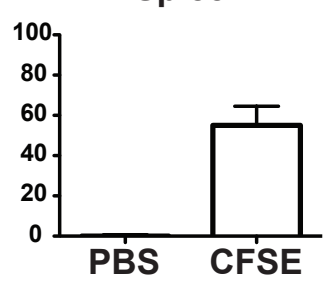

LN

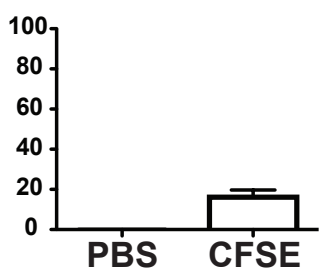

Tumour

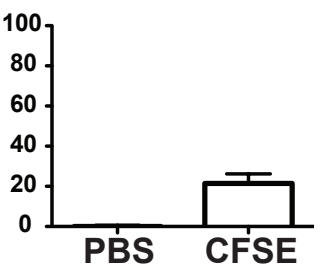

Figure 3.12: Blood cells reperesent a small proportion of the tumour immune infiltrate. C57BL/6 mice bearing 14 day B16F1 tumours were injected i.v. with CFDASE solution or PBS. 25 min later the indicated tissues were removed and stained for flow cytometry analysis. (A) Representative histograms for CFSE staining of live cells in the indicated organs. (B) The percentage of CFSE+ cells among live cells is shown as mean $+\mathrm{SD}$ for 5-10 mice per group. Data are pooled from two independent experiments. 
cells in these tumour samples were from the tumour tissue and that the flow cytometric analysis of the tumour-infiltrating immune cells was only minimally affected by contamination with CD45+ immune cells residing in the tumour vasculature.

A.
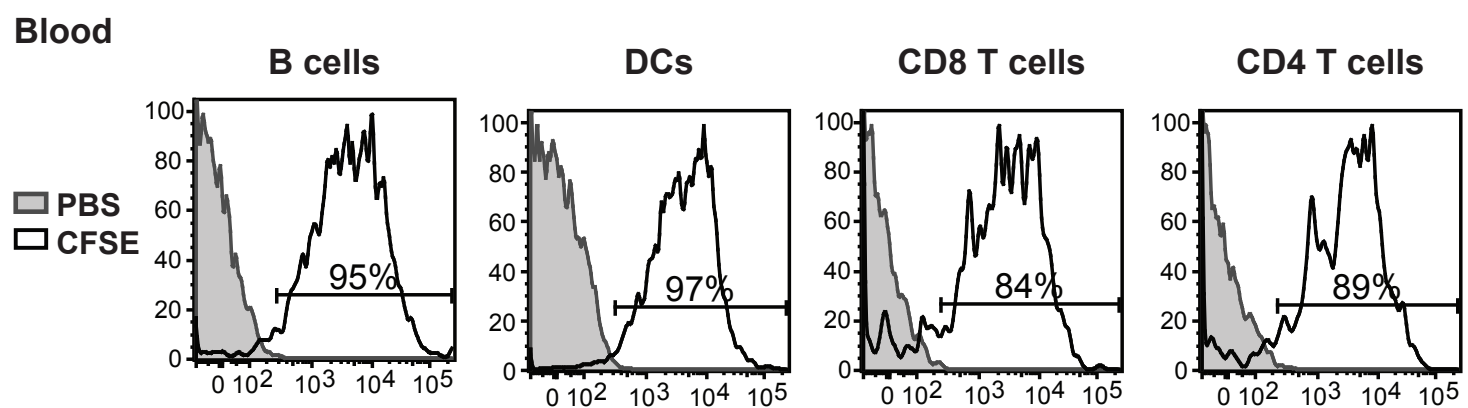

Tumour
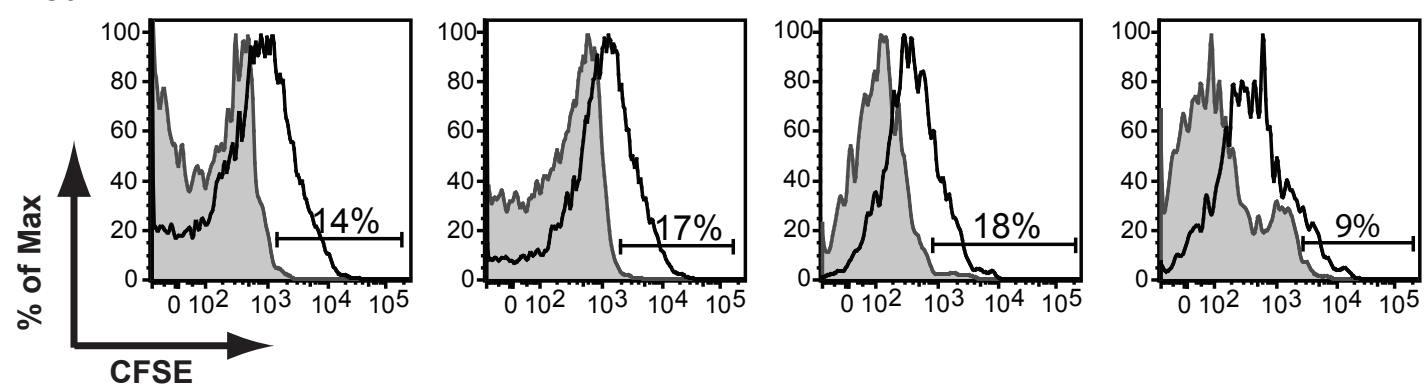

B.

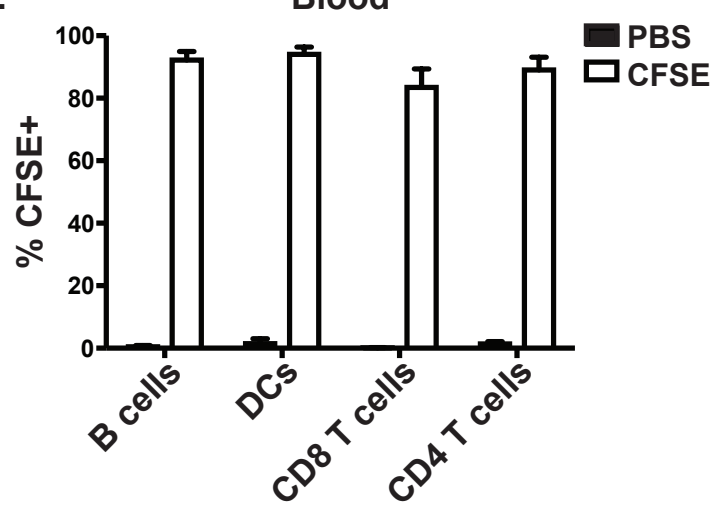

C.

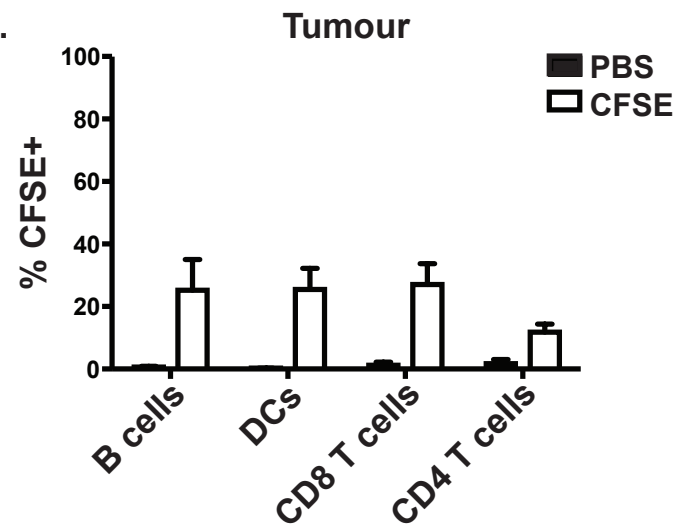

Figure 3.13: Blood cells do not skew cell type analysis of the tumour immune infiltrate by flow cytometry. C57BL/6 mice bearing 14 day B16F1 tumours were injected i.v. with CFDA-SE solution or PBS. 25 min later the indicated tissues were removed and stained for flow cytometry analysis. (A) Representative histograms for CFSE staining of the indicated cell types are shown. B and $\mathrm{T}$ cells were gated as live (DAPI-) lymphocytes (by FSC and SSC) and B220+, CD4+ or CD8+, respectively. DCs were gated as live CD11c+ cells. (B) The percentages of CFSE + cell populations in the blood are shown. (C) The frequency of CFSE+ cells of the indicated cell types in tumours is shown. Bar graphs represent mean $+\mathrm{SD}$ for 5-10 mice per group. Data are pooled from two independent experiments. 
While the small percentage of CFSE labelled immune cells in tumours indicates that immune cells in the blood did not impact on flow cytometric analysis of the tumour-immune infiltrate, it was still possible that the few blood cell in these samples consisted predominantly of selected cell types (such as B cells or T cells) and would therefore skew the analysis of these cell types in the tumour. To investigate if any cell types were preferentially labelled with CFSE in tumour samples, B cells, T cells and DCs were analysed individually.

When mice were injected with CFDA-SE, all cell types were found to be labelled equally in blood (Figure 3.13 A top panels \& B). The fraction of CFSE+ cells was much lower in tumours, but appeared to be similar among all the analysed cell types (Figure $3.13 \mathrm{~A}$ bottom panels \& C). Thus, leukocytes residing in the tumour vasculature do not appear to skew analysis of immune infiltrate in tumours by flow cytometry.

\subsubsection{DCs from tumours can be activated by adjuvants $e x$ vivo}

As DCs had been confirmed to infiltrate tumours and a range of adjuvants had been shown to activate BMDCs in vitro, we next wished to investigate whether DCs from tumours could respond to adjuvant stimulation ex vivo.

To address this question, total tumour cell suspensions, which included DCs, were incubated with different adjuvants ex vivo. After $24 \mathrm{~h}$, the phenotype of DCs was analysed. Exposure to adjuvants did not significantly alter the proportion of DCs among the total cells from the tumour cell suspension compared to controls that were cultured with medium only (Figure 3.14 A). All adjuvants induced up-regulation of MHC II and CD86 expression (Figure 3.14 B \& D). CD40 levels on DCs also increased after stimulation with all adjuvants except MSU and Poly IC (Figure 3.14 C).

As observed with BMDCs, MSU appeared to be the weakest adjuvant, as it only resulted in a small increase in MHC II and CD86 expression and failed to induce CD40 up-regulation (Figure 3.14 B-D). This was comparable to the effect of Poly IC, although Poly IC stimulation resulted in higher levels of MHC II than MSU exposure (Figure 3.14). LPS alone or 
in combination with Poly IC induced high levels of CD40 and MHC II and resulted in the most pronounced up-regulation of CD86. CpG, mycobacteria and mycobacteria + MSU also considerably increased expression of all three activation markers, but the extent of CD86 up-regulation was lower compared to LPS.

Therefore, DCs from tumours appeared to be activated by all tested adjuvants. However, the combination of stimuli did not enhance activation marker expression further compared to the individual adjuvants.

To further investigate the potential of adjuvants to activate DCs and other immune cells in the tumour cell suspensions, cytokines in the culture supernatant were also analysed. Only very low levels of IL-12p70 were detected and only MSU + M. smegmatis stimulation resulted in IL-12p70 levels significantly above the assay limit (Figure 3.15A). IL-10 levels were not significantly different from the detection threshold in any condition tested (Figure 3.15 B). In contrast, secretion of high amounts of the pro-inflammatory cytokines IL-6 and TNF $\alpha$ was induced with a range of adjuvants (Figure $3.15 \mathrm{C} \& \mathrm{D}$ ). CpG, M. smegmatis, BCG, mycobacteria + MSU and Poly IC + LPS induced substantial levels of IL-6 (Figure 3.15 C). The high cytokine levels observed in response to Poly IC + LPS are especially remarkable, as the single adjuvants induced no significant IL-6 secretion. The adjuvants that induced IL-6 release also stimulated secretion of $\mathrm{TNF} \alpha$ (Figure $3.15 \mathrm{D}$ ). However, high levels of $\mathrm{TNF} \alpha$ were only detected in response to mycobacteria or mycobacteria + MSU.

Taken together, these data indicate that DC in tumours can respond to adjuvant stimulation as evidenced by surface-marker up-regulation. In addition, the observed production of IL-6 and $\mathrm{TNF} \alpha$ in response to adjuvants indicates that DCs and/or other cell types in the tumour cell suspension were activated to induce a pro-inflammatory milieu. IL-10 could not be detected either in the absence or in the presence of adjuvants, indicating that IL-10 is not produced at significant levels in B16F1 melanomas. While production of the proinflammatory cytokines IL- 6 and $\mathrm{TNF} \alpha$ was readily induced by various adjuvants, IL-12 was not produced in large quantities and could in fact only be detected in samples stimulated with MSU + M. smegmatis. 
A.

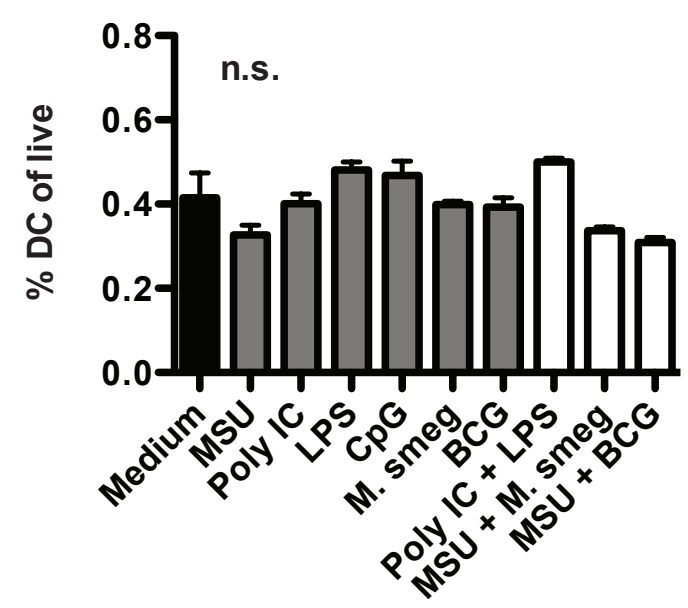

C.

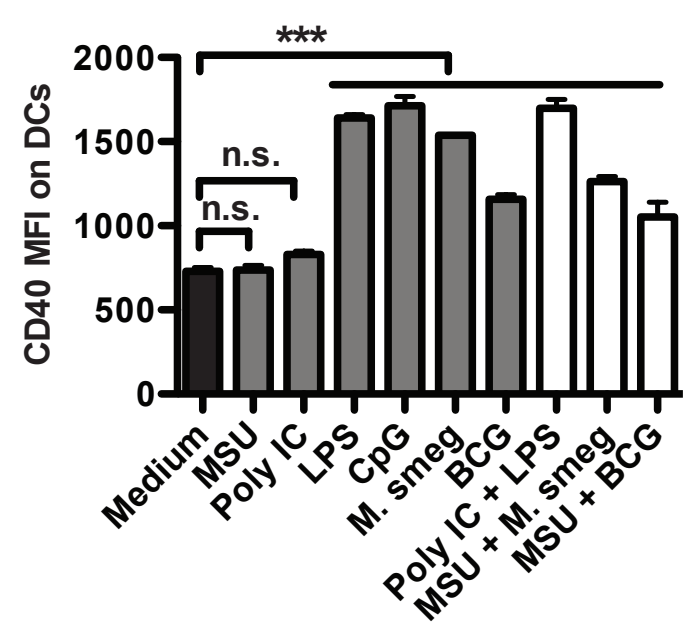

B.

MHC II

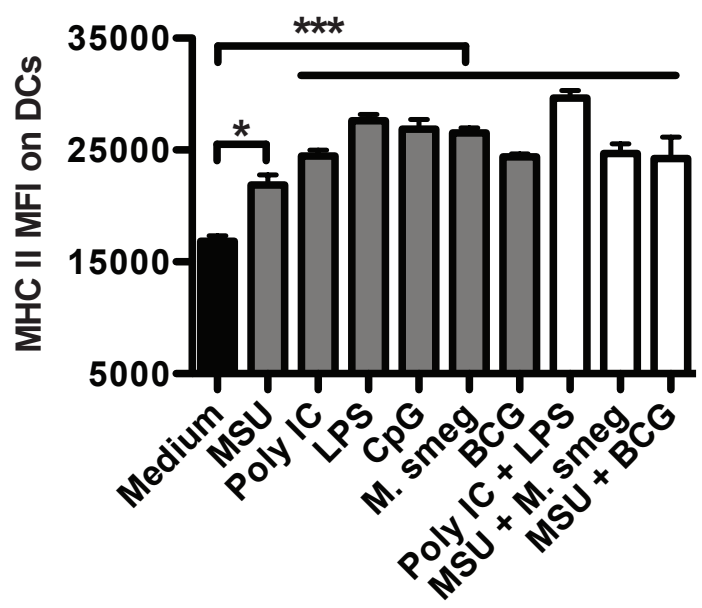

D.

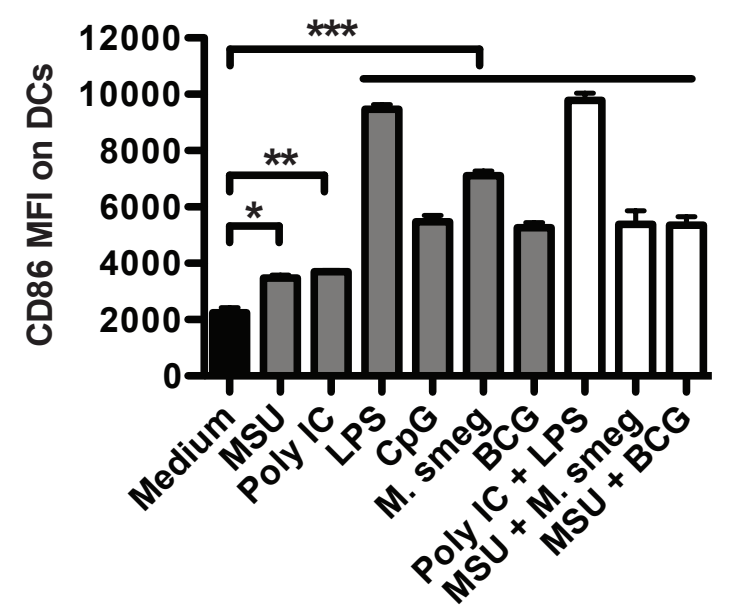

Figure 3.14: DCs from B16F1 tumours can be activated by exposure to adjuvants ex vivo. Day 14 B16F1 tumours were excised and processed into single cell suspension. $5 \times 10^{6}$ cells per well were then seeded into 6 -well plates and stimulated with adjuvants. The following concentrations were used: MSU $250 \mathrm{\mu g} / \mathrm{mL}$; LPS $100 \mathrm{ng} / \mathrm{mL}$; CpG $2 \mu \mathrm{g} / \mathrm{mL}$; Poly IC $25 \mu \mathrm{g} / \mathrm{mL}$; M. smegmatis (M. smeg) and BCG $0.33 \times 10^{6} \mathrm{CFU} / \mathrm{mL}$. After $24 \mathrm{~h}$ culture, cells were harvested and analysed by flow cytometry. DCs were identified as described in Figure 3.10. (A) The frequency of DCs among all live cells is shown. (B-C) The expression of MHC II, CD40 and CD86 on the surface of DCs is shown as median fluorescence intensity (MFI). Data from three samples per condition are graphed as mean $+\mathrm{SE}$. ${ }^{*} p<0.05,{ }^{* *} p<0.01,{ }^{* * *} p<0.001$ as assessed by the Kruskal-Wallis test with Dunn's post test; n.s. $=$ not significant. Data are from one of two experiments with comparable results. 
A.

IL-12p70

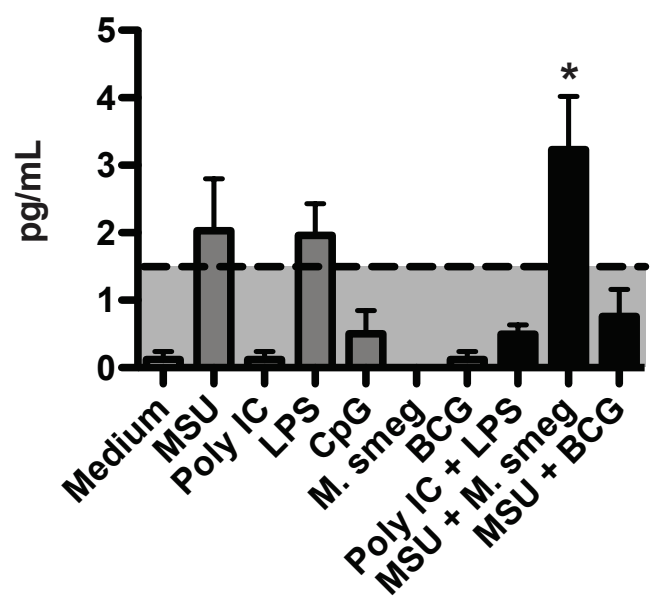

C.

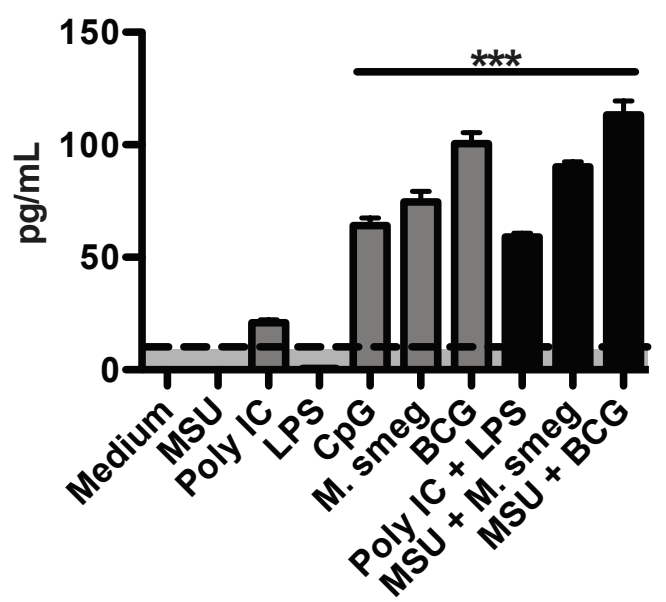

B.

IL-10

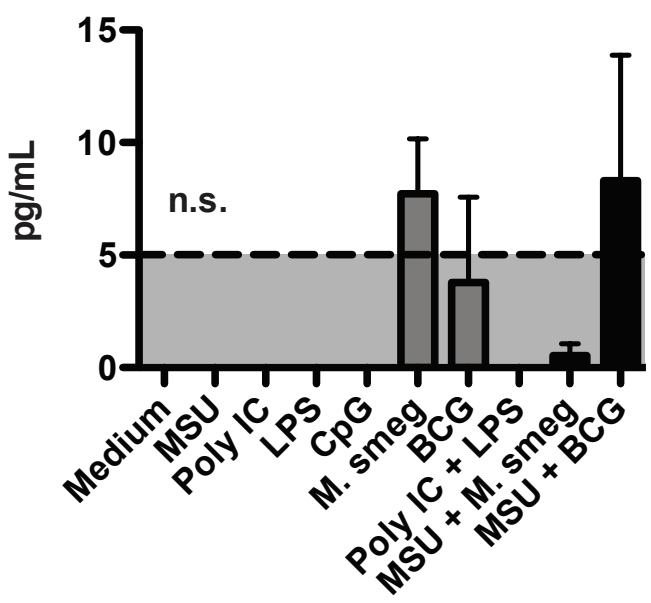

D.

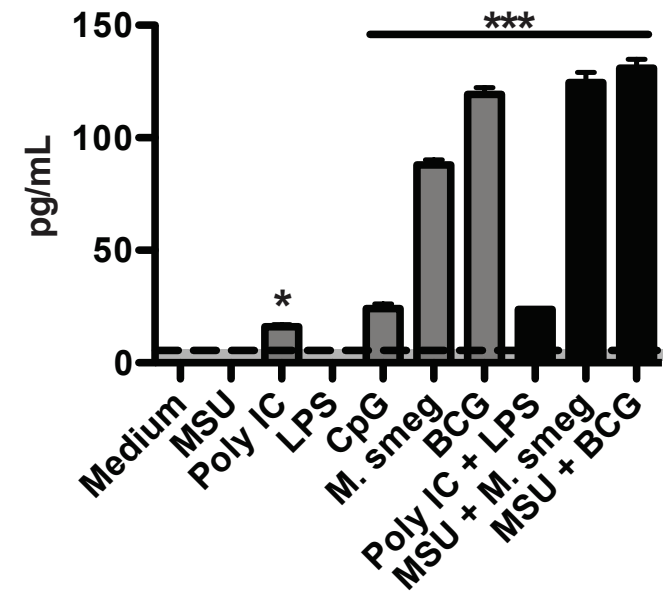

Figure 3.15: Adjuvant treatment induces secretion of pro-inflammatory cytokines in B16F1 tumours ex vivo. The supernatants of the B16F1 cell suspensions stimulated with adjuvants for $24 \mathrm{~h}$ ex vivo as described in Figure 3.14 were analysed for the indicated cytokines using a multiplex bead assay. (A-D) The concentrations of IL-12p70, TNF $\alpha$, IL- 6 and IL-10 are shown as mean + SE. The dotted line indicates the detection threshold of the assay. ${ }^{*} p<0.05$ and ${ }^{* * *} p<0.001$ compared to medium control as determined by Kruskal-Wallis with Dunn's post test; n.s. = not significant. Data are from one of two experiments with comparable results. 


\subsection{Discussion}

The aim of this chapter was to identify adjuvants for the activation of tumour-infiltrating DCs. To this end, adjuvants were tested for their ability to induce DC activation and polarise them towards secreting a Th1-inducing cytokine profile. A strong Th1 bias is thought to be important for the activation and full licencing of CTLs [274] and could also contribute to anti-tumour immunity by activating NK cells and tumour-infiltrating macrophages to become highly tumoricidal $[426,427]$.

\section{Timing of BMDCs activation by adjuvants}

In vitro experiments showed that BMDCs up-regulated activation marker expression in response to adjuvants. While several published studies have previously assessed BMDC phenotype and cytokine production in vitro after $20 \mathrm{~h}$ to $24 \mathrm{~h}$ of activation [306, 341, 428], different stimuli have been found to induce DC activation with different kinetics [420]. All the adjuvants compared in this study, however, induced some activation of both GM-CSF/IL-4 and Flt3L differentiated DCs already after $6 \mathrm{~h}$ of adjuvant stimulation, with maximal expression of the activation markers CD40 and CD86 observed after $20 \mathrm{~h}$ (Figures $3.1 \& 3.4$ ). Longer exposure of BMDCs to adjuvants did not enhance activation marker expression further and in the case of Flt3L-DCs actually appeared to lead to a slight decrease in CD40 and CD86 levels. In addition, prolonged adjuvant stimulation of DCs has been reported to induce DC exhaustion, with down-regulation of cytokine production, especially of IL-12, and increased DC cell death [380, 429]. Therefore, further experiments to compare different adjuvants were conducted after $20 \mathrm{~h}$ stimulation to enable concomitant assessment of surface marker up-regulation and cytokine production.

\section{Activation of GM-CSF/IL-4 DCs by adjuvants}

The ability of adjuvants to induce DC activation as assessed by up-regulation of the expression of MHC II and the co-stimulatory molecules CD40, CD80 and CD86 varied between 
adjuvants and DC types tested. In addition, some adjuvants only affected the expression of some surface markers.

GM-CSF/IL-4 DCs showed the highest levels of activation marker expression in response to LPS, M. smegmatis, MSU + M. smegmatis and LPS + Poly IC. The ability of the TLR4 ligand LPS to activate BMDCs is well established [64, 306, 341, 377, 420]. Mycobacteria express multiple PAMPs, enabling them to be recognised via TLR2, TLR4, TLR9 and NOD2 [328, 329], and M. smegmatis has previously been reported to activate monocyte-derived DCs in vitro [430].

The finding that Poly IC did not activate GM-CSF/IL-4 DCs is in line with the limited expression of its receptor TLR3 in this DC subset [368]. However, while some studies find that Poly IC fails to activate GM-CSF/IL-4 DCs [341], others report DC activation, which was dependent on the engagement of the cytosolic receptor MDA-5 rather than TLR3. [368]. These discrepancies may be due to the length of the Poly IC molecules used, as Poly IC of $>2 \mathrm{kbp}$ length is preferentially recongnised by MDA-5 and may therefore activate GM-CSF/IL-4 DCs via this receptor. Shorter Poly IC molecules, such as the low molecular weight preparation used in this study, bind to RIG-I [367] and not MDA-5, which may be the reason we did not see activation of GM-CSF/IL-4 DCs in response to Poly IC stimulation.

MSU also failed to activate GM-CSF/IL-4 DCs, despite a report in the literature showing that MSU can activate GM-CSF/IL-4 DCs [64]. This does not appear to be due to a lack of MSU internalisation, as DCs incubated with MSU showed higher SSC profiles, which is suggestive of the increased granularity following particle uptake. MSU sensing requires the NLRP3 inflammasome which upon activation recruits and activates caspase-1, which in turn processes IL-1 $\beta$ to its active form [431]. NLRP3 is highly expressed in macrophages and monocytes and has also been reported in GM-CSF/IL-4 BMDCs and Flt3L cDCs [359, 431]. In addition, Shi et al. reported CD86 up-regulation on GM-CSF/IL-4 DCs in response to MSU stimulation [64]. However, they did not state the dose of MSU used in their experiment. Although three different doses of MSU were used in this study, the highest dose may still have been insufficient to induce DC activation. 
MSU did, however, enhance GM-CSF/IL-4 DC activation when it was combined with M. smegmatis at doses at which neither MSU nor M. smegmatis alone had any effect on BMDC activation. In contrast, LPS + Poly IC did not enhance DC surface marker expression over the levels seen with LPS alone. As the observed levels of surface markers with LPS alone were already very high, this lack of further up-regulation with the addition of Poly IC may be due to the dose of $100 \mathrm{ng} / \mathrm{mL}$ LPS saturating the response.

Cytokine production from GM-CSF/IL-4 DCs reflected the levels of DC activation seen by surface marker expression. The pro-inflammatory cytokines IL- $1 \alpha$, IL- $1 \beta$, IL- 6 and TNF $\alpha$ as well as the Th1 promoting cytokine IL-12 were mainly induced by M. smegmatis, MSU + M. smegmatis, LPS and LPS + Poly IC. MSU + M. smegmatis enhanced the levels of all cytokines over those of the individual adjuvants at the same concentration, while LPS + Poly IC only increased IL-12 secretion compared to LPS alone. This is in line with data from Napolitani et al., who showed synergistic triggering of IL-12 production by adjuvant combinations and also reported induction of IL- $1 \beta$, IL- 6 and TNF $\alpha$ mRNA in response to LPS [377]. Mycobacteria have previously been reported to induce secretion of small amounts of IL- $1 \beta$ from BM macrophages [354]. It is well established that macrophages secrete IL- $1 \beta$ in response to MSU [353]. However, macrophages differentiated in vitro require priming with TLR ligands to induce formation of pro-IL1 $\beta$ to respond to NLR agonists such as MSU $[432,433]$. The MSU used in this study was tested for endotoxin contamination and found to be virtually endotoxin-free. It is conceivable that BMDCs require TLR signals in addition to NLR signals for release of IL- $1 \beta$ and this may be the reason why MSU stimulation induces IL-1 $\beta$ secretion only in combination with $M$. smegmatis. In addition to inducing pro-inflammatory cytokines and IL-12, M. smegmatis, LPS and LPS + Poly IC also stimulated secretion of considerable amounts of IL-10. In contrast, MSU + M. smegmatis enhanced only pro-inflammatory cytokines and IL-12 without inducing IL-10. Therefore, the addition of MSU may suppress IL-10 induction from mycobacteria and lead to a more pronounced pro-inflammatory cytokine milieu. 


\section{Activation of Flt3L DCs by adjuvants}

Flt3L DCs comprise three subsets, namely pDCs, CD24+ cDCs and CD11b+ cDCs [422], which differ in their responsiveness to different adjuvants. pDCs have been shown to express high levels of TLR7 and TLR9, but low amounts of TLR2, TLR3, TLR4 and NLRP3 [291, 359]. In line with this receptor expression pattern, they were not activated by the TLR4 agonist LPS, the TLR3 agonist Poly IC or the NLRP3 stimulus MSU, but up-regulated activation markers in response to the TLR9 agonist CpG. Mycobacteria, which provide TLR2, TLR4, TLR9 and NOD2 ligands, failed to activate pDCs [328, 329]. However, the combination of MSU + M.smegmatis induced strong activation of pDCs and even higher activation marker expression than CpG. Mycobacteria may provide the TLR signals necessary to enable cytokine production in response to the NLRP3 stimulus MSU, similar to the requirement for pre-stimulation of macrophages with TLR to enable NLR responses [432, 433]. In addition, synergy between TLR and NLR signalling has previously been described for NOD1 and NOD2 ligands and TLRs both in B cells and DCs [357, 434] and synergistic signalling induced by mycobacterial ligands + MSU may result pDC activation, while the signals provided by each individual stimulus were not sufficient.

In contrast to Flt3L pDCs, both CD24+ and CD11b + cDCs responded to all adjuvants tested with up-regulation of activation markers. MSU led to the lowest level of cDC activation overall, but had varied effects on the different activation markers. MSU exposure resulted in less pronounced up-regulation of CD40 and CD86 than other adjuvants, but induced CD80 to levels comparable to LPS, Poly IC, CpG and mycobacteria. It also stimulated MHC II expression to very high levels, especially in CD11b+ DCs. All other individual adjuvants induced considerable up-regulation of all activation markers in both cDC subsets. This is consistent with expression of the respective TLRs and NLRP3 in cDCs [291,359] and reports of responsiveness of Flt3L DCs to LPS, CpG and Poly IC [306, 428]. Cytokines secreted by one subset directly responding to adjuvant stimulation may also have contributed indirectly to the activation of other DCs in the same culture. As the response of the three DC subsets to the different adjuvants was in line with their reported receptor expression, indirect activation by cytokines does not appear to be a major factor in these experiments. 
However, to assess the contribution of indirect activation via other DCs in the same culture, the three Flt3L DC subsets would need to be sorted prior to adjuvant stimulation.

Combination of MSU and mycobacteria enhanced expression of CD40 and CD80, but had little effect on CD86 and MHC II expression, which were already expressed at very high levels in response to the individual stimuli. Addition of LPS + Poly IC to DC cultures somewhat enhanced CD40, CD80 and CD86 expression. However, the increase in activation marker expression with all combinations was not synergistic, but rather additive.

The induction of cytokine secretion from Flt3L DCs also largely reflected the surface activation profiles, with all adjuvants apart from MSU and Poly IC inducing secretion of high levels of TNF $\alpha$ and IL-6. IL-12 was also induced by most stimuli and Poly IC, MSU + M. smegmatis and LPS + Poly IC activation led to the most significant production of IL-12. In line with these findings, Brawand et al. examined pDCs and cDCs from Flt3L cultures separately and found that both subsets produced some IL-12 in response to Poly IC and CpG, but LPS induced IL-12 mainly from cDCs [428]. IL-12 secretion after combined stimulation with LPS + Poly IC was increased 2-fold over the single adjuvants, indicating that this was an additive effect of the higher dose of adjuvant, rather than a synergistic increase in IL-12 production as reported from adjuvant combinations in GM-CSF/IL-4 DCs [377]. Alternatively, the increase in IL-12 may be due to production of this cytokine by different DC subsets in response to LPS and Poly IC. Interestingly, LPS failed to induce IL-1 $\beta$ production in Flt3L DCs and only mycobacteria alone or in combination with MSU induced significant amounts of this cytokine.

Overall, the cytokine levels detected in Flt3L cultures were lower compared to what was observed with GM-CSF/IL-4 DC supernatants. This is likely related to the total number of DCs in each well, as 8-10 x $10^{5}$ DCs per well could be recovered from GM-CSF/IL-4 cultures, while Flt3L cultures yielded only $2-3 \times 10^{5}$ DCs per well.

Taken together, the results from in vitro activated BMDCs revealed large differences in the responsiveness of the different DC types to adjuvants, which were mainly linked to the differential receptor expression in these cells [291, 359, 420-422]. LPS + Poly IC induced the highest level of activation marker expression and cytokine production in Flt3L cDCs 
and GM-CSF/IL-4 DCs. MSU + M. smegmatis also induced pronounced activation in both Flt3L cDCs and pDCs and also activated GM-CSF/IL-4 DCs without inducing secretion of IL-10. These adjuvant combinations therefore seemed most promising for in vivo experiments.

\section{Characterisation of tumour-infiltrating DCs}

DCs are associated with both human and murine tumours [311-313], but the degree of DC infiltration varies considerably with the type of tumour. Ovarian cancer, for example, recruits many DCs to the tumour site and in 8-week old tumours DCs constitute $30 \%$ of the total cell mass [435]. In contrast, prostate tumours in TRAMP mice show very low DC infiltration [436].

We analysed the infiltration of DCs and their phenotype in murine B16F1 melanomas to determine whether it would be feasible to target these tumour-infiltrating DCs by in vivo application of adjuvants. DCs were identified by flow cytometry as CD45+ CD11c+ MHC II+ cells and represented 20-30\% of the tumour-infiltrating immune cells. Some of the tumour-infiltrating DCs expressed co-stimulatory molecules, but the extent of CD40, CD86 and MHC II expression was much lower compared to LN DCs. This is not surprising, as tissue resident DCs generally display a non-activated phenotype compared to LN DCs [294]. Furthermore, tumours can also secrete cytokines and growth factors that inhibit DCs and render them immuno-suppressive [324, 437, 438].

When the phenotype of the tumour-infiltrating DCs was examined in more detail, $80 \%$ were found to be CD11b+ and $5 \%$ were B220+. MHC II expression was much lower on the B220+ population compared to the CD11b+ DCs. These results are similar to the analysis of the immune infiltrate of the B16.OVA melanoma model conducted by Stoitzner et al. [314]. They report that $23 \%$ of the tumour-immune infiltrate consists of CD11c+ DCs, which are $80 \% \mathrm{CD} 11 \mathrm{~b}+$. In addition, they find a small population of $\mathrm{B} 220+\mathrm{pDCs}$ and virtually no Langerin+ DCs in these tumours. In the steady-state, pDCs reside mainly in lymphoid tissues [281]. However, pDCs can be recruited to sites of inflammation [439] and have previously been reported to infiltrate other tumours [311, 312, 316]. 
Although DCs constituted up to $30 \%$ of the immune infiltrate in B16F1 tumours, overall immune cells accounted for only $5 \%$ of live cells in these tumours, so that the total frequency of DCs was only $1.5 \%$ of the live cells in tumours. Monocytes also accounted for $30 \%$ of the immune infiltrate, while macrophages, B cells, T cells and NK cells each represented only $5-10 \%$ of the immune cells in tumours. While the frequency of B cells and T cells was comparable to the results of Stoitzner et al., they found $40 \%$ of the immune infiltrate to be macrophages [314]. This may be mainly due to the identification of macrophages solely on the basis of F4/80 expression in this study, which also showed $40 \%$ overlap with the CD11c+ DC population, while in the current analysis only non-DC, F4/80 ${ }^{\text {high }}$ FSC $^{\text {high }}$ cells were identified as macrophages.

\section{Validation of flow-cytometry to assess the tumour immune infiltrate}

The unexpected presence of B cells and high infiltration of monocytes raised concerns that the analysis of tumour-infiltrating immune cells may be skewed by contaminating blood cells. Protocols for flow cytometric study of other organs such as lung or liver frequently use perfusion with saline to flush blood cells from these organs and thus exclude them from the sample [440, 441]. Perfusion of B16F1 tumours, however, was not successful, as tumours either ruptured or were not properly perfused. This is likely due to the poor vasculature combined with central necrosis in these tumours [442]. In addition to their generally soft texture, this makes B16F1 melanomas highly susceptible to breaking apart during perfusion [442]. Therefore, tumours were prepared for flow cytometry without perfusion.

To assess the degree of contamination by blood leukocytes, mice were injected with CFDASE to label all blood cells with CFSE prior to the excision of tumours. While $>95 \%$ of blood cells were labelled with CFSE, only $15 \%$ of the leukocytes in tumours were CFSE+. Furthermore, all assessed cell types were affected equally, thus excluding a skewing of the analysis of the relative infiltration of different immune cell types in tumours by blood leukocytes. The percentage of CFSE + cells in tumours was similar to the staining observed in LNs in this study and also in a published report [425]. Labelling in spleens was much 
higher with $60 \%$ CFSE+ leukocytes. This may be due either to leakage of the dye from blood vessels into the organ or to trafficking of immune cells from the blood into the organ. Thus, this method may even overestimate the amount of blood contamination in tumours, as some staining may be due to leakage or recent infiltration of immune cells from the blood to the tumour site. These data indicate that blood leukocytes do not affect the analysis of the tumour immune-infiltrate by flow cytometry and validate this method for further experiments.

\section{Activation of tumour-infiltrating DCs ex vivo}

The DCs in B16F1 tumours expressed low levels of activation markers and the antigenpresentation molecule MHC II. Previous studies indicate that tumour-infiltrating DCs can present antigen to activate anti-tumour $\mathrm{T}$ cell responses $[311,319]$. However, DCs in the study by Chaux et al. were purified and therefore removed from any suppressive factors made by the tumour cells before they were assessed for their ability to stimulate $\mathrm{T}$ cell proliferation ex vivo [319]. While Preynat-Seauve and co-workers investigated T cell proliferation in vivo, they did this using purified, antigen-pulsed tumour-infiltrating DCs that were injected into tumour-free mice [311], again separating the DCs from any ongoing influence of the tumour. Another study also reported that DCs only stimulated naïve T cell proliferation ex vivo when they were loaded with antigen and exposed to powerful activation cocktails (TNF $\alpha$ + anti-IL-10 + CpG) [435]. In contrast, stimulation with the TLR4 ligand LPS was not sufficient to overcome the inability of tumour-infiltrating DCs to stimulate T cell responses ex vivo [314]. It was therefore important to determine, whether tumour-infiltrating DCs could still respond to all or selected adjuvants ex vivo in the presence of tumour cells.

Complete tumour cell suspensions cultured with adjuvants for $24 \mathrm{~h}$ showed activation of DCs in response to all tested stimuli, as assessed by activation marker up-regulation. LPS and LPS + Poly IC induced the highest levels of CD86 and also resulted in increased expression of CD40 and MHC II. MSU and Poly IC were the weakest adjuvants, as they only induced an increase in MHC II with limited up-regulation of CD86 and no enhancement of CD40 expression. This response pattern is similar to what was observed upon adjuvant stimulation 
of GM-CSF/IL-4 DCs. Together with the high levels of CD11b expression, this indicates that tumour-infiltrating DCs may be similar to inflammatory monocyte-derived DCs, as modelled in the GM-CSF/IL-4 cultures [306]. In addition, these results suggest that DCs in tumours may still respond to adjuvants. However, as the tissue structure was disrupted in the ex vivo cultures, interactions between immune and tumour cells may have been altered and thus the DCs may have been less suppressed than in the intact tumours.

Surface marker expression is not a good correlate of the immuno-stimulatory properties of DCs, as tolerogenic DCs can also express high levels of these molecules [284]. When cytokine production was analysed, high levels of IL-6 and TNF $\alpha$ were found in tumour cell suspensions stimulated with CpG, mycobacteria with or without MSU and Poly IC + LPS. However, LPS alone, which induced considerable surface marker up-regulation on DCs, failed to induce any significant cytokine production. IL-12 was generally very low and the only significant amount was detected in cultures stimulated with MSU + M. smegmatis. Overall, the detected cytokine levels were about 10-fold lower than what had been observed in BMDC cultures. This is in line with DCs in tumours representing ca. $30 \%$ of the $5 \%$ immune infiltrate, and thus total tumour cultures containing about 75,000 DCs as compared to $>1$ million DCs in BMDC cultures. In addition, it is possible that other immune cells present in these mixed cultures consumed cytokines that were produced by DCs, thus leading to low or undetectable levels. On the other hand, DCs may not have been the only source of the detected cytokines, as macrophages and monocytes also express receptors for the tested adjuvants and may have contributed to the cytokine levels observed in total cultures.

Taken together, these results indicate that DCs and possibly other cell types in tumours retain the ability to respond to adjuvant stimulation by increasing expression of co-stimulatory molecules and production of pro-inflammatory cytokines. This suggests that adjuvant treatment at the tumour site may be able to overcome DC unresponsiveness and enable them to initiate successful anti-tumour immune responses. 


\section{Conclusions}

All adjuvants assessed in this study activated BMDCs. However, the levels of co-stimulatory molecules and cytokine production induced varied both among different adjuvants and DC populations. Combination of adjuvants had additive rather than synergistic effects. LPS + Poly IC appeared to induce the most potent activation of GM-CSF/IL-4 DCs, while MSU + M. smegmatis activated all three subsets of steady-state DCs and led to production of proinflammatory cytokines and IL-12 without inducing IL-10.

DCs were found to comprise $30 \%$ of the immune infiltrate in murine B16F1 melanomas, were mainly CD11b+ and showed low expression of co-stimulatory molecules and MHC II. Monocytes were also found at a high frequency in these tumours.

Flow cytometry as a method to study tumour-infiltrating immune cells was validated by CFSE-labelling of all blood cells, showing that blood leukocytes did not affect the analysis.

Lastly, DCs from tumours were found to retain responsiveness to adjuvant stimulation ex vivo. A range of adjuvants induced activation-marker up-regulation on DCs and production of pro-inflammatory cytokines from tumour cell suspensions.

In summary, these results indicate that targeting DCs at the tumour site with adjuvants and polarising them towards an immuno-stimulatory phenotype may be a valuable strategy to improve anti-tumour immunity. 

Chapter 4

Treatment with specific adjuvants

delays tumour growth and activates DCs in vivo 


\subsection{Introduction}

DCs have been identified in the immune infiltrate of many murine and human tumours [311313]. While some studies found that tumour-infiltrating DCs can present antigen ex vivo or in vitro to activate anti-tumour $\mathrm{T}$ cell responses [311,319], later research showed that they are incapable of presenting tumour-derived antigen in the absence of ex vivo antigen loading [314].

This failure of tumour-infiltrating DCs to activate $\mathrm{T}$ cells has been largely attributed to their insufficient maturation [314]. Tumours can secrete cytokines and growth factors such as IL-10 and VEGF that inhibit DCs [324], thus inducing DCs to suppress immunity and thereby to favour tumour growth $[222,326]$. However, DCs in regressing tumours express maturation markers and co-localise with cytotoxic T cells [4]. Furthermore, tumour-induced inhibition of DCs can be overcome by the addition of maturation factors [322, 443].

Adjuvants are known for their ability to activate and mature DCs [444]. In the previous chapter, both bone-marrow derived DCs and ex vivo stimulated tumour-infiltrating DCs were confirmed to respond to adjuvant exposure with up-regulation of activation markers and production of inflammatory cytokines. However, the response to adjuvant administration in vivo may differ from in vitro results, as bio-availability, stability and uptake by other cell types may reduce the exposure of DCs to the adjuvant. This is reflected in different dosing reported in the literature, where $1 \mu \mathrm{g} / \mathrm{mL}$ CpG was effective in activating DCs in vitro [341], but $100 \mu g$ to $180 \mu \mathrm{g}$ CpG given in several injections of $100 \mu \mathrm{L}$ were required to achieve sufficient DC activation to promote tumour growth inhibition in vivo [403, 445].

While a range of adjuvants had been identified to activate DCs in vitro, fewer have been examined for their ability to induce anti-tumour responses in vivo. BCG has been used for the treatment of bladder cancer in patients since 1976 [9] and was even found to be superior to chemotherapy [10], but little is known about its mechanism of action or its effects on DCs in vivo [401]. Several reports show anti-tumour effects of CpG administration [11, 403, 445]. In response to CpG increased DC migration to dLNs and up-regulation of activation markers on DCs is reported $[11,445]$. However, the relative contribution of tumour-infiltrating and LN resident DCs as well as the importance of different DCs subsets, or even other antigen- 
presenting cells such as macrophages, remains to be determined.

DC subtypes show varying and opposing roles in the tumour-response. In ovarian cancer and breast cancer, pDC infiltration has been linked with poor prognosis, whereas myeloid DCs did not correlate with disease outcome $[312,316]$. In addition, DC subsets differ in their ability to induce cytotoxic $\mathrm{T}$ cell responses to externally acquired antigen and to activate CD4 T cells $[258,259]$.

Furthermore, the effect of multiple adjuvant administrations, as necessary to achieve anti-tumour effects in vivo [403], on DC activation is poorly defined. Repeated adjuvant exposure may increase DC activation and migration, or it may also cause tolerance via feedback regulation [446]. DC exhaustion, death and down-regulation of cytokine production has also been reported after prolonged LPS exposure [380]. 


\subsection{Aims}

In Chapter 3, it was established that murine melanomas were infiltrated by DC that could be activated by adjuvants ex vivo. The purpose of the experiments described in this chapter was to establish which adjuvants were effective in inhibiting tumour growth in vivo and to determine whether anti-tumour activity correlated with activation of DCs. The hypothesis was that adjuvants would activate tumour-infiltrating DCs and induce them to migrate to the tumour-draining LNs, where the DCs would then initiate the anti-tumour immune response.

The specific aims were:

- To asses whether peri-tumoral administration of some or all studied adjuvants had anti-tumour activity in vivo

- To investigate whether adjuvant administration activated antigen-presenting cells, in particular DCs in tumours

- To assess whether adjuvant treatment increased the number or activation of DCs in the tumour-draining LNs and which DC subpopulations were affected

- To investigate which systemic or local cytokines were induced by adjuvant treatment

- To determine whether multiple adjuvant treatments had sustained effects on DCs in tumours or draining LNs 


\subsection{Results}

\subsubsection{Selected adjuvants have anti-tumour activity in vivo}

To assess the anti-tumour activity of adjuvants in vivo, the well established transplantable murine melanoma B16F1 was used. This tumour is derived from a spontaneous melanoma in immuno-competent mice that grows quickly in syngeneic wild-type hosts and can thus be considered a poorly immunogenic tumour that has escaped the immune response. This is similar to the situation in patients with progressing malignancy. B16 tumours therefore are a good model to develop immunotherapies with potential clinical application. Tumour cells were injected s.c. into the flank of immuno-competent, syngeneic wild-type C57BL/6 mice (C57 mice). After tumours had become established to a palpable size, adjuvant treatment was administered at the tumour site.

Peritumoral administration of $\mathrm{CpG}$ has previously been reported to be superior to intravenous or distant subcutaneous application [445]. In addition, peritumoral treatment was chosen over intratumoral injection. The rapid growth of B16 mouse melanomas is associated with progressively worse vascularisation and increasing central necrosis [442]. In addition, tumour infiltrate and in particular DCs are mainly localised in the periphery of the tumours [447]. Therefore, peritumoral adjuvant treatment was deemed best to maximise exposure of immune cells to the adjuvants while minimising side effects through rupture of tumour tissue.

Four peritumoral adjuvant injections were administered every second day as several studies reported a requirement for multiple treatments to reduce tumour growth by adjuvant administration in a range of tumour models $[11,12,403]$. As Poly IC doses of $10 \mu \mathrm{g}$ to $200 \mu \mathrm{g}$ have been used for in vivo administrations [12, 345], an intermediate dose of $50 \mathrm{\mu g}$ per administration was chosen. Kunikata et al. found that 6 injections of $10 \mu \mathrm{g}$ CpG or 3 injections of $30 \mu \mathrm{g}$ CpG were effective to reduce B16 melanoma growth [403]. As four administrations were used in this study, $20 \mu \mathrm{g}$ CpG were given per administration. Mycobacteria were injected at $2-4 \times 10^{6} \mathrm{CFU}$, and LPS was given at $20 \mathrm{\mu g}$ per administration on the basis of published literature [448]. 
PBS

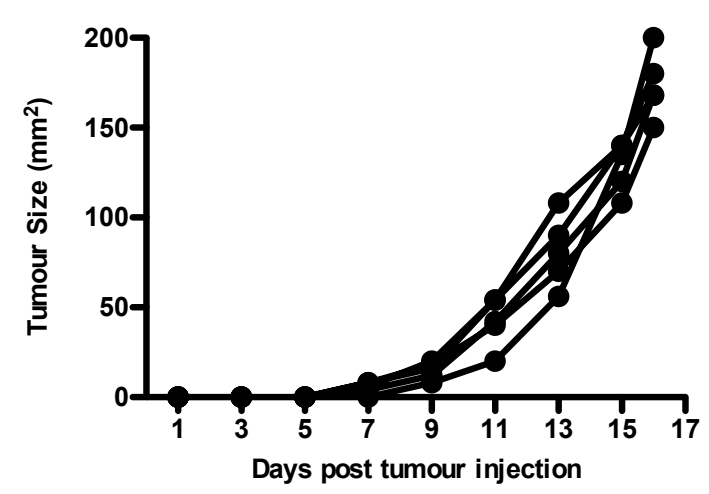

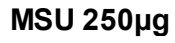

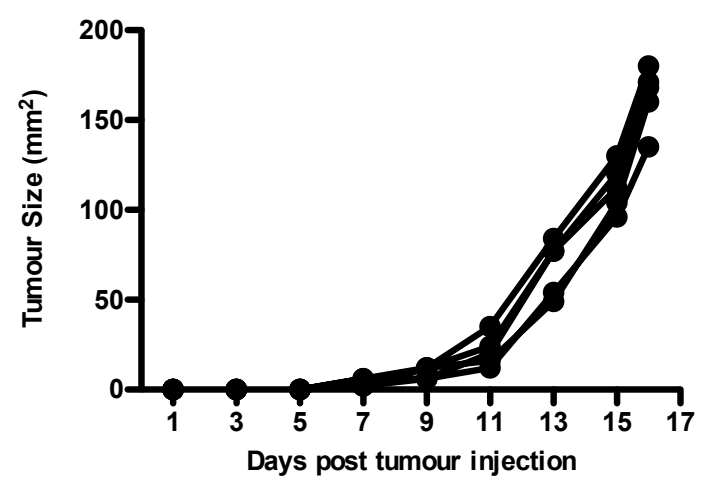

MSU $100 \mu \mathrm{g}$

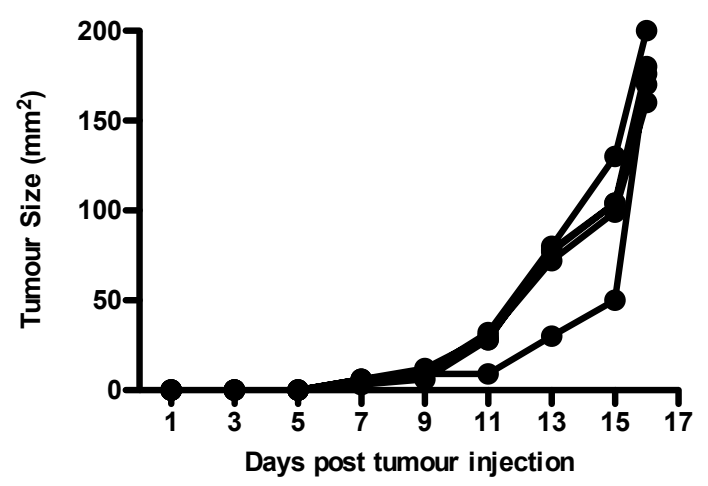

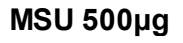

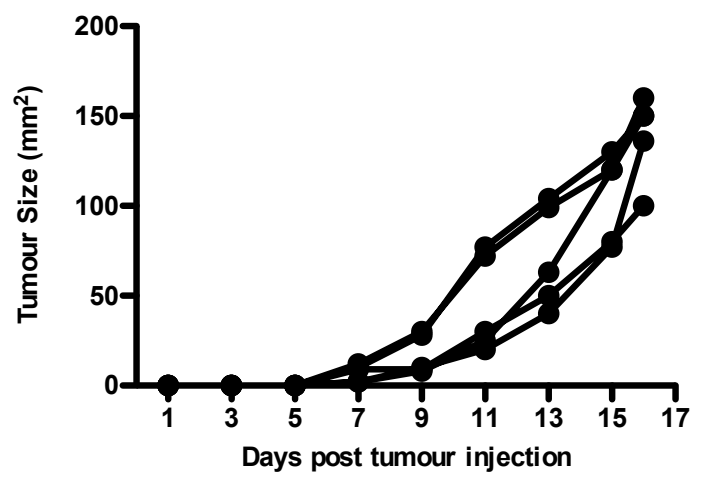

Figure 4.1: Effect of different MSU doses on B16F1 tumour growth. Groups of 5 C57 mice bearing established B16F1 tumours were injected around the tumour with $100 \mathrm{\mu g}$, $250 \mathrm{\mu g}$ or $500 \mathrm{\mu g}$ MSU on day 7, 911 and 13. Tumour growth as measured with callipers is shown for individual mice. Mean tumour size at day 15 and 16 is significantly different $\left({ }^{* *} p<0.01\right)$ between mice treated with $500 \mu \mathrm{g}$ MSU compared to PBS controls as assessed by two-way ANOVA with Bonferroni's post test.

MSU crystals, thought to be an endogenous danger signal [64], have not previously been used as an adjuvant to treat murine tumours. Therefore a titration using $100 \mu \mathrm{g}, 250 \mu \mathrm{g}$ or $500 \mu \mathrm{g}$ for each of the four administrations was performed to determine whether MSU had an anti-tumour effect. $100 \mu \mathrm{g}$ and $250 \mu \mathrm{g}$ MSU did not alter the growth of B16F1 tumours (Figure 4.1). Tumour growth of mice treated with $500 \mu \mathrm{g}$ MSU per administration was more variable and in an initial experiment these mice had slightly smaller tumours on day 15 and 16 compared to PBS controls (Figure 4.1). However, when data from 3 experiments analysing the effect of peri-tumoral treatments with $500 \mu \mathrm{g}$ MSU were compiled, no difference in tumour growth was observed between saline controls and MSU treated mice (Figure 4.2 A, top left panel). This suggests that the slight difference observed in the first experiment 
Table 4.1: Number of mice per treatment group and experiment for B16F1 tumour growth

\begin{tabular}{|c|c|c|c|c|c|}
\hline & \multicolumn{5}{|c|}{ Experiment number } \\
\hline & 38 & 40 & 50 & 52 & 56 \\
\hline PBS & 5 & 5 & 5 & 5 & 5 \\
\hline LPS & & & 5 & 5 & \\
\hline M. smegmatis & 5 & & & 5 & \\
\hline MSU & 5 & 5 & 5 & & \\
\hline BCG & 4 & & & 5 & \\
\hline $\mathrm{MSU}+\mathrm{BCG}$ & & & & 5 & \\
\hline CpG & 5 & & & 5 & \\
\hline Poly IC & & & 5 & 5 & 5 \\
\hline MSU + M. smegmatis & 5 & 5 & & 5 & 5 \\
\hline Poly IC + LPS & & & 5 & 5 & \\
\hline Poly IC + M. smegmatis & & & 5 & & \\
\hline Poly IC + MSU & & & 5 & & \\
\hline
\end{tabular}

may have been due to individual variation between mice rather than representing a true benefit from MSU treatment. Overall, MSU alone appears to have no effect in the B16F1 tumour model.

Several experiments were performed to compare the effects of saline, MSU, LPS, CpG, Poly IC, M. smegmatis and BCG on B16F1 tumour growth. As there is increasing evidence that combination of stimuli can enhance DC activation [377], a range of adjuvant combinations was also tested. The number of mice in each treatment group and experiment is summarised in Table 4.1. Figure 4.2 shows tumour growth and survival. While tumours became palpable and treatment was initiated at days 7 to 9 , days 0 to 2 were set as day 0 so that the treatment start day became "day 7" for all experiments for easier comparison.

Peri-tumoral injection of $4 \times 10^{6}$ live CFU of M. smegmatis likewise did not affect B16F1 tumour growth (Figure 4.2 A, top left panel). Strikingly, the combination of $2 \mathrm{x} 10^{6}$ live CFU of M. smegmatis and $250 \mu \mathrm{g}$ MSU significantly delayed tumour growth and prolonged survival (Figure 4.2 A, top left panel \& 4.2 B). This effect was specific to M. smegmatis, as BCG had no effect either alone or in combination with MSU (Figure $4.2 \mathrm{~A}$, top right panel \& $4.2 \mathrm{~B}$ ). 
A.
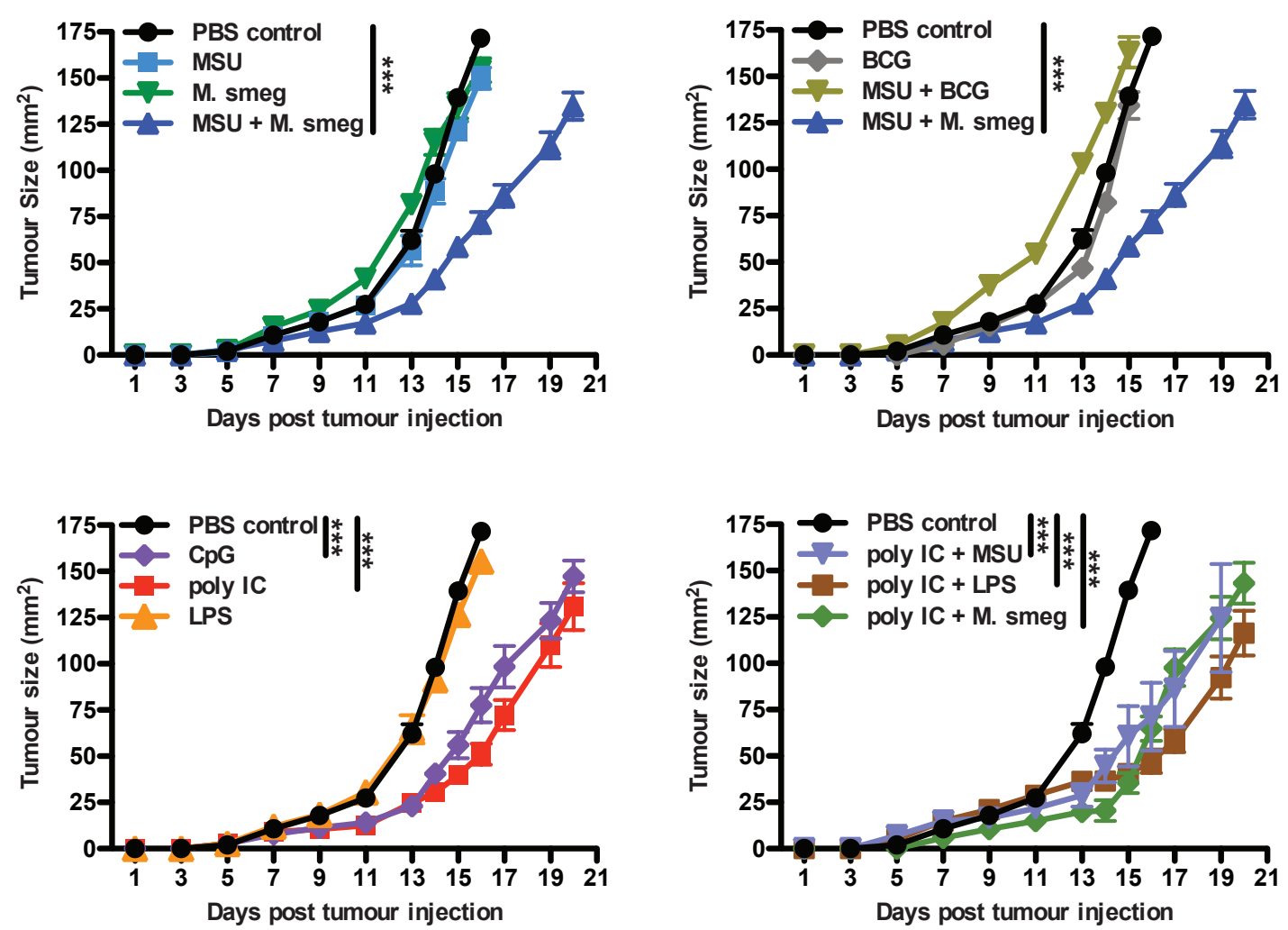

B.

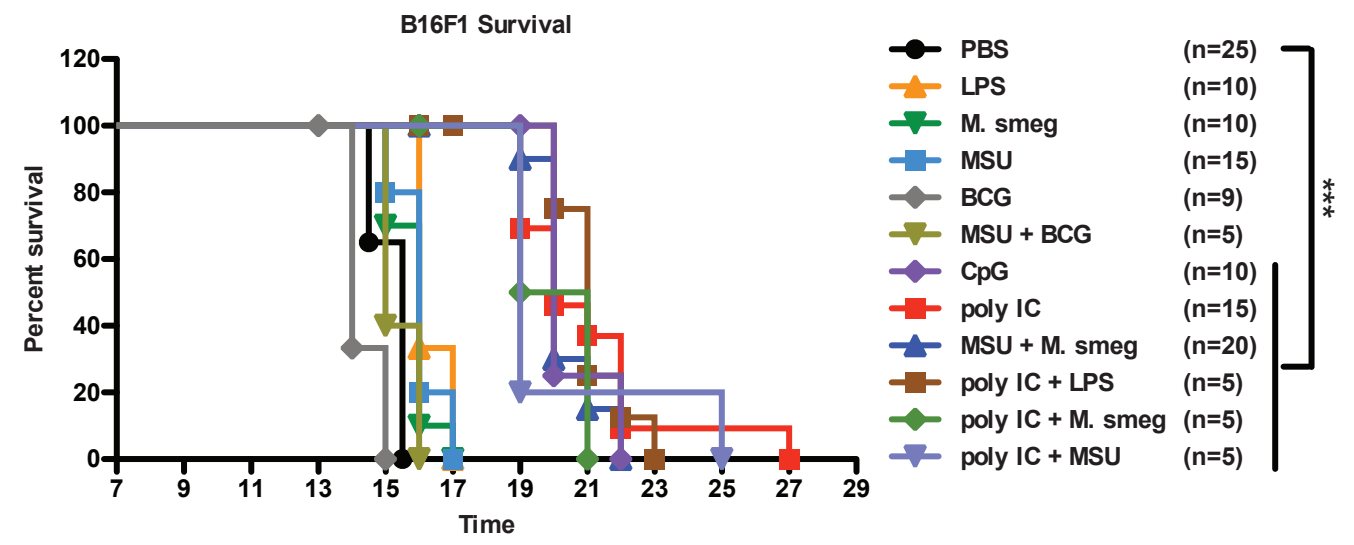

Figure 4.2: Some adjuvants delay tumour growth when administered peritumorally. C57 mice bearing established B16F1 tumours were injected around the tumour with the indicated adjuvants on day 7, 911 and 13. (A) Tumour growth of 5-25 mice per group is shown as mean $\pm \mathrm{SE}$. Differences between treatment groups as assessed to two-way ANOVA with Bonferroni's post test are significant from day 11 and are indicated by ${ }^{* *} p<0.01 * * * p<0.001$. (B) Survival of the B16F1 bearing, adjuvant-treated mice in (A) is depicted. ${ }^{* * *} p<0.001$ compared to PBS control according to Log-Rank-Test with Bonferroni's adjustment for multiple comparisons. Data are pooled from 5 independent experiments as illustrated in table 4.1. 
In line with reports in the literature [12, 403], $20 \mu \mathrm{g}$ CpG or $50 \mu \mathrm{g}$ Poly IC showed anti-tumour activity, delaying tumour growth and increasing survival to a similar degree as MSU + M. smegmatis (Figure 4.2 A, left panels \& $4.2 \mathrm{~B}$ ). Administration of $20 \mu \mathrm{g}$ LPS per treatment had no effect on tumour growth or survival (Figure 4.2 A, bottom left panel \& 4.2 B). Furthermore, combinations of $50 \mu \mathrm{g}$ Poly IC with $250 \mu \mathrm{g}$ MSU, $20 \mu \mathrm{g}$ LPS or $2 \mathrm{x} 10^{6}$ live CFU of M. smegmatis did not improve the tumour growth delay over the effect observed with $50 \mathrm{\mu g}$ Poly IC alone (Figure $4.2 \mathrm{~A}$, bottom right panel \& $4.2 \mathrm{~B}$ ).

In summary, none of the adjuvants tested could halt tumour growth or induce tumour regression of B16F1 melanomas when administered around the established tumours. However, treatment with Poly IC, CpG or a combination of MSU + M. smegmatis resulted in a significant delay of tumour growth and prolonged survival.

\subsubsection{Effect of one treatment with adjuvants on tumour- infiltrating APCs}

Poly IC and MSU + M. smegmatis, which were effective in delaying tumour growth, as well as the ineffective adjuvants LPS, M. smegmatis and MSU were chosen for analysis of their effects on the immune system in vivo. We compared the immune responses elicited by effective and ineffective adjuvants to dissect required responses from unnecessary or even detrimental effects of the adjuvants. CpG was excluded from further study, as its success in clinical trials has been reported to be very limited [14] and the expression of its receptor TLR9 differs considerably between mouse and human DC subpopulations [291, 449].

To investigate whether adjuvants activated DCs or other APCs in the tumour, the composition of the immune infiltrate and activation marker expression following a single adjuvant administration was analysed by flow cytometry. A common gating strategy was used in all staining panels to identify CD45+ immune cells in the tumour cell suspensions (Figure $4.3 \mathrm{~A}$ ). First, singlet events were gated on FSC and SSC area versus height (Figure 4.3 A, left panels). Subsequently, live cells in tumours were gated on the basis of viability-dye exclusion (DAPI-) and immune cells were identified as CD45 positive (Figure $4.3 \mathrm{~A}$, right panels). 
A.
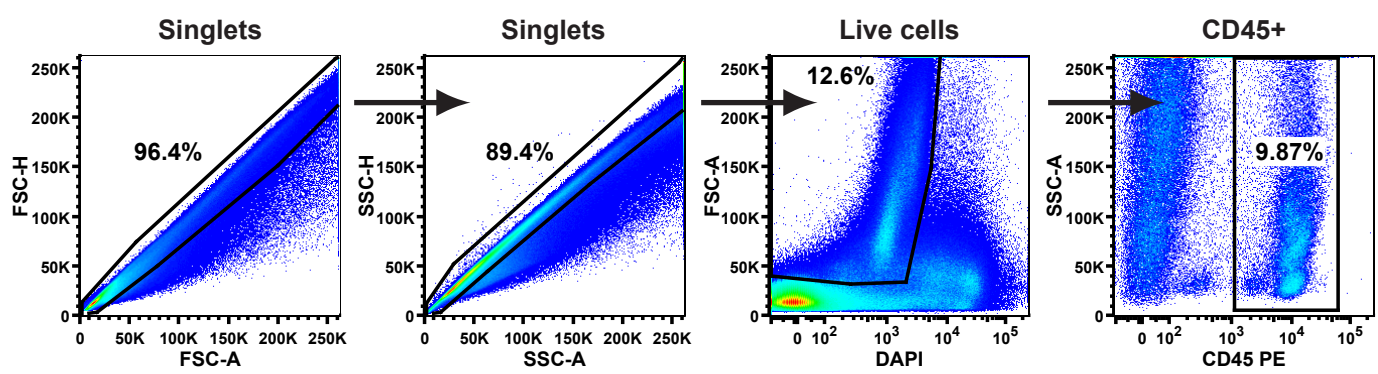

B. CD45 PE

\section{CD45 PE-TR+}
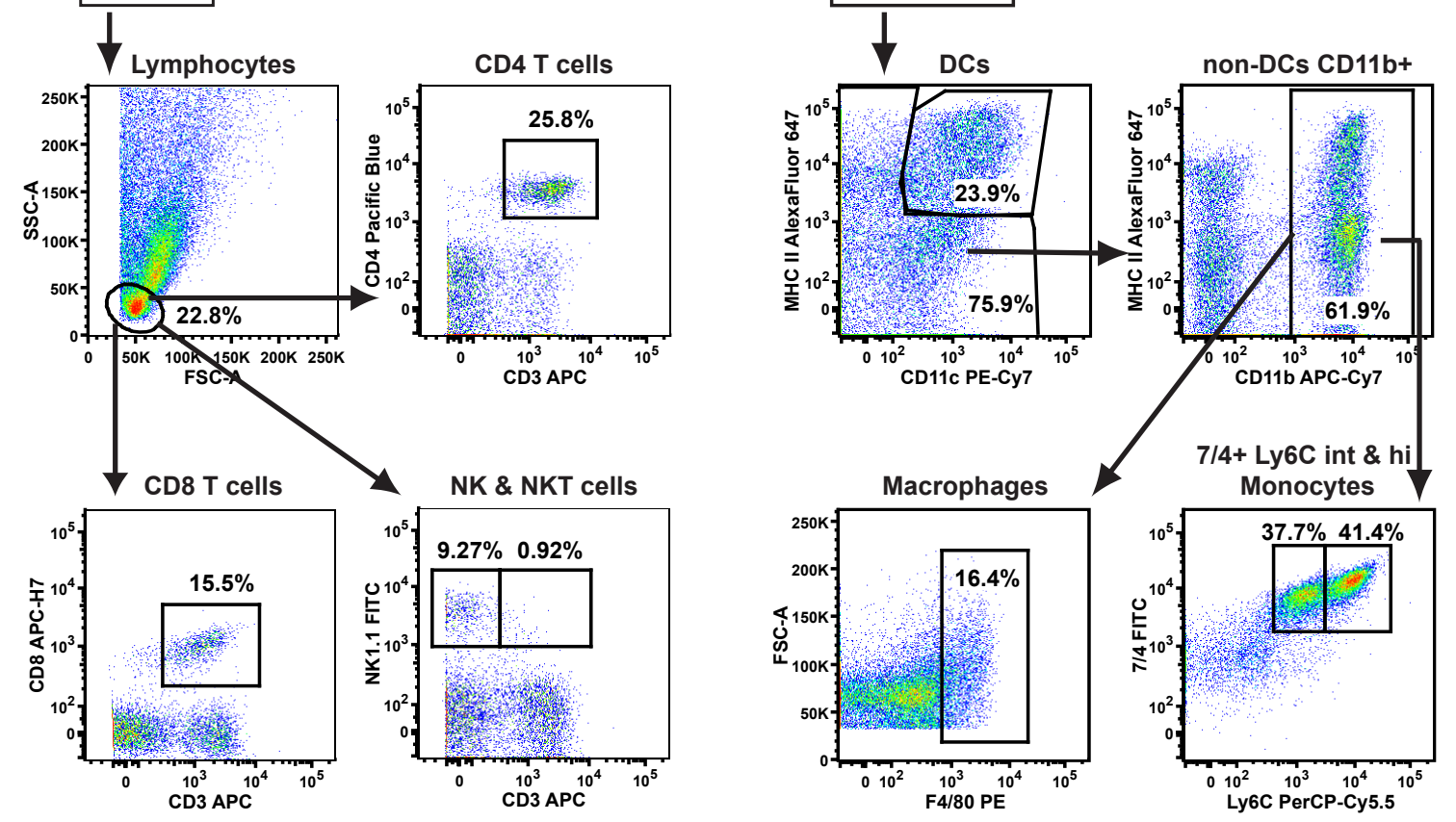

Figure 4.3: Representative gating of lymphoid and myeloid immune cells in tumours. C57 mice bearing established B16F1 tumours were injected around the tumour with the indicated adjuvants on day $7.24 \mathrm{~h}$ later tumours were analysed by multicolour flow cytometry. (A) Singlets were gated on the basis of FSC and SSC area (-A) versus height $(-\mathrm{H})$. Subsequently, live cells were identified on the basis of viability dye (DAPI) exclusion and immune cells were gated as CD45+. (B) In one multicolour panel, lymphocytes were gated on the basis of FSC-A and SSC-A and further divided into CD3+CD4+ T cells, CD3+CD8 $+\mathrm{T}$ cells, CD3+NK1.1+ NKT cells and CD3-NK1.1+ NK cells. (C) Myeloid populations were analysed in a separate staining panel. DCs were identified as CD11c+ MHCII+. All cells outside the DC gate were further gated on CD11b expression, followed by F4/80 and $\mathrm{FSC}^{\text {hi }}$ for macrophages and $7 / 4$ and Ly6C for two populations of monocytes. In some experiments, additional antibodies to evaluate cell activation status or cytokine production were included (shown in relevant figures) 
Two separate staining panels were then used to analyse either CD4 T cells, CD8 T cells and NK cells (Figure 4.3 B) or DCs, monocytes and macrophages (Figure 4.3 C). Within the CD45+ population, DCs were gated as CD11c+ MHC II+ cells. About $60 \%$ of the remaining CD45+ CD11c- cells expressed the myeloid marker CD11b. Macrophages were then identified within the CD45+ CD11c- CD11b+ population as F4/80 high FSC-A ${ }^{\text {high }}$ cells. CD45+ CD11c- CD11b + cells also comprised two populations of $7 / 4+$ monocytes, which expressed either high or intermediate levels of Ly6C (Figure $4.3 \mathrm{C}$ ).

In response to a single adjuvant administration, the frequency of immune cells in the tumours tended to increase (Figure $4.4 \mathrm{~A}$ ). This was highly significant for tumours treated with M. smegmatis or MSU+ M. smegmatis, where the proportion of CD45+ cells doubled (Figure $4.4 \mathrm{~A}$ ). In line with the results obtained for large, untreated B16F1 tumours with a different staining panel shown in Figure 3.11, the majority of tumour-infiltrating immune cells were monocytes and DCs (Figure 4.4 B). This general pattern was not altered in response to adjuvant treatment.

A.

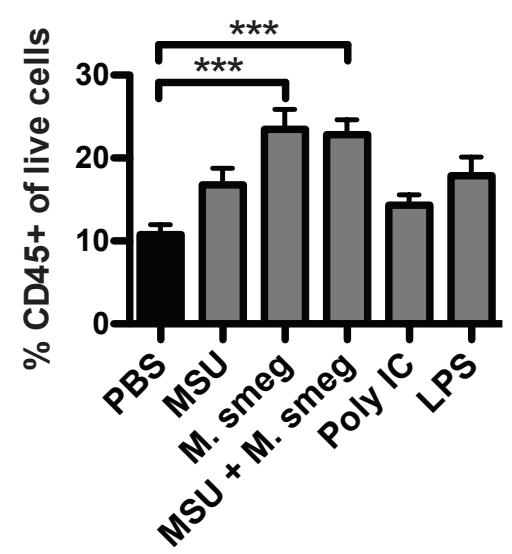

B.

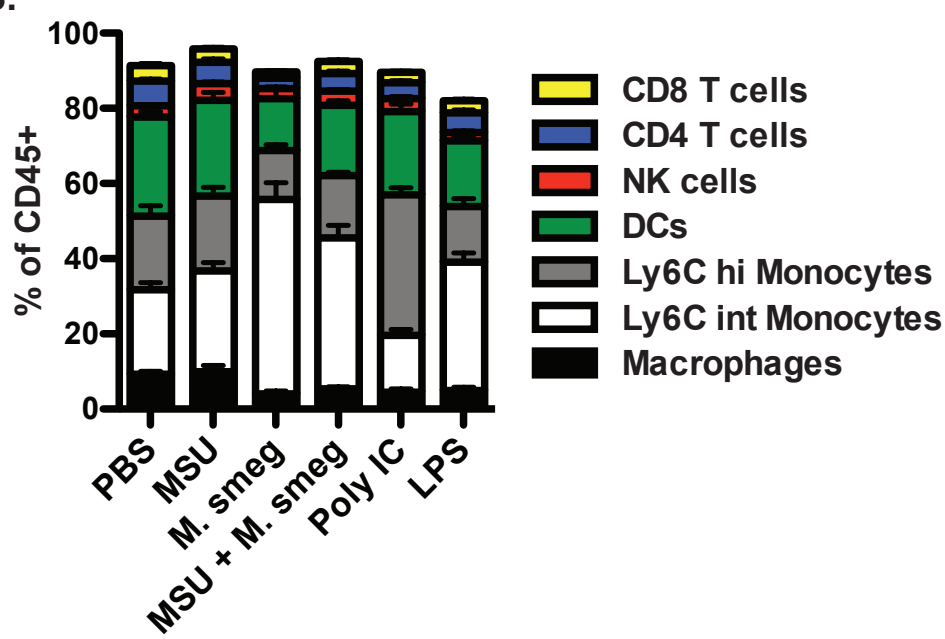

Figure 4.4: Some adjuvants affect immune infiltrate in tumours after a single adjuvant treatment. C57 mice bearing established B16F1 tumours were injected around the tumour with the indicated adjuvants on day $7.24 \mathrm{~h}$ later tumours were analysed by flow cytometry. All cell types were identified as shown in figure 4.3. (A) The percentage of CD45+ immune infiltrate among the total cells including tumour cells is shown. (B) Proportions of the different immune cell types that comprise the immune infiltrate are shown. Data are expressed as mean $+\mathrm{SE}$ and are combined from two independent experiments with 5 mice per group each. Statistical difference is indicated by ${ }^{* * *} p<0.001$ according to oneway ANOVA with Tukey's post test. 
However, the total proportion of monocytes among the CD45+ cells increased in response to M. smegmatis, MSU + M. smegmatis and Poly IC treatment (Figure 4.4 B). Subdividing the monocytes into the Ly6C $\mathrm{C}^{\mathrm{int}}$ and $\mathrm{Ly} 6 \mathrm{C}^{\text {hi }}$ populations revealed that while M. smegmatis alone or in combination with MSU lead to a considerable increase in the frequency of Ly6C ${ }^{\text {int }}$ monocytes in the CD45+ population, Poly IC treatment tripled the frequency of Ly6 $\mathrm{C}^{\text {hi }}$ monocytes (Figure $4.4 \mathrm{~B}$ ).

Overall, after a single adjuvant treatment, there was no correlation between the increase in CD $45+$ cells in the tumours (Figure $4.4 \mathrm{~A}$ ) or the increase in monocytes among immune cells (Figure $4.4 \mathrm{~B}$ ) with the activity of adjuvants to delay tumour growth (Figure 4.2).

To account for the overall increase in immune infiltration in some treatment groups and prevent the considerable changes in monocyte infiltration from masking the effects of adjuvants on the other tumour-infiltrating immune cells, all populations were also plotted as a percentage of all live cells (Figures for myeloid cells are shown below; Figures for T cells and NK cells are included in Chapter 5).

When the frequencies of the two monocyte populations were plotted as percentage of live cells, the increase of monocytes in response to M. smegmatis, MSU+ M. smegmatis and Poly IC became even more clear (Figure $4.5 \mathrm{~A}$ ). In addition to M. smegmatis and MSU+ M. smegmatis, LPS also led to an increase in the tumour-infiltrating Ly6C ${ }^{\text {int }}$ monocytes, although this was less pronounced (Figure $4.5 \mathrm{~A}$, left panel). Ly6Chi monocytes were indeed only affected by Poly IC administration (Figure $4.5 \mathrm{~A}$, right panel). Of note, a proportion of both monocyte populations expressed low levels of the DC marker CD11c, but not MHC II (Figure 4.5 B and compare monocyte gating in Figure 4.3 C).

This suggests that peri-tumoral administration of M. smegmatis with or without MSU and Poly IC attracts inflammatory monocytes to the tumour site, which may then up-regulate CD11c and MHC II expression and differentiate into DCs. 
A.

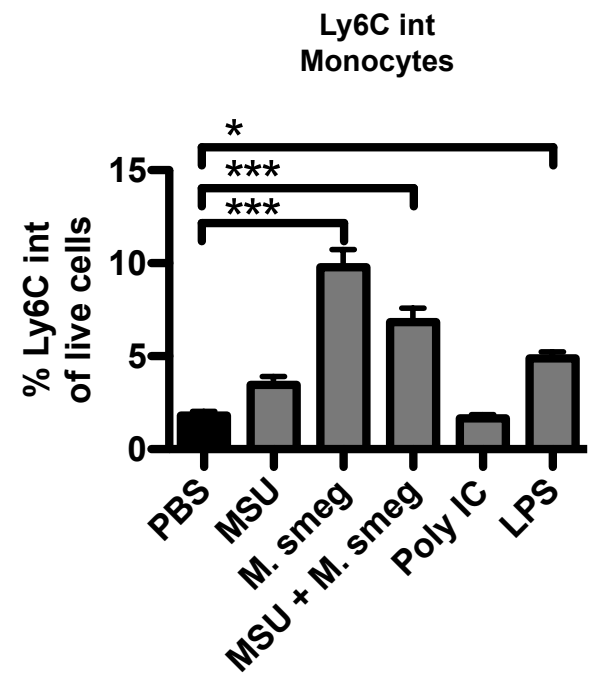

B.
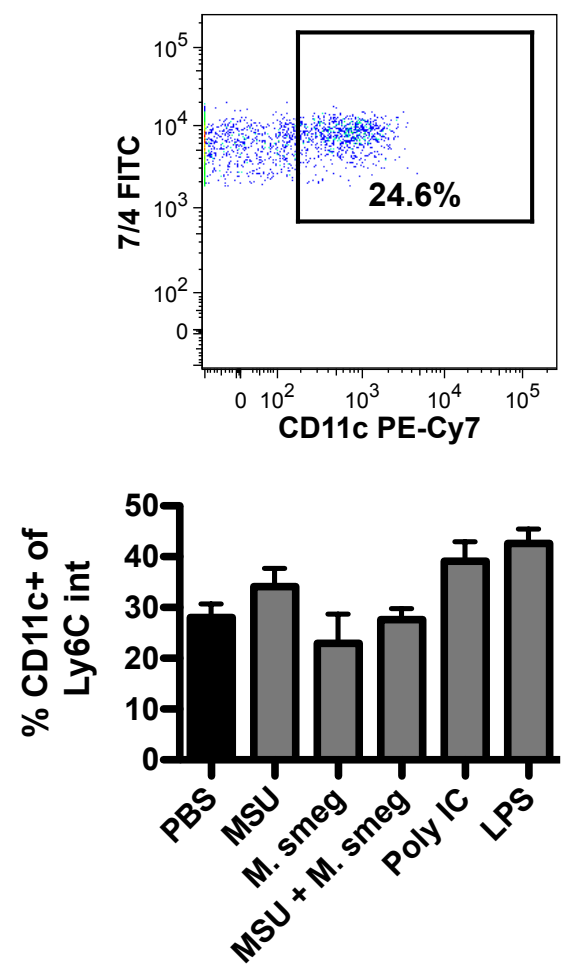

Ly6C hi

Monocytes
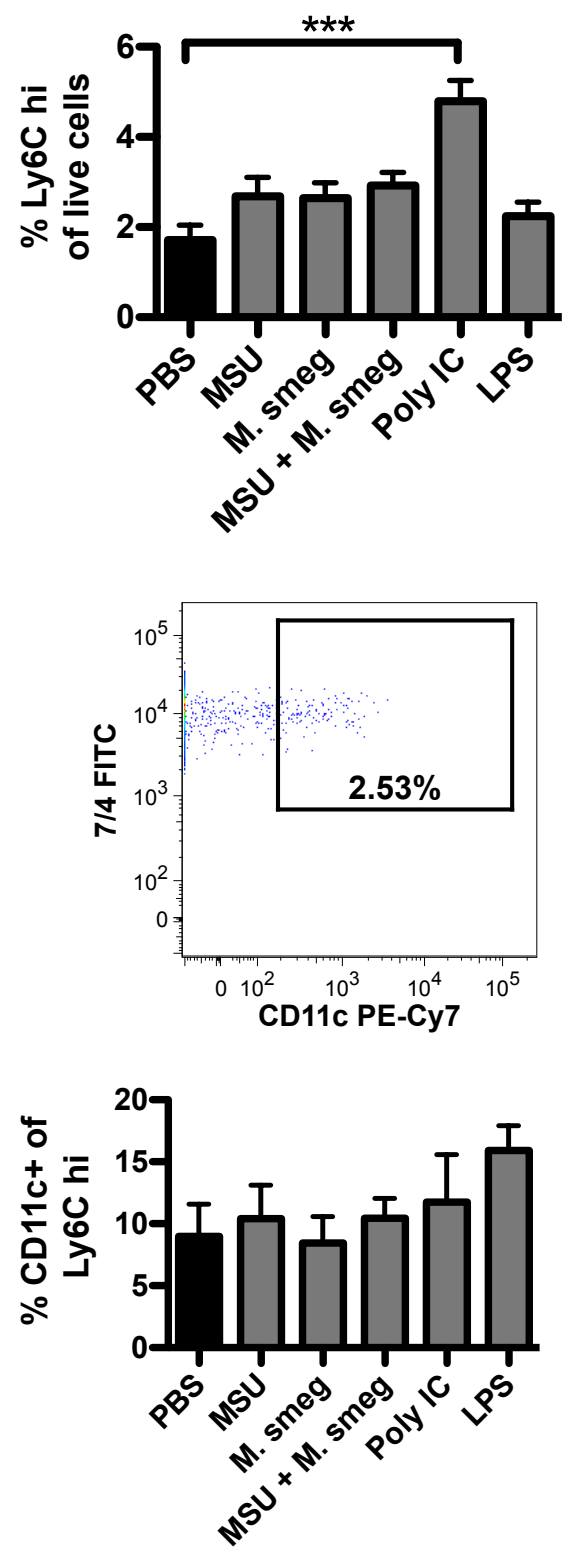

Figure 4.5: Some adjuvants increase monocyte infiltrate in tumours after a single treatment]. C57 mice bearing established B16F1 tumours were injected around the tumour with the indicated adjuvants on day $7.24 \mathrm{~h}$ later tumours were analysed by flow cytometry. CD11b $+7 / 4+$ Ly6C $^{\text {hi }}$ and CD11b $+7 / 4+$ Ly6C $^{\text {int }}$ monocytes were identified as shown in figure 4.3. (A) The percentage of monocyte populations among total live cells is shown. (B) Expression of the DC marker CD11c on the two monocyte populations is illustrated in representative flow plots and the frequency of CD11c+ monocytes is graphed. Bar graphs show means $+\mathrm{SE}$ and are combined from two independent experiments with 5 mice per group each. Statistical significance as assessed by to one-way ANOVA with Tukey's post test is indicated by ${ }^{*} p<0.05$ and ${ }^{* * *} p<0.001$. 
A.

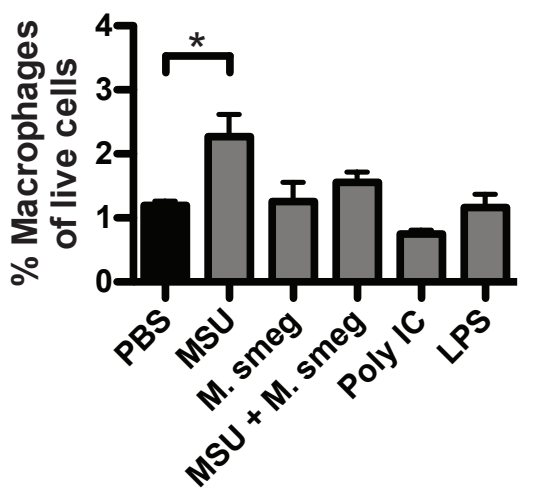

C.

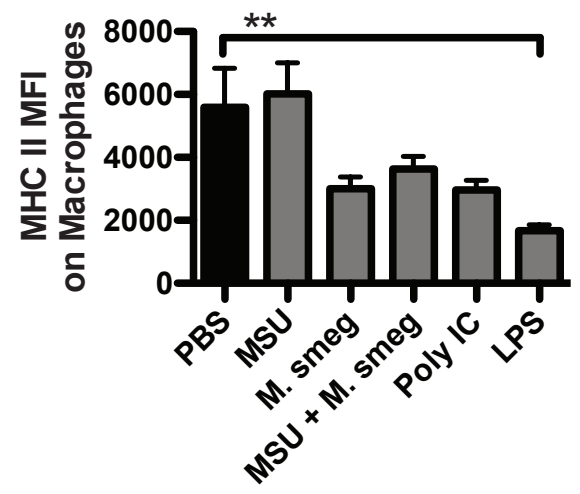

B.

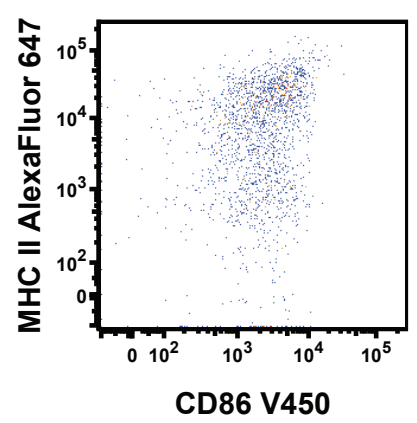

Figure 4.6: Macrophages in tumours remain largely unaffected by one adjuvant treatment. C57 mice bearing established B16F1 tumours were injected around the tumour with the indicated adjuvants on day $7.24 \mathrm{~h}$ after the last treatment, tumours were excised and analysed by flow cytometry. Macrophages were identified as shown in Figure 4.3 C. (A) The percentage of macrophages in tumours is graphed. (B) Representative flow plot of MHC II and CD86 expression on macrophages. (C) The levels of the activation markers MHC II and CD86 on macrophages are quantified as median flourescence intensity (MFI). Data in bar graphs are shown as mean $+\mathrm{SE}$ and are combined from two independent experiments with 5 mice per group each. Statistical significance is indicated by ${ }^{*} p<0.05$ and ${ }^{* *} p<0.01$ as assessed to one-way ANOVA with Tukey's post test. n.s. = not significant. 
Both DCs and macrophages express receptors for the studied adjuvants and may act as APCs if they become sufficiently activated. The frequency of macrophages among live cells was not significantly changed in any of the adjuvant-treated groups, apart from the MSU treated group, in which more macrophages were present (Figure $4.6 \mathrm{~A}$ ). In addition, adjuvant treatment did not increase the activation of macrophages in tumours, as assessed by their expression of the activation markers MHC II and CD86 (Figure 4.6 B \& C). MHC II expression was even reduced in response to LPS administration (Figure 4.6 C, left panel). This suggests that macrophages were neither attracted to the tumour site, nor activated to become effective APCs by adjuvant treatment.

The effect of a single adjuvant treatment on tumour-infiltrating DCs was also investigated. The frequency of DCs among live cells increased slightly in response to MSU, MSU + M. smegmatis and Poly IC, but this trend was not statistically significant (Figure 4.7 A). Analysis of the surface phenotype of the tumour-infiltrating DCs showed that about a third of them expressed considerable levels of the activation markers CD86 and MHC II even in saline treated mice (Figure $4.7 \mathrm{~B}$, left panel). However, as judged by these markers, tumour-infiltrating DCs were not activated by adjuvant treatment (Figure $4.7 \mathrm{C}$ ). To investigate whether adjuvant administration induced a change in the types of DCs found in the tumour, expression of CD11b, Ly6C and 7/4 was analysed. Nealry all tumour-infiltrating DCs expressed CD11b and the frequency of CD11b+ DCs was not affected by adjuvant treatment (Figure 4.7 B, middle panel \& $4.7 \mathrm{C}$, left panel). About $15 \%$ of DCs also co-expressed the monocyte markers $7 / 4$ and Ly6C, suggesting that they had recently differentiated from monocytes (Figure $4.7 \mathrm{D}$, right panel). Although the frequency of monocyte-derived DCs appeared to be increased in the Poly IC treated tumours, this was not statistically significant (Figure $4.7 \mathrm{D}$, right panel).

In summary, adjuvants did not increase the frequency or activation of DCs or macrophages in tumours $24 \mathrm{~h}$ after the first treatment. However, M. smegmatis, MSU + M. smegmatis and Poly IC administration resulted in a higher frequency of monocytes within the tumours. Some monocytes were found to express the DC marker CD11c, while some DC expressed the monocyte markers $7 / 4$ and Ly6C. This suggests that monocytes may be differentiating into DCs in the tumour. 
A.

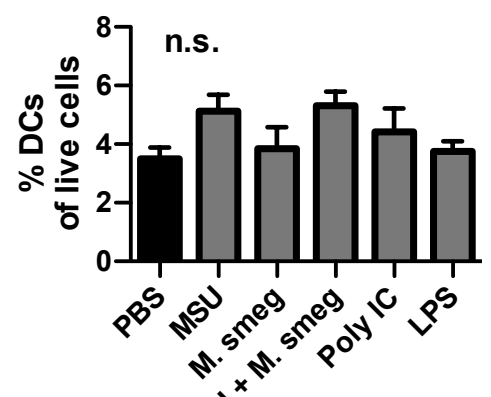

B.
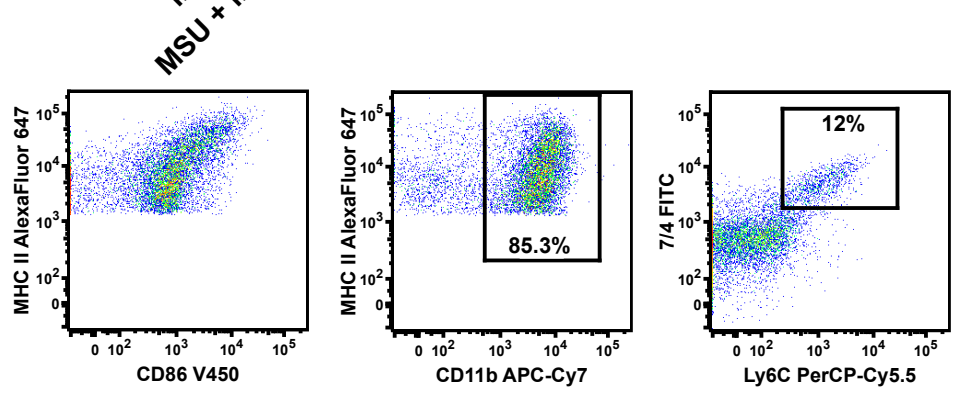

C.
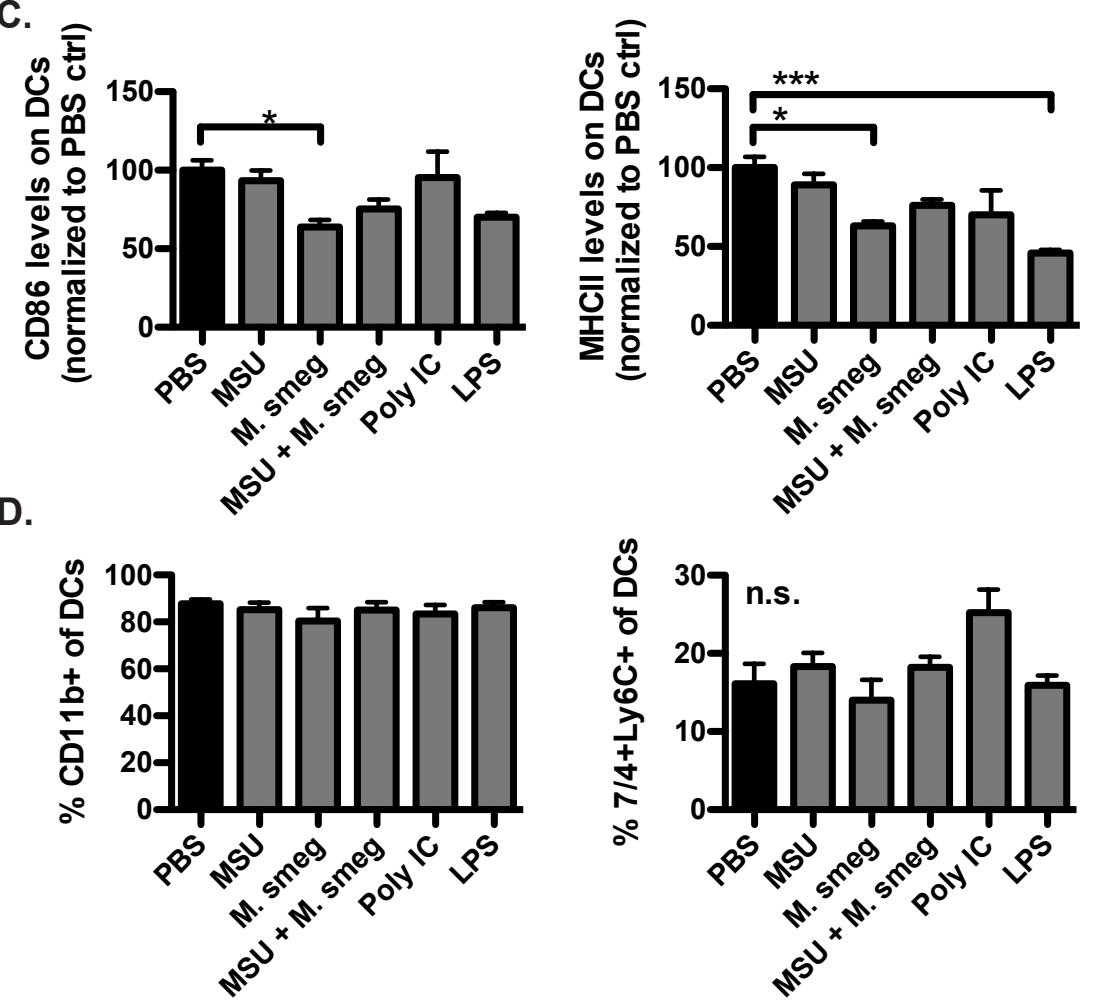

Figure 4.7: DCs in tumours are not activated after a single adjuvant treatment. C57 mice bearing established B16F1 tumours were injected around the tumour with the indicated adjuvants on day 7. $24 \mathrm{~h}$ later DCs in tumours were analysed by flow cytometry. DCs were identified as described in Figure 4.3. (A) The percentage of DCs among total live cells in tumours is shown. (B) Representative flow plots illustrate the surface expression of CD86, MHC II, CD11b, 7/4 and Ly6C on DCs in a saline treated tumour. (C) Expression of the activation markers CD86 and MHC II on DCs is graphed as mean fluorescence intensity (MFI). (D) The frequency of CD11b+ (left panel) and 7/4+Ly6C+ DCs (right panel) is shown. Data in bar graphs are shown as mean $+\mathrm{SE}$ and are combined from two independent experiments with 5 mice per group each. MFI values are normalized to the PBS control in each experiment. ${ }^{*} p<0.05$ and ${ }^{* * *} p<0.001$ as assessed by one-way ANOVA with Tukey's post test. n.s. $=$ not significant. 


\subsubsection{A single adjuvant administration affects DCs in tumour-draining LNs}

Several reports have demonstrated that bone marrow-derived DCs cross-present tumor antigen to $\mathrm{CD} 8 \mathrm{~T}$ cells in the lymph node $[310,450]$. While there was no difference in DC frequency or activation in the tumours 1 day after adjuvant treatment, DCs from tumours may already have migrated to the dLNs by that time to present antigen to $\mathrm{T}$ cells and initiate the anti-tumour response.

To investigate the effect of adjuvants on DCs in tumour-draining LNs, LNs were excised one day after a single adjuvant administration and DCs were analysed by flow cytometry. Singlet live cells were gated similar to the strategy shown in Figure 4.3. Subsequently, DCs were gated as CD11 $\mathrm{c}^{\text {hi }} \mathrm{MHC}$ II ${ }^{\mathrm{hi}}$ cells and DC subpopulations were identified on the basis of CD11b, CD8, B220 and Ly6C 7/4 expression (Figure 4.8 A).

After a single administration of MSU + M. smegmatis, Poly IC or LPS, LNs were doubled in size compared to PBS controls (Figure 4.8 B). While the frequency of DCs was significantly increased only in response to Poly IC treatment (Figure $4.8 \mathrm{C}$ ), a slight increase in DC percentage together with considerably larger LNs resulted in significantly higher numbers of DCs in the dLNs of Poly IC, MSU + M. smegmatis and LPS treated animals (Figure $4.8 \mathrm{D})$. When the different DC sub-populations were examined individually, CD11b+ DCs were significantly increased in these groups $(\mathrm{p}<0.05)$, with a corresponding decrease in the percentage of CD8+ DCs (Figure $4.8 \mathrm{E}$ ). The frequency of B220+ pDCs was not significantly altered (Figure $4.8 \mathrm{E}$ ).

The increase in the number of total DCs and the percentage of CD11b + DCs was seen both with the effective adjuvants Poly IC and MSU + M. smegmatis and also with the ineffective LPS treatment. Thus, there was no correlation of DC numbers or frequency of CD11b+ DCs with treatment benefit.

As some adjuvants attracted monocytes to the tumour site that seemed to differentiate into DCs, we investigated whether these adjuvants induced monocyte-derived inflammatory DCs to appear in the dLNs. In naïve mice or saline treated controls, less than $3 \%$ of DCs 
A.

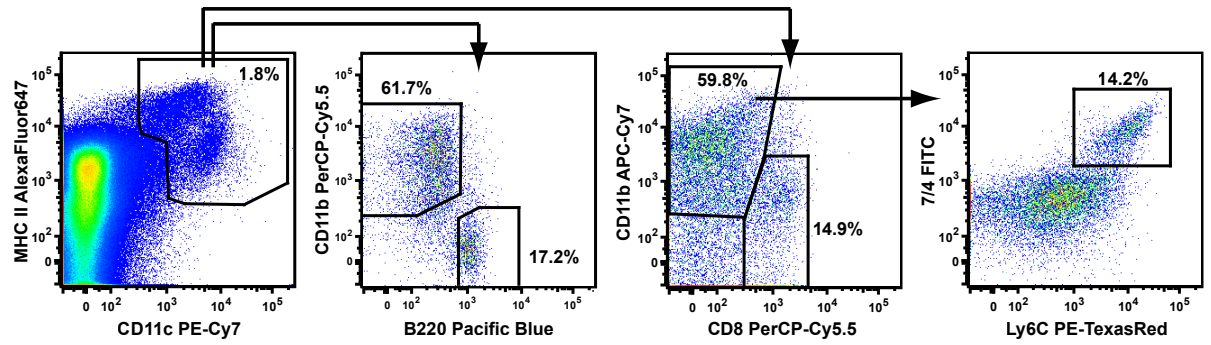

B.

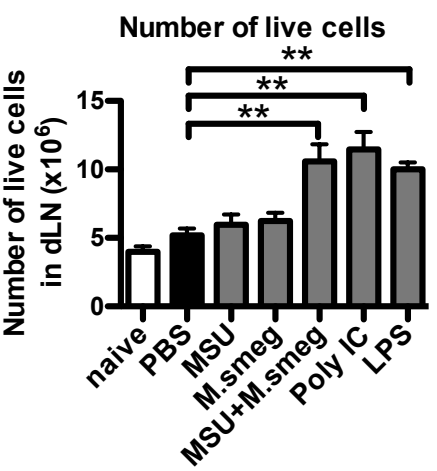

C.

Percentage of DCs

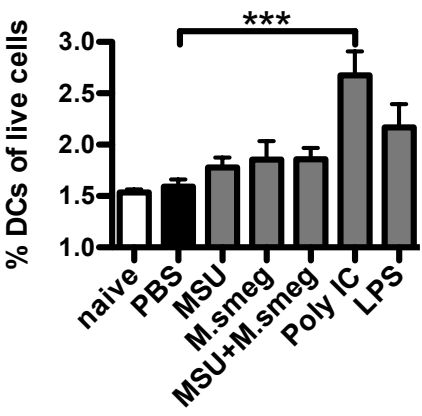

D.

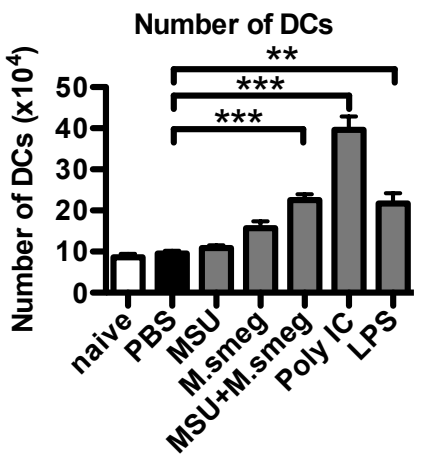

E.

DC populations

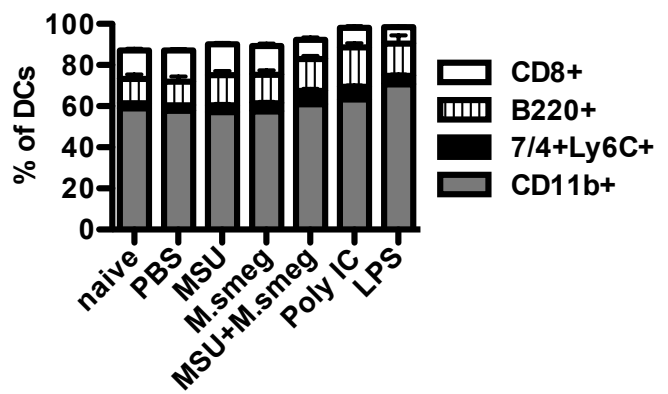

F.

Inflammatory DCs
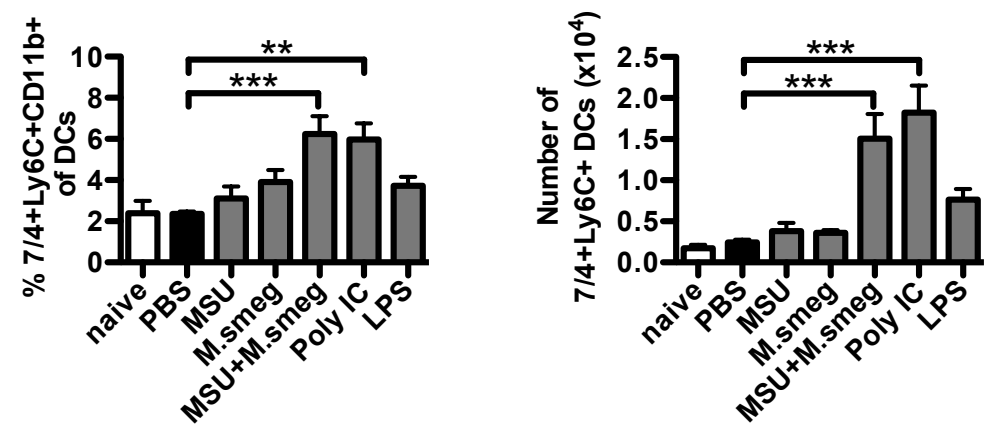

Figure 4.8: DC numbers and subsets in dLN change after a single adjuvant treatment. C57 mice bearing established B16F1 tumours were injected around the tumour with the indicated adjuvants on day $7.24 \mathrm{~h}$ later DCs in dLNs were analysed by flow cytometry. Gating on singlets and live cells was performed as described in Figure 4.3. Subsequently, $\mathrm{CD} 11 \mathrm{c}+\mathrm{MHCII}+\mathrm{DCs}$ and CD11b,$+ \mathrm{CD} 8+, \mathrm{B} 220+$ and Ly6C $+7 / 4+$ DC subtypes were identified as shown in representative flow plots in (A). (B) The total number of live cells in combined tumour-draining inguinal and axillary LNs as determined by trypan-blue exclusion on a haemocytometer is graphed. (C \& $\mathbf{D})$ The percentage and number of DCs in dLN is shown. (E)Total DCs comprise CD11b+7/4-Ly6C- (CD11b+), CD8+CD11b- (CD8+), B220+CD11b- $(\mathrm{B} 220+)$ and CD11b+7/4+Ly6C+ $(7 / 4+\mathrm{Ly} 6 \mathrm{C}+)$ DCs. (F) The frequency and number of inflammatory DCs $(\mathrm{CD} 11 \mathrm{~b}+7 / 4+\mathrm{Ly} 6 \mathrm{C}+)$ is shown. Data in bar graphs are shown as mean $+\mathrm{SE}$ and are combined from two independent experiments with 5 mice per group each. ${ }^{*} p<0.05,{ }^{* *} p<0.01$ and ${ }^{* * *} p<0.001$ as assessed by one-way ANOVA with Tukey's post test. 
expressed the inflammatory DC markers $7 / 4$ and Ly6C (Figure $4.8 \mathrm{E} \mathrm{\&} \mathrm{F}$, left panel). Poly IC and MSU + M. smegmatis treatments significantly increased both the percentage and numbers of inflammatory monocyte-derived DCs in dLNs (Figure $4.8 \mathrm{~F}$ ). Although LPS and M. smegmatis had also increased the frequency of monocytes in the tumours (Figure $4.5 \mathrm{~A}$ ), these adjuvants did not significantly affect inflammatory monocyte-derived DCs in dLN (Figure $4.8 \mathrm{~F}$ ).

Thus, the ability of adjuvants to delay tumour growth in vivo correlated with an increase in inflammatory DCs in the tumour-draining LNs after a single adjuvant administration.

Next, we examined the expression of activation markers on the DCs in tumour-draining LNs. Poly IC induced up-regulation of MHC II, CD40 and CD86 on the total DC population (Figure 4.9 A). However, LPS administration resulted in a similar increase in CD40 and CD86 levels, while MSU + M. smegmatis did not alter activation marker expression (Figure $4.9 \mathrm{~A}$ ).

A.
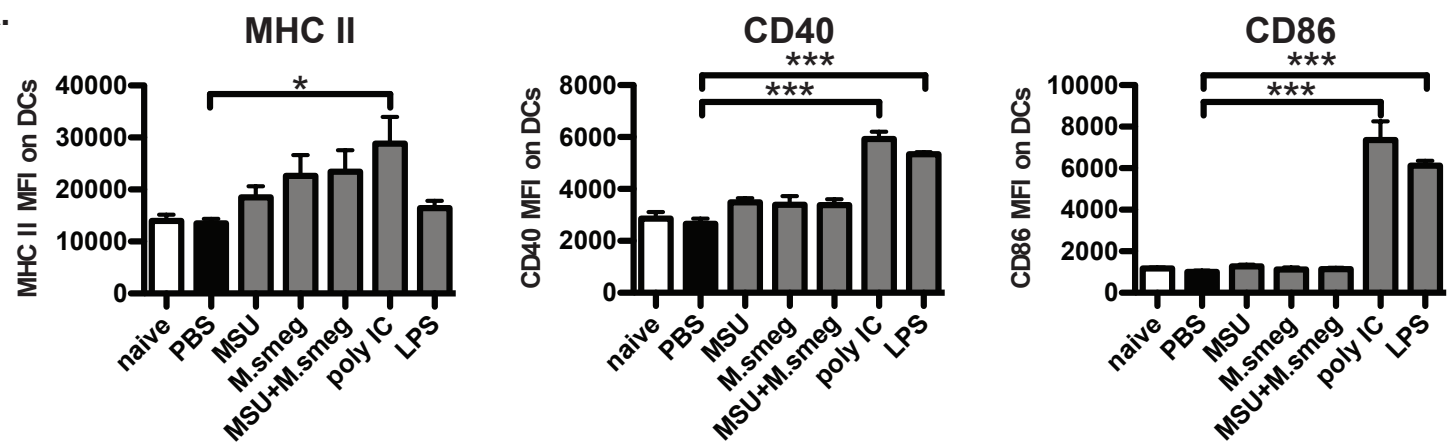

B.
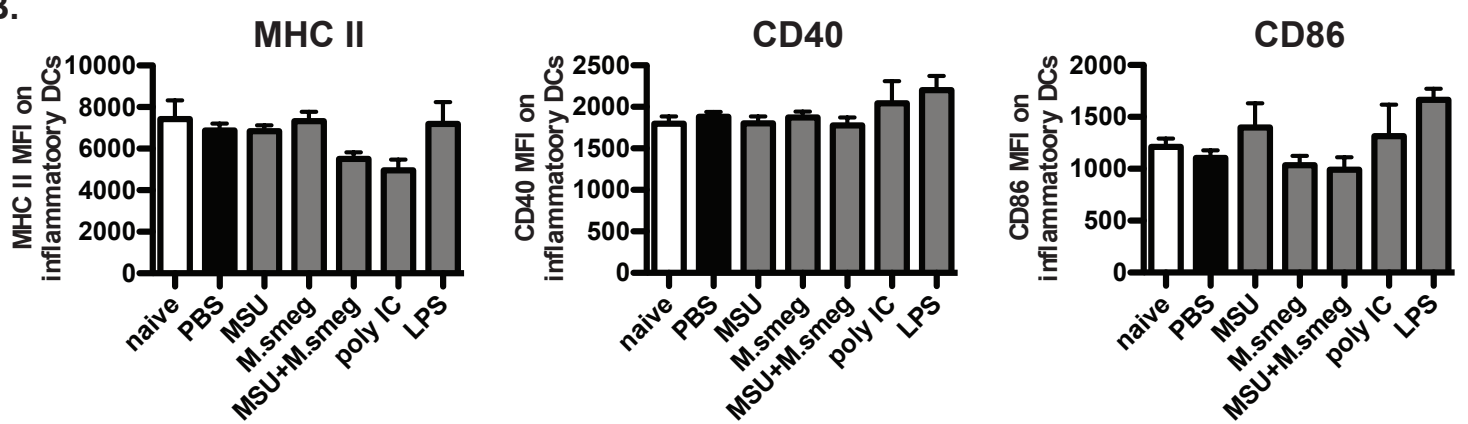

Figure 4.9: Some adjuvants activate DCs in dLN after a single administration. Data refer to the same experiment described in Figure 4.8. Expression of the activation markers MHC II, CD40 and CD86 is shown as median fluorescence intensity (MFI) on all DCs (A) or on inflammatory $7 / 4+\mathrm{Ly} 6 \mathrm{C}+\mathrm{CD} 11 \mathrm{~b}+\mathrm{DCs}(\mathbf{B})$. Data are shown as mean $+\mathrm{SE}$ and are combined from two independent experiments with 5 mice per group each. ${ }^{*} p<0.05$ and ${ }^{* * *} p<0.001$ as assessed by one-way ANOVA with Tukey's post test. 
As the inflammatory monocyte-derived DCs that correlated with treatment benefit only represented a small proportion of the total DCs, they were analysed separately for activation marker up-regulation. Inflammatory monocyte-derived DCs expressed lower levels of all activation markers examined compared to the total DC population (Figure 4.9, compare B to A). Adjuvant treatment did not lead to significant changes in the expression of any activation marker on inflammatory DCs (Figure 4.9 B).

In addition to high levels of peptide antigen presented on MHC molecules and co-stimulatory signals delivered via CD80 and CD86, cytokine production by DCs is also critical to activate potent $\mathrm{T}$ cell responses. In particular, IL-12 is required for the successful induction of a Th1 profile and cytotoxic CD8 T cells [451-453]. Therefore, the ability of peri-tumoral adjuvant administration to stimulate the ability of LN DCs to produce cytokines was investigated. $24 \mathrm{~h}$ after adjuvant administration, LNs were processed into single cell suspensions and cultured for $6 \mathrm{~h}$ in the presence of Golgi-Stop without re-stimulation. Golgi-Stop blocks the secretion of cytokines, which increases their detectability by intracellular staining. FMO staining controls and representative flow plots are shown in Figure 4.10 A.

DCs were found to either co-express IL-12 and TNF- $\alpha$ or to express TNF- $\alpha$ alone (Figure $4.10 \mathrm{~A} \& \mathrm{~B})$. Treatment with $\mathrm{CpG}$ or $\mathrm{MSU}+$ M. smegmatis significantly increased the percentage of cytokine producing DCs (Figure 4.10 B).

In summary, DCs in tumour-draining LNs were already affected by a single administration of adjuvant. However, while MSU + M. smegmatis, Poly IC and LPS treatments all resulted in higher DC numbers (Figure 4.8) and Poly IC and LPS also increased DC activation (Figure 4.9), these readouts did not correlate with the anti-tumour activity of adjuvants. In contrast, only the adjuvants that delayed tumour growth were found to induce accumulation of inflammatory monocyte-derived DCs in dLNs (Figure $4.8 \mathrm{~F}$ ) and they also increased the capacity of LN DCs to produce IL-12 and TNF- $\alpha$ (Figure 4.10). 
A.
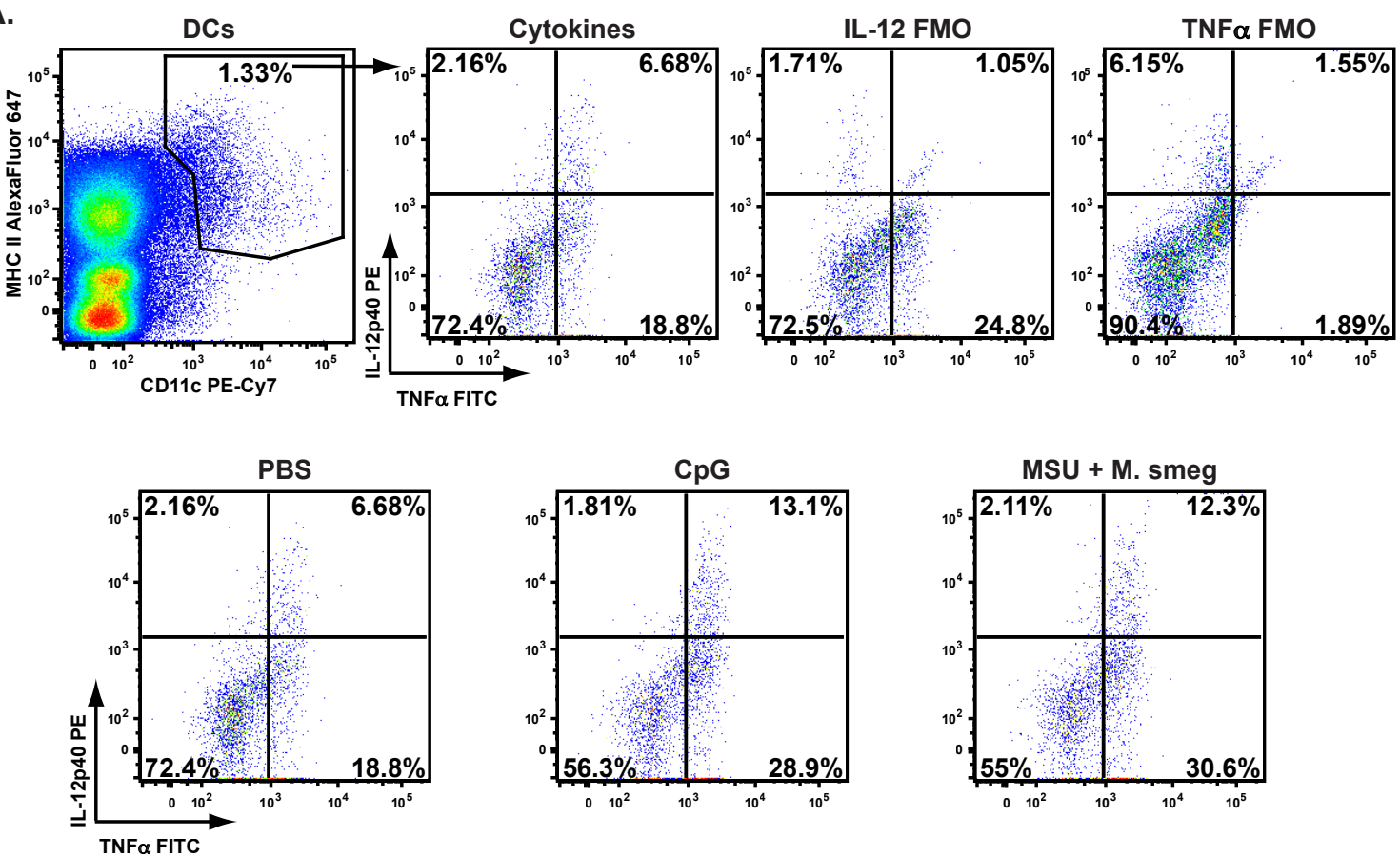

B.

IL-12+TNF $\alpha$ -

IL-12+ TNF $\alpha+$

IL-12-TNF $\alpha+$
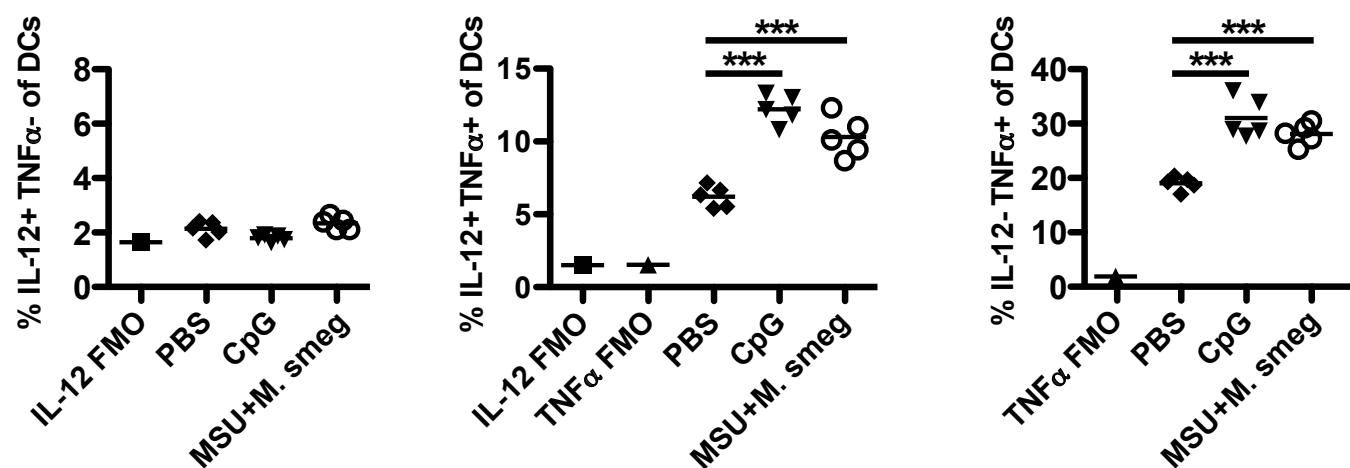

Figure 4.10: DC in dLN increase their capacity to produce cytokines after a single adjuvant treatment. C57 mice bearing established B16F1 tumours were injected around the tumour with the indicated adjuvants on day 7. $24 \mathrm{~h}$ later dLNs were excised and cultured for $6 \mathrm{~h}$ with Golgi-Stop, followed by intracellular cytokine staining for IL-12 and TNF- $\alpha$. FMO are staining controls using the same antibodies as in the stained samples but replacing the cytokine antibody with the appropriate isotype control. (A) DCs were gated on live singlets and then on CD11c+MHC II+ cells. Cytokine gating including staining controls and representative samples from indicated treatment groups are shown. (B) Scatter plots represent the frequency of single (IL-12 or TNF $\alpha$ ) or double positive (IL-12+TNF $\alpha+$ ) cells among all DCs. Data are shown as mean $+\mathrm{SE}$ and are from one representative experiment out of two independent experiments with 5 and 3 mice per group, respectively. ${ }^{* * *} p<0.001$ as assessed to one-way ANOVA with Tukey's post test. 


\subsubsection{A single peri-tumoral adjuvant treatment induces sys- temic cytokine release}

To further investigate the early response to adjuvant administration, the systemic release of cytokines was analysed in the serum.

As the kinetics of cytokine levels in the serum may vary for different cytokines or adjuvants, cytokine levels were analysed at $1 \mathrm{~h}, 3 \mathrm{~h}$ and $24 \mathrm{~h}$ after the first adjuvant administration in tumour bearing mice. Saline treated, tumour-bearing mice and naïve controls had low or undetectable levels of all cytokines apart from IL-1 $\alpha$, which showed considerable constitutive expression in all mice (Figure 4.11).

IFN- $\gamma$ is known to be important for the anti-tumour response to B16F1 melanomas and can be released by T cells or NK cells [454]. Only Poly IC and MSU + M. smegmatis induced significant levels of IFN- $\gamma$ at $3 \mathrm{~h}$ and $1 \mathrm{~h}$, respectively (Figure 4.11, top left panel). This rapid spike of IFN- $\gamma$ suggests that it was released through activation of effector cells rather than induced de novo.

GM-CSF is involved in the generation of granulocytes and monocytes from the bone-marrow. Its role in tumour immunology is ambiguous, with both tumour-promoting and tumourinhibitory effects described in the literature [455], sometimes in a dose-dependent manner [456]. In response to a single peri-tumoral adjuvant treatment, serum GM-CSF was only elevated in some mice and GM-CSF induction was not significant for any adjuvant and time point analysed (Figure 4.11, top right panel).

The serum levels of the pro-inflammatory cytokines IL- $1 \alpha$, IL- $1 \beta$, IL- 6 and TNF $\alpha$ were also examined. IL- $1 \alpha$ was constitutively present in all mice and its level was not altered in response to adjuvant treatment (Figure 4.11, left upper middle panel). In contrast, IL-1 $\beta$ was very low in control mice and increased in response to MSU $+M$. smegmatis at the $1 \mathrm{~h}$ time point (Figure 4.11, right upper middle panel). A number of Poly IC treated mice also had elevated serum levels of IL-1 $\beta$ at $1 \mathrm{~h}$ and $3 \mathrm{~h}$ after the adjuvant treatment, but the response was more variable and not significant. 

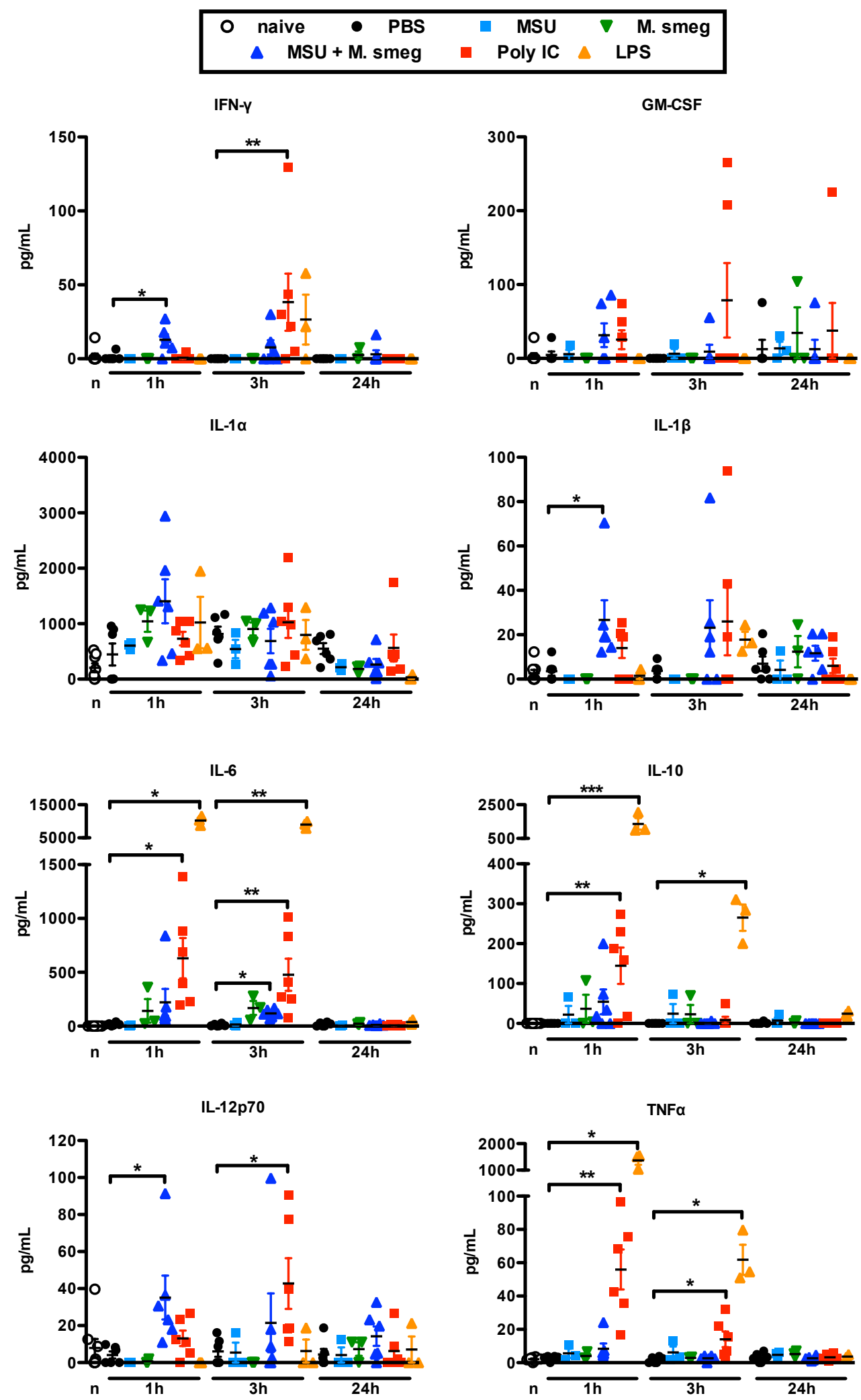

Figure 4.11: Peri-tumoral administration of some adjuvants increases serum cytokine concentrations. C57 mice bearing established B16F1 tumours were injected around the tumour with the indicated adjuvants on day $7.1 \mathrm{~h}, 3 \mathrm{~h}$ and $24 \mathrm{~h}$ later, serum samples were taken from mice and were analysed for the indicated cytokines using a multiplex bead assay. Data are shown with mean $\pm \mathrm{SE}$ and are pooled from 2 independent experiments with 0 or 3 mice per condition. ${ }^{*} p<0.05,{ }^{* *} p<0.01$ and ${ }^{* * *} p<0.001$ as analysed by the Kruskal-Wallis test with Dunn's post test. 
A moderate increase in IL-6 and $\mathrm{TNF} \alpha$ was observed in response to Poly IC and MSU + M. smegmatis, while peri-tumoral LPS treatment resulted in more than 10-fold higher levels of these cytokines (Figure 4.11, lower middle panels). In addition, LPS induced IL-6 and $\mathrm{TNF} \alpha$ levels were still elevated at $3 \mathrm{~h}$, and dropped back to baseline at $24 \mathrm{~h}$.

Interestingly, the anti-inflammatory cytokine IL-10 followed a very similar pattern compared to IL-6 and TNF $\alpha$ (Figure 4.11, lower left panel). Poly IC induced moderate IL-10 release at $1 \mathrm{~h}$, with levels down to baseline at $3 \mathrm{~h}$. Again, LPS induced very large amounts of IL-10 already at $1 \mathrm{~h}$, dropping somewhat at $3 \mathrm{~h}$ and returning to baseline levels again at $24 \mathrm{~h}$. This indicates that effective adjuvants only induce moderate amounts of proand anti-inflammatory cytokines, while ineffective adjuvants either induce neither (MSU, M. smegmatis) or large amounts of both (LPS).

Lastly, IL-12p70 induction was analysed. This cytokine is critical for the induction of a Th1 profile and the licensing of cytotoxic CD8 T cells [451-453]. IL-12p70 serum levels were significantly elevated at $1 \mathrm{~h}$ in response to MSU $+M$. smegmatis and at $3 \mathrm{~h}$ after Poly IC administration (Figure 4.11, lower right panel). Other adjuvants did not affect IL-12p70 levels at the studied time points.

In summary, only effective adjuvants induced a significant increase in the serum levels of IFN- $\gamma$ and IL-12p70. Poly IC and MSU + M. smegmatis were also associated with a moderate elevation of pro-inflammatory cytokines, while M. smegmatis or MSU did not induce any serum cytokines. In contrast, LPS induced large amounts of both pro-inflammatory cytokines and anti-inflammatory IL-10. 


\subsubsection{Local induction of cytokines by one peri-tumoral adju- vant administration}

The release of pro-inflammatory factors is tightly controlled and many cytokines, chemokines and enzymes involved in inflammation and host defense are only released locally. Therefore, the expression of a large panel of inflammatory factors in response to MSU + M. smegmatis, Poly IC or LPS treatment was analysed on a RNA level using a 96-well-plate quantitative real-time PCR array (see Appendix C). The results from these experiments showed that many genes were expressed at low levels and did not change more than 2-fold in response to adjuvant administration. G-CSF, CCL3, IL- $1 \alpha$, IL-1 $\beta$, IL-6 and iNOS were among the genes that showed the highest induction in response to MSU $+M$. smegmatis treatment. Poly IC also substantially up-regulated IL-6 expression, but had a lower impact on G-CSF, CCL3, IL- $1 \alpha$, IL-1 $\beta$ and iNOS. Instead Poly IC induced considerable expression of the chemokines CXCL-10 and CXCL-11. Strikingly, LPS induced even higher up-regulation of nearly all studied factors than MSU + M. smegmatis or Poly IC. However, it also increased GM-CSF and IL-10 expression to very high levels.

To validate the initial gene expression data, at least three biological replicates, non-reverse transcribed controls and water controls were necessary. In addition, a comparison between saline, MSU, M. smegmatis, MSU + M. smegmatis, Poly IC and LPS treated tumours at several time points after adjuvant administration was desirable. To enable the analysis of all of these samples, a few targets from the 96-well array were chosen for individual qPCR assays. Genes that were highly expressed, highly up-regulated or differentially affected by the tested adjuvants were selected. The growth factor G-CSF and the pro-inflammatory cytokine IL-6 showed the biggest increase in expression in the data obtained from the 96 -well assay and both were also differentially regulated in response to the different adjuvants. GM-CSF was highly expressed already at baseline and also showed different kinetics of up-regulation between MSU $+M$. smegmatis and LPS. IL-10 was chosen as an anti-inflammatory factor, iNOS was included as a marker of classical macrophage activation and CXCL10 was selected as a representative $\mathrm{T}$ cell attracting chemokine. Moreover, IL-10, iNOS and CXCL10 all exhibited differential expression in response to adjuvant treatment in the 96-well assay. 
The data obtained with individual qPCR analyses was largely in line with the previous array-based results. The $18 \mathrm{~S}$ samples of all conditions had CT values of 8-12, whereas reactions with non-reverse transcribed RNA samples and water had CT values $>30$. For all target transcripts, water controls and reactions performed with non-reverse transcribed RNA samples remained blank $(\mathrm{CT}>40)$, indicating that there was no significant contamination of reagents or genomic DNA present in samples.

GM-CSF was up-regulated in response to MSU, M. smegmatis or MSU $+M$. smegmatis (Figure 4.12, top left panel). However, at $24 \mathrm{~h}$, MSU + M. smegmatis induced GM-CSF levels had dropped down to baseline again. Poly IC did not affect GM-CSF expression. In contrast, LPS treatment led to a large increase in GM-CSF expression that kept increasing at $24 \mathrm{~h}$.

G-CSF showed a similar pattern to GM-CSF, with Poly IC only initially inducing some up-regulation and initial induction by MSU + M. smegmatis slowly declining over time (Figure 4.12, top right panel). Again, the induction of gene expression was highest for LPS and continued to increase at $24 \mathrm{~h}$.

The kinetics of IL-10 expression were more varied, either showing an initial induction followed by a decrease in response to MSU, induction with a drop at the $6 \mathrm{~h}$ time point after M. smegmatis or MSU + M. smegmatis treatment, or a peak at $6 \mathrm{~h}$ in response to Poly IC or LPS (Figure 4.12, left middle panel). Consistent with serum cytokine levels, LPS induced the highest and most persistent increase in IL-10 RNA.

IL-6 expression also reflected the serum cytokine data, with LPS causing a more than 100fold increase in RNA levels, while the other adjuvant only showed a up to 10-fold induction with varied kinetics (Figure 4.12, right middle panel).

Interestingly, iNOS levels were initially down-regulated by MSU and M. smegmatis and only increased at $24 \mathrm{~h}$ in response to mycobacteria, while an 8-fold induction of iNOS was observed at all time points following MSU + M. smegmatis administration (Figure 4.12, left bottom panel). Once again, LPS induced the biggest increase in expression levels. 
GM-CSF

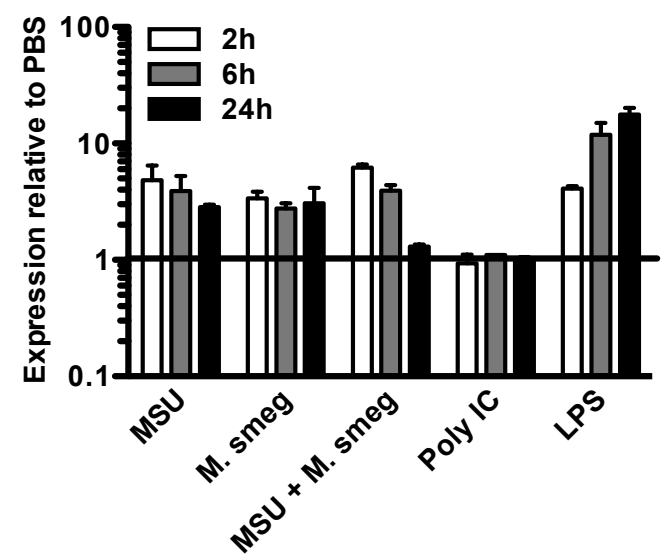

IL-10

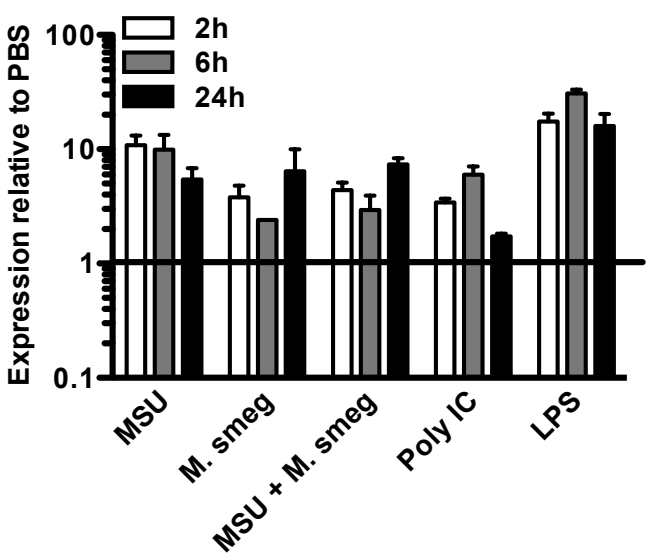

iNOS

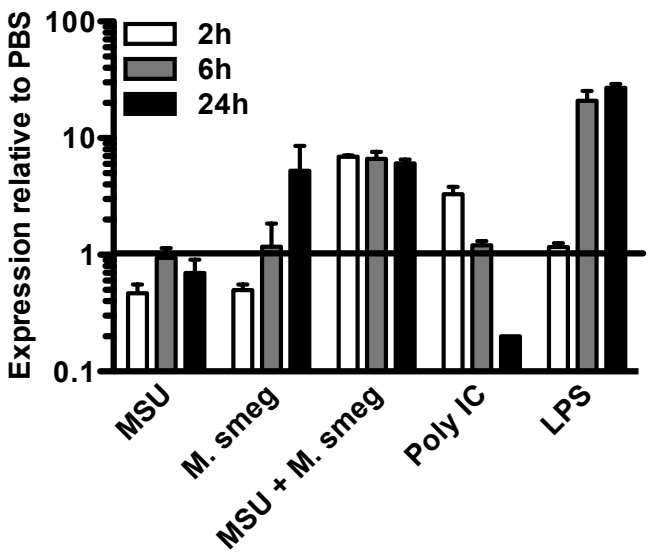

G-CSF

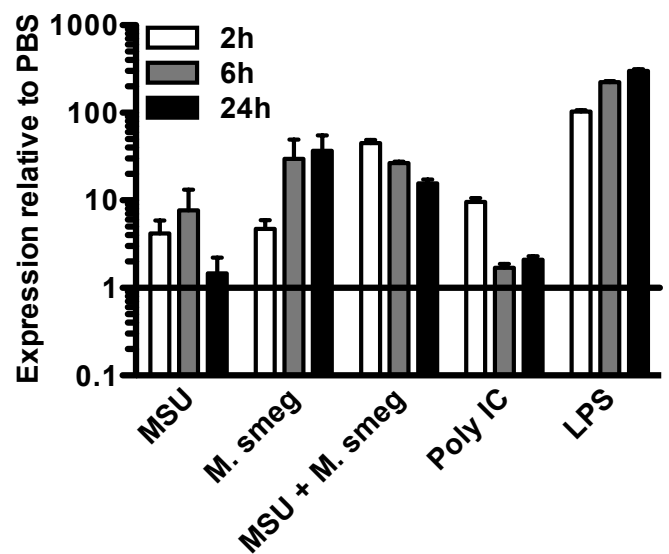

IL-6

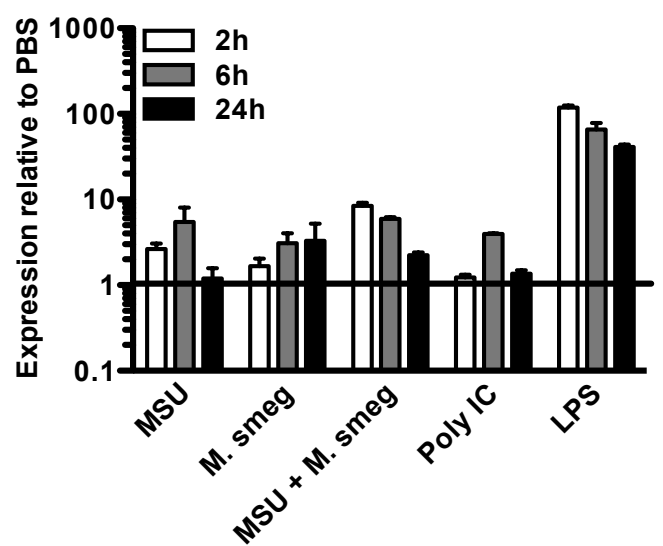

CXCL10

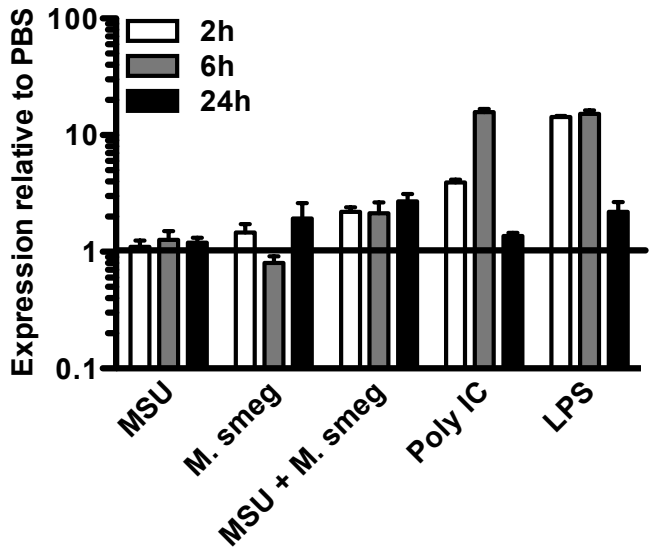

Figure 4.12: Changes in the expression of immune-related molecules in tumours after one adjuvant treatment. C57 mice bearing established B16F1 tumours were injected around the tumour with the indicated adjuvants on day $7.1 \mathrm{~h}, 6 \mathrm{~h}$ and $24 \mathrm{~h}$ later, tumours were excised and RNA was extracted. Following reverse transcription, expression of the indicated transcripts was analysed by real-time PCR. Data were normalized to $18 \mathrm{~S}$ and compared to the PBS treated groups $(\Delta \Delta \mathrm{CT})$. Relative expression levels from 3 mice per condition are shown as mean + SE. 
Unlike all of the other targets, CXCL10 was not up-regulated to a considerably higher extent by LPS, but instead Poly IC and LPS induced similar levels (Figure 4.12, right bottom panel). Both adjuvants up-regulated CXCL10 expression 15-fold, whereas MSU + M. smegmatis induced a much smaller, but sustained increase in CXCL10.

Altogether, the effective adjuvants Poly IC and MSU + M. smegmatis resulted in a moderate local up-regulation of the pro-inflammatory cytokine IL-6. The growth factors GM-CSF and G-CSF showed an initial spike in induction with MSU + M. smegmatis, which declined over time, while Poly IC only induced some G-CSF at the $2 \mathrm{~h}$ time point. Furthermore, both effective adjuvants induced low levels of IL-10. The induction of iNOS was largely limited to MSU + M. smegmatis, while CXCL10 was mainly induced by Poly IC. LPS, in contrast, induced much higher levels of the pro-inflammatory IL-6 and iNOS, as well as the growth factors G-CSF and GM-CSF, but also up-regulated IL-10 to a greater extent than the other adjuvants. Thus, a moderate amount of pro-inflammatory mediators and growth factors coupled with lower levels of IL-10 appears to be beneficial for the induction of an anti-tumour response by adjuvant treatment.

\subsubsection{Changes in DCs in tumour-draining LNs are sustained after repeated adjuvant administration}

Multiple adjuvant administrations may promote further activation of DCs and even higher numbers of inflammatory monocyte-derived DCs. However, repeated adjuvant exposure may also have the opposite effect, making DCs refractory to further adjuvant activation and reducing the induction of inflammatory cell types via feedback regulation $[446,457]$.

To examine the effect of repeated adjuvant administration, DCs in tumour-draining LNs were analysed one day after the 4 th peri-tumoral adjuvant treatment. LNs were still enlarged in Poly IC and MSU + M. smegmatis treated animals (Figure 4.13 A \& Figure 4.8 B). After 4 administrations, M. smegmatis treatment also induced an increase in LN size, while the initial LN enlargement in response to LPS was not sustained after 4 administrations (Figure 4.13 A \& Figure 4.8 B). After 4 adjuvant treatments, the frequency of DCs was similar in all groups (Figure 4.13 B), but due to the overall LN hyperplasia, a larger number 
of DCs was present in M. smegmatis, MSU + M. smegmatis and Poly IC treated groups (Figure $4.13 \mathrm{C}$ ).

Inflammatory monocyte-derived DCs remained increased after 4 administrations of Poly IC and MSU + M. smegmatis compared to saline treated controls (Figure 4.13 D, right panel). In fact, their frequency rose from $6 \%$ of DCs after one adjuvant treatment to $10-15 \%$ of all DCs following 4 adjuvant administrations (Figure 4.13 D \& Figure 4.8 F). While a single M. smegmatis dose had not altered DC numbers or induced inflammatory monocytederived DCs, after 4 administrations its effect was similar to the response induced by MSU + M. smegmatis (Figure 4.13 D \& Figure 4.8 F). The higher percentage of inflammatory
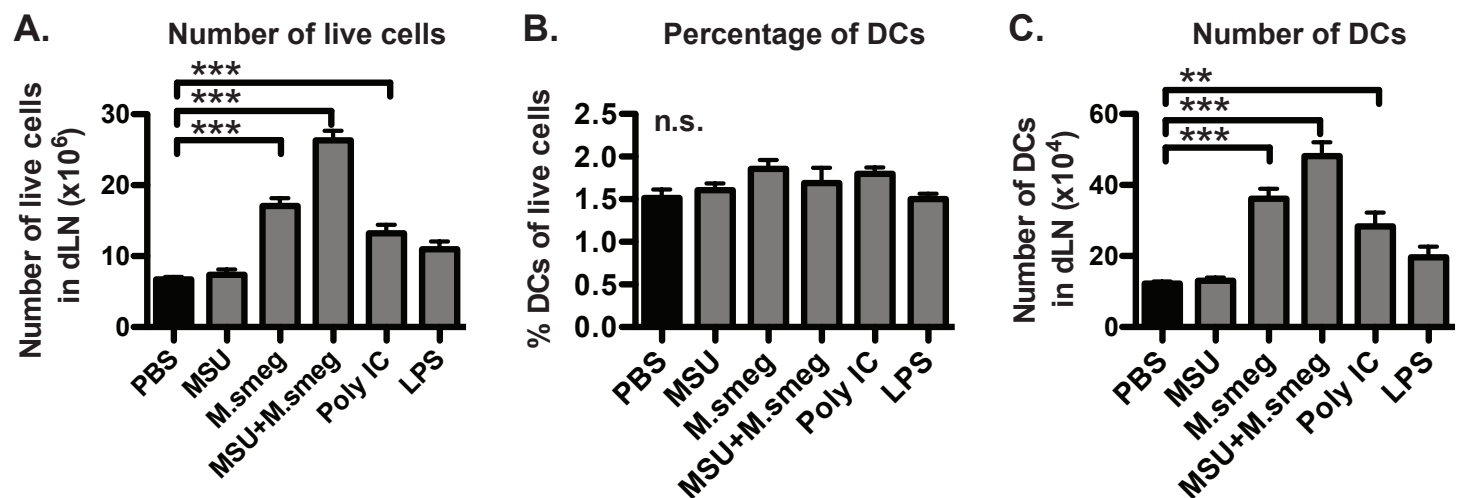

D.
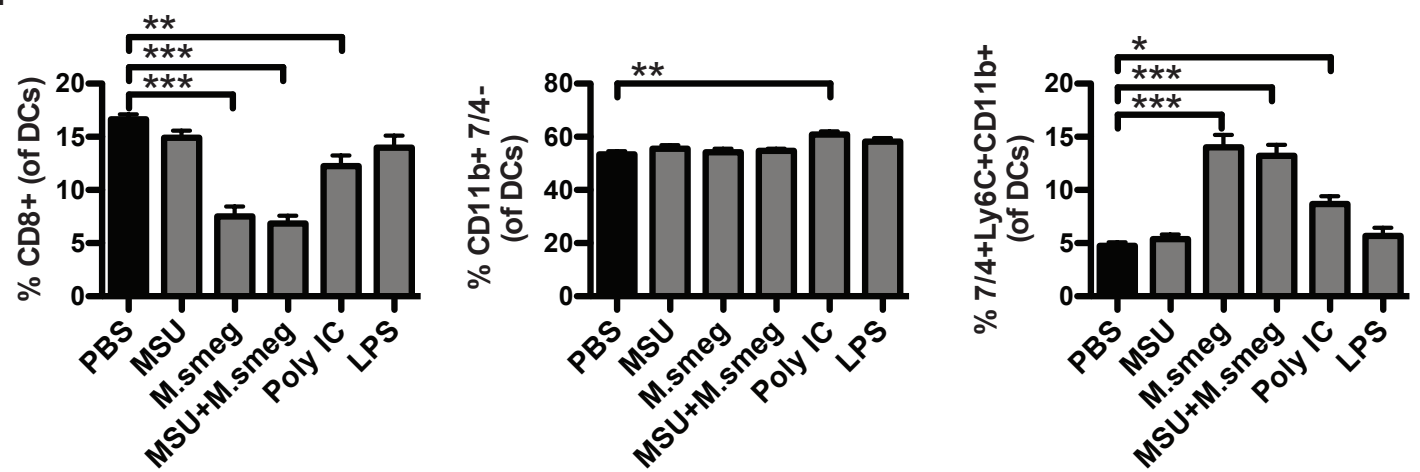

Figure 4.13: MSU + M. smegmatis and Poly IC continue to induce inflammatory DCs in dLNs after 4 treatments. C57 mice bearing established B16F1 tumours were injected around the tumour with the indicated adjuvants on day 7, 9, 11 and 13 . One day after the last treatment DCs in dLNs were analysed by flow cytometry. DCs were identified as CD11c+MHCII+ live cells as shown in Figure 4.8. (A) The total number of live cells as determined by trypan-blue exclusion on a haemocytometer in pooled inguinal and axillary dLN is graphed. The percentages (B) and numbers (C) of DCs in dLNs are shown. (D) Bar graphs depict the percemtages of CD8+CD11b- (CD8+), CD11b+7/4Ly6C- $(\mathrm{CD} 11 \mathrm{~b}+)$ and CD11b $+7 / 4+\mathrm{Ly} 6 \mathrm{C}+(7 / 4+\mathrm{Ly} 6 \mathrm{C}+)$ DCs among total DCs. Data are shown as mean $+\mathrm{SE}$ and are combined from two independent experiments with 5 mice per group each. ${ }^{*} p<0.05,{ }^{* *} p<0.01$ and ${ }^{* * *} p<0.001$ as assessed by one-way ANOVA with Tukey's post test. 
monocyte-derived DCs in M. smegmatis, MSU + M. smegmatis and Poly IC treated groups was accompanied by a decrease in the frequency of CD8+ DCs, whereas the CD11b $+7 / 4$ Ly6C- population was largely unaffected (Figure 4.13 D).

To further evaluate whether repeated adjuvant exposure had activating or tolerising effects on the DCs in tumour-draining LNs, their expression of activation markers was also analysed. When the whole DC population was examined, no change in MHC II expression was observed (Figure 4.14 A, left panel). Only Poly IC induced up-regulation of CD86 (Figure 4.14 A, middle panel). In addition, a slight increase in CD40 expression occurred after $M$. smegmatis treatment (Figure $4.14 \mathrm{~A}$, right panel). Although a single LPS administration had induced substantial up-regulation of CD86 and CD40 expression on DCs, 4 LPS treatments did not appear to activate DCs and even led to slightly reduced CD40 levels compared to saline treated controls (Figure 4.14 A \& Figure 4.9 A).

While inflammatory DCs were already detectable in dLNs after a single administration of Poly IC or MSU + M. smegmatis, they did not display a more activated phenotype than the few inflammatory DCs found in saline treated mice (Figure 4.9 B). Repeated adjuvant administration still did not alter MHC II expression by inflammatory DCs (Figure 4.14 B, left panel). However, 4 doses of Poly IC induced significant up-regulation of CD86 on inflammatory DCs, while M. smegmatis alone or in combination with MSU increased the expression of CD40 (Figure 4.14 B, middle and right panels \& Figure $4.14 \mathrm{C}$ ).

These data suggest that one dose of Poly IC and MSU + M. smegmatis was sufficient to induce inflammatory monocyte-derived DCs in tumour-draining LNs, but not to activate them. Repeated administration continued to promote monocyte-derived inflammatory DC formation and migration to the dLNs. In addition, 4 treatments with Poly IC and MSU + M. smegmatis enhanced the activation state of inflammatory monocyte-derived DCs in dLNs. In contrast, LPS strongly increased DC numbers and activation in dLNs only after a single administration, but did not affect DCs when given repeatedly.

Overall, repeated LPS administration appears to abrogate its ability to activate DCs, while multiple treatments with Poly IC and MSU $+M$. smegmatis resulted in even higher numbers and increased activation of inflammatory monocyte-derived DCs. 
A. all LN DCs
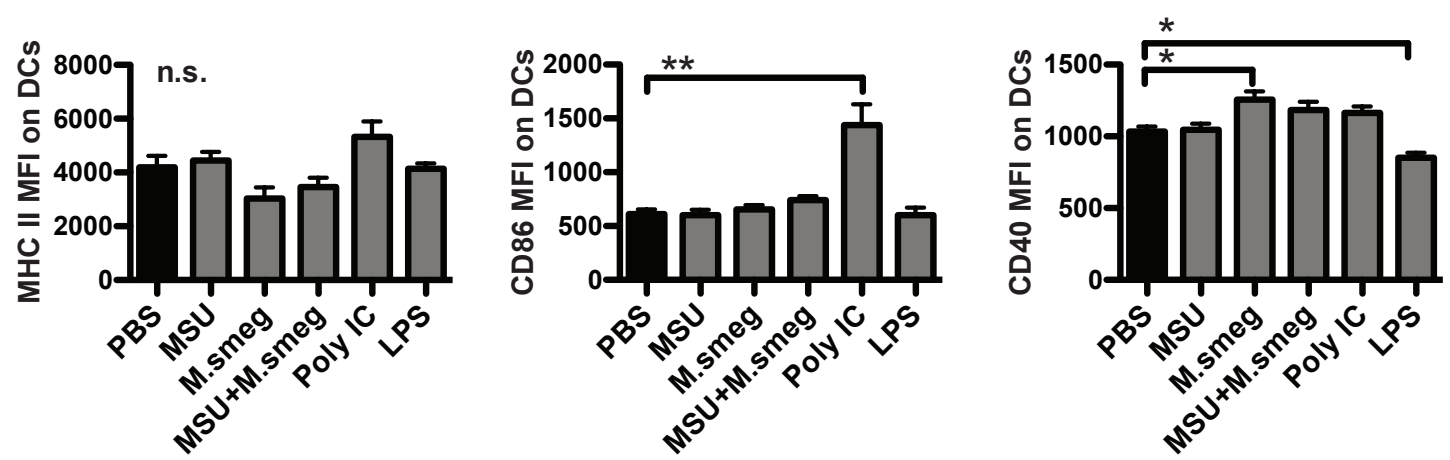

B. inflammatory LN DCs
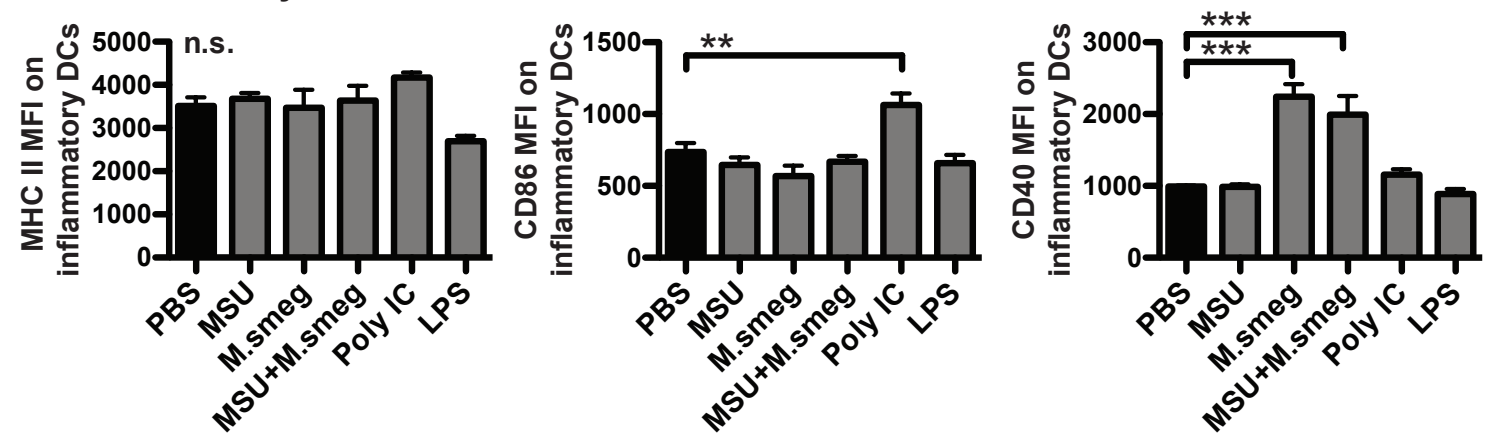

C. inflammatory LN DCs
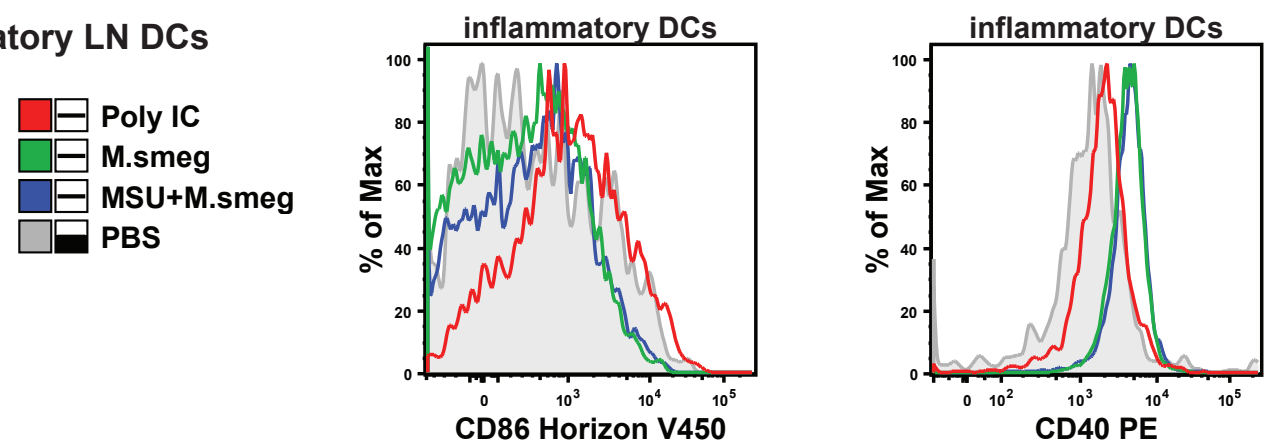

Figure 4.14: Inflammatory DCs in dLN are activated by some adjuvants after 4 treatments. C57 mice bearing established B16F1 tumours were injected around the tumour with the indicated adjuvants on day 7, 9, 11 and 13. One day after the last treatment DCs in dLNs were analysed by flow cytometry. All DCs were identified as CD11c+MHCII+ live cells and inflammatory DCs were identified as CD11b+7/4+Ly6C+. See Figure 4.8 for a representative gating strategy. Levels of the activation markers MHC II, CD86 and CD40 on the surface of DCs are depicted as median fluorescence intensity (MFI) on total DCs (A) and inflammatory DCs (B). For selected treatment groups, representative histograms for expression of CD86 and CD40 on inflammatory DCs are given. Data in bar graphs are shown as mean $+\mathrm{SE}$ and are combined from two independent experiments with 5 mice per group each. Statistical difference is indicated by ${ }^{*} p<0.05,{ }^{* *} p<0.01$ and ${ }^{* * *} p<0.001$ according to one-way ANOVA with Tukey's post test. 


\subsubsection{Impact of repeated adjuvant administration on mono- cytes and DCs in tumours}

The most likely origin of the monocyte-derived DCs observed in dLNs after multiple administrations of Poly IC and MSU + M. smegmatis is the adjuvant-treated tumour site. These adjuvants were found to induce inflammatory factors in the tumour and increase tumourinfiltration of monocytes already after one adjuvant administration. Repeated adjuvant injection to the tumour site may continue to attract immune cells.

A.

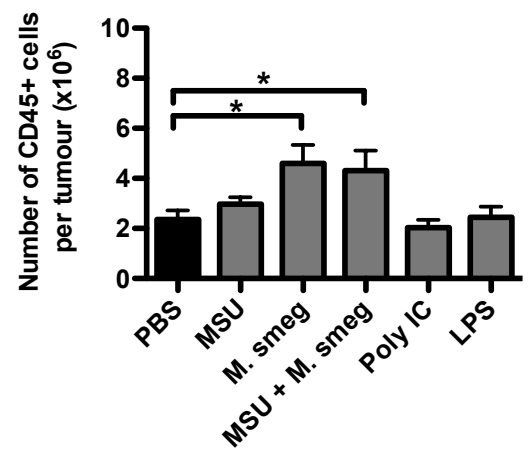

B.

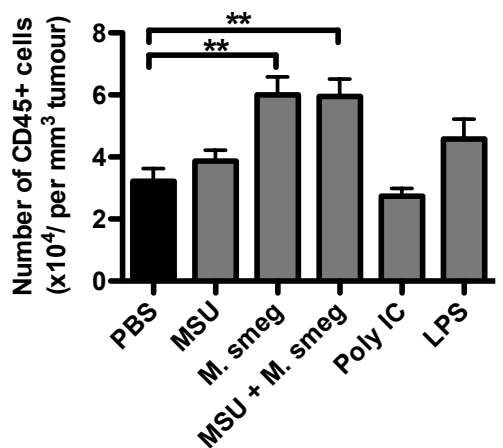

Day 16

(after 4 treatments)

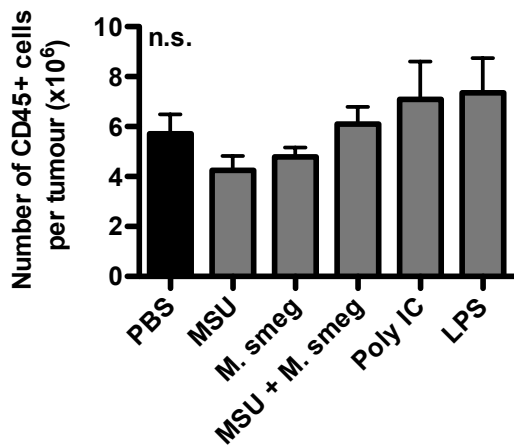

Total

cells

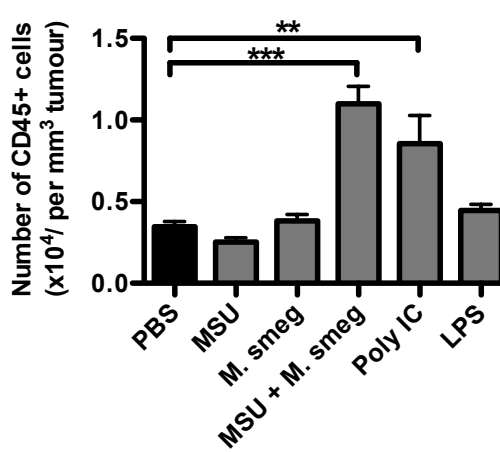

Immune

cells per

tumour

volume

Figure 4.15: Number of immune cells in tumours. C57 mice bearing established B16F1 tumours were injected around the tumour with the indicated adjuvants on day 7 or on day 7, 9, 11 and 13. One day after the last treatment, tumours were measured, excised and processed into single cell suspension. Live cells were counted on a hemocytometer by trypan-blue exclusion. CD45+ immune cells were identified by flow cytometry as described in Figure 4.3. (A) The total number of CD45+ immune cells in each tumour was calculated. (B) The immune infiltrate is expressed relative to tumour volume (million CD45+ cells per $\mathrm{mm}^{3}$ tumour mass). Bar graphs show means $+\mathrm{SE}$ and are combined from two independent experiments with 5 mice per group each. ${ }^{*} p<0.05,{ }^{* *} p<0.01$ and ${ }^{* * *} p<0.001$ as assessed by to one-way ANOVA with Tukey's post test. 
To compare the total numbers of tumour-infiltrating immune cells in B16F1 tumours after one and four adjuvant treatments, tumour cell suspensions were again analysed by flow cytometry and live cells were counted by trypan-blue exclusion. Small tumours one day after the first adjuvant treatment were associated with 2 million immune cells. Similar to the result obtained for the percentage of CD45+ immune cells among live cells (Figure 4.4), both $M$. smegmatis and MSU + M. smegmatis increased the total number of immune cells in each tumour (Figure $4.15 \mathrm{~A}$, left panel). As tumours were all of similar size, the same pattern was seen when the immune cell infiltrate was expressed in relation to tumour volume (Figure 4.15 B, left panel).

After 4 adjuvant administrations, the tumour sizes were more varied, with tumours treated with Poly IC or MSU + M. smegmatis being considerably smaller than the tumours in other treatment groups (Figure 4.2). The total number of immune cells per tumour at day 14 was not significantly different between treatment groups (Figure $4.15 \mathrm{~A}$, right panel). However, compared to the total amount of immune cells present at the tumour site at day 8 , there was a 3-fold increase in the immune infiltrate in saline treated controls at day 14 (Figure $4.15 \mathrm{~A}$ ). In the mycobacteria treated groups that had already experienced an influx of immune cells at day 8 , the effect was less pronounced. The density of the immune infiltrate in small tumours was about 3 million per $\mathrm{mm}^{3}$ in saline controls, but decreased to 0.3 million per $\mathrm{mm}^{3}$ as the tumours progressed (Figure $4.15 \mathrm{~B}$ ). This indicates that while immune cells continued to infiltrate the tumours between days 8 and 14, the tumour cells outgrew the immune infiltrate. This still occurred in Poly IC and MSU + M. smegmatis treated tumours, but to a lesser degree, as the density of immune cells on day 14 was 1 million per $\mathrm{mm}^{3}, 3$-fold higher than in saline controls (Figure 4.15 B, right panel).

Monocytes accounted for a major portion of the tumour immune-infiltrate (Figure 4.4). A single administration of $M$. smegmatis, MSU + M. smegmatis or LPS increased the frequency of Ly6 $\mathrm{C}^{\text {int }}$ monocytes in tumours (Figure 4.5). After 4 adjuvant administrations, only combination treatment with MSU $+M$. smegmatis enhanced recruitment of Ly6C ${ }^{\text {int }}$ monocytes to the tumour (Figure $4.16 \mathrm{~A}$, left panel).

$\mathrm{MSU}+$ M. smegmatis and LPS also increased the percentage of Ly6C $\mathrm{Ch}^{\mathrm{i}}$ monocytes in tumours (Figure $4.16 \mathrm{~A}$, right panel). In contrast to the significant increase in tumour 
A.
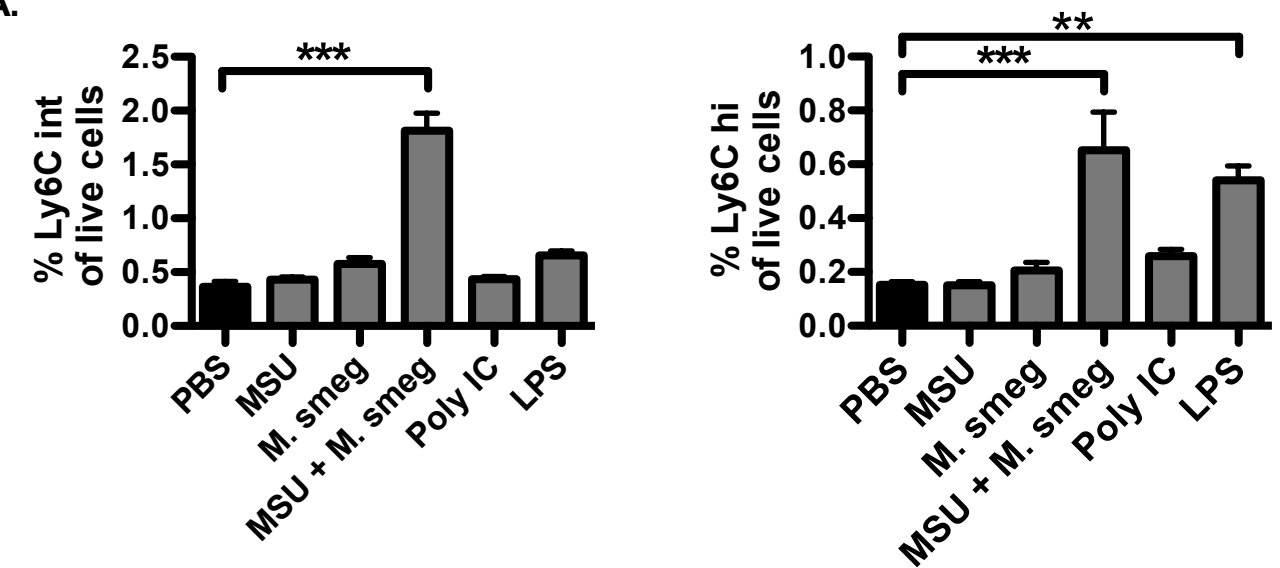

B.
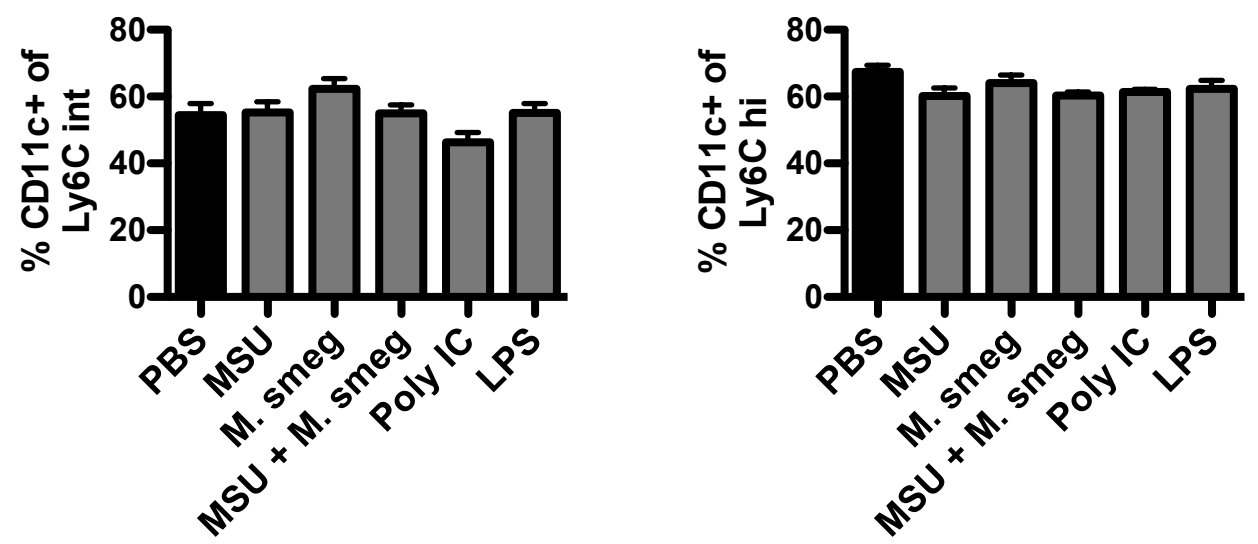

Figure 4.16: Inflammatory monocytes remain increased after $4 \mathrm{MSU}+M$. smegmatis treatments. C57 mice bearing established B16F1 tumours were injected around the tumour with the indicated adjuvants on day 7, 9, 11 and 13. One day after the last treatment monocytes in tumours were analysed by flow cytometry. Monocytes were identified as described in Figure 4.3 and CD11c expression was analysed as shown in Figure 4.5. (A) The percentage of CD11b $+7 / 4+\mathrm{Ly}_{6} \mathrm{C}^{\text {int }}$ and $\mathrm{CD} 11 \mathrm{~b}+7 / 4+\mathrm{Ly}_{6 \mathrm{C}}{ }^{\text {hi }}$ monocytes among live cells in tumours is shown. (B) A fraction of both monocyte populations expresses the DC marker CD11c but not MHC II. Data are graphed as mean + SE and are combined from two independent experiments with 5 mice per group each. ${ }^{* *} p<0.01$ and ${ }^{* * *} p<0.001$ as assessed by one-way ANOVA with Tukey's post test.

infiltration of Ly6C $\mathrm{C}^{\text {hi }}$ monocytes after a single Poly IC dose, repeated Poly IC administration did not affect monocyte infiltrate (Figure 4.16 A).

A proportion of both monocyte populations was positive for the DC marker CD11c, but did not express MHC II (Figure4.16 B). CD11c expression of monocytes was generally higher in day 14 tumours than in small tumours on day 8 (Figure 4.16 B \& Figure 4.5 B). Similar to what was observed after one treatment, the percentage of monocytes that expressed CD11c was not altered by adjuvant treatment. 
Overall, after 4 adjuvant treatments, monocyte infiltration into tumours was mainly increased in response to $\mathrm{MSU}+M$. smegmatis, but not to Poly IC. Therefore, monocyte infiltration after repeated adjuvant administration did not correlate with anti-tumour activity of the adjuvants.

Similar to the effect on monocytes, after four adjuvant treatments the percentage and number of DCs in tumours was only increased by MSU + M. smegmatis (Figure $4.17 \mathrm{~A}$ ). In addition, tumour-infiltrating DCs did not display enhanced activation in response to repeated adjuvant exposure (Figure $4.17 \mathrm{~B}$ ). In line with the data obtained after a single adjuvant treatment, more than $80 \%$ of the DCs in saline treated tumours expressed CD11b and about $20 \%$ of DCs were $7 / 4+\mathrm{Ly} 6 \mathrm{C}+\mathrm{CD} 11 \mathrm{~b}+$ (Figure $4.17 \mathrm{C}$ ). The frequency of CD11b+ DCs was neither affected by one nor by multiple adjuvant treatments (Figure $4.17 \mathrm{C}$, left panel \& Figure 4.7 D, left panel). In contrast, repeated exposure to all adjuvants apart from MSU resulted in a significant increase in the percentage of monocyte-derived inflammatory (7/4+Ly6C+) DCs in tumours (Figure $4.17 \mathrm{~A})$.

This suggests that repeated administration of all adjuvants apart from MSU created a sufficiently inflammatory milieu at the tumour site to enhance the differentiation of monocytes into DCs. However, only Poly IC and MSU + M. smegmatis treatment induced early accumulation of monocyte-derived DCs in the dLNs, where they may activate the anti-tumour $\mathrm{T}$ cell response.

To investigate the ability of tumour-infiltrating DCs to produce TNF $\alpha$ and IL-12, tumour cell suspensions were cultured for $6 \mathrm{~h}$ with Golgi-Stop, followed by intracellular cytokine staining and flow cytometry analysis. In saline treated controls, less than $1 \%$ of DCs produced TNF $\alpha$ or IL-12 (Figure 4.18). TNF $\alpha$ production was significantly enhanced after 4 administrations of MSU + M. smegmatis, but was not altered by any of the other adjuvants (Figure $4.18 \mathrm{~A} \& \mathrm{~B}$ ). In response to MSU + M. smegmatis treatments, DCs also expressed higher levels of $\mathrm{TNF} \alpha$ as illustrated by the increased staining intensity (Figure $4.18 \mathrm{~A}$, histogram).

In contrast, repeated administration of MSU + M. smegmatis, Poly IC and LPS all resulted in a higher frequency of IL-12+ DCs, but none of the adjuvants increased the IL-12+ 
A.
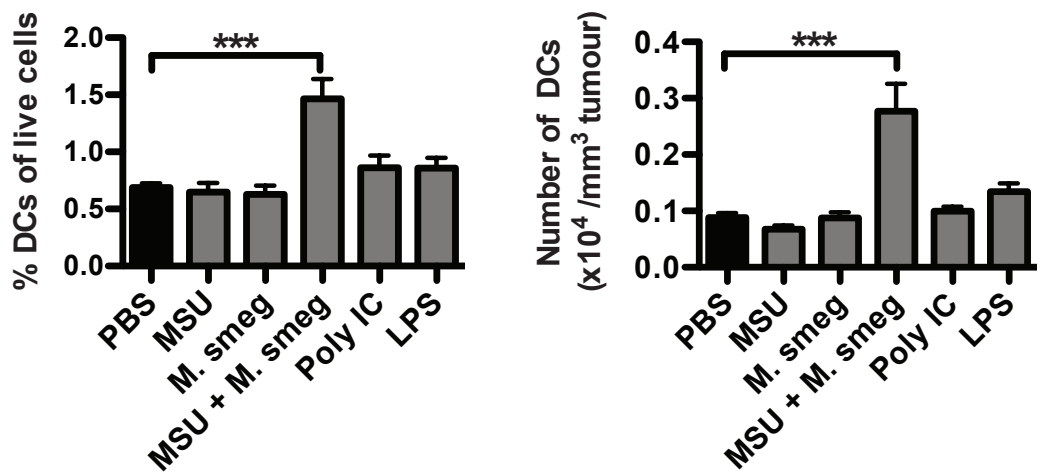

B.
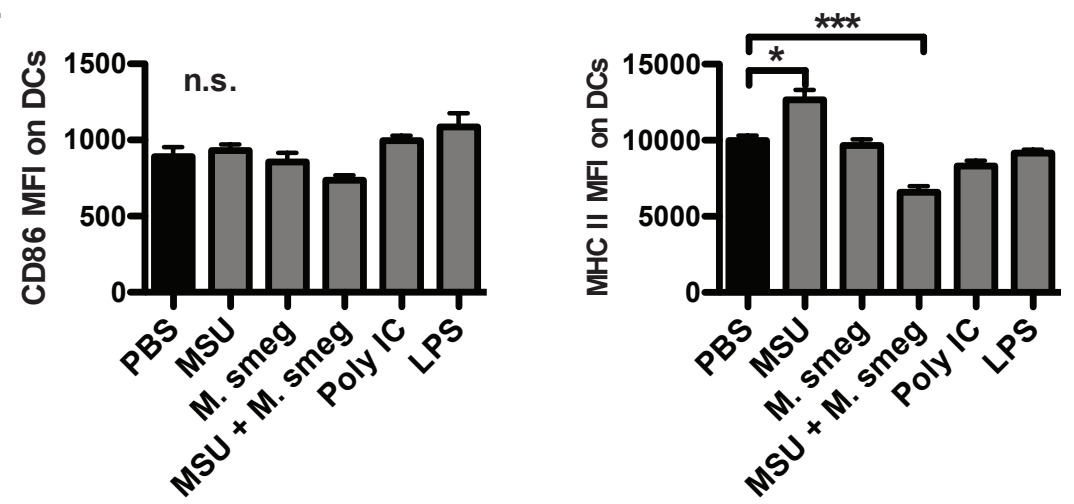

C.
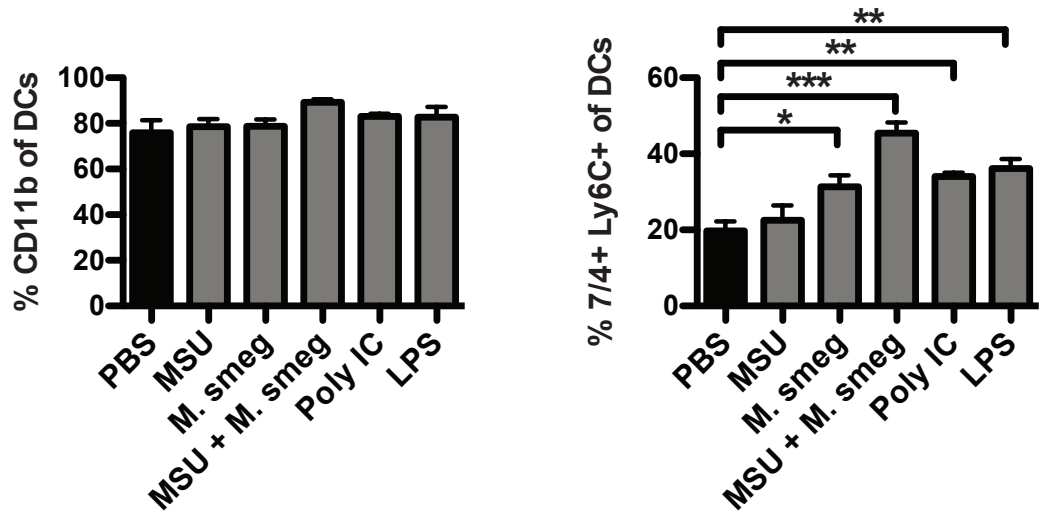

Figure 4.17: Inflammatory DCs are increased in tumours after 4 adjuvant treatments. C57 mice bearing established B16F1 tumours were injected around the tumour with the indicated adjuvants on day 7, 9, 11 and 13. One day after the last treatment DCs in tumours were analysed by flow cytometry. DCs were identified as described in Figure 4.3 and the $7 / 4+\mathrm{Ly} 6 \mathrm{C}+$ population was gated as shown in Figure 4.8. (A) The percentage of DCs among live cells and the number of DCs per tumour volume is shown. (B) Expression levels of the activation markers CD86 and MHC II on DCs are depicted as median fluorescence intensity (MFI). (C) The frequency of CD11b+7/4-Ly6C- (CD11b+) DCs and $\mathrm{CD} 11 \mathrm{~b}+7 / 4+\mathrm{Ly} 6 \mathrm{C}+(7 / 4+\mathrm{Ly} 6 \mathrm{C}+) \mathrm{DCs}$ is shown. Data are expressed as mean $+\mathrm{SE}$ and are combined from two independent experiments with 5 mice per group each. ${ }^{*} p<0.05$, ${ }^{* *} p<0.01$ and ${ }^{* * *} p<0.001$ as assessed by one-way ANOVA with Tukey's post test. n.s. $=$ not significant. 
A.
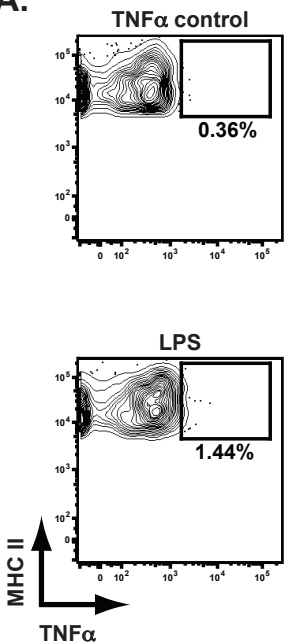

C.
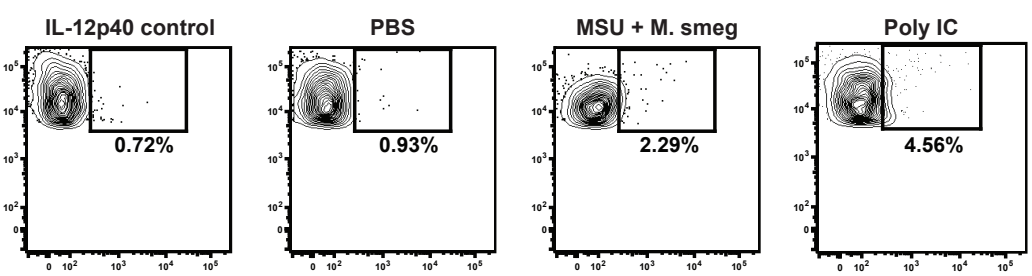

D.
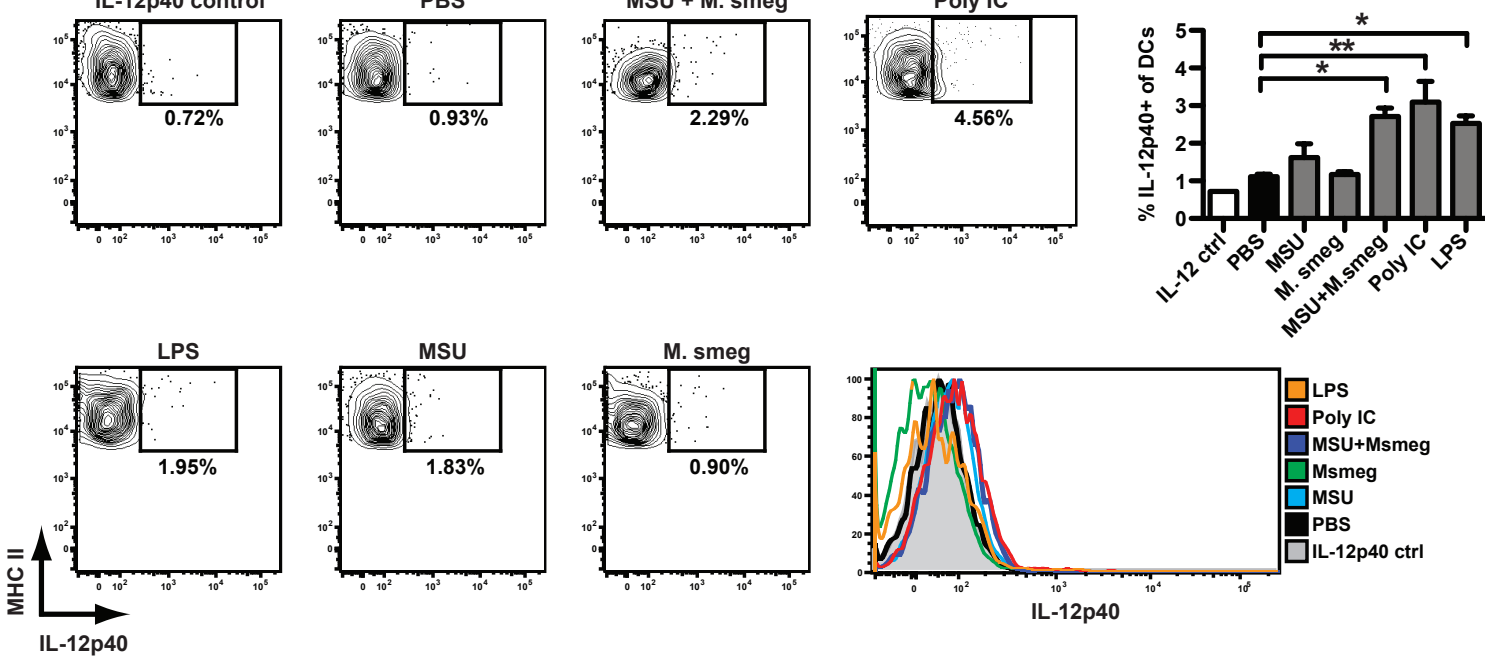

Figure 4.18: Effective adjuvants increase the capacity of tumour-DCs to produce pro-inflammatory cytokines. C57 mice bearing established B16F1 tumours were injected around the tumour with the indicated adjuvants on day 7, 9, 11 and 13. One day after the last treatment tumours were excised and cultured for $6 \mathrm{~h}$ with Golgi-Stop. Cytokine production capacity was analysed by intracellular cytokine staining for IL-12 and TNF $\alpha$. DCs were identified as described in Figure 4.3. In staining controls the same antibodies as in the stained samples were used but the cytokine antibody was replaced with the appropriate isotype control. (A) and (C) show representative flow plots and a histogram for TNF $\alpha$ staining and IL-12 staining in DCs, respectively (B) The percentage of TNF $\alpha+$ DCs and (D) IL-12 + DCs is shown as mean + SE. Data are combined from two independent experiments with 5 mice per group each. ${ }^{*} p<0.05,{ }^{* *} p<0.01$ and ${ }^{* * *} p<0.001$ as assessed by one-way ANOVA with Tukey's post test. 
fraction over $5 \%$ (Figure $4.18 \mathrm{C} \& \mathrm{D}$ ). Poly IC and MSU + M. smegmatis treatments also enhanced the level of IL-12 produced in each DC, as seen by the increase in IL-12 staining intensity (Figure 4.18 C, histogram).

In summary, multiple administrations of the effective adjuvants Poly IC and MSU + M. smegmatis resulted in increased density of immune infiltrate in smaller tumours, indicating that the recruited immune cells were partially controlling tumour growth. While monocyte and DC infiltration continued to be enhanced by repeated exposure MSU $+M$. smegmatis, neither cell type was affected by repeated Poly IC administration. However, both effective adjuvants induced higher frequencies of inflammatory monocyte-derived DCs in tumours and tumour-draining LNs and increased cytokine production in tumour-infiltrating DCs.

This suggests that effective adjuvants induce an inflammatory milieu at the tumour site that attracts monocyte infiltration, promotes differentiation of monocytes into DCs and induces activation and migration of monocyte-derived inflammatory DCs to dLNs, where they may initiate the anti-tumour $\mathrm{T}$ cell response.

\subsection{Discussion}

\section{Effects of adjuvant treatment on tumour growth}

The aim of the experiments described in this chapter was to assess the ability of different adjuvants to activate DCs in vivo and induce a successful anti-tumour immune response. The in vitro experiments with BMDCs and tumour-infiltrating DCs had shown that Poly IC + LPS and MSU + M. smegmatis were superior to other adjuvants in activating DCs and inducing Th1 cytokines. While both of these combinations showed anti-tumour activity in vivo, Poly IC + LPS was not superior to Poly IC treatment by itself and LPS did not affect tumour growth. In addition, MSU only exhibited anti-tumour activity in combination with M. smegmatis, but not with BCG, while both adjuvant combinations were equally effective in vitro. Therefore the activity of adjuvants in vitro and in vivo differed in several aspects. This may be due to the interaction of adjuvants with other cell types not present in the in vitro culture systems, the effects of repeated administration and possible feedback regulation 
mechanisms, and different bioavailability of adjuvants due to solubility, metabolism and uptake by various cell types in the animals.

The combination of MSU + M. smegmtis was not previously described in a tumour-immunotherapy setting. However, individually MSU and M. smegmatis have been reported to show anti-tumour activity in the E.G7-OVA model in separate studies [361, 458]. This tumour is highly immunogenic and spontaneous regression was observed in a considerable fraction of mice in the study by $\mathrm{Hu}$ et al., which could be enhanced by injection of a mixture of soluble uric acid and crystalline MSU. However, MSU was ineffective against the parental EL4 tumour line that lacks the foreign OVA protein and is therefore less immunogenic. This is in line with the findings in this chapter that MSU alone has little activity against the poorly immunogenic B16F1 tumour and also correlated with low levels of DC activation induced by MSU. Likewise, M. smegmatis alone showed no anti-tumour effect in the B16F1 model, although it was able to induce BMDC activation in vitro. However, the level of BMDC activation in vitro with $M$. smegmatis alone was lower compared to the combination of M. smegmatis + MSU and this may not be sufficient to induce a successful response against the aggressive B16F1 tumour.

It is intriguing that MSU + M. smegmatis and MSU + BCG had very comparable effects on BMDCs in vitro, but MSU + BCG completely lacked anti-tumour activity in vivo. This may be due to BCG being derived from a pathogen and therefore having evolved strategies to avoid the immune response. In human monocyte-derived DCs, BCG has been shown to suppress IL-12 production and instead induced high amounts of IL-10 [459]. Furthermore, BCG is preferentially taken up by neutrophils at the injection site [460] and the anti-BCG T cell response is only initiated one week after s.c. injection of $\mathrm{BCG}$, peaking at three weeks [461]. This indicates that DCs may not initially encounter BCG and only become sufficiently exposed to activate antigen-specific $\mathrm{T}$ cells after prolonged periods of time. Moreover, a recent publication showed that BCG was only effective against a murine orthotopic bladder cancer if either three weekly instillations were given, or BCG-specific T cells were present prior to intravesical BCG therapy [402]. Therefore, with the regimen used in the B16F1 model BCG + MSU may activate the anti-tumour immune response too late to induce a discernible effect on tumour growth. 
LPS administration also failed to induce an effective anti-tumour response in the B16F1 model. LPS injection at the tumour site has been variously reported to achieve tumour regression in murine and rat gliomas $[462,463]$, to have no effect on tumour growth in 3LL lung cancer model [464] and even to enhance tumour progression of murine fibrosarcomas [448]. This discrepancy may be caused by different responsiveness of various tumour models to LPS treatment. In addition, the studies that reported tumour regression used very high doses of $0.3 \mathrm{mg}$ to $2.0 \mathrm{mg}$ LPS per injection, which are similar to the amounts used to induce endotoxic shock [465]. The lower dose of LPS chosen in this study to avoid potentially harmful systemic inflammation apparently was insufficient to activate successful anti-tumour immunity, despite the observed activation of BMDCs in vitro and DCs in dLNs in vivo. Possible reasons for this will be discussed below.

Poly IC and CpG both activated BMDCs in vitro and exhibited good anti-tumour activity in the B16F1 model. Kawarada et al. reported similar anti-tumour effetcs for $\mathrm{CpG}$ in a range of tumour models, including a B16 melanoma [11]. Intratumoral injections of Poly IC were previously reported to delay the tumour growth in a murine mesothelioma model [12]. Both studies found that the adjuvant effect depended on CD8 T cells and in the case of CpG also on NK cells. However, the exact mechanism by which the immune response was activated has only partially been elucidated. As mentioned above, CpG was not pursued in this study, as its receptor TLR9 is expressed differently in humans and mice and clinical trials have been disappointing [14, 291, 449].

The effects of Poly IC, LPS, MSU + M. smegmatis combination and individual agonists on DC activation in vivo were investigated in more detail, to elucidate how some adjuvants successfully activated the anti-tumour immune response, while others failed, as discussed below.

\section{Effects of adjuvant treatment on DCs in vivo}

Contrary to our expectations, none of the adjuvants induced activation of DCs at the tumour site as assessed by surface marker up-regulation. It has been shown that skin-resident DCs become activated and start to appear in the dLNs as early as $8 \mathrm{~h}$ post infection or adjuvant 
application [296]. It is therefore likely that 2 days after adjuvant treatment, when DC activation was analysed, any DCs activated at the tumour site had already migrated to the dLNs. However, DC activation in the dLNs did not correlate with treatment success, as LPS administration activated DCs to a similar extent as Poly IC, but MSU + M. smegmatis did not induce up-regulation of surface markers on LN DCs. A single treatment with Poly IC, MSU + M. smegmatis or LPS led to LN enlargement, indicating that the immune cells in dLNs reacted to these adjuvants. This resulted in an higher numbers of all cell types, including DCs.

Repeated administration of Poly IC and MSU + M. smegmatis resulted in a further increase in LN size, whereas the initial LN hyperplasia was not sustained after four treatments with LPS. In addition, the activation marker profile of DCs in repeatedly LPS treated LNs was similar to DCs in PBS controls. This indicates that the DC activation and LN reactivity induced by LPS administration is transient and not sustained after several treatments.

When the DC populations in dLNs were examined in more detail, a subset of DCs expressing Ly6C and 7/4 was found to increase in dLNs of Poly IC and MSU + M. smegmatis treated animals. The expression of Ly6C, 7/4, CD11c and MHC II on these cells is consistent with inflammatory monocyte-derived DCs $[466,467]$. Poly IC and MSU + M. smegmatis also induced an accumulation of inflammatory monocytes at the tumour site already after a single administration. The frequency and numbers of inflammatory DCs remained elevated in dLNs in response to Poly IC and MSU $+M$. smegmatis and these DCs also showed enhanced activation after several treatments. This indicates that successful adjuvants induced inflammation at the tumour site that resulted in the recruitment of inflammatory monocytes. Some of these tumour-infiltrating monocytes expressed low levels of the DC marker CD11c, indicating that they may be in the process of differentiating into inflammatory DCs. In addition, Poly IC and MSU + M. smegmatis treated tumours had elevated numbers of inflammatory DCs after four adjuvant applications and inflammatory DCs were increased in the dLNs both after one and several adjuvant treatments. Inflammatory DCs have previously been shown to mediate the initiation of $\mathrm{T}$ cell responses against Leishmania major [307] and can cross-prime CD8 T cells in response to administration of antigen with adjuvant at skin and mucosal sites [468]. In addition, inflammatory monocyte-derived 
DCs accumulate in dLNs in response to successful M. smegmatis treatment of E.G7-OVA tumours, albeit only 8 days after the initiation of $M$. smegmatis treatment [458]. This suggests that M. smegmatis can induce inflammatory monocyte-derived DCs similar to MSU + M. smegmatis, but that the response takes longer to develop.

LPS treatment, in contrast, only seemed to induce part of this response. LPS induced sustained accumulation of monocytes and after several applications also of inflammatory DCs at the tumour site. However, inflammatory DCs never increased in the dLNs in response to LPS, indicating that LPS failed to induce full differentiation of monocytes into DCs and their migration to dLN. This finding is in line with a report by Rotta et al., who find that LPS injection together with particulate antigens blocks the conversion of monocytes to migratory DCs and consequently results in very weak T cell responses [469].

\section{Adjuvant-induced monocyte recruitment to the tumour site}

As discussed above, it is likely that the monocytes recruited to the tumour tissue differentiate into DCs and thereby promote the anti-tumour immune response. However, their surface phenotype is also consistent with them being myeloid-derived suppressor cells (MDSCs) [196]. Without analysis of their functional capacity, this possibility cannot be fully excluded. The highest level of monocyte-accumulation was found in MSU + M. smegmatis treated tumours and Poly IC likewise induced a considerable influx of monocytes into tumours. These adjuvant treatments successfully delayed tumour growth and correlated with the accumulation of monocyte-derived inflammatory DCs in dLNs, indicating that the recruited monocytes were not suppressing the immune response. Several reports have described the induction of MDSCs in the spleens of tumour-bearing mice [470]. In a comparative analysis by Youn et al., EL4 thymomas resulted in MDSCs accounting for over $20 \%$ of all splenocytes, whereas B16F10 melanomas only induced about 4\% MDSCs [196]. In the present study, both the frequencies and numbers of monocytic and granulocytic monocytes were similar between naïve and tumour bearing, saline-treated mice (see Appendix D). This indicated that the B16F1 melanomas were not inducing MDSCs in the spleen. Moreover, when spleen monocytes were purified and assessed for their ability to suppress polycolonal 
T cell expansion in vitro in a well established assay of MDSC function [196, 471, 472], no difference in the suppressive ability of moncytes from naïve, saline-treated tumour bearing and adjuvant-treated tumour bearing animals was found (see Appendix D). This further supports that neither the tumour nor any of the adjuvant treatments induced MDSCs in the spleens. In addition, the adjuvant treatments that led to monocyte recruitment to the tumours (MSU + M. smegmatis, Poly IC and LPS) resulted in even lower numbers of monocytes in the spleens. It was previously thought that monocytes were recruited to sites of inflammation mainly from the blood and that emergency haematopoiesis in response to inflammation increased their availability. However, a recent publication by Swirski et al. demonstrated that the spleen is a main reservoir for monocytes that are recruited to inflammatory sites in a CCR2-independent, angiotensin II driven manner [473]. As the decrease in monocyte-numbers in the spleen corresponds to the increase of monocytes at the tumour site after adjuvant treatment, the spleen appears to be the major source of these cells.

\section{Systemic and local induction of cytokines}

To elucidate further how MSU + M. smegmatis and Poly IC successfully activated DCs to induce an anti-tumour immune response in vivo, while LPS only transiently activated DCs and failed to achieve a subsequent anti-tumour effect, cytokines and growth factors produced systemically and locally in response to adjuvant administration were analysed.

Rapid systemic release of IFN $\gamma$ and IL-12 were observed only in response to the successful treatments Poly IC and MSU $+M$. smegmatis. IFN $\gamma$ can be produced by NK cells and activated T cells with NK cells producing high levels early during the course of infection [474]. Therefore, NK cells and T cells are likely to be the source of the observed IFN $\gamma$ in the serum of adjuvant treated mice. While T cells have been reported to express TLRs [475], their functional role remains controversial [476]. A study by Caramalho et al. described LPS activating Tregs via TLR4 [475], while later reports found Treg activation was due to TLR2 and not mediated by TLR4 activation [477]. In addition, non-Treg CD4 T cells express low amounts of TLR mRNA and and mRNA content does not always correlate with receptor expression, as TLR expression is tightly regulated at a protein level [478]. Furthermore, 
T cells and NK cells do not express NLRP3, while DCs and macrophages highly express both TLRs and NLRs $[291,359]$. IFN $\gamma$ induction in NK cells and T cells is generally mediated by Stat4 signalling in response to IL-12 $[479,480]$. Both IL-12 and TNF $\alpha$ production was increased in LN DCs in response to $\mathrm{CpG}$ and $\mathrm{MSU}+M$. smegmatis treatment. It is therefore likely that NK cells and T cells do not directly respond to adjuvants, but are instead activated to release IFN $\gamma$ by IL-12, which is secreted from DCs in response to adjuvant treatment.

IL-1 $\beta$ was significantly elevated only in response to MSU $+M$. smegmatis, which also induced some secretion of IL-6. Poly IC induced higher levels of IL-6 in serum than MSU + M. smegmatis and also systemically increased TNF $\alpha$. While IL-1 $\beta$ is mainly produced by DCs and macrophages, TNF $\alpha$ and IL-6 can be secreted a range of cell types [481, 482]. In response to LPS, about 10-fold higher levels of the pro-inflammatory cytokines IL-6 and TNF $\alpha$ were detected, compared to Poly IC. At the same time, LPS treatment resulted in very high serum concentrations of the regulatory cytokine IL-10. This pronounced systemic induction of IL-6 and IL-10 also correlated with a local increase in IL-6 and IL-10 mRNA in LPS treated tumours. Together, these findings indicate that successful adjuvants such as Poly IC and MSU + M. smegmatis induce a limited release of pro-inflammatory factors, while LPS induces very strong inflammation coupled with feedback regulation and and overall suppression of the immune response. This hypothesis is supported by previous reports that LPS stimulates high levels of IL-10 production in macrophages and DCs [483, 484] and induces IL-10 producing Tregs [485]. Furthermore, LPS induced IL-10 can lead to unresponsiveness to further stimulation with LPS and even other TLR ligands, partly via degradation of the signalling molecule MyD88 [446, 486].

The growth factors GM-CSF and G-CSF are both involved in hematopoiesis and can be released from a range of cells in response to inflammation [487]. While G-CSF mainly mobilises neutrophils from the BM, GM-CSF induces the release of myeloid cells from the BM, but has also been implicated in activation and survival of myeloid cells and maturation of DCs. In response to adjuvant treatments, GM-CSF was not significantly elevated in the serum. However, GM-CSF mRNA was transiently up-regulated at the tumour site by MSU + M. smegmatis treatment. LPS administration led to much higher expression of GM-CSF 
mRNA compared to the other adjuvants, which continued to increase at 24 hours. The substantial induction of GM-CSF mRNA at the tumour site without detection of GM-CSF protein in the serum may reflect generally higher levels of this growth factor in inflamed or stressed tissues compared to serum [488].

The role of GM-CSF in tumour immunity remains unclear, with reports from the literature showing anti-tumour, as well as pro-tumour effects. When B16F10 melanoma cells were engineered to secrete GM-CSF, DC recruitment to the tumour site and tumour rejection were observed [489]. Several clinical trials that used tumour cells transduced to secreted GM-CSF or vaccination with tumour peptides and GM-CSF reported increased anti-tumour immunity [490-493]. However, some of these studies did not compare the GM-CSF effect to the vaccine without GM-CSF [492]. Furthermore, when the vaccines with and without GM-CSF were compared, some trials reported an inhibitory effect of GM-CSF [494, 495]. Suppressive effects of high-dose GM-CSF were found to be due to induction of MDSCs [204] and a shift from NO to arginase I and urea production [496, 497]. Together, these studies indicate that high levels of GM-CSF may favour tumour progression, whereas low levels of GM-CSF may activate anti-tumour activity. Therefore, the low levels of GM-CSF and G-CSF induction observed in response to Poly IC and MSU + M. smegmatis may be beneficial, while the levels induced by LPS may be too high to stimulate the anti-tumour immune response.

While a lack of NO production is associated with the inhibitory function of MDSCs [496], the production of NO and ROS from macrophages in tumours has been shown to be tumoricidal and enhance anti-tumour immunity [498]. Intratumoral IL-12 therapy is also reported to induce NO production and to increase the expression of the NO producing enzyme iNOS. However, this negatively regulated the anti-tumour immune response and inhibition of iNOS activity increased the anti-tumour effect of IL-12 [499]. The role of iNOS in the anti-tumour immune response therefore remains controversial. In the B16F1 tumours, Poly IC only induced iNOS mRNA transiently at 2 hours and at 24 hours resulted in a substantial decrease. MSU + M. smegmatis treatment led to a sustained 8-fold increase of iNOS, whereas LPS induced a progressive over 30-fold increase in iNOS mRNA. This indicates that iNOS may either not be critical for the response to adjuvant treatment or that low or transient in- 
duction may be sufficient or even more beneficial, than the high levels associated with LPS treatment.

The analysis of CXCL10 expression revealed a limited increase with MSU + M. smegmatis treatment and a transient induction with Poly IC and LPS. CXCL10 has been implicated in the recruitment of activated $\mathrm{T}$ cells to sites of inflammation and tumours $[346,500]$. Therefore, induction of CXCL10 at the tumour site may enable the infiltration of activated CD8 T cells and enhance anti-tumour immunity.

In summary, the analysis of cytokine induction in response to adjuvant treatment showed that both Poly IC and MSU + M. smegmatis increased IL-12 and IFN $\gamma$ production. IL-12 is critical for the activation of Th1 responses and CTLs [451-453] and enhances IFN $\gamma$ production from NK cells and T cells $[479,480]$. IFN $\gamma$ can activate macrophages to become tumoricidal [498] and has been shown to be critical for the control of B16 melanomas [501]. Both Poly IC and MSU + M. smegmatis induced limited secretion of pro-inflammatory cytokines and hematopoietic growth factors. In contrast, LPS administration resulted in very high levels of pro-inflammatory mediators, coupled with equally high levels of the regulatory cytokine IL-10. This suggests that moderate inflammation may be beneficial for the induction of anti-tumour immunity, while a very high level of inflammatory mediators may result in feedback regulation with the induction of suppressive cytokines and may thus fail to stimulate the anti-tumour immune response. 


\section{Conclusions}

Repeated peri-tumoral administration of Poly IC, CpG and MSU + M. smegmatis delayed the growth of B16F1 tumours. In contrast, MSU, M. smegmatis, LPS, BCG or MSU + BCG had no effect on tumour growth. Combination of Poly IC with LPS, M. smegmatis or MSU did not lead to further tumour growth delay compared to Poly IC alone.

None of the adjuvants showed activation of DCs at the tumour site and while up-regulation of activation markers on DCs in dLNs was observed in response to Poly IC and LPS, this did not correlate with treatment success. Rather, accumulation of inflammatory monocytederived DCs in dLNs and tumours was associated with adjuvants that delayed tumour growth. These DCs may have differentiated from monocytes that were recruited to the tumour site already after a single adjuvant administration.

Poly IC and MSU + M. smegmatis adjuvants also induced systemic release of the Th1 inducing cytokine IL-12 and the Th1 effector cytokine IFN $\gamma$, indicating that these adjuvants induced Th1 polarisation. Induction of a limited amount of the pro-inflammatory cytokines IL-1 $\beta$, IL-6 and TNF $\alpha$ was also observed, whereas LPS induced very high levels of both pro-inflammatory cytokines and anti-inflammatory IL-10.

Taken together, successful adjuvants appear to induce limited inflammation coupled with the release of Th1 cytokines. They recruit monocytes to the tumour site and may induce them to differentiate into mature DCs. Inflammatory monocyte-derived DCs then migrate to the tumour-draining LNs, where they can initiate the anti-tumour response.

Repeated adjuvant administration enhanced or sustained the effects of Poly IC and MSU + M. smegmatis on the induction and activation of monocyte-derived inflammatory DCs, while DC activation in response to LPS was transient and repeated LPS treatment seemed to decrease DC activation in dLNs. 



\section{Chapter 5}

\section{Effective adjuvants activate both \\ innate and adaptive effector cells}




\section{$5.1 \quad$ Introduction}

Both CD8 T cells and NK cells are cytotoxic and have the capacity to directly eliminate tumour cells as discussed in more detail in the general introduction. Studies that all used the same adjuvant, $\mathrm{CpG}$, in different murine tumour models reported that the anti-tumour immune response was mediated either solely by CD8 T cells [502], solely by NK cells [503] or dependent on both CD8 T cells and NK cells [11, 504]. Another group using Poly IC administration at the tumour site in a mesothelioma model concluded that CD8 $\mathrm{T}$ cells, but not NK cells, were critical for the anti-tumour response [12]. The importance of CD8 T cells and NK cells in the anti-tumour effect of local Poly IC treatment in B16 melanoma has not been established yet. As the combination of $\mathrm{MSU}+$ M. smegmatis has not previously been used as an adjuvant in tumour immunotherapy, the requirement for different effector cell types in this treatment also remains to be determined.

Several different ways in which adjuvant treatment can improve effector cell activity have been described. Adjuvant treatment can activate DCs in tumours and tumour-draining LNs, enabling them to prime tumour-specific CD8 T cells [318]. DC activation can be further enhanced by anti-IL10 receptor treatment, which results in a potent CD8 T cell and CD4 T cell dependent anti-tumour response [322]. Increased recruitment of CD8 T cells and NK cells to the tumour site frequently contributes to the beneficial effect of adjuvant treatment $[345,503,504]$. Despite this, effector cell recruitment is not altered by adjuvant administration in a study by Currie et al., but cytokine production and lytic activity of effector cells already present at the tumour site prior to adjuvant treatment are improved instead [12]. The different effects of adjuvants on immune effector cells are not mutually exclusive, and enhanced effector cell function, such as increased target cell lysis, can be observed in conjunction with increased effector cell recruitment [503].

While the requirement for CD8 T cells and NK cells is readily examined in murine tumour models, the assessment of the role of CD4 $\mathrm{T}$ cells in the adjuvant induced anti-tumour response is more complex. In studies that used depletion of the total CD4 T cell population to assess their importance for an adjuvant-induced anti-tumour effect, CD4 T cells were either found to be required [322], or to be dispensable [12]. CD4 T helper cells have been 
shown to improve anti-tumour responses [505], as they can enhance the priming of cytotoxic CD8 T cells via 'licensing' of DCs $[272,273]$. In addition, CD4 T cells have been implicated in increasing CD8 $\mathrm{T}$ cell recruitment to the tumour and enhancing their effector function in situ [506]. Recent work by Quezada et al. suggests that CD4 T cells may even become cytotoxic and directly eliminate tumour cells [71]. However, the CD4 T cell population consists not only of effector cells but also comprises Tregs. Tregs are known to suppress anti-tumour immune responses as evidenced by the correlation of high Treg numbers with worse disease outcome in tumour-bearing individuals $[45,46,172]$. In addition, numerous studies have shown that depletion of Tregs in mice [173, 507] and humans [508] enhances the anti-tumour immune response. 


\subsection{Aims}

The purpose of the experiments described in this chapter was to establish which effects adjuvant treatment had on CD4 T cells, CD8 T cells and NK cells. The hypothesis was that CD8 T cells and/or NK cells would be required for the elimination of tumour cells, and that adjuvant treatment with Poly IC and MSU + M. smegmatis would increase their priming, function or recruitment into tumours. Furthermore, CD4 T helper cells would probably be required to provide help for the initiation of CD8 $\mathrm{T}$ cell responses, but CD4 Tregs might suppress the anti-tumour functions of effector cells.

The specific aims were:

- To asses whether treatment of B16OVA tumours with effective adjuvants increased the proliferation of OVA-specific CD8 and CD4 T cells in vivo

- To investigate whether effective adjuvants increased recruitment of CD4 T cells, CD8 T cells and NK cells to the tumour, or changed their expression of activation markers, or cytokine production capacity

- To determine whether CD8 T cells from animals treated with effective adjuvants were sufficient to confer tumour protection when transferred to naïve recipients

- To determine which effector cell types were necessary for the anti-tumour effect in vivo

- To assess whether combination of adjuvant treatment with Treg depletion could be used to enhance the anti-tumour effect 


\subsection{Results}

\subsubsection{Effect of adjuvant treatment on in vivo $\mathrm{T}$ cell proliferation}

To investigate if adjuvant treatment could increase priming of CD8 and CD4 T cells, an OVA expressing B16 melanoma was used in conjunction with adoptive transfer of OVA-specific OTI or OTII T cells. In order to track CD8 T cell proliferation in vivo, naïve CD8 T cells from OTI congenic donors were purified and labelled with CFSE. This resulted in > 95\% pure OTI cells (Figure 5.1 A), which were then transferred into B16.OVA bearing animals. Three days after a single adjuvant administration at the tumour site, OTI T cell proliferation was assessed by CFSE dilution in tumour-draining and non-draining LNs (Figure $5.1 \mathrm{~B}$ ). Representative proliferation histograms for the different adjuvant treatments are shown in Figure 5.1 C.

Nearly half of the OTI cells had divided at least once in response to the OVA-expressing tumour, even in the absence of adjuvant treatment (Figure 5.1 C and 5.2 B). Proliferation and proportion of OTI cells among CD8 T cells were much lower in LNs contralateral to the tumour site compared to tumour-draining LNs, indicating that the presence of antigen was required for OTI proliferation and that the observed proliferation was tumour-dependent (Figure $5.2 \mathrm{~A} \& \mathrm{~B}$ ).

The proliferation of OTI cells was slightly enhanced by adjuvants, but only MSU + M. smegmatis treatment resulted in a significant increase in the frequency of OTI among CD8 T cells and in the proportion of divided OTI (Figure 5.2 A \& B). As the tumour-draining LNs were considerably larger in adjuvant treated animals, especially in response to MSU + M. smegmatis and Poly IC (Figure 4.8), the number of total OTI T cells as well as divided OTI cells was substantially increased in these groups (Figure $5.2 \mathrm{C} \& \mathrm{D}$ ). This may be due to augmented retention of all $\mathrm{T}$ cells, including antigen-specific CD8 $\mathrm{T}$ cells, in the tumour-draining LNs under the local inflammatory conditions established by Poly IC and $\mathrm{MSU}+$ M. smegmatis treatment. 
A. OTI T cell purity

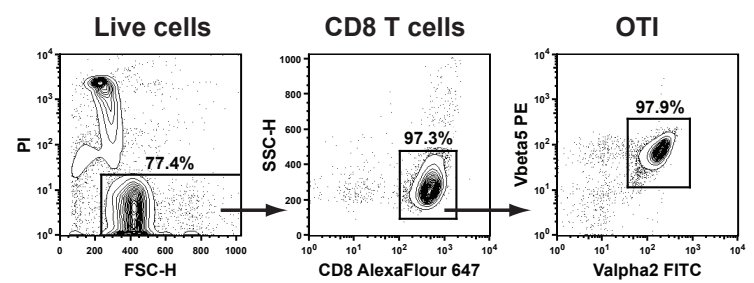

B.

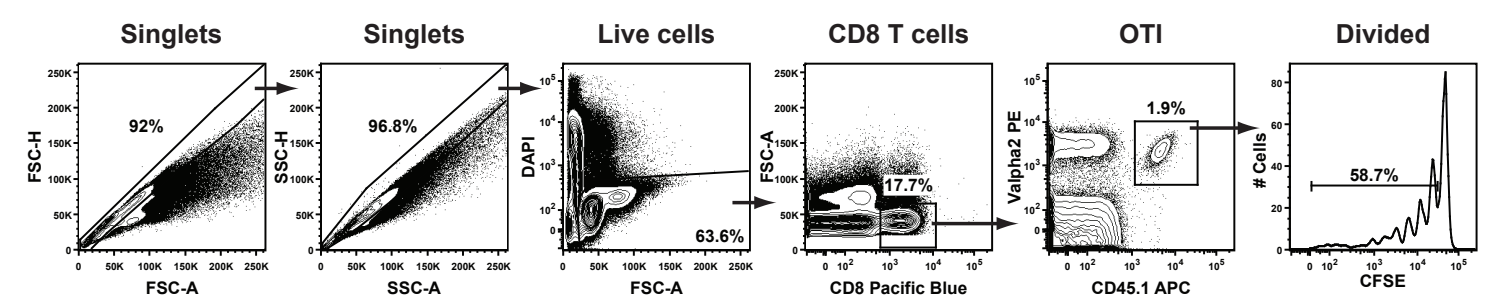

C.

OTI proliferation in tumour-draining LN
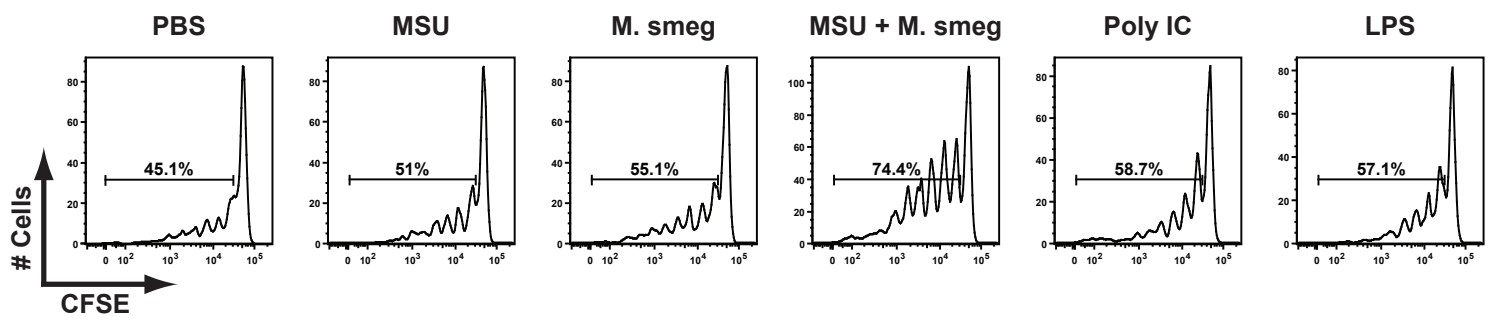

Figure 5.1: Adjuvant treatment enhances tumour-antigen specific CD8 $\mathrm{T}$ cell proliferation. CD8 T cells were purified from LNs and spleens of OTI congenic mice by magnetic sorting, labelled with CFSE and adoptively transferred into C57 mice bearing established B16.OVA tumours (day 8). One day later, mice were given a single adjuvant injection around the tumour. OTI proliferation was assessed by CFSE dilution in tumour-draining and contralateral LNs three days after adjuvant treatment. (A) The purity of OTI T cells before transfer into C57 mice is illustrated by flow plots. (B) The flow cytometry gating strategy identifying OTI T cells as singlet live CD8+CD45.1+V $\alpha 2+$ cells and divided cells based on CFSE dilution is shown. (C) Representative CFSE dilution profiles for the different adjuvant treatments in dLNs are depicted.

The increased proliferation together with the higher numbers of OTI T cells observed in tumour-draining LNs of Poly IC and MSU + M. smegmatis treated mice indicate that these adjuvants increase recruitment and priming of antigen-specific CD8 $\mathrm{T}$ cells in the tumour-draining LNs.

CD4 $\mathrm{T}$ cell proliferation was assessed using a similar experimental strategy as for CD8 T cells. CD4 T cells were purified from OTII congenic mice (Figure 5.3 A) and transferred into mice bearing subcutaneous B16.OVA tumours. Five days after a single adjuvant administra- 
A.

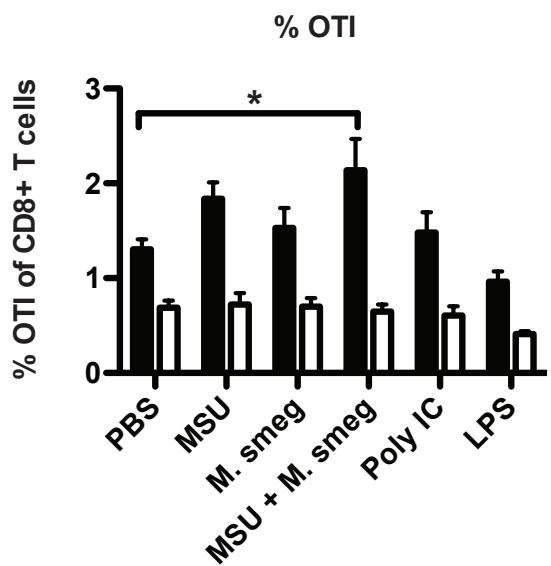

B.

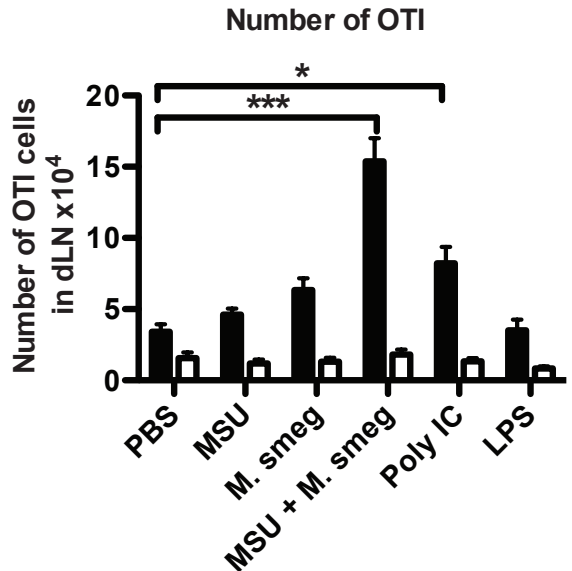

B.

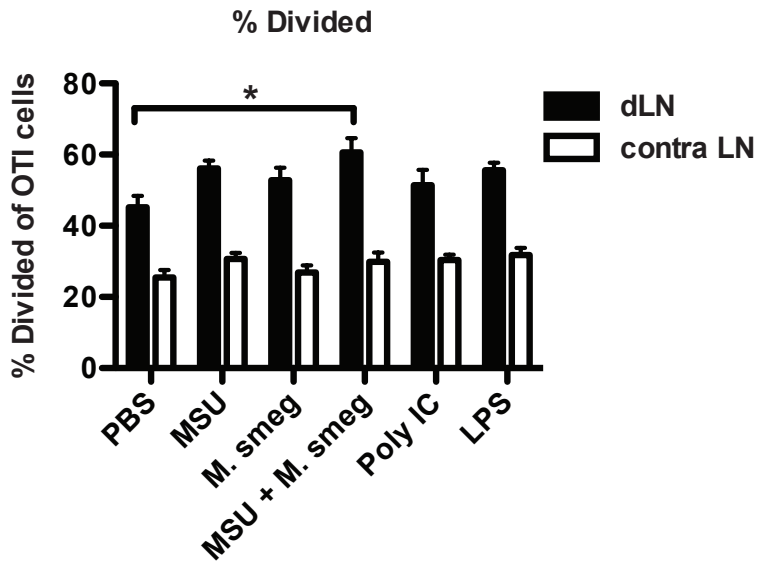

C. Number of divided OTI

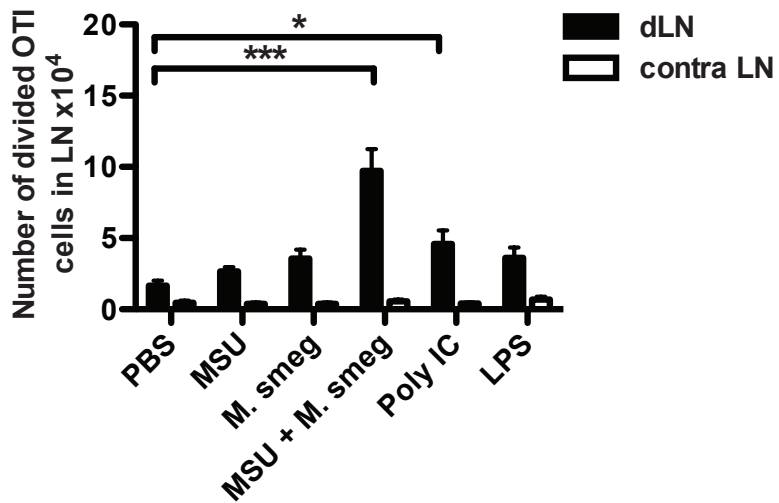

Figure 5.2: Successful adjuvants result in increased numbers of tumour-specific CD8 $\mathbf{T}$ cells in dLN. OTI T cell proliferation was assessed as described in Figure 5.1. The frequency $(\mathbf{A})$ and number $(\mathbf{C})$ of OTI T cells after the indicated adjuvant treatments is shown. The proportion (B) and number (D) of divided OTI T cells in response to adjuvant administration is depicted. Data are shown as mean $+\mathrm{SE}$ and are pooled from 2 independent experiments, with 5 mice per group each.

$\mathrm{dLN}=$ tumour-draining LNs; contra $\mathrm{LN}=\mathrm{LNs}$ contralateral to the tumour. ${ }^{*} p<0.05$, $* * * \quad p<0.001$ according to one-way ANOVA with Tukey's post test.

tion, OTII proliferation was analysed by CFSE dilution (Figure $5.3 \mathrm{~B}$ ). In the absence of adjuvant treatment, no proliferation of OTII T cells was observed (Figure 5.3 C \& 5.4 B). MSU, M. smegmatis, LPS, and Poly IC all failed to induce OTII proliferation (Figure 5.3 C \& 5.4 B) and in fact the frequency and number of OTII cells was similar in tumour-draining and non-draining LNs in some of these groupsd (Figure 5.4 A \& C). MSU + M. smegmatis, by contrast, induced substantial OTII proliferation, with up to $35 \%$ of OTII cells in dLNs having divided (Figure 5.3 C \& $5.4 \mathrm{~B}$ ). This significant level of OTII proliferation in the MSU + M. smegmatis treated mice was also reflected in a large increase in the number 
A.

OTII T cell purity

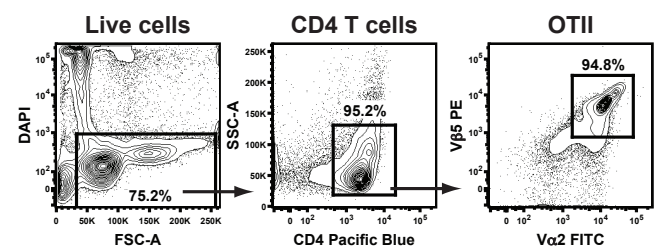

B.

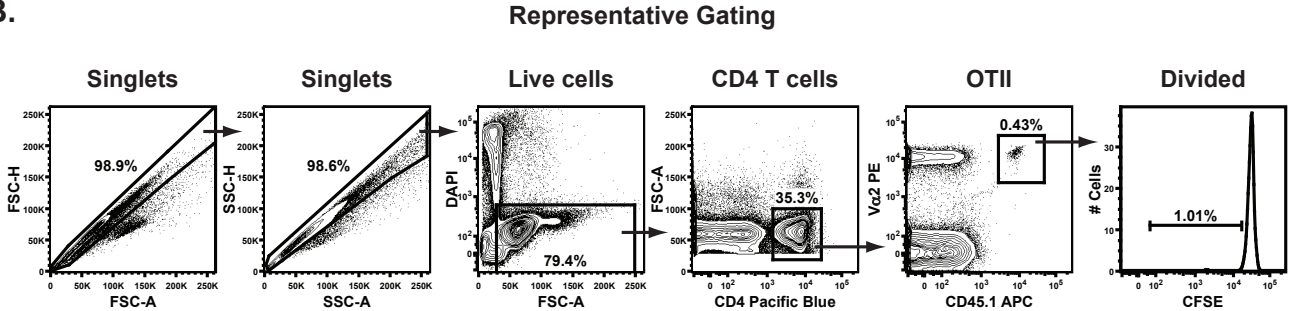

C.

OTII proliferation in tumour-draining LN
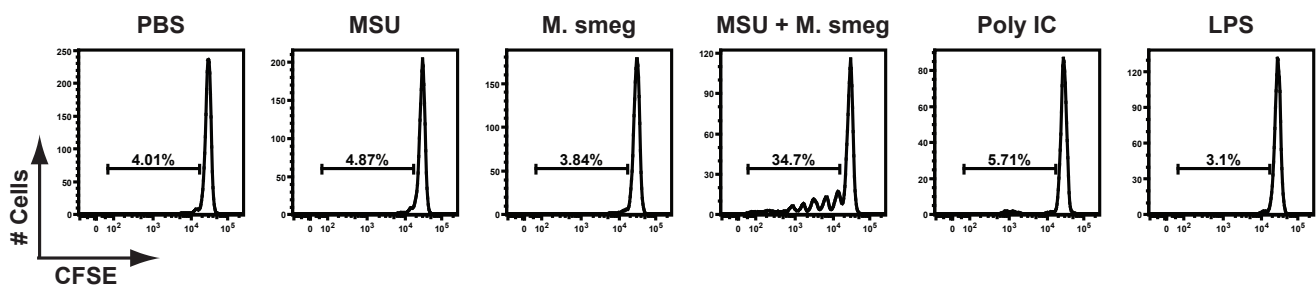

Figure 5.3: Only MSU + M. smegmatis treatment stimulates proliferation of tumour-antigen specific CD4 $\mathbf{T}$ cells in $\mathbf{d L N}$. CD4 $\mathrm{T}$ cells were purified from LNs and spleens of OTII congenic mice by magnetic sorting, labelled with CFSE and adoptively transferred into C57 mice bearing established B16.OVA tumours (day 8). One day later, mice were given a single adjuvant injection around the tumour. OTII proliferation was assessed by CFSE dilution in tumour-draining and contralateral LNs five days after adjuvant treatment. (A) The purity of OTII T cells before transfer into C57 mice is illustrated by flow plots. (B) The flow cytometry gating strategy identifying OTII T cells as singlet live CD4+CD45.1+V $\alpha 2+$ cells and divided cells based on CFSE dilution is shown. (C) Representative CFSE dilution profiles for the different adjuvant treatments in dLNs are depicted.

of total and divided OTII cells (Figure $5.4 \mathrm{C} \& \mathrm{D}$ ). Despite this, the frequency of OTII cells among all CD4 T cells was not increased in dLNs of MSU + M. smegmatis treated animals (Figure 5.4 A). This could be caused by proliferation of endogenous CD4 T cells in response to other tumour antigens or general retention of $\mathrm{T}$ cells in the dLNs, which would result in an increase in all CD4 T cells in dLN, thereby masking the OTII expansion. This would be in line with the overall increase in LN size in response to $\mathrm{MSU}+M$. smegmatis treatment. It is unlikely that OTII T cells proliferating in MSU + M. smegmatis treated animals were responding directly to any antigen of M. smegmatis that could be cross-reactive 
A.

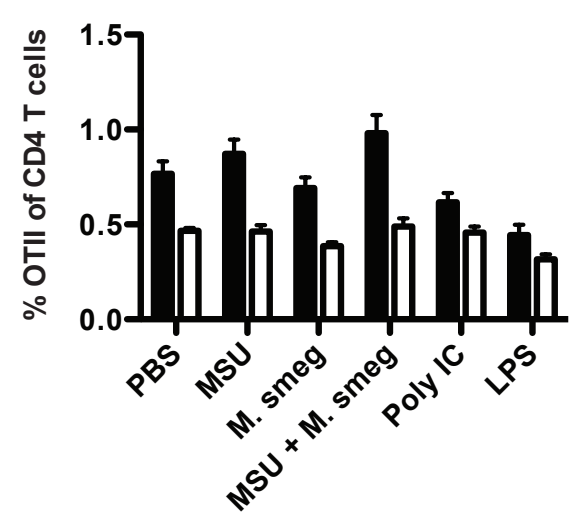

C.

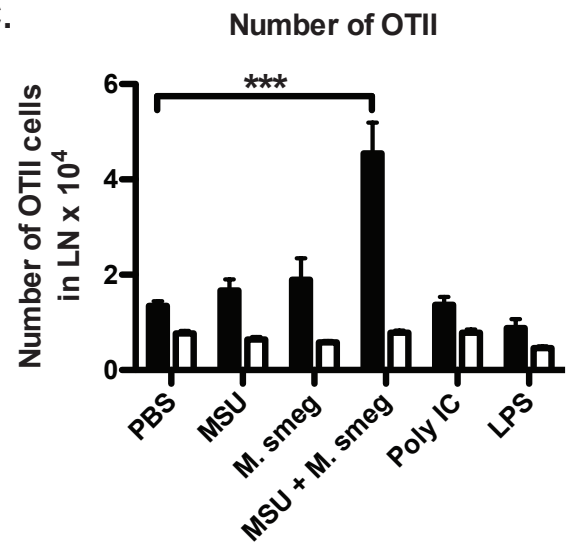

B.

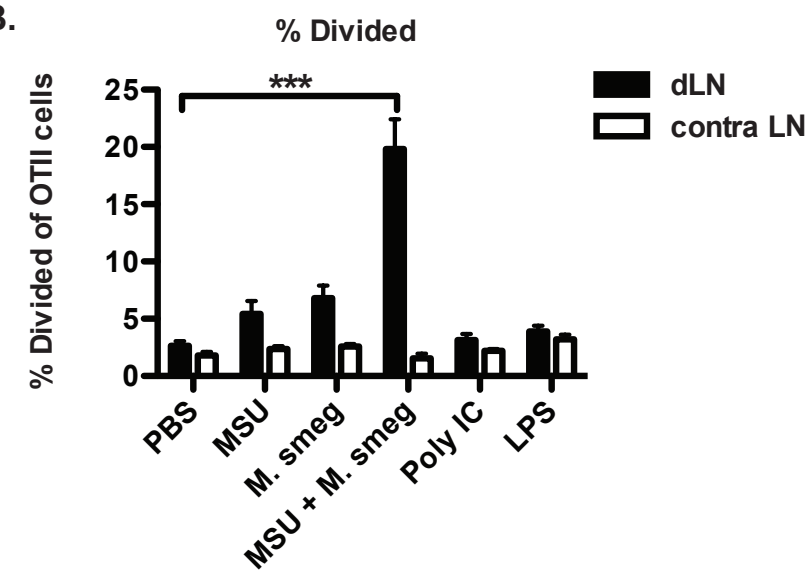

D.

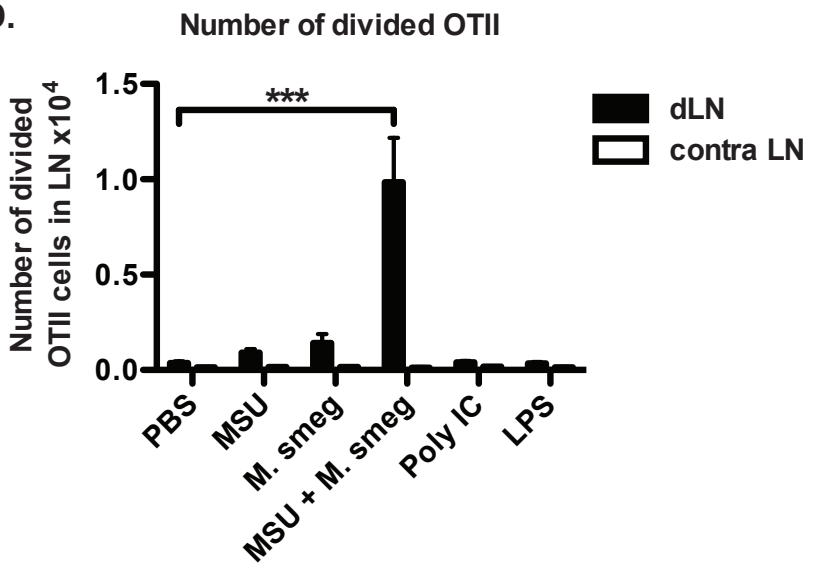

Figure 5.4: MSU + M. smegmatis treatment increases tumour-specific CD4 $\mathbf{T}$ cell proliferation and numbers in $\mathbf{d L N}$. OTII T cell proliferation was assessed as described in Figure 5.3. The frequency $(\mathbf{A})$ and number $(\mathbf{C})$ of OTII T cells after the indicated adjuvant treatments is shown. The proportion (B) and number (D) of divided OTII T cells in response to adjuvant administration is depicted. Data are shown as mean $+\mathrm{SE}$ and are pooled from 2 independent experiments with 5 mice per group each.

$\mathrm{dLN}=$ tumour-draining LNs; contra $\mathrm{LN}=\mathrm{LNs}$ contralateral to the tumour. ${ }^{* * *} p<0.001$ according to one-way ANOVA with Tukey's post test.

to OVA, as the response only occurred after $\mathrm{MSU}+M$. smegmatis treatment and not in response to $M$. smegmatis alone.

These data indicate that adjuvant treatment can induce proliferation of CD4 T cells specific for a model antigen expressed in tumours. However, the response was limited to MSU + M. smegmatis and not observed in any other adjuvant treated group, including Poly IC treated mice. Therefore only MSU + M. smegmatis treatment induced tumour-specific CD4 T cell priming, while both MSU + M. smegmatis and Poly IC augmented CD8 T cell priming. 


\subsubsection{A single adjuvant treatment has a minor impact on effector cell numbers and phenotype in tumours}

In addition to enhancing $\mathrm{T}$ cells priming via increased $\mathrm{DC}$ activation, adjuvants can also induce local inflammation, thus attracting effector cells to the tumour site. As shown in Figure 4.12, Poly IC, MSU + M. smegmatis and also LPS induced expression of pro-inflammatory cytokines and chemokines in the tumour. To determine if this led to recruitment of effector cells to the tumour site, the infiltration of CD4 T cells, CD8 T cells and NK cells was analysed after a single adjuvant administration.

While there was a trend towards a higher proportion of CD4 T cells and CD8 T cells in response to MSU + M. smegmatis treatment, this was not statistically significant (Fig. 5.5 A left and middle panels). However, a single MSU + M. smegmatis administration induced a significant increase in the frequency of NK cells (Fig. 5.5 A right panel). Separate treatments with either MSU or $M$. smegmatis resulted in a slightly smaller, not statistically significant increase in NK cell frequency. In contrast, Poly IC did not change the proportion of any of the effector cells analysed (Fig. 5.5 A), but instead led to up-regulation of the early activation marker CD69 on CD8 T cells and NK cells (Fig. 5.5 B middle and right panels). A similar increase in CD69 levels on NK cells was observed in response to LPS (Fig. 5.5 B, right panel). On CD4 T cells, a minor up-regulation of CD69 levels was seen with MSU + M. smegmatis treatment (Fig. 5.5 B left panel).

Overall, a single adjuvant treatment did not cause major changes in effector cell recruitment or activation in tumours. MSU $+M$. smegmatis resulted in increased NK cell infiltration, while Poly IC led to enhanced activation of intra-tumoral CD8 T cells and NK cells. However, MSU and $M$. smegmatis alone attracted nearly as many NK cells as MSU $+M$. smegmatis. Furthermore, LPS activated NK cells to a similar degree as Poly IC, but none of these adjuvants were effective in delaying tumour growth (Figure 4.2). Therefore, these early changes in tumour-infiltrating effector cell populations do not seem to be sufficient to achieve the observed anti-tumour growth effect. 
A.

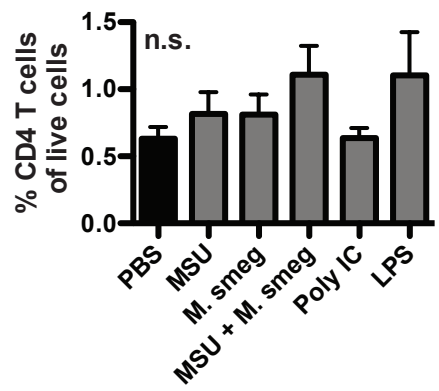

B.

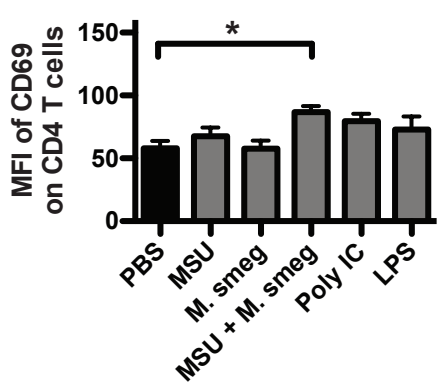

CD8 T cells
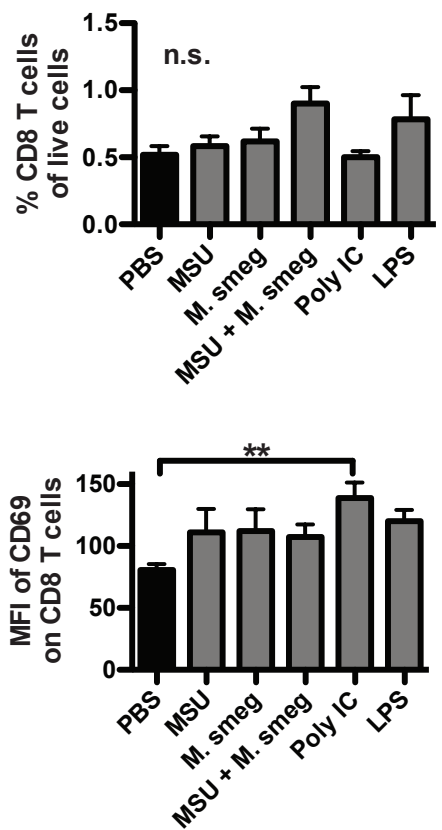

NK cells
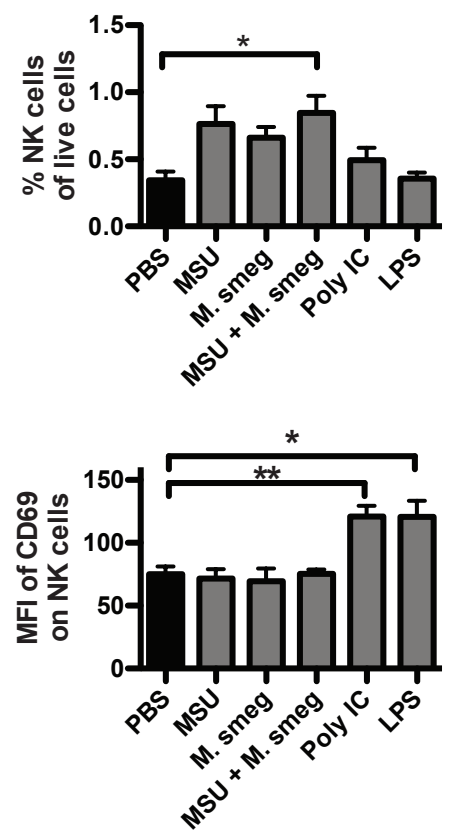

Figure 5.5: A single adjuvant treatment leads to minor changes in intratumoral effector cells. C57 mice bearing B16F1 tumours were injected peri-tumorally with the indicated adjuvants on day 7 . One day later, effector cells in tumours were analysed by flow cytometry and gated as in Figure 4.3. (A) Frequency of CD4 T cells, CD8 T cells and NK cells among live cells is shown. (B) The expression of CD69 on these cells is shown as median fluorescence intensity (MFI). Data are shown as mean $+\mathrm{SE}$ and are pooled from two independent experiments with 5 mice per group each. ${ }^{*} p<0.05,{ }^{* *} p<0.01$ as assessed by one-way ANOVA with Tukey's post test. 


\subsubsection{Repeated adjuvant treatment increases infiltration and activation of effector cells in tumours}

The changes observed in effector cell populations in tumours after a single adjuvant treatment had been minor and didn't show a clear correlation with the capacity of each adjuvant to induce a delay in tumour-growth. T cells are known to require several days of expansion in LNs and only begin to exit the LN and migrate to the effector tissue $48 \mathrm{~h}$ after antigen encounter [509]. In mice treated with Poly IC or MSU $+M$. smegmatis, increased priming of CD8 T cells had been observed in dLN 3 days after treatment (Figure 5.1). Therefore, $\mathrm{T}$ cells primed in response to adjuvant treatment may only become apparent in the tumours at later time points. In addition, repeated adjuvant administration and consequently sustained release of pro-inflammatory and chemoattractant factors may be required for optimal attraction of $\mathrm{T}$ cells and NK cells to the tumours.

Thus, the tumour-infiltrating immune cells were analysed after four adjuvant administra-

tions. Tumours from Poly IC and MSU + M. smegmatis treated mice showed an increase in total immune cells (Figure 5.6 A). The overall composition of the immune infiltrate was also altered, with more monocytes infiltrating M. smegmatis, MSU + M. smegmatis and LPS treated tumours (Figure 5.6 B). Poly IC treated tumours showed quite a marked increase in the frequency of NK cells and CD8 T cells among all immune cells (Figure 5.6 B). To take the overall increase in immune infiltrate into account, the infiltration of effector cells is also shown as a fraction of all live cells, including tumour cells (Figure $5.7 \mathrm{~A}$ ). MSU + M. smegmatis treatment more than doubled the infiltration of CD4 T cells, CD8 T cells and NK cells. A similar effect was seen after Poly IC administration, although the increase in CD4 T cells was less pronounced and not statistically significant (Figure $5.7 \mathrm{~A}$ ).

Taken together, these results show a greater infiltration of immune cells in tumours treated 4 times with adjuvants that delayed tumour growth. CD8 T cells and NK cells were markedly increased after Poly IC or MSU $+M$. smegmatis administration and more CD4 T cells were observed in MSU + M. smegmatis treated tumours.

In addition to increased recruitment of immune cells to the tumour site, adjuvants may also 
A.

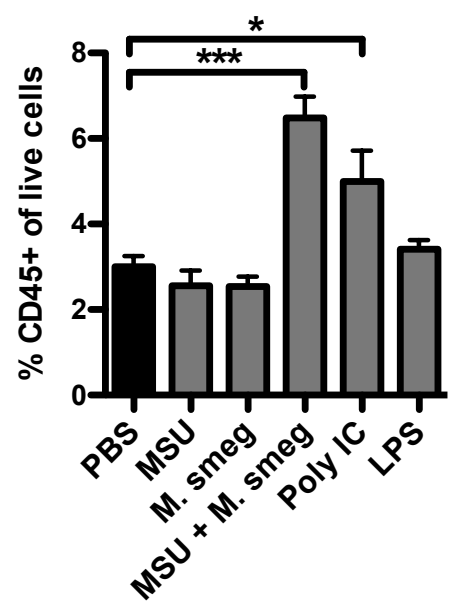

B.

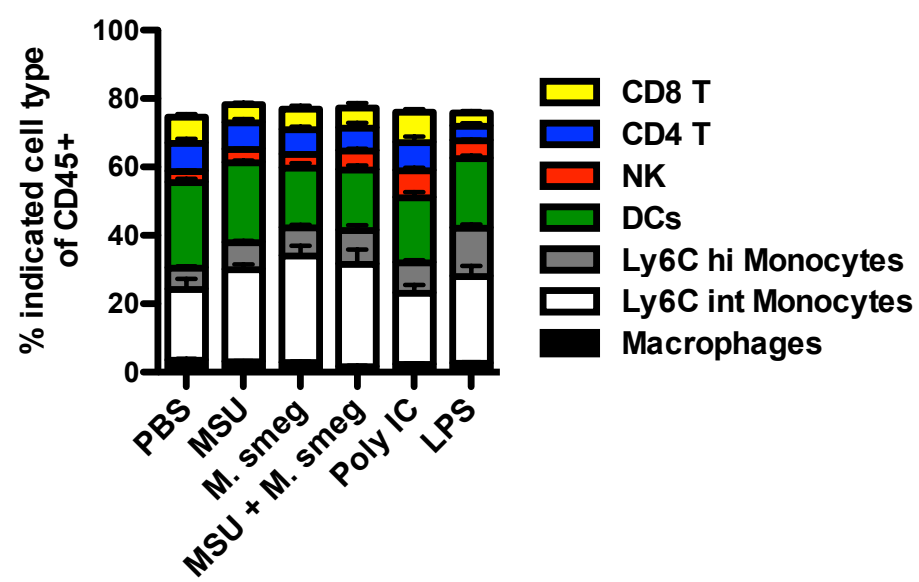

Figure 5.6: Repeated treatment with effective adjuvants substantially increases immune infiltrate in tumours and alters its composition. C57 mice bearing established B16F1 tumours were injected around the tumour with the indicated adjuvants on day 7, 9, 11 and 14. One day after the last treatment, tumours were analysed by flow cytometry. All cell types were identified as shown in Figure 4.3. (A) Percentage of CD45+ immune infiltrate among the total cells including tumour cells is shown. (B) Proportions of the different immune cell types that comprise the immune infiltrate are shown. Data are expressed as mean $+\mathrm{SE}$ and are combined from two independent experiments with 5 mice per group each. Statistical difference is indicated by ${ }^{* *} p<0.01$ and ${ }^{* * *} p<0.001$ according to one-way ANOVA with Tukey's post test.

exert their anti-tumour effects via increasing the activation of effector cells in the tumour. Therefore, we investigated the expression levels of the early activation marker CD69 and the exhaustion marker PD-1 on T cells and NK cells in tumours. MSU + M. smegmatis treatment resulted in up-regulation of CD69 on both CD4 and CD8 T cells, but not on NK cells (Figure 5.7 B). All other treatments did not significantly alter CD69 expression on any of the studied cell types (Figure 5.7 B). PD-1 expression was generally very low on NK cells and did not change in response to adjuvant administration (Figure 5.7 C right panel). Similarly, CD4 T cell expression of PD-1 were not affected (Figure 5.7 C left panel). CD8 T cells, however, showed a down-regulation of PD-1 in MSU, M. smegmatis or MSU + M. smegmatis treated tumours, which was highly significant in the group that received the combined adjuvant treatment (Figure $5.7 \mathrm{C}$, middle panel). In contrast, expression of PD-1 seemed to be increased after Poly IC administration, although this change was not statistically significant (Figure $5.7 \mathrm{C}$, middle panel). The surface marker expression profiles therefore indicate that only MSU + M. smegmatis treatment increased CD4 and CD8 T cell activation and at the same time reduced CD8 T cell exhaustion. 
In summary, both Poly IC and MSU + M. smegmatis administration increased effector cell infiltration in tumours after 4 treatments (Figure $5.6 \& 5.7$ ). Only MSU + M. smegmatis up-regulated expression of the activation marker CD69 and at the same time down-regulated the exhaustion marker PD-1 on CD8 T cells (Figure 5.7).

\subsubsection{Repeated adjuvant treatment increases the capacity of effector cells in tumours to produce pro-inflammatory cytokines}

To further determine the function of the tumour-infiltrating effector cells in response to adjuvant treatment, their capacity to produce pro-inflammatory cytokines was investigated. To this end, tumour cell suspensions were incubated with Golgi-Stop ex vivo without restimulation, followed by intracellular staining for IFN $\gamma$ and $\mathrm{TNF} \alpha$. T cells and NK cells were identified as previously described (see Figure 4.3) and appropriate staining controls were used to designate cytokine-positive cells.

In the absence of adjuvant treatment, less than $2 \%$ of CD8 T cells in tumours showed intracellular staining for IFN $\gamma$ or $\operatorname{TNF} \alpha$. MSU + M. smegmatis, Poly IC and LPS were found to increase the percentage of IFN $\gamma$ positive CD8 T cells up to 4-fold (Fig.5.8 A \& B). In addition, the intensity of the IFN $\gamma$ staining was markedly higher in CD8 T cells from MSU + M. smegmatis and Poly IC treated tumours (Figure 5.8 A histogram), indicating that each cell was also producing higher amounts of this cytokine. TNF $\alpha$ producing CD8 T cells were also increased 5-fold by MSU + M. smegmatis treatment (Figure 5.8 C \& D) and showed higher TNF $\alpha$ staining intensity (Figure $5.8 \mathrm{C}$ histogram). All other adjuvants did not have a significant effect on $\mathrm{TNF} \alpha$ production by $\mathrm{CD} 8 \mathrm{~T}$ cells (Figure $5.8 \mathrm{C} \& \mathrm{D}$ ).

As observed for CD8 T cells, MSU + M. smegmatis and Poly IC treatments also increased the percentage of NK cells producing IFN $\gamma$ (Figure 5.9 A \& B). The intensity of IFN $\gamma$ staining was also considerably higher after MSU + M. smegmatis administration and slightly enhanced with Poly IC (Figure 5.9 A histogram). Conversely to their effect on TNF $\alpha$ production in CD8 T cells, MSU + M. smegmatis didn`t alter the percentage or intensity 
A.

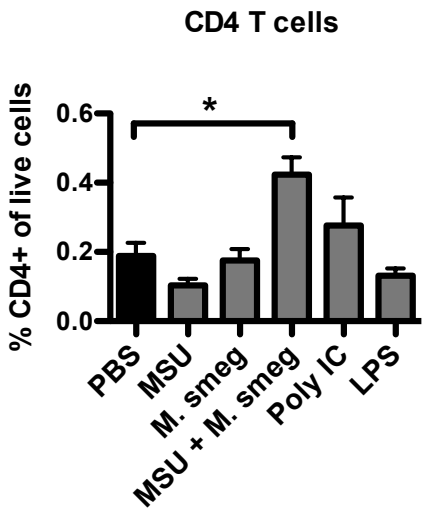

B.

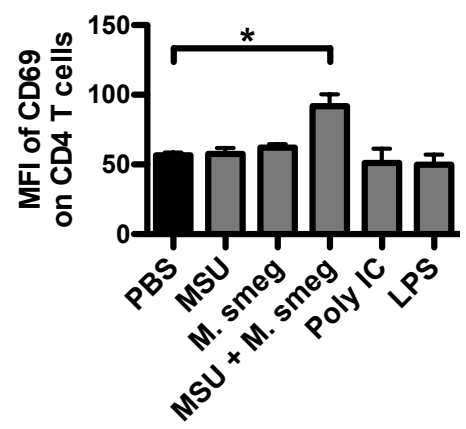

C.

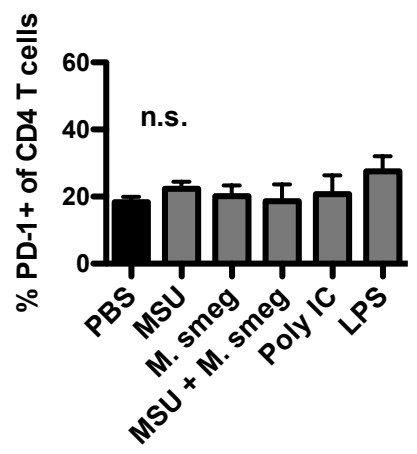

CD8 T cells
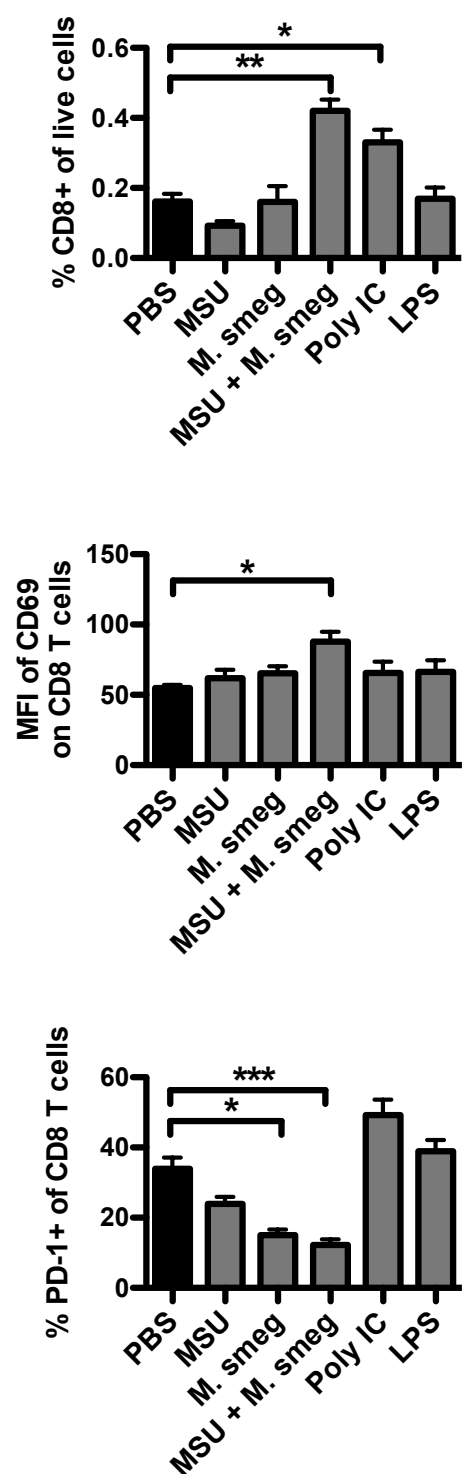

NK cells
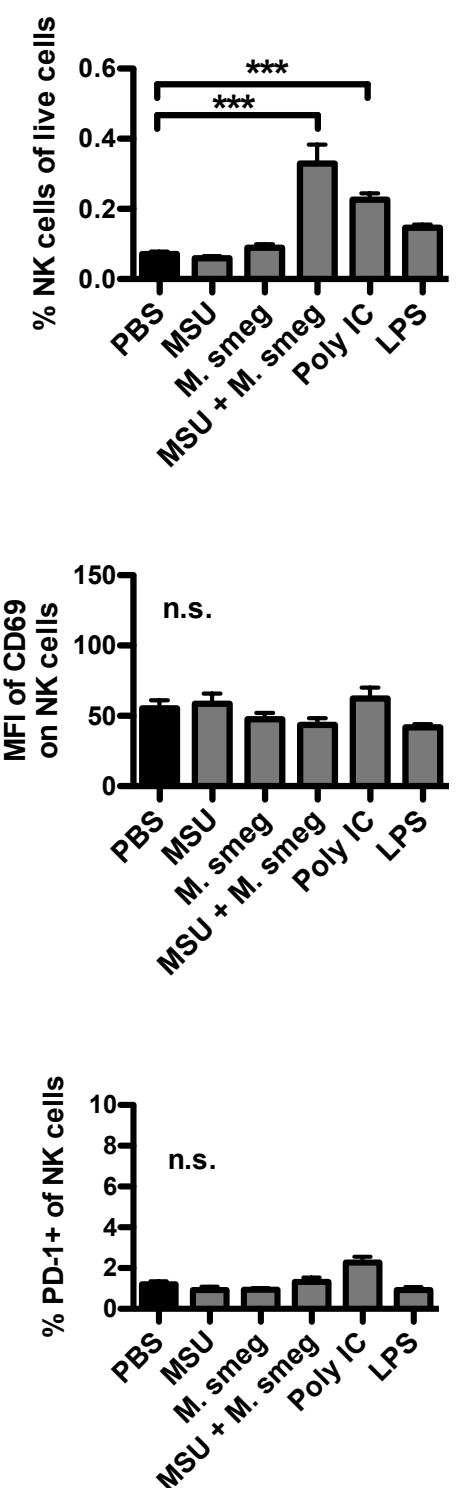

Figure 5.7: Repeated treatment with effective adjuvants substantially increases the percentage of intratumoral effector cells and changes their phenotype. C57 mice bearing established B16F1 tumours were injected around the tumour with the indicated adjuvants on day 7, 9, 11 and 14. One day after the last treatment, tumours were analysed by flow cytometry. All cell types were identified as shown in Figure 4.3. (A) Percentage of CD4 T cells, CD8 T cells and NK cells among all live cells in tumours is shown. (B \& C) Expression level of the activation marker CD69 (B) and the exhaustion marker PD-1 (C) on CD4 T cells, CD8 T cells and NK cells is shown as median fluorescence intensity (MFI). Data are expressed as mean $+\mathrm{SE}$ and are combined from two independent experiments with 5 mice per group each. ${ }^{*} p<0.05,{ }^{* *} p<0.01$ and ${ }^{* * *} p<0.001$ as assessed by the Kruskal-Wallis test with Dunn's post test; n.s. = not significant. 
A.
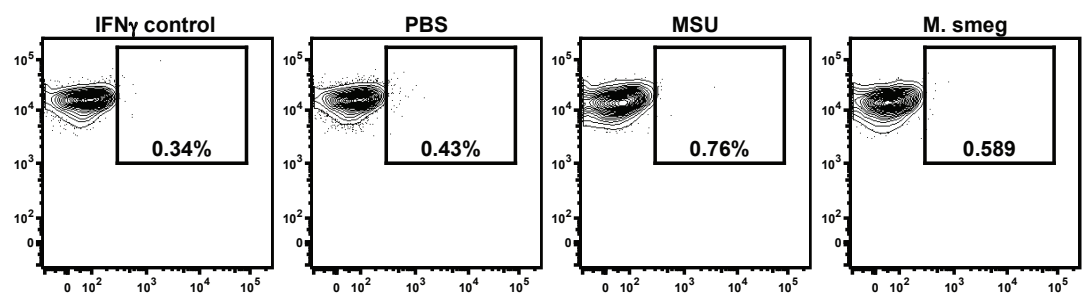

B.
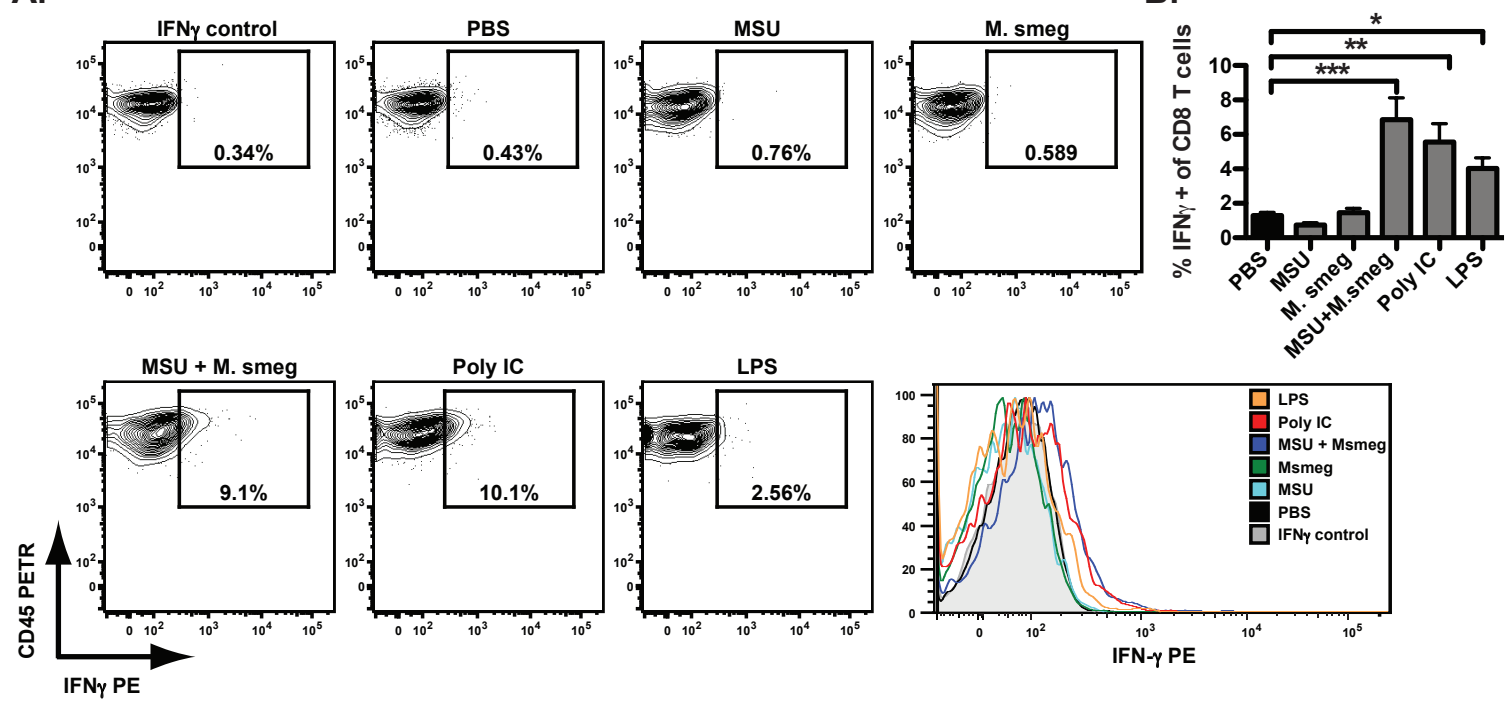

C.
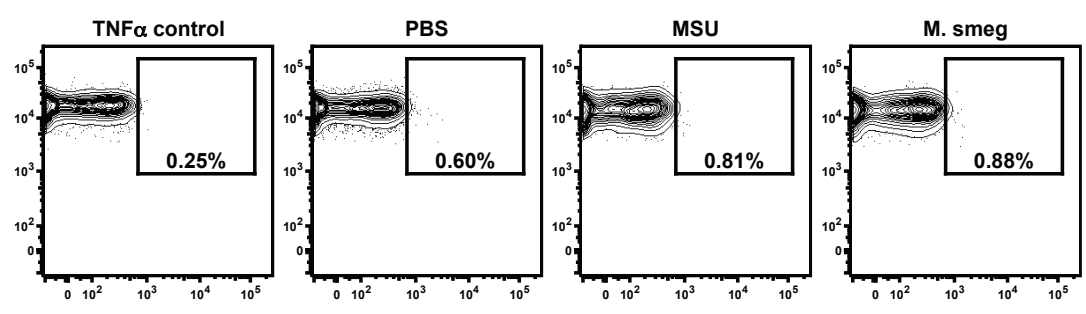

D.
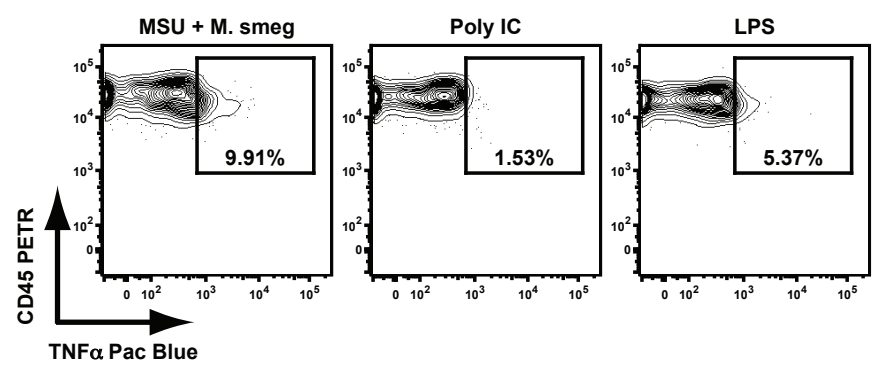

Figure 5.8: Repeated treatment with effective adjuvants increases the capacity of intra-tumoral CD8 T cells to produce pro-inflammatory cytokines. C57 mice bearing established B16F1 tumours were injected around the tumour with the indicated adjuvants on day 7, 9,11 and 14. One day after the last treatment, tumours were excised and cell suspensions were incubated for $6 \mathrm{~h}$ with Golgi-Stop. Intracellular staining for cytokines and surface staining was performed and analysed by flow cytometry. CD8 T cells were identified as shown in Figure 4.3. (A \& C) Representative flow plots show the percentage of IFN $\gamma$ and TNF $\alpha$ positive CD8 T cells, while histograms depict the intensity of cytokine staining. Staining controls are FMOs with isotype antibody for the analysed cytokine. (B \& D) Cytokine producing cells are shown as percentage of CD8 T cells in tumours. Data are expressed as mean $+\mathrm{SE}$ and are combined from two independent experiments with 5 mice per group each. ${ }^{*} p<0.05,{ }^{* *} p<0.01$ and ${ }^{* * *} p<0.001$ as assessed by one-way ANOVA with Tukey's post test. 
A.
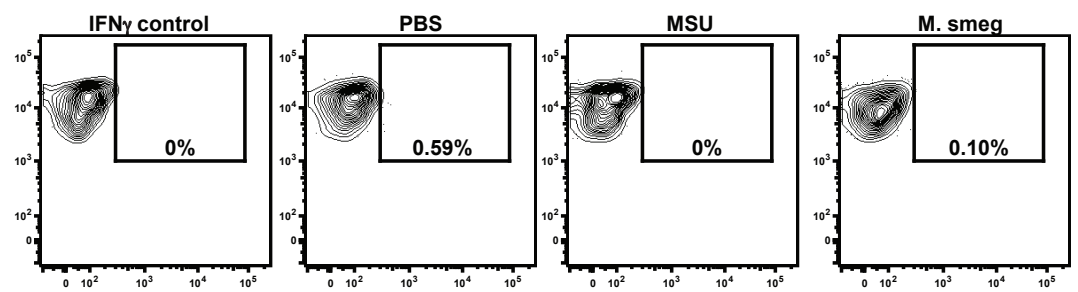

B.
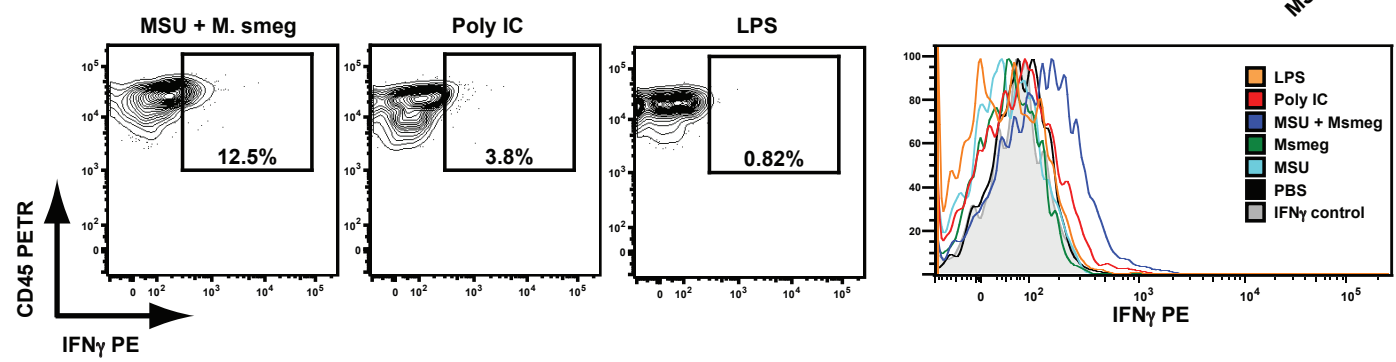

C.
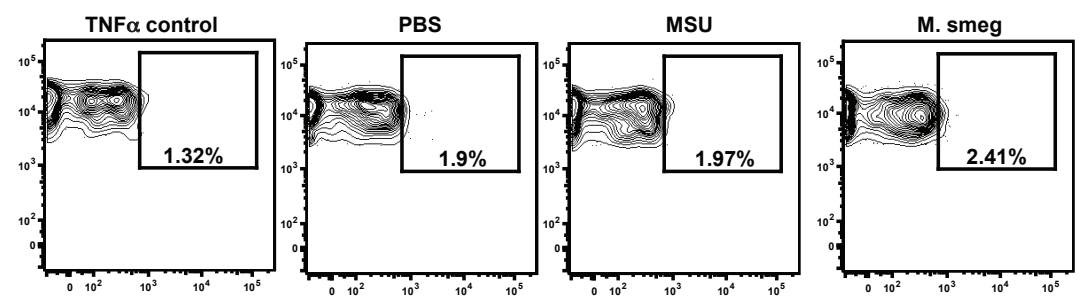

D.
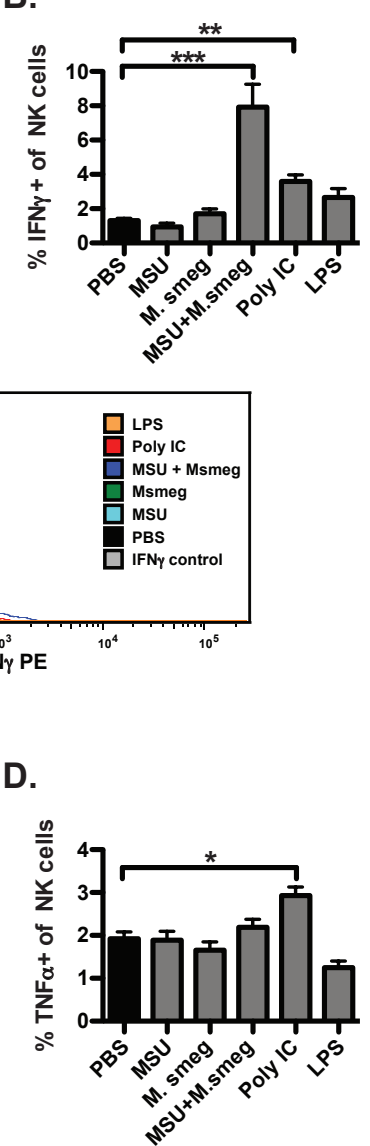
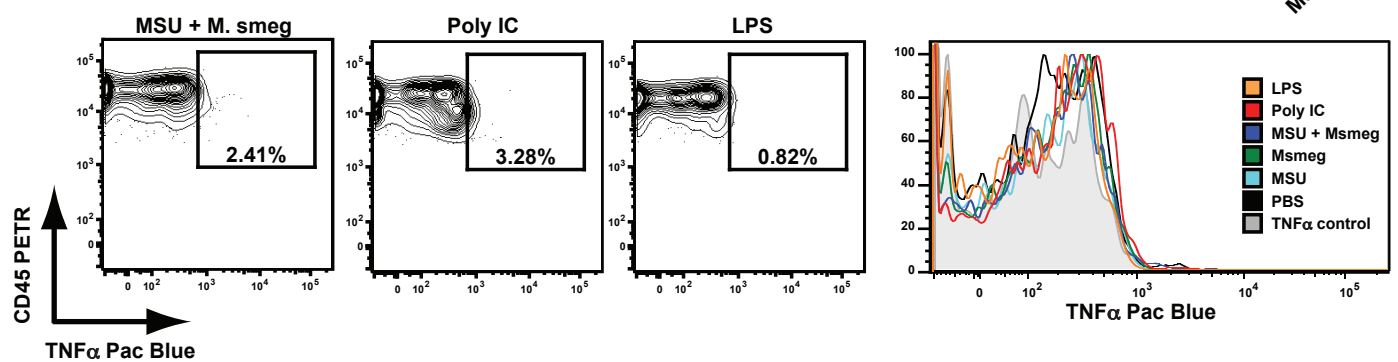

Figure 5.9: Repeated treatment with effective adjuvants enhances the capacity of intra-tumoral NK cells to produce pro-inflammatory cytokines. Data refer to the same experiment shown in Figure 5.8. NK cells were identified as shown in Figure 4.3. (A \& C) Representative flow plots show the percentage of IFN $\gamma$ and TNF $\alpha$ positive NK cells and histograms depict the intensity of cytokine staining. Staining controls are FMOs with isotype antibody for the analysed cytokine. (B \& D) Cytokine producing cells are shown as percentage of NK cells in tumours. Data are expressed as mean $+\mathrm{SE}$ and are combined from two independent experiments with 5 mice per group each. ${ }^{*} p<0.05,{ }^{* *} p<0.01$ and ${ }^{* * *} p<0.001$ according to one-way ANOVA with Tukey's post test. 
of TNF $\alpha$ positive NK cells, while Poly IC treatment resulted in a moderate increase in the amount of $\mathrm{TNF} \alpha$ producing NK cells Figure $5.9 \mathrm{~B} \& \mathrm{D})$.

Altogether, the analysis of intracellular cytokine staining showed that IFN $\gamma$ from CD8 T cells as well as NK cells was enhanced by both Poly IC and MSU + M. smegmatis administration (Figure 5.8 \& 5.9). MSU + M. smegmatis treatment also resulted in substantially greater $\mathrm{TNF} \alpha$ production form $\mathrm{CD} 8 \mathrm{~T}$ cells, while Poly IC increased the percentage of $\mathrm{TNF} \alpha$ positive NK cells (Figure $5.8 \& 5.9$ ).

Thus, Poly IC and MSU + M. smegmatis may exert their anti-tumour effects by improving effector cell function through a combination of greater priming in dLNs (see Section 5.3.1), increased recruitment to the tumour site (see Section 5.3.3), as well as enhancing functional activity, such as pro-inflammatory cytokine production.

\subsubsection{T cells from adjuvant-treated mice delay onset of tu- mour growth in naïve recipients}

As described in Chapter 1.1.4 \& 1.1.5 CD8 T cells have the capacity to directly eliminate tumour cells $[22,510]$. The main anti-tumour functions of CD4 T cells are thought to be the provision of help to enhance CD8 T cell priming [511] and the production of pro-inflammatory cytokines, especially those with direct anti-tumour activity such as IFN $\gamma$ and $\mathrm{TNF} \alpha[498,512]$. In addition, recent reports indicate that CD4 $\mathrm{T}$ cells may also have the capacity to directly kill tumour cells [71]. To assess if T cells activated through adjuvant administration were sufficient to confer tumour-protection, total $\mathrm{T}$ cells were isolated from spleens of adjuvant-treated animals and transferred into naïve recipients.

In line with previous results (Figure 4.2), Poly IC and MSU + M. smegmatis treated tumours were smaller than saline treated controls or tumours treated with MSU or M. smegmatis alone (Figure $5.10 \mathrm{~A}$ ). Magnetic enrichment of splenic T cells by depletion of other cell types yielded greater than $80 \%$ pure T cells, with about $40 \%$ CD8 T cells and $60 \%$ CD4 T cells (Figure 5.10 B). After i.v. transfer, these cells could be readily identified in the blood of recipient mice by flow cytometry analysis by gating on CD3 and the congenic marker CD45.2 
A.

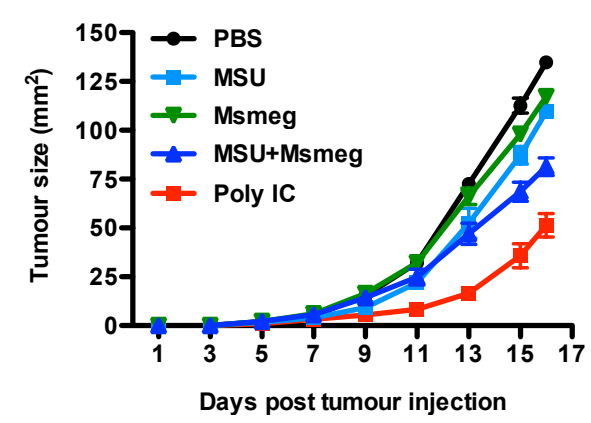

C.

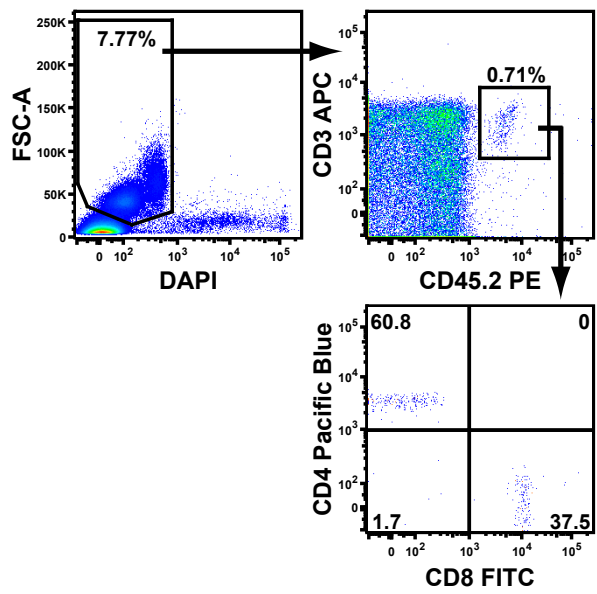

B.
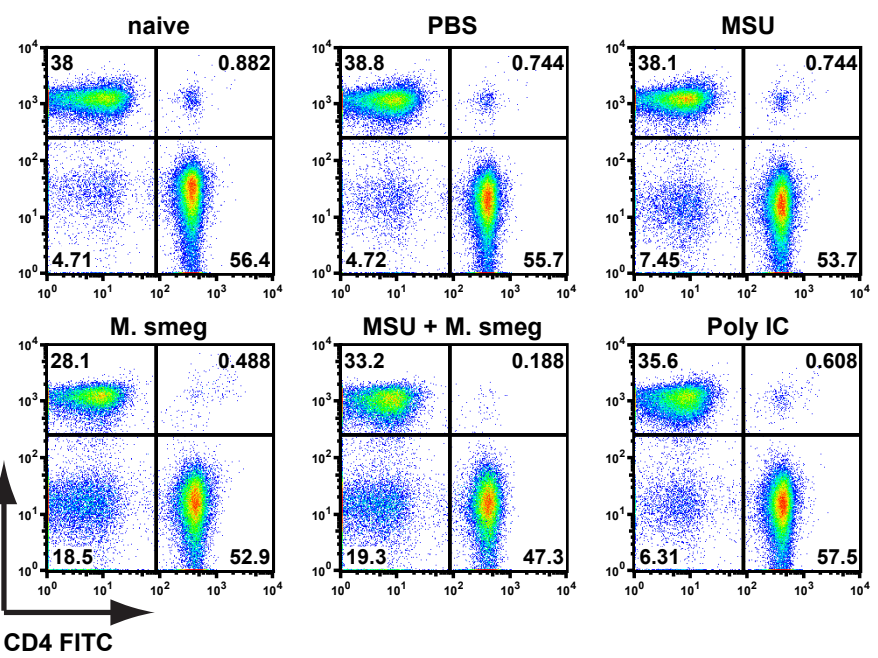

D.

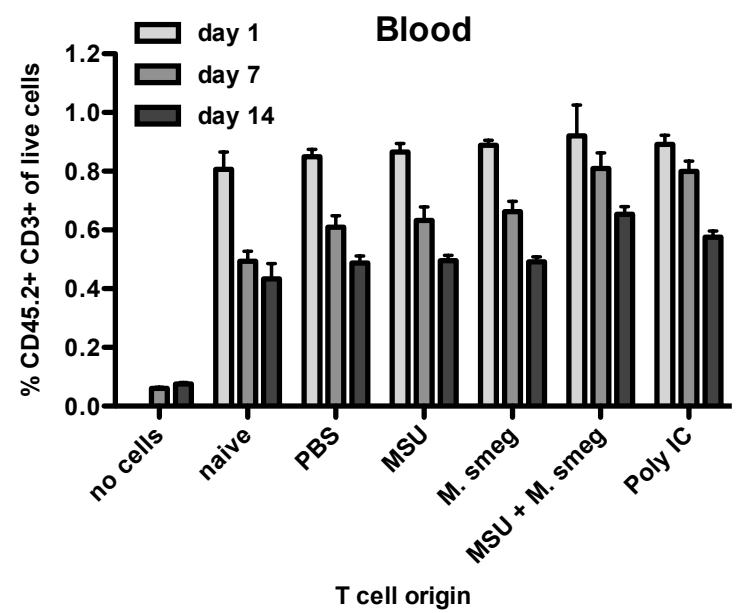

Figure 5.10: Adoptive transfer of splenic $\mathrm{T}$ cells from adjuvant treated tumourbearing mice into naïve recipients. C57 mice bearing established B16F1 tumours were injected around the tumour with the indicated adjuvants on day 9, 11, 13 and 15 . On day 16 total $\mathrm{T}$ cells from their spleens were enriched by depletion of other cells types with magnetic beads as described in section 2.2.5.1 and $7 \times 10^{6}$ cells were injected i.v. into B6 recipients. One day later, recipients or naïve mice were challenged with $1 \times 10^{5} \mathrm{~B} 16 \mathrm{~F} 1$ tumours cells s.c. into the flank. (A) Tumour growth of 5 donor mice per group is shown as mean \pm SE. (B) Flow plots depict the percentage of CD4 and CD8 T cells before transfer into recipients. (C) Representative gating for the identification of transferred $\mathrm{T}$ cells in the blood of recipients is shown. (D) The percentage of transferred $\mathrm{T}$ cells in the blood of recipients 1, 7, and 14 days after transfer is shown as mean $+\mathrm{SE}$ for 5 mice per group.

(Figure $5.10 \mathrm{C}$ ). The ratio of CD4 to CD8 T cells was maintained one day after adoptive transfer (Figure $5.10 \mathrm{C}$ ). The percentage of transferred $\mathrm{T}$ cells in the blood of recipient mice was initially just below $1 \%$ in all groups (Figure 5.10 D). However, with time the transferred $\mathrm{T}$ cells decreased to $0.5-0.7 \%$ of live cells in the blood of all recipient mice. $\mathrm{T}$ cells from Poly IC and MSU + M. smegmatis were slightly more abundant one and two weeks after 
transfer compared with $\mathrm{T}$ cells from naïve or saline treated tumour-bearing donors.

Mice that had received no T cells or $7 \times 10^{6} \mathrm{~T}$ cells from naïve mice or saline-treated tumourbearing donors all developed palpable tumours by day 6 (Figure $5.11 \mathrm{~A}$ ). This is in line with tumour appearance in other experiments (see Figure 4.2). Mice that had received $\mathrm{T}$ cells from adjuvant treated donors experienced a delay in the onset of tumour growth, with mice receiving T cells from Poly IC or MSU + M. smegmatis treated donors developing tumours only at day 8-10 (Figure 5.11 A). This was a significant benefit in tumour-free survival. In addition, tumour growth rates were slightly slower in mice that had received $\mathrm{T}$ cells from adjuvant treated mice, showing the same pattern in recipients as in donors (Figure $5.11 \mathrm{~B}$ ). However, the transferred $\mathrm{T}$ cells were not sufficient to confer tumour protection and once tumours became apparent, they all grew progressively (Figure 5.11).

The delay in tumour onset and slower growth in mice that received $\mathrm{T}$ cells from adjuvant treated, tumour-bearing donors indicates that these $\mathrm{T}$ cells exert some anti-tumour function. The lack of protection in all mice despite transfer of $7 \times 10^{6} \mathrm{~T}$ cells illustrates the aggressiveness of the B16F1 melanoma. As no selection for tumour-specific T cells was possible in the current model, it is possible that the number of tumour-specific $\mathrm{T}$ cells isolated from donor spleens was still too low to achieve tumour protection.

To investigate the potential of higher $\mathrm{T}$ cell numbers to confer tumour-protection, another $\mathrm{T}$ cell transfer experiment was performed. Graded numbers of T cells from $\mathrm{MSU}+M$. smegmatis treated mice were compared to T cells from saline treated control animals or no T cells. $\mathrm{T}$ cell donor mice treated by peri-tumoral adjuvant injection had significantly smaller tumours than saline treated control animals (Figure 5.12 A). After magnetic depletion of other cells, T cells from saline and MSU + M. smegmatis treated mice were $88.3 \%$ and $83.0 \%$ pure, respectively (Figure 5.12 B). 20 million T cells from saline treated donors and 10, 20 and 40million T cells from MSU + M. smegmatis treated mice were transferred into recipients by i.v. injection. The numbers of transferred $\mathrm{T}$ cells were reflected in their percentage among live cells in the blood of recipients, with 10 million transferred cells resulting in $1 \%$, 20 million resulting in $2 \%$ and 40 million leading to $3.7 \%$ of donor cells among live cells (Figure $5.12 \mathrm{C}$ ). In the course of 2 weeks, the $\mathrm{T}$ cell percentages in blood declined in all recipients to roughly half the initial amount, which is similar to the decrease observed in 
A.

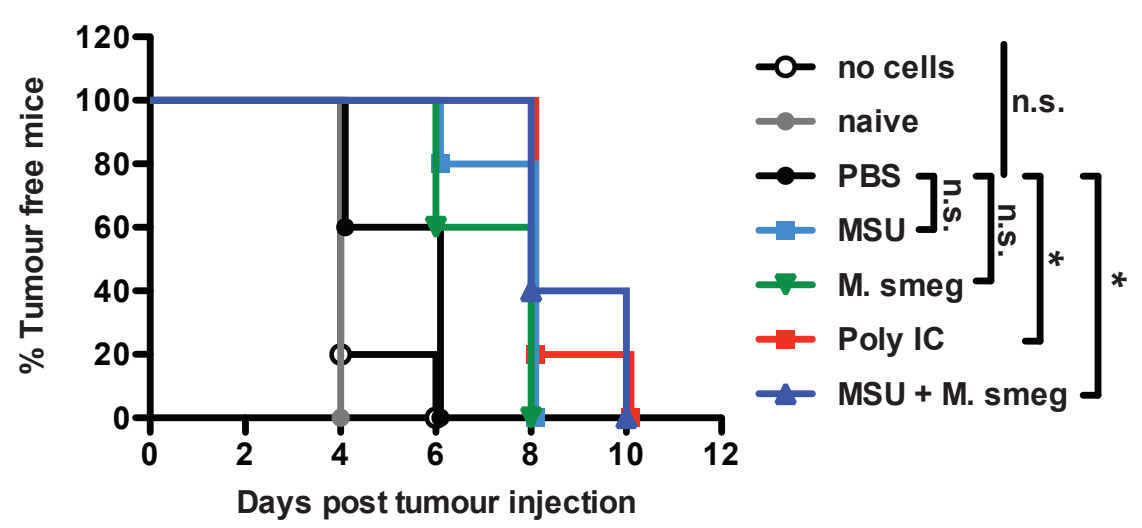

B.

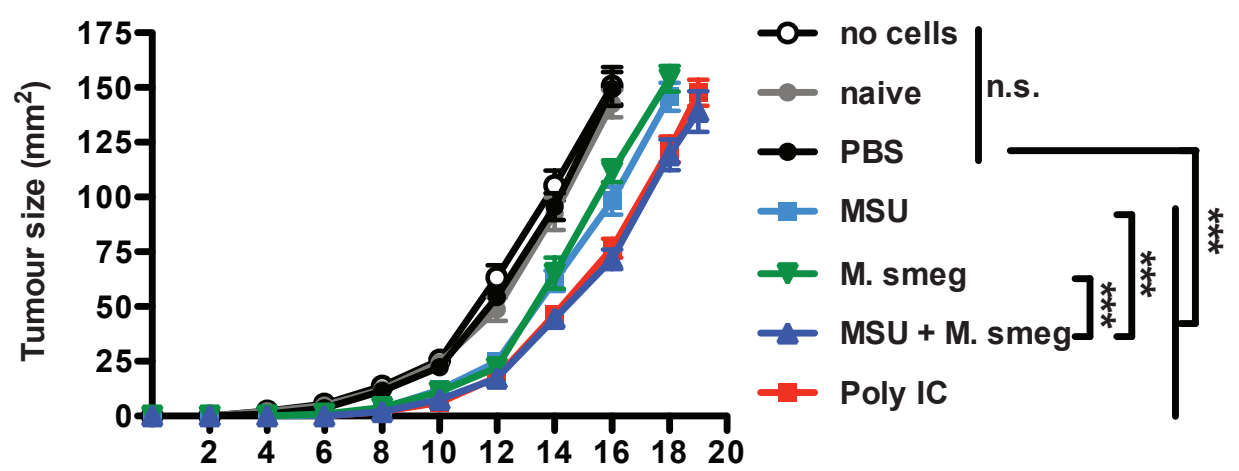

Days after tumour injection

Figure 5.11: Adoptive transfer of splenic $T$ cells from adjuvant treated tumourbearing mice delays onset of tumour growth. The recipients of $\mathrm{T}$ cells from the mice shown in Figure 5.10 were monitored for tumour growth. (A) The time until the first palpable tumour was detected in each mouse is graphed as percentage of tumour-free survival. n.s. $=$ not significant, ${ }^{*} p<0.05$ as compared by Log-Rank-Test with Bonferroni's adjustment for multiple comparisons. (B) Mean tumour sizes \pm SE for 5 recipients per group are shown. n.s. $=$ not significant, ${ }^{* * *} p<0.001$ as compared by two-way ANOVA with Bonferroni's post test.

the previous experiment.

As observed in the previous experiment, mice that received $\mathrm{T}$ cells from adjuvant treated donors showed a delayed onset of tumour growth (Figure 5.12 D \& E). However, despite the higher numbers of transferred $\mathrm{T}$ cells, complete tumour protection was not achieved and all mice still developed tumours (Figure 5.12 D \& E). Moreover, while tumour growth was again slower in mice that had received $\mathrm{T}$ cells from adjuvant treated donors, the growth rates did not differ between mice receiving increasing numbers of T cells (Figure $5.12 \mathrm{D}$ ). In addition, the delay in the onset of tumour growth was similar in all groups that received 
A.

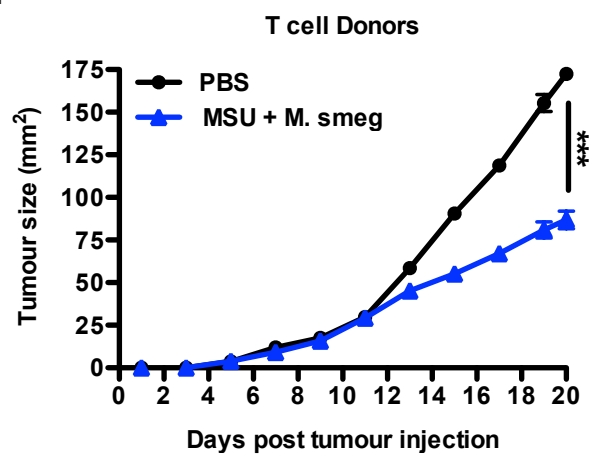

D.

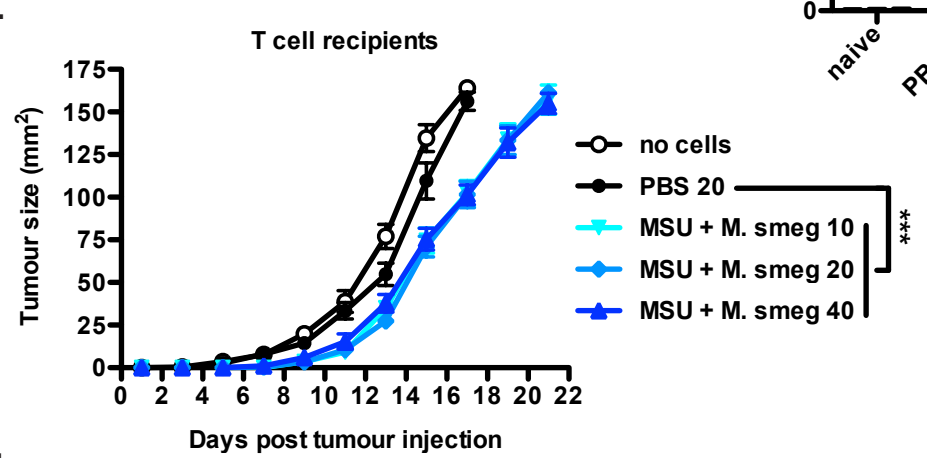

E.

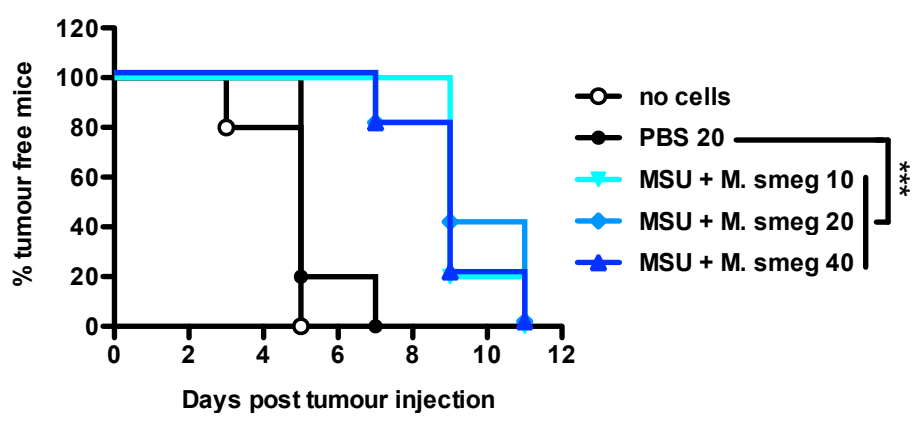

B.

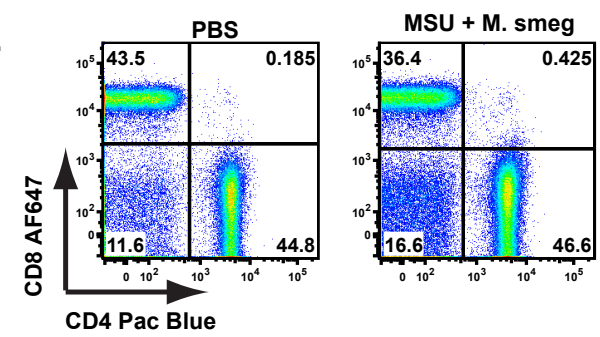

C.

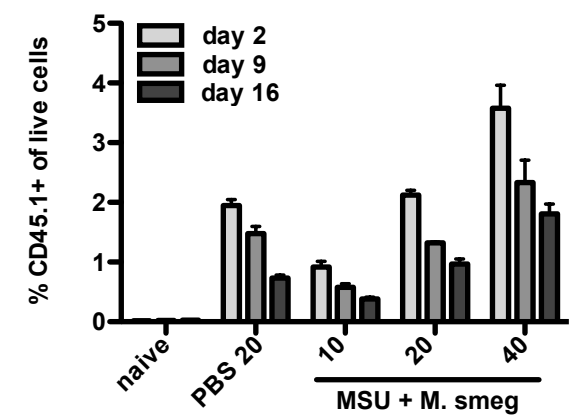

Figure 5.12: Increasing the numbers of adoptively transferred splenic $T$ cells from adjuvant treated tumour-bearing mice does not improve tumour protection in recipients. $\mathrm{B} 6$ mice bearing established $\mathrm{B} 16 \mathrm{~F} 1$ tumours were injected around the tumour with the indicated adjuvants on day 9, 11, 13, 15, 17 and 19. On day 20 total T cells from their spleens were enriched by depletion of other cells types with magnetic beads as described in section 2.2.5.1 and graded numbers of $\mathrm{T}$ cells were injected i.v. into C57 recipients. One day later, recipients or naïve mice were challenged with $1 \times 10^{5}$ B16F1 tumours cells s.c. into the flank. (A) Tumour growth in groups of 5 donor mice in the PBS and 15 mice in the MSU + M. smegmatis group is shown as mean \pm SE. (B) Flow plots depict the percentage of CD4 and CD8 T cells before transfer into recipients. (C) The percentage of transferred $\mathrm{T}$ cells in the blood of recipients 2,9 , and 16 days after transfer is shown as mean + SE for 5 mice per group. (D) The tumour sizes of 5 recipients per group are shown as mean \pm SE. (E) Time to the appearance of palpable tumours is graphed as percentage of tumour-free survival. Numbers 10, 20 and 40 indicate million transferred cells. ${ }^{* * *} p<0.001$ by two-way ANOVA with Bonferroni's post test for tumour growth and Log-Rank-Test with Bonferroni's adjustment for multiple comparisons for tumour-free survival. 
T cells from MSU + M. smegmatis treated donors and could not be improved by transferring higher numbers of $\mathrm{T}$ cells (Figure $5.12 \mathrm{E}$ ).

These data indicate that total $\mathrm{T}$ cells from the spleens of adjuvant treated mice do have anti-tumour function, as they significantly delayed onset of tumour growth. However, they are not sufficient to confer tumour protection. This may be due to low numbers of tumour-antigen-specific $\mathrm{T}$ cells in spleens. Alternatively, dLN or tumours themselves may be a more appropriate source of donor $\mathrm{T}$ cells. Furthermore, $\mathrm{T}$ cells alone may not be sufficient to confer tumour protection, but may need to act in concert with other effector cells, such as NK cells. In addition, T cells in recipients may not migrate to the tumour site at high rates, but local inflammation after adjuvant treatment may be needed to induce $\mathrm{T}$ cell recruitment. Lastly, $\mathrm{T}$ cell preparations used for transfer experiments contained both CD4 and CD8 T cells. CD4 T cells were included to potentially provide help to CD8 T cells or contribute to the anti-tumour effect through cytokine production. However, the CD4 T cell fraction may also have contained Tregs, which may have counteracted the anti-tumour activity of CD8 T cells. Further experiments will be required to address these possibilities.

\subsubsection{Adaptive immune cells are required for the anti-tumour effect of adjuvants}

The data presented in the previous section indicate that $\mathrm{T}$ cells play a role in the anti-tumour response induced by successful adjuvants. However, they are not sufficient to induce complete tumour protection. To address the question of whether $\mathrm{T}$ cells are required for the anti-tumour effect in response to adjuvant treatment, experiments were carried out in knockout mice lacking adaptive immune cells.

In the absence of treatment, B16F1 tumours in Rag1-/- mice, which lack B and T cells, grew similar to tumours in C57 wild-type animals (Figure 5.13). However, in Rag1-/- mice, the delay in tumour growth in response to peritumoral injection and survival benefit in response to MSU + M. smegmatis treatment was completely abolished (Figure 5.13 A \& B). The effect of Poly IC administration was also diminished in the Rag1-/- animals, but treated mice 
still experienced a significant delay in tumour growth compared to saline treated controls

(Figure $5.13 \mathrm{~A}$ ). This translated into a slight survival benefit for Rag1-/- mice treated with Poly IC compared to saline controls (Figure 5.13 B).

A.

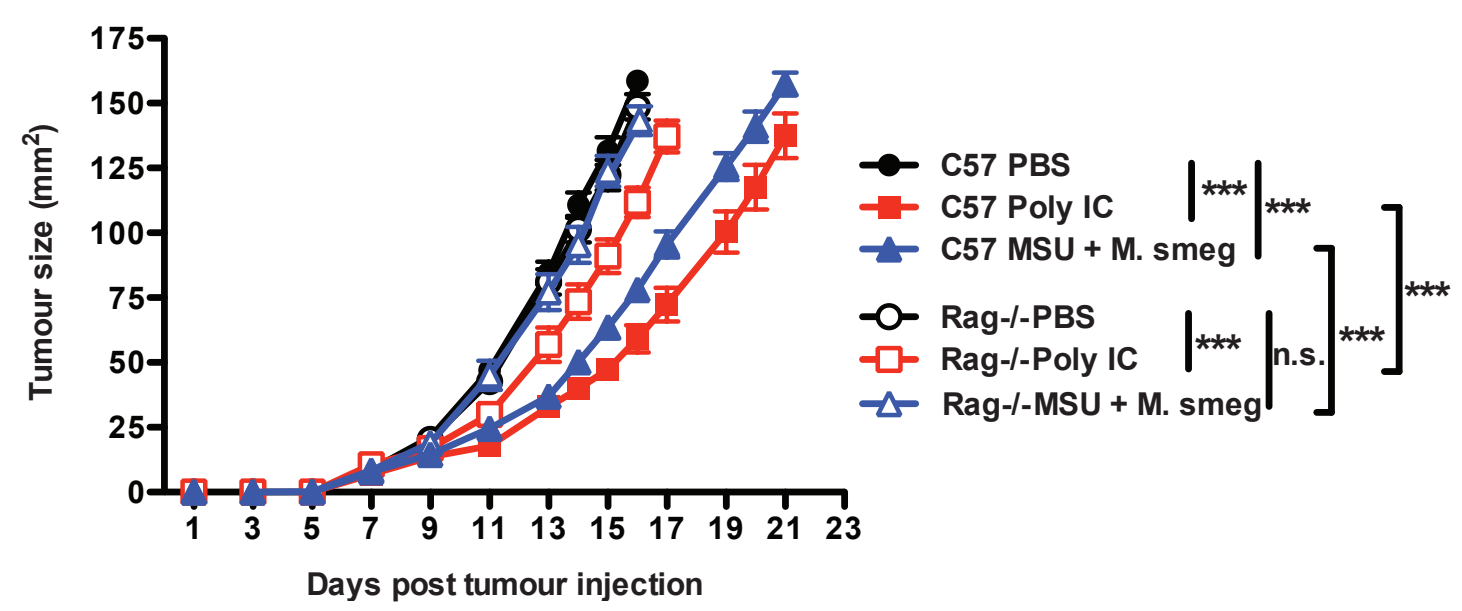

B.

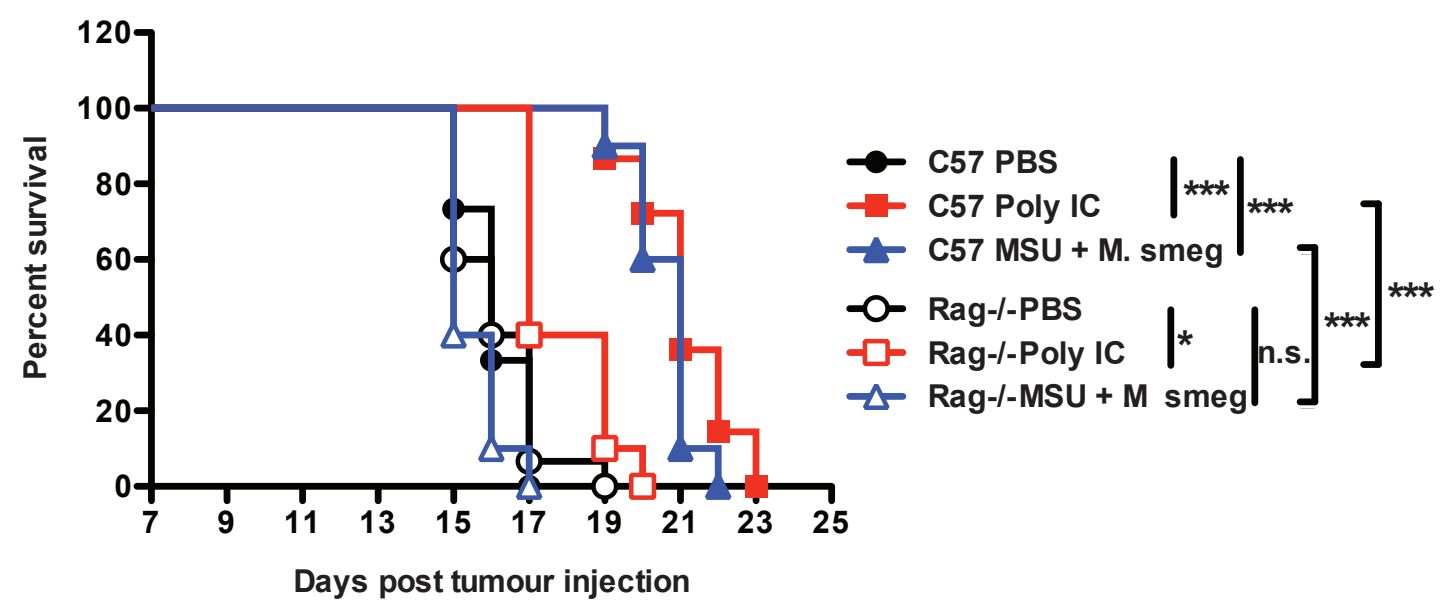

Figure 5.13: The anti-tumour effect of Poly IC and MSU + M. smegmatis treatment requires adaptive immunity. C57 mice or Rag1-/- mice bearing established B16F1 tumours were injected around the tumour with the indicated adjuvants on day 9, 11, 13 and 15. (A) Tumour growth of 10 mice per group is shown as mean \pm SE. Significant differences between treatment groups according to two-way ANOVA with Bonferroni's post test starting from day 11 are indicated by ${ }^{* * *} p<0.001 ;$ n.s. = not significant. (B) Survival of the B16F1 bearing mice treated with adjuvants is depicted. Statistical significance between treatment groups is indicated by ${ }^{*} p<0.05,{ }^{* * *} p<0.001$ as assessed by the Log-Rank-Test with Bonferroni's adjustment for multiple comparisons; n.s. = not significant. Data are pooled from 2 independent experiments with 5 mice per group each. 
These data indicate that the tumour growth delay in response to $\mathrm{MSU}+M$. smegmatis treatment is completely dependent on adaptive immunity. Poly IC also requires adaptive immune cells to exhibit its full anti-tumour effect, but some activity is retained in the absence of these cells. This could be due to effects on innate immune cells, which are not affected by Rag1 deletion, such as NK cells.

\subsubsection{Both adaptive and innate immune cells are necessary for the anti-tumour effect of adjuvants}

The required adaptive cells most likely mediating the anti-tumour effect are $\mathrm{T}$ cells, and in particular CD8 $\mathrm{T}$ cells. To further investigate the relative contribution of CD4 T cells, CD8 $\mathrm{T}$ cells and NK cells to the anti-tumour response induced by Poly IC and MSU + M. smegmatis treatment, C57 wild-type mice were depleted of these cell types by antibody administration. In addition, the role of Tregs was investigated via depletion of CD25 positive cells. Prior to the tumour injection, mice were given i.p. injections with antibodies against CD4, CD8, NK1.1 or CD25 and depletion of the target cell types was monitored in blood throughout the tumour experiment as shown in Figure 5.14. Depletion of CD8 T cells, CD4 T cells and NK cells was nearly complete and was maintained for two weeks (Figure 5.14 B, C \& E). T cells were presumed to be depleted instead of simply having down-regulated or masked their CD4 or CD8 antigen, as nearly all CD3+ T cells in the CD8 depleted mice were CD4+ and conversely most CD3+ T cells in the CD4 depleted mice were CD8+ (Figure 5.14 B \& C). In the blood of NK1.1-depleted mice, no substantial increase in DX5+NK1.1- cells was observed, indicating that NK cells were also depleted and not just NK1.1 low (Figure 5.14 E). Tregs were depleted using the well-established anti-CD25 clone PC61 [513] and were monitored in blood using the anti-CD25 clone 7D4 to prevent underestimating the frequency of Tregs through masking of CD25 by bound PC61. With this method, Tregs were found to be strongly reduced and their low frequency was maintained throughout the experiment (Figure 5.14 D).

DCs were only minimally affected by anti-CD4 or anti-CD8 antibody treatment. In a control experiment, CD4+ and CD8 $\alpha+$ DCs were transiently reduced in spleens and LNs 
A.
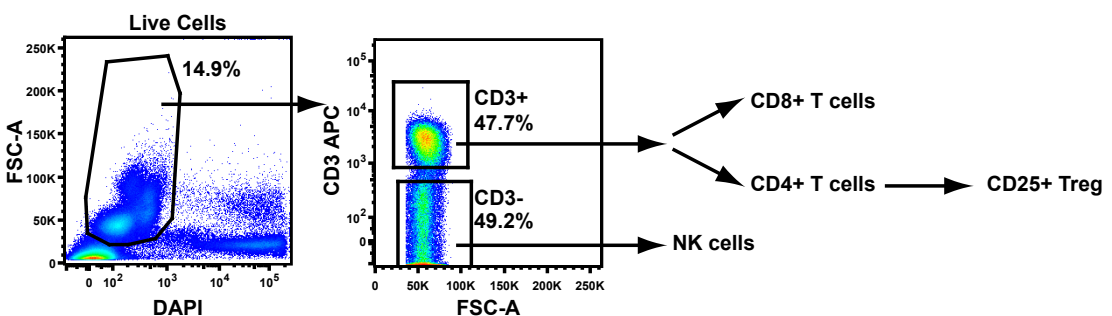

B.
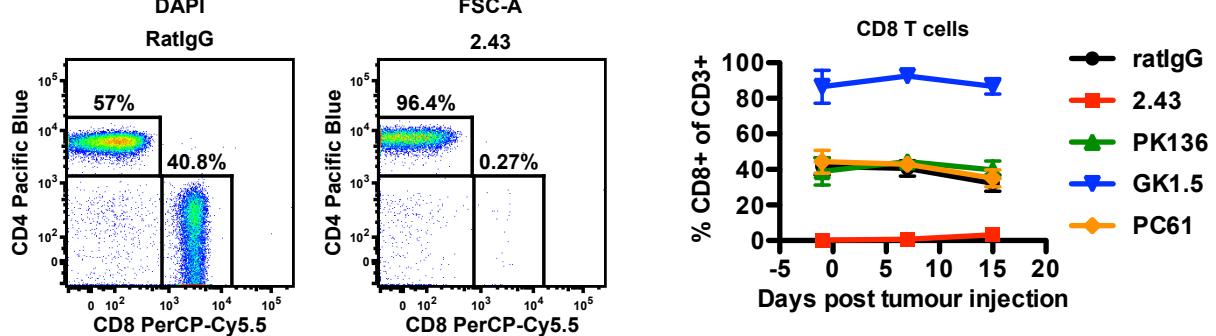

C.

RatigG

GK1.5
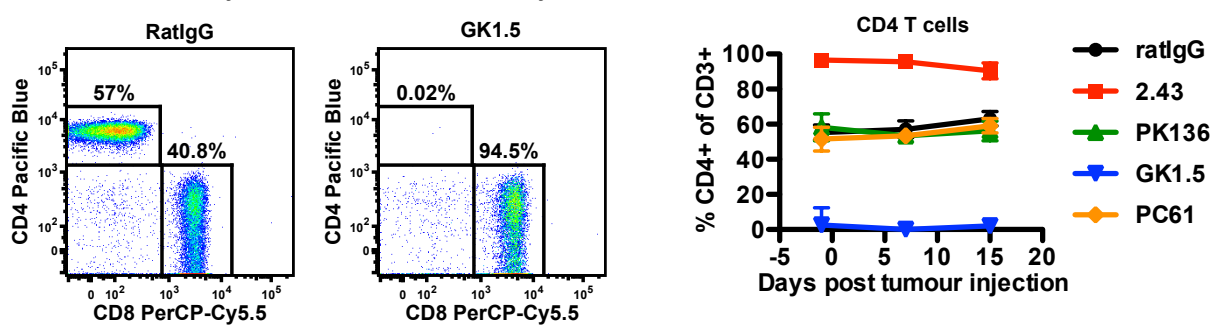

D.
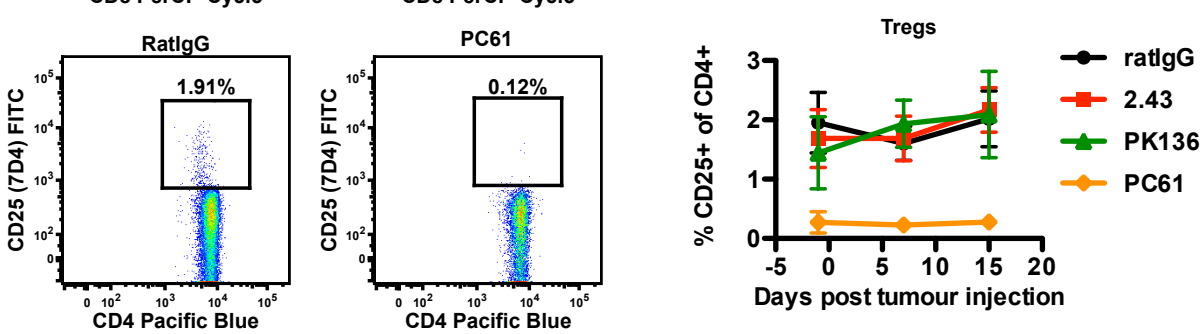

E.
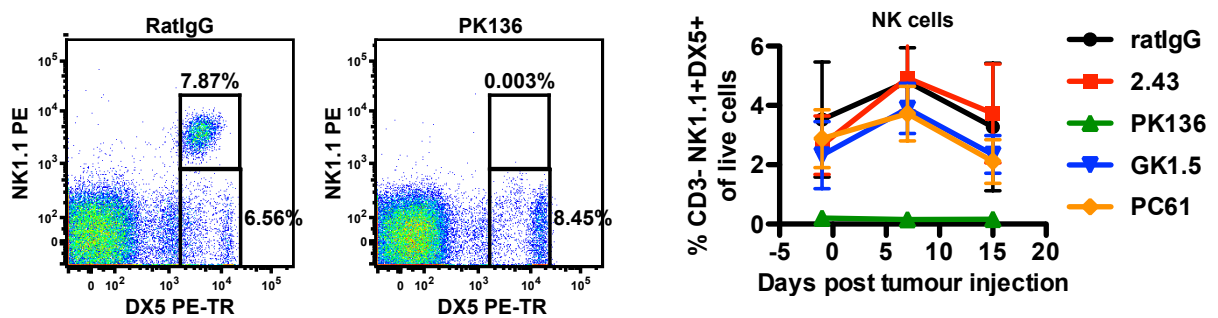

Figure 5.14: Depletion of CD4 $T$ cells, CD8 $T$ cells, Tregs and NK cells in tumour-bearing adjuvant-treated mice. C57 mice were depleted of CD8 T cells, CD4 T cells, Tregs and NK cells by i.p. injection of $250 \mu \mathrm{g}$ of 2.43, GK1.5, PC61 or PK136 antibody, respectively, on day -2 and -1 . On day 0 mice were injected with $10^{5}$ B16F1 tumour cells. Cell depletion was monitored in the blood of treated mice by flow cytometry. (A) Live cells were identified by DAPI exclusion, followed by gating on CD3- to identify NK cells or CD3+ T cells. (B-E) Representative flow plots of one mouse treated with control rat IgG and one mouse that received depleting antibody are shown with the gating for (B) CD8 T cells, (C) CD4 T cells, (D) Tregs and (E) NK cells. Graphs on the right depict the frequency of the cell type of interest in the different groups on days $-1,7$ and 15 as mean \pm SD. 
A.

B16F1 day 15

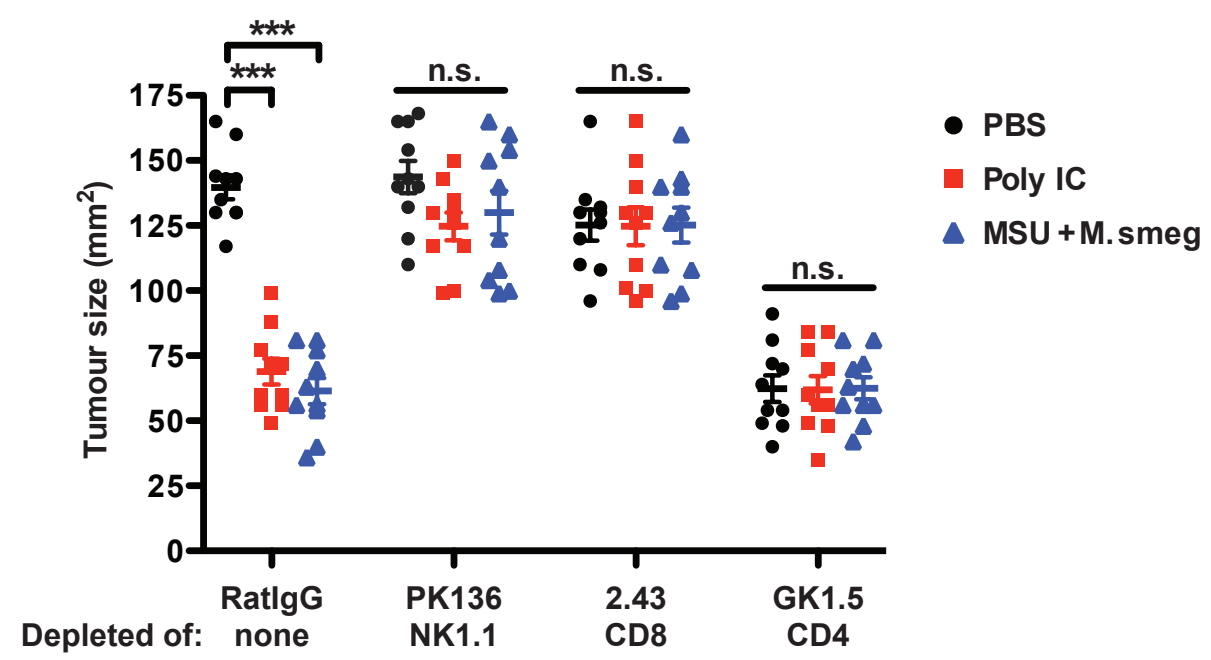

B.

\section{B16F1}

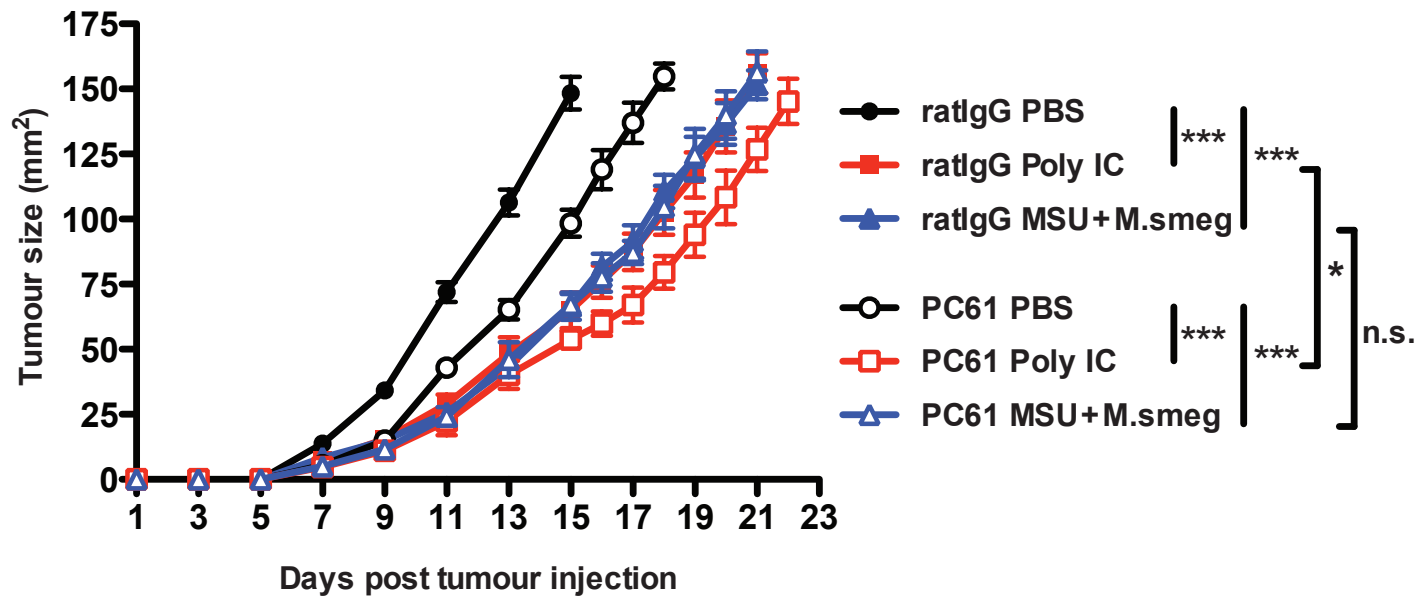

Figure 5.15: Both CD8 $\mathrm{T}$ cells and NK cells are required for the anti-tumour effect of Poly IC and MSU + M. smegmatis treatment. C57 mice depleted of the indicated cell types as shown in Figure 5.14 on day -2 and -1 were injected s.c with $10^{5}$ B16F1 tumour cells on day 0. On day 7, 9, 11 and 13, mice were treated with PBS, Poly IC or MSU $+M$. smegmatis. (A) Tumour sizes and mean $+\mathrm{SE}$ are shown on day 15 when control mice had large tumours. ${ }^{* * *} p<0.001$ as assessed by one-way ANOVA with Tukey's post test; n.s. = not significant. Data are pooled from two independent experiments with 5 mice per group. (B) Tumour growth of mice injected with control antibody or PC61 to deplete Tregs and treated with PBS, Poly IC or MSU + M. smegmatis is shown as mean \pm SE. ${ }^{* * *} p<0.001$ from day 9 and ${ }^{*} p<0.05$ from day 16 as assessed by two-way ANOVA with Bonferroni's post test; n.s. = not significant. Data are from one experiment with 5 mice per group. 
one day after antibody administration, but their numbers were restored to the same level as in control-antibody treated mice at day 7 (data not shown). Thus there is no significant change in DC populations of CD4 and CD8 T cell depleted mice at the time of adjuvant treatment.

The absence of NK1.1+ cells or CD8 T cells completely abrogated the effect of Poly IC and MSU + M. smegmatis treatment on tumour growth and after two weeks all mice had large tumours similar in size to non-adjuvant-treated, non-depleted controls (Figure 5.15 A). When CD4 T cells were depleted, even control mice that had only received saline treatment had smaller tumours and there was no further improvement with adjuvant treatment (Figure 5.15 A). This may be due to the depletion of Tregs when the whole CD4 T cell population is targeted.

Therefore, the effect of Treg depletion on B16F1 tumour growth with adjuvant treatment was examined. In line with previous findings [447], depletion of Tregs without any additional adjuvant treatment reduced tumour growth (Figure $5.15 \mathrm{~B}$ ). Adjuvant treatment with Poly IC and MSU + M. smegmatis was effective in Treg-depleted mice and delayed tumour growth compared to non-treated, Treg-depleted controls (Figure 5.15 B). However, $\mathrm{MSU}+$ M. smegmatis treatment in conjunction with Treg depletion did not show any additional benefit over MSU + M. smegmatis treatment alone (Figure 5.15 B). In contrast, Poly IC treated mice that were also depleted of Tregs experienced an additional slight delay in tumour growth over Poly IC administration alone (Figure 5.15 B).

The results of the antibody depletion experiments show that both CD8+ and NK1.1+ cells are required for the anti-tumour effect of Poly IC and MSU + M. smegmatis treatment. As tumour growth was still delayed by adjuvant treatment in mice that had Tregs depleted but not in mice in which all CD4 T cells were depleted, this may suggest that CD4 T cells are also involved in the adjuvant-induced anti-tumour response. The observation that Treg depletion conferred additional anti-tumour benefits only to Poly IC but not MSU + M. smegmatis treated mice indicates that if these adjuvant treatments were to be used in the clinic, only Poly IC would benefit from use in combination with Treg depletion. MSU + M. smegmatis treatment, on the other hand, may have a direct effect on Tregs, so that depletion of Tregs does not enhance the anti-tumour response. 


\subsubsection{MSU + M. smegmatis reduces Tregs and induces CD4 $\mathrm{T}$ cell activation in tumours}

To study the effect of MSU + M. smegmatis treatment on Tregs, mice that express GFP under the FoxP3 promoter were injected with B16F1 tumours and treated with Poly IC or MSU + M. smegmatis. After 4 adjuvant treatments, CD45+ immune cells in tumours were gated as shown in Figure 4.3. Subsequently, Tregs were identified as CD4+ Foxp3-GFP+ immune cells (Figure 5.16 A). C57 mice were used as GFP negative controls. The frequency of Tregs among the tumour-infiltrating immune cells was similar between PBS and Poly IC treated mice, but was reduced following MSU + M. smegmatis treatment (Figure $5.16 \mathrm{~B}$ ). To account for the increased infiltration of CD45+ immune cells found in Poly IC and MSU + M. smegmatis treated tumours, the frequency of Tregs was also graphed as percentage of all live cells (Figure 5.16 C).

Even though the infiltration of total CD4 T cells was higher in response to $\mathrm{MSU}+M$. smegmatis administration (Figure 5.7 A), the frequency of Tregs was reduced (Figure 5.16 C).

Furthermore, the expression of CD25 and FoxP3 on CD4 T cells was analysed as shown in Figure 5.17. In PBS and Poly IC treated mice, the majority of CD4 T cells in tumours that expressed CD25 were also FoxP3-GFP positive and conversely, most FoxP3-GFP positive cells were also CD25 positive (Figure $5.17 \mathrm{~B}$ ).

In MSU + M. smegmatis treated tumours, however, a population of CD25 positive FoxP3GFP negative CD4 T cells was observed (Figure $5.17 \mathrm{~B}$ ). The frequency of this cell population was variable in individual mice but was significantly higher than in saline treated controls (Figure 5.17 C). CD25 is expressed both on Tregs and on activated T cells [514]. Therefore this CD25+FoxP3-GFP- population may represent activated CD4 T cells.

Taken together, these results indicate that MSU + M. smegmatis reduced the infiltration of Tregs into tumours. At the same time, a population of CD25+FoxP3-GFP+ CD4 T cells was induced. Those effects were specific to MSU + M. smegmatis adjuvant treatment and not observed in response to Poly IC. 
A.
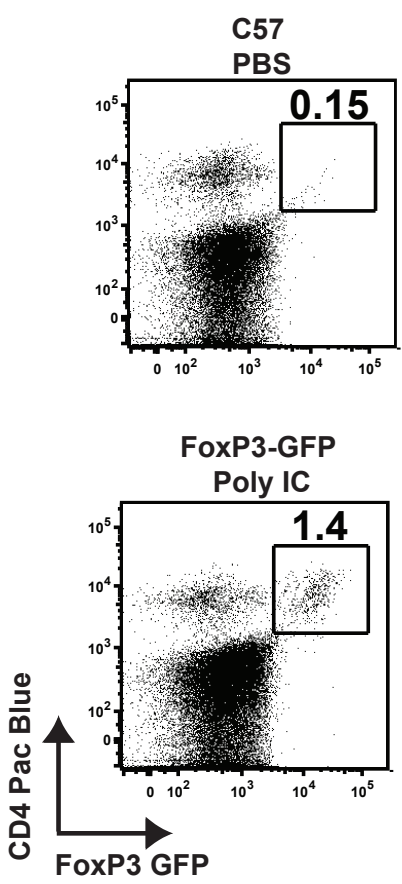

B.

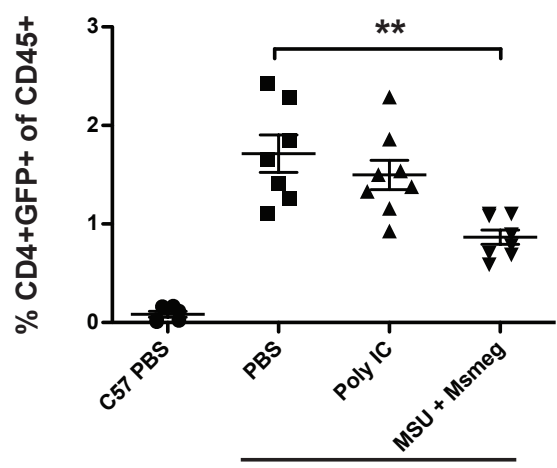

FoxP3-GFP MSU+M.smeg

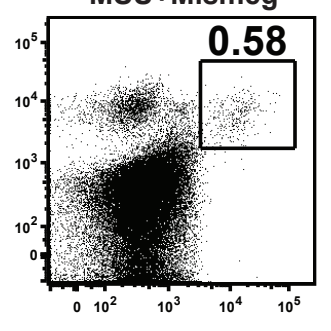

C.

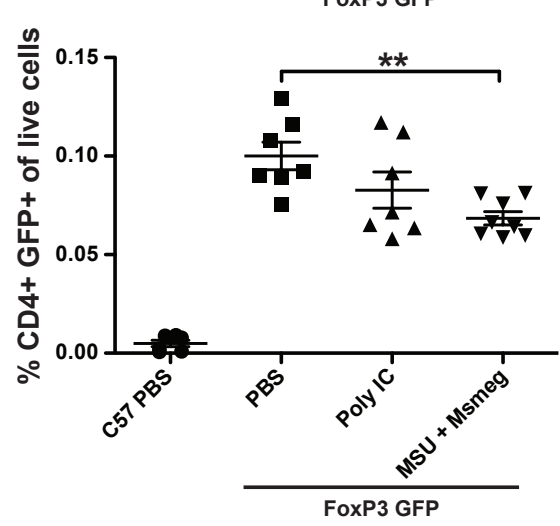

Figure 5.16: MSU + M. smegmatis but not Poly IC treatment reduces the frequency of Tregs in tumours. C57 and FoxP3-GFP mice bearing established B16F1 tumours were injected around the tumour with PBS, Poly IC or MSU + M. smegmatis on day 7, 911 and 13. On day 14, tumours were excised and analysed by flow cytometry. (A) CD45+ immune cells were gated as in Figure 4.3 and subsequently Tregs were identified as $\mathrm{CD} 4+\mathrm{FoxP} 3-\mathrm{GFP}+$. Representative flow plots from control and adjuvant treated mice are shown. (B) The frequency of Treg among CD45+ immune cells is graphed with mean \pm SE. (C) The percentage of Tregs among all live cells in tumours is shown with mean $\pm \mathrm{SE}$. ${ }^{* *} p<0.01$ as assessed by one-way ANOVA with Tukey's post test. Data are pooled from 2 independent experiments with 3-5 mice per group. 
A.
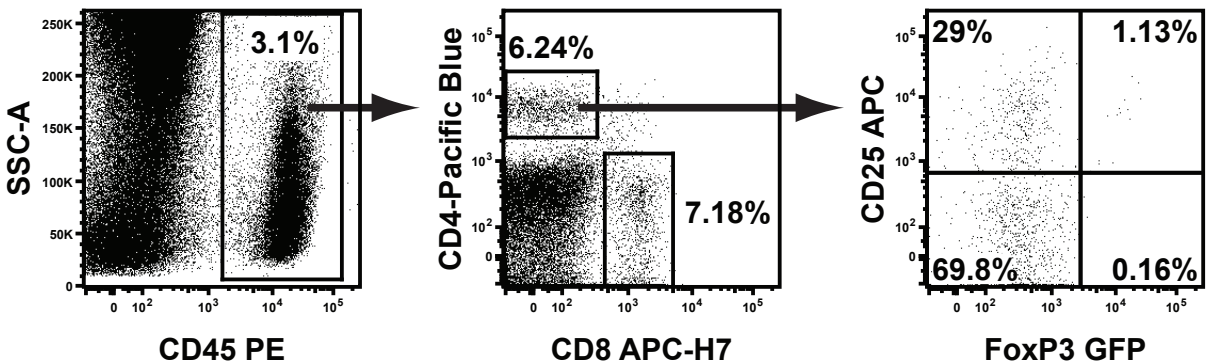

B.

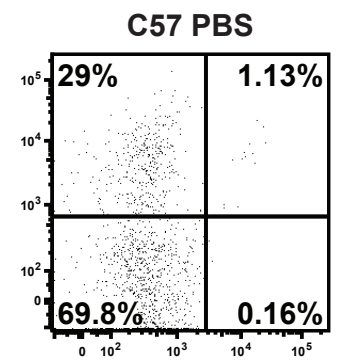

FoxP3-GFP PBS

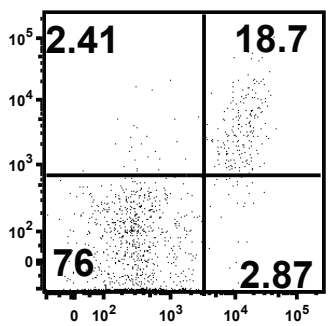

C.

FoxP3-GFP

Poly IC

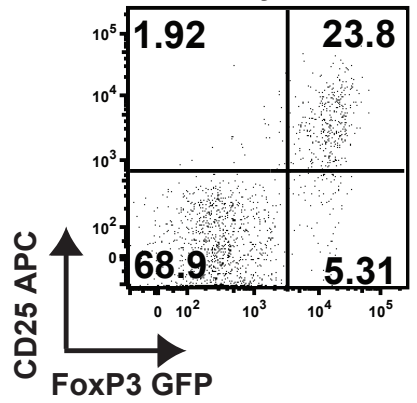

FoxP3-GFP

MSU+M.smeg
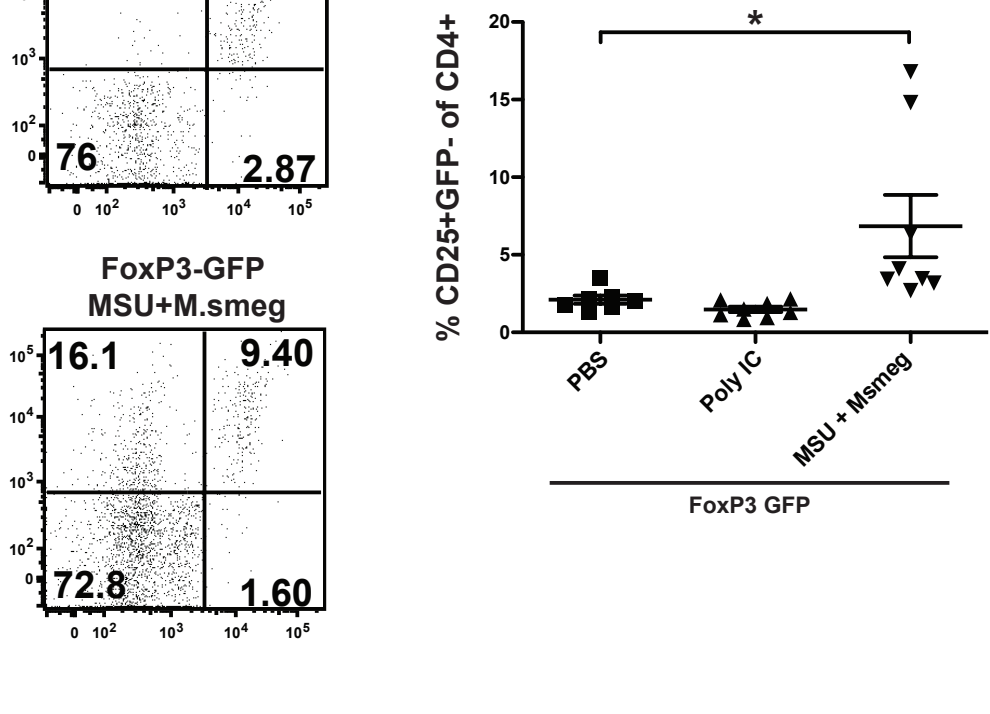

Figure 5.17: MSU + M. smegmatis but not Poly IC treatment induces CD25+FoxP3- CD4 $\mathbf{T}$ cells in tumours. In the same experiment described in Figure 5.16, CD25 and FoxP3 expression on tumour-infiltrating CD4 T cells was analysed. (A) Live singlet cells were gated as in Figure 4.3 and subsequently CD45+ CD4+ cells were identified and analysed for CD25 and FoxP3-GFP expression. (B) Representative flow plots for CD25 and FoxP3-GFP on CD4 T cells from control and adjuvant treated mice are shown. (C) The percentage of CD25+FoxP3-GFP- among CD4 T cells in tumours is shown with mean \pm SE. ${ }^{*} p<0.05$ as assessed by one-way ANOVA with Tukey's post test. Data are pooled from 2 independent experiments with $3-5$ mice per group. 


\subsection{Discussion}

The results described in Chapter 4 show that treatments with Poly IC and MSU $+M$. smegmatis can delay tumour growth and induce the accumulation of inflammatory monocytederived DCs in dLNs. The aim of the experiments described in this chapter was to determine the different effector cell types involved in the anti-tumour response elicited by these adjuvants and investigate how the proliferation, recruitment and function of T cells and NK cells was affected.

\section{Effects of adjuvant treatment on $\mathrm{T}$ cell proliferation in vivo}

Few studies have analysed the effect of adjuvant treatment at the tumour site on $\mathrm{T}$ cell proliferation. One report by VanOosten et al. found that CpG enhanced the proliferation of tumour-antigen specific CD8 T cells, as well as slightly improved their in vivo cytotoxicity [515]. When our study examined the proliferation of OTI T cells in dLNs of adjuvant treated B16.OVA tumours, a slight increase in the total percentage of OTI cells as well as of divided OTI cells was seen only in response to $\mathrm{MSU}+$ M. smegmatis. However, both $\mathrm{MSU}+$ M. smegmatis and Poly IC treatment led to significantly higher numbers of OTI and divided OTI cells in dLNs. This correlated with an overall enlargement of dLNs in these groups. As the adjuvant treatment given did not include any tumour-antigens, but instead aimed at activating DCs presenting endogenous tumour antigen, it is possible that other CD8 T cells were also proliferating in the dLNs. This would result in an overall increase in the numbers of CD8 T cells with a range of tumour-specific TCRs, but the percentages of $\mathrm{T}$ cells with different specificity would remain largely unaffected.

MSU + M. smegmatis treatment was the only adjuvant examined that induced CD4 T cell proliferation. Similar to what was observed for CD8 T cells, the percentage of OTII cells among all CD4 $\mathrm{T}$ cells in the dLNs of adjuvant treated B16.OVA tumours remained unchanged. The numbers of OTII cells, as well as the numbers and frequency of divided OTII cells increased considerably in response to $\mathrm{MSU}+M$. smegmatis. If adjuvant treatment induced a polyclonal CD4 T cell response, other CD4 T cells besides OTII cells may also 
be proliferating in dLNs, thereby masking any expansion of OTII cells in the CD4 population.

A lack of OTII proliferation in the dLNs of mice bearing OVA-expressing tumours including the colon carcinomas CMT93 and MC38, the T-cell lymphoma EL-4, and several transfected B16 melanomas was previously described [321, 447]. At the same time, some CD8 T cell proliferation was always detectable. Furthermore, the lack of CD4 T cell activation could not be overcome by inducing tumour cell death with chemotherapeutic agents or FasL. Moreover, intra-tumoral injection of LPS $+\mathrm{CD} 40$ ligand, TNF $\alpha, \mathrm{CFA}$ and even a cocktail of LPS, CpG, Poly IC, Peptidoglycan and Lipoteichoic acid failed to induce CD4 T cell proliferation [321]. These findings are in line with the lack of OTII cell proliferation in response to LPS, Poly IC or mycobacteria observed in this study and make the ability of the combination of $\mathrm{MSU}+$ M. smegmatis to stimulate OTII proliferation very remarkable.

The increased proliferation of tumour-antigen specific T cells in dLNs after Poly IC and MSU + M. smegmatis treatment indicates that these adjuvants enhanced the capacity of DCs to activate tumour-specific T cells. However, proliferation of $\mathrm{T}$ cells does not always result in the successful generation of an effector response. Instead, the proliferation can be abortive, followed by the establishment of $\mathrm{T}$ cell tolerance [516]. This is characterised by the failure of these $\mathrm{T}$ cells to acquire effector functions such as cytotoxicity and cytokine production, followed by deletion [237]. Phenotypically, T cells undergoing tolerance up-regulate CD69 and CD44 in a similar manner to T cells becoming effector cells, but only partially down-regulate CD62L [237]. As the proliferating T cells in this study showed clear down-regulation of CD62L, they are likely to be differentiating into effector cells.

\section{Accumulation of effector cells in adjuvant-treated tumours}

Adjuvant administration appeared to cause some inflammation at the tumour site as evidenced by the induction of pro-inflammatory cytokines such as TNF $\alpha$ and IL-6. Inflammation is known to lead to increased expression of adhesion molecules on the endothelial cells of blood vessels, as well as cytokines in the inflamed tissue that promote the influx of immune cells from the blood [517]. Therefore, the recruitment of NK cells and T cells to the tumour 
site in response to adjuvant administration was analysed. A single adjuvant administration only had a minor impact on the percentage of effector cells as well as their activation state. Poly IC and MSU + M. smegmatis enhanced CD8 T cell proliferation 3 days after the first adjuvant administration. If $\mathrm{T}$ cells needed to be activated de novo and expand in the dLNs they would be expected to migrate to the tumour site at the earliest 3-5 days after priming and would not be detectable 2 days after the first adjuvant treatment. Alternatively, more sustained inflammation may be required for the recruitment of effector cells to the tumour site.

After four adjuvant treatments, the frequency of CD8 $\mathrm{T}$ cells was doubled and that of NK cells tripled in MSU + M. smegmatis and Poly IC treated tumours, compared to saline controls. CD4 T cell infiltration was only significantly increased in response to MSU + M. smegmatis. The total numbers of CD45+ immune cells in these groups also increased compared to the earlier time point (see Chapter 4) and the percentages of effector cells among the immune infiltrate remained constant or increased as well. This indicates that $\mathrm{T}$ cells and NK cells are actively recruited to the tumour site and not just less outgrown by the melanoma mass in the smaller, Poly IC and MSU + M. smegmatis treated tumours. The increase in tumour-infiltrating CD4 T cells only in the MSU + M. smegmatis treated tumours is in line with the previous result that only this adjuvant combination elicited CD4 T cell proliferation. Studies of the adjuvant effect of $\mathrm{CpG}$ administration have previously reported an increase in CD8 and CD4 T cell infiltration at the tumour site, which was implicated in the anti-tumour effect $[403,504]$. However, Currie et al. found that Poly IC did not enhance CD8 T cell accumulation at the tumour site, despite the requirement for CD8 T cells in its anti-tumour function [12]. The discrepancy between the data of Currie et al. with the data obtained in this study may be due to the different tumour models employed. Furthermore, Currie et al. analysed the immune infiltrate 3 days after initiation of adjuvant treatment. This is more similar to the early time point of analysis after one adjuvant treatment in this study, at which no significant increase in CD8 T cell infiltration was observed either.

While the delay in recruitment of $\mathrm{T}$ cells is likely due to the time they needed to expand in dLNs, the concurrent delay in NK cell infiltration appears to suggest that prolonged adjuvant stimulation was generally needed to promote access of effector cells to the tumour site. 
NK cells are called "natural" as they do not require priming to exert their effector functions. Rather, the integration of signals from activating and inhibitory surface receptors on the NK cell determines its action [518]. However, it has now become clear that NK cells can also interact with DCs. This cross-talk leads to increased production of IFN $\gamma$ from NK cells, enhanced target cell lysis and improved anti-tumour activity [227]. Furthermore, in viral infections NK cells have been found to proliferate, with the Ly49H+ subset peaking at day 6 after infection [519]. Thus, it is possible that NK cells also need to be activated by DCs and maybe even proliferate before they infiltrate the tumour site and that this leads to the observed delay in DC recruitment. However, a need for sustained local adjuvant stimulation to increase inflammation, chemokine secretion and endothelial adhesion molecules to promote access of effector cells to the tumour site may also contribute to the late increase in NK cell and T cell infiltration.

In addition to proliferation and recruitment of effector cells to the tumour site, their activation state as assessed by the expression of surface activation and inhibitory markers was also analysed. Repeated administration of MSU + M. smegmatis slightly increased the expression of CD69 on tumour-infiltrating T cells. In addition, M. smegmatis and MSU + M. smegmatis both resulted in down-regulation of PD-1 on CD8 T cells in tumours. In contrast, Poly IC did not induce any significant change in CD69 or PD-1 levels. PD-1 is an inhibitory molecule expressed on $\mathrm{T}$ cells that is normally involved in the maintenance of peripheral tolerance by blocking the TCR-driven motility arrest and subsequent T cell activation [520]. The corresponding ligand, PD-L1, is not detectable in normal tissues, but is highly expressed in tumour cells, including melanoma [146]a. PD-1 ligation on T cells leads to T cell apoptosis and PD-L1+ tumours grow more aggressively in vivo [146]. Therefore, blocking antibodies against PD-1 or PD-L1 have been tested in immunotherapy models and were shown to increase the anti-tumour effect of CpG administration, as well as DC vaccination combined with adoptive $\mathrm{T}$ cell transfer $[521,522]$. The down-regulation of PD-1 on CD8 T cells in response to M. smegmatis and MSU + M. smegmatis suggests that these T cells may be less susceptible to suppression by the tumour and therefore may have superior anti-tumour activity. 


\section{Cytokine production by effector cells in the tumour}

The production of IFN $\gamma$ and $\mathrm{TNF} \alpha$ by tumour-infiltrating CD8 $\mathrm{T}$ cells and NK cell was examined by intra-cellular cytokine staining. In the absence of adjuvant treatment, very few cells produced any of these cytokines. The frequency and level of $\mathrm{TNF} \alpha$ production was increased in response to MSU $+M$. smegmatis, while Poly IC treatment augmented the percentage of $\mathrm{TNF} \alpha+\mathrm{NK}$ cells. This suggests that MSU + M. smegmatis had a more profound impact on T cells, while Poly IC preferentially activated NK cells. Both adjuvants enhanced the capacity of effector cells to secrete TNF $\alpha$, suggesting that these effector cells were more activated than in saline treated controls.

The frequency and level of IFN $\gamma$ production in both CD8 T cells and NK cells was increased to a similar extent after four administrations of Poly IC or MSU + M. smegmatis. IFN $\gamma$ was shown to be critical for the control of spontaneous and chemically induced tumours, as well as transplantable B16 melanomas [20, 501]. The anti-tumour effect of IFN $\gamma$ is thought to be due to up-regulation of MHC I on the tumour cells, which renders them more immunogenic and susceptible to CD8 T cell mediated killing [20]. Increased IFN $\gamma$ production in T cells has previously been reported in response to $\mathrm{CpG}$ adjuvant treatment [523]. Furthermore, the increase in IFN $\gamma$ production by $\mathrm{CD} 8 \mathrm{~T}$ cells in response to Poly IC is in line with data from Currie et al., who find that enhanced cytoxicity of CD8 T cells coupled with an increase in MHC I expression in adjuvant-treated tumours, probably due to enhanced IFN $\gamma$ secretion, is the main mechanism of tumour rejection in response to Poly IC treatment [12].

\section{Tumour-protective capacity of adoptively transferred $\mathbf{T}$ cells from adjuvant treated mice}

Several studies have used adoptive transfer of $\mathrm{T}$ cells from treated tumour-bearing mice into naïve hosts to dissect their protective function against tumour challenge [12, 403, 524]. All reports used $\mathrm{T}$ cells purified from the spleens of treated animals and transferred high numbers $\left(5 \times 10^{6}-5 \times 10^{7}\right) \mathrm{T}$ cells i.v. into recipients.

Kunikata et al. reported that the full protection potential was only achieved when both 
CD8 $\mathrm{T}$ cells and CD4 T cells were co-transferred [403]. In addition, a different group investigating the anti-tumour potential of adoptively transferred, antigen-specific $\mathrm{T}$ cells reported that co-transfer of CD4 T cells with CD8 T cells was dispensable for CD8 T cell cytotoxicity, but required for the maintenance of CD8 T cells and anti-tumour activity [69]. Furthermore, in a viral infection model of mucosal sites, CD4 T cell help was critical for the access of CD8 T cells to the infected tissue [500].

In light of those studies, CD4 and CD8 T cells were purified from spleens of adjuvant treated animals and transferred into naïve, CD45 congenic hosts. Subsequent tumour challenge revealed that $\mathrm{T}$ cells from all adjuvant treated mice mediated a slight delay in tumour onset, but only T cells from Poly IC and MSU + M. smegmatis treated donors conferred a slight survival benefit. However, none of the recipient mice was protected from tumour growth. As $\mathrm{T}$ cells could not be selected for tumour-specificity prior to transfer, the number of tumour-specific $\mathrm{T}$ cells within the $7 \times 10^{6}$ transferred $\mathrm{T}$ cells may have been too low to achieve tumour protection. In a subsequent experiment, graded numbers of 10,20 and $40 \times 10^{6}$ $\mathrm{T}$ cells from MSU $+M$. smegmatis treated donors were transferred. While the relative numbers of transferred $\mathrm{T}$ cells were reflected in the percentage of CD45 congenic T cells detected in the blood of recipient mice, this increase in numbers still resulted in a similar delay in onset of tumour growth in all recipients of $\mathrm{MSU}+$ M. smegmatis $\mathrm{T}$ cells.

Several factors could be causing this lack of tumour protection. Firstly, the spleen may not be the ideal source of tumour-specific T cells in the adjuvant treated mice. Proliferation of $\mathrm{T}$ cells was observed in tumour-draining but not contra-lateral LNs and increased infiltration of $\mathrm{T}$ cells to the tumour site also occurred in response to adjuvant administration. Therefore, most tumour-specific T cells may be recruited to the tumour site and dLNs and few may be circulating or resident in the spleens.

Secondly, the number of transferred $\mathrm{T}$ cells may be too low to confer tumour protection. In a gp33 expressing B16 melanoma model, a study by Perret et al. showed that 1-2 x 107 in vitro activated, antigen-specific CD8 T cells were required to confer tumour protection, while 1-3 x $10^{6}$ cells only resulted in a variable degree of delay of the onset of tumour growth [385]. For p-mel CD8 T cells, which recognise the endogenous tumour-antigen gp100 and have a lower avidity than OVA or gp33 specific T cells, even $10^{7}$ activated $\mathrm{T}$ cells were not 
sufficient to prevent the growth of B16 melanomas [384]. Instead, additional vaccination with antigen plus adjuvant and IL-2 delivery was required to achieve tumour protection [384]. These publications suggest that the transfer of $7-40 \times 10^{6}$ splenic $\mathrm{T}$ cell from adjuvanttreated donors may indeed be insufficient for anti-tumour activity in the B16 melanoma model.

Thirdly, the transfer of total $\mathrm{T}$ cells is likely to have included Tregs within the CD4+ population. These may have counteracted the anti-tumour activity of effector CD4 and CD8 T cells in the recipient mice. At the same time, NK cells were excluded by depletion of NK1.1+ cells prior to transfer. As both T cell and NK cell infiltrate increased in Poly IC and $\mathrm{MSU}+$ M. smegmatis treated tumours, these effector cell populations may work together to exert their anti-tumour activity and T cells may be less effective in the absence of activated NK cells.

Lastly, even with co-transfer of CD4 and CD8 T cells, access of T cells to the tumour may have been limited in the absence of local adjuvant administration and inflammation. A similar restriction was described by Currie et al., who report that $5.5 \times 10^{6}$ transferred $\mathrm{T}$ cells from Poly IC treated donors only delayed tumour growth in recipients with small established tumours when Poly IC was also administered intra-tumorally in the recipients [12]. Even adjacent tumours that shared dLNs but did not receive Poly IC injections were unaffected by the transferred $\mathrm{T}$ cells.

Together, these results suggest that a relatively low number of transferred $\mathrm{T}$ cells in combination with the lack of local adjuvant stimulation in the recipients was likely to be the main reason that transferred $\mathrm{T}$ cells only conferred a delay in the onset of tumour growth and no tumour protection. In addition, the small but significant effect indicates that the transferred $\mathrm{T}$ cells do have some anti-tumour activity. 


\section{Requirement of $T$ cells and NK cells for adjuvant-induced tumour-growth delay}

To further investigate the requirement for adaptive immune cells, as well as examine the contribution of CD4 T cells, CD8 T cells and NK cells in more detail, tumour growth experiments were performed in Rag1-/- mice and C57BL/6 mice depleted of these cell subsets. MSU + M. smegmatis treatment did not delay tumour growth at all in Rag1-/- mice, as well as CD8 T cell and NK cell depleted animals. Likewise, the effect of Poly IC treatment was largely abolished in Rag1-/- mice and completely abolished in the absence of CD8 T cells and NK cells. This indicates that both CD8 T cells and NK cells were required for Poly IC and MSU + M. smegmatis to exert their adjuvant activity. The slight delay of tumour growth in Rag1-/- mice in response to Poly IC administration is likely mediated by NK cells, which are not affected by the absence of Rag1. In CD8 T cell depleted animals, Poly IC may still have activated NK cells, but at the same time Tregs are present in these mice and can suppress anti-tumour activity.

The tumour growth in CD4 $\mathrm{T}$ cells depleted adjuvant treated mice is more difficult to interpret, as CD4 $\mathrm{T}$ cell depletion already slowed tumour growth to a similar extent as adjuvant treatment. This may be due to the depletion of Tregs within the CD4 population. CD4+CD25+ Tregs have previously been associated with more aggressive growth of B16 melanomas [447]. If the only effect of the CD4 $\mathrm{T}$ cell population in the adjuvant treated tumours was the suppression of the immune response, an even improved outcome with adjuvant treatment that activated anti-tumour CD8 T cells and NK cells would be expected. As no further delay in tumour growth was observed with Poly IC and MSU + M. smegmatis treatment in CD4 depleted animals, this suggests that CD4 T cells may also be required to contribute to the anti-tumour effects of these adjuvants. While Currie et al. reported that the anti-tumour effect of Poly IC was solely dependent on CD8 T cells and independent of CD4 T cells, they had not included controls of tumour-bearing, cell depleted mice without adjuvant treatment [12]. As CD4 depleted mice already showed similar tumour growth as adjuvant treated mice, the omission of this control may mask the possible role of CD4 T cells. The different requirement for NK cells, on the other hand, may rather be due to the different 
tumour models in this study and the work by Currie et al.. Studies investigating the requirement of $\mathrm{CD} 8 \mathrm{~T}$ cells and $\mathrm{NK}$ cells in response to $\mathrm{CpG}$ treatment have variably reported tumour elimination solely by CD8 T cells [502], solely by NK cells [503] or by both CD8 T cells and NK cells $[11,504]$. The tumour models employed in those studies all differed and may be differentially susceptible to NK cell mediated and CD8 T cell mediated recognition and killing.

\section{Role of Tregs in the adjuvant-induced anti-tumour response}

The role of Tregs in the adjuvant treated tumours was investigated in more detail. Treg depletion using the anti-CD25 antibody PC61 resulted in delayed tumour growth without additional adjuvant treatment. However, tumour development in these mice was still faster than in CD4 $\mathrm{T}$ cell depleted animals. This may be due to the the incomplete depletion of Tregs by the PC61 antibody. $80 \%$ of CD25+CD4+ T cells remained depleted throughout the experiment, leaving ca. $20 \%$ unaffected. In addition, not all CD4+ Tregs express CD25 and approximately $12 \%$ of FoxP3+CD4+ Tregs were shown to be CD25- [411]. Moreover, activated T cells also express CD25 [167] and while anti-CD25 antibody was given one week prior to adjuvant treatment a minimal impact on activated $\mathrm{T}$ cells cannot be entirely excluded. Nevertheless, when CD25 depleted mice were given peri-tumoral adjuvant injections, Poly IC and MSU + M. smegmatis treated tumours grew slower compared to saline treated, CD25 depleted tumours. Furthermore, the anti-tumour effect of Poly IC combined with CD25 depletion was significantly better than Poly IC administration on its own. This indicates that Poly IC treatment does not modulate the function of Tregs but can be further improved by depleting Tregs. In contrast, MSU + M. smegmatis treated, CD25 depleted tumours developed precisely as non-CD25 depleted MSU + M. smegmatis treated B16 melanomas. This result suggested that MSU + M. smegmatis may directly affect Tregs in some way and therefore not gain additional benefit from Treg depletion.

FoxP3-GFP mice were employed to study Tregs in tumours in more detail. FoxP3 expression has been shown to strictly correlate with the suppressive function of Tregs, irrespective of CD25 expression [411]. Using FoxP3-GFP mice, a small fraction (2\%) of tumour-infiltrating 
immune cells were identified to be Tregs in saline controls. The percentage of Tregs was unaltered in Poly IC treated tumours, but reduced by nearly half in response to MSU + M. smegmatis administration. In addition, the majority of Tregs in PBS or Poly IC treated tumours co-expressed CD25 and FoxP3. In MSU + M. smegmatis tumours, a significant fraction of CD4 T cells was found to be CD25+ but FoxP3-. The percentage of these cells varied from $5-15 \%$ of all tumour-infiltrating CD4 T cells. Their lack of FoxP3-GFP indicates that they are not Tregs, but rather activated CD4 T cells, which also express high levels of CD25. Thus, MSU + M. smegmatis appears to considerably increase the ratio of activated CD4 T cells to Tregs. Few other immunotherapies, such as the combination of cyclophosphamide with IL-12 and a synthetic bacterial lipoprotein (TLR1/TLR2 agonist) have previously been shown to increase the infiltration of effector $\mathrm{T}$ cells while at the same time reducing Tregs $[525,526]$. In patients with ovarian cancer as well as hepatocellular carcinoma, a high effector cell to Treg ratio has been associated with a more favourable prognosis $[44,527]$. Thus, the ability of an adjuvant treatment to skew the effector cell : Treg ratio to favour tumour rejection may be clinically relevant. 


\section{Conclusions}

Both Poly IC and MSU + M. smegmatis treatment required CD8 T cells and NK cells to exhibit anti-tumour activity. CD4 T cells may also be involved in the anti-tumour response to adjuvant treatment.

The activation of effector cells appeared to occur at several levels. The numbers of tumour-specific CD8 $\mathrm{T}$ cells that proliferated in tumour-dLNs were increased with Poly IC and MSU + M. smegmatis treatment. In addition, the infiltration of immune cells, especially of CD8 T cells and NK cells in tumours was enhanced after 4 adjuvant applications. The capacity of tumour-infiltrating effector cells to produce $\operatorname{IFN} \gamma$ and $\mathrm{TNF} \alpha$ was also augmented by both adjuvant treatments.

While CD8 T cells were required for anti-tumour activity in response to Poly IC and MSU + M. smegmatis adjvuant treatment, adoptively transferred T cells from treated donors were not sufficient to confer tumour protection to recipients. However, a significant delay in the onset of tumour growth suggests that these $\mathrm{T}$ cells exert some anti-tumour function.

CD4 T cells were only significantly affected by MSU + M. smegmatis treatment. The percentage and number of proliferating, tumour-antigen specific CD4 T cells increased. In addition, a higher frequency of CD4 T cells was observed to infiltrate MSU + M. smegmatis treated B16 melanomas. At the same time, the infiltration of Tregs was decreased and a population of CD25+ FoxP3- activated CD4 T cells accumulated in tumours in response to $\mathrm{MSU}+$ M. smegmatis administration.

Thus, Poly IC and MSU + M. smegmatis both require innate as well as adaptive effector cells to delay tumour growth. Their effects on CD8 T cells and NK cells are comparable, whereas only MSU + M. smegmatis alters CD4 T cell proliferation, infiltration and effector to Treg ratio. 




\section{Chapter 6}

\section{Optimisation of adjuvant}

administration to delay tumour growth and reduce metastases 


\subsection{Introduction}

Several studies have demonstrated a requirement for multiple adjuvant administrations to elicit potent anti-tumour effects $[11,12,403]$. While a single exposure to adjuvant-activated DCs is sufficient to induce $\mathrm{T}$ cell responses in naïve mice, persistent adjuvant signals are required to break established $\mathrm{T}$ cell tolerance and induce anti-tumour immunity [528].

Repeated exposure to adjuvants can boost the immune responses, but it can also induce feedback regulation and tolerance to future activation by the same and even different adjuvants, as shown for LPS and Poly IC $[446,529,530]$. The induction of immune activation as opposed to tolerance to repeated administrations depends on the adjuvant dose, with super-low doses $(0.05 \mathrm{ng} / \mathrm{mL}$ to $0.5 \mathrm{ng} / \mathrm{mL}$ LPS $)$ enhancing activation, low doses $(>100 \mathrm{ng} / \mathrm{mL}$ LPS in vitro; $50 \mu$ g Poly IC i.p. weekly) inducing tolerance and high doses ( $1 \mathrm{ng} / \mathrm{mL}$ to $100 \mathrm{ng} / \mathrm{mL}$ LPS in vitro; $20 \mu \mathrm{g}$ Poly IC i.p. weekly) favouring immune activation $[529,530]$. In addition, the adjuvant dose encountered by immune cells is also likely to vary according to the administration route and the persistence of individual adjuvants at the injection site.

It has also been reported that the tolerance to a secondary adjuvant administration is transient [457]. Therefore treatment schedules that allow for rest days between the adjuvant administrations have widely been employed [11, 403, 531]. However, a study directly comparing the effect of different treatment regimens with Poly IC found that a higher frequency of Poly IC administrations was superior to treatments at 3-day intervals [12].

In addition to eliciting the maximal anti-tumour effect in vivo, adjuvants also need to be safe for clinical application. While Poly IC and MSU have already been used in clinical trials without major side effects $[15,360]$, live mycobacteria are more problematic. Live BCG instillation has been successfully used in the treatment of superficial bladder cancer for decades [10]. However, a moderate level of toxicity with local severe inflammation is associated with the treatment, and a number of patients subsequently develop systemic BCG infection with granuloma formation $[532,533]$. While M. smegmatis is a non-pathogenic mycobacterium [534], heat-killed preparations may still be preferable over live organisms provided that effectiveness is retained. 
In the previous chapter, both Poly IC and MSU + M. smegmatis treatments were found to have considerable anti-tumour activity against the B16F1 melanoma when given peritumorally (Figure 4.2). In addition, a report comparing different administration routes for CpG clearly demonstrated that peri-tumoral injection is the most effective way of adjuvant delivery [445]. However, in a clinical setting, the tumor mass is not always accessible for adjuvant injection. Therefore, most clinical trials have injected adjuvants such as BCG subcutaneously $[535,536]$, albeit with limited success compared to initial reports of intralesional application [537]. The anti-tumour activity of Poly IC and MSU + M. smegmatis applied at a site distant from the tumour remains to be determined.

Death of cancer patients is rarely caused by the primary tumour, and in breast cancer patients more than $90 \%$ of deaths are due to metastases [538]. Metastatic tumours are often not accessible to surgery and fail to respond to therapies that were initially successful in halting primary tumour progression [538]. It is now clear that metatstatic cancer cells cells differ from primary tumor cells in their protein expression profiles, as well as in their response to therapeutic agents $[539,540]$. Immunotherapy may be a viable strategy to target metastases, as immune cells can migrate to all sites in the body to seek out and eliminate metastatic tumour cells. Indeed, $\mathrm{CpG}$ and Poly IC immunotherapy has previously been shown to control experimental metastases in mice via activated $\mathrm{T}$ cells [403] or NK cells [541], respectively. CpG administration could also prevent spontaneous brain metastasis from orthotopic breast carcinomas [542], while the effect of Poly IC and MSU + M. smegmatis treatment on spontaneous metastases from orthotopic tumours remains to be established. 


\subsection{Aims}

Results described in Chapter 4 showed that four peri-tumoral administrations of Poly IC or $\mathrm{MSU}+$ M. smegmatis delayed the growth of B16F1 tumours. The experiments described in this chapter were conducted to optimise the administration of these two effective adjuvants to achieve the best possible anti-tumour effect. In addition, it was evaluated whether dead M. smegmatis could be used instead of live bacteria to increase safety. Furthermore, their effect on primary growth and metastasis formation in other tumour models was investigated. As the anti-tumour activity of these adjuvants was mediated by innate and adaptive immune effector cells (Chapter 5), we hypothesized that they would have similar anti-tumour activity in different tumour models. Metastasis formation may either be reduced by immune effector cells activated in response to Poly IC and $\mathrm{MSU}+M$. smegmatis treatment or may be increased by the inflammation induced by adjuvant administration.

The specific aims of the work described in this chapter were:

- To identify the optimal number of adjuvant administrations and the best treatment schedule to achieve the maximal anti-tumour effect

- To determine whether heat-killed M. smegmatis in combination with MSU could delay tumour growth and activate immune cells in a manner similar to live M. smegmatis $+\mathrm{MSU}$

- To assess whether the adjuvant treatment had to be administered at the tumour site to induce an anti-tumour response

- To investigate whether peri-tumoral adjuvant treatments were effective in reducing primary tumour growth in other tumour models

- To assess whether Poly IC and MSU + M. smegmatis treatment enhanced or reduced the formation of lung metastases in the $4 \mathrm{~T} 1$ orthotopic breast carcinoma model 


\subsection{Results}

\subsubsection{Optimisation of the number and frequency of treat- ments with Poly IC and MSU + M. smegmatis}

Results presented in previous chapters have shown that four peri-tumoral administrations of Poly IC and MSU + M. smegmatis effective in delaying tumour growth. To identify, whether this number of treatments was required and to establish whether more than four treatments could improve the effect, mice bearing B16F1 melanomas were treated with each adjuvant 1-6 times and tumour growth was recorded. The results are shown in Figure 6.1.

Poly IC required at least two administrations to show any anti-tumour activity (Figure 6.1 A). The more treatments were given, the more tumour growth was delayed. However, the differences between 3-6 treatments were small, and no significant improvement was observed by increasing the number of treatments from 4 to 6 . MSU $+M$. smegmatis elicited a small delay in tumour growth already after a single administration (Figure 6.1 B). There was a marked improvement in the anti-tumour activity if 4 treatments were given, but no significant further enhancement with 4-6 administrations.

These results indicate that both Poly IC and $\mathrm{MSU}+M$. smegmatis treatments require multiple administrations to reach their maximal anti-tumour activity and that increasing the number of treatments is beneficial for the anti-tumour effect. In addition, there was no evidence of a tolerising effect of repeated adjuvant administration.

Different treatment schedules were also evaluated for their potential to enhance the anti-tumour activity of Poly IC and MSU + M. smegmatis (Figure 6.2). Daily treatment of established tumours with Poly IC for two weeks did not further delay tumour growth compared to 4 treatments every second day (Figure 6.2 B). Commencing Poly IC treatment before tumours became palpable (day 3) did not significantly reduce tumour growth overall compared to 4 treatments (Figure $6.2 \mathrm{~B}$ ). However, from day 15 to day 19, mice treated earlier had significantly smaller tumours, indicating that there was a transient enhancement of the anti-tumour response. 
A.

Poly IC

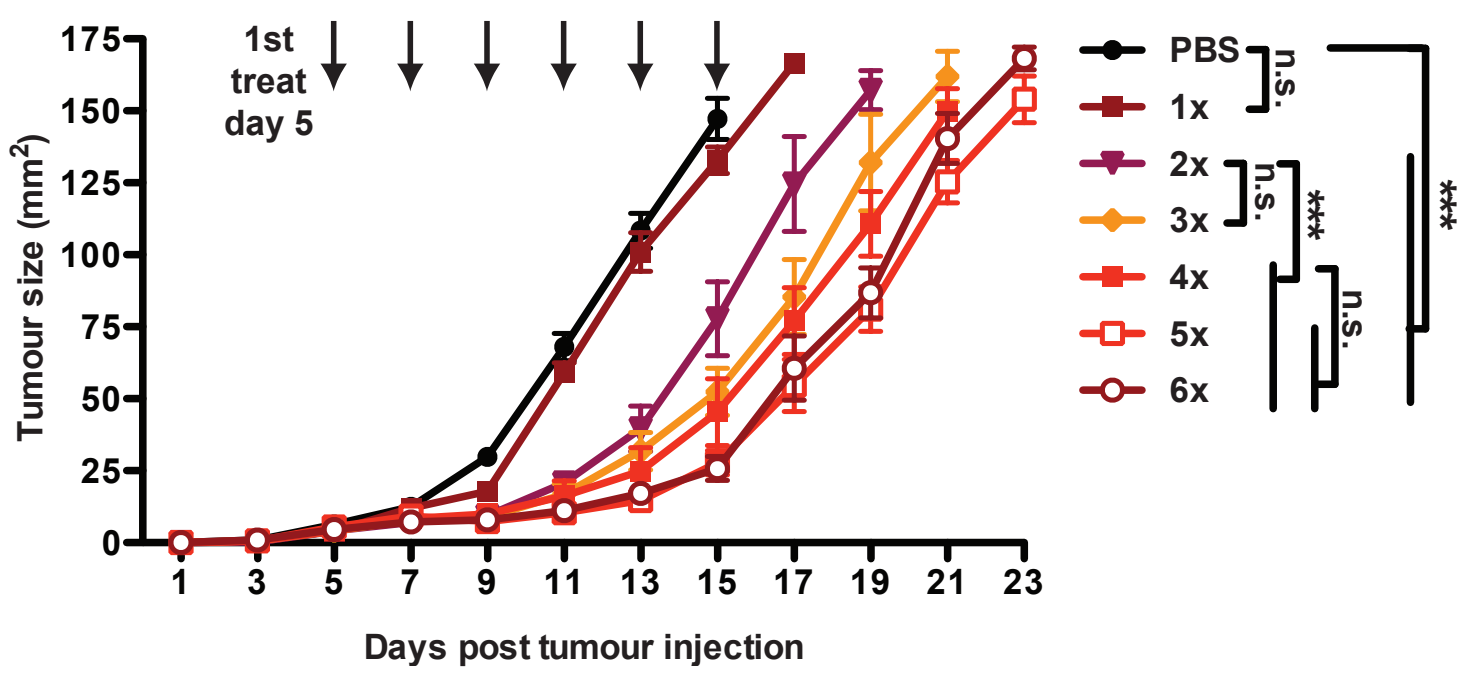

B.

MSU + M. smeg

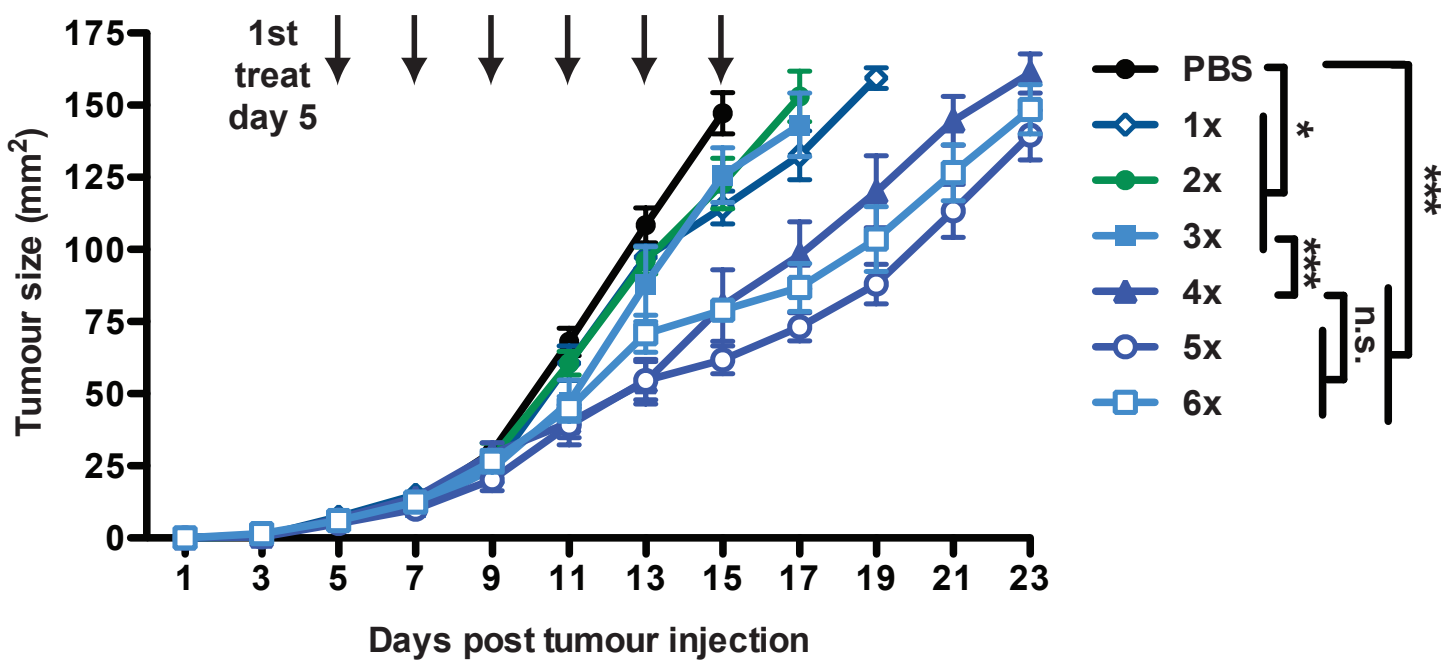

Figure 6.1: Repeated administration increases the anti-tumour effect of Poly IC and MSU + M. smegmatis treatments. C57 mice bearing small B16F1 tumours were injected around the tumour with Poly IC (A) or MSU + M. smegmatis (B) 1 to 6 times (1x to $6 \mathrm{x}$ ) starting from day 5. For multiple treatments, adjuvants were injected every second day as indicated by the arrows. Tumour size is shown as mean $\pm \mathrm{SE} .{ }^{*} p<0.05$ and ${ }^{* * *}$ $p<0.001$ from day 9 (Poly IC) or day 13 (MSU + M. smegmatis) as assessed by two-way ANOVA with Bonferroni's post test; n.s. = not significant. Data are from one experiment with 5 mice per group. 
A.

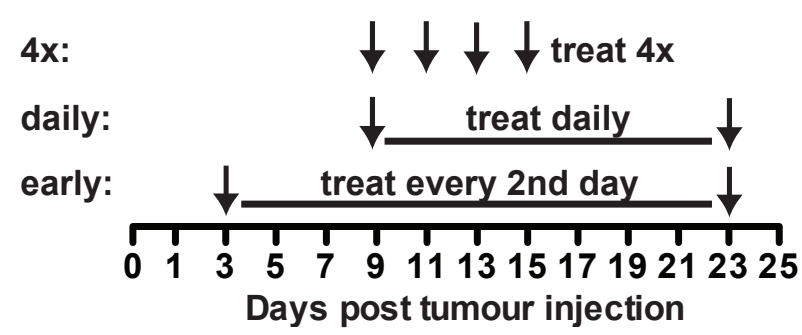

B.

Poly IC

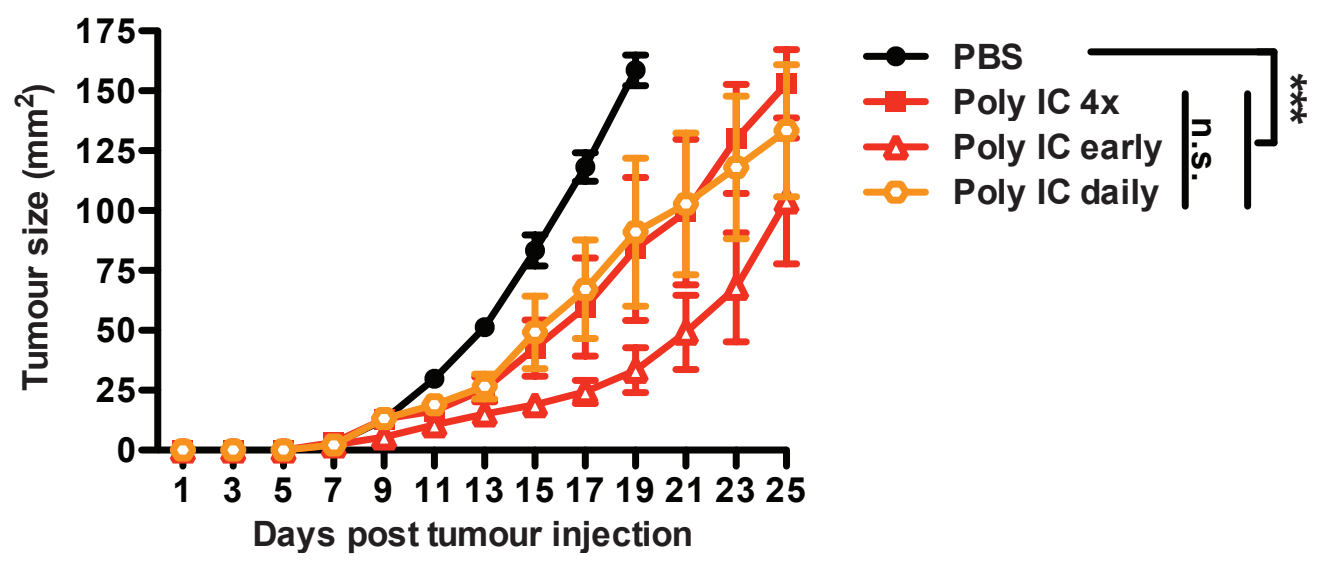

C.

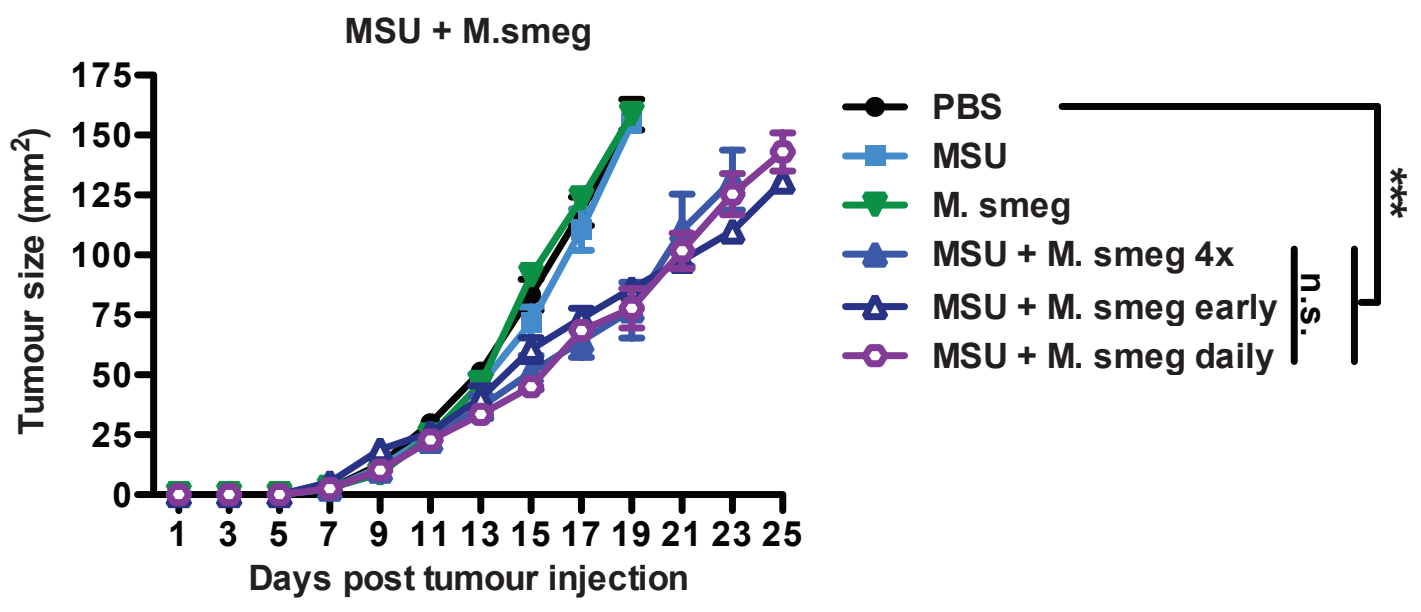

Figure 6.2: More frequent or earlier start of adjuvant administration does not significantly improve anit-tumour activity. C57 mice were injected with $10^{5}$ B16F1 tumour cells s.c. Poly IC or MSU + M. smegmatis treatments were given as illustrated in the schematic in (A). Tumour growth in Poly IC treated mice (B) and MSU + M. smegmatis treated animals $(\mathbf{C})$ is shown as mean \pm SE. ${ }^{*} p<0.05$ and ${ }^{* * *} p<0.001$ from day 11 (Poly IC) or day 15 (MSU + M. smegmatis) as assessed by two-way ANOVA with Bonferroni's post test; n.s. = not significant. Data are from one experiment with 5 mice per group. 
Earlier or more frequent application of MSU + M. smegmatis treatment did not alter tumour growth compared to the schedule of 4 treatments every second day also used in previous experiments (Figure 6.2 C).

Overall, the results from these experiments indicate that a higher number of adjuvant treatments (4-6) can boost their anti-tumour activity and does not appear to induce tolerance. However, increasing the frequency of dosing to daily applications didn't improve the anti-tumour effect and earlier start of adjuvant treatment was only found to have a transient benefit for Poly IC but not for MSU + M. smegmatis adjuvant treatment.

\subsubsection{Heat-killed and live $M$. smegmatis + MSU both activate anti-tumour immunity}

To improve the safety of MSU $+M$. smegmatis adjuvant treatment for potential application in a clinical setting, it would be desirable to use dead mycobacteria or extracts from mycobacteria to avoid the possibility of infections in immune-compromised patients. Therefore, the effect of MSU + dead M. smegmatis was compared to MSU + live M. smegmatis.

When four adjuvant treatments were given peri-tumorally after palpable B16F1 tumours had developed, dead M. smegmatis + MSU showed the same anti-tumour activity as live M. smegmatis + MSU (Figure 6.3). Both the delay in tumour growth and increase in survival were very similar in the two groups.

The effects of dead M. smegmatis + MSU and live M. smegmatis + MSU on the immune system were also compared. A single administration of dead M. smegmatis + MSU resulted in similar recruitment of CD45+ immune cells to the tumour site as with live M. smegmatis + MSU treatment (Figure 6.4 A). The relative percentages of the different immune cell populations infiltrating the tumour were also affected in a similar manner with live and dead M. smegmatis + MSU (Figure 6.4 B). Both monocyte populations increased at this early time point relative to other immune cells in tumours. Combined with the overall higher percentage of CD45+ immune cells at the tumour site, this translated into a similar increase in $\mathrm{Ly} 6 \mathrm{C}^{\text {hi }}$ and $\mathrm{Ly} 6 \mathrm{C}^{\text {int }}$ monoctyes at the tumour site in response to both live and 
dead M. smegmatis + MSU (Figure 6.4 C \& D).

Dead and live M. smegmatis + MSU also resulted in similar immune activation, as measured by the increase in dLN size and accordingly in DC numbers in dLNs after a single adjuvant treatment (Figure 6.5 A). Moreover, dead M. smegmatis + MSU also induced inflammatory monocyte-derived DCs in dLNs similar to live M. smegmatis + MSU (Figure 6.5 B).

A.

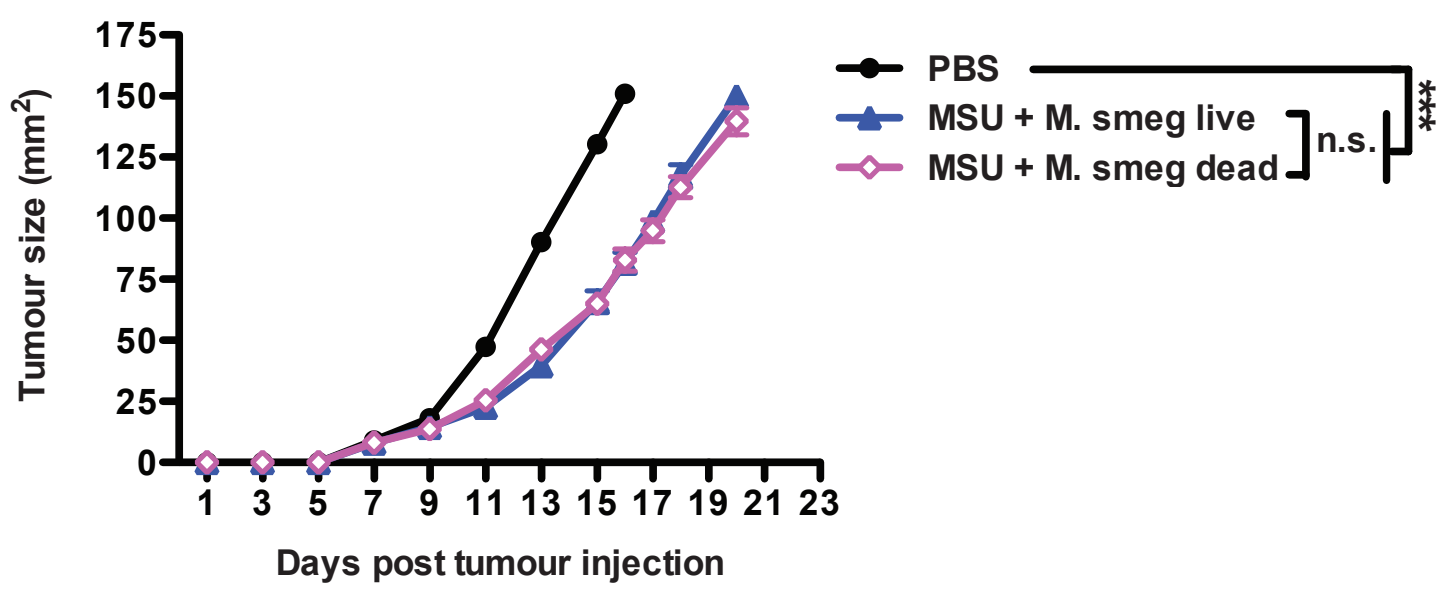

B.

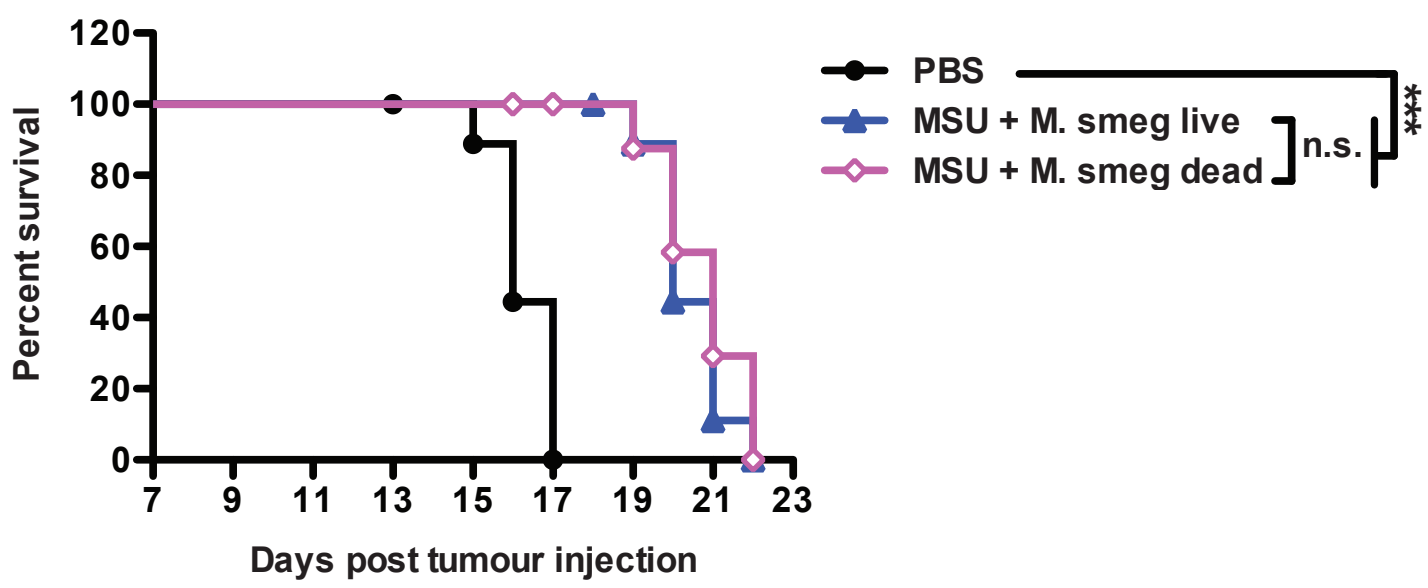

Figure 6.3: Live and dead M. smegmatis in combination with MSU are equally effective at delaying tumour progression. C57 mice bearing established B16F1 tumours were injected around the tumour with MSU + live M. smegmatis or MSU + M. smegmatis that had been heat killed for $60 \mathrm{~min}$ at $70^{\circ} \mathrm{C}$ (MSU + M. smeg dead) on day 7, 9 11 and 13. (A) Tumour growth is shown as mean \pm SE. Significant differences between treatment groups according to two-way ANOVA with Bonferroni's post test starting from day 11 are indicated by ${ }^{* * *} p<0.001$. (B) Survival of the B16F1 bearing mice treated with adjuvants in $(\mathrm{A})$ is depicted. ${ }^{* * *} p<0.001$ as assessed by Log-Rank-Test with Bonferroni's adjustment for multiple comparisons. n.s. = not significant. Data are pooled from 2 independent experiments with 5 mice per group each. 
Thus, dead M. smegmatis + MSU had the same capacity to delay tumour growth, prolong survival, increase monocyte infiltration at the tumour site and induce inflammatory monocyte-derived DCs in the dLNs as live M. smegmatis + MSU. This indicates that MSU + M. smegmatis treatment activates the anti-tumour immune response regardless of the viability of the mycobacteria.

A.

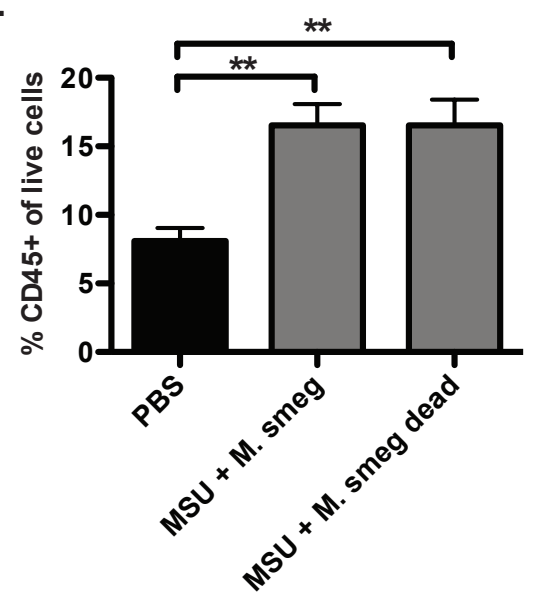

C.

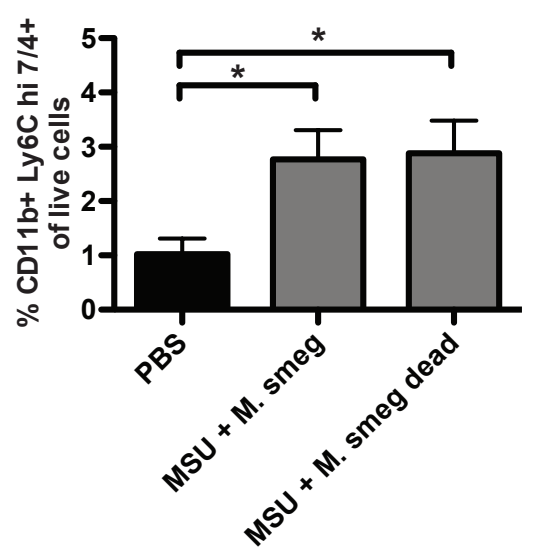

B.

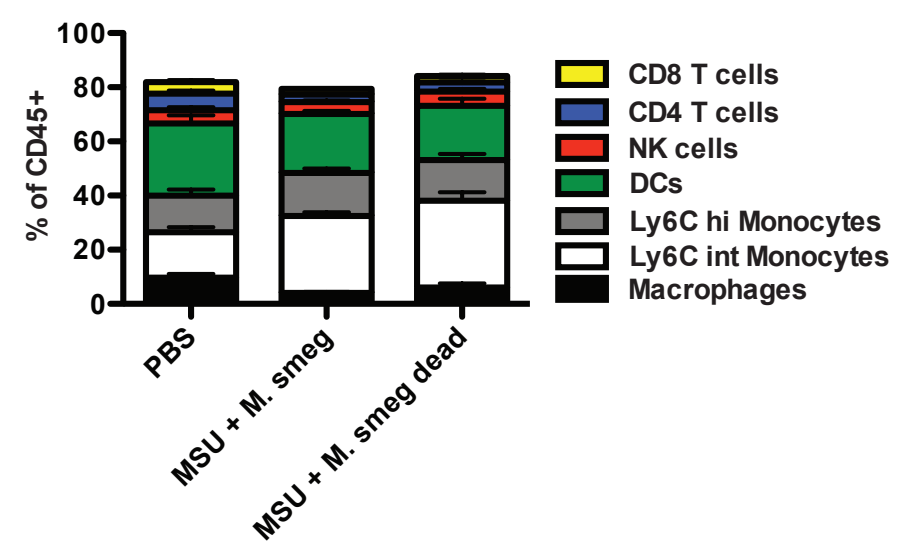

D.

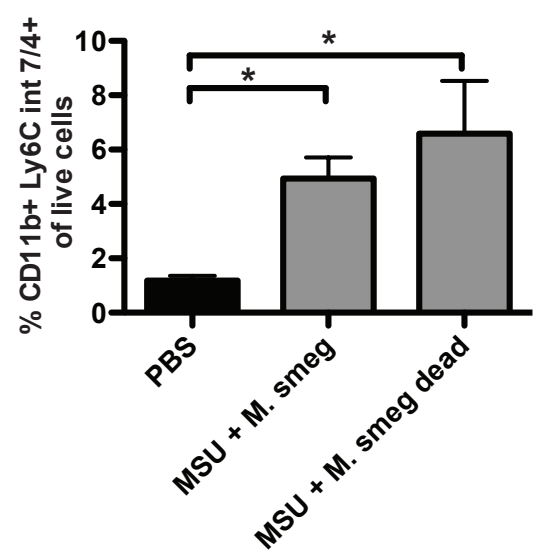

Figure 6.4: A single treatment with dead $M$. smegmatis + MSU has the same effects on intratumoral immune cells as live $M$. smegmatis + MSU. C57 mice bearing established B16F1 tumours were injected once around the tumour with either live M. smegmatis + MSU (MSU + M. smeg) or dead M. smegmatis + MSU (MSU + M. smeg dead). One day later, immune cells in tumours were analysed by flow cytometry as described in Figure 4.3. (A) The frequency of CD45+ cells in tumours is shown. (B) The percentages of different immune cell types among the total CD45+ immune infiltrate is graphed. (C) \& (D) The frequencies of Ly6 $\mathrm{C}^{\text {hi }}$ and Ly6 $\mathrm{C}^{\text {int }}$ monocytes among all live cells in tumours are shown. All data are presented as mean \pm SE. ${ }^{*} p<0.05{ }^{* *} p<0.01$ as assessed by one-way ANOVA with Tukey's post test. Data are from 1 experiment with 5 mice per group. 
A.

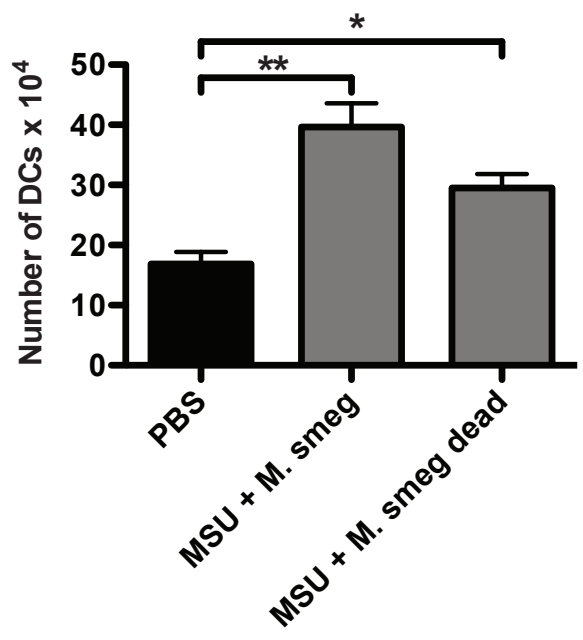

B.

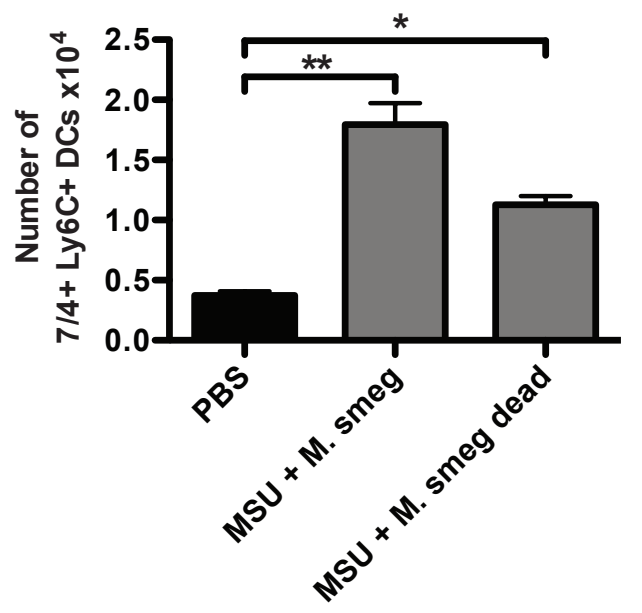

Figure 6.5: Heat killed $M$. smegmatis + MSU increases the number of inflammatory DCs in dLN similar to live $M$. smegmatis + MSU. Data are from the same experiment described in Figure 6.4. DCs in dLNs were analysed by flow cytometry as described in Figure 4.8. The total numbers of DCs (A) and the numbers of inflammatory monocyte-derived DCs $(7 / 4+\mathrm{Ly} 6 \mathrm{C}+)(\mathrm{B})$ in pooled axillary and inguinal dLNs are shown as mean \pm SE. ${ }^{*} p<0.05{ }^{* *} p<0.01$ as assessed by one-way ANOVA with Tukey's post test. Data are from 1 experiment with 5 mice per group.

\subsubsection{Effect of Poly IC and MSU + M. smegmatis treatment contralateral to the tumour site}

As Poly IC and MSU + M. smegmatis injections activated the anti-tumour immune response without the inclusion of any tumour antigens in the treatment, we hypothesized that they needed to be administered at the tumour site to be effective. To test this hypothesis, both adjuvant treatments were either given peri-tumorally, or injected on the flank contralateral to the tumour site.

Poly IC and MSU + M. smegmatis treatment around the tumours delayed tumour growth and prolonged survival of mice as observed in previous experiments (Figure 6.6 \& Figures 4.2, 6.1, 6.2). Poly IC had very little anti-tumour activity when it was administered s.c. contralateral to the tumour (Figure 6.6). In contrast, MSU + M. smegmatis treatment on the contralateral flank elicited a similar anti-tumour effect as peri-tumoral MSU + M. smegmatis administration (Figure 6.6). This result was unexpected and the phenomenon was investigated further in the following experiments. 
A.

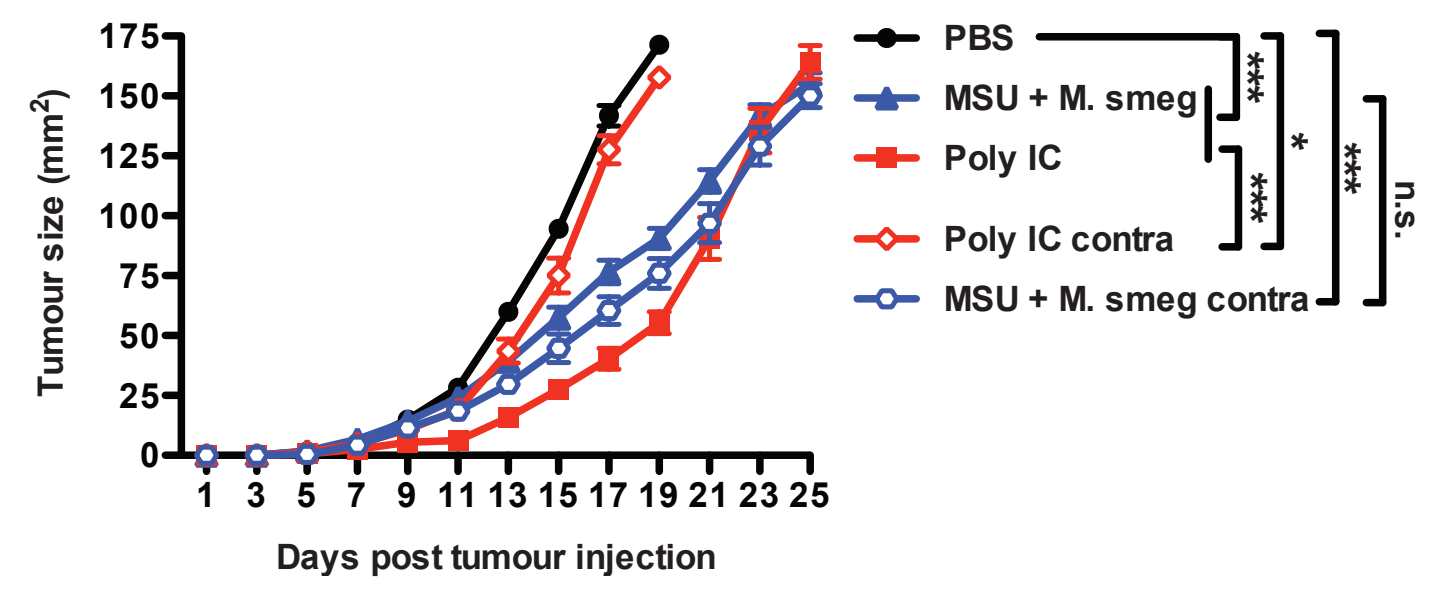

B.

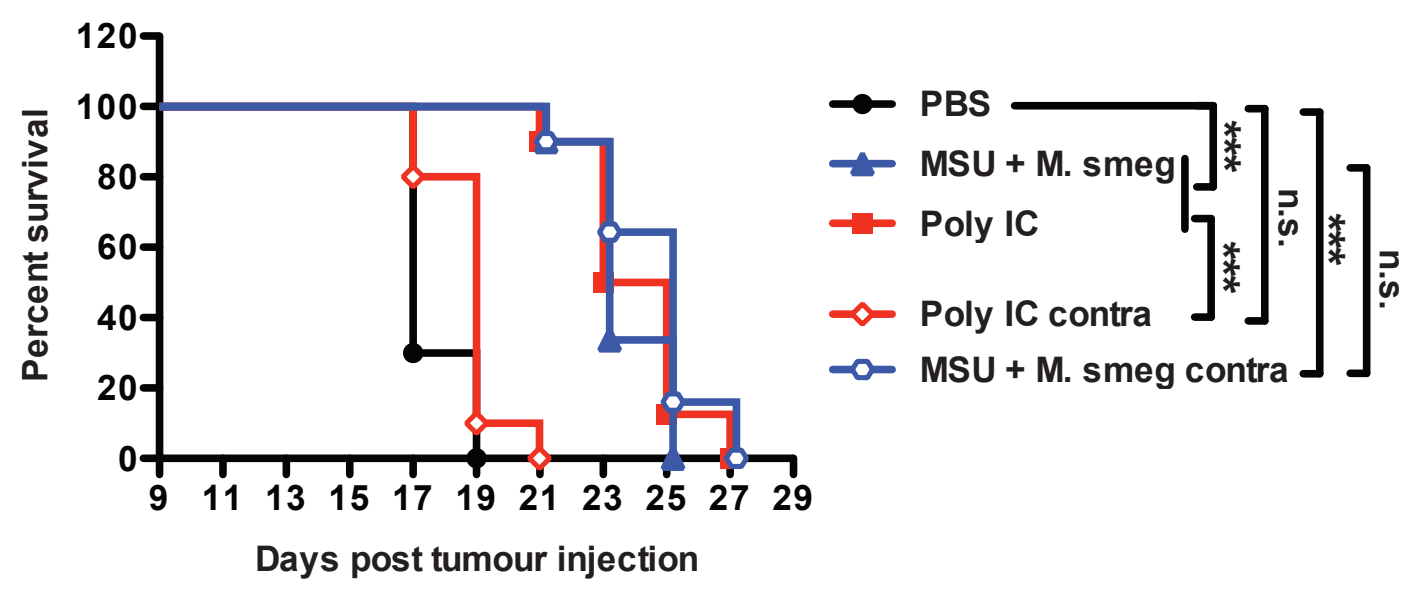

Figure 6.6: Poly IC must be administered at the tumour site while MSU + $M$. smegmatis is effective even if injected into the contralateral flank. C57 mice bearing established B16F1 tumours were treated with Poly IC or MSU + M. smegmatis either around the tumour or s.c. on the contralateral flank (contra) on day 7, 911 and 13. (A) Tumour growth is shown as mean \pm SE. Significant differences between treatment groups according to two-way ANOVA with Bonferroni's post test starting from day 13 are indicated by ${ }^{*} p<0.05$ and ${ }^{* * *} p<0.001$. (B) The survival of the treated mice is depicted. ${ }^{* * *} p<0.001$ as assessed by the Log-Rank-Test with Bonferroni's adjustment for multiple comparisons. n.s. $=$ not significant. Data are pooled from 2 independent experiments with 5 mice per group each. 


\subsubsection{Contralateral MSU + M. smegmatis treatment re- quires adaptive immmunity}

To assess if the anti-tumour effect of contralateral MSU + M. smegmatis treatment required adaptive immunity like the response to peri-tumoral MSU + M. smegmatis administration, tumour growth experiments were conducted in Rag1-/- mice that lack adaptive immune cells.

A.

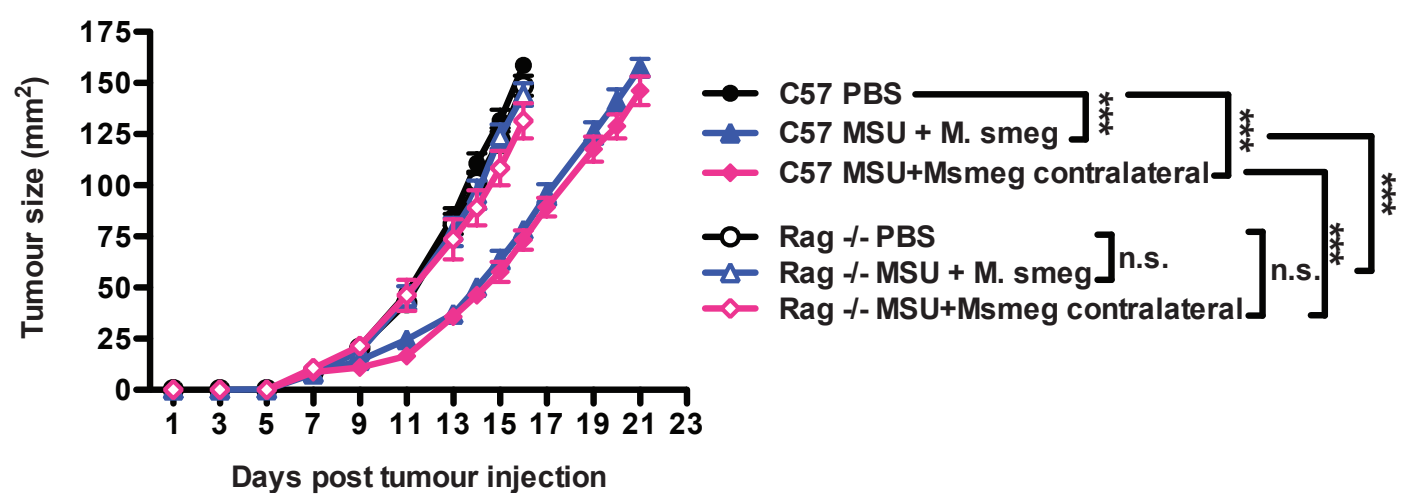

B.

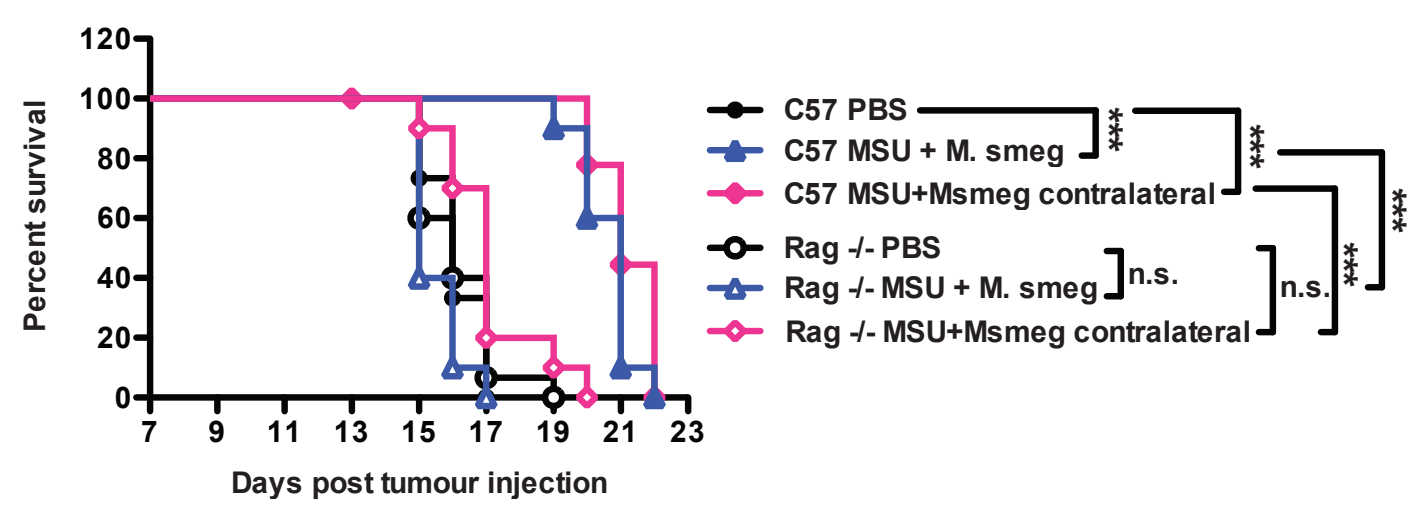

Figure 6.7: MSU + M. smegmatis contralateral treatment requires adaptive immunity. C57 mice or Rag1-/- mice bearing established B16F1 tumours were treated with $\mathrm{MSU}+$ M. smegmatis either around the tumour or s.c. on the contralateral flank on day 7 , 911 and 13. (A) Tumour growth is shown as mean tumour size \pm SE. Significant differences between treatment groups according to two-way ANOVA with Bonferroni's post test starting from day 11 are indicated by ${ }^{* * *} p<0.001$. (B) The survival of the treated mice is graphed. ${ }^{* * *} p<0.001$ as assessed by the Log-Rank-Test with Bonferroni's adjustment for multiple comparisons. n.s. $=$ not significant. Data are pooled from 2 independent experiments with 5 mice per group each. 
In line with the result from the previous experiment, contralateral MSU + M. smegmatis treatment delayed tumour growth just like peri-tumoral MSU + M. smegmatis treatment in C57 wild type mice (Figure 6.7). Furthermore, the adjuvant effect was completely abolished in Rag1-/- mice in both treatment groups (Figure 6.7). These data show that contralateral MSU + M. smegmatis treatment also requires adaptive immune cells to induce its anti-tumour effect and suggests that the two treatments engage similar immune effector mechanisms.

\subsubsection{Differential immune response to contralateral and peri- tumoral MSU + M. smegmatis treatment}

A single administration of contralateral or peri-tumoral MSU + M. smegmatis adjuvant had distinct effects on the tumour immune infiltrate. Contralateral administration did not induce the marked influx of immune cells to the tumour site (Figure 6.8 A). The recruitment of Ly6 $\mathrm{C}^{\text {hi }}$ and Ly6 $\mathrm{C}^{\mathrm{int}}$ monocytes likewise did not occur in response to contralateral MSU + M. smegmatis treatment (Figure 6.8 C \& D). Therefore, the relative percentages of different immune cells in the tumours of mice that had received contralateral MSU $+M$. smegmatis administration more closely resembled those found in saline treated controls than those in peri-tumorally treated tumours (Figure 6.8 B).

The analysis of the LNs draining the tumour site furthermore showed that they were only slightly enlarged after contralateral MSU + M. smegmatis treatment (Figure 6.9 A). Correspondingly, the number of total DC, as well as inflammatory monocyte-derived DCs was not significantly increased in tumour-draining LNs, although there was a trend towards higher numbers of inflammatory monocyte-derived DCs (Figure 6.9 B \& C). However, LNs contralateral to the tumour site but draining the area of contralateral MSU + M. smegmatis administration clearly reacted to the presence of adjuvant becoming enlarged (Figure 6.9 A). The DC populations in the contralateral LNs were not analysed.

To assess the capacity of DCs in LNs draining either the tumour or the adjuvant administration site to induce proliferation of tumour-antigen specific T cells, B16 tumours expressing the model antigen OVA in combination with adoptive transfer of CFSE-labelled, 
A.

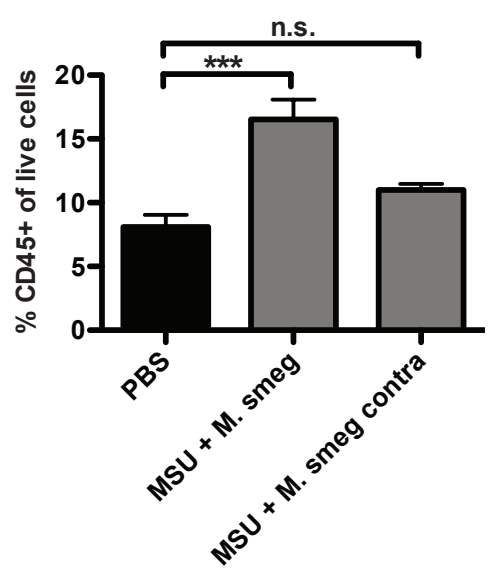

C.

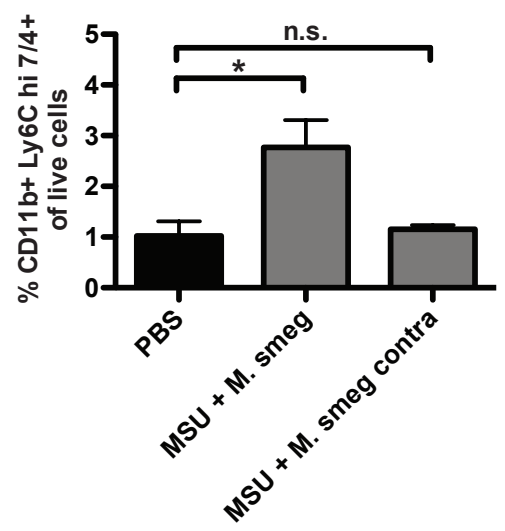

B.

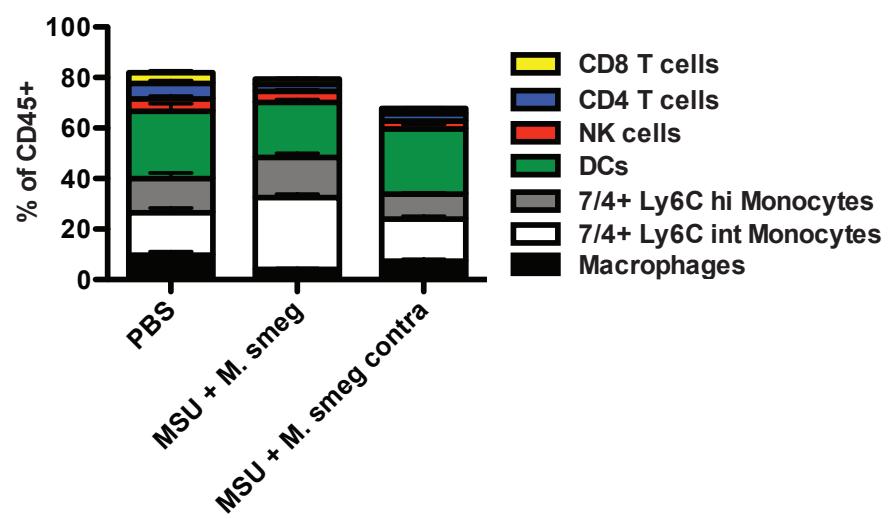

D.

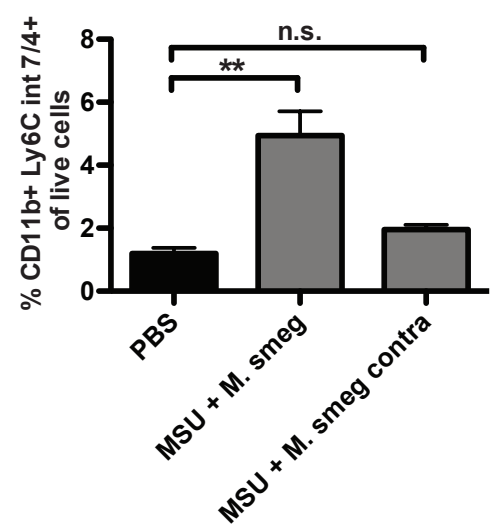

Figure 6.8: A single MSU $+M$. smegmatis contralateral treatment does not affect the immune infiltrate in tumours. C57 mice bearing established B16F1 tumours were injected once either around the tumour or s.c. on the contralateral flank with MSU + M. smegmatis. One day later, immune cells in tumours were analysed by flow cytometry as described in Figure 4.3. (A) The frequency of CD45+ cells in tumours is shown. (B) The percentages of different immune cell types among the total CD45+ immune infiltrate is graphed. (C) \& (D) The frequencies of Ly6C ${ }^{\text {hi }}$ and Ly6C ${ }^{\text {int }}$ monocytes among all live cells in tumours are shown. All data are presented as mean \pm SE. ${ }^{*} p<0.05,{ }^{* *} p<0.01$ and ${ }^{* * *} p<0.001$ as assessed by one-way ANOVA with Tukey's post test; n.s. not significant. Data are from 1 experiment with 5 mice per group.

OVA-specific CD8 T cells (OTI) or CD4 T cells (OTII) were used.

Although contralateral MSU + M. smegmatis administration had not significantly increased DC numbers in tumour-draining LNs, the proliferation of OTI cells in these LNs was enhanced (Figure 6.10 A, left panel). The numbers of divided and total OTI cells in tumourdraining LNs in response to contralateral MSU + M. smegmatis treatment were also higher compared to PBS treated controls, but still considerably lower than in mice treated with peri-tumoral MSU + M. smegmatis (Figure 6.10 A, middle \& right panels). A significant 
A.
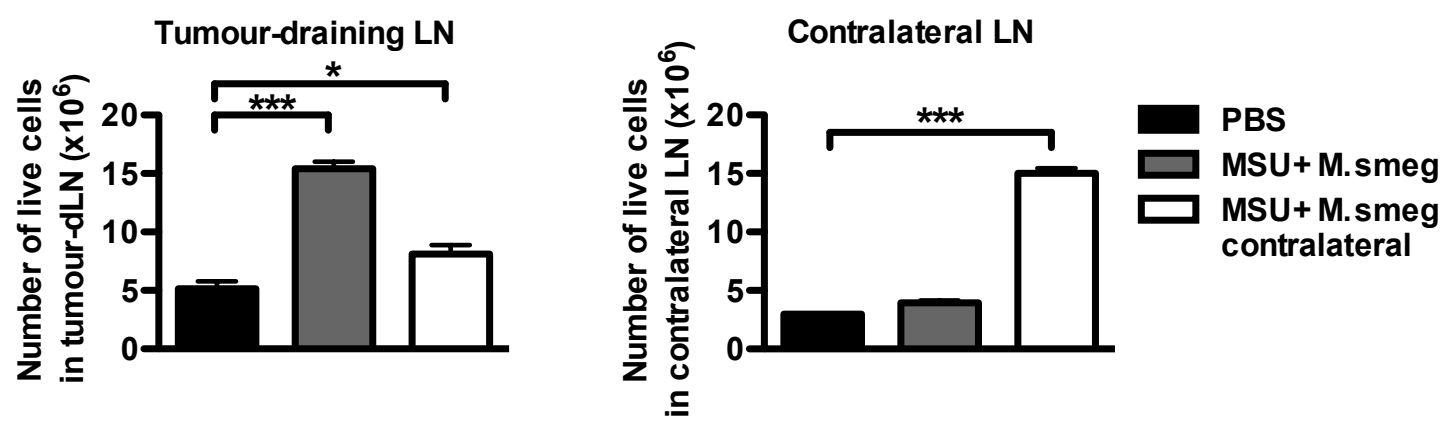

B.

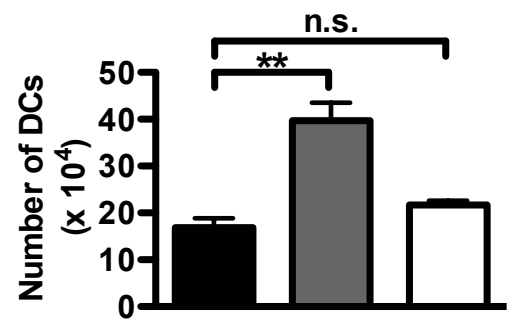

C.

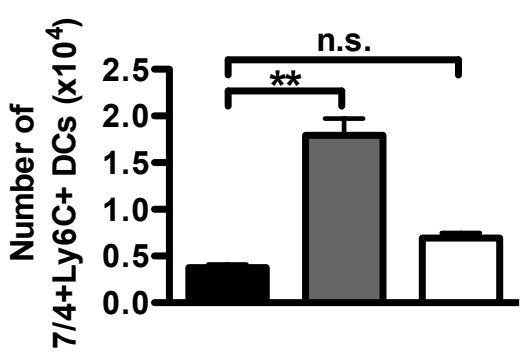

Figure 6.9: A single MSU $+M$. smegmatis contralateral treatment does not affect DCs in tumour-draining LN. Data are from the same experiment described in Figure 6.8. DCs in dLNs were analysed by flow cytometry as described in Figure 4.8. (A) The number of live cells in pooled axillary and inguinal tumour-draining and contralateral LNs is shown. (B) \& (C) The numbers of total DCs and the numbers of inflammatory monocyte-derived DCs $(7 / 4+\mathrm{Ly} 6 \mathrm{C}+)$ in tumour-draining LNs are shown as mean $\pm \mathrm{SE}$. ${ }^{*} p<0.05,{ }^{* *} p<0.01$ and ${ }^{* * *} p<0.001$ as assessed by one-way ANOVA with Tukey's post test. Data are from 1 experiment with 5 mice per group.

number of total and divided OTI cells was also observed in the contralateral LNs draining the MSU + M. smegmatis administration site (Figure 6.10 A, middle \& right panels).

The percentage of divided OTII cells in tumour-draining LNs was also significantly enhanced by contralateral MSU + M. smegmatis treatment (Figure $6.10 \mathrm{~B}$, left panel), but total numbers and numbers of divided OTII were not elevated in tumour-draining LNs of contralaterally treated compared to PBS injected mice (Figure $6.10 \mathrm{~B}$, middle \& right panels). However, a substantial proportion of OTII cells in contralateral LNs was found to be divided after contralateral MSU + M. smegmatis administration (Figure 6.10 B, left panel). In line with the enlargement of contralateral LNs in response to contralateral MSU + M. smegmatis treatment (Figure 6.9 A, right panel), this translated into significantly higher numbers of total OTII cells and divided OTII cells in these LNs (Figure $6.10 \mathrm{~B}$, middle \& right panels). 
A.

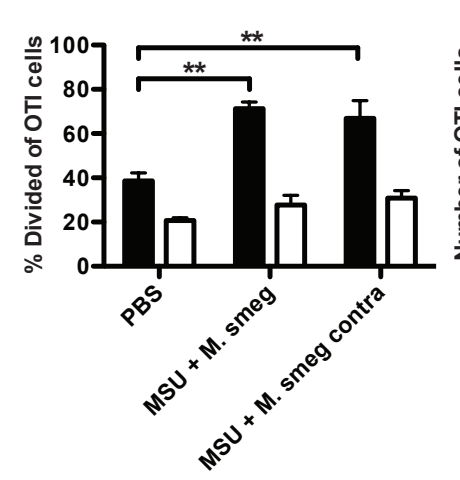

B.

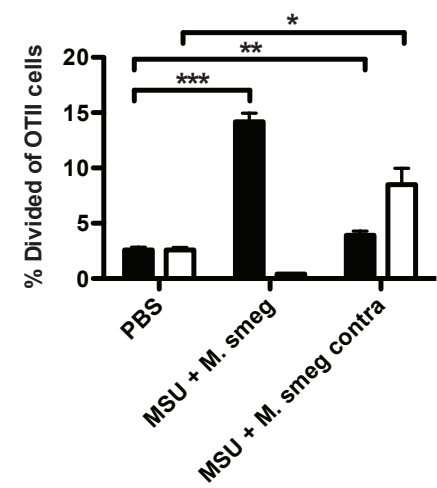

OTI in vivo proliferation
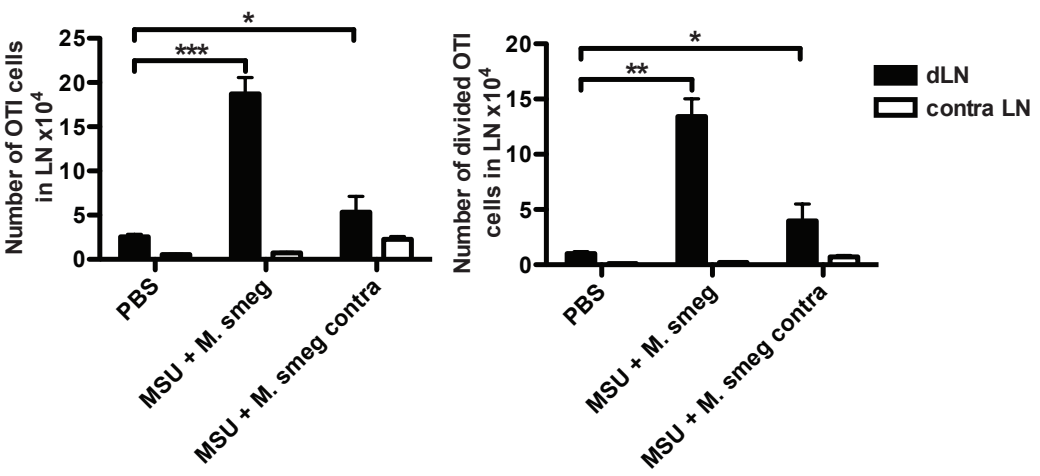

OTII in vivo proliferation

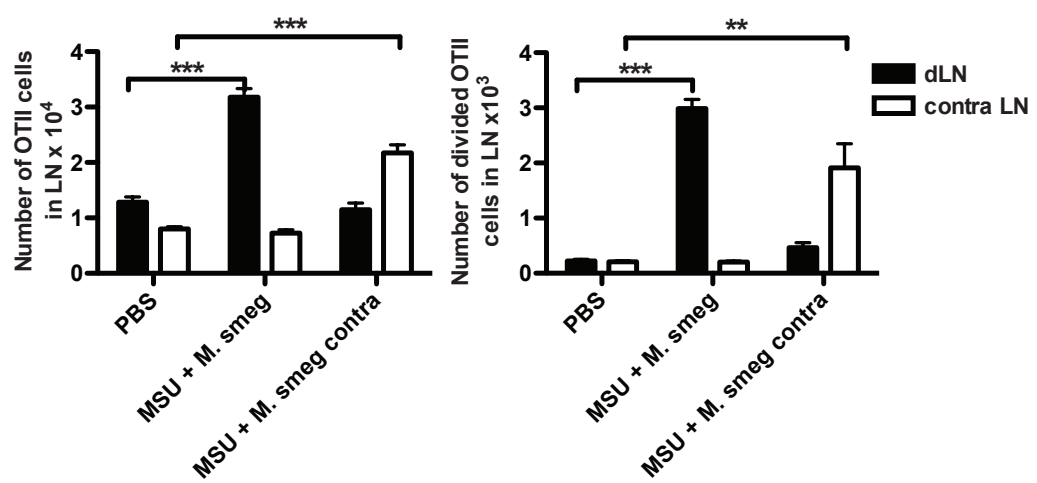

Figure 6.10: MSU + M. smegmatis contralateral treatment induces proliferation of tumour specific CD4 and CD8 T cells. CD4 T cells and CD8 T cells were purified by magnetic sorting from LNs and spleens of OTII or OTI congenic mice, respectively, and labelled with CFSE. OTI cells were $>95 \%$ CD8+V $\alpha 2+\mathrm{V} \beta 5+$ and OTII cells were $>90 \%$ $\mathrm{CD} 4+\mathrm{V} \alpha 2+\mathrm{V} \beta 5+$ as assessed by flow cytometry. Labelled $\mathrm{T}$ cells were then adoptively transferred into C57 mice bearing established B16.OVA tumours (day 8). One day later, mice were given a single MSU + M. smegmatis injection either around the tumour or s.c. on the contralateral flank. (A) OTI proliferation was assessed by CFSE dilution in tumourdraining and contralateral LNs 3 days after adjuvant treatment. The percentage and number of divided OTI cells and the total number of OTI cells is shown. (B) OTII proliferation was assessed 5 days after adjuvant treatment. The percentage and number of divided OTII cells and the total number of OTII cells is graphed. ${ }^{*} p<0.05$ and ${ }^{* * *} p<0.001$ as assessed by one-way ANOVA with Tukey's post test. Data are pooled from 2 independent experiments with 5 mice per group each.

These data indicate that a single contralateral administration of MSU $+M$. smegmatis induces both CD4 and CD8 T cells specific for tumour antigens to proliferate in the tumour-draining LNs. However, a large number of divided CD4 T cells was also observed in contralateral LNs. 
A.

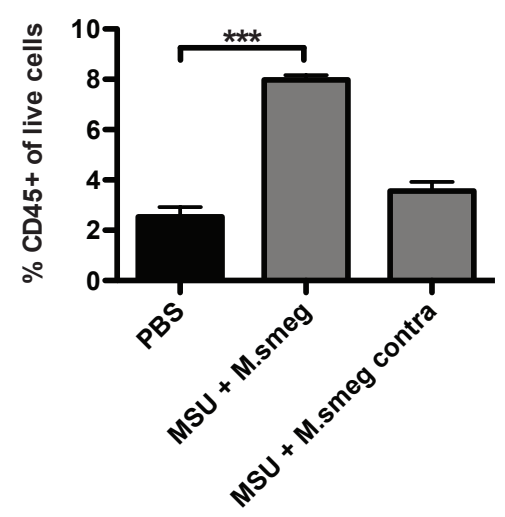

C.

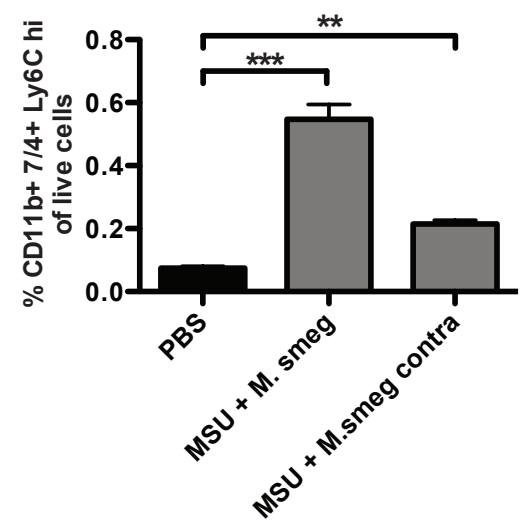

B.

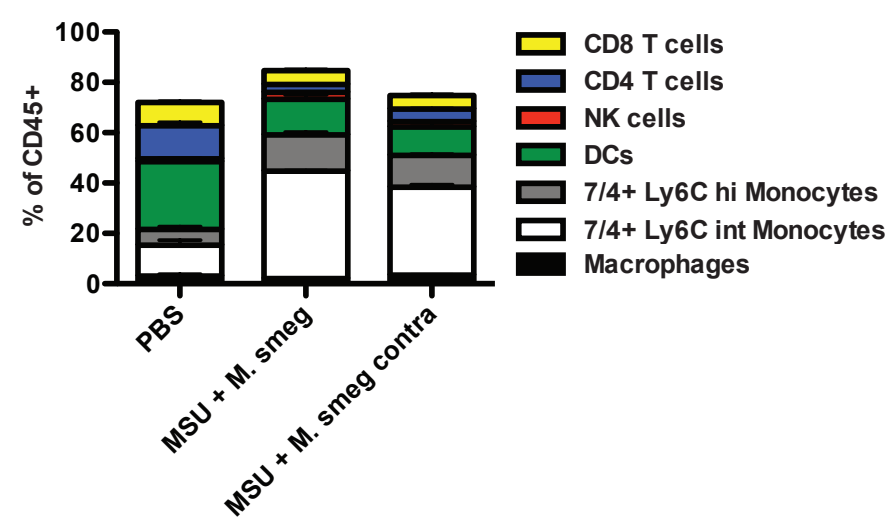

D.

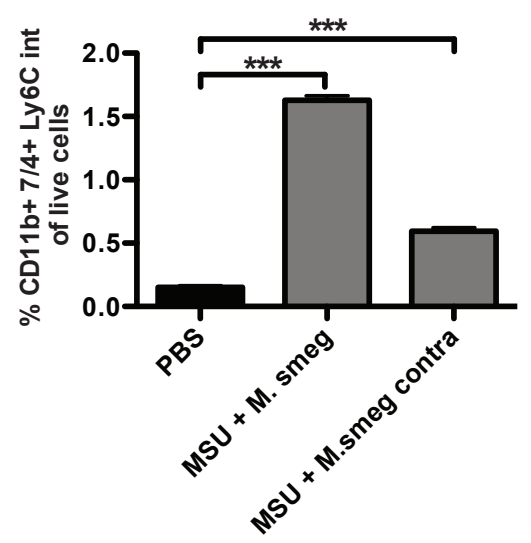

Figure 6.11: Repeated MSU + M. smegmatis contralateral treatment has similar effects on the composition of the immune infiltrate in tumours as peritumoral administration. C57 mice bearing established B16F1 tumours were injected either around the tumour or s.c. on the contralateral flank with MSU + M. smegmatis on days 7, 9, 11 and 13 . One day later, immune cells in tumours were analysed by flow cytometry as described in Figure 4.3. (A) The frequency of CD45+ cells in tumours is shown. (B) The percentages of different immune cell types among the total CD45+ immune infiltrate is graphed. (C) \& (D) The frequencies of Ly6Chi and Ly6C ${ }^{\text {int }}$ monocytes among all live cells in tumours are shown. All data are presented as mean \pm SE. ${ }^{*} p<0.05,{ }^{* *} p<0.01$ and ${ }^{* * *} p<0.001$ as assessed by one-way ANOVA with Tukey's post test; n.s. not significant. Data are combined from 2 experiments with 5 mice per group each.

The effect of four treatments with MSU + M. smegmatis, either around the tumour or on the contralateral flank, on the immune infiltrate in tumours was analysed by flow cytometry. Contralateral administration of MSU + M. smegmatis induced only a slight, non-significant increase in the overall infiltration of immune cells into the tumours (Figure 6.11 A). The recruitment of Ly6C $\mathrm{C}^{\mathrm{hi}}$ and $\mathrm{Ly} 6 \mathrm{C}^{\mathrm{int}}$ monocytes was observed both after peri-tumoral and after contralateral treatment, albeit to a lesser extent in the contralaterally treated group 
(Figure 6.11 C \& D). After multiple adjuvant administrations, the overall composition of the immune infiltrate in mice that received contralateral MSU $+M$. smegmatis resembled more closely that of peri-tumorally treated mice than that of saline treated controls (Figure 6.11 B).

Overall these results show that contralateral and peri-tumoral administration of MSU + M. smegmatis differ in their early effects on tumour infiltrate and DCs in tumour-draining LNs. However, both application routes induce the proliferation of tumour-specific $\mathrm{T}$ cells and lead to similar infiltration of monocytes in tumours at later time points.

\subsubsection{The capacity of adjuvants delay primary tumour growth is similar in melanoma, thymoma and breast carcinoma models}

To investigate whether the use of Poly IC and MSU + M. smegmatis adjuvants was also applicable to other solid tumours in mice, the impact of adjuvant treatment on the growth of the subcutaneous murine thymoma E.G7-OVA in C57 mice, and of the orthotopic breast carcinoma 4T1 in BALB/c mice, was evaluated. To this end, established tumours were treated every second day by peri-tumoral adjuvant injection as for the B16F1 model. E.G7-OVA tumours were treated 6-times, as an increased number of administrations had appeared to be beneficial in the B16F1 model (Figure 6.1).

In the E.G7-OVA model, Poly IC and MSU + M. smegmatis administration resulted in a significant delay of tumour growth and prolonged survival of tumour bearing mice (Figure 6.12). This was in line with the results obtained in the murine B16F1 melanoma (Figure 4.2). Furthermore, LPS had no significant effect on tumour growth or survival in either model (Figure 6.12 and Figure 4.2). However, treatments with M. smegmatis or MSU alone were effective in delaying tumour growth and increasing survival in the E.G7-OVA thymoma bearing mice (Figure 6.12). This contrasts with their lack of anti-tumour activity against B16F1 melanomas. The combination of MSU with M. smegmatis was still superior in delaying tumour growth and especially in prolonging survival compared to the individual 
A.

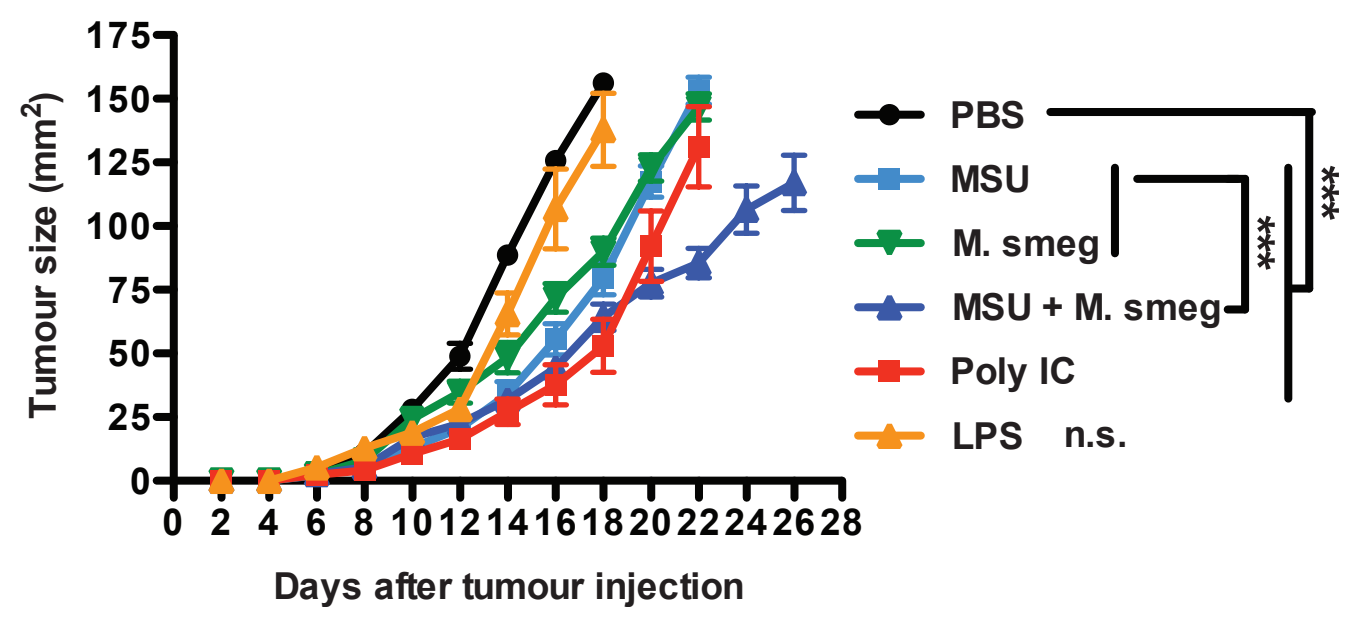

B.

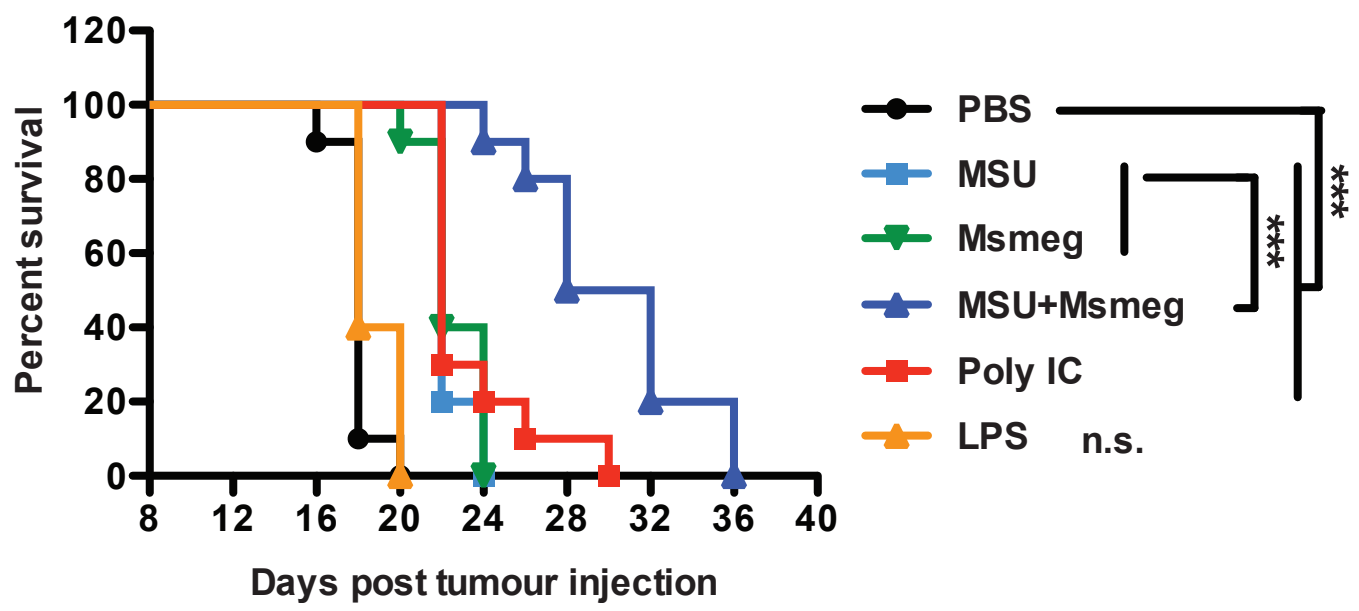

Figure 6.12: Adjuvant treatment in a thymoma model is effective at delaying tumour growth. C57 mice bearing established s.c. E.G7-OVA tumours were injected around the tumour with the indicated adjuvants on day $6,8,10,12,14$ and 16 . Tumour growth was measured with callipers and is graphed as mean \pm SE in (A). Significant differences between treatment groups as assessed by two-way ANOVA with Bonferroni's post test starting from day 10 are indicated by ${ }^{* * *} p<0.001$. (B) Survival of the adjuvant treated mice is shown. ${ }^{* * *} p<0.001$ as assessed by the Log-Rank-Test with Bonferroni's adjustment for multiple comparisons. Data are pooled from 2 independent experiments with 5 mice per group. 
adjuvants (Figure 6.12).

To extend this study to an orthotopic tumour model and investigate the adjuvant effects in a different mouse strain, BALB/c mice were injected with $4 \mathrm{~T} 1$ breast carcinoma cells into the mammary fat pad. After tumours became palpable, they were treated with peritumoral adjuvant injections. As the mammary fat pad can only tolerate lower injection volumes without tissue disruption, adjuvants were twice as concentrated in half the volume, and were administered only 4-times.

As observed in the melanoma and thymoma models, Poly IC and MSU + M. smegmatis showed potent anti-tumour activity, significantly reducing tumour growth and increasing survival by more than 10 days (Figure 6.13). Futhermore, LPS and M. smegmatis were ineffective (Figure 6.13). Administration of MSU alone delayed growth of the 4T1 tumours, but did not have a significant impact on survival (Figure 6.13).

In summary, the effect of the peri-tumoral adjuvant treatments on tumour growth was similar in the melanoma, thymoma and breast carcinoma models. Poly IC and MSU + M. smegmatis consistently induced the most pronounced delay in tumour growth and significantly prolonged the survival of animals in all tumour models. LPS treatment failed to elicit any significant anti-tumour effect in all models. Separate treatments with M. smegmatis or MSU showed some activity against the E.G7-OVA thymoma, while only MSU had a slight effect on $4 \mathrm{~T} 1$ tumour growth and neither of these treatments was effective against the B16F1 melanoma. 
A.

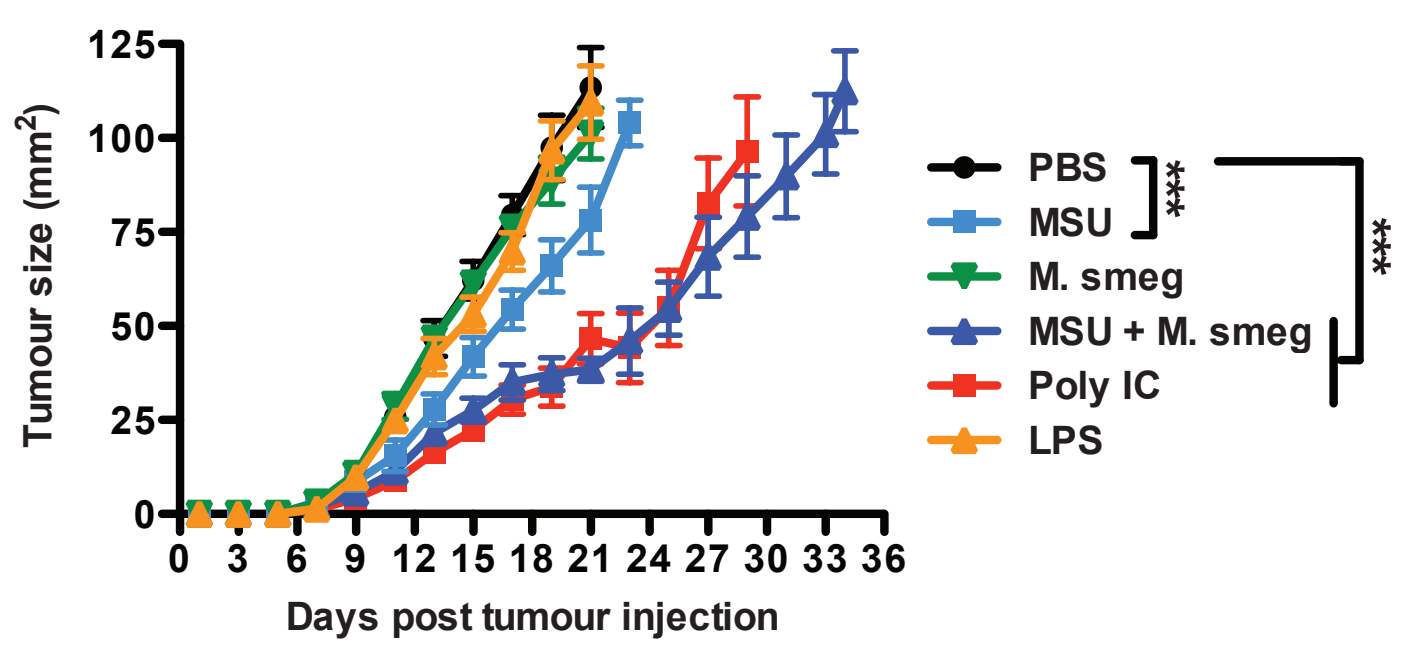

B.

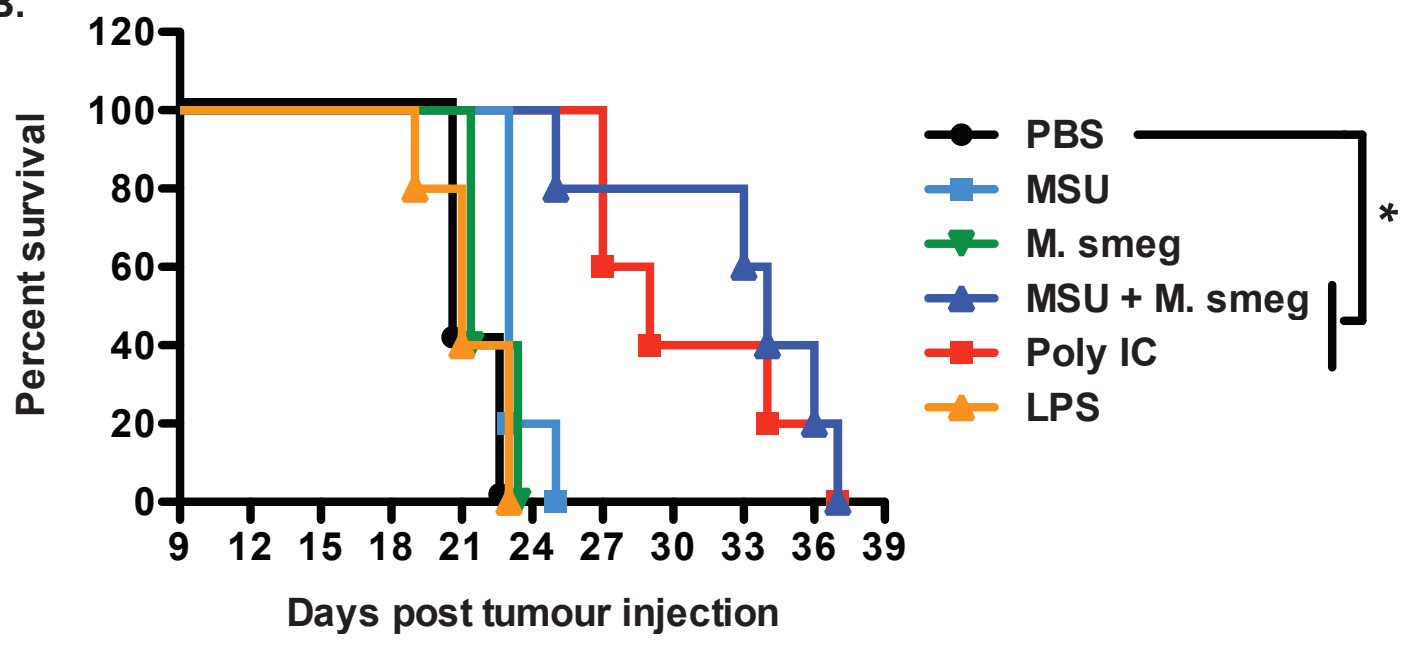

Figure 6.13: Treatments with selected adjuvants delay tumour growth in an orthotopic breast carcinoma model similar to their effect against melanoma. $\mathrm{BALB} / \mathrm{c}$ mice were injected with $4 \mathrm{~T} 1$ breast carcinoma cells into the mammary fat pad and tumours were left to establish a palpable mass. On day 7, 9, 11 and 13 the indicated adjuvants were administered peri-tumorally. (A) Tumour growth as measured with callipers is shown as mean \pm SE. Significant differences between treatment groups as assessed by twoway ANOVA with Bonferroni's post test starting from day 11 are indicated by ${ }^{* * *} p<0.001$. (B) Survival of the adjuvant treated, tumour-bearing mice is graphed. ${ }^{*} p<0.05$ as assessed by the Log-Rank-Test with Bonferroni's adjustment for multiple comparisons. Data are pooled from 1 experiment with 5 mice per group. 


\subsubsection{Peri-tumoral Poly IC and MSU + M. smegmatis treat- ments reduce metastases}

Both Poly IC and MSU + M. smegmatis cause some inflammation as evidenced by the induction of pro-inflammatory cytokines, recruitment of monocytes and appearance of monocytederived inflammatory DCs at the tumour site. As inflammation has been shown to enhance metastasis formation [543], it was important to determine the effect of these adjuvants on the metastatic load.

To investigate the effect of adjuvant treatment on spontaneous metastases from an orthotopic tumour, the 4T1 breast carcinoma model was used. Primary 4T1 tumours spontaneously metastasize to lymph nodes, brain, bone, liver and lung [544, 545]. BALB/c mice bearing established 4T1 tumours in their mammary fat pads were treated by peri-tumoral administration of Poly IC or MSU + M. smegmatis. When the tumours of the saline treated control animals reached the maximal ethical tumour size, mice in all treatment groups were sacrificed and their lungs were analysed for the presence of metastases.

Primary tumour growth was reduced in mice that received Poly IC or MSU + M. smegmatis treatment (Figure 6.14 A). The total counts of metastatic lung colonies in PBS treated control mice were quite variable and ranged from 2,000 to 20,000 per mouse (Figure 6.14 B). The total number of metastases was reduced by both Poly IC and MSU $+M$. smegmatis treatment (Figure 6.14 B). To account for any differences in total lung size, metastases were also expressed as counts per lung weight, showing a similar reduction in response to adjuvant treatment as total metastases counts (Figure 6.14 C). To establish whether the reduced size of the primary tumours in Poly IC or MSU $+M$. smegmatis treated groups alone led to the lower lung metastatic load observed, the correlation between tumour weight and total lung colony counts was analysed in saline-treated controls. Mice with very similar tumour sizes showed a large spread of metastatic load (Figure 6.14 A \& B) and no correlation between tumour size and metastasis counts was evident $\left(R^{2}=0.07\right)$. In addition, Pulaski et al. show that in the $4 \mathrm{~T} 1$ model, lung metastases formation is a very early even that already occurs in tumours of $2 \mathrm{~mm}$ to $3 \mathrm{~mm}$ diameter [546]. This suggests 
A.

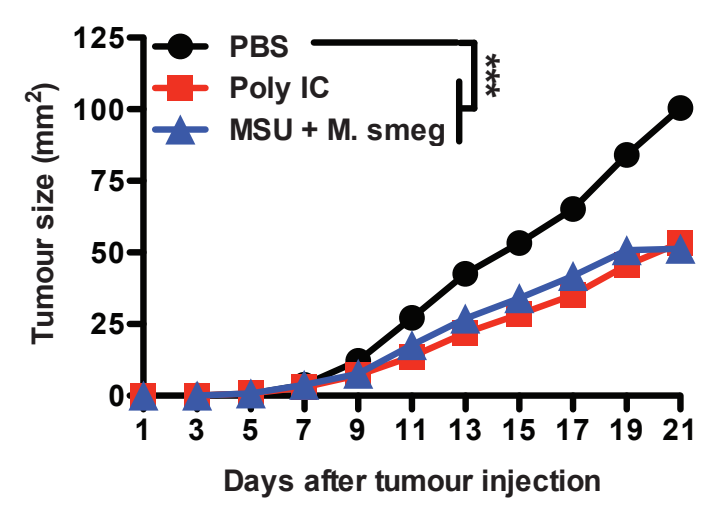

C.

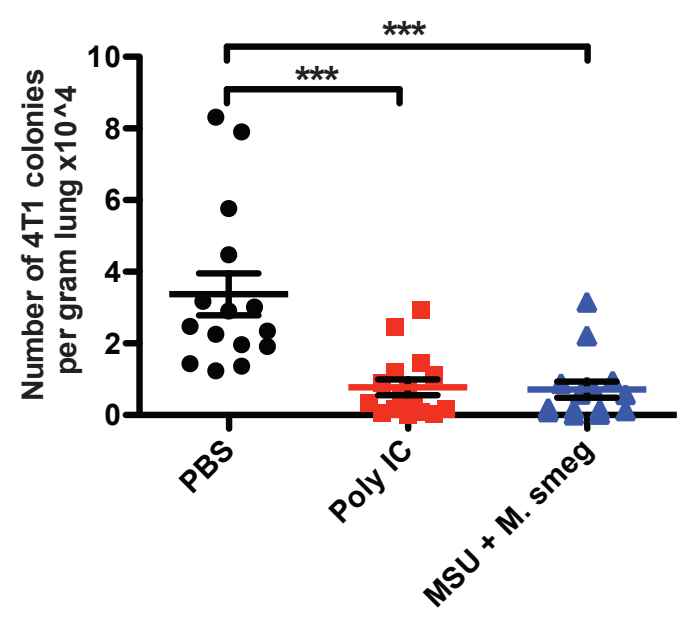

B.

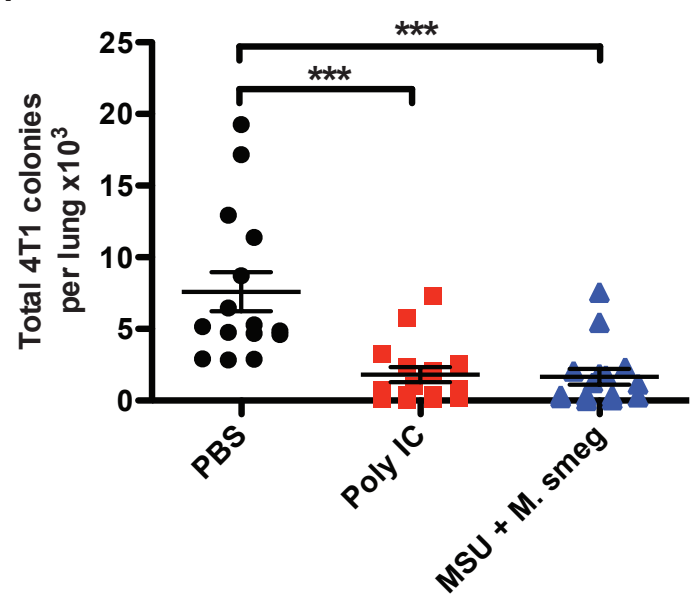

D.

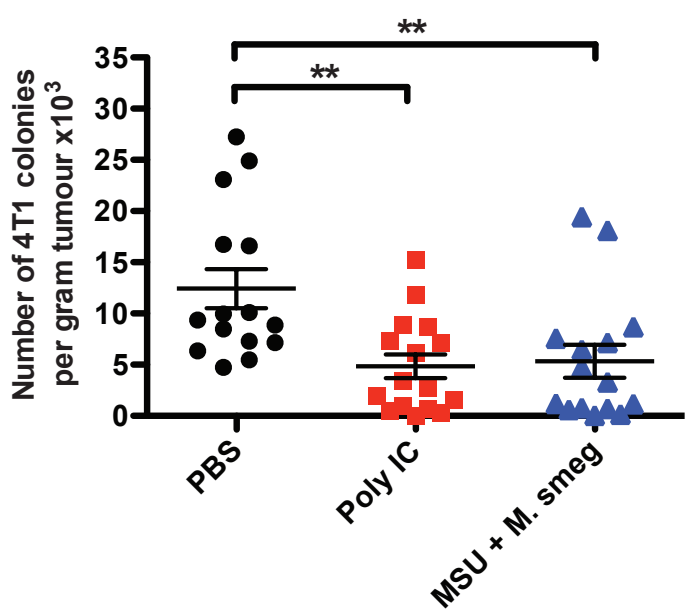

Figure 6.14: Treatment with Poly IC or MSU + M. smegmatisreduces metastases. BALB/c mice bearing established $4 \mathrm{~T} 1$ breast carcinomas in the mammary fat pad were treated by peri-tumoral injection of the indicated adjuvants on day 7, 9, 11 and 13. Tumour size was measured with callipers and mice were sacrificed when saline treated controls developed large tumours. Lung metastases were analysed by culturing lung cell suspensions for two weeks in the presence of 6-thioguanine. Subsequently, 4T1 tumour cell colonies were stained and counted. (A) Tumour growth is shown as mean \pm SE. Significant differences between treatment groups as assessed by two-way ANOVA with Bonferroni's post test starting from day 11 are indicated by $* * * p<0.001$. (B-D) Metastatic lung colonies are shown as total counts (B), counts per lung weight (C) or counts per tumour weight (D). Individual values and means $\pm \mathrm{SE}$ are shown for each group. ${ }^{* *} p<0.01$ and ${ }^{* * *} p<0.001$ as assessed by the Kruskal-Wallis test with Dunn's post test. Data are pooled from 2 independent experiments with 6 and 8 mice per group, respectively.

that lung metastases form relatively independently of primary tumour size. Furthermore, even when the number of metastases per tumour weight were calculated, Poly IC and MSU + M. smegmatis treated mice still have significantly lower metastasis counts compared to saline treated controls (Figure 6.14 D). 
Together, these data indicate that Poly IC and MSU $+M$. smegmatis do not increase the formation of metastases. In contrast, both adjuvant treatments appeared to reduce the metastatic load in lungs even when the smaller size of primary tumours in these groups was taken into account.

\subsection{Discussion}

The aim of the experiments described in this chapter was to optimise the treatment schedule and route for adjuvant administration. In addition, it was evaluated whether live M. smegmatis could be replaced with dead M. smegmatis in the MSU combination treatment to improve safety. The study was also extended to other tumour models, including the assessment of the effect of adjuvant treatment on metastases.

\section{Requirement for several adjuvant treatments for tumour growth delay}

At least two administrations of Poly IC, and 4 administrations of MSU + M. smegmatis, were required to delay growth of the B16F1 melanoma and increasing the number of Poly IC and MSU + M. smegmatis treatments improved the anti-tumour effect. When more than 4 doses were given, there was still a trend towards further benefit, however this was not statistically significant. These results are in line with studies on CpG and Poly IC that reported the requirement of repeated administration for adjuvants to exhibit significant anti-tumour activity [11, 12, 403]. Furthermore, this result suggests that multiple doses of Poly IC and MSU + M. smegmatis boost the anti-tumour immune response. Moreover, they do not appear to induce feedback regulation that inhibits their activity.

The kinetics of the anti-tumour effect of Poly IC and MSU + M. smegmatis differed. Poly IC inhibited tumour progression directly after the first application and tumours started to grow out as soon as treatment was stopped. In MSU $+M$. smegmatis treated mice, an anti-tumour effect only became apparent several days after the first adjuvant administration, but tumours still grew at a slower rate even after treatment was ceased. This may be due to 
different retention and availability of these adjuvants. Poly IC is a small, soluble molecule. This may allow Poly IC to be rapidly taken up and bind to its intracellular sensors TLR3 and RIG-I, thus activating downstream responses quickly after administration. In contrast, MSU and M. smegmatis are both comparably large, particulate adjuvants, and it may take longer for them to be taken up and processed to expose their TLR and NOD ligands. On the other hand, these adjuvants both accumulated at the treatment site. This may lead to small tumours with MSU and M. smegmatis build-up appearing bigger than they actually are when measured with callipers. The persistence of MSU and M. smegmatis at the tumour site may also provide a more sustained stimulus that keeps activating immune cells locally even after treatment has stopped. Currie et al. also observed that Poly IC only showed anti-tumour activity during the adjuvant treatment schedule and that tumours grew out as soon as treatment stopped [12]. In addition, Yang et al. have shown that persistent TLR signals were required for reversal of Treg mediated CD8 T cell tolerance, and for anti-tumour immunity in a lymphoma model [528]. These findings all indicate that repeated stimulation with PAMPs or DAMPs may be required to break tolerance and promote the anti-tumour response.

The different treatment schedules tested in this study did not differ significantly in their ability to slow B16F1 melanoma growth. While MSU + M. smegmatis did not appear to be more effective when treatment was started earlier or given more often, smaller tumour size may have been masked by accumulation of the adjuvant around the B16F1 tumours. With Poly IC administration, a trend was observed that earlier start of the adjuvant treatment may improve its anti-tumour effect. This is in line with previously published work in a mesothelioma model [12]. Other immunotherapy approaches using adoptive transfer of tumour-specific $\mathrm{T}$ cells, also reported a greater anti-tumour effect when the $\mathrm{T}$ cells were transferred into tumour-bearing mice early [547]. This highlights that early intervention appears generally to be preferable to achieve the best anti-tumour response. 


\section{Comparable anti-tumour activity of dead and live $M$. smeg- matis + MSU}

In experiments in previous chapters, live M. smegmatis was used in combination with MSU to induce anti-tumour immunity. The live mycobacteria BCG are currently used in patients with superficial bladder cancer with considerable success $[10,548]$. However, about $3 \%$ of bladder cancer patients treated with BCG developed systemic mycobacterial infection with granuloma formation [533]. This raises safety concerns for the use of live M. smegmatis. While M. smegmatis is not derived from a pathogen and may therefore pose a lower risk for infection than BCG, it may still be desirable to use heat-killed preparations instead of live bacteria. Therefore, the use of dead M. smegmatis + MSU for the treatment of murine B16F1 melanoma was investigated. Tumour growth delay and survival benefit were similar irrespective of the viability of $M$. smegmatis. In addition, the early recruitment of immune cells to the tumour, and in particular of monocytes, as well as the accumulation of inflammatory monocyte-derived DCs in dLNs were comparable between dead and live M. smegmatis + MSU. A previous study by Martino et al. has also shown that human monocytes were activated in a similar manner by live and heat-killed M. smegmatis [430]. Furthermore, M. smegmatis provides multiple PAMPs, including TLRs, Dectin-1 ligands and NOD2 ligands, which can activate the immune system [549-551]. Together, this indicates that the immuno-stimulatory properties of M. smegmatis do not depend on its viability and that dead M. smegmatis can be used to replace live M. smegmatis to enhance adjuvant safety.

Other studies have used extracts from mycobacteria to stimulate the anti-tumour immune response $[552,553]$. While it may be possible to purify the components of $M$. smegmatis that act as immuno-stimulators in this study, the use of whole mycobacteria may be superior. Intact mycobacteria present a particulate source of multiple PAMPs at once. Particulate substances are preferentially taken up by DCs and shuttled to the dLNs as opposed to soluble compounds, which are mainly found in neutrophils [554]. Moreover, the immune-stimulatory properties of adjuvants such as alum have been attributed to their ability to form a depot, leading to slow, prolonged release of antigen [555]. Thus, whole M. smegmatis, which was 
observed to persist at the injection site, may provide a sustained activation stimulus that is preferentially detected by DCs. Furthermore, concomitant triggering of multiple PRRs within the same DC by multiple mycobacterial PAMPs may provide synergistic signals and thereby increase DC activation [377].

\section{Effectiveness of contra-lateral treatment with MSU + M. smeg- matis but not Poly IC}

Previous studies on $\mathrm{CpG}$ have shown that administration of adjuvant at the tumour site was superior to i.v., i.p., or distant s.c. injection $[445,556]$. However, in cancer patients, the tumour site is not always accessible for adjuvant treatment. It would therefore be desirable for adjuvants to exhibit anti-tumour activity even when they are applied to a distant site. In this study, Poly IC delayed B16F1 tumour growth when administered peri-tumorally, but not when given on the contralateral flank. Other reports of Poly IC adjuvant treatment have reported activity against a s.c. tumour with intra-tumoral injection [12], as well as i.p. and i.v. administration when high doses were used [557]. This indicates that Poly IC can be effective when given at the tumour site, i.v. or i.p. but not when administered at a distant s.c. location. In contrast to Poly IC, MSU + M. smegmatis treatment showed similar anti-tumour activity both with peri-tumoral and contralateral application routes. It may therefore be feasible to activate the anti-tumour immune response with MSU + M. smegmatis in cases where tumours are not accessible for adjuvant injection.

The pronounced anti-tumour effect of contra-laterally administered MSU + M. smegmatis was unexpected and the underlying activation of immune cells was investigated in more detail. As for peri-tumoral MSU + M. smegmatis administration, contra-lateral treatment required adaptive immune cells to delay tumour growth. However, in contrast to peritumoral MSU + M. smegmatis there was no marked influx of CD45+ immune cells to the tumour site after a single dose of contra-lateral adjuvant injection, and the composition of immune infiltrate at that time point rather resembled the situation in PBS-treated controls. Furthermore, the contra-lateral LNs showed a more pronounced increase in size than the tumour-draining LNs in response to contra-lateral MSU + M. smegmatis treatment. In- 
creased proliferation of tumour-antigen specific CD8 and CD4 T cells was observed both after peri-tumoral and contra-lateral MSU + M. smegmatis treatment. After 4 adjuvant treatments, the percentage of CD45+ immune cells in tumours was still lower in the contralateral compared to the peri-tumoral treatment group, but the composition was similar in both groups, with a significant increase in inflammatory monocytes. This suggests that while the initial response differs depending on the site of adjuvant administration, repeated administrations activate tumour-specific $\mathrm{T}$ cells that are required to control tumour growth in either case.

At this stage, the exact mechanism by which contra-lateral MSU + M. smegmatis treatment activates tumour-specific T cells is not clear. As the treatment-draining LNs show a more profound increase in size than the tumour-draining LNs, MSU + M. smegmatis appears to induce some local, tumour-independent immune activation. However, CD8 T cells proliferation specific for tumour-model-antigen OVA was mainly observed in tumour-draining LNs even when MSU + M. smegmatis was given on the contra-lateral flank. Divided OVAspecific CD4 T cells (OTII) were detected both in the tumour-draining and the contra-lateral LNs. One possibility would be that M. smegmatis cross-reacts with the CD4 T cell epitope of OVA and that this antigen induces OTII cell proliferation. This appears unlikely, as the same treatment effect was observed in B16F1 tumours that do not express OVA. However, tumour-specific CD4 T cell proliferation could not be assessed in this situation and therefore the possibility of antigenic mimicry between M. smegmatis and OVA cannot be excluded. As the percentage and number of divided, OTII cells was also higher in tumour-draining LNs of mice that received contra-lateral MSU + M. smegmatis compared to saline controls, OTII T cells may have proliferated in tumour-draining LNs and subsequently been attracted to the inflamed treatment-draining LNs. This effect may be more profound with OTII compared to OTI T cells, as they were assessed 2 days later, allowing for a longer period of egress from the LN where proliferation had occurred. If this scenario were true, it would suggest that MSU + M. smegmatis systemically activates the immune system to favour the anti-tumour response. MSU + M. smegmatis increased the serum levels of IL-12, IFN $\gamma$, IL-1 $\beta$ and IL-6, as described in Chapter 4. A systemic induction of pro-inflammatory and Th1 cytokines may be a potential mechanism by which MSU + M. smegmatis could 
promote the induction of anti-tumour immunity even at distant sites.

\section{Effectiveness of MSU + M. smegmatis and Poly IC in other tumour models}

Adjuvant treatment was also effective in a s.c. model of murine thymoma and an orthotopic model breast carcinoma model. In both of these tumour models, as well as in the murine melanoma model, Poly IC and $\mathrm{MSU}+M$. smegmatis induced the most profound tumour growth delay and the best survival benefit compared to other adjuvants. LPS was not effective against any of the studied tumours. The response to M. smegmatis or MSU administration was more varied. Neither adjuvant delayed tumour growth in the B16F1 model (as described in Chapter 4), whereas both adjuvants moderately delayed the growth of the E.G7-OVA thymoma. MSU also slightly slowed the growth of $4 \mathrm{~T} 1$ breast carcinomas but failed to increase survival of $4 \mathrm{~T} 1$ bearing mice. These results suggest that effective adjuvants activate the anti-tumour response in a range of models and therefore may be applicable to a broad range of different solid tumours. According to the literature, CpG similarly activates anti-tumour immunity in many different tumour models [11, 403, 504]. While the ensuing immune response after CpG treatment may be similar, the relative importance of the activation of innate and adaptive effector cells appears to differ depending on the studied tumour model, as differential requirements for either only CD8 T cells [502], only NK cells [503] or both CD8 T cells and NK cells $[11,504]$ have been reported.

Inherent properties of the different tumour models may also account for the observed anti-tumour activity of M. smegmatis treatment and MSU administration against the E.G7OVA tumours, whereas these adjuvants were not effective in the melanoma model. In line with the results in this study, E.G7-OVA has previously been reported to respond to adjuvant treatment with M. smegmatis [458] and MSU [361]. E.G7-OVA appears to be more immunogenic than the B16.OVA melanoma, as E.G7-OVA tumour cells constitutively secrete high amounts of the foreign OVA protein and also express high levels of MHC I [406], compared to low levels of OVA and MHC I expression by B16.OVA tumour cells [558]. Furthermore, 100-fold lower numbers of adoptively transferred OTI T cells were required 
to prevent tumour growth of s.c. injected E.G7-OVA compared to B16.OVA tumour cells $[385,559]$. The ability of $M$. smegmatis alone to induce an anti-tumour response in the E.G7-OVA model indicates that M. smegmatis can activate anti-tumour immunity. However, in the more aggressive B16 melanoma model, only the combination of $M$. smegmatis and MSU was effective. Together with the later accumulation of inflammatory monocytederived DCs in the dLNs of mice treated with M. smegmatis alone compared to animals that received MSU $+M$. smegmatis, these results suggest that MSU somehow accelerates the immune-stimulatory effects of M. smegmatis.

\section{Reduction of metastatic load with MSU + M. smegmatis and Poly IC treatment}

The 4T1 breast carcinoma model also enabled the assessment of spontaneous lung metastases from the primary tumour. Both Poly IC and MSU + M. smegmatis reduced the metastatic load, even when the readout was adjusted for the smaller tumour size in the adjuvant treated mice. Due to constraints in mouse availability, a direct comparison of metastases in saline and adjuvant treated tumours left to develop to the same size could not be carried out. These experiments would help clarify whether Poly IC and MSU + M. smegmatis can reduce the metastatic burden irrespective of primary tumour size.

Previous reports also showed a role for adjuvant treatment in preventing lung metastases. However, these studies used lung colonies after i.v. injection of tumour cells as an experimental model of metastasis. While these experiments do not model the egress of tumour cells from the primary site, they model the seeding of metastatic cells in distant tissues. Using such an i.v. model of metastases, Kunikata et al. have shown that CpG administration at a s.c. tumour site can inhibit lung colonisation by identical tumour cells [403]. This protection could be transmitted to naïve recipients when both CD4 and CD8 T cells were adoptively transferred. In a similar approach, Lee et al. found fewer lung colonies of a mammary tumour cell line when Poly IC was injected i.p. repeatedly over a period of several weeks [560]. The elimination of tumour cells in response to Poly IC was at least partially NK cell dependent. These studies highlight that adjuvants can inhibit seeding 
of metastatic cells at distant sites and that this control may involve both NK cells and T cells. As Poly IC and MSU + M. smegmatis were found to activate both T cells and NK cells in this study, both effector populations may be involved in the observed reduction of pulmonary metastases.

A more recent report using an orthotopic model of breast carcinoma also showed that CpG administration at the primary breast tumour site could prevent new formation of brain metastases and slow progression of very small implanted brain tumours [542]. However, CpG treatment did not alter the growth of more established brain tumours in this model, despite $\mathrm{T}$ cell infiltration. In this study, a reduction of metastatic load was found in response to treatment of established primary tumours with Poly IC and MSU + M. smegmatis. It is likely that some metastases were already present at the start of the adjuvant treatment, as metastatic spread of the breast carcinoma cells occurs already when tumours are $2 \mathrm{~mm}$ to $3 \mathrm{~mm}$ in diameter [546]. A number of mice that received Poly IC or MSU + M. smegmatis treatment had no detectable metastatic lung colonies three weeks after tumour inoculation. This suggests that adjuvant treatment with Poly IC and MSU + M. smegmatis may have prevented further formation of metastases, and possibly also reduced micrometastases already established in the lung at the start of adjuvant treatment. Enumeration of the lung colonies before the start of adjuvant treatment would help clarify whether Poly IC and MSU + M. smegmatis can indeed reduce already established metastases. While further experiments are required to investigate the precise mechanism of action, the reduction of the metastatic load in Poly IC and MSU + M. smegmatis treated mice suggests that these adjuvants may be beneficial for use both in primary and metastatic malignancies.

\section{Conclusions}

Increasing the number of Poly IC and MSU + M. smegmatis administrations improved tumour growth delay. However, after 4-6 doses the treatment effect appeared to plateau and did not significantly improve further. Altering the treatment schedule to start adjuvant administration earlier, or give adjuvant injections daily instead of every second day, did not significantly alter tumour growth compared to the original schedule of 4 treatments 
every other day. A trend towards better treatment effect with earlier administration was observed. Overall these results suggest that repeated adjuvant administration improves the anti-tumour effect and that early treatment start may be beneficial.

Heat-killed M. smegmatis + MSU had the same effect on tumour growth, survival, early immune infiltration into the tumour and DCs in dLNs as live M. smegmatis + MSU. This indicates that active infection of cells by $M$. smegmatis or production of compounds by viable bacteria is not necessary for its immuno-stimulatory activity. Therefore, the safer dead mycobacterial preparation may be used to develop the MSU + M. smegmatis adjuvant further for an application in the clinic.

Contra-lateral administration of Poly IC was ineffective, whereas contra-lateral treatment with MSU + M. smegmatis delayed tumour growth similar to peri-tumoral administration. This appeared to be likely due to systemic activation of the immune response. Tumourantigen specific $\mathrm{T}$ cell proliferation was observed and the anti-tumour activity was dependent on adaptive immune cells. These findings suggest that MSU + M. smegmatis may be a suitable adjuvant in situations where tumours are not accessible for direct adjuvant treatment.

Adjuvant treatment was also found to be effective in a murine thymoma model, as well as an orthotopic breast carcinoma model, in addition to the murine melanoma model investigated in detail throughout this study. Poly IC and MSU + M. smegmatis were the most effective adjuvants in delaying tumour growth and prolonging survival in all of these models. In the breast carcinoma model, spontaneous metastases to the lung were also reduced by these adjuvants.

Collectively these findings show that Poly IC and MSU $+M$. smegmatis are promising adjuvants that may be broadly applicable against a range of solid tumours and even in metastatic disease. 

Chapter 7

\section{General discussion}


The aim of this thesis was to investigate the use of natural adjuvants to stimulate the anti-tumour immune response. All tested adjuvants could activate BMDCs in vitro. Murine melanomas were found to be infiltrated by DCs, which were capable to respond to adjuvant stimulation ex vivo. When the different adjuvants were tested for their ability to affect tumour growth in vivo, peri-tumoral administration of CpG, Poly IC and MSU + M. smegmatis delayed tumour growth. In contrast, LPS, M. smegmatis, BCG, MSU and MSU + BCG did not alter tumour growth. We investigated the effects of the successful adjuvants Poly IC and MSU + M. smegmatis on the immune cells in the tumour and the dLN in more detail and compared them to the ineffective treatments with individually administered MSU or M. smegmatis, as well as LPS. A working model of how these adjuvants affect immune cells is shown in Figure 7.1.

\subsection{Cytokines induced by adjuvant treatment}

All adjuvants up-regulated pro-inflammatory cytokine expression at the tumour site within $24 \mathrm{~h}$ of administration. However, only Poly IC, MSU + M. smegmatis and LPS treatment resulted in a systemic increase of cytokine levels in the blood. IFN $\gamma$ and IL-12 were only significantly elevated in response to Poly IC and MSU + M. smegmatis. LPS, on the other hand, induced much higher levels of pro-inflammatory cytokines such as IL-6 and TNF $\alpha$ both at the tumour site and systemically. At the same time, LPS also induced very high levels of the regulatory cytokine IL-10, whereas the other adjuvants only moderately upregulated IL-10. These findings suggest that M. smegmatis and MSU individually fail to cause sufficient inflammation, whereas LPS increases pro-inflammatory factors so much that regulatory mechanisms become activated that result in production of IL-10 and subsequent dampening of the inflammatory response. This is in line with the response of dLN DCs to LPS. dLN DCs transiently up-regulated activation markers after 1 treatment but appeared even less activated than saline controls after 4 doses of LPS. Short-term activation followed by depletion of splenic DCs $48 \mathrm{~h}$ after LPS administration was previously described [285]. A reduction of the dose of LPS may be able to overcome these effects, but it may be difficult to find a dose at which the immuno-stimulatory and regulatory properties of LPS can be separated. 


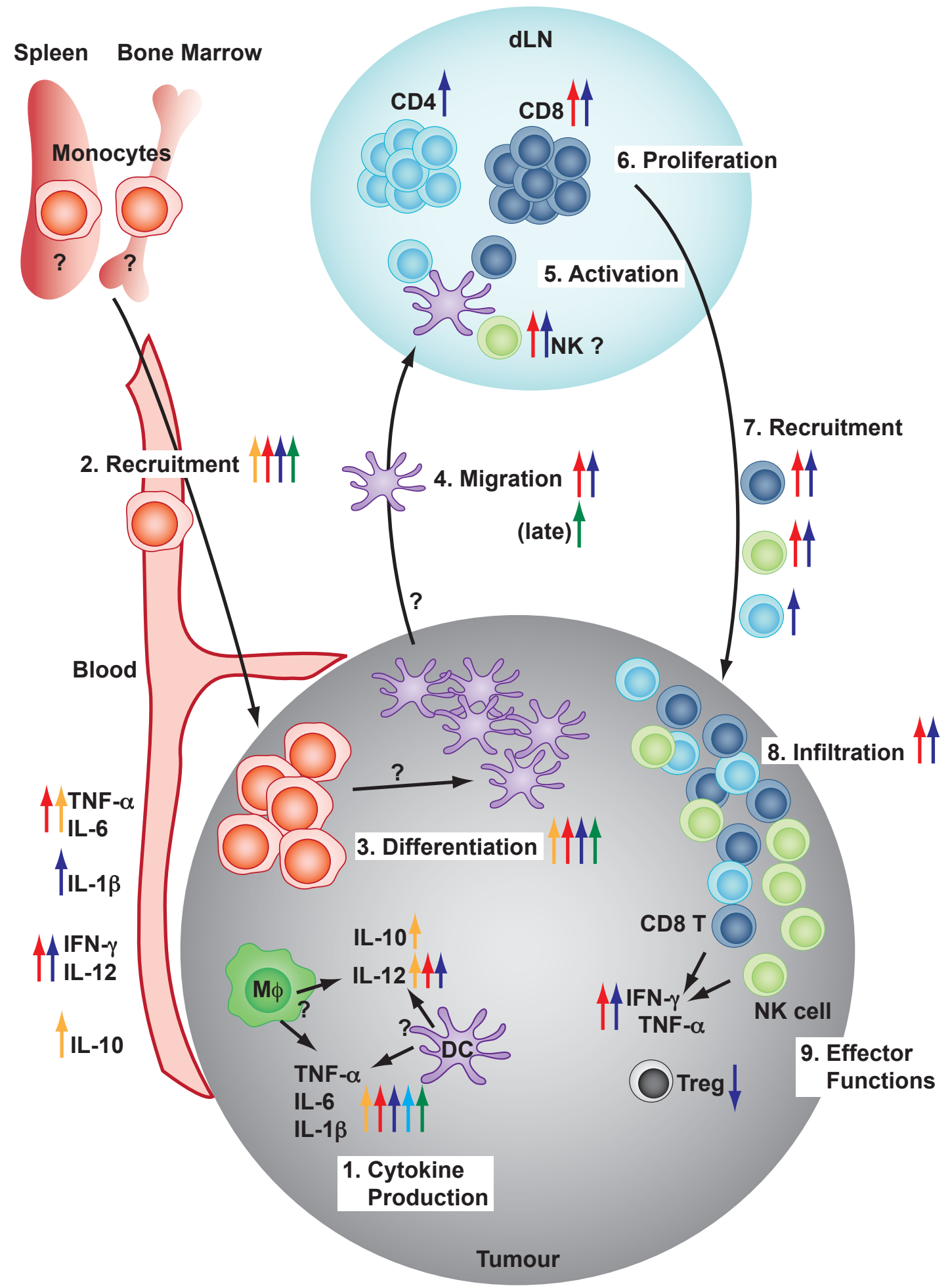

\begin{tabular}{|lllll|}
\hline $\begin{array}{l}\text { Peri-tumoral } \\
\text { adjuvants: }\end{array}$ & LPS Poly IC MSU M.smegmatis & $\begin{array}{l}\text { MSU + } \\
\text { M. smegmatis }\end{array}$ & $\begin{array}{l}\text { Increase } \\
\downarrow \text { Decrease }\end{array}$ \\
\hline
\end{tabular}

Figure 7.1: Working model of the effects of adjuvant treatments on the anti-tumour immune response. 
While previous publications have established the importance of IL-12 and IFN $\gamma$ in the control of tumour growth $[20,561]$, the relative importance of these and other cytokines for the anti-tumour effect of the adjuvants used in this study remains to be determined. Cytokine or cytokine-receptor deficient mice, as well as administration of blocking antibodies for different factors could be used to establish which cytokines are required for the anti-tumour response elicited by the different adjuvants. However, pro-inflammatory cytokines such as IL-6, TNF $\alpha$ and IL-1 $\beta$ partly overlap in their functions and in the transcription factors and down-stream responses they induce. Therefore deficiency or blocking of only one of these molecules may not show a detectable phenotype and simultaneous inhibition of two or all of them may be required to abrogate the anti-tumour effect of Poly IC and MSU + M. smegmatis.

DCs and macrophages are likely to be the main source of TNF $\alpha$, IL-6, IL-1 $\beta$, IL-10 and IL-12 in response to adjuvant treatment. They express the PRRs required to detect LPS, Poly IC, MSU and M. smegmatis [291, 359] and both have the capacity to produce these cytokines. T cells and NK cells may contribute to the secretion of TNF $\alpha$ and IFN $\gamma$, probably in response to the inflammatory milieu created by adjuvants treatment or interaction with activated DCs and macrophages. They are not expected to respond to the adjuvants directly, as they only express low levels of TLRs and no NLRP3 [359, 478].

Intracellular cytokine staining of tumour-infiltrating leukocytes after 4 adjuvant administrations showed that MSU + M. smegmatis increased the production of TNF $\alpha$ in DCs and CD8 T cells, while Poly IC enhanced TNF $\alpha$ levels in NK cells. In addition, MSU + M. smegmatis, Poly IC and LPS increased IL-12p40 staining in DCs and IFN $\gamma$ in CD8 T cells. MSU + M. smegmatis and Poly IC also increased IFN $\gamma$ levels in NK cells. As the cells were not re-stimulated before staining, these results are likely to reflect the actual cytokine production by these cells in the tumour. To confirm that cytokines were also secreted, it may be possible to isolate the various tumour-infiltrating immune cell types by fluorescenceactivated cell sorting (FACS) and assess their cytokine production in cell culture supernatant ex vivo. However, as the anti-tumour response induced by adjuvants is probably polyclonal, it would not be possible to re-stimulate the cells with a specific antigen and thus cytokine levels may be very low. Furthermore, the FACS procedure subjects cells to mechanical 
stress, which has been shown to activate DCs [562] and may therefore alter their cytokine production profiles. Therefore, we chose not to pursue these experiments, as they would require many mice to obtain sufficient numbers of tumour-infiltrating immune cells and may provide limited additional information on their cytokine secretion capacity.

\subsection{Accumulation of monocytes and monocyte-derived DCs in response to adjuvants}

Treatment with all adjuvants apart from MSU resulted in recruitment of monocytes to the tumour site, which was already observed at $48 \mathrm{~h}$ after the first adjuvant administration. Under inflammatory conditions, monocytes can be mobilised from the BM [563]. However, a recent report also identified a major reservoir of monocytes in the spleen, which was released to migrate to tissues upon injury [473]. The percentage and number of Ly6C $\mathrm{C}^{\mathrm{hi}}$ and Ly6 $\mathrm{C}^{\text {int }}$ monocytes in spleens was decreased after 4 peri-tumoral treatments with MSU + M. smegmatis, Poly IC and LPS. Administration of M. smegmatis also resulted in a lower frequency of Ly6C $\mathrm{C}^{\text {int }}$, but not Ly6C $\mathrm{C}^{\text {hi }}$ monocytes. This may suggest that the monocytes recruited to the tumour site by adjuvant treatments were mobilised from the spleen. It would be interesting to investigate whether monocytes were also decreased in the BM after adjuvant treatment. Furthermore, experiments on splenectomised animals could confirm whether the spleen was the source of the monocytes recruited to tumours.

Monocytes that enter tissues can differentiate into macrophages or DCs [564]. After 4 administrations of these adjuvants, an increased frequency of CD11c+MHCII $+7 / 4+\mathrm{Ly} 6 \mathrm{C}+$ cells, consistent with inflammatory monocyte derived DCs [466, 467], was observed in tumours. Monocyte-derived DCs may take up tumour-antigen, migrate to dLNs and stimulate anti-tumour T cell responses. The antigen-specific CD8 T cell response to OVA + Poly IC and other adjuvants has previously been shown to be partly dependent on monocyte-derived DCs [468]. Moreover, inflammatory monocyte-derived DCs have previously been shown to mediate the initiation of $\mathrm{T}$ cell responses against Leishmania major [307]. Thus, inflammatory monocyte-derived DCs can prime functional CD8 T cell responses in vaccination and infection models. In this study, inflammatory monocyte-derived DCs accumulated in 
tumour-dLNs already after one administration of Poly IC and MSU + M. smegmatis. On its own, M. smegmatis elevated their frequency in dLNs after 4 administrations, whereas they were never observed in dLNs in response to LPS. This suggests that Poly IC and MSU + M. smegmatis effectively and rapidly induced inflammatory monocyte-derived DCs to migrate to the dLNs. This response was delayed when M. smegmatis was used alone. In contrast, LPS resulted in the appearance of inflammatory monocyte-derived DCs at the tumour site but not in the dLNs. This may be due to a failure of LPS to induce migration of inflammatory monocyte-derived DCs to the dLNs or limited survival of these DCs in dLNs. As intradermal injection of LPS doses $>1 \mu \mathrm{g}$ have previously been shown to block the conversion of monocytes into fully migratory DCs [469], a lack of migration after LPS treatment appears more likely.

As the observation of monocyte-derived inflammatory DCs at the tumour site and dLNs is only correlative, there is a possibility that the inflammatory DCs in dLNs did not migrate from tumours but were instead recruited to dLNs directly from the blood, as previously shown in a viral infection model [308]. To investigate their origin, it may be possible to use a tumour transfected to express a fluorescent protein, such as GFP. If inflammatory DCs migrate from the tumour to stimulate the $\mathrm{T}$ cell response in dLNs, they would be expected to carry tumour antigen and therefore be GFP + in such a model. Such a result could not rule out the possibility of antigen-transfer to these DCs in the dLNs, but would support their migration from the tumour site. Furthermore the requirement of DCs for the anti-tumour effect could be established using CD11c-DTR BM chimeric mice, in which CD11c+ DCs that bear the human diphteria toxin receptor (DTR) can be depleted long-term by repeated administration of diphteria toxin $[565,566]$.

The role of monocytes has frequently been investigated by depletion with an antibody against Ly6C/Ly6G (anti-Gr-1, clone RB6-8C5) [567, 568]. However, this antibody also depletes neutrophils [199]. Furthermore, a recent publication suggests that it does not actually deplete monocytes but rather masks the Ly6C/Ly6G epitope in vivo and alters cell function [569]. Therefore, the anti-Gr-1 antibody may not be suitable to assess the impact of monocytes in adjuvant induced anti-tumour immune responses. Another antibody that specifically targets only Ly6C, clone AL-21, has been developed for flow cytometry, but has 
not previously been reported for cell depletion. If AL-21 proved suitable to deplete Ly6C+ cells in vivo, it may be useful to study the role of Ly6C ${ }^{\text {hi }}$ monocytes. In addition, monocytes use chemokine receptors for entry at the site of inflammation. CCR2, CCR5 and CX3CR1 have all been implicated in their recruitment [570], with CX3CR1 attributed a crucial role in the recruitment of monocytes that turn into inflammatory DCs [571]. Therefore, chemokine receptor deficient mice may be useful to establish the importance of monocyte infiltration at the tumour site for the adjuvant induced anti-tumour response.

\subsection{Potential of tumour-infiltrating monocytes to function as MDSCs}

It is important to address the role of monocytes in this model in more detail, as the surface phenotype of the CD11b+7/4+Ly6C+ monocytes identified in this study is also consistent with MDSCs [196, 470]. Most studies of MDSCs focus on the spleen, where these cells accumulate in tumour-bearing animals [196]. However, MDSCs are variably induced by different tumours, and B16F10 melanoma was found to only moderately increase MDSCs from $2 \%$ to about $5 \%$ of spleen cells [196]. In contrast, EL4 thymomas, CT26 colon carcinomas and 4T1 breast carcinomas resulted in MDSCs comprising 20-30\% of all live cells in spleens [196]. Using the B16F1 melanoma, no significant increase in Ly6C ${ }^{\text {hi }}$ Ly6G- monocytic and Ly6 ${ }^{\mathrm{lo}} \mathrm{Ly} 6 \mathrm{G}^{\text {hi }}$ granulocytic CD11b+ cells was found in the spleen of saline treated tumourbearing compared to naïve mice. In mice treated with $\mathrm{MSU}+M$. smegmatis, Poly IC and LPS, the number of both subsets was even significantly decreased. Furthermore, the presence of tumours has been reported to increase the suppressive ability of splenic MDSCs $[572,573]$. However, as shown in Appendix D of this study, enriched Ly6Chi Ly6G-CD11b+ and Ly6C ${ }^{\text {lo }} \mathrm{Ly} 6 \mathrm{G}^{\text {hi }} \mathrm{CD} 11 \mathrm{~b}+$ cells from naïve and B16F1 tumour bearing mice had a similar, moderate capacity to suppress $\mathrm{T}$ cell proliferation in vitro. This suggests that neither the tumour itself nor adjuvant treatment induced MDSCs in the spleen. Moreover, the highest infiltration of Ly6C ${ }^{\text {hi }} \mathrm{CD} 11 \mathrm{~b}+$ and $\mathrm{Ly} 6 \mathrm{C}^{\mathrm{int}} \mathrm{CD} 11 \mathrm{~b}+$ cells was seen in $\mathrm{MSU}+M$. smegmatis treated tumours and was associated with a significant delay in their progression. $\mathrm{MSU}+$ M. smegmatis and Poly IC were also the most successful adjuvants in delaying 
tumour growth and increasing survival in the E.G7-OVA and 4T1 tumour models, which are both known to induce high amounts of MDSCs [196]. This further supports the hypothesis that the observed myeloid cells infiltrating MSU + M. smegmatis and Poly IC treated tumours were not suppressive MDSCs but rather inflammatory monocytes that could differentiate into immuno-stimulatory inflammatory DCs and possibly M1-like macrophages. A previous study on a nano-particulated adjuvant also reported accumulation of cells with MDSC phenotype that lacked suppressive function [574]. Although the numbers of tumourinfiltrating immune cells are relatively low, it may be possible to isolate the $\mathrm{Ly} 6 \mathrm{C}^{\mathrm{hi}} \mathrm{CD} 11 \mathrm{~b}+$ and Ly6C ${ }^{\text {int }} \mathrm{CD} 11 \mathrm{~b}+$ cells by FACS from pooled, treated B16.OVA tumours and assess their ability to suppress or induce naïve OVA-specific T cell proliferation. This assay would determine if the tumour-infiltrating monocytes preferentially exert suppressive or stimulatory functions.

\subsection{Activation of adaptive and innate effector cells by adju- vant treatment}

The accumulation of inflammatory monocyte-derived DCs in dLNs of tumours treated once with Poly IC or MSU + M. smegmatis also correlated with an increase in the number of divided tumour-antigen specific CD $8 \mathrm{~T}$ cells. This suggests that both of these adjuvants enhanced CD8 T cell proliferation. MSU + M. smegmatis also enhanced CD4 T cell proliferation. In addition, the dLNs in these groups were enlarged already after a single adjuvant application and dLNs remained large after 4 adjuvant treatments. This indicates sustained reactivity of the dLNs in response to Poly IC and MSU $+M$. smegmatis. NK cells have also been reported to interact with DCs in LNs and DCs and NK cells can reciprocally activate each other $[230,575,576]$. While DC-NK interaction was not investigated in this study, increased production of IFN $\gamma$ and $\mathrm{TNF} \alpha$ by NK cells in tumours indicated that NK cells were more activated in response to adjuvant treatment. Furthermore, Poly IC and MSU + M. smegmatis enhanced infiltration of NK cells and CD8 T cells into B16F1 tumours. In addition, CD8 $\mathrm{T}$ cell production of cytokines was also augmented by both adjuvants. MSU + M. smegmatis also significantly increased CD4 T cell infiltration, while 
at the same time reducing Tregs. Studies in Rag1 deficient and cell-depleted mice revealed that both NK cells and CD8 T cells were required for the anti-tumour effect of Poly IC and MSU + M. smegmatis. The results for CD4 T cells were less conclusive, but suggested that CD4 T cells may also play a role in adjuvant-induced anti-tumour immunity. Collectively, these data indicate that increased $\mathrm{T}$ cell activation and proliferation in dLNs, together with enhanced infiltration of T cells and NK cells as well as augmented cytokine production by these effector cells results is sufficient to delay tumour growth. Most likely, CD8 T cells and NK cells kill tumour cells directly by exocytosis of cytotoxic granules and death-receptor pathways [577]. Tumours that are sensitive to TNF $\alpha$ or IFN $\gamma$ may also be eliminated via secretion of these cytokines by CD8 T cells and NK cells [24, 117]. However, CD8 T cells especially may need several days to proliferate and then migrate to the tumour, which may allow the aggressive B16F1 tumours to progress. A previous study of adoptively transferred tumour-specific $\mathrm{T}$ cell has shown elegantly that small tumours can be eliminated even in the presence of large tumours, but that large tumour masses cannot be controlled [547]. Therefore, tumour cells may grow faster than the immune response can eliminate them, resulting in the observed delay in tumour progression by adjuvant treatment, but not tumour regression or eradication. To improve the outcome of adjuvant-induced immunotherapy, it would therefore need to be combined with treatments that debulk large tumour masses, such as surgery or chemotherapy. For combination with chemotherapeuty, it will be important to avoid agents that induce immuno-suppression, and instead select drugs that can enhance anti-tumour immunity [578].

\subsection{Implications of the findings of this thesis}

\subsubsection{Tumour-infiltrating monocytes may exert suppressive or stimulating functions}

The outcome of the functional assessment of Ly6C ${ }^{\text {hi }} \mathrm{CD} 11 \mathrm{~b}+$ and $\mathrm{Ly} 6 \mathrm{C}^{\mathrm{int}} \mathrm{CD} 11 \mathrm{~b}+$ cells has important implications for the assessment of MDSCs as well as the applicability of adjuvant treatments in the clinic. If these cells were found to have no effect on $\mathrm{T}$ cell proliferation or 
were even stimulatory, the results of studies assessing MDSC solely by surface staining may need to be re-assessed. Furthermore, it would make "less-suppressive" MDSC a common feature of successful adjuvants. CpG has previously been reported to partly restore T cell proliferation in the presence of MDSC and to increase expression of CD11c and MHC II on MDSCs [472]. This suggests that MDSCs have a considerable level of plasticity and can be harnessed for anti-tumour immunotherapy if activated with the appropriate stimuli.

On the other hand, if Ly6C ${ }^{\text {hi }} \mathrm{CD} 11 \mathrm{~b}+$ and $\mathrm{Ly} 6 \mathrm{C}^{\mathrm{int}} \mathrm{CD} 11 \mathrm{~b}+$ cells were suppressive MDSCs, adjuvant treatments with Poly IC or MSU + M. smegmatis would benefit from combination with a therapy that depletes MDSCs or inhibits their function. A study by Serafini et al. indicates that Phosphodiesterase-5 inhibitors, such as Sildenafil, Tadalafil and Vardenafil, which are clinically used to treat erectile dysfunction, blocked MDSC function by down-regulating arginase 1 and nitric oxide synthase 2 [579]. A synthetic triterpenoid, bardoxolone-methyl, was also found to inhibit MDSC function, delay tumour growth in mice and restore $\mathrm{T}$ cell proliferation of cancer patients, via reduction of ROS from MDSCs [580]. It may be beneficial to combine therapies that inhibit MDSC function with antibodies that block $\mathrm{T}$ cell inhibition to release tumour-specific effector cells from all suppressive influences. Blocking antibodies against inhibitory molecules on $\mathrm{T}$ cells are already in clinical use and show promising anti-tumour activity.

\subsubsection{Monocyte-derived DCs in tumour immunotherapy}

In the current study, DC activation as assessed by up-regulation of surface markers was not detected in tumours after adjuvant treatment. Only production of IL-12 and TNF $\alpha$ could be somewhat enhanced by Poly IC and MSU + M. smegmatis. However, DC numbers in dLNs were increased and especially the rapid accumulation of monocyte-derived inflammatory DCs in dLNs correlated with a delay in tumour growth. This suggests that it may not be possible to activate tumour-resident DCs by adjuvant application in situ, and induce them to migrate to the dLNs and stimulate the anti-tumour response. Rather, newly differentiated, monocyte-derived DCs may have the capacity to fulfil this function. While further work is needed to confirm this hypothesis as outlined above, if it were true, it would 
have consequences for future studies on DC activation in tumours. Inflammatory monocytederived DCs may be a better correlate of successful activation of the immune response than global up-regulation of activation markers on LN DCs. It might therefore be interesting to study these inflammatory DCs in more detail also in other tumour-immunotherapy approaches. In addition, several molecules expressed by DCs are being investigated to target antigens to specific DC populations to increase vaccine efficiency [581]. If monocyte-derived inflammatory DCs are really particularly suited to induce anti-tumour immunity in a milieu of tumour-immune-suppression and tolerance, they may be a viable target for vaccine-based tumour-immunotherapy. However, more research would be needed to identify unique surface molecules of these cells suitable for specific targeting.

\subsubsection{Potential for the combination of adjuvant treatment with blockade of immuno-suppression}

Therapies combining immune stimulation with the blockade of immuno-suppressive mechanisms are promising strategies to break tolerance and increase anti-tumour immunity. While methods to inhibit MDSCs have started to be developed, Tregs have already been targeted in several clinical trials. Antibodies to the inhibitory molecule CTLA-4 on T cells can enhance anti-tumour immunity in mice [394, 395]. And the human anti-CTLA-4 antibody Ipilimumab has been used in several clinical trials and was approved by the FDA in 2011 $[6,7]$. While some complete responses were observed, mainly in melanoma patients, Ipilimumab has been associated with severe autoimmune side effects, which frequently require treatment with steroids or $\mathrm{TNF} \alpha$ blockade, and may limit its use [6, 7]. PD-1 is another inhibitory molecule expressed by $\mathrm{T}$ cells [520] that has been linked to $\mathrm{T}$ cell tolerance and CD8 T cell exhaustion $[269,582]$. PD-1+ T cells from melanoma patients have found to be dysfunctional [270], probably due to expression of PD-L1 by the melanoma cells [583] and could be rescued by PD-1 blockade [270]. An antibody for use in humans has been developed and a phase I trial involving 296 patients has just been completed [8]. The results of this trial were very promising, with $20-25 \%$ of patients with diverse types of tumours showing objective, durable responses. In patients with PD-L1+ tumours, the response rate 
was even as high as $36 \%$. While some patients experienced grade III/IV toxicities, the authors conclude that these could be managed and would not preclude clinical use. Phase II studies are currently underway and phase III trials are planned. In mice, combination of anti-PD-1 or anti-CTLA-4 therapy with CpG adjuvant therapy led to tumour eradication and increased survival [521]. Therefore, combination of Poly IC or MSU + M. smegmatis with anti-CTLA-4 antibody may enhance the anti-tumour effect. However, MSU + M. smegmatis may not benefit from combination with anti-PD-1 antibody, as the percentage of PD-1+ CD8 T cells in B16F1 melanomas was already reduced by treatment with $\mathrm{MSU}+$ M. smegmatis. In contrast, CD8 T cells in Poly IC treated tumours were PD-1+ and their function may be enhanced with anti-PD-1 blocking antibody.

\subsection{Summary and conclusions}

Peri-tumoral treatments with Poly IC and MSU + M. smegmatis delayed tumour growth and increased the survival of tumour bearing mice. The effects of adjuvant treatment on the immune system are summarised in Figure 7.1. Poly IC and MSU + M. smegmatis caused systemic release of IL-12 and IFN $\gamma$ within hours of administration and also induced local up-regulation of cytokines at the tumour site. In contrast, ineffective adjuvants either failed to induce cytokines (MSU, M. smegmatis) or induced very high levels of pro-inflammatory cytokines accompanied by substantial levels of IL-10 (LPS). In addition, the induction of successful anti-tumour immunity by Poly IC and MSU + M. smegmatis correlated with the infiltration of Ly6C+ monocytes into the adjuvant-treated tumours and accumulation of monocyte-derived inflammatory DCs in dLNs. These findings suggest that moderate levels of inflammation may be needed to stimulate anti-tumour immunity. Furthermore, rather than activating DCs already present in the tumour, the adjuvants used in this thesis appear to act via recruiting monocytes to the tumour site and inducing their differentiation into DCs that can initiate anti-tumour responses in dLNs.

Poly IC and MSU + M. smegmatis treatment increased T cell proliferation, infiltration of T cells and NK cells into tumours and cytokine production by effector cells. The activation of both innate and adaptive effector cells is advantageous, as NK cells and T cells may 
cooperate in rejecting tumours with different levels of MHC I expression. In addition, the adaptive anti-tumour immune response resulting from adjuvant treatment is likely to be polyclonal, limiting tumour immune escape via loss of antigen expression.

The strong combined innate and adaptive response induced by Poly IC and MSU + M. smegmatis delayed the growth of several different murine tumours in two different mouse strains. Furthermore, not only primary tumour growth but also metastatic load was reduced by adjuvant treatment. Therefore Poly IC and MSU + M. smegmatis may be effective against a wide range of cancers, including aggressive tumours with low immunogenicity, and may even be beneficial in the treatment of metastatic disease.

The advantage of using adjuvants like Poly IC or MSU + M. smegmatis as anti-cancer therapy is that these treatments should be cheap, easy to administer and do not require special expertise or intensive labour in manufacturing. Adjuvants are already in clinical use for the treatment of specific cancers. Creams containing the TLR7 agonist Imiquimod are applied topically to treat skin cancers including squamous cell carcinoma and superficial malignant melanoma. Superficial bladder cancer can be treated by intravesical instillations of BCG, which was found to be superior to other treatments including chemotherapy. Thus, adjuvants can be used for successful cancer-immunotherapy.

However, both Imiquimod and BCG show activity only in selected malignancies. One possibility for the effectiveness of BCG against bladder cancer but not melanoma may be that urine contains uric acid, which can form MSU crystals. Thus, BCG may act together with MSU to stimulate anti-tumour immunity in the bladder, but may fail to activate the immune system in other sites that lack uric acid. Both BCG and Imiquimod also need to be applied at the tumour site to be effective. In contrast, MSU + M. smegmatis showed considerable anti-tumour activity even when given s.c. at a site distant from the tumour. This suggests that MSU $+M$. smegmatis treatment may not be limited by the accessibility of tumours for direct injection and may thus be applicable in a wide range of patients.

The safety of Poly IC and MSU administration has already been established in clinical trials. While M. smegmatis is not currently used in the clinic, the related mycobacterium BCG is a standard therapy for superficial bladder cancer. In contrast to BCG, M. smegmatis is 
not derived from a pathogen and should therefore pose an even smaller risk for patients. Moreover, heat-killed M. smegmatis was found to be as effective as live M. smegmatis in the combination treatment with MSU and the use of heat-killed preparations would eliminate any risk of infection.

While further work is required to elucidate the role of monocytes and monocyte-derived DCs in the anti-tumour response induced by Poly IC and MSU + M. smegmatis, these adjuvants are promising candidates for the development of successful immunotherapy regimens in patients. In addition, the findings of this thesis provide insights into the factors involved in the generation of a successful anti-tumour immune response by adjuvant treatment. Understanding this process in more detail will help to select and develop the treatments that are most effective in stimulating anti-tumour immunity.

\section{7 $\quad$ Future directions}

This thesis has characterised a range of cytokines and changes in monocyte- and DC populations that accompany the activation of NK cells and $\mathrm{T}$ cells that exert anti-tumour function. However, further work is needed to clarify which of these factors are required for the anti-tumour response and how they contribute to anti-tumour immunity. Several suggestions for experiments to address these questions are briefly outlined below:

\section{I) Which cytokines are required and which cell types produce them?}

Knock-out mice and blocking antibodies against a range of cytokines, including IFN $\gamma$, IL-12, TNF $\alpha$ and IL-1 $\beta$ have been described. Cytokine deficient mice could be used to determine which cytokines are required for the adjuvant-induced anti-tumour response, while blocking antibodies could be employed to determine at which time points these cytokines were necessary. Simultaneous blocking or knock-out of several cytokines may be required to reveal the importance of cytokines that share overlapping functions, such as the pro-inflammatory $\mathrm{TNF} \alpha$ and IL-1 $\beta$. Studies in reporter mice that express fluorescent proteins concomitantly with cytokines may help to determine which cells produce each cytokine in the course of the anti-tumour response. 
Are DCs required and which subset migrates from tumours and presents antigen in dLNs? DCs can be depleted long- or short-term using CD11c-DTR BM-chimeras to establish whether DCs are required for the anti-tumour immune response throughout treatment or only at specific stages. Furthermore, adjuvant treated mice bearing GFP-expressing tumours could be used to assess antigen-uptake by different DC subsets and to confirm antigen-transport to dLNs. To establish which DC subset presents antigen and is capable of activating tumour-specific T cells in dLNs, DC subsets from dLNs of adjuvant-treated OVA-expressing tumours could be sorted by FACS and analysed for their ability to induce OTI and OTII T cells proliferation ex vivo.

Are tumour-infiltrating monocytes immuno-suppressive or stimulatory?

All methods for the abrogation of monocytes to assess their function have limitations. Anti-Gr-1 antibodies deplete neutrophils in addition to monocytes. The chemokine receptors CCR2 and CX3CR1 are crucial for monocyte recruitment during inflammation and CX3CR1 in particular was required for the influx of monocytes that differentiate into inflammatory DCs. Therefore, experiments in CCR2 and CX3CR1 deficient mice may help to clarify the role of monocyte infiltration at the tumour site. The effect of tumour-infiltrating monocytes on T cells could also be directly assessed by FACS purification of the monocytes and analysis of their capacity to induce $\mathrm{T}$ cell suppression or proliferation ex vivo.

Adjuvant treatment in vivo has a multitude of direct and indirect effects on many immune cells. The observation that LPS induces many of the cytokines seen with Poly IC and $\mathrm{MSU}+$ M. smegmatis administration and considerable transient DC activation in dLNs, but fails to delay tumour growth, highlights the need to understand the adjuvant induced immune response in more detail. The dissection of beneficial, neutral and detrimental effects of adjuvant administration on the immune system will greatly aid the development of successful adjuvant-based cancer immunotherapy. 


\section{References}

[1] Ferlay J, Shin HR, Bray F, Forman D, Mathers C, and Parkin DM. Estimates of worldwide burden of cancer in 2008: GLOBOCAN 2008. Int J Cancer, 127(12):2893-917, 2010 .

[2] Aly HAA. Cancer therapy and vaccination. J Immunol Methods, 382(1-2):1-23, 2012.

[3] Weiner LM, Dhodapkar MV, and Ferrone S. Monoclonal antibodies for cancer immunotherapy. Lancet, 373(9668):1033-40, 2009.

[4] Movassagh M, Spatz A, Davoust J, Lebecque S, Romero P, Pittet M, Rimoldi D, Liénard D, Gugerli O, Ferradini L, Robert C, Avril MF, Zitvogel L, and Angevin E. Selective accumulation of mature DC-Lamp + dendritic cells in tumor sites is associated with efficient T-cell-mediated antitumor response and control of metastatic dissemination in melanoma. Cancer Res, 64(6):2192-8, 2004.

55] Cheever MA and Higano CS. PROVENGE (Sipuleucel-T) in prostate cancer: the first FDA-approved therapeutic cancer vaccine. Clin Cancer Res, 17(11):3520-6, 2011.

[6] Phan GQ, Weber JS, and Sondak VK. CTLA-4 blockade with monoclonal antibodies in patients with metastatic cancer: surgical issues. Ann Surg Oncol, 15(11):3014-21, 2008.

[7] Weber J. Ipilimumab: controversies in its development, utility and autoimmune adverse events. Cancer Immunol Immunother, 58(5):823-30, 2009.

[8] Topalian SL, Hodi FS, Brahmer JR, Gettinger SN, Smith DC, McDermott DF, Powderly JD, Carvajal RD, Sosman JA, Atkins MB, Leming PD, Spigel DR, Antonia SJ, Horn L, Drake CG, Pardoll DM, Chen L, Sharfman WH, Anders RA, Taube JM, McMiller TL, Xu H, Korman AJ, Jure-Kunkel M, Agrawal S, McDonald D, Kollia GD, Gupta A, Wigginton JM, and Sznol M. Safety, Activity, and Immune Correlates of Anti-PD-1 Antibody in Cancer. New Engl J Med, 2012.

[9] Morales A, Eidinger D, and Bruce AW. Intracavitary Bacillus Calmette-Guerin in the treatment of superficial bladder tumors. J Urol, 116(2):180-3, 1976.

[10] Sylvester RJ, van der MEIJDEN APM, and Lamm DL. Intravesical bacillus CalmetteGuerin reduces the risk of progression in patients with superficial bladder cancer: a metaanalysis of the published results of randomized clinical trials. J Urol, 168(5):1964-70, 2002.

[11] Kawarada Y, Ganss R, Garbi N, Sacher T, Arnold B, and Hämmerling GJ. NKand CD8(+) $\mathrm{T}$ cell-mediated eradication of established tumors by peritumoral injection of CpG-containing oligodeoxynucleotides. J Immunol, 167(9):5247-53, 2001.

[12] Currie AJ, van der Most RG, Broomfield SA, Prosser AC, Tovey MG, and Robinson BWS. Targeting the effector site with IFN-alphabeta-inducing TLR ligands reactivates tumor-resident CD8 T cell responses to eradicate established solid tumors. J Immunol, 180(3):1535-44, 2008.

[13] Broomfield SA, van der Most RG, Prosser AC, Mahendran S, Tovey MG, Smyth MJ, Robinson BWS, and Currie AJ. Locally administered TLR7 agonists drive systemic antitumor immune responses that are enhanced by anti-CD40 immunotherapy. J Immunol, 182(9):5217-24, 2009.

[14] Weber JS, Zarour H, Redman B, Trefzer U, O'Day S, van den Eertwegh AJM, Marshall E, and Wagner S. Randomized phase 2/3 trial of CpG oligodeoxynucleotide PF3512676 alone or with dacarbazine for patients with unresectable stage III and IV melanoma. Cancer, 115(17):3944-54, 2009.

[15] Caskey M, Lefebvre F, Filali-Mouhim A, Cameron MJ, Goulet JP, Haddad EK, Breton G, Trumpfheller C, Pollak S, Shimeliovich I, Duque-Alarcon A, Pan L, 
Nelkenbaum A, Salazar AM, Schlesinger SJ, Steinman RM, and Sékaly RP. Synthetic double-stranded RNA induces innate immune responses similar to a live viral vaccine in humans. J Exp Med, 2011.

[16] Salazar AM, Levy HB, Ondra S, Kende M, Scherokman B, Brown D, Mena H, Martin N, Schwab K, Donovan D, Dougherty D, Pulliam M, Ippolito M, Graves M, Brown H, and Ommaya A. Long-term treatment of malignant gliomas with intramuscularly administered polyinosinic-polycytidylic acid stabilized with polylysine and carboxymethylcellulose: an open pilot study. Neurosurgery, 38(6):1096-103; discussion 1103-4, 1996.

[17] Novak N, Yu CF, Bieber T, and Allam JP. Toll-like receptor 7 agonists and skin. Drug News Perspect, 21(3):158-65, 2008.

[18] Ehrlich P. Über den jetzigen Stand der Karzinomforschung. Nederlands Tijdscrift Voor Geneeskunde, 5:273-290, 1909.

[19] Burnet M. Cancer: a biological approach. III. Viruses associated with neoplastic conditions. IV. Practical applications. $\operatorname{Br}$ Med J, 1(5023):841-7, 1957.

[20] Kaplan DH, Shankaran V, Dighe AS, Stockert E, Aguet M, Old LJ, and Schreiber RD. Demonstration of an interferon gamma-dependent tumor surveillance system in immunocompetent mice. Proc Natl Acad Sci USA, 95(13):7556-61, 1998.

[21] Shankaran V, Ikeda H, Bruce AT, White JM, Swanson PE, Old LJ, and Schreiber RD. IFNgamma and lymphocytes prevent primary tumour development and shape tumour immunogenicity. Nature, 410(6832):1107-11, 2001.

[22] Smyth MJ, Thia KY, Street SE, MacGregor D, Godfrey DI, and Trapani JA. Perforin-mediated cytotoxicity is critical for surveillance of spontaneous lymphoma. J Exp Med, 192(5):755-60, 2000.

[23] Gresser I, Bourali C, Lévy JP, Fontaine-Brouty-Boyé D, and Thomas MT. Increased survival in mice inoculated with tumor cells and treated with interferon preparations. Proc Natl Acad Sci USA, 63(1):51-7, 1969.

[24] Dighe AS, Richards E, Old LJ, and Schreiber RD. Enhanced in vivo growth and resistance to rejection of tumor cells expressing dominant negative IFN gamma receptors. Immunity, 1(6):447-56, 1994.

[25] Street SE, Cretney E, and Smyth MJ. Perforin and interferon-gamma activities independently control tumor initiation, growth, and metastasis. Blood, 97(1):192-7, 2001.

[26] Shinkai Y, Rathbun G, Lam KP, Oltz EM, Stewart V, Mendelsohn M, Charron J, Datta M, Young F, and Stall AM. RAG-2-deficient mice lack mature lymphocytes owing to inability to initiate V(D)J rearrangement. Cell, 68(5):855-67, 1992.

[27] Smyth MJ, Crowe NY, and Godfrey DI. NK cells and NKT cells collaborate in host protection from methylcholanthrene-induced fibrosarcoma. Int Immunol, 13(4):459-63, 2001.

[28] Smyth MJ, Thia KY, Street SE, Cretney E, Trapani JA, Taniguchi M, Kawano T, Pelikan SB, Crowe NY, and Godfrey DI. Differential tumor surveillance by natural killer (NK) and NKT cells. J Exp Med, 191(4):661-8, 2000.

[29] Girardi M, Oppenheim DE, Steele CR, Lewis JM, Glusac E, Filler R, Hobby P, Sutton B, Tigelaar RE, and Hayday AC. Regulation of cutaneous malignancy by gammadelta T cells. Science, 294(5542):605-9, 2001.

[30] Frisch M, Biggar RJ, Engels EA, Goedert JJ, and Group ACMRS. Association of cancer with AIDS-related immunosuppression in adults. J Am Med Assoc, 285(13):1736-45, 2001.

[31] Chaturvedi AK, Pfeiffer RM, Chang L, Goedert JJ, Biggar RJ, and Engels EA. Elevated risk of lung cancer among people with AIDS. AIDS, 21(2):207-13, 2007. 
[32] Vajdic CM, McDonald SP, McCredie MRE, van Leeuwen MT, Stewart JH, Law M, Chapman JR, Webster AC, Kaldor JM, and Grulich AE. Cancer incidence before and after kidney transplantation. J Am Med Assoc, 296(23):2823-31, 2006.

[33] Jiang Y, Villeneuve PJ, Wielgosz A, Schaubel DE, Fenton SSA, and Mao Y. The incidence of cancer in a population-based cohort of Canadian heart transplant recipients. $\mathrm{Am}$ $J$ Transplant, 10(3):637-45, 2010.

[34] Strauss DC and Thomas JM. Transmission of donor melanoma by organ transplantation. Lancet Oncol, 11(8):790-6, 2010.

[35] Coley WB. The Treatment of Inoperable Sarcoma by Bacterial Toxins (the Mixed Toxins of the Streptococcus erysipelas and the Bacillus prodigiosus). Proc R Soc Med, 3(Surg Sect):1-48, 1910.

[36] Carey TE, Takahashi T, Resnick LA, Oettgen HF, and Old LJ. Cell surface antigens of human malignant melanoma: mixed hemadsorption assays for humoral immunity to cultured autologous melanoma cells. Proc Natl Acad Sci USA, 73(9):3278-82, 1976.

[37] Reuschenbach M, von Knebel Doeberitz M, and Wentzensen N. A systematic review of humoral immune responses against tumor antigens. Cancer Immunol Immunother, 58(10):1535-44, 2009.

[38] Weide B, Zelba H, Derhovanessian E, Pflugfelder A, Eigentler TK, Giacomo AMD, Maio M, Aarntzen EHJG, de Vries IJM, Sucker A, Schadendorf D, Büttner P, Garbe C, and Pawelec G. Functional T Cells Targeting NY-ESO-1 or Melan-A Are Predictive for Survival of Patients With Distant Melanoma Metastasis. J Clin Oncol, 30(15):1835-41, 2012.

[39] Ferradini L, Mackensen A, Genevée C, Bosq J, Duvillard P, Avril MF, and Hercend T. Analysis of T cell receptor variability in tumor-infiltrating lymphocytes from a human regressive melanoma. Evidence for in situ $\mathrm{T}$ cell clonal expansion. J Clin Invest, 91(3):1183-90, 1993.

[40] Zorn E and Hercend T. A natural cytotoxic T cell response in a spontaneously regressing human melanoma targets a neoantigen resulting from a somatic point mutation. Eur. J. Immunol., 29(2):592-601, 1999.

[41] Clemente CG, Mihm MC, Bufalino R, Zurrida S, Collini P, and Cascinelli N. Prognostic value of tumor infiltrating lymphocytes in the vertical growth phase of primary cutaneous melanoma. Cancer, 77(7):1303-10, 1996.

[42] Pagès F, Berger A, Camus M, Sanchez-Cabo F, Costes A, Molidor R, Mlecnik B, Kirilovsky A, Nilsson M, Damotte D, Meatchi T, Bruneval P, Cugnenc PH, Trajanoski Z, Fridman WH, and Galon J. Effector memory T cells, early metastasis, and survival in colorectal cancer. N Engl J Med, 353(25):2654-66, 2005.

[43] Galon J, Costes A, Sanchez-Cabo F, Kirilovsky A, Mlecnik B, Lagorce-Pagès C, Tosolini M, Camus M, Berger A, Wind P, Zinzindohoué F, Bruneval P, Cugnenc PH, Trajanoski Z, Fridman WH, and Pagès F. Type, density, and location of immune cells within human colorectal tumors predict clinical outcome. Science, 313(5795):1960-4, 2006 .

[44] Sato E, Olson SH, Ahn J, Bundy B, Nishikawa H, Qian F, Jungbluth AA, Frosina D, Gnjatic S, Ambrosone C, Kepner J, Odunsi T, Ritter G, Lele S, Chen YT, Ohtani H, Old LJ, and Odunsi K. Intraepithelial CD8+ tumor-infiltrating lymphocytes and a high $\mathrm{CD} 8+$ /regulatory $\mathrm{T}$ cell ratio are associated with favorable prognosis in ovarian cancer. Proc Natl Acad Sci USA, 102(51):18538-43, 2005.

[45] Liu F, Lang R, Zhao J, Zhang X, Pringle GA, Fan Y, Yin D, Gu F, Yao Z, and Fu L. CD8 cytotoxic $\mathrm{T}$ cell and FOXP3 regulatory $\mathrm{T}$ cell infiltration in relation to breast cancer survival and molecular subtypes. Breast Cancer Res Treat, 130(2):645-55, 2011. 
[46] Curiel TJ, Coukos G, Zou L, Alvarez X, Cheng P, Mottram P, Evdemon-Hogan M, Conejo-Garcia JR, Zhang L, Burow M, Zhu Y, Wei S, Kryczek I, Daniel B, Gordon A, Myers L, Lackner A, Disis ML, Knutson KL, Chen L, and Zou W. Specific recruitment of regulatory $\mathrm{T}$ cells in ovarian carcinoma fosters immune privilege and predicts reduced survival. Nat Med, 10(9):942-9, 2004.

[47] Badoual C, Hans S, Rodriguez J, Peyrard S, Klein C, Agueznay NEH, Mosseri V, Laccourreye O, Bruneval P, Fridman WH, Brasnu DF, and Tartour E. Prognostic value of tumor-infiltrating CD4+ T-cell subpopulations in head and neck cancers. Clin Cancer Res, 12(2):465-72, 2006.

[48] Yotnda P, Firat H, Garcia-Pons F, Garcia Z, Gourru G, Vernant JP, Lemonnier FA, Leblond V, and Langlade-Demoyen P. Cytotoxic T cell response against the chimeric p210 BCR-ABL protein in patients with chronic myelogenous leukemia. J Clin Invest, 101(10):2290-6, 1998.

[49] Hoffmann TK, Arsov C, Schirlau K, Bas M, Friebe-Hoffmann U, Klussmann JP, Scheckenbach K, Balz V, Bier H, and Whiteside TL. T cells specific for HPV16 E7 epitopes in patients with squamous cell carcinoma of the oropharynx. Int J Cancer, 118(8):1984$91,2006$.

[50] Chan PKS, Liu SJ, Cheung TH, Yeo W, Ngai SM, Cheung JLK, Chong P, and Man S. T-cell response to human papillomavirus type 58 L1, E6, And E7 peptides in women with cleared infection, cervical intraepithelial neoplasia, or invasive cancer. Clin Vaccine Immunol, 17(9):1315-21, 2010.

[51] Stebbing J, Bourboulia D, Johnson M, Henderson S, Williams I, Wilder N, Tyrer M, Youle M, Imami N, Kobu T, Kuon W, Sieper J, Gotch F, and Boshoff C. Kaposi's sarcoma-associated herpesvirus cytotoxic T lymphocytes recognize and target Darwinian positively selected autologous K1 epitopes. J Virol, 77(7):4306-14, 2003.

[52] van der Bruggen P, Zhang Y, Chaux P, Stroobant V, Panichelli C, Schultz ES, Chapiro J, Eynde BJVD, Brasseur F, and Boon T. Tumor-specific shared antigenic peptides recognized by human T cells. Immunol Rev, 188:51-64, 2002.

[53] Figueiredo DLA, Mamede RCM, Spagnoli GC, Silva WA, Zago M, Neder L, Jungbluth AA, and Saggioro FP. High expression of cancer testis antigens MAGE-A, MAGEC1/CT7, MAGE-C2/CT10, NY-ESO-1, and gage in advanced squamous cell carcinoma of the larynx. Head Neck, 33(5):702-7, 2011.

[54] Kawakami Y, Dang N, Wang X, Tupesis J, Robbins PF, Wang RF, Wunderlich JR, Yannelli JR, and Rosenberg SA. Recognition of shared melanoma antigens in association with major HLA-A alleles by tumor infiltrating T lymphocytes from 123 patients with melanoma. J Immunother, 23(1):17-27, 2000.

[55] Peoples GE, Goedegebuure PS, Smith R, Linehan DC, Yoshino I, and Eberlein TJ. Breast and ovarian cancer-specific cytotoxic T lymphocytes recognize the same HER2/neuderived peptide. Proc Natl Acad Sci USA, 92(2):432-6, 1995.

[56] Groh V, Rhinehart R, Secrist H, Bauer S, Grabstein KH, and Spies T. Broad tumor-associated expression and recognition by tumor-derived gamma delta T cells of MICA and MICB. Proc Natl Acad Sci USA, 96(12):6879-84, 1999.

[57] Porgador A, Mandelboim O, Restifo NP, and Strominger JL. Natural killer cell lines kill autologous beta2-microglobulin-deficient melanoma cells: implications for cancer immunotherapy. Proc Natl Acad Sci USA, 94(24):13140-5, 1997.

[58] Matzinger P. Tolerance, danger, and the extended family. Annu Rev Immunol, 12:991-1045, 1994.

[59] Santini SM, Lapenta C, Logozzi M, Parlato S, Spada M, Pucchio TD, and Belardelli F. Type I interferon as a powerful adjuvant for monocyte-derived dendritic cell 
development and activity in vitro and in Hu-PBL-SCID mice. J Exp Med, 191(10):1777-88, 2000.

[60] Picaud S, Bardot B, Maeyer ED, and Seif I. Enhanced tumor development in mice lacking a functional type I interferon receptor. J Interferon Cytokine Res, 22(4):457-62, 2002.

[61] Dunn GP, Bruce AT, Sheehan KCF, Shankaran V, Uppaluri R, Bui JD, Diamond MS, Koebel CM, Arthur C, White JM, and Schreiber RD. A critical function for type I interferons in cancer immunoediting. Nat Immunol, 6(7):722-9, 2005.

[62] Ito N, DeMarco RA, Mailliard RB, Han J, Rabinowich H, Kalinski P, Stolz DB, Zeh HJ, and Lotze MT. Cytolytic cells induce HMGB1 release from melanoma cell lines. J Leukoc Biol, 81(1):75-83, 2007.

[63] Aymeric L, Apetoh L, Ghiringhelli F, Tesniere A, Martins I, Kroemer G, Smyth MJ, and Zitvogel L. Tumor cell death and ATP release prime dendritic cells and efficient anticancer immunity. Cancer Res, 70(3):855-8, 2010.

[64] Shi Y, Evans JE, and Rock KL. Molecular identification of a danger signal that alerts the immune system to dying cells. Nature, 425(6957):516-21, 2003.

[65] Srikrishna G and Freeze HH. Endogenous damage-associated molecular pattern molecules at the crossroads of inflammation and cancer. Neoplasia, 11(7):615-28, 2009.

[66] Tesniere A, Apetoh L, Ghiringhelli F, Joza N, Panaretakis T, Kepp O, Schlemmer F, Zitvogel L, and Kroemer G. Immunogenic cancer cell death: a key-lock paradigm. Curr Opin Immunol, 20(5):504-11, 2008.

[67] Pandolfi F, Boyle LA, Trentin L, Oliva A, and Kurnick JT. T cell receptor gene rearrangements and cytotoxic activities of clones isolated from tumour-infiltrating lymphocytes (TIL) from melanoma patients. Clin Exp Immunol, 95(1):141-7, 1994.

[68] Keene JA and Forman J. Helper activity is required for the in vivo generation of cytotoxic T lymphocytes. J Exp Med, 155(3):768-82, 1982.

[69] Marzo AL, Kinnear BF, Lake RA, Frelinger JJ, Collins EJ, Robinson BW, and Scott B. Tumor-specific CD4+ T cells have a major "post-licensing" role in CTL mediated anti-tumor immunity. J Immunol, 165(11):6047-55, 2000.

[70] Broom JK, Lew AM, Azukizawa H, Kenna TJ, Leggatt GR, and Frazer IH. Antigenspecific CD4 cells assist CD8 T-effector cells in eliminating keratinocytes. J Invest Dermatol, 130(6):1581-9, 2010.

[71] Quezada SA, Simpson TR, Peggs KS, Merghoub T, Vider J, Fan X, Blasberg R, Yagita H, Muranski P, Antony PA, Restifo NP, and Allison JP. Tumor-reactive CD4(+) T cells develop cytotoxic activity and eradicate large established melanoma after transfer into lymphopenic hosts. J Exp Med, 207(3):637-50, 2010.

[72] Gabizon A, Leibovich SJ, and Goldman R. Contrasting effects of activated and nonactivated macrophages and macrophages from tumor-bearing mice on tumor growth in vivo. $J$ Natl Cancer Inst, 65(5):913-20, 1980.

[73] Mosser DM and Edwards JP. Exploring the full spectrum of macrophage activation. Nat Rev Immunol, 8(12):958-69, 2008.

[74] Ohri CM, Shikotra A, Green RH, Waller DA, and Bradding P. Macrophages within NSCLC tumour islets are predominantly of a cytotoxic M1 phenotype associated with extended survival. Eur Respir J, 33(1):118-26, 2009.

[75] Utsugi T, Schroit AJ, Connor J, Bucana CD, and Fidler IJ. Elevated expression of phosphatidylserine in the outer membrane leaflet of human tumor cells and recognition by activated human blood monocytes. Cancer Res, 51(11):3062-6, 1991. 
[76] Elnemr A, Ohta T, Yachie A, Fushida S, Ninomiya I, Nishimura GI, Yamamoto M, Ohkuma S, and Miwa K. N-ethylmaleimide-enhanced phosphatidylserine externalization of human pancreatic cancer cells and immediate phosphatidylserine-mediated phagocytosis by macrophages. Int J Oncol, 16(6):1111-6, 2000.

[77] Ushio Y, Yamamoto N, Sanchez-Bueno A, and Yoshida R. Failure to reject an allografted tumor after elimination of macrophages in mice. Microbiol Immunol, 40(7):489-98, 1996.

[78] Brantley EC, Guo L, Zhang C, Lin Q, Yokoi K, Langley RR, Kruzel E, Maya M, Kim SW, Kim SJ, Fan D, and Fidler IJ. Nitric oxide-mediated tumoricidal activity of murine microglial cells. Transl Oncol, 3(6):380-8, 2010.

[79] Miguel RDV, Cherpes TL, Watson LJ, and McKenna KC. CTL induction of tumoricidal nitric oxide production by intratumoral macrophages is critical for tumor elimination. $J$ Immunol, 185(11):6706-18, 2010.

[80] Russell JH and Ley TJ. Lymphocyte-mediated cytotoxicity. Annu Rev Immunol, 20:323-70, 2002.

[81] van den Broek ME, Kägi D, Ossendorp F, Toes R, Vamvakas S, Lutz WK, Melief CJ, Zinkernagel RM, and Hengartner $\mathbf{H}$. Decreased tumor surveillance in perforindeficient mice. J Exp Med, 184(5):1781-90, 1996.

[82] Hishii M, Kurnick JT, Ramirez-Montagut T, and Pandolfi F. Studies of the mechanism of cytolysis by tumour-infiltrating lymphocytes. Clin Exp Immunol, 116(3):388-94, 1999.

[83] Mori S, Jewett A, Murakami-Mori K, Cavalcanti M, and Bonavida B. The participation of the Fas-mediated cytotoxic pathway by natural killer cells is tumor-cell-dependent. Cancer Immunol Immunother, 44(5):282-90, 1997.

[84] Kline J, Zhang L, Battaglia L, Cohen KS, and Gajewski TF. Cellular and molecular requirements for rejection of B16 melanoma in the setting of regulatory $\mathrm{T}$ cell depletion and homeostatic proliferation. J Immunol, 188(6):2630-42, 2012.

[85] Zeytun A, Hassuneh M, Nagarkatti M, and Nagarkatti PS. Fas-Fas ligand-based interactions between tumor cells and tumor-specific cytotoxic T lymphocytes: a lethal twoway street. Blood, 90(5):1952-9, 1997.

[86] Cretney E, Takeda K, Yagita H, Glaccum M, Peschon JJ, and Smyth MJ. Increased susceptibility to tumor initiation and metastasis in TNF-related apoptosis-inducing liganddeficient mice. J Immunol, 168(3):1356-61, 2002.

[87] Takeda K, Hayakawa Y, Smyth MJ, Kayagaki N, Yamaguchi N, Kakuta S, Iwakura Y, Yagita H, and Okumura K. Involvement of tumor necrosis factor-related apoptosisinducing ligand in surveillance of tumor metastasis by liver natural killer cells. Nat Med, $7(1): 94-100,2001$

[88] Chang CI, Liao JC, and Kuo L. Macrophage arginase promotes tumor cell growth and suppresses nitric oxide-mediated tumor cytotoxicity. Cancer Res, 61(3):1100-6, 2001.

[89] Bromberg JF, Horvath CM, Wen Z, Schreiber RD, and Darnell JE. Transcriptionally active Stat1 is required for the antiproliferative effects of both interferon alpha and interferon gamma. Proc Natl Acad Sci USA, 93(15):7673-8, 1996.

[90] Detjen KM, Farwig K, Welzel M, Wiedenmann B, and Rosewicz S. Interferon gamma inhibits growth of human pancreatic carcinoma cells via caspase-1 dependent induction of apoptosis. Gut, 49(2):251-62, 2001.

[91] Gresser I, Maury C, Vignaux F, Haller O, Belardelli F, and Tovey MG. Antibody to mouse interferon alpha/beta abrogates resistance to the multiplication of Friend erythroleukemia cells in the livers of allogeneic mice. J Exp Med, 168(4):1271-91, 1988. 
[92] Sato K, Hida S, Takayanagi H, Yokochi T, Kayagaki N, Takeda K, Yagita H, Okumura K, Tanaka N, Taniguchi T, and Ogasawara K. Antiviral response by natural killer cells through TRAIL gene induction by IFN-alpha/beta. Eur J Immunol, 31(11):3138-46, 2001.

[93] Hiroishi K, Tüting T, and Lotze MT. IFN-alpha-expressing tumor cells enhance generation and promote survival of tumor-specific CTLs. J Immunol, 164(2):567-72, 2000.

[94] Lasfar A, Lewis-Antes A, Smirnov SV, Anantha S, Abushahba W, Tian B, Reuhl K, Dickensheets H, Sheikh F, Donnelly RP, Raveche E, and Kotenko SV. Characterization of the mouse IFN-lambda ligand-receptor system: IFN-lambdas exhibit antitumor activity against B16 melanoma. Cancer Research, 66(8):4468-77, 2006.

[95] Sato A, Ohtsuki M, Hata M, Kobayashi E, and Murakami T. Antitumor activity of IFN-lambda in murine tumor models. J Immunol, 176(12):7686-94, 2006.

[96] Kacha AK, Fallarino F, Markiewicz MA, and Gajewski TF. Cutting edge: spontaneous rejection of poorly immunogenic P1.HTR tumors by Stat6-deficient mice. J Immunol, 165(11):6024-8, 2000.

[97] Liu J, Xiang Z, and Ma X. Role of IFN regulatory factor-1 and IL-12 in immunological resistance to pathogenesis of $\mathrm{N}$-methyl-N-nitrosourea-induced $\mathrm{T}$ lymphoma. J Immunol, 173(2):1184-93, 2004 .

[98] Langowski JL, Zhang X, Wu L, Mattson JD, Chen T, Smith K, Basham B, McClanahan T, Kastelein RA, and Oft M. IL-23 promotes tumour incidence and growth. Nature, 442(7101):461-5, 2006.

[99] Smedt TD, Mechelen MV, Becker GD, Urbain J, Leo O, and Moser M. Effect of interleukin-10 on dendritic cell maturation and function. Eur J Immunol, 27(5):1229-35, 1997.

[100] Lissoni P, Rovelli F, Tisi E, Brivio F, Ardizzoia A, Barni S, Tancini G, Saudelli M, Cesana E, and Viganò MG. Relation between macrophage and T helper-2 lymphocyte functions in human neoplasms: neopterin, interleukin-10 and interleukin-6 blood levels in early or advanced solid tumors. J Biol Regul Homeost Agents, 9(4):146-9, 1995.

[101] Yue FY, Dummer R, Geertsen R, Hofbauer G, Laine E, Manolio S, and Burg G. Interleukin-10 is a growth factor for human melanoma cells and down-regulates HLA class-I, HLA class-II and ICAM-1 molecules. Int J Cancer, 71(4):630-7, 1997.

[102] Swann JB, Vesely MD, Silva A, Sharkey J, Akira S, Schreiber RD, and Smyth MJ. Demonstration of inflammation-induced cancer and cancer immunoediting during primary tumorigenesis. Proc Natl Acad Sci USA, 105(2):652-6, 2008.

[103] García-Hernández ML, Hernández-Pando R, Gariglio P, and Berumen J. Interleukin-10 promotes B16-melanoma growth by inhibition of macrophage functions and induction of tumour and vascular cell proliferation. Immunology, 105(2):231-43, 2002.

[104] Ivanović V, Demajo M, Krtolica K, Krajnović M, Konstantinović M, Baltić V, Prtenjak G, Stojiljković B, Breberina M, Nesković-Konstantinović Z, NikolićVukosavljević D, and Dimitrijević B. Elevated plasma TGF-beta1 levels correlate with decreased survival of metastatic breast cancer patients. Clin Chim Acta, 371(1-2):191-3, 2006.

[105] Wikström P, Stattin P, Franck-Lissbrant I, Damber JE, and Bergh A. Transforming growth factor beta1 is associated with angiogenesis, metastasis, and poor clinical outcome in prostate cancer. Prostate, 37(1):19-29, 1998.

[106] Oft M, Heider KH, and Beug H. TGFbeta signaling is necessary for carcinoma cell invasiveness and metastasis. Curr Biol, 8(23):1243-52, 1998.

[107] Birnbaum DJ, Mamessier E, and Birnbaum D. The emerging role of the TGF tumor suppressor pathway in pancreatic cancer. Cell Cycle, 11(4):683-6, 2012. 
[108] Seijo ER, Song H, Lynch MA, Jennings R, Qong X, Lazaridis E, Muro-Cacho C, Weghorst CM, and Muñoz-Antonia T. Identification of genetic alterations in the TGFbeta type II receptor gene promoter. Mutat Res, 483(1-2):19-26, 2001.

[109] Janda E, Lehmann K, Killisch I, Jechlinger M, Herzig M, Downward J, Beug $\mathbf{H}$, and Grünert $\mathbf{S}$. Ras and TGF[beta] cooperatively regulate epithelial cell plasticity and metastasis: dissection of Ras signaling pathways. J Cell Biol, 156(2):299-313, 2002.

[110] Martin-Orozco N, Muranski P, Chung Y, Yang XO, Yamazaki T, Lu S, Hwu P, Restifo NP, Overwijk WW, and Dong C. T helper 17 cells promote cytotoxic T cell activation in tumor immunity. Immunity, 31(5):787-98, 2009.

[111] Teng MWL, Andrews DM, McLaughlin N, von Scheidt B, Ngiow SF, Möller A, Hill GR, Iwakura Y, Oft M, and Smyth MJ. IL-23 suppresses innate immune response independently of IL-17A during carcinogenesis and metastasis. Proc Natl Acad Sci USA, 107(18):8328-33, 2010.

[112] Wang L, Yi T, Kortylewski M, Pardoll DM, Zeng D, and Yu H. IL-17 can promote tumor growth through an IL-6-Stat3 signaling pathway. J Exp Med, 206(7):1457-64, 2009.

[113] Kryczek I, Wu K, Zhao E, Wei S, Vatan L, Szeliga W, Huang E, Greenson J, Chang A, Roliński J, Radwan P, Fang J, Wang G, and Zou W. IL-17+ regulatory T cells in the microenvironments of chronic inflammation and cancer. J Immunol, 186(7):4388-95, 2011.

[114] Yang XO, Nurieva R, Martinez GJ, Kang HS, Chung Y, Pappu BP, Shah B, Chang SH, Schluns KS, Watowich SS, Feng XH, Jetten AM, and Dong C. Molecular antagonism and plasticity of regulatory and inflammatory T cell programs. Immunity, 29(1):44-56, 2008.

[115] Tartar DM, van Morlan AM, and Zaghouani H. T-regulatory/T-helper 17: promiscuous signals and fear of commitment. J Immunother, 1(1):27-9, 2009.

[116] Moore RJ, Owens DM, Stamp G, Arnott C, Burke F, East N, Holdsworth H, Turner L, Rollins B, Pasparakis M, Kollias G, and Balkwill F. Mice deficient in tumor necrosis factor-alpha are resistant to skin carcinogenesis. Nat Med, 5(7):828-31, 1999.

[117] Cordero JB, Macagno JP, Stefanatos RK, Strathdee KE, Cagan RL, and Vidal M. Oncogenic Ras diverts a host TNF tumor suppressor activity into tumor promoter. Dev Cell, 18(6):999-1011, 2010.

[118] Torisu H, Ono M, Kiryu H, Furue M, Ohmoto Y, Nakayama J, Nishioka Y, Sone S, and Kuwano M. Macrophage infiltration correlates with tumor stage and angiogenesis in human malignant melanoma: possible involvement of TNFalpha and IL-1alpha. Int J Cancer, 85(2):182-8, 2000.

[119] Shime H, Matsumoto M, Oshiumi H, Tanaka S, Nakane A, Iwakura Y, Tahara H, Inoue $\mathbf{N}$, and Seya $\mathbf{T}$. Toll-like receptor 3 signaling converts tumor-supporting myeloid cells to tumoricidal effectors. Proc Natl Acad Sci USA, 109(6):2066-71, 2012.

[120] Lo CW, Chen MW, Hsiao M, Wang S, Chen CA, Hsiao SM, Chang JS, Lai TC, Rose-John S, Kuo ML, and Wei LH. IL-6 trans-signaling in formation and progression of malignant ascites in ovarian cancer. Cancer Res, 71(2):424-34, 2011.

[121] Becker C, Fantini MC, Wirtz S, Nikolaev A, Lehr HA, Galle PR, Rose-John S, and Neurath MF. IL-6 signaling promotes tumor growth in colorectal cancer. Cell Cycle, $4(2): 217-20,2005$.

[122] Naugler WE, Sakurai T, Kim S, Maeda S, Kim K, Elsharkawy AM, and Karin M. Gender disparity in liver cancer due to sex differences in MyD88-dependent IL-6 production. Science, 317(5834):121-4, 2007.

[123] Diehl S and Rincón M. The two faces of IL-6 on Th1/Th2 differentiation. Mol Immunol, 39(9):531-6, 2002. 
[124] Haabeth OAW, Lorvik KB, Hammarström C, Donaldson IM, Haraldsen G, Bogen B, and Corthay A. Inflammation driven by tumour-specific Th1 cells protects against B-cell cancer. Nat Commun, 2:240, 2011.

[125] Hsiao YW, Liao KW, Hung SW, and Chu RM. Tumor-infiltrating lymphocyte secretion of IL-6 antagonizes tumor-derived TGF-beta 1 and restores the lymphokine-activated killing activity. J Immunol, 172(3):1508-14, 2004.

[126] Hsiao YW, Liao KW, Chung TF, Liu CH, Hsu CD, and Chu RM. Interactions of host IL-6 and IFN-gamma and cancer-derived TGF-beta1 on MHC molecule expression during tumor spontaneous regression. Cancer Immunol Immunother, 57(7):1091-104, 2008.

[127] Krelin Y, Voronov E, Dotan S, Elkabets M, Reich E, Fogel M, Huszar M, Iwakura Y, Segal S, Dinarello CA, and Apte RN. Interleukin-1beta-driven inflammation promotes the development and invasiveness of chemical carcinogen-induced tumors. Cancer Res, 67(3):1062-71, 2007.

[128] Ghiringhelli F, Apetoh L, Tesniere A, Aymeric L, Ma Y, Ortiz C, Vermaelen K, Panaretakis T, Mignot G, Ullrich E, Perfettini JL, Schlemmer F, Tasdemir E, Uhl M, Génin P, Civas A, Ryffel B, Kanellopoulos J, Tschopp J, André F, Lidereau R, Mclaughlin NM, Haynes NM, Smyth MJ, Kroemer G, and Zitvogel L. Activation of the NLRP3 inflammasome in dendritic cells induces IL-1beta-dependent adaptive immunity against tumors. Nat Med, 15(10):1170-8, 2009.

[129] Grivennikov SI, Greten FR, and Karin M. Immunity, inflammation, and cancer. Cell, 140(6):883-99, 2010.

[130] Jäger E, Ringhoffer M, Karbach J, Arand M, Oesch F, and Knuth A. Inverse relationship of melanocyte differentiation antigen expression in melanoma tissues and CD8+ cytotoxic-T-cell responses: evidence for immunoselection of antigen-loss variants in vivo. Int J Cancer, 66(4):470-6, 1996.

[131] Hofbauer GF, Kamarashev J, Geertsen R, Böni R, and Dummer R. Melan A/MART1 immunoreactivity in formalin-fixed paraffin-embedded primary and metastatic melanoma: frequency and distribution. Melanoma Res, 8(4):337-43, 1998.

[132] Khong HT, Wang QJ, and Rosenberg SA. Identification of multiple antigens recognized by tumor-infiltrating lymphocytes from a single patient: tumor escape by antigen loss and loss of MHC expression. J Immunother, 27(3):184-90, 2004.

[133] Smahel M, Tejklova P, Smahelova J, Polakova I, and Mackova J. Mutation in the immunodominant epitope of the HPV16 E7 oncoprotein as a mechanism of tumor escape. Cancer Immunol Immunother, 57(6):823-31, 2008.

[134] Singh R and Paterson Y. Immunoediting sculpts tumor epitopes during immunotherapy. Cancer Res, 67(5):1887-92, 2007.

[135] Restifo NP, Marincola FM, Kawakami Y, Taubenberger J, Yannelli JR, and Rosenberg SA. Loss of functional beta 2-microglobulin in metastatic melanomas from five patients receiving immunotherapy. J Natl Cancer Inst, 88(2):100-8, 1996.

[136] Stölzel F, Hackmann K, Kuithan F, Mohr B, Füssel M, Oelschlägel U, Thiede C, Röllig C, Platzbecker U, Schetelig J, Illmer T, Schaich M, Seliger B, Hartmann A, Baretton G, Zietz C, Ehninger G, Schrock E, and Bornhäuser M. Clonal evolution including partial loss of human leukocyte antigen genes favoring extramedullary acute myeloid leukemia relapse after matched related allogeneic hematopoietic stem cell transplantation. Transplantation, 93(7):744-9, 2012.

[137] Baba T, Hanagiri T, Takenoyama M, Shiota H, Kuroda K, Shigematsu Y, Ichiki $\mathbf{Y}$, Uramoto $\mathbf{H}$, So T, and Yasumoto K. Identification of a lung cancer antigen evading CTL attack due to loss of human leukocyte antigen (HLA) class I expression. Cancer Sci, 101(10):2115-20, 2010. 
[138] Atkins D, Breuckmann A, Schmahl GE, Binner P, Ferrone S, Krummenauer F, Störkel S, and Seliger B. MHC class I antigen processing pathway defects, ras mutations and disease stage in colorectal carcinoma. Int J Cancer, 109(2):265-73, 2004.

[139] Dunn GP, Sheehan KCF, Old LJ, and Schreiber RD. IFN unresponsiveness in LNCaP cells due to the lack of JAK1 gene expression. Cancer Res, 65(8):3447-53, 2005.

[140] Holdenrieder S, Stieber P, Peterfi A, Nagel D, Steinle A, and Salih HR. Soluble MICB in malignant diseases: analysis of diagnostic significance and correlation with soluble MICA. Cancer Immunol Immunother, 55(12):1584-9, 2006.

[141] Salih HR, Rammensee HG, and Steinle A. Cutting edge: down-regulation of MICA on human tumors by proteolytic shedding. J Immunol, 169(8):4098-102, 2002.

[142] Shin MS, Kim HS, Lee SH, Park WS, Kim SY, Park JY, Lee JH, Lee SK, Lee SN, Jung SS, Han JY, Kim H, Lee JY, and Yoo NJ. Mutations of tumor necrosis factor-related apoptosis-inducing ligand receptor 1 (TRAIL-R1) and receptor 2 (TRAIL-R2) genes in metastatic breast cancers. Cancer Res, 61(13):4942-6, 2001.

[143] Takahashi H, Feuerhake F, Kutok JL, Monti S, Cin PD, Neuberg D, Aster JC, and Shipp MA. FAS death domain deletions and cellular FADD-like interleukin 1beta converting enzyme inhibitory protein (long) overexpression: alternative mechanisms for deregulating the extrinsic apoptotic pathway in diffuse large B-cell lymphoma subtypes. Clin Cancer Res, 12(11 Pt 1):3265-71, 2006.

[144] Lajmanovich A, Irisarri M, Molens JP, Pasquier MA, Sotto JJ, Bensa JC, Leroux $\mathbf{D}$, and Plumas J. Impairment of death-inducing signalling complex formation in CD95resistant human primary lymphoma B cells. Br J Haematol, 124(6):746-53, 2004.

[145] Hinz S, Trauzold A, Boenicke L, Sandberg C, Beckmann S, Bayer E, Walczak H, Kalthoff $\mathbf{H}$, and Ungefroren $\mathbf{H}$. Bcl-XL protects pancreatic adenocarcinoma cells against CD95- and TRAIL-receptor-mediated apoptosis. Oncogene, 19(48):5477-86, 2000.

[146] Dong H, Strome SE, Salomao DR, Tamura H, Hirano F, Flies DB, Roche PC, Lu J, Zhu G, Tamada K, Lennon VA, Celis E, and Chen L. Tumor-associated B7-H1 promotes T-cell apoptosis: a potential mechanism of immune evasion. Nat Med, 8(8):793-800, 2002.

[147] Uyttenhove C, Pilotte L, Théate I, Stroobant V, Colau D, Parmentier N, Boon T, and den Eynde BJV. Evidence for a tumoral immune resistance mechanism based on tryptophan degradation by indoleamine 2,3-dioxygenase. Nat Med, 9(10):1269-74, 2003.

[148] Frumento G, Rotondo R, Tonetti M, Damonte G, Benatti U, and Ferrara GB. Tryptophan-derived catabolites are responsible for inhibition of $\mathrm{T}$ and natural killer cell proliferation induced by indoleamine 2,3-dioxygenase. J Exp Med, 196(4):459-68, 2002.

[149] Curti A, Pandolfi S, Valzasina B, Aluigi M, Isidori A, Ferri E, Salvestrini V, Bonanno G, Rutella S, Durelli I, Horenstein AL, Fiore F, Massaia M, Colombo MP, Baccarani M, and Lemoli RM. Modulation of tryptophan catabolism by human leukemic cells results in the conversion of CD25- into CD25+ T regulatory cells. Blood, 109(7):2871-7, 2007.

[150] Mehal WZ, Sheikh SZ, Gorelik L, and Flavell RA. TGF-beta signaling regulates CD8+ $\mathrm{T}$ cell responses to high- and low-affinity TCR interactions. Int Immunol, 17(5):531-8, 2005.

[151] Gorelik L, Constant S, and Flavell RA. Mechanism of transforming growth factor betainduced inhibition of T helper type 1 differentiation. J Exp Med, 195(11):1499-505, 2002.

[152] Gorelik L and Flavell RA. Immune-mediated eradication of tumors through the blockade of transforming growth factor-beta signaling in T cells. Nat Med, 7(10):1118-22, 2001.

[153] Doedens AL, Stockmann C, Rubinstein MP, Liao D, Zhang N, DeNardo DG, Coussens LM, Karin M, Goldrath AW, and Johnson RS. Macrophage expression of 
hypoxia-inducible factor-1 alpha suppresses T-cell function and promotes tumor progression. Cancer Res, 70(19):7465-75, 2010.

[154] Biswas SK, Gangi L, Paul S, Schioppa T, Saccani A, Sironi M, Bottazzi B, Doni A, Vincenzo B, Pasqualini F, Vago L, Nebuloni M, Mantovani A, and Sica A. A distinct and unique transcriptional program expressed by tumor-associated macrophages (defective NF-kappaB and enhanced IRF-3/STAT1 activation). Blood, 107(5):2112-22, 2006.

[155] Tiemessen MM, Jagger AL, Evans HG, van Herwijnen MJC, John S, and Taams LS. CD4+CD25+Foxp3+ regulatory $\mathrm{T}$ cells induce alternative activation of human monocytes/macrophages. Proc Natl Acad Sci USA, 104(49):19446-51, 2007.

[156] Qian BZ and Pollard JW. Macrophage diversity enhances tumor progression and metastasis. Cell, 141(1):39-51, 2010.

[157] Bingle L, Brown NJ, and Lewis CE. The role of tumour-associated macrophages in tumour progression: implications for new anticancer therapies. J Pathol, 196(3):254-65, 2002.

[158] Ueno T, Toi M, Saji H, Muta M, Bando H, Kuroi K, Koike M, Inadera H, and Matsushima K. Significance of macrophage chemoattractant protein-1 in macrophage recruitment, angiogenesis, and survival in human breast cancer. Clin Cancer Res, 6(8):3282-9, 2000.

[159] Goede V, Brogelli L, Ziche M, and Augustin HG. Induction of inflammatory angiogenesis by monocyte chemoattractant protein-1. Int J Cancer, 82(5):765-70, 1999.

[160] Skobe M, Hamberg LM, Hawighorst T, Schirner M, Wolf GL, Alitalo K, and Detmar M. Concurrent induction of lymphangiogenesis, angiogenesis, and macrophage recruitment by vascular endothelial growth factor-C in melanoma. Am J Pathol, 159(3):893-903, 2001.

[161] Hagemann T, Wilson J, Burke F, Kulbe H, Li NF, Plüddemann A, Charles K, Gordon S, and Balkwill FR. Ovarian cancer cells polarize macrophages toward a tumorassociated phenotype. J Immunol, 176(8):5023-32, 2006.

[162] Eubank TD, Roda JM, Liu H, O'Neil T, and Marsh CB. Opposing roles for HIF-1 and HIF-2 in the regulation of angiogenesis by mononuclear phagocytes. Blood, 117(1):323-32, 2011.

[163] Bronte V, Serafini P, Santo CD, Marigo I, Tosello V, Mazzoni A, Segal DM, Staib C, Lowel M, Sutter G, Colombo MP, and Zanovello P. IL-4-induced arginase 1 suppresses alloreactive T cells in tumor-bearing mice. J Immunol, 170(1):270-8, 2003.

[164] Brunkow ME, Jeffery EW, Hjerrild KA, Paeper B, Clark LB, Yasayko SA, Wilkinson JE, Galas D, Ziegler SF, and Ramsdell F. Disruption of a new forkhead/wingedhelix protein, scurfin, results in the fatal lymphoproliferative disorder of the scurfy mouse. Nat Genet, 27(1):68-73, 2001.

[165] Williams LM and Rudensky AY. Maintenance of the Foxp3-dependent developmental program in mature regulatory $\mathrm{T}$ cells requires continued expression of Foxp3. Nat Immunol, $8(3): 277-84,2007$.

[166] Allan SE, Crome SQ, Crellin NK, Passerini L, Steiner TS, Bacchetta R, Roncarolo MG, and Levings MK. Activation-induced FOXP3 in human T effector cells does not suppress proliferation or cytokine production. Int Immunol, 19(4):345-54, 2007.

[167] Sakaguchi S, Sakaguchi N, Asano M, Itoh M, and Toda M. Immunologic self-tolerance maintained by activated $\mathrm{T}$ cells expressing IL-2 receptor alpha-chains (CD25). Breakdown of a single mechanism of self-tolerance causes various autoimmune diseases. J Immunol, 155(3):1151-64, 1995.

[168] Asano M, Toda M, Sakaguchi N, and Sakaguchi S. Autoimmune disease as a consequence of developmental abnormality of a T cell subpopulation. J Exp Med, 184(2):387-96, 1996. 
[169] Chen W, Jin W, Hardegen N, Lei KJ, Li L, Marinos N, McGrady G, and Wahl SM. Conversion of peripheral CD4+CD25- naive T cells to CD4+CD25+ regulatory T cells by TGF-beta induction of transcription factor Foxp3. J Exp Med, 198(12):1875-86, 2003.

[170] Grauer OM, Nierkens S, Bennink E, Toonen LWJ, Boon L, Wesseling P, Sutmuller RPM, and Adema GJ. CD4+FoxP3+ regulatory T cells gradually accumulate in gliomas during tumor growth and efficiently suppress antiglioma immune responses in vivo. Int $J$ Cancer, 121(1):95-105, 2007.

[171] Andaloussi AE and Lesniak MS. CD4+ CD25+ FoxP3+ T-cell infiltration and heme oxygenase-1 expression correlate with tumor grade in human gliomas. J Neurooncol, 83(2):145$52,2007$.

[172] Fu J, Xu D, Liu Z, Shi M, Zhao P, Fu B, Zhang Z, Yang H, Zhang H, Zhou C, Yao J, Jin L, Wang H, Yang Y, Fu YX, and Wang FS. Increased regulatory T cells correlate with CD8 T-cell impairment and poor survival in hepatocellular carcinoma patients. Gastroenterology, 132(7):2328-39, 2007.

[173] Onizuka S, Tawara I, Shimizu J, Sakaguchi S, Fujita T, and Nakayama E. Tumor rejection by in vivo administration of anti-CD25 (interleukin-2 receptor alpha) monoclonal antibody. Cancer Res, 59(13):3128-33, 1999.

[174] Valzasina B, Piconese S, Guiducci C, and Colombo MP. Tumor-induced expansion of regulatory $\mathrm{T}$ cells by conversion of $\mathrm{CD} 4+\mathrm{CD} 25-$ lymphocytes is thymus and proliferation independent. Cancer Res, 66(8):4488-95, 2006.

[175] Moo-Young TA, Larson JW, Belt BA, Tan MC, Hawkins WG, Eberlein TJ, Goedegebuure PS, and Linehan DC. Tumor-derived TGF-beta mediates conversion of CD4+Foxp3+ regulatory $\mathrm{T}$ cells in a murine model of pancreas cancer. J Immunother, $32(1): 12-21,2009$.

[176] Cederbom L, Hall H, and Ivars F. CD4+CD25+ regulatory T cells down-regulate costimulatory molecules on antigen-presenting cells. Eur J Immunol, 30(6):1538-43, 2000.

[177] Thornton AM and Shevach EM. CD4+CD25+ immunoregulatory T cells suppress polyclonal T cell activation in vitro by inhibiting interleukin 2 production. J Exp Med, 188(2):28796, 1998.

[178] Oberle N, Eberhardt N, Falk CS, Krammer PH, and Suri-Payer E. Rapid suppression of cytokine transcription in human CD4+CD25 T cells by CD4+Foxp3+ regulatory $\mathrm{T}$ cells: independence of IL-2 consumption, TGF-beta, and various inhibitors of TCR signaling. $J$ Immunol, 179(6):3578-87, 2007.

[179] Chen ML, Pittet MJ, Gorelik L, Flavell RA, Weissleder R, von Boehmer H, and Khazaie K. Regulatory $\mathrm{T}$ cells suppress tumor-specific CD8 T cell cytotoxicity through TGF-beta signals in vivo. Proc Natl Acad Sci USA, 102(2):419-24, 2005.

[180] Mempel TR, Pittet MJ, Khazaie K, Weninger W, Weissleder R, von Boehmer H, and von Andrian UH. Regulatory T cells reversibly suppress cytotoxic T cell function independent of effector differentiation. Immunity, 25(1):129-41, 2006.

[181] Cao X, Cai SF, Fehniger TA, Song J, Collins LI, Piwnica-Worms DR, and Ley TJ. Granzyme B and perforin are important for regulatory $\mathrm{T}$ cell-mediated suppression of tumor clearance. Immunity, 27(4):635-46, 2007.

[182] Tadokoro CE, Shakhar G, Shen S, Ding Y, Lino AC, Maraver A, Lafaille JJ, and Dustin ML. Regulatory T cells inhibit stable contacts between CD4+ T cells and dendritic cells in vivo. J Exp Med, 203(3):505-11, 2006.

[183] Boissonnas A, Scholer-Dahirel A, Simon-Blancal V, Pace L, Valet F, Kissenpfennig A, Sparwasser T, Malissen B, Fetler L, and Amigorena S. Foxp3+ T cells induce perforin-dependent dendritic cell death in tumor-draining lymph nodes. Immunity, 32(2):266$78,2010$. 
[184] Deaglio S, Dwyer KM, Gao W, Friedman D, Usheva A, Erat A, Chen JF, Enjyoji K, Linden J, Oukka M, Kuchroo VK, Strom TB, and Robson SC. Adenosine generation catalyzed by CD39 and CD73 expressed on regulatory T cells mediates immune suppression. J Exp Med, 204(6):1257-65, 2007.

[185] Nakamura K, Kitani A, and Strober W. Cell contact-dependent immunosuppression by CD4(+)CD25(+) regulatory $\mathrm{T}$ cells is mediated by cell surface-bound transforming growth factor beta. J Exp Med, 194(5):629-44, 2001.

[186] Tai N, Yasuda H, Xiang Y, Zhang L, Rodriguez-Pinto D, Yokono K, Sherwin R, Wong FS, Nagata M, and Wen L. IL-10-conditioned dendritic cells prevent autoimmune diabetes in NOD and humanized HLA-DQ8/RIP-B7.1 mice. Clin Immunol, 139(3):336-49, 2011.

[187] Chaput N, Darrasse-Jèze G, Bergot AS, Cordier C, Ngo-Abdalla S, Klatzmann D, and Azogui O. Regulatory T cells prevent CD8 T cell maturation by inhibiting CD4 Th cells at tumor sites. J Immunol, 179(8):4969-78, 2007.

[188] Pandiyan P, Zheng L, Ishihara S, Reed J, and Lenardo MJ. CD4+CD25+Foxp3+ regulatory $\mathrm{T}$ cells induce cytokine deprivation-mediated apoptosis of effector CD4+ $\mathrm{T}$ cells. Nat Immunol, 8(12):1353-62, 2007.

[189] Gondek DC, Lu LF, Quezada SA, Sakaguchi S, and Noelle RJ. Cutting edge: contactmediated suppression by CD4+CD25+ regulatory cells involves a granzyme B-dependent, perforin-independent mechanism. J Immunol, 174(4):1783-6, 2005.

[190] Grossman WJ, Verbsky JW, Barchet W, Colonna M, Atkinson JP, and Ley TJ. Human $\mathrm{T}$ regulatory cells can use the perforin pathway to cause autologous target cell death. Immunity, 21(4):589-601, 2004.

[191] Ghiringhelli F, Ménard C, Terme M, Flament C, Taieb J, Chaput N, Puig PE, Novault S, Escudier B, Vivier E, Lecesne A, Robert C, Blay JY, Bernard J, Caillat-Zucman S, Freitas A, Tursz T, Wagner-Ballon O, Capron C, Vainchencker W, Martin F, and Zitvogel L. CD4+CD25+ regulatory T cells inhibit natural killer cell functions in a transforming growth factor-beta-dependent manner. J Exp Med, 202(8):1075-85, 2005.

[192] Smyth MJ, Teng MWL, Swann J, Kyparissoudis K, Godfrey DI, and Hayakawa Y. CD4+CD25+ T regulatory cells suppress NK cell-mediated immunotherapy of cancer. $J$ Immunol, 176(3):1582-7, 2006.

[193] Zea AH, Rodriguez PC, Atkins MB, Hernandez C, Signoretti S, Zabaleta J, McDermott D, Quiceno D, Youmans A, O'Neill A, Mier J, and Ochoa AC. Arginaseproducing myeloid suppressor cells in renal cell carcinoma patients: a mechanism of tumor evasion. Cancer Res, 65(8):3044-8, 2005.

[194] Schmielau J and Finn OJ. Activated granulocytes and granulocyte-derived hydrogen peroxide are the underlying mechanism of suppression of t-cell function in advanced cancer patients. Cancer Res, 61(12):4756-60, 2001.

[195] Movahedi K, Guilliams M, den Bossche JV, den Bergh RV, Gysemans C, Beschin A, Baetselier PD, and Ginderachter JAV. Identification of discrete tumor-induced myeloid-derived suppressor cell subpopulations with distinct T cell-suppressive activity. Blood, 111(8):4233-44, 2008.

[196] Youn JI, Nagaraj S, Collazo M, and Gabrilovich DI. Subsets of myeloid-derived suppressor cells in tumor-bearing mice. J Immunol, 181(8):5791-802, 2008.

[197] Pekarek LA, Starr BA, Toledano AY, and Schreiber H. Inhibition of tumor growth by elimination of granulocytes. J Exp Med, 181(1):435-40, 1995. 
[198] Kusmartsev S, Nefedova Y, Yoder D, and Gabrilovich DI. Antigen-specific inhibition of CD8+ T cell response by immature myeloid cells in cancer is mediated by reactive oxygen species. J Immunol, 172(2):989-99, 2004.

[199] Daley JM, Thomay AA, Connolly MD, Reichner JS, and Albina JE. Use of Ly6Gspecific monoclonal antibody to deplete neutrophils in mice. J Leukoc Biol, 83(1):64-70, 2008.

[200] Greifenberg V, Ribechini E, Rössner S, and Lutz MB. Myeloid-derived suppressor cell activation by combined LPS and IFN-gamma treatment impairs DC development. Eur $J$ Immunol, 39(10):2865-76, 2009.

[201] Dolcetti L, Peranzoni E, Ugel S, Marigo I, Gomez AF, Mesa C, Geilich M, Winkels G, Traggiai E, Casati A, Grassi F, and Bronte V. Hierarchy of immunosuppressive strength among myeloid-derived suppressor cell subsets is determined by GM-CSF. Eur $J$ Immunol, 40(1):22-35, 2010.

[202] Melani C, Chiodoni C, Forni G, and Colombo MP. Myeloid cell expansion elicited by the progression of spontaneous mammary carcinomas in c-erbB-2 transgenic BALB/c mice suppresses immune reactivity. Blood, 102(6):2138-45, 2003.

[203] Bronte V, Chappell DB, Apolloni E, Cabrelle A, Wang M, Hwu P, and Restifo NP. Unopposed production of granulocyte-macrophage colony-stimulating factor by tumors inhibits CD8+ T cell responses by dysregulating antigen-presenting cell maturation. $J \mathrm{Im}$ munol, 162(10):5728-37, 1999.

[204] Serafini P, Carbley R, Noonan KA, Tan G, Bronte V, and Borrello I. High-dose granulocyte-macrophage colony-stimulating factor-producing vaccines impair the immune response through the recruitment of myeloid suppressor cells. Cancer Res, 64(17):6337-43, 2004.

[205] Gabrilovich D, Ishida T, Oyama T, Ran S, Kravtsov V, Nadaf S, and Carbone DP. Vascular endothelial growth factor inhibits the development of dendritic cells and dramatically affects the differentiation of multiple hematopoietic lineages in vivo. Blood, 92(11):4150-66, 1998.

[206] Kusmartsev S, Eruslanov E, Kübler H, Tseng T, Sakai Y, Su Z, Kaliberov S, Heiser A, Rosser C, Dahm P, Siemann D, and Vieweg J. Oxidative stress regulates expression of VEGFR1 in myeloid cells: link to tumor-induced immune suppression in renal cell carcinoma. J Immunol, 181(1):346-53, 2008.

[207] Menetrier-Caux C, Montmain G, Dieu MC, Bain C, Favrot MC, Caux C, and Blay JY. Inhibition of the differentiation of dendritic cells from CD34(+) progenitors by tumor cells: role of interleukin-6 and macrophage colony-stimulating factor. Blood, 92(12):4778-91, 1998.

[208] Huang B, Lei Z, Zhao J, Gong W, Liu J, Chen Z, Liu Y, Li D, Yuan Y, Zhang GM, and Feng ZH. CCL2/CCR2 pathway mediates recruitment of myeloid suppressor cells to cancers. Cancer Lett, 252(1):86-92, 2007.

[209] Mazzoni A, Bronte V, Visintin A, Spitzer JH, Apolloni E, Serafini P, Zanovello $\mathbf{P}$, and Segal DM. Myeloid suppressor lines inhibit T cell responses by an NO-dependent mechanism. J Immunol, 168(2):689-95, 2002.

[210] Gallina G, Dolcetti L, Serafini P, Santo CD, Marigo I, Colombo MP, Basso G, Brombacher F, Borrello I, Zanovello P, Bicciato S, and Bronte V. Tumors induce a subset of inflammatory monocytes with immunosuppressive activity on CD8+ T cells. J Clin Invest, 116(10):2777-90, 2006.

[211] Terabe M, Matsui S, Park JM, Mamura M, Noben-Trauth N, Donaldson DD, Chen W, Wahl SM, Ledbetter S, Pratt B, Letterio JJ, Paul WE, and Berzofsky JA. Transforming growth factor-beta production and myeloid cells are an effector mechanism through which CD1d-restricted T cells block cytotoxic T lymphocyte-mediated tumor immunosurveillance: abrogation prevents tumor recurrence. J Exp Med, 198(11):1741-52, 2003. 
[212] Rodriguez PC, Zea AH, Culotta KS, Zabaleta J, Ochoa JB, and Ochoa AC. Regulation of T cell receptor CD3zeta chain expression by L-arginine. J Biol Chem, 277(24):21123-9, 2002 .

[213] Rodriguez PC, Quiceno DG, and Ochoa AC. L-arginine availability regulates Tlymphocyte cell-cycle progression. Blood, 109(4):1568-73, 2007.

[214] Bingisser RM, Tilbrook PA, Holt PG, and Kees UR. Macrophage-derived nitric oxide regulates $\mathrm{T}$ cell activation via reversible disruption of the Jak3/STAT5 signaling pathway. $J$ Immunol, 160(12):5729-34, 1998.

[215] Harari O and Liao JK. Inhibition of MHC II gene transcription by nitric oxide and antioxidants. Curr Pharm Des, 10(8):893-8, 2004.

[216] Allione A, Bernabei P, Bosticardo M, Ariotti S, Forni G, and Novelli F. Nitric oxide suppresses human T lymphocyte proliferation through IFN-gamma-dependent and IFNgamma-independent induction of apoptosis. J Immunol, 163(8):4182-91, 1999.

[217] Saio M, Radoja S, Marino M, and Frey AB. Tumor-infiltrating macrophages induce apoptosis in activated CD8 $(+) \mathrm{T}$ cells by a mechanism requiring cell contact and mediated by both the cell-associated form of TNF and nitric oxide. J Immunol, 167(10):5583-93, 2001.

[218] Vickers SM, MacMillan-Crow LA, Green M, Ellis C, and Thompson JA. Association of increased immunostaining for inducible nitric oxide synthase and nitrotyrosine with fibroblast growth factor transformation in pancreatic cancer. Arch Surg, 134(3):245-51, 1999.

[219] Ekmekcioglu S, Ellerhorst J, Smid CM, Prieto VG, Munsell M, Buzaid AC, and Grimm EA. Inducible nitric oxide synthase and nitrotyrosine in human metastatic melanoma tumors correlate with poor survival. Clin Cancer Res, 6(12):4768-75, 2000.

[220] Bronte V, Kasic T, Gri G, Gallana K, Borsellino G, Marigo I, Battistini L, Iafrate M, Prayer-Galetti T, Pagano F, and Viola A. Boosting antitumor responses of $\mathrm{T}$ lymphocytes infiltrating human prostate cancers. J Exp Med, 201(8):1257-68, 2005.

[221] Nagaraj S, Gupta K, Pisarev V, Kinarsky L, Sherman S, Kang L, Herber DL, Schneck J, and Gabrilovich DI. Altered recognition of antigen is a mechanism of CD8+ T cell tolerance in cancer. Nat Med, 13(7):828-35, 2007.

[222] Ghiringhelli F, Puig PE, Roux S, Parcellier A, Schmitt E, Solary E, Kroemer G, Martin F, Chauffert B, and Zitvogel L. Tumor cells convert immature myeloid dendritic cells into TGF-beta-secreting cells inducing CD4+CD25+ regulatory $\mathrm{T}$ cell proliferation. $J$ Exp Med, 202(7):919-29, 2005.

[223] Young MR, Wright MA, Matthews JP, Malik I, and Prechel M. Suppression of T cell proliferation by tumor-induced granulocyte-macrophage progenitor cells producing transforming growth factor-beta and nitric oxide. J Immunol, 156(5):1916-22, 1996.

[224] Banchereau J and Steinman RM. Dendritic cells and the control of immunity. Nature, 392(6673):245-52, 1998

[225] Hawiger D, Inaba K, Dorsett Y, Guo M, Mahnke K, Rivera M, Ravetch JV, Steinman RM, and Nussenzweig MC. Dendritic cells induce peripheral T cell unresponsiveness under steady state conditions in vivo. J Exp Med, 194(6):769-79, 2001.

[226] Guermonprez P, Valladeau J, Zitvogel L, Théry C, and Amigorena S. Antigen presentation and T cell stimulation by dendritic cells. Annu Rev Immunol, 20:621-67, 2002.

[227] Fernandez NC, Lozier A, Flament C, Ricciardi-Castagnoli P, Bellet D, Suter M, Perricaudet M, Tursz T, Maraskovsky E, and Zitvogel L. Dendritic cells directly trigger NK cell functions: cross-talk relevant in innate anti-tumor immune responses in vivo. Nat Med, 5(4):405-11, 1999. 
[228] Gerosa F, Baldani-Guerra B, Nisii C, Marchesini V, Carra G, and Trinchieri G. Reciprocal activating interaction between natural killer cells and dendritic cells. J Exp Med, 195(3):327-33, 2002.

[229] Watt SV, Andrews DM, Takeda K, Smyth MJ, and Hayakawa Y. IFN-gammadependent recruitment of mature CD27(high) NK cells to lymph nodes primed by dendritic cells. J Immunol, 181(8):5323-30, 2008.

[230] Prestwich RJ, Errington F, Steele LP, Ilett EJ, Morgan RSM, Harrington KJ, Pandha HS, Selby PJ, Vile RG, and Melcher AA. Reciprocal human dendritic cellnatural killer cell interactions induce antitumor activity following tumor cell infection by oncolytic reovirus. J Immunol, 183(7):4312-21, 2009.

[231] Jiang W, Swiggard WJ, Heufler C, Peng M, Mirza A, Steinman RM, and Nussenzweig MC. The receptor DEC-205 expressed by dendritic cells and thymic epithelial cells is involved in antigen processing. Nature, 375(6527):151-5, 1995.

[232] Sallusto F, Cella M, Danieli C, and Lanzavecchia A. Dendritic cells use macropinocytosis and the mannose receptor to concentrate macromolecules in the major histocompatibility complex class II compartment: downregulation by cytokines and bacterial products. J Exp Med, 182(2):389-400, 1995.

[233] Regnault A, Lankar D, Lacabanne V, Rodriguez A, Théry C, Rescigno M, Saito T, Verbeek S, Bonnerot C, Ricciardi-Castagnoli P, and Amigorena S. Fcgamma receptor-mediated induction of dendritic cell maturation and major histocompatibility complex class I-restricted antigen presentation after immune complex internalization. J Exp Med, $189(2): 371-80,1999$.

[234] e Sousa CR, Stahl PD, and Austyn JM. Phagocytosis of antigens by Langerhans cells in vitro. J Exp Med, 178(2):509-19, 1993.

[235] e Sousa CR. Dendritic cells in a mature age. Nat Rev Immunol, 6(6):476-83, 2006.

[236] Liu K, Iyoda T, Saternus M, Kimura Y, Inaba K, and Steinman RM. Immune tolerance after delivery of dying cells to dendritic cells in situ. J Exp Med, 196(8):1091-7, 2002.

[237] Hernandez J, Aung S, Redmond WL, and Sherman LA. Phenotypic and functional analysis of $\mathrm{CD} 8(+) \mathrm{T}$ cells undergoing peripheral deletion in response to cross-presentation of self-antigen. J Exp Med, 194(6):707-17, 2001.

[238] Jonuleit H, Schmitt E, Schuler G, Knop J, and Enk AH. Induction of interleukin 10producing, nonproliferating $\mathrm{CD} 4(+) \mathrm{T}$ cells with regulatory properties by repetitive stimulation with allogeneic immature human dendritic cells. J Exp Med, 192(9):1213-22, 2000.

[239] Steinman RM and Nussenzweig MC. Avoiding horror autotoxicus: the importance of dendritic cells in peripheral T cell tolerance. Proc Natl Acad Sci USA, 99(1):351-8, 2002.

[240] Nakahara T, Urabe K, Fukagawa S, Uchi H, Inaba K, Furue M, and Moroi Y. Engagement of human monocyte-derived dendritic cells into interleukin (IL)-12 producers by IL-1beta + interferon (IFN)-gamma. Clin Exp Immunol, 139(3):476-82, 2005.

[241] Cella M, Engering A, Pinet V, Pieters J, and Lanzavecchia A. Inflammatory stimuli induce accumulation of MHC class II complexes on dendritic cells. Nature, 388(6644):782-7, 1997.

[242] Sallusto F, Schaerli P, Loetscher P, Schaniel C, Lenig D, Mackay CR, Qin S, and Lanzavecchia A. Rapid and coordinated switch in chemokine receptor expression during dendritic cell maturation. Eur J Immunol, 28(9):2760-9, 1998.

[243] Pedersen AE, Gad M, Kristensen NN, Haase C, Nielsen CH, and Claesson MH. Tolerogenic dendritic cells pulsed with enterobacterial extract suppress development of colitis in the severe combined immunodeficiency transfer model. Immunology, 121(4):526-32, 2007. 
[244] Koch F, Stanzl U, Jennewein P, Janke K, Heufler C, Kämpgen E, Romani N, and Schuler G. High level IL-12 production by murine dendritic cells: upregulation via MHC class II and CD40 molecules and downregulation by IL-4 and IL-10. J Exp Med, 184(2):741-6, 1996.

[245] e Sousa CR, Hieny S, Scharton-Kersten T, Jankovic D, Charest H, Germain RN, and Sher A. In vivo microbial stimulation induces rapid CD40 ligand-independent production of interleukin 12 by dendritic cells and their redistribution to T cell areas. J Exp Med, 186(11):1819-29, 1997.

[246] Chamorro S, García-Vallejo JJ, Unger WWJ, Fernandes RJ, Bruijns SCM, Laban S, Roep BO, 't Hart BA, and van Kooyk Y. TLR triggering on tolerogenic dendritic cells results in TLR2 up-regulation and a reduced proinflammatory immune program. J Immunol, 183(5):2984-94, 2009.

[247] Radhakrishnan S, Wiehagen KR, Pulko V, Keulen VV, Faubion WA, Knutson KL, and Pease LR. Induction of a Th1 response from Th2-polarized T cells by activated dendritic cells: dependence on TCR:peptide-MHC interaction, ICAM-1, IL-12, and IFN-gamma. J Immunol, 178(6):3583-92, 2007.

[248] Singh RP. Immunoregulation of cytokines in infectious diseases (leprosy), future strategies. Nihon Hansenbyo Gakkai Zasshi, 67(2):263-8, 1998.

[249] Nurieva RI, Chung Y, Hwang D, Yang XO, Kang HS, Ma L, hong Wang Y, Watowich SS, Jetten AM, Tian Q, and Dong C. Generation of T follicular helper cells is mediated by interleukin-21 but independent of $\mathrm{T}$ helper 1, 2, or 17 cell lineages. Immunity, 29(1):138-49, 2008.

[250] Zhu J, Yamane H, and Paul WE. Differentiation of effector CD4 T cell populations. Annu Rev Immunol, 28:445-89, 2010.

[251] Banchereau J, Briere F, Caux C, Davoust J, Lebecque S, Liu YJ, Pulendran B, and Palucka K. Immunobiology of dendritic cells. Annu Rev Immunol, 18:767-811, 2000.

[252] Mach B, Steimle V, Martinez-Soria E, and Reith W. Regulation of MHC class II genes: lessons from a disease. Annu Rev Immunol, 14:301-31, 1996.

[253] Ramachandra L, Simmons D, and Harding CV. MHC molecules and microbial antigen processing in phagosomes. Curr Opin Immunol, 21(1):98-104, 2009.

[254] Harding CV and Ramachandra L. Presenting exogenous antigen to T cells. Curr Protoc Immunol, Chapter 16:Unit 16.2, 2010.

[255] Heath WR, Belz GT, Behrens GMN, Smith CM, Forehan SP, Parish IA, Davey GM, Wilson NS, Carbone FR, and Villadangos JA. Cross-presentation, dendritic cell subsets, and the generation of immunity to cellular antigens. Immunol Rev, 199:9-26, 2004.

[256] Bevan MJ. Cross-priming for a secondary cytotoxic response to minor H antigens with H-2 congenic cells which do not cross-react in the cytotoxic assay. J Exp Med, 143(5):1283-8, 1976.

[257] Lin ML, Zhan Y, Villadangos JA, and Lew AM. The cell biology of cross-presentation and the role of dendritic cell subsets. Immunol Cell Biol, 86(4):353-62, 2008.

[258] den Haan JM, Lehar SM, and Bevan MJ. CD8(+) but not CD8(-) dendritic cells crossprime cytotoxic T cells in vivo. J Exp Med, 192(12):1685-96, 2000.

[259] Pooley JL, Heath WR, and Shortman K. Cutting edge: intravenous soluble antigen is presented to CD4 T cells by CD8- dendritic cells, but cross-presented to CD8 T cells by CD8+ dendritic cells. J Immunol, 166(9):5327-30, 2001.

[260] Bedoui S, Whitney PG, Waithman J, Eidsmo L, Wakim L, Caminschi I, Allan RS, Wojtasiak M, Shortman K, Carbone FR, Brooks AG, and Heath WR. Cross- 
presentation of viral and self antigens by skin-derived CD103+ dendritic cells. Nat Immunol, 10(5):488-95, 2009.

[261] Acuto O and Michel F. CD28-mediated co-stimulation: a quantitative support for TCR signalling. Nat Rev Immunol, 3(12):939-51, 2003.

[262] Lenschow DJ, Walunas TL, and Bluestone JA. CD28/B7 system of T cell costimulation. Annu Rev Immunol, 14:233-58, 1996.

[263] McAdam AJ, Schweitzer AN, and Sharpe AH. The role of B7 co-stimulation in activation and differentiation of CD4+ and CD8+ T cells. Immunol Rev, 165:231-47, 1998.

[264] Linsley PS, Brady W, Urnes M, Grosmaire LS, Damle NK, and Ledbetter JA. CTLA-4 is a second receptor for the B cell activation antigen B7. J Exp Med, 174(3):561-9, 1991.

[265] Chambers CA, Kuhns MS, Egen JG, and Allison JP. CTLA-4-mediated inhibition in regulation of T cell responses: mechanisms and manipulation in tumor immunotherapy. Annu Rev Immunol, 19:565-94, 2001.

[266] Freeman GJ, Long AJ, Iwai Y, Bourque K, Chernova T, Nishimura H, Fitz LJ, Malenkovich N, Okazaki T, Byrne MC, Horton HF, Fouser L, Carter L, Ling V, Bowman MR, Carreno BM, Collins M, Wood CR, and Honjo T. Engagement of the PD-1 immunoinhibitory receptor by a novel B7 family member leads to negative regulation of lymphocyte activation. J Exp Med, 192(7):1027-34, 2000.

[267] Latchman Y, Wood CR, Chernova T, Chaudhary D, Borde M, Chernova I, Iwai Y, Long AJ, Brown JA, Nunes R, Greenfield EA, Bourque K, Boussiotis VA, Carter LL, Carreno BM, Malenkovich N, Nishimura H, Okazaki T, Honjo T, Sharpe AH, and Freeman GJ. PD-L2 is a second ligand for PD-1 and inhibits T cell activation. Nat Immunol, 2(3):261-8, 2001.

[268] Keir ME, Liang SC, Guleria I, Latchman YE, Qipo A, Albacker LA, Koulmanda M, Freeman GJ, Sayegh MH, and Sharpe AH. Tissue expression of PD-L1 mediates peripheral T cell tolerance. J Exp Med, 203(4):883-95, 2006.

[269] Barber DL, Wherry EJ, Masopust D, Zhu B, Allison JP, Sharpe AH, Freeman GJ, and Ahmed R. Restoring function in exhausted CD8 T cells during chronic viral infection. Nature, 439(7077):682-7, 2006.

[270] Fourcade J, Sun Z, Benallaoua M, Guillaume P, Luescher IF, Sander C, Kirkwood JM, Kuchroo V, and Zarour HM. Upregulation of Tim-3 and PD-1 expression is associated with tumor antigen-specific CD8+ T cell dysfunction in melanoma patients. J Exp Med, 207(10):2175-86, 2010.

[271] Quezada SA, Jarvinen LZ, Lind EF, and Noelle RJ. CD40/CD154 interactions at the interface of tolerance and immunity. Annu Rev Immunol, 22:307-28, 2004.

[272] Schoenberger SP, Toes RE, van der Voort EI, Offringa R, and Melief CJ. T-cell help for cytotoxic T lymphocytes is mediated by CD40-CD40L interactions. Nature, 393(6684):4803,1998 .

[273] Bennett SR, Carbone FR, Karamalis F, Flavell RA, Miller JF, and Heath WR. Help for cytotoxic-T-cell responses is mediated by CD40 signalling. Nature, 393(6684):478-80, 1998.

[274] Curtsinger JM, Schmidt CS, Mondino A, Lins DC, Kedl RM, Jenkins MK, and Mescher MF. Inflammatory cytokines provide a third signal for activation of naive CD4+ and CD8+ T cells. J Immunol, 162(6):3256-62, 1999.

[275] Mescher MF, Curtsinger JM, Agarwal P, Casey KA, Gerner M, Hammerbeck CD, Popescu F, and Xiao Z. Signals required for programming effector and memory development by CD8+ T cells. Immunol Rev, 211:81-92, 2006. 
[276] Hou WS and Parijs LV. A Bcl-2-dependent molecular timer regulates the lifespan and immunogenicity of dendritic cells. Nat Immunol, 5(6):583-9, 2004.

[277] Degli-Esposti MA and Smyth MJ. Close encounters of different kinds: dendritic cells and NK cells take centre stage. Nat Rev Immunol, 5(2):112-24, 2005.

[278] Yang J, Huck SP, McHugh RS, Hermans IF, and Ronchese F. Perforin-dependent elimination of dendritic cells regulates the expansion of antigen-specific CD8+ $\mathrm{T}$ cells in vivo. Proc Natl Acad Sci USA, 103(1):147-52, 2006.

[279] Kim TW, Hung CF, Ling M, Juang J, He L, Hardwick JM, Kumar S, and Wu TC. Enhancing DNA vaccine potency by coadministration of DNA encoding antiapoptotic proteins. J Clin Invest, 112(1):109-17, 2003.

[280] Kim TW, Lee JH, He L, Boyd DAK, Hardwick JM, Hung CF, and Wu TC. Modification of professional antigen-presenting cells with small interfering RNA in vivo to enhance cancer vaccine potency. Cancer Res, 65(1):309-16, 2005.

[281] Shortman K and Liu YJ. Mouse and human dendritic cell subtypes. Nat Rev Immunol, $2(3): 151-61,2002$.

[282] Vremec D, Pooley J, Hochrein H, Wu L, and Shortman K. CD4 and CD8 expression by dendritic cell subtypes in mouse thymus and spleen. J Immunol, 164(6):2978-86, 2000.

[283] Vremec D and Shortman K. Dendritic cell subtypes in mouse lymphoid organs: crosscorrelation of surface markers, changes with incubation, and differences among thymus, spleen, and lymph nodes. J Immunol, 159(2):565-73, 1997.

[284] Villadangos JA and Schnorrer P. Intrinsic and cooperative antigen-presenting functions of dendritic-cell subsets in vivo. Nat Rev Immunol, 7(7):543-55, 2007.

[285] Smedt TD, Pajak B, Muraille E, Lespagnard L, Heinen E, Baetselier PD, Urbain J, Leo $\mathbf{O}$, and Moser $\mathbf{M}$. Regulation of dendritic cell numbers and maturation by lipopolysaccharide in vivo. J Exp Med, 184(4):1413-24, 1996.

[286] Farrand KJ, Dickgreber N, Stoitzner P, Ronchese F, Petersen TR, and Hermans IF. Langerin + CD8alpha+ dendritic cells are critical for cross-priming and IL-12 production in response to systemic antigens. J Immunol, 183(12):7732-42, 2009.

[287] Bachem A, Güttler S, Hartung E, Ebstein F, Schaefer M, Tannert A, Salama A, Movassaghi K, Opitz C, Mages HW, Henn V, Kloetzel PM, Gurka S, and Kroczek RA. Superior antigen cross-presentation and XCR1 expression define human CD11c+CD141+ cells as homologues of mouse CD8+ dendritic cells. J Exp Med, 207(6):1273-81, 2010.

[288] Poulin LF, Salio M, Griessinger E, Anjos-Afonso F, Craciun L, Chen JL, Keller AM, Joffre O, Zelenay S, Nye E, Moine AL, Faure F, Donckier V, Sancho D, Cerundolo V, Bonnet D, and e Sousa CR. Characterization of human DNGR-1+ BDCA3+ leukocytes as putative equivalents of mouse CD8alpha+ dendritic cells. J Exp Med, 207(6):1261-71, 2010.

[289] Schulz O, Diebold SS, Chen M, Näslund TI, Nolte MA, Alexopoulou L, Azuma YT, Flavell RA, Liljeström P, and e Sousa CR. Toll-like receptor 3 promotes crosspriming to virus-infected cells. Nature, 433(7028):887-92, 2005.

[290] Doxsee CL, Riter TR, Reiter MJ, Gibson SJ, Vasilakos JP, and Kedl RM. The immune response modifier and Toll-like receptor 7 agonist S-27609 selectively induces IL-12 and TNF-alpha production in CD11c+CD11b+CD8- dendritic cells. J Immunol, 171(3):1156-63, 2003.

[291] Edwards AD, Diebold SS, Slack EMC, Tomizawa H, Hemmi H, Kaisho T, Akira S, and e Sousa CR. Toll-like receptor expression in murine DC subsets: lack of TLR7 expression by CD8 alpha+ DC correlates with unresponsiveness to imidazoquinolines. Eur $J$ Immunol, 33(4):827-33, 2003. 
[292] Merad M, Ginhoux F, and Collin M. Origin, homeostasis and function of Langerhans cells and other langerin-expressing dendritic cells. Nat Rev Immunol, 8(12):935-47, 2008.

[293] Valladeau J, Ravel O, Dezutter-Dambuyant C, Moore K, Kleijmeer M, Liu Y, Duvert-Frances V, Vincent C, Schmitt D, Davoust J, Caux C, Lebecque S, and Saeland S. Langerin, a novel C-type lectin specific to Langerhans cells, is an endocytic receptor that induces the formation of Birbeck granules. Immunity, 12(1):71-81, 2000.

[294] Bursch LS, Wang L, Igyarto B, Kissenpfennig A, Malissen B, Kaplan DH, and Hogquist KA. Identification of a novel population of Langerin+ dendritic cells. J Exp Med, 204(13):3147-56, 2007.

[295] Ginhoux F, Collin MP, Bogunovic M, Abel M, Leboeuf M, Helft J, Ochando J, Kissenpfennig A, Malissen B, Grisotto M, Snoeck H, Randolph G, and Merad M. Blood-derived dermal langerin+ dendritic cells survey the skin in the steady state. J Exp Med, 204(13):3133-46, 2007.

[296] Allan RS, Waithman J, Bedoui S, Jones CM, Villadangos JA, Zhan Y, Lew AM, Shortman K, Heath WR, and Carbone FR. Migratory dendritic cells transfer antigen to a lymph node-resident dendritic cell population for efficient CTL priming. Immunity, 25(1):15362, 2006.

[297] Colonna M, Trinchieri G, and Liu YJ. Plasmacytoid dendritic cells in immunity. Nat Immunol, 5(12):1219-26, 2004.

[298] Liu YJ. IPC: professional type 1 interferon-producing cells and plasmacytoid dendritic cell precursors. Annu Rev Immunol, 23:275-306, 2005.

[299] Iwasaki A and Medzhitov R. Regulation of adaptive immunity by the innate immune system. Science, 327(5963):291-5, 2010.

[300] Grouard G, Rissoan MC, Filgueira L, Durand I, Banchereau J, and Liu YJ. The enigmatic plasmacytoid T cells develop into dendritic cells with interleukin (IL)-3 and CD40ligand. J Exp Med, 185(6):1101-11, 1997.

[301] Inaba K, Inaba M, Romani N, Aya H, Deguchi M, Ikehara S, Muramatsu S, and Steinman RM. Generation of large numbers of dendritic cells from mouse bone marrow cultures supplemented with granulocyte/macrophage colony-stimulating factor. J Exp Med, 176(6):1693-702, 1992.

[302] Thurner B, Haendle I, Röder C, Dieckmann D, Keikavoussi P, Jonuleit H, Ben$\operatorname{der}$ A, Maczek C, Schreiner D, von den Driesch P, Bröcker EB, Steinman RM, Enk A, Kämpgen E, and Schuler G. Vaccination with mage-3A1 peptide-pulsed mature, monocyte-derived dendritic cells expands specific cytotoxic $\mathrm{T}$ cells and induces regression of some metastases in advanced stage IV melanoma. J Exp Med, 190(11):1669-78, 1999.

[303] O'Rourke MGE, Johnson M, Lanagan C, See J, Yang J, Bell JR, Slater GJ, Kerr BM, Crowe B, Purdie DM, Elliott SL, Ellem KAO, and Schmidt CW. Durable complete clinical responses in a phase I/II trial using an autologous melanoma cell/dendritic cell vaccine. Cancer Immunol Immunother, 52(6):387-95, 2003.

[304] Avigan D, Vasir B, Gong J, Borges V, Wu Z, Uhl L, Atkins M, Mier J, McDermott D, Smith T, Giallambardo N, Stone C, Schadt K, Dolgoff J, Tetreault JC, Villarroel M, and Kufe D. Fusion cell vaccination of patients with metastatic breast and renal cancer induces immunological and clinical responses. Clin Cancer Res, 10(14):4699-708, 2004.

[305] Markovic SN, Dietz AB, Greiner CW, Maas ML, Butler GW, Padley DJ, Bulur PA, Allred JB, Creagan ET, Ingle JN, Gastineau DA, and Vuk-Pavlovic S. Preparing clinical-grade myeloid dendritic cells by electroporation-mediated transfection of in vitro amplified tumor-derived mRNA and safety testing in stage IV malignant melanoma. J Transl Med, 4:35, 2006. 
[306] Xu Y, Zhan Y, Lew AM, Naik SH, and Kershaw MH. Differential development of murine dendritic cells by GM-CSF versus Flt3 ligand has implications for inflammation and trafficking. J Immunol, 179(11):7577-84, 2007.

[307] León B, López-Bravo M, and Ardavín C. Monocyte-derived dendritic cells formed at the infection site control the induction of protective $\mathrm{T}$ helper 1 responses against Leishmania. Immunity, 26(4):519-31, 2007.

[308] Nakano H, Lin KL, Yanagita M, Charbonneau C, Cook DN, Kakiuchi T, and Gunn MD. Blood-derived inflammatory dendritic cells in lymph nodes stimulate acute T helper type 1 immune responses. Nat Immunol, 10(4):394-402, 2009.

[309] Zhan Y, Xu Y, Seah S, Brady JL, Carrington EM, Cheers C, Croker BA, Wu L, Villadangos JA, and Lew AM. Resident and monocyte-derived dendritic cells become dominant IL-12 producers under different conditions and signaling pathways. J Immunol, 185(4):2125-33, 2010.

[310] Huang AY, Golumbek P, Ahmadzadeh M, Jaffee E, Pardoll D, and Levitsky H. Role of bone marrow-derived cells in presenting MHC class I-restricted tumor antigens. Science, 264(5161):961-5, 1994 .

[311] Preynat-Seauve O, Schuler P, Contassot E, Beermann F, Huard B, and French LE. Tumor-infiltrating dendritic cells are potent antigen-presenting cells able to activate $\mathrm{T}$ cells and mediate tumor rejection. J Immunol, 176(1):61-7, 2006.

[312] Treilleux I, Blay JY, Bendriss-Vermare N, Ray-Coquard I, Bachelot T, Guastalla JP, Bremond A, Goddard S, Pin JJ, Barthelemy-Dubois C, and Lebecque S. Dendritic cell infiltration and prognosis of early stage breast cancer. Clin Cancer Res, 10(22):7466$74,2004$.

[313] Sandel MH, Dadabayev AR, Menon AG, Morreau H, Melief CJM, Offringa R, van der Burg SH, van Rhijn CMJ, Ensink NG, Tollenaar RAEM, van de Velde CJH, and Kuppen PJK. Prognostic value of tumor-infiltrating dendritic cells in colorectal cancer: role of maturation status and intratumoral localization. Clin Cancer Res, 11(7):257682,2005 .

[314] Stoitzner P, Green LK, Jung JY, Price KM, Atarea H, Kivell B, and Ronchese F. Inefficient presentation of tumor-derived antigen by tumor-infiltrating dendritic cells. Cancer Immunol Immunother, 57(11):1665-73, 2008.

[315] Vermi W, Bonecchi R, Facchetti F, Bianchi D, Sozzani S, Festa S, Berenzi A, Cella M, and Colonna M. Recruitment of immature plasmacytoid dendritic cells (plasmacytoid monocytes) and myeloid dendritic cells in primary cutaneous melanomas. J Pathol, 200(2):255$68,2003$.

[316] Labidi-Galy SI, Sisirak V, Meeus P, Gobert M, Treilleux I, Bajard A, Combes JD, Faget J, Mithieux F, Cassignol A, Tredan O, Durand I, Ménétrier-Caux C, Caux C, Blay JY, Ray-Coquard I, and Bendriss-Vermare N. Quantitative and functional alterations of plasmacytoid dendritic cells contribute to immune tolerance in ovarian cancer. Cancer Res, 71(16):5423-34, 2011.

[317] Ladányi A, Kiss J, Somlai B, Gilde K, Fejos Z, Mohos A, Gaudi I, and Tímár J. Density of DC-LAMP $(+)$ mature dendritic cells in combination with activated $\mathrm{T}$ lymphocytes infiltrating primary cutaneous melanoma is a strong independent prognostic factor. Cancer Immunol Immunother, 56(9):1459-69, 2007.

[318] Furumoto K, Soares L, Engleman EG, and Merad M. Induction of potent antitumor immunity by in situ targeting of intratumoral DCs. J Clin Invest, 113(5):774-83, 2004.

[319] Chaux P, Hammann A, Martin F, and Martin M. Surface phenotype and functions of tumor-infiltrating dendritic cells: CD8 expression by a cell subpopulation. Eur J Immunol, 23(10):2517-25, 1993. 
[320] Gabrilovich DI, Ciernik IF, and Carbone DP. Dendritic cells in antitumor immune responses. I. Defective antigen presentation in tumor-bearing hosts. Cell Immunol, 170(1):101$10,1996$.

[321] Preynat-Seauve O, Contassot E, Schuler P, Piguet V, French LE, and Huard B. Extralymphatic tumors prepare draining lymph nodes to invasion via a T-cell cross-tolerance process. Cancer Res, 67(10):5009-16, 2007.

[322] Vicari AP, Chiodoni C, Vaure C, Ait-Yahia S, Dercamp C, Matsos F, Reynard O, Taverne C, Merle P, Colombo MP, O'Garra A, Trinchieri G, and Caux C. Reversal of tumor-induced dendritic cell paralysis by CpG immunostimulatory oligonucleotide and anti-interleukin 10 receptor antibody. J Exp Med, 196(4):541-9, 2002.

[323] Zou W, Machelon V, Coulomb-L'Hermin A, Borvak J, Nome F, Isaeva T, Wei S, Krzysiek R, Durand-Gasselin I, Gordon A, Pustilnik T, Curiel DT, Galanaud P, Capron F, Emilie D, and Curiel TJ. Stromal-derived factor-1 in human tumors recruits and alters the function of plasmacytoid precursor dendritic cells. Nat Med, 7(12):1339-46, 2001.

[324] Pinzon-Charry A, Maxwell T, and López JA. Dendritic cell dysfunction in cancer: a mechanism for immunosuppression. Immunol Cell Biol, 83(5):451-61, 2005.

[325] Liu Q, Zhang C, Sun A, Zheng Y, Wang L, and Cao X. Tumor-educated CD11bhighIalow regulatory dendritic cells suppress $\mathrm{T}$ cell response through arginase I. $J$ Immunol, 182(10):6207-16, 2009.

[326] von Bergwelt-Baildon MS, Popov A, Saric T, Chemnitz J, Classen S, Stoffel MS, Fiore F, Roth U, Beyer M, Debey S, Wickenhauser C, Hanisch FG, and Schultze JL. CD25 and indoleamine 2,3-dioxygenase are up-regulated by prostaglandin E2 and expressed by tumor-associated dendritic cells in vivo: additional mechanisms of T-cell inhibition. Blood, 108(1):228-37, 2006.

[327] Freund J. The mode of action of immunologic adjuvants. Bibl Tuberc, 10:130-48, 1956.

[328] Bafica A, Scanga CA, Feng CG, Leifer C, Cheever A, and Sher A. TLR9 regulates Th1 responses and cooperates with TLR2 in mediating optimal resistance to Mycobacterium tuberculosis. J Exp Med, 202(12):1715-24, 2005.

[329] Ferwerda G, Girardin SE, Kullberg BJ, Bourhis LL, de Jong DJ, Langenberg DML, van Crevel R, Adema GJ, Ottenhoff THM, der Meer JWMV, and Netea MG. NOD2 and toll-like receptors are nonredundant recognition systems of Mycobacterium tuberculosis. PLoS Pathog, 1(3):279-85, 2005.

[330] Kang JY, Nan X, Jin MS, Youn SJ, Ryu YH, Mah S, Han SH, Lee H, Paik SG, and Lee JO. Recognition of lipopeptide patterns by Toll-like receptor 2-Toll-like receptor 6 heterodimer. Immunity, 31(6):873-84, 2009.

[331] Jin MS, Kim SE, Heo JY, Lee ME, Kim HM, Paik SG, Lee H, and Lee JO. Crystal structure of the TLR1-TLR2 heterodimer induced by binding of a tri-acylated lipopeptide. Cell, 130(6):1071-82, 2007.

[332] Beutler B. Tlr4: central component of the sole mammalian LPS sensor. Curr Opin Immunol, 12(1):20-6, 2000.

[333] Smith KD, Andersen-Nissen E, Hayashi F, Strobe K, Bergman MA, Barrett SLR, Cookson BT, and Aderem A. Toll-like receptor 5 recognizes a conserved site on flagellin required for protofilament formation and bacterial motility. Nat Immunol, 4(12):1247-53, 2003.

[334] Yarovinsky F, Zhang D, Andersen JF, Bannenberg GL, Serhan CN, Hayden MS, Hieny S, Sutterwala FS, Flavell RA, Ghosh S, and Sher A. TLR11 activation of dendritic cells by a protozoan profilin-like protein. Science, 308(5728):1626-9, 2005. 
[335] Alexopoulou L, Holt AC, Medzhitov R, and Flavell RA. Recognition of doublestranded RNA and activation of NF-kappaB by Toll-like receptor 3. Nature, 413(6857):732-8, 2001.

[336] Diebold SS, Kaisho T, Hemmi H, Akira S, and e Sousa CR. Innate antiviral responses by means of TLR7-mediated recognition of single-stranded RNA. Science, 303(5663):1529-31, 2004.

[337] Heil F, Hemmi H, Hochrein H, Ampenberger F, Kirschning C, Akira S, Lipford G, Wagner H, and Bauer S. Species-specific recognition of single-stranded RNA via toll-like receptor 7 and 8. Science, 303(5663):1526-9, 2004.

[338] Lund JM, Alexopoulou L, Sato A, Karow M, Adams NC, Gale NW, Iwasaki A, and Flavell RA. Recognition of single-stranded RNA viruses by Toll-like receptor 7 . Proc Natl Acad Sci USA, 101(15):5598-603, 2004.

[339] Hemmi H, Takeuchi O, Kawai T, Kaisho T, Sato S, Sanjo H, Matsumoto M, Hoshino K, Wagner H, Takeda K, and Akira S. A Toll-like receptor recognizes bacterial DNA. Nature, 408(6813):740-5, 2000.

[340] Kawai T and Akira S. TLR signaling. Semin Immunol, 19(1):24-32, 2007.

[341] Dearman RJ, Cumberbatch M, Maxwell G, Basketter DA, and Kimber I. Tolllike receptor ligand activation of murine bone marrow-derived dendritic cells. Immunology, 126(4):475-84, 2009.

[342] Konno R, Dobbelaere KO, Godeaux OO, Tamura S, and Yoshikawa H. Immunogenicity, reactogenicity, and safety of human papillomavirus 16/18 AS04-adjuvanted vaccine in Japanese women: interim analysis of a phase II, double-blind, randomized controlled trial at month 7. Int J Gynecol Cancer, 19(5):905-11, 2009.

[343] Hemmi H, Kaisho T, Takeuchi O, Sato S, Sanjo H, Hoshino K, Horiuchi T, Tomizawa H, Takeda $\mathbf{K}$, and Akira S. Small anti-viral compounds activate immune cells via the TLR7 MyD88-dependent signaling pathway. Nat Immunol, 3(2):196-200, 2002.

[344] Hartmann G, Weeratna RD, Ballas ZK, Payette P, Blackwell S, Suparto I, Rasmussen WL, Waldschmidt M, Sajuthi D, Purcell RH, Davis HL, and Krieg AM. Delineation of a $\mathrm{CpG}$ phosphorothioate oligodeoxynucleotide for activating primate immune responses in vitro and in vivo. J Immunol, 164(3):1617-24, 2000.

[345] Chin AI, Miyahira AK, Covarrubias A, Teague J, Guo B, Dempsey PW, and Cheng G. Toll-like receptor 3-mediated suppression of TRAMP prostate cancer shows the critical role of type I interferons in tumor immune surveillance. Cancer Res, 70(7):2595-603, 2010.

[346] Zhu X, Fallert-Junecko BA, Fujita M, Ueda R, Kohanbash G, Kastenhuber ER, McDonald HA, Liu Y, Kalinski P, Reinhart TA, Salazar AM, and Okada H. PolyICLC promotes the infiltration of effector $\mathrm{T}$ cells into intracranial gliomas via induction of CXCL10 in IFN-alpha and IFN-gamma dependent manners. Cancer Immunol Immunother, 59(9):1401-9, 2010.

[347] Williams A, Flavell RA, and Eisenbarth SC. The role of NOD-like Receptors in shaping adaptive immunity. Curr Opin Immunol, 22(1):34-40, 2010.

[348] Martinon F, Burns K, and Tschopp J. The inflammasome: a molecular platform triggering activation of inflammatory caspases and processing of proIL-beta. Mol Cell, 10(2):417-26, 2002 .

[349] Arend WP, Palmer G, and Gabay C. IL-1, IL-18, and IL-33 families of cytokines. Immunol Rev, 223:20-38, 2008. 
[350] Mariathasan S, Newton K, Monack DM, Vucic D, French DM, Lee WP, RooseGirma M, Erickson S, and Dixit VM. Differential activation of the inflammasome by caspase-1 adaptors ASC and Ipaf. Nature, 430(6996):213-8, 2004.

[351] Sutterwala FS, Mijares LA, Li L, Ogura Y, Kazmierczak BI, and Flavell RA. Immune recognition of Pseudomonas aeruginosa mediated by the IPAF/NLRC4 inflammasome. J Exp Med, 204(13):3235-45, 2007.

[352] Mariathasan S, Weiss DS, Newton K, McBride J, O'Rourke K, Roose-Girma M, Lee WP, Weinrauch Y, Monack DM, and Dixit VM. Cryopyrin activates the inflammasome in response to toxins and ATP. Nature, 440(7081):228-32, 2006.

[353] Martinon F, Pétrilli V, Mayor A, Tardivel A, and Tschopp J. Gout-associated uric acid crystals activate the NALP3 inflammasome. Nature, 440(7081):237-41, 2006.

[354] Eisenbarth SC, Colegio OR, O'Connor W, Sutterwala FS, and Flavell RA. Crucial role for the Nalp3 inflammasome in the immunostimulatory properties of aluminium adjuvants. Nature, 453(7198):1122-6, 2008.

[355] Schroder K, Zhou R, and Tschopp J. The NLRP3 Inflammasome: A Sensor for Metabolic Danger? Science, 327(5963):296-300, 2010.

[356] Zhou R, Tardivel A, Thorens B, Choi I, and Tschopp J. Thioredoxin-interacting protein links oxidative stress to inflammasome activation. Nat Immunol, 11(2):136-40, 2010.

[357] Tada H, Aiba S, Shibata KI, Ohteki T, and Takada H. Synergistic effect of Nod1 and Nod2 agonists with toll-like receptor agonists on human dendritic cells to generate interleukin12 and $\mathrm{T}$ helper type 1 cells. Infect Immun, 73(12):7967-76, 2005.

[358] Asano J, Tada H, Onai N, Sato T, Horie Y, Fujimoto Y, Fukase K, Suzuki A, Mak TW, and Ohteki T. Nucleotide oligomerization binding domain-like receptor signaling enhances dendritic cell-mediated cross-priming in vivo. J Immunol, 184(2):736-45, 2010.

[359] Guarda G, Zenger M, Yazdi AS, Schroder K, Ferrero I, Menu P, Tardivel A, Mattmann C, and Tschopp J. Differential expression of NLRP3 among hematopoietic cells. J Immunol, 186(4):2529-34, 2011.

[360] Sakamaki I, Inai K, and Tsutani H. Safety of intradermal injection of monosodium urate crystals as a vaccine carrier in volunteers. Nucleos Nucleot Nucl, 30(12):1077-1084, 2011.

[361] Hu DE, Moore AM, Thomsen LL, and Brindle KM. Uric acid promotes tumor immune rejection. Cancer Res, 64(15):5059-62, 2004.

[362] Yoneyama M, Kikuchi M, Natsukawa T, Shinobu N, Imaizumi T, Miyagishi M, Taira K, Akira S, and Fujita T. The RNA helicase RIG-I has an essential function in double-stranded RNA-induced innate antiviral responses. Nat Immunol, 5(7):730-7, 2004.

[363] Hornung V, Ellegast J, Kim S, Brzózka K, Jung A, Kato H, Poeck H, Akira S, Conzelmann KK, Schlee M, Endres S, and Hartmann G. 5'-Triphosphate RNA is the ligand for RIG-I. Science, 314(5801):994-7, 2006.

[364] Yoneyama M, Kikuchi M, Matsumoto K, Imaizumi T, Miyagishi M, Taira K, Foy E, Loo YM, Gale M, Akira S, Yonehara S, Kato A, and Fujita T. Shared and unique functions of the DExD/H-box helicases RIG-I, MDA5, and LGP2 in antiviral innate immunity. J Immunol, 175(5):2851-8, 2005.

[365] Baril M, Racine ME, Penin F, and Lamarre D. MAVS dimer is a crucial signaling component of innate immunity and the target of hepatitis C virus NS3/4A protease. J Virol, 83(3):1299-311, 2009.

[366] Honda K and Taniguchi T. IRFs: master regulators of signalling by Toll-like receptors and cytosolic pattern-recognition receptors. Nat Rev Immunol, 6(9):644-58, 2006. 
[367] Kato H, Takeuchi O, Mikamo-Satoh E, Hirai R, Kawai T, Matsushita K, Hiiragi A, Dermody TS, Fujita T, and Akira S. Length-dependent recognition of double-stranded ribonucleic acids by retinoic acid-inducible gene-I and melanoma differentiation-associated gene 5. J Exp Med, 205(7):1601-10, 2008.

[368] Jelinek I, Leonard JN, Price GE, Brown KN, Meyer-Manlapat A, Goldsmith PK, Wang Y, Venzon D, Epstein SL, and Segal DM. TLR3-specific double-stranded RNA oligonucleotide adjuvants induce dendritic cell cross-presentation, CTL responses, and antiviral protection. J Immunol, 186(4):2422-9, 2011.

[369] Kumar H, Koyama S, Ishii KJ, Kawai T, and Akira S. Cutting edge: cooperation of IPS-1- and TRIF-dependent pathways in poly IC-enhanced antibody production and cytotoxic T cell responses. J Immunol, 180(2):683-7, 2008.

[370] Wang Y, Cella M, Gilfillan S, and Colonna M. Cutting edge: polyinosinic:polycytidylic acid boosts the generation of memory CD8 T cells through melanoma differentiation-associated protein 5 expressed in stromal cells. J Immunol, 184(6):2751-5, 2010.

[371] Robinson MJ, Osorio F, Rosas M, Freitas RP, Schweighoffer E, Gross O, Verbeek JS, Ruland J, Tybulewicz V, Brown GD, Moita LF, Taylor PR, and e Sousa CR. Dectin-2 is a Syk-coupled pattern recognition receptor crucial for Th17 responses to fungal infection. J Exp Med, 206(9):2037-51, 2009

[372] Conti HR, Shen F, Nayyar N, Stocum E, Sun JN, Lindemann MJ, Ho AW, Hai JH, Yu JJ, Jung JW, Filler SG, Masso-Welch P, Edgerton M, and Gaffen SL. Th17 cells and IL-17 receptor signaling are essential for mucosal host defense against oral candidiasis. J Exp Med, 206(2):299-311, 2009.

[373] Agrawal S, Agrawal A, Doughty B, Gerwitz A, Blenis J, Dyke TV, and Pulendran B. Cutting edge: different Toll-like receptor agonists instruct dendritic cells to induce distinct Th responses via differential modulation of extracellular signal-regulated kinase-mitogenactivated protein kinase and c-Fos. J Immunol, 171(10):4984-9, 2003.

[374] Iwasaki A and Medzhitov R. Toll-like receptor control of the adaptive immune responses. Nat Immunol, 5(10):987-95, 2004.

[375] Fritz JH, Bourhis LL, Sellge G, Magalhaes JG, Fsihi H, Kufer TA, Collins C, Viala J, Ferrero RL, Girardin SE, and Philpott DJ. Nod1-mediated innate immune recognition of peptidoglycan contributes to the onset of adaptive immunity. Immunity, 26(4):445-59, 2007.

[376] Ishii KJ, Kawagoe T, Koyama S, Matsui K, Kumar H, Kawai T, Uematsu S, Takeuchi O, Takeshita F, Coban C, and Akira S. TANK-binding kinase-1 delineates innate and adaptive immune responses to DNA vaccines. Nature, 451(7179):725-9, 2008.

[377] Napolitani G, Rinaldi A, Bertoni F, Sallusto F, and Lanzavecchia A. Selected Tolllike receptor agonist combinations synergistically trigger a $\mathrm{T}$ helper type 1-polarizing program in dendritic cells. Nat Immunol, 6(8):769-76, 2005.

[378] Zhu Q, Egelston C, Vivekanandhan A, Uematsu S, Akira S, Klinman DM, Belyakov IM, and Berzofsky JA. Toll-like receptor ligands synergize through distinct dendritic cell pathways to induce T cell responses: implications for vaccines. Proc Natl Acad Sci USA, 105(42):16260-5, 2008.

[379] Luft T, Jefford M, Luetjens P, Toy T, Hochrein H, Masterman KA, Maliszewski CR, Shortman K, Cebon J, and Maraskovsky E. Functionally distinct dendritic cell (DC) populations induced by physiologic stimuli: prostaglandin $\mathrm{E}(2)$ regulates the migratory capacity of specific DC subsets. Blood, 100(4):1362-72, 2002.

[380] Camporeale A, Boni A, Iezzi G, Degl'Innocenti E, Grioni M, Mondino A, and Bellone M. Critical impact of the kinetics of dendritic cells activation on the in vivo induction of tumor-specific T lymphocytes. Cancer Res, 63(13):3688-94, 2003. 
[381] Carbone FR, Belz GT, and Heath WR. Transfer of antigen between migrating and lymph node-resident DCs in peripheral T-cell tolerance and immunity. Trends Immunol, 25(12):655-8, 2004 .

[382] Smith CM, Wilson NS, Waithman J, Villadangos JA, Carbone FR, Heath WR, and Belz GT. Cognate CD4(+) T cell licensing of dendritic cells in CD8(+) T cell immunity. Nat Immunol, 5(11):1143-8, 2004.

[383] Vulink A, Radford KJ, Melief CJM, and Hart DNJ. Dendritic cells in cancer immunotherapy. Adv Cancer Res, 99:363-407, 2008.

[384] Overwijk WW, Theoret MR, Finkelstein SE, Surman DR, de Jong LA, VythDreese FA, Dellemijn TA, Antony PA, Spiess PJ, Palmer DC, Heimann DM, Klebanoff CA, Yu Z, Hwang LN, Feigenbaum L, Kruisbeek AM, Rosenberg SA, and Restifo NP. Tumor regression and autoimmunity after reversal of a functionally tolerant state of self-reactive CD8+ T cells. J Exp Med, 198(4):569-80, 2003.

[385] Perret R and Ronchese F. Effector CD8+ T cells activated in vitro confer immediate and long-term tumor protection in vivo. Eur. J. Immunol., 38(10):2886-95, 2008.

[386] Rosenberg S, Packard B, Aebersold P, Solomon D, Topalian S, Toy S, Simon P, Lotze M, Yang J, Seipp C, Simpson C, Carter C, Bock S, Schwartzentruber D, Wei J, and White D. Use of tumour-infiltrating lymphocytes and interleukin-2 in the immunotherapy of patients with metastatic melanoma - a preliminary report. New Engl J Med, 319(25):1676-1680, 1988.

[387] Dudley ME, Wunderlich JR, Robbins PF, Yang JC, Hwu P, Schwartzentruber DJ, Topalian SL, Sherry R, Restifo NP, Hubicki AM, Robinson MR, Raffeld M, Duray P, Seipp CA, Rogers-Freezer L, Morton KE, Mavroukakis SA, White DE, and Rosenberg SA. Cancer regression and autoimmunity in patients after clonal repopulation with antitumor lymphocytes. Science, 298(5594):850-4, 2002.

[388] Gattinoni L, Finkelstein SE, Klebanoff CA, Antony PA, Palmer DC, Spiess PJ, Hwang LN, Yu Z, Wrzesinski C, Heimann DM, Surh CD, Rosenberg SA, and Restifo NP. Removal of homeostatic cytokine sinks by lymphodepletion enhances the efficacy of adoptively transferred tumor-specific CD8+ T cells. J Exp Med, 202(7):907-12, 2005.

[389] Morgan RA, Dudley ME, Wunderlich JR, Hughes MS, Yang JC, Sherry RM, Royal RE, Topalian SL, Kammula US, Restifo NP, Zheng Z, Nahvi A, de Vries CR, Rogers-Freezer LJ, Mavroukakis SA, and Rosenberg SA. Cancer regression in patients after transfer of genetically engineered lymphocytes. Science, 314(5796):126-9, 2006.

[390] Sallusto F and Lanzavecchia A. Efficient presentation of soluble antigen by cultured human dendritic cells is maintained by granulocyte/macrophage colony-stimulating factor plus interleukin 4 and downregulated by tumor necrosis factor alpha. J Exp Med, 179(4):1109-18, 1994.

[391] López MN, Pereda C, Segal G, Muñoz L, Aguilera R, González FE, Escobar A, Ginesta A, Reyes D, González R, Mendoza-Naranjo A, Larrondo M, Compán A, Ferrada C, and Salazar-Onfray F. Prolonged survival of dendritic cell-vaccinated melanoma patients correlates with tumor-specific delayed type IV hypersensitivity response and reduction of tumor growth factor beta-expressing T cells. J Clin Oncol, 27(6):945-52, 2009 .

[392] Timmerman JM, Czerwinski DK, Davis TA, Hsu FJ, Benike C, Hao ZM, Taidi B, Rajapaksa R, Caspar CB, Okada CY, van Beckhoven A, Liles TM, Engleman EG, and Levy R. Idiotype-pulsed dendritic cell vaccination for B-cell lymphoma: clinical and immune responses in 35 patients. Blood, 99(5):1517-26, 2002.

[393] Kantoff PW, Higano CS, Shore ND, Berger ER, Small EJ, Penson DF, Redfern CH, Ferrari AC, Dreicer R, Sims RB, Xu Y, Frohlich MW, Schellhammer PF, and 
Investigators IS. Sipuleucel-T immunotherapy for castration-resistant prostate cancer. The New England journal of medicine, 363(5):411-22, 2010.

[394] Leach DR, Krummel MF, and Allison JP. Enhancement of antitumor immunity by CTLA-4 blockade. Science, 271(5256):1734-6, 1996.

[395] van Elsas A, Hurwitz AA, and Allison JP. Combination immunotherapy of B16 melanoma using anti-cytotoxic T lymphocyte-associated antigen 4 (CTLA-4) and granulocyte/macrophage colony-stimulating factor (GM-CSF)-producing vaccines induces rejection of subcutaneous and metastatic tumors accompanied by autoimmune depigmentation. $J$ Exp Med, 190(3):355-66, 1999.

[396] Brahmer JR, Tykodi SS, Chow LQM, Hwu WJ, Topalian SL, Hwu P, Drake CG, Camacho LH, Kauh J, Odunsi K, Pitot HC, Hamid O, Bhatia S, Martins R, Eaton K, Chen S, Salay TM, Alaparthy S, Grosso JF, Korman AJ, Parker SM, Agrawal S, Goldberg SM, Pardoll DM, Gupta A, and Wigginton JM. Safety and Activity of Anti-PD-L1 Antibody in Patients with Advanced Cancer. The New England journal of medicine, 2012.

[397] Fyfe G, Fisher R, Rosenberg SA, Sznol M, Parkinson D, and Louie A. Results of the treatment of 255 patients with metastatic renal-cell carcinoma who received high-dose recombinant interleukin-2 therapy. J Clin Oncol, 13(3):688-696, 1995.

[398] Smith FO, Downey SG, Klapper JA, Yang JC, Sherry RM, Royal RE, Kammula US, Hughes MS, Restifo NP, Levy CL, White DE, Steinberg SM, and Rosenberg SA. Treatment of metastatic melanoma using interleukin-2 alone or in conjunction with vaccines. Clin Cancer Res, 14(17):5610-5618, 2008.

[399] McDermott DF. The application of high-dose interleukin-2 for metastatic renal cell carcinoma. Med Oncol, 26:13-17, 2009.

[400] Bekaii-Saab TS, Roda JM, Guenterberg KD, Ramaswamy B, Young DC, Ferketich AK, Lamb TA, Grever MR, Shapiro CL, and Carson WE. A phase I trial of paclitaxel and trastuzumab in combination with interleukin-12 in patients with HER2/neu-expressing malignancies. Mol Cancer Ther, 8(11):2983-2991, 2009.

[401] Chade DC, Shariat SF, and Dalbagni G. Intravesical Therapy for Urothelial Carcinoma of the Urinary Bladder: A Critical Review. Int Braz J Urol, 35(6):640-650, 2009.

[402] Biot C, Rentsch CA, Gsponer JR, Birkhäuser FD, Jusforgues-Saklani H, Lemaître F, Auriau C, Bachmann A, Bousso P, Demangel C, Peduto L, Thalmann GN, and Albert ML. Preexisting BCG-Specific T Cells Improve Intravesical Immunotherapy for Bladder Cancer. Science Translational Medicine, 4(137):137ra72, 2012.

[403] Kunikata N, Sano K, Honda M, Ishii K, Matsunaga J, Okuyama R, Takahashi K, Watanabe H, Tamura G, Tagami H, and Terui T. Peritumoral CpG oligodeoxynucleotide treatment inhibits tumor growth and metastasis of B16F10 melanoma cells. J Invest Dermatol, 123(2):395-402, 2004.

[404] Getting SJ, Flower RJ, Parente L, de Medicis R, Lussier A, Woliztky BA, Martins MA, and Perretti M. Molecular determinants of monosodium urate crystal-induced murine peritonitis: a role for endogenous mast cells and a distinct requirement for endothelial-derived selectins. J Pharmacol Exp Ther, 283(1):123-30, 1997.

[405] Lugade AA, Moran JP, Gerber SA, Rose RC, Frelinger JG, and Lord EM. Local radiation therapy of B16 melanoma tumors increases the generation of tumor antigen-specific effector cells that traffic to the tumor. J Immunol, 174(12):7516-23, 2005.

[406] Moore MW, Carbone FR, and Bevan MJ. Introduction of soluble protein into the class I pathway of antigen processing and presentation. Cell, 54(6):777-85, 1988. 
[407] Aslakson CJ and Miller FR. Selective events in the metastatic process defined by analysis of the sequential dissemination of subpopulations of a mouse mammary tumor. Cancer Res, 52(6):1399-405, 1992.

[408] Stockert E, Boyse EA, Obata Y, Ikeda H, Sarkar NH, and Hoffman HA. New mutant and congenic mouse stocks expressing the murine leukemia virus-associated thymocyte surface antigen GIX. J Exp Med, 142(2):512-7, 1975.

[409] Hogquist KA, Jameson SC, Heath WR, Howard JL, Bevan MJ, and Carbone FR. T cell receptor antagonist peptides induce positive selection. Cell, 76(1):17-27, 1994.

[410] Barnden MJ, Allison J, Heath WR, and Carbone FR. Defective TCR expression in transgenic mice constructed using cDNA-based alpha- and beta-chain genes under the control of heterologous regulatory elements. Immunol Cell Biol, 76(1):34-40, 1998.

[411] Fontenot JD, Rasmussen JP, Williams LM, Dooley JL, Farr AG, and Rudensky AY. Regulatory $\mathrm{T}$ cell lineage specification by the forkhead transcription factor foxp3. Immunity, 22(3):329-41, 2005.

[412] Mombaerts P, Iacomini J, Johnson RS, Herrup K, Tonegawa S, and Papaioannou VE. RAG-1-deficient mice have no mature B and T lymphocytes. Cell, 68(5):869-77, 1992.

[413] Adam C, King S, Allgeier T, Braumüller H, Lüking C, Mysliwietz J, Kriegeskorte A, Busch DH, Röcken M, and Mocikat R. DC-NK cell cross talk as a novel CD4+ T-cell-independent pathway for antitumor CTL induction. Blood, 106(1):338-44, 2005.

[414] Semmling V, Lukacs-Kornek V, Thaiss CA, Quast T, Hochheiser K, Panzer U, Rossjohn J, Perlmutter P, Cao J, Godfrey DI, Savage PB, Knolle PA, Kolanus W, Förster I, and Kurts C. Alternative cross-priming through CCL17-CCR4-mediated attraction of CTLs toward NKT cell-licensed DCs. Nat Immunol, 11(4):313-20, 2010.

[415] Castellino F, Huang AY, Altan-Bonnet G, Stoll S, Scheinecker C, and Germain RN. Chemokines enhance immunity by guiding naive CD8+ $\mathrm{T}$ cells to sites of CD4+ T cell-dendritic cell interaction. Nature, 440(7086):890-5, 2006.

[416] Hedayat M, Netea MG, and Rezaei N. Targeting of Toll-like receptors: a decade of progress in combating infectious diseases. Lancet Infect Dis, 11(9):702-12, 2011.

[417] Shortman K and Naik SH. Steady-state and inflammatory dendritic-cell development. Nat Rev Immunol, 7(1):19-30, 2007.

[418] Vremec D, Lieschke GJ, Dunn AR, Robb L, Metcalf D, and Shortman K. The influence of granulocyte/macrophage colony-stimulating factor on dendritic cell levels in mouse lymphoid organs. Eur J Immunol, 27(1):40-4, 1997.

[419] McKenna HJ, Stocking KL, Miller RE, Brasel K, Smedt TD, Maraskovsky E, Maliszewski CR, Lynch DH, Smith J, Pulendran B, Roux ER, Teepe M, Lyman SD, and Peschon JJ. Mice lacking flt3 ligand have deficient hematopoiesis affecting hematopoietic progenitor cells, dendritic cells, and natural killer cells. Blood, 95(11):3489-97, 2000.

[420] Brasel K, Smedt TD, Smith JL, and Maliszewski CR. Generation of murine dendritic cells from flt3-ligand-supplemented bone marrow cultures. Blood, 96(9):3029-39, 2000.

[421] Naik SH, Sathe P, Park HY, Metcalf D, Proietto AI, Dakic A, Carotta S, O'Keeffe M, Bahlo M, Papenfuss A, Kwak JY, Wu L, and Shortman K. Development of plasmacytoid and conventional dendritic cell subtypes from single precursor cells derived in vitro and in vivo. Nat Immunol, 8(11):1217-26, 2007.

[422] Naik SH, Proietto AI, Wilson NS, Dakic A, Schnorrer P, Fuchsberger M, Lahoud MH, O'Keeffe M, xiang Shao Q, feng Chen W, Villadangos JA, Shortman K, and Wu L. Cutting edge: generation of splenic CD8+ and CD8- dendritic cell equivalents in Fms-like tyrosine kinase 3 ligand bone marrow cultures. J Immunol, 174(11):6592-7, 2005. 
[423] Jiang HR, Muckersie E, Robertson M, Xu H, Liversidge J, and Forrester JV. Secretion of interleukin-10 or interleukin-12 by LPS-activated dendritic cells is critically dependent on time of stimulus relative to initiation of purified DC culture. J Leukoc Biol, 72(5):978-85, 2002.

[424] Martin WJ, Shaw O, Liu X, Steiger S, and Harper JL. MSU crystal-recruited noninflammatory monocytes differentiate into M1-like pro-inflammatory macrophages in a peritoneal murine model of gout. Arthritis Rheum, 2011.

[425] Becker HM, Chen M, Hay JB, and Cybulsky MI. Tracking of leukocyte recruitment into tissues of mice by in situ labeling of blood cells with the fluorescent dye CFDA SE. J Immunol Methods, 286(1-2):69-78, 2004.

[426] Street D, Kaufmann AM, Vaughan A, Fisher SG, Hunter M, Schreckenberger C, Potkul RK, Gissmann L, and Qiao L. Interferon-gamma enhances susceptibility of cervical cancer cells to lysis by tumor-specific cytotoxic T cells. Gynecol Oncol, 65(2):265-72, 1997.

[427] Douvas GS, Looker DL, Vatter AE, and Crowle AJ. Gamma interferon activates human macrophages to become tumoricidal and leishmanicidal but enhances replication of macrophage-associated mycobacteria. Infect Immun, 50(1):1-8, 1985.

[428] Brawand P, Fitzpatrick DR, Greenfield BW, Brasel K, Maliszewski CR, and Smedt TD. Murine plasmacytoid pre-dendritic cells generated from Flt3 ligand-supplemented bone marrow cultures are immature APCs. J Immunol, 169(12):6711-9, 2002.

[429] Langenkamp A, Messi M, Lanzavecchia A, and Sallusto F. Kinetics of dendritic cell activation: impact on priming of TH1, TH2 and nonpolarized T cells. Nat Immunol, 1(4):3116,2000 .

[430] Martino A, Sacchi A, Volpe E, Agrati C, Santis RD, Pucillo LP, Colizzi V, and Vendetti S. Non-pathogenic Mycobacterium smegmatis induces the differentiation of human monocytes directly into fully mature dendritic cells. J Clin Immunol, 25(4):365-75, 2005.

[431] Sutterwala FS, Ogura Y, Szczepanik M, Lara-Tejero M, Lichtenberger GS, Grant EP, Bertin J, Coyle AJ, Galán JE, Askenase PW, and Flavell RA. Critical role for NALP3/CIAS1/Cryopyrin in innate and adaptive immunity through its regulation of caspase1. Immunity, 24(3):317-27, 2006.

[432] Netea MG, Nold-Petry CA, Nold MF, Joosten LAB, Opitz B, van der Meer JHM, van de Veerdonk FL, Ferwerda G, Heinhuis B, Devesa I, Funk CJ, Mason RJ, Kullberg BJ, Rubartelli A, der Meer JWMV, and Dinarello CA. Differential requirement for the activation of the inflammasome for processing and release of IL-1beta in monocytes and macrophages. Blood, 113(10):2324-35, 2009.

[433] Netea MG, van de Veerdonk FL, Kullberg BJ, der Meer JWMV, and Joosten LAB. The role of NLRs and TLRs in the activation of the inflammasome. Expert Opin Biol Ther, 8(12):1867-72, 2008.

[434] Petterson T, Jendholm J, Månsson A, Bjartell A, Riesbeck K, and Cardell LO. Effects of NOD-like receptors in human B lymphocytes and crosstalk between NOD1/NOD2 and Toll-like receptors. J Leukoc Biol, 89(2):177-87, 2011.

[435] Conejo-Garcia JR, Benencia F, Courreges MC, Kang E, Mohamed-Hadley A, Buckanovich RJ, Holtz DO, Jenkins A, Na H, Zhang L, Wagner DS, Katsaros D, Caroll R, and Coukos G. Tumor-infiltrating dendritic cell precursors recruited by a betadefensin contribute to vasculogenesis under the influence of Vegf-A. Nat Med, 10(9):950-8, 2004.

[436] Ciavarra RP, Brown RR, Holterman DA, Garrett M, Glass WF, Wright GL, Schellhammer PF, and Somers KD. Impact of the tumor microenvironment on host 
infiltrating cells and the efficacy of flt3-ligand combination immunotherapy evaluated in a treatment model of mouse prostate cancer. Cancer Immunol Immunother, 52(9):535-45, 2003.

[437] Aspord C, Pedroza-Gonzalez A, Gallegos M, Tindle S, Burton EC, Su D, Marches F, Banchereau J, and Palucka AK. Breast cancer instructs dendritic cells to prime interleukin 13-secreting CD4+ T cells that facilitate tumor development. J Exp Med, 204(5):1037$47,2007$.

[438] Krempski J, Karyampudi L, Behrens MD, Erskine CL, Hartmann L, Dong H, Goode EL, Kalli KR, and Knutson KL. Tumor-infiltrating programmed death receptor$1+$ dendritic cells mediate immune suppression in ovarian cancer. J Immunol, 186(12):6905-13, 2011.

[439] Wollenberg A, Wagner M, Günther S, Towarowski A, Tuma E, Moderer M, Rothenfusser S, Wetzel S, Endres S, and Hartmann G. Plasmacytoid dendritic cells: a new cutaneous dendritic cell subset with distinct role in inflammatory skin diseases. J Invest Dermatol, 119(5):1096-102, 2002.

[440] Lukens JR, Dolina JS, Kim TS, Tacke RS, and Hahn YS. Liver is able to activate naïve CD8+ $\mathrm{T}$ cells with dysfunctional anti-viral activity in the murine system. PLoS ONE, 4(10):e7619, 2009.

[441] Martin J and White IN. Preparation of rat lung cells for flow cytometry. Methods Mol Biol, 80:363-8, 1992.

[442] Fallowfield ME. Blood flow distribution within transplantable tumours in the mouse. Eur $J$ Cancer Clin Oncol, 25(12):1683-8, 1989.

[443] Chiodoni C, Paglia P, Stoppacciaro A, Rodolfo M, Parenza M, and Colombo MP. Dendritic cells infiltrating tumors cotransduced with granulocyte/macrophage colonystimulating factor (GM-CSF) and CD40 ligand genes take up and present endogenous tumorassociated antigens, and prime naive mice for a cytotoxic T lymphocyte response. J Exp Med, $190(1): 125-33,1999$.

[444] Coffman RL, Sher A, and Seder RA. Vaccine adjuvants: putting innate immunity to work. Immunity, 33(4):492-503, 2010.

[445] Nierkens S, den Brok MH, Roelofsen T, Wagenaars JAL, Figdor CG, Ruers TJ, and Adema GJ. Route of administration of the TLR9 agonist CpG critically determines the efficacy of cancer immunotherapy in mice. PLoS ONE, 4(12):e8368, 2009.

[446] de Vos AF, Pater JM, van den Pangaart PS, de Kruif MD, van 't Veer C, and van der Poll T. In vivo lipopolysaccharide exposure of human blood leukocytes induces cross-tolerance to multiple TLR ligands. J Immunol, 183(1):533-42, 2009.

[447] Ataera H, Hyde E, Price KM, Stoitzner P, and Ronchese F. Murine melanomainfiltrating dendritic cells are defective in antigen presenting function regardless of the presence of CD4CD25 regulatory T cells. PLoS ONE, 6(3):e17515, 2011.

[448] Nelson M and Nelson DS. II. Tumor growth at sites of inflammation induced by mitogens in mice. Am J Pathol, 104(2):125-31, 1981.

[449] Hornung V, Rothenfusser S, Britsch S, Krug A, Jahrsdörfer B, Giese T, Endres S, and Hartmann G. Quantitative expression of toll-like receptor 1-10 mRNA in cellular subsets of human peripheral blood mononuclear cells and sensitivity to CpG oligodeoxynucleotides. $J$ Immunol, 168(9):4531-7, 2002.

[450] van Mierlo GJD, Boonman ZFHM, Dumortier HMH, den Boer AT, Fransen MF, Nouta J, van der Voort EIH, Offringa R, Toes REM, and Melief CJM. Activation of dendritic cells that cross-present tumor-derived antigen licenses CD8+ CTL to cause tumor eradication. J Immunol, 173(11):6753-9, 2004. 
[451] Athie-Morales V, Smits HH, Cantrell DA, and Hilkens CMU. Sustained IL-12 signaling is required for Th1 development. J Immunol, 172(1):61-9, 2004.

[452] Xu S, Koski GK, Faries M, Bedrosian I, Mick R, Maeurer M, Cheever MA, Cohen PA, and Czerniecki BJ. Rapid high efficiency sensitization of CD8 $+\mathrm{T}$ cells to tumor antigens by dendritic cells leads to enhanced functional avidity and direct tumor recognition through an IL-12-dependent mechanism. J Immunol, 171(5):2251-61, 2003.

[453] Gupta S, Boppana R, Mishra GC, Saha B, and Mitra D. Interleukin-12 is necessary for the priming of CD4+ T cells required during the elicitation of HIV-1 gp120-specific cytotoxic T-lymphocyte function. Immunology, 124(4):553-61, 2008.

[454] Böhm W, Thoma S, Leithäuser F, Möller P, Schirmbeck R, and Reimann J. T cellmediated, IFN-gamma-facilitated rejection of murine B16 melanomas. J Immunol, 161(2):897908, 1998.

[455] Parmiani G, Castelli C, Pilla L, Santinami M, Colombo MP, and Rivoltini L. Opposite immune functions of GM-CSF administered as vaccine adjuvant in cancer patients. Ann Oncol, 18(2):226-32, 2007.

[456] Li J, Bouton-Verville H, Holmes LM, Burgin KE, Jakubchak S, Yu X, Wagner TE, and Wei Y. Inhibition or promotion of tumor growth by granulocyte-macrophage colony stimulating factor derived from engineered tumor cells is dose-dependent. Anticancer Res, 24(5A):2717-21, 2004.

[457] Rosenbaum JT and Mandell RB. The effect of endotoxin and endotoxin tolerance on inflammation induced by mycobacterial adjuvant. Yale J Biol Med, 56(4):293-301, 1983.

[458] Rich FJ, Kuhn S, Hyde EJ, Harper JL, Ronchese F, and Kirman JR. Induction of $\mathrm{T}$ cell responses and recruitment of an inflammatory dendritic cell subset following tumor immunotherapy with Mycobacterium smegmatis. Cancer Immunol Immunother, 2012.

[459] Gagliardi MC, Teloni R, Giannoni F, Pardini M, Sargentini V, Brunori L, Fattorini L, and Nisini R. Mycobacterium bovis Bacillus Calmette-Guerin infects DC-SIGN- dendritic cell and causes the inhibition of IL-12 and the enhancement of IL-10 production. J Leukoc Biol, 78(1):106-13, 2005.

[460] Abadie V, Badell E, Douillard P, Ensergueix D, Leenen PJM, Tanguy M, Fiette L, Saeland S, Gicquel B, and Winter N. Neutrophils rapidly migrate via lymphatics after Mycobacterium bovis BCG intradermal vaccination and shuttle live bacilli to the draining lymph nodes. Blood, 106(5):1843-50, 2005.

[461] Begum D, Umemura M, Yahagi A, Okamoto Y, Hamada S, Oshiro K, and Matsuzaki G. Accelerated induction of mycobacterial antigen-specific CD8+ T cells in the Mycobacterium tuberculosis-infected lung by subcutaneous vaccination with Mycobacterium bovis bacille Calmette-Guérin. Immunology, 128(4):556-63, 2009.

[462] Chicoine MR, Won EK, and Zahner MC. Intratumoral injection of lipopolysaccharide causes regression of subcutaneously implanted mouse glioblastoma multiforme. Neurosurgery, 48(3):607-14; discussion 614-5, 2001.

[463] Mariani CL, Rajon D, Bova FJ, and Streit WJ. Nonspecific immunotherapy with intratumoral lipopolysaccharide and zymosan A but not GM-CSF leads to an effective antitumor response in subcutaneous RG-2 gliomas. J Neurooncol, 85(3):231-40, 2007.

[464] Zhang Y, Luo F, Cai Y, Liu N, Wang L, Xu D, and Chu Y. TLR1/TLR2 agonist induces tumor regression by reciprocal modulation of effector and regulatory T cells. J Immunol, 186(4):1963-9, 2011.

[465] Han C, Jin J, Xu S, Liu H, Li N, and Cao X. Integrin CD11b negatively regulates TLRtriggered inflammatory responses by activating Syk and promoting degradation of MyD88 and TRIF via Cbl-b. Nat Immunol, 11(8):734-42, 2010. 
[466] Taylor PR, Brown GD, Geldhof AB, Martinez-Pomares L, and Gordon S. Pattern recognition receptors and differentiation antigens define murine myeloid cell heterogeneity ex vivo. Eur. J. Immunol., 33(8):2090-7, 2003.

[467] Auffray C, Sieweke MH, and Geissmann F. Blood monocytes: development, heterogeneity, and relationship with dendritic cells. Annu Rev Immunol, 27:669-92, 2009.

[468] Borgne ML, Etchart N, Goubier A, Lira SA, Sirard JC, van Rooijen N, Caux C, Aït-Yahia S, Vicari A, Kaiserlian D, and Dubois B. Dendritic cells rapidly recruited into epithelial tissues via CCR6/CCL20 are responsible for CD8+ T cell crosspriming in vivo. Immunity, 24(2):191-201, 2006.

[469] Rotta G, Edwards EW, Sangaletti S, Bennett C, Ronzoni S, Colombo MP, Steinman RM, Randolph GJ, and Rescigno M. Lipopolysaccharide or whole bacteria block the conversion of inflammatory monocytes into dendritic cells in vivo. J Exp Med, 198(8):1253-63, 2003.

[470] Gabrilovich DI and Nagaraj S. Myeloid-derived suppressor cells as regulators of the immune system. Nat Rev Immunol, 9(3):162-74, 2009.

[471] Haile LA, Gamrekelashvili J, Manns MP, Korangy F, and Greten TF. CD49d is a new marker for distinct myeloid-derived suppressor cell subpopulations in mice. J Immunol, 185(1):203-10, 2010.

[472] Zoglmeier C, Bauer H, Nörenberg D, Wedekind G, Bittner P, Sandholzer N, Rapp M, Anz D, Endres S, and Bourquin C. CpG blocks immunosuppression by myeloidderived suppressor cells in tumor-bearing mice. Clin Cancer Res, 17(7):1765-75, 2011.

[473] Swirski FK, Nahrendorf M, Etzrodt M, Wildgruber M, Cortez-Retamozo V, Panizzi P, Figueiredo JL, Kohler RH, Chudnovskiy A, Waterman P, Aikawa E, Mempel TR, Libby P, Weissleder R, and Pittet MJ. Identification of Splenic Reservoir Monocytes and Their Deployment to Inflammatory Sites. Science, 325(5940):612-616, 2009.

[474] Thäle C and Kiderlen AF. Sources of interferon-gamma (IFN-gamma) in early immune response to Listeria monocytogenes. Immunobiology, 210(9):673-83, 2005.

[475] Caramalho I, Lopes-Carvalho T, Ostler D, Zelenay S, Haury M, and Demengeot J. Regulatory $\mathrm{T}$ cells selectively express toll-like receptors and are activated by lipopolysaccharide. J Exp Med, 197(4):403-11, 2003.

[476] Sutmuller RPM, Morgan ME, Netea MG, Grauer O, and Adema GJ. Toll-like receptors on regulatory T cells: expanding immune regulation. Trends Immunol, 27(8):38793, 2006.

[477] Sutmuller RPM, den Brok MHMGM, Kramer M, Bennink EJ, Toonen LWJ, Kullberg BJ, Joosten LA, Akira S, Netea MG, and Adema GJ. Toll-like receptor 2 controls expansion and function of regulatory T cells. J Clin Invest, 116(2):485-94, 2006.

[478] Chuang TH and Ulevitch RJ. Triad3A, an E3 ubiquitin-protein ligase regulating Toll-like receptors. Nat Immunol, 5(5):495-502, 2004.

[479] Kobayashi M, Fitz L, Ryan M, Hewick RM, Clark SC, Chan S, Loudon R, Sherman F, Perussia B, and Trinchieri G. Identification and purification of natural killer cell stimulatory factor (NKSF), a cytokine with multiple biologic effects on human lymphocytes. $J$ Exp Med, 170(3):827-45, 1989.

[480] Thierfelder WE, van Deursen JM, Yamamoto K, Tripp RA, Sarawar SR, Carson RT, Sangster MY, Vignali DA, Doherty PC, Grosveld GC, and Ihle JN. Requirement for Stat4 in interleukin-12-mediated responses of natural killer and T cells. Nature, 382(6587):171-4, 1996.

[481] Tracey KJ and Cerami A. Tumor necrosis factor: a pleiotropic cytokine and therapeutic target. Annu Rev Med, 45:491-503, 1994. 
[482] Snick JV. Interleukin-6: an overview. Annu Rev Immunol, 8:253-78, 1990.

[483] Iyer SS, Ghaffari AA, and Cheng G. Lipopolysaccharide-mediated IL-10 transcriptional regulation requires sequential induction of type I IFNs and IL-27 in macrophages. J Immunol, 185(11):6599-607, 2010.

[484] Bogunovic D, Manches O, Godefroy E, Yewdall A, Gallois A, Salazar AM, Marie I, Levy DE, and Bhardwaj N. TLR4 engagement during TLR3-induced proinflammatory signaling in dendritic cells promotes IL-10-mediated suppression of antitumor immunity. Cancer Res, 71(16):5467-76, 2011.

[485] den Haan JMM, Kraal G, and Bevan MJ. Cutting edge: Lipopolysaccharide induces IL-10-producing regulatory CD4+ T cells that suppress the CD8+ T cell response. J Immunol, 178(9):5429-33, 2007.

[486] Chang J, Kunkel SL, and Chang CH. Negative regulation of MyD88-dependent signaling by IL-10 in dendritic cells. Proc Natl Acad Sci USA, 106(43):18327-32, 2009.

[487] Hamilton JA. GM-CSF in inflammation and autoimmunity. Trends Immunol, 23(8):403-8, 2002.

[488] Sheridan JW and Metcalf D. Studies on the bone marrow colony stimulating factor (CSF): relation of tissue CSF to serum CSF. J Cell Physiol, 80(1):129-40, 1972.

[489] Mach N, Gillessen S, Wilson SB, Sheehan C, Mihm M, and Dranoff G. Differences in dendritic cells stimulated in vivo by tumors engineered to secrete granulocyte-macrophage colony-stimulating factor or Flt3-ligand. Cancer Res, 60(12):3239-46, 2000.

[490] Soiffer R, Lynch T, Mihm M, Jung K, Rhuda C, Schmollinger JC, Hodi FS, Liebster L, Lam P, Mentzer S, Singer S, Tanabe KK, Cosimi AB, Duda R, Sober A, Bhan A, Daley J, Neuberg D, Parry G, Rokovich J, Richards L, Drayer J, Berns A, Clift S, Cohen LK, Mulligan RC, and Dranoff G. Vaccination with irradiated autologous melanoma cells engineered to secrete human granulocyte-macrophage colony-stimulating factor generates potent antitumor immunity in patients with metastatic melanoma. Proc Natl Acad Sci USA, 95(22):13141-6, 1998.

[491] Simons JW, Mikhak B, Chang JF, DeMarzo AM, Carducci MA, Lim M, Weber CE, Baccala AA, Goemann MA, Clift SM, Ando DG, Levitsky HI, Cohen LK, Sanda MG, Mulligan RC, Partin AW, Carter HB, Piantadosi S, Marshall FF, and Nelson WG. Induction of immunity to prostate cancer antigens: results of a clinical trial of vaccination with irradiated autologous prostate tumor cells engineered to secrete granulocytemacrophage colony-stimulating factor using ex vivo gene transfer. Cancer Res, 59(20):5160-8, 1999.

[492] Scheibenbogen C, Schmittel A, Keilholz U, Allgäuer T, Hofmann U, Max R, Thiel E, and Schadendorf D. Phase 2 trial of vaccination with tyrosinase peptides and granulocytemacrophage colony-stimulating factor in patients with metastatic melanoma. J Immunother, $23(2): 275-81,2000$.

[493] Ullenhag GJ, Frödin JE, Jeddi-Tehrani M, Strigård K, Eriksson E, Samanci A, Choudhury A, Nilsson B, Rossmann ED, Mosolits S, and Mellstedt H. Durable carcinoembryonic antigen (CEA)-specific humoral and cellular immune responses in colorectal carcinoma patients vaccinated with recombinant CEA and granulocyte/macrophage colonystimulating factor. Clin Cancer Res, 10(10):3273-81, 2004.

[494] von Mehren M, Arlen P, Gulley J, Rogatko A, Cooper HS, Meropol NJ, Alpaugh RK, Davey M, McLaughlin S, Beard MT, Tsang KY, Schlom J, and Weiner LM. The influence of granulocyte macrophage colony-stimulating factor and prior chemotherapy on the immunological response to a vaccine (ALVAC-CEA B7.1) in patients with metastatic carcinoma. Clin Cancer Res, 7(5):1181-91, 2001. 
[495] Rosenberg SA, Yang JC, Schwartzentruber DJ, Hwu P, Marincola FM, Topalian SL, Restifo NP, Sznol M, Schwarz SL, Spiess PJ, Wunderlich JR, Seipp CA, Einhorn JH, Rogers-Freezer L, and White DE. Impact of cytokine administration on the generation of antitumor reactivity in patients with metastatic melanoma receiving a peptide vaccine. J Immunol, 163(3):1690-5, 1999.

[496] Mills CD, Shearer J, Evans R, and Caldwell MD. Macrophage arginine metabolism and the inhibition or stimulation of cancer. J Immunol, 149(8):2709-14, 1992.

[497] Liu Y, Ginderachter JAV, Brys L, Baetselier PD, Raes G, and Geldhof AB. Nitric oxide-independent CTL suppression during tumor progression: association with arginaseproducing (M2) myeloid cells. J Immunol, 170(10):5064-74, 2003.

[498] Hung K, Hayashi R, Lafond-Walker A, Lowenstein C, Pardoll D, and Levitsky H. The central role of $\mathrm{CD} 4(+) \mathrm{T}$ cells in the antitumor immune response. J Exp Med, 188(12):2357-68, 1998.

[499] Egilmez NK, Harden JL, Virtuoso LP, Schwendener RA, and Kilinc MO. Nitric oxide short-circuits interleukin-12-mediated tumor regression. Cancer Immunol Immunother, 60(6):839-45, 2011.

[500] Nakanishi Y, Lu B, Gerard C, and Iwasaki A. CD8(+) T lymphocyte mobilization to virus-infected tissue requires CD4(+) T-cell help. Nature, 462(7272):510-3, 2009.

[501] Prévost-Blondel A, Neuenhahn M, Rawiel M, and Pircher H. Differential requirement of perforin and IFN-gamma in CD8 T cell-mediated immune responses against B16.F10 melanoma cells expressing a viral antigen. Eur J Immunol, 30(9):2507-15, 2000.

[502] Heckelsmiller K, Rall K, Beck S, Schlamp A, Seiderer J, Jahrsdörfer B, Krug A, Rothenfusser S, Endres S, and Hartmann G. Peritumoral CpG DNA elicits a coordinated response of $\mathrm{CD} 8 \mathrm{~T}$ cells and innate effectors to cure established tumors in a murine colon carcinoma model. J Immunol, 169(7):3892-9, 2002.

[503] Mastini C, Becker PD, Iezzi M, Curcio C, Musiani P, Forni G, Cavallo F, and Guzmán CA. Intramammary application of non-methylated-CpG oligodeoxynucleotides $(\mathrm{CpG})$ inhibits both local and systemic mammary carcinogenesis in female BALB/c Her-2/neu transgenic mice. Curr Cancer Drug Targets, 8(3):230-42, 2008.

[504] Hamzah J, Altin JG, Herringson T, Parish CR, Hämmerling GJ, O’Donoghue H, and Ganss R. Targeted liposomal delivery of TLR9 ligands activates spontaneous antitumor immunity in an autochthonous cancer model. J Immunol, 183(2):1091-8, 2009.

[505] Antony PA, Piccirillo CA, Akpinarli A, Finkelstein SE, Speiss PJ, Surman DR, Palmer DC, Chan CC, Klebanoff CA, Overwijk WW, Rosenberg SA, and Restifo NP. CD8+ T cell immunity against a tumor/self-antigen is augmented by CD4+ T helper cells and hindered by naturally occurring T regulatory cells. J Immunol, 174(5):2591-601, 2005.

[506] Bos R and Sherman LA. CD4+ T-cell help in the tumor milieu is required for recruitment and cytolytic function of CD8+ T lymphocytes. Cancer Res, 70(21):8368-77, 2010.

[507] Golgher D, Jones E, Powrie F, Elliott T, and Gallimore A. Depletion of CD25+ regulatory cells uncovers immune responses to shared murine tumor rejection antigens. Eur $J$ Immunol, 32(11):3267-75, 2002.

[508] Dannull J, Su Z, Rizzieri D, Yang BK, Coleman D, Yancey D, Zhang A, Dahm P, Chao N, Gilboa E, and Vieweg J. Enhancement of vaccine-mediated antitumor immunity in cancer patients after depletion of regulatory T cells. J Clin Invest, 115(12):3623-33, 2005.

[509] Mempel TR, Henrickson SE, and Andrian UHV. T-cell priming by dendritic cells in lymph nodes occurs in three distinct phases. Nature, 427(6970):154-9, 2004. 
[510] Valmori D, Scheibenbogen C, Dutoit V, Nagorsen D, Asemissen AM, Rubio-Godoy V, Rimoldi D, Guillaume P, Romero P, Schadendorf D, Lipp M, Dietrich PY, Thiel E, Cerottini JC, Liénard D, and Keilholz U. Circulating Tumor-reactive CD8 $(+)$ $\mathrm{T}$ cells in melanoma patients contain a CD45RA(+)CCR7(-) effector subset exerting ex vivo tumor-specific cytolytic activity. Cancer Res, 62(6):1743-50, 2002.

[511] Ossendorp F, Mengedé E, Camps M, Filius R, and Melief CJ. Specific T helper cell requirement for optimal induction of cytotoxic $\mathrm{T}$ lymphocytes against major histocompatibility complex class II negative tumors. J Exp Med, 187(5):693-702, 1998.

[512] Fransen L, der Heyden JV, Ruysschaert R, and Fiers W. Recombinant tumor necrosis factor: its effect and its synergism with interferon-gamma on a variety of normal and transformed human cell lines. Eur J Cancer Clin Oncol, 22(4):419-26, 1986.

[513] McNeill A, Spittle E, and Bäckström BT. Partial depletion of CD69low-expressing natural regulatory $\mathrm{T}$ cells with the anti-CD25 monoclonal antibody PC61. Scand J Immunol, 65(1):63-9, 2007.

[514] Couper KN, Lanthier PA, Perona-Wright G, Kummer LW, Chen W, Smiley ST, Mohrs M, and Johnson LL. Anti-CD25 antibody-mediated depletion of effector T cell populations enhances susceptibility of mice to acute but not chronic Toxoplasma gondii infection. J Immunol, 182(7):3985-94, 2009.

[515] VanOosten RL and Griffith TS. Activation of tumor-specific CD8+ T Cells after intratumoral Ad5-TRAIL/CpG oligodeoxynucleotide combination therapy. Cancer Res, 67(24):11980-90, 2007.

[516] Kurts C, Kosaka H, Carbone FR, Miller JF, and Heath WR. Class I-restricted crosspresentation of exogenous self-antigens leads to deletion of autoreactive CD8 $(+)$ T cells. J Exp Med, 186(2):239-45, 1997.

[517] Garrood T, Lee L, and Pitzalis C. Molecular mechanisms of cell recruitment to inflammatory sites: general and tissue-specific pathways. Rheumatology (Oxford), 45(3):250-60, 2006.

[518] Smyth MJ, Cretney E, Kelly JM, Westwood JA, Street SEA, Yagita H, Takeda K, van Dommelen SLH, Degli-Esposti MA, and Hayakawa Y. Activation of NK cell cytotoxicity. Mol Immunol, 42(4):501-10, 2005.

[519] Dokun AO, Kim S, Smith HR, Kang HS, Chu DT, and Yokoyama WM. Specific and nonspecific NK cell activation during virus infection. Nat Immunol, 2(10):951-6, 2001.

[520] Fife BT, Pauken KE, Eagar TN, Obu T, Wu J, Tang Q, Azuma M, Krummel MF, and Bluestone JA. Interactions between PD-1 and PD-L1 promote tolerance by blocking the TCR-induced stop signal. Nat Immunol, 10(11):1185-92, 2009.

[521] Mangsbo SM, Sandin LC, Anger K, Korman AJ, Loskog A, and Tötterman TH. Enhanced tumor eradication by combining CTLA-4 or PD-1 blockade with CpG therapy. J Immunother, 33(3):225-35, 2010.

[522] Pilon-Thomas S, Mackay A, Vohra N, and Mulé JJ. Blockade of programmed death ligand 1 enhances the therapeutic efficacy of combination immunotherapy against melanoma. J Immunol, 184(7):3442-9, 2010.

[523] Lysaght J, Jarnicki AG, and Mills KHG. Reciprocal effects of Th1 and Treg cell inducing pathogen-associated immunomodulatory molecules on anti-tumor immunity. Cancer Immunol Immunother, 56(9):1367-79, 2007.

[524] Ding Y, Seow SV, Huang CH, Liew LM, Lim YC, Kuo IC, and Chua KY. Coadministration of the fungal immunomodulatory protein FIP-Fve and a tumour-associated antigen enhanced antitumour immunity. Immunology, 128(1 Suppl):e881-94, 2009.

[525] Medina-Echeverz J, Fioravanti J, Zabala M, Ardaiz N, Prieto J, and Berraondo 
P. Successful colon cancer eradication after chemoimmunotherapy is associated with profound phenotypic change of intratumoral myeloid cells. J Immunol, 186(2):807-15, 2011.

[526] Zhang Y, Luo F, Cai Y, Liu N, Wang L, Xu D, and Chu Y. TLR1/TLR2 agonist induces tumor regression by reciprocal modulation of effector and regulatory T cells. J Immunol, 186(4):1963-9, 2011.

[527] Gao Q, Qiu SJ, Fan J, Zhou J, Wang XY, Xiao YS, Xu Y, Li YW, and Tang ZY. Intratumoral balance of regulatory and cytotoxic $\mathrm{T}$ cells is associated with prognosis of hepatocellular carcinoma after resection. J Clin Oncol, 25(18):2586-93, 2007.

[528] Yang Y, Huang CT, Huang X, and Pardoll DM. Persistent Toll-like receptor signals are required for reversal of regulatory T cell-mediated CD8 tolerance. Nat Immunol, 5(5):508-15, 2004.

[529] Dyck DG, Driedger SM, Nemeth R, Osachuk TA, and Greenberg AH. Conditioned tolerance to drug-induced (poly I:C) natural killer cell activation: effects of drug-dosage and context-specificity parameters. Brain Behav Immun, 1(3):251-66, 1987.

[530] Morris M and Li L. Molecular mechanisms and pathological consequences of endotoxin tolerance and priming. Arch Immunol Ther Exp (Warsz), 60(1):13-8, 2012.

[531] Sharma S, Karakousis CP, Takita H, Shin K, and Brooks SP. Intra-tumoral injection of $\mathrm{CpG}$ results in the inhibition of tumor growth in murine Colon-26 and B-16 tumors. Biotechnol Lett, 25(2):149-53, 2003.

[532] Orihuela E, Herr HW, Pinsky CM, and Whitmore WF. Toxicity of intravesical BCG and its management in patients with superficial bladder tumors. Cancer, 60(3):326-33, 1987.

[533] Steg A, Leleu C, Debré B, Boccon-Gibod L, and Sicard D. Systemic bacillus CalmetteGuerin infection in patients treated by intravesical BCG therapy for superficial bladder cancer. Prog Clin Biol Res, 310:325-34, 1989.

[534] Faldt J, Dahlgren C, and Ridell M. Difference in neutrophil cytokine production induced by pathogenic and non-pathogenic mycobacteria. APMIS, 110(9):593-600, 2002.

[535] Czarnetzki BM, Macher E, Suciu S, Thomas D, Steerenberg PA, and Rümke P. Long-term adjuvant immunotherapy in stage I high risk malignant melanoma, comparing two BCG preparations versus non-treatment in a randomised multicentre study (EORTC Protocol 18781). Eur J Cancer, 29A(9):1237-42, 1993.

[536] Agarwala SS, Neuberg D, Park Y, and Kirkwood JM. Mature results of a phase III randomized trial of bacillus Calmette-Guerin (BCG) versus observation and BCG plus dacarbazine versus BCG in the adjuvant therapy of American Joint Committee on Cancer Stage I-III melanoma (E1673): a trial of the Eastern Oncology Group. Cancer, 100(8):1692-8, 2004.

[537] Morton DL, Eilber FR, Holmes EC, Hunt JS, Ketcham AS, Silverstein MJ, and Sparks FC. BCG immunotherapy of malignant melanoma: summary of a seven-year experience. Ann Surg, 180(4):635-43, 1974.

[538] Weigelt B, Peterse JL, and van 't Veer LJ. Breast cancer metastasis: markers and models. Nat Rev Cancer, 5(8):591-602, 2005.

[539] Hao X, Sun B, Hu L, Lähdesmäki H, Dunmire V, Feng Y, Zhang SW, Wang H, Wu C, Wang H, Fuller GN, Symmans WF, Shmulevich I, and Zhang W. Differential gene and protein expression in primary breast malignancies and their lymph node metastases as revealed by combined cDNA microarray and tissue microarray analysis. Cancer, 100(6):111022,2004 .

[540] Padera TP, Kuo AH, Hoshida T, Liao S, Lobo J, Kozak KR, Fukumura D, and Jain RK. Differential response of primary tumor versus lymphatic metastasis to VEGFR-2 
and VEGFR-3 kinase inhibitors cediranib and vandetanib. Molecular Cancer Therapeutics, $7(8): 2272-9,2008$.

[541] Cohen SA, Tzung SP, Doerr RJ, and Goldrosen MH. Role of asialo-GM1 positive liver cells from athymic nude or polyinosinic-polycytidylic acid-treated mice in suppressing colon-derived experimental hepatic metastasis. Cancer Res, 50(6):1834-40, 1990.

[542] Xiong Z, Gharagozlou S, Vengco I, Chen W, and Ohlfest JR. Effective CpG immunotherapy of breast carcinoma prevents but fails to eradicate established brain metastasis. Clin Cancer Res, 14(17):5484-93, 2008.

[543] Kim S, Takahashi H, Lin WW, Descargues P, Grivennikov S, Kim Y, Luo JL, and Karin M. Carcinoma-produced factors activate myeloid cells through TLR2 to stimulate metastasis. Nature, 457(7225):102-6, 2009.

[544] Pulaski BA and Ostrand-Rosenberg S. Reduction of established spontaneous mammary carcinoma metastases following immunotherapy with major histocompatibility complex class II and B7.1 cell-based tumor vaccines. Cancer Res, 58(7):1486-93, 1998.

[545] Lelekakis M, Moseley JM, Martin TJ, Hards D, Williams E, Ho P, Lowen D, Javni J, Miller FR, Slavin J, and Anderson RL. A novel orthotopic model of breast cancer metastasis to bone. Clin Exp Metastasis, 17(2):163-70, 1999.

[546] Pulaski BA, Terman DS, Khan S, Muller E, and Ostrand-Rosenberg S. Cooperativity of Staphylococcal aureus enterotoxin B superantigen, major histocompatibility complex class II, and CD80 for immunotherapy of advanced spontaneous metastases in a clinically relevant postoperative mouse breast cancer model. Cancer Res, 60(10):2710-5, 2000.

[547] Hanson HL, Donermeyer DL, Ikeda H, White JM, Shankaran V, Old LJ, Shiku H, Schreiber RD, and Allen PM. Eradication of established tumors by CD8+ T cell adoptive immunotherapy. Immunity, 13(2):265-76, 2000.

[548] Böhle A, Jocham D, and Bock PR. Intravesical bacillus Calmette-Guerin versus mitomycin C for superficial bladder cancer: a formal meta-analysis of comparative studies on recurrence and toxicity. J Urol, 169(1):90-5, 2003.

[549] Wieland CW, Knapp S, Florquin S, de Vos AF, Takeda K, Akira S, Golenbock DT, Verbon A, and van der Poll T. Non-mannose-capped lipoarabinomannan induces lung inflammation via toll-like receptor 2. Am J Respir Crit Care Med, 170(12):1367-74, 2004.

[550] Coulombe F, Divangahi M, Veyrier F, de Léséleuc L, Gleason JL, Yang Y, Kelliher MA, Pandey AK, Sassetti CM, Reed MB, and Behr MA. Increased NOD2-mediated recognition of N-glycolyl muramyl dipeptide. J Exp Med, 206(8):1709-16, 2009.

[551] Yadav M and Schorey JS. The beta-glucan receptor dectin-1 functions together with TLR2 to mediate macrophage activation by mycobacteria. Blood, 108(9):3168-75, 2006.

[552] Morales A, Chin JL, and Ramsey EW. Mycobacterial cell wall extract for treatment of carcinoma in situ of the bladder. J Urol, 166(5):1633-7; discussion 1637-8, 2001.

[553] Vosika GJ, Schmidtke JR, Goldman A, Ribi E, Parker R, and Gray GR. Intralesional immunotherapy of malignant melanoma with Mycobacterium smegmatis cell wall skeleton combined with trehalose dimycolate (P3). Cancer, 44(2):495-503, 1979.

[554] de Veer M, Kemp J, Chatelier J, Elhay MJ, and Meeusen ENT. The kinetics of soluble and particulate antigen trafficking in the afferent lymph, and its modulation by aluminum-based adjuvant. Vaccine, 28(40):6597-602, 2010.

[555] Lambrecht BN, Kool M, Willart MAM, and Hammad H. Mechanism of action of clinically approved adjuvants. Curr Opin Immunol, 21(1):23-9, 2009.

[556] Lou Y, Liu C, Lizée G, Peng W, Xu C, Ye Y, Rabinovich BA, Hailemichael Y, 
Gelbard A, Zhou D, Overwijk WW, and Hwu P. Antitumor activity mediated by CpG: the route of administration is critical. J Immunother, 34(3):279-88, 2011.

[557] Black PL, Hartmann D, Pennington R, Phillips H, Schneider M, Tribble HR, and Talmadge JE. Effect of tumor burden and route of administration on the immunotherapeutic properties of polyinosinic-polycytidylic acid stabilized with poly-L-lysine in carboxymethyl cellulose [Poly(I,C)-LC]. Int J Immunopharmacol, 14(8):1341-53, 1992.

[558] Brown DM, Fisher TL, Wei C, Frelinger JG, and Lord EM. Tumours can act as adjuvants for humoral immunity. Immunology, 102(4):486-97, 2001.

[559] Connolly MK, Clair JMS, Bedrosian AS, Malhotra A, Vera V, Ibrahim J, Henning J, Pachter HL, Bar-Sagi D, Frey AB, and Miller G. Distinct populations of metastasesenabling myeloid cells expand in the liver of mice harboring invasive and preinvasive intraabdominal tumor. J Leukoc Biol, 87(4):713-25, 2010.

[560] Lee AE, Rogers LA, Longcroft JM, and Jeffery RE. Reduction of metastasis in a murine mammary tumour model by heparin and polyinosinic-polycytidylic acid. Clin Exp Metastasis, $8(2): 165-71,1990$

[561] Tsung K, Meko JB, Peplinski GR, Tsung YL, and Norton JA. IL-12 induces T helper 1-directed antitumor response. J Immunol, 158(7):3359-65, 1997.

[562] Gallucci S, Lolkema M, and Matzinger P. Natural adjuvants: endogenous activators of dendritic cells. Nat Med, 5(11):1249-55, 1999.

[563] Serbina NV and Pamer EG. Monocyte emigration from bone marrow during bacterial infection requires signals mediated by chemokine receptor CCR2. Nat Immunol, 7(3):311-7, 2006.

[564] Geissmann F, Jung S, and Littman DR. Blood monocytes consist of two principal subsets with distinct migratory properties. Immunity, 19(1):71-82, 2003.

[565] Jung S, Unutmaz D, Wong P, Sano GI, los Santos KD, Sparwasser T, Wu S, Vuthoori S, Ko K, Zavala F, Pamer EG, Littman DR, and Lang RA. In vivo depletion of CD11c+ dendritic cells abrogates priming of CD8+ T cells by exogenous cellassociated antigens. Immunity, 17(2):211-20, 2002.

[566] Probst HC, Tschannen K, Odermatt B, Schwendener R, Zinkernagel RM, and Broek MVD. Histological analysis of CD11c-DTR/GFP mice after in vivo depletion of dendritic cells. Clin Exp Immunol, 141(3):398-404, 2005.

[567] Robben PM, LaRegina M, Kuziel WA, and Sibley LD. Recruitment of Gr-1+ monocytes is essential for control of acute toxoplasmosis. J Exp Med, 201(11):1761-9, 2005.

[568] Morales JK, Kmieciak M, Graham L, Feldmesser M, Bear HD, and Manjili MH. Adoptive transfer of HER2/neu-specific T cells expanded with alternating gamma chain cytokines mediate tumor regression when combined with the depletion of myeloid-derived suppressor cells. Cancer immunology, immunotherapy : CII, 58(6):941-53, 2009.

[569] Ribechini E, Leenen PJM, and Lutz MB. Gr-1 antibody induces STAT signaling, macrophage marker expression and abrogation of myeloid-derived suppressor cell activity in BM cells. Eur. J. Immunol., 39(12):3538-51, 2009.

[570] Tacke F, Alvarez D, Kaplan TJ, Jakubzick C, Spanbroek R, Llodra J, Garin A, Liu J, Mack M, van Rooijen N, Lira SA, Habenicht AJ, and Randolph GJ. Monocyte subsets differentially employ CCR2, CCR5, and CX3CR1 to accumulate within atherosclerotic plaques. J Clin Invest, 117(1):185-94, 2007.

[571] Auffray C, Fogg DK, Narni-Mancinelli E, Senechal B, Trouillet C, Saederup N, Leemput J, Bigot K, Campisi L, Abitbol M, Molina T, Charo I, Hume DA, Cumano A, Lauvau G, and Geissmann F. CX3CR1+ CD115+ CD135+ common 
macrophage/DC precursors and the role of CX3CR1 in their response to inflammation. J Exp Med, 206(3):595-606, 2009.

[572] Pilon-Thomas S, Nelson N, Vohra N, Jerald M, Pendleton L, Szekeres K, and Ghansah T. Murine pancreatic adenocarcinoma dampens SHIP-1 expression and alters MDSC homeostasis and function. PLoS ONE, 6(11):e27729, 2011.

[573] Kim YS, Kim YJ, Lee JM, Kim EK, Park YJ, Choe SK, Ko HJ, and Kang CY. Functional changes in myeloid-derived suppressor cells (MDSCs) during tumor growth: FKBP51 contributes to the regulation of the immunosuppressive function of MDSCs. J Immunol, 188(9):4226-34, 2012.

[574] Fernández A, Mesa C, Marigo I, Dolcetti L, Clavell M, Oliver L, Fernández LE, and Bronte V. Inhibition of tumor-induced myeloid-derived suppressor cell function by a nanoparticulated adjuvant. J Immunol, 186(1):264-74, 2011.

[575] Ferlazzo G, Morandi B, D’Agostino A, Meazza R, Melioli G, Moretta A, and Moretta L. The interaction between NK cells and dendritic cells in bacterial infections results in rapid induction of NK cell activation and in the lysis of uninfected dendritic cells. Eur J Immunol, 33(2):306-13, 2003.

[576] Andrews DM, Andoniou CE, Scalzo AA, van Dommelen SLH, Wallace ME, Smyth MJ, and Degli-Esposti MA. Cross-talk between dendritic cells and natural killer cells in viral infection. Mol Immunol, 42(4):547-55, 2005.

[577] Shresta S, Pham CT, Thomas DA, Graubert TA, and Ley TJ. How do cytotoxic lymphocytes kill their targets? Curr Opin Immunol, 10(5):581-7, 1998.

[578] Zitvogel L, Apetoh L, Ghiringhelli F, and Kroemer G. Immunological aspects of cancer chemotherapy. Nat Rev Immunol, 8(1):59-73, 2008.

[579] Serafini P, Meckel K, Kelso M, Noonan K, Califano J, Koch W, Dolcetti L, Bronte V, and Borrello I. Phosphodiesterase-5 inhibition augments endogenous antitumor immunity by reducing myeloid-derived suppressor cell function. J Exp Med, 203(12):2691-702, 2006.

[580] Nagaraj S, Youn JI, Weber H, Iclozan C, Lu L, Cotter MJ, Meyer C, Becerra CR, Fishman M, Antonia S, Sporn MB, Liby KT, Rawal B, Lee JH, and Gabrilovich DI. Anti-inflammatory triterpenoid blocks immune suppressive function of MDSCs and improves immune response in cancer. Clin Cancer Res, 16(6):1812-23, 2010.

[581] Idoyaga J, Lubkin A, Fiorese C, Lahoud MH, Caminschi I, Huang Y, Rodriguez A, Clausen BE, Park CG, Trumpfheller C, and Steinman RM. Comparable T helper 1 (Th1) and CD8 T-cell immunity by targeting HIV gag p24 to CD8 dendritic cells within antibodies to Langerin, DEC205, and Clec9A. Proc Natl Acad Sci USA, 108(6):2384-9, 2011.

[582] Probst HC, McCoy K, Okazaki T, Honjo T, and van den Broek M. Resting dendritic cells induce peripheral CD8+ T cell tolerance through PD-1 and CTLA-4. Nat Immunol, 6(3):280-6, 2005.

[583] Taube JM, Anders RA, Young GD, Xu H, Sharma R, McMiller TL, Chen S, Klein AP, Pardoll DM, Topalian SL, and Chen L. Colocalization of inflammatory response with B7-h1 expression in human melanocytic lesions supports an adaptive resistance mechanism of immune escape. Science Translational Medicine, 4(127):127ra37, 2012. 
Appendix 


\section{A LSRII specifications}

Table A.1: LSR II Lasers

\begin{tabular}{|l|l|l|}
\hline Number & Wavelength & Power and Type \\
\hline 1 & $355 \mathrm{~nm}$ & $20 \mathrm{~mW}$ solid state (UV) \\
\hline 2 & $488 \mathrm{~nm}$ & $100 \mathrm{~mW}$ solid state (blue) \\
\hline 3 & $532 \mathrm{~nm}$ & $150 \mathrm{~mW}$ Pulsed diode (green) \\
\hline 4 & $405 \mathrm{~nm}$ & $50 \mathrm{~mW}$ CUBE diode laser (violet) \\
\hline 5 & $640 \mathrm{~nm}$ & $40 \mathrm{~mW}$ CUBE diode laser (red) \\
\hline
\end{tabular}

Table A.2: LSR II Detectors

\begin{tabular}{|l|l|l|l|}
\hline Name & Laser & $\begin{array}{l}\text { Wavelength } \\
\text { range }\end{array}$ & Dyes \\
\hline U670LP & 1 & $\leq 670 \mathrm{~nm}$ & Hoechst Red \\
\hline U450/50 & 1 & $425-475 \mathrm{~nm}$ & DAPI, Live/Dead Fixable Blue \\
\hline B685/35 & 2 & $667-702 \mathrm{~nm}$ & PerCP, PE-Cy5.5 \\
\hline B515/20 & 2 & $505-525 \mathrm{~nm}$ & AlexaFluor488, GFP, CFSE, FITC \\
\hline B488/10 & 2 & $478-498 \mathrm{~nm}$ & SSC \\
\hline G780/60 & 3 & $750-810 \mathrm{~nm}$ & PE-Cy7 \\
\hline G695/40 & 3 & $675-715 \mathrm{~nm}$ & PerCP-Cy5.5, PE-Alexa Fluor700, PE-Cy5.5 \\
\hline G660/40 & 3 & $640-680 \mathrm{~nm}$ & PE-Cy5, PE-Alexa Fluor 647 \\
\hline G610/20 & 3 & $600-620 \mathrm{~nm}$ & PI, PE-Texas Red \\
\hline G575/25 & 3 & $562-580 \mathrm{~nm}$ & PE \\
\hline V705/70 & 4 & $670-740 \mathrm{~nm}$ & Qdot 705 \\
\hline V660/20 & 4 & $650-670 \mathrm{~nm}$ & Qdot 655 \\
\hline V605/40 & 4 & $585-625 \mathrm{~nm}$ & Qdot 605 \\
\hline V560/40 & 4 & $540-580 \mathrm{~nm}$ & Pacific Orange \\
\hline V525/50 & 4 & $500-550 \mathrm{~nm}$ & AmCyan \\
\hline V450/50 & 4 & $425-475 \mathrm{~nm}$ & Pacific Blue, HorizonV450 \\
\hline R780/60 & 5 & $750-810 \mathrm{~nm}$ & APC-Cy7 \\
\hline R710//50 & 5 & $685-735 \mathrm{~nm}$ & Alexa Fluor 700 \\
\hline R670/14 & 5 & $663-677 \mathrm{~nm}$ & APC, AlexaFluor 647 \\
\hline
\end{tabular}




\section{B Distribution of cell frequencies and tumour sizes}

Day 13

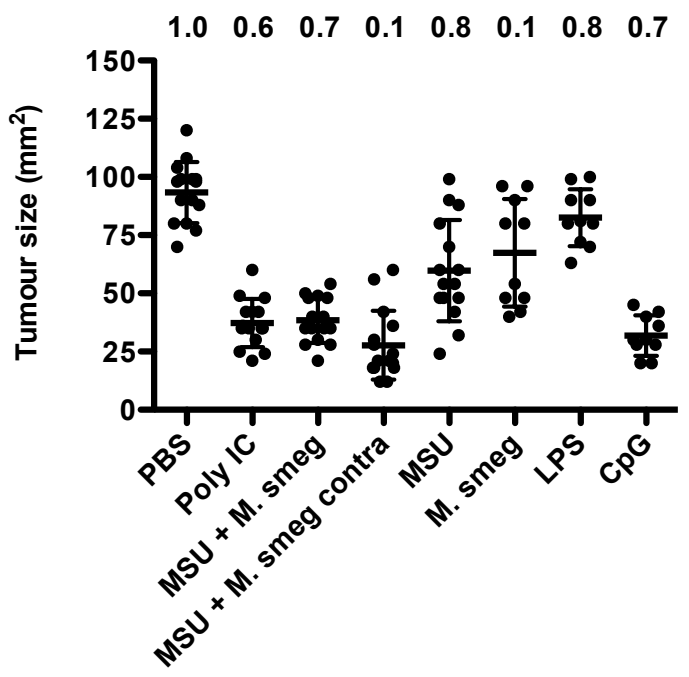

Day 15

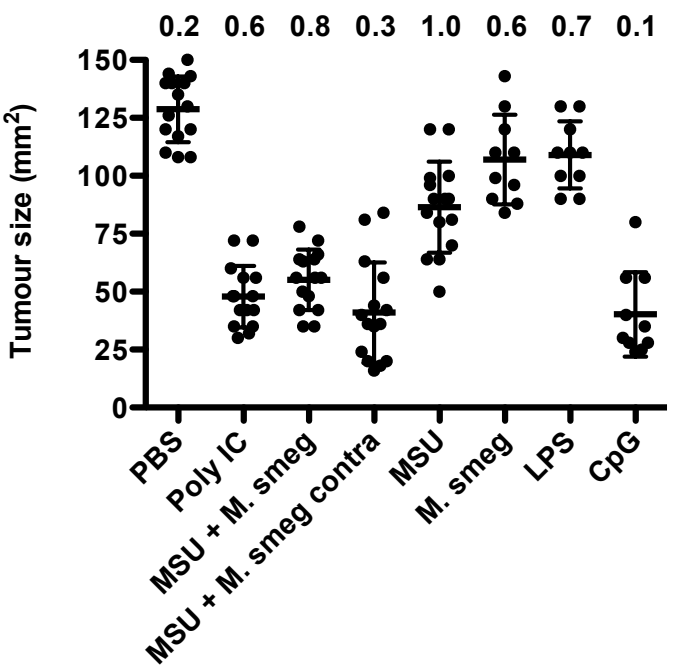

Figure B.1: B16F1 tumour sizes are consistent with Gaussian distribution. C57 mice bearing established B16F1 tumours were injected around the tumour with the indicated adjuvants on day $7,9,11$ and 13 . Tumour sizes at day 13 and 15 are shown as mean + SE and are pooled from 2-3 experiments with 5 mice per group. The D'Agostino Pearson test for normality was performed and $\mathrm{p}$ values are reported on the top line. $p>0.05$ is considered to be consistent with Gaussian distribution.

A.

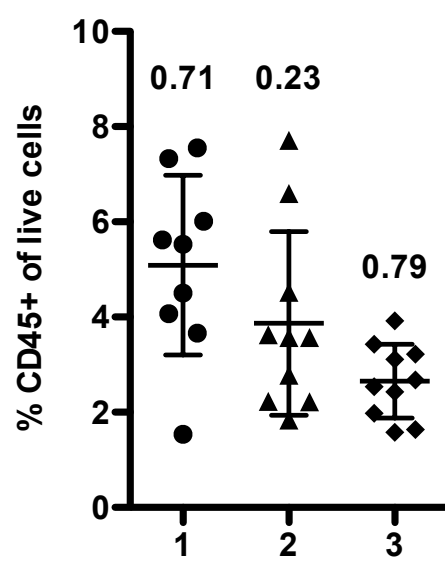

Experiment
B. Immune cell types

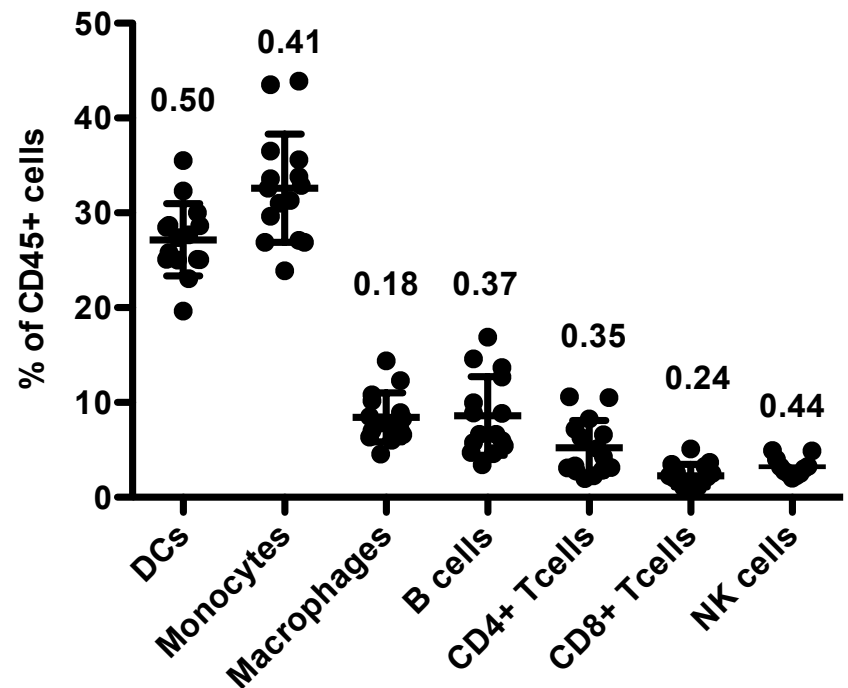

Figure B.2: Infiltration of immune cells into tumours is consistent with Gaussian distribution. The immune infiltrate of $\mathrm{B} 16 \mathrm{~F} 1$ tumours from $\mathrm{C} 57$ mice was analysed as described in Figure 3.11. The D'Agostino Pearson test for normality was performed and $\mathrm{p}$ values are reported on the top line. $p>0.05$ is considered to be consistent with Gaussian distribution. 
A.

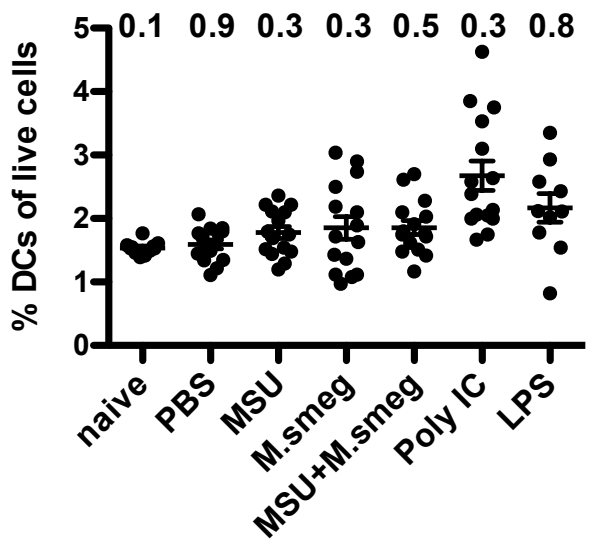

C.

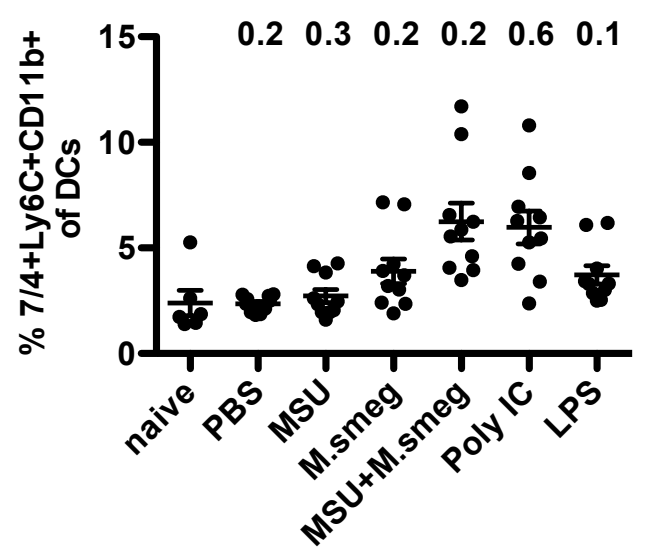

B.

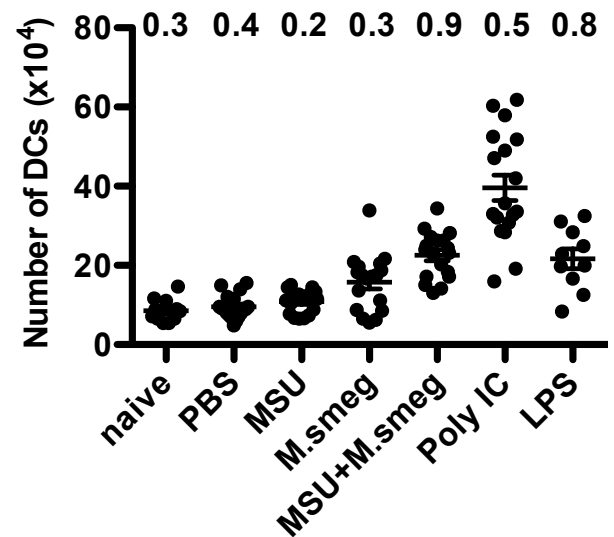

D.

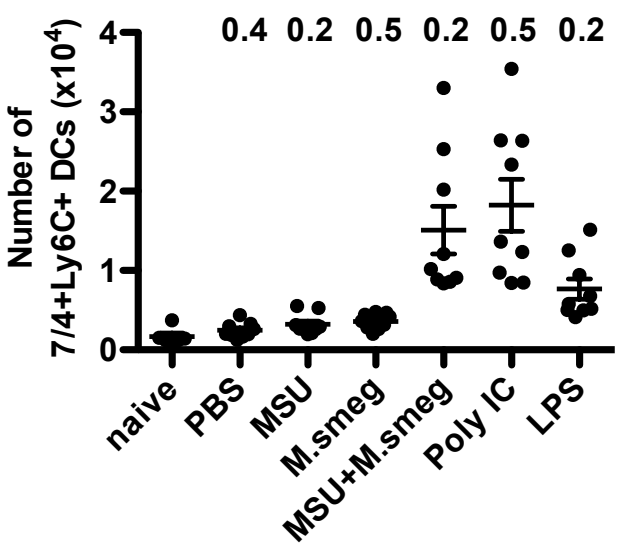

Figure B.3: Accumulation of DCs in dLNs is consistent with Gaussian distribution. The DCs in dLNs of B16F1 tumours treated once with the indicated adjuvants was analysed as described in Figure 4.8. The D'Agostino Pearson test for normality was performed and $\mathrm{p}$ values are reported on the top line. $p>0.05$ is considered to be consistent with Gaussian distribution. 


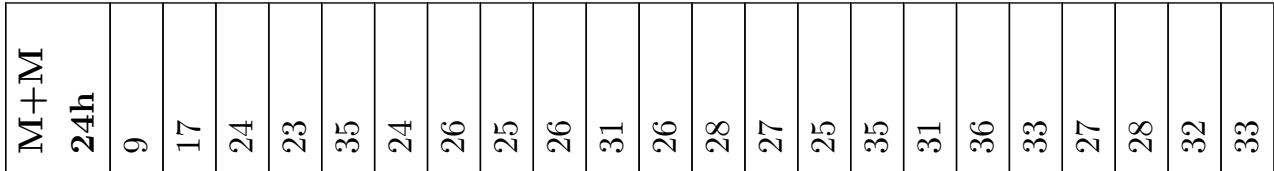
芝

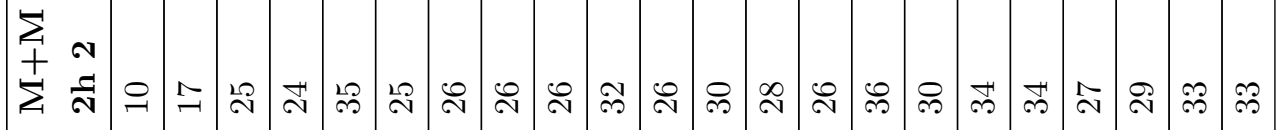

$\sum_{+}^{N}$

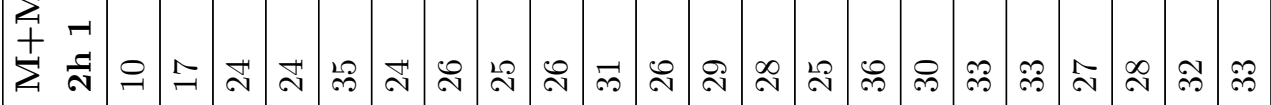

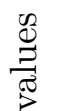

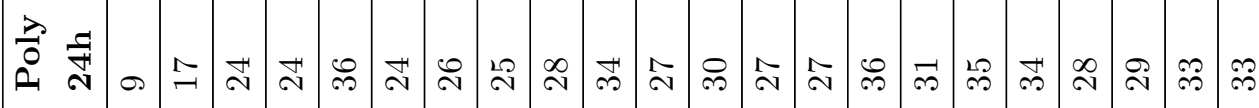

兒

$\begin{array}{lll}0 & 0 \\ 0 & 0 & 0 \\ 0 & 0 & 0\end{array}$

穴

菅

药

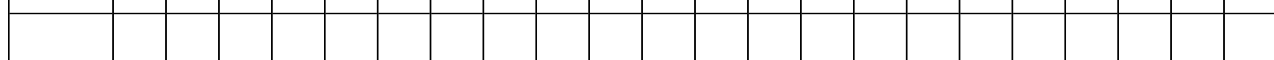

匀

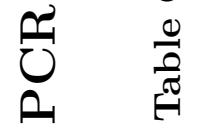

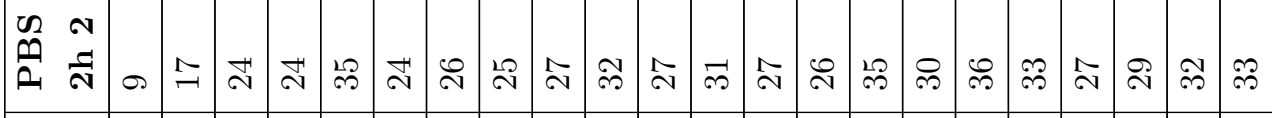

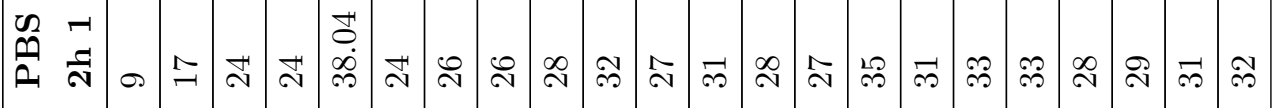

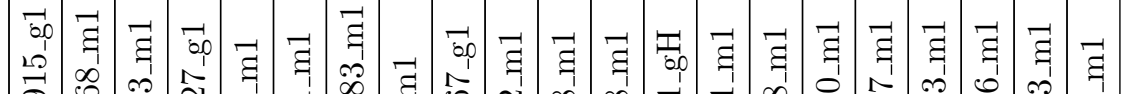

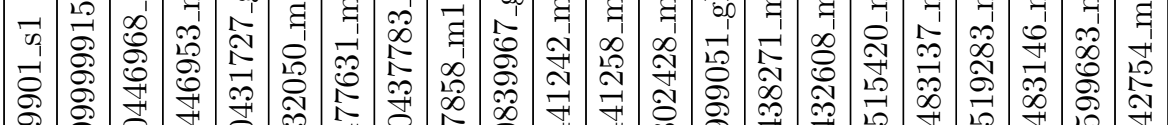

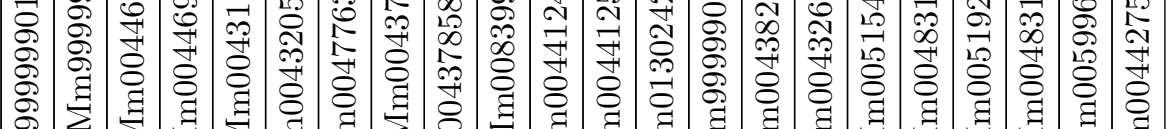




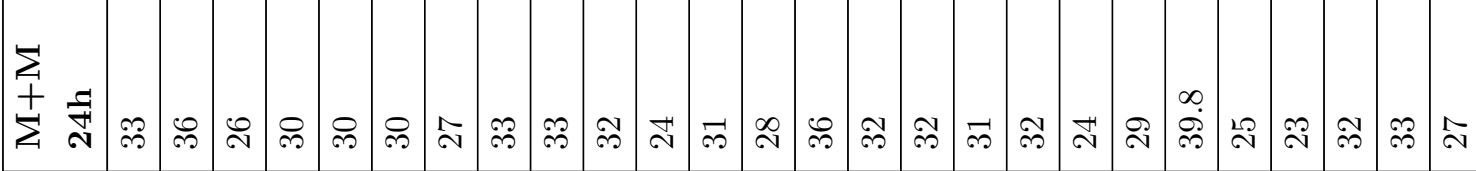 \\ $\sum_{+}$

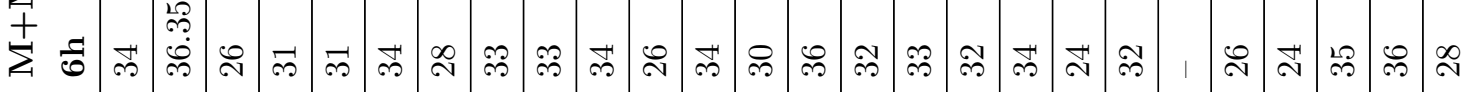 \\ $\sum_{\substack{+i}}^{2}$

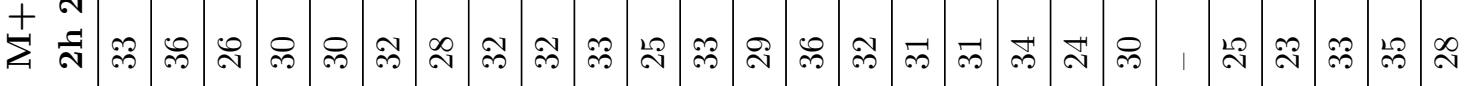 \\ $\sum_{+\infty}^{\infty}$

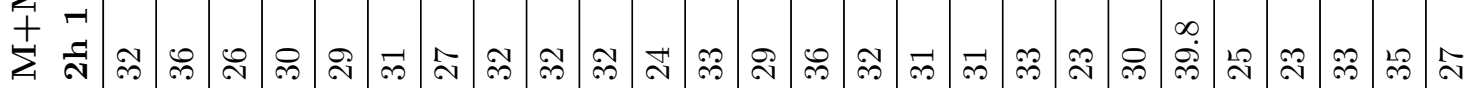

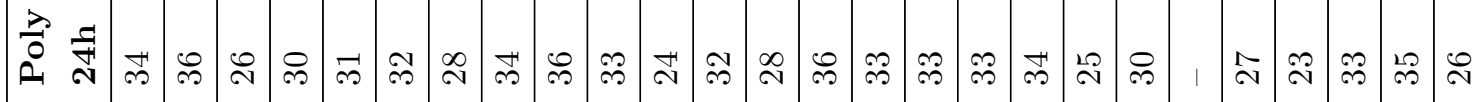

可

专

热

祭 ন

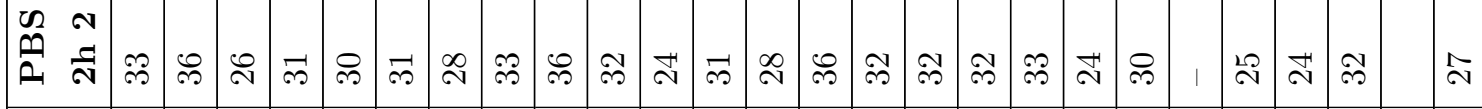

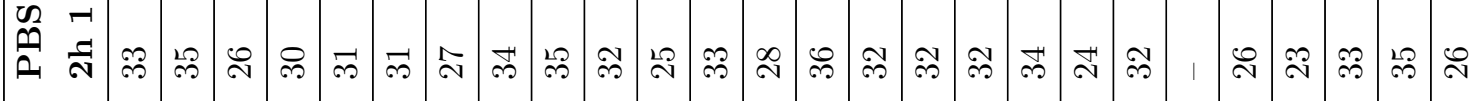

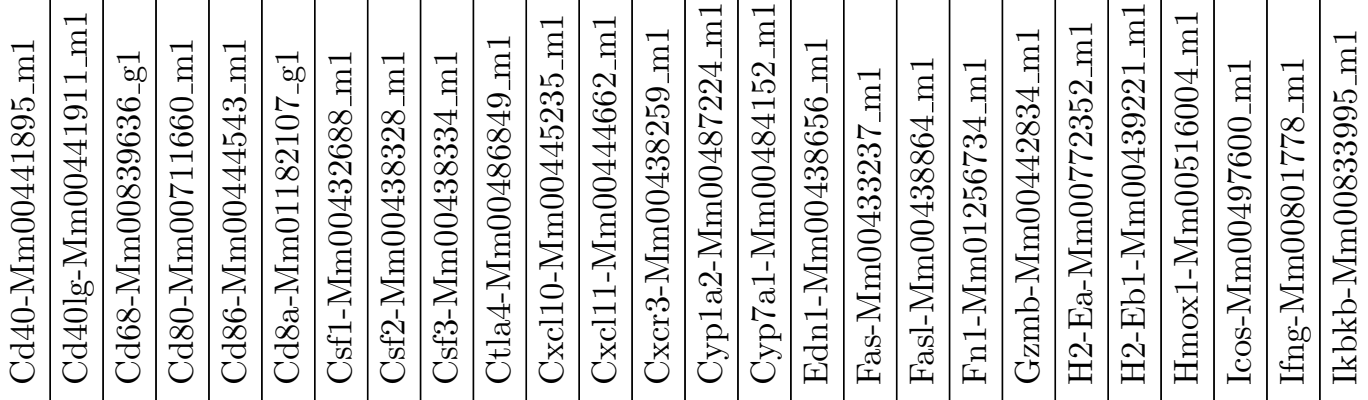




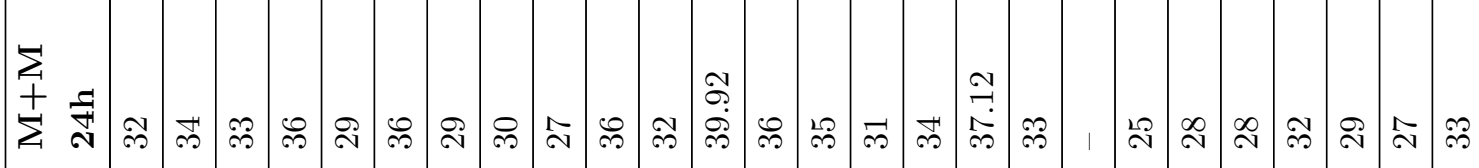

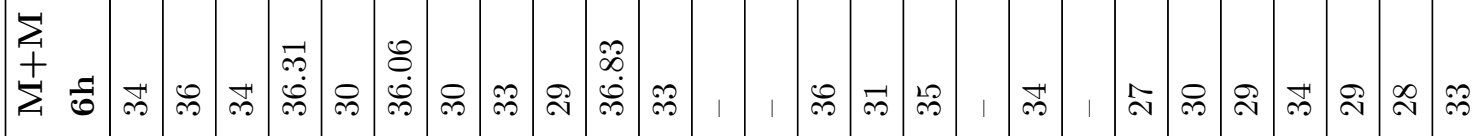

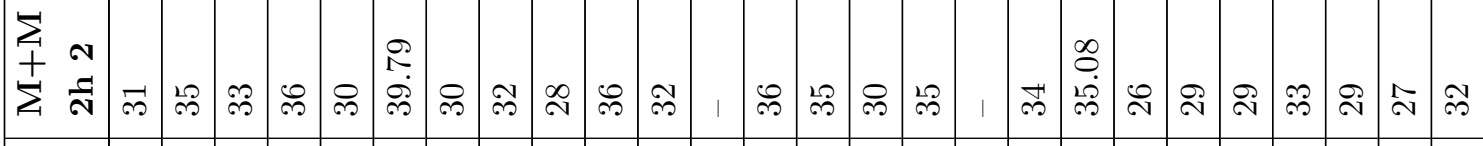

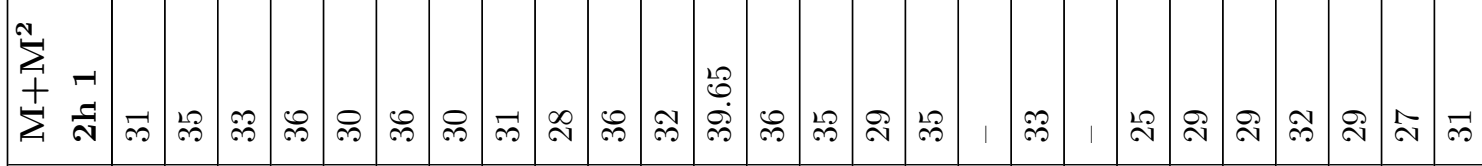

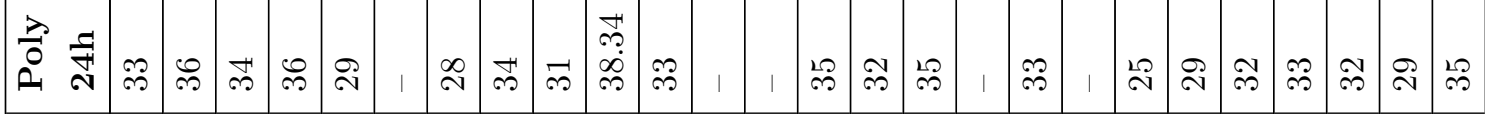

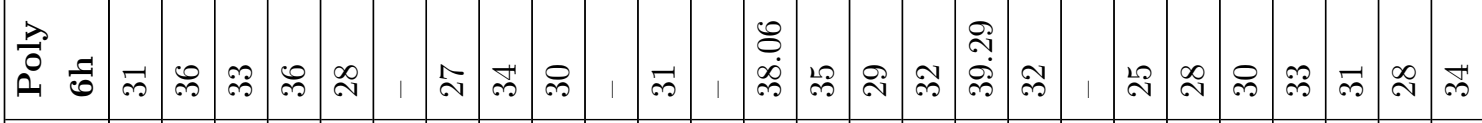

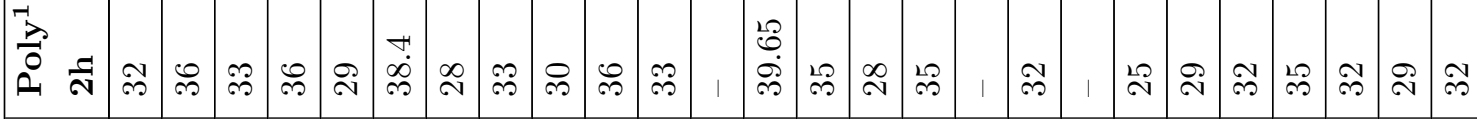

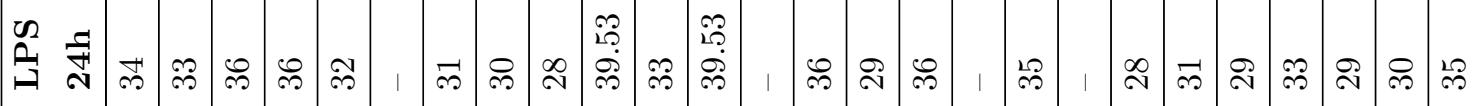

勾

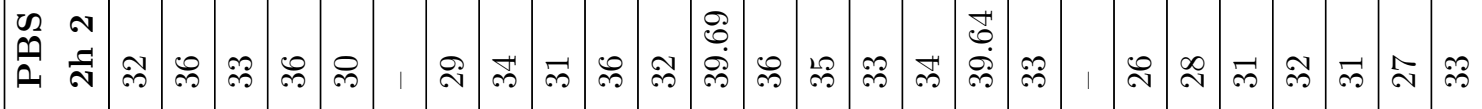

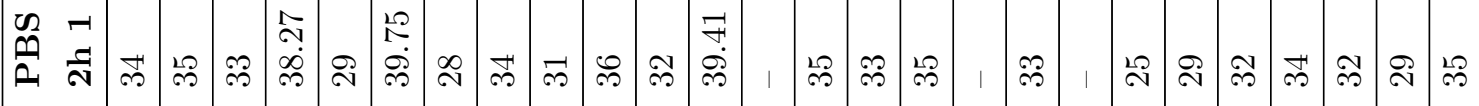

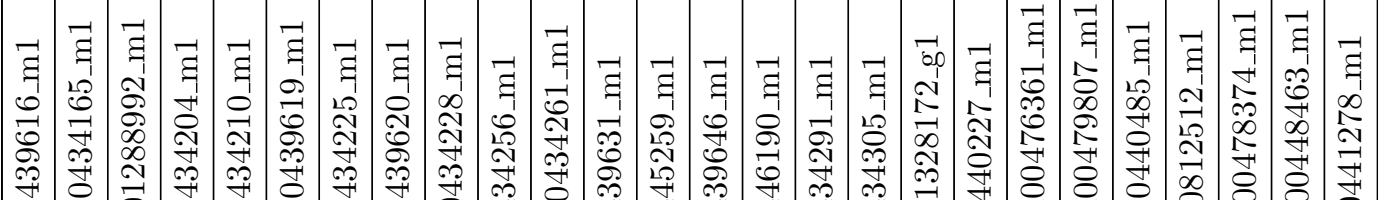

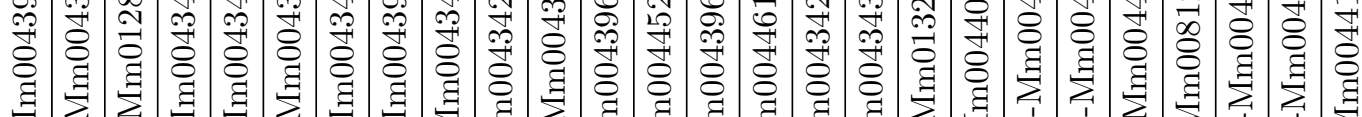

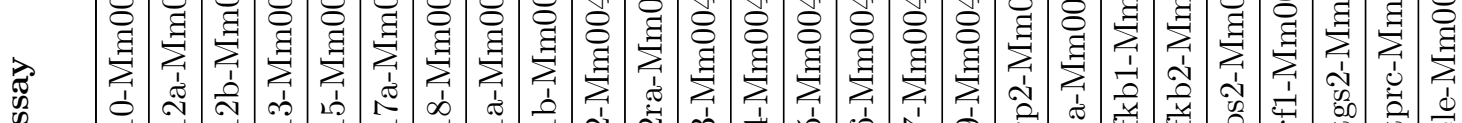

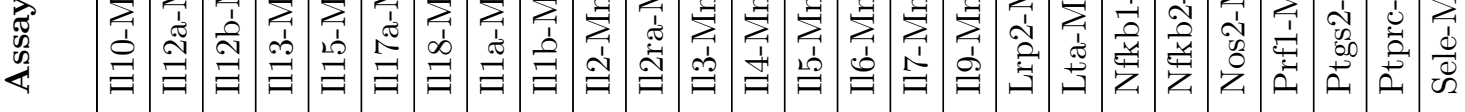




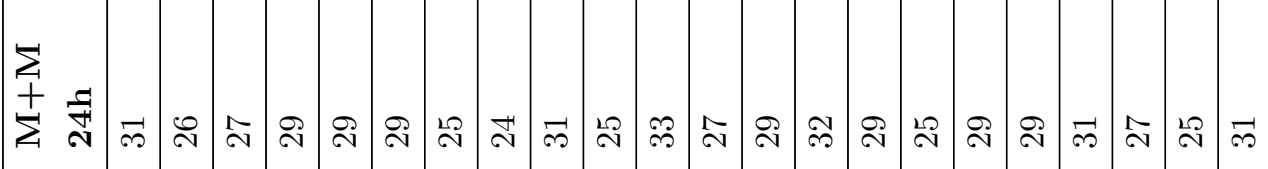 \\ $\sum_{+}$

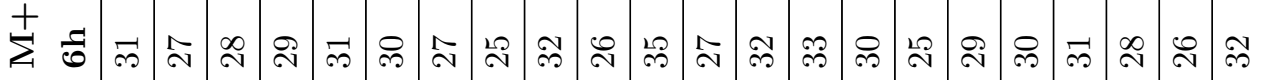

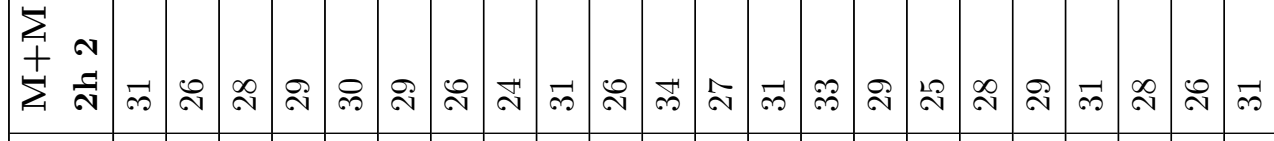 \\ $\sum_{\substack{1 \\ i}}^{N}$

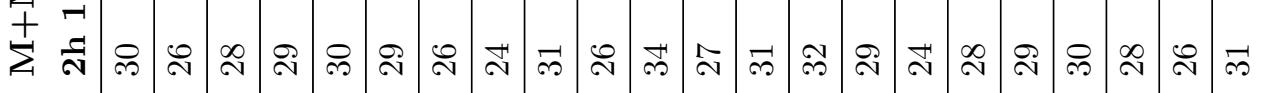

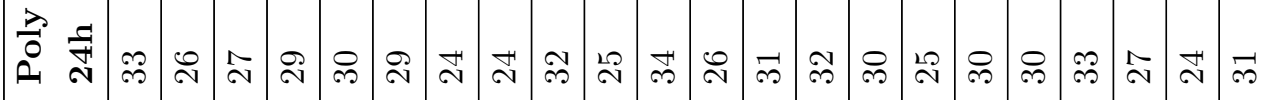

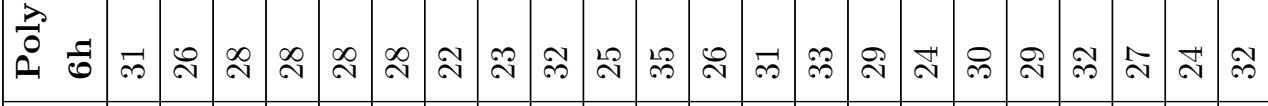

高竞

鱼

育

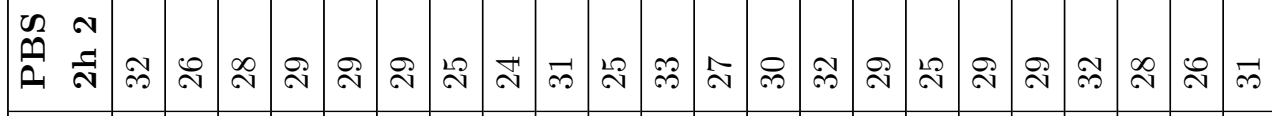

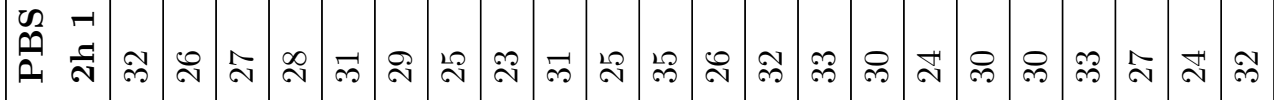

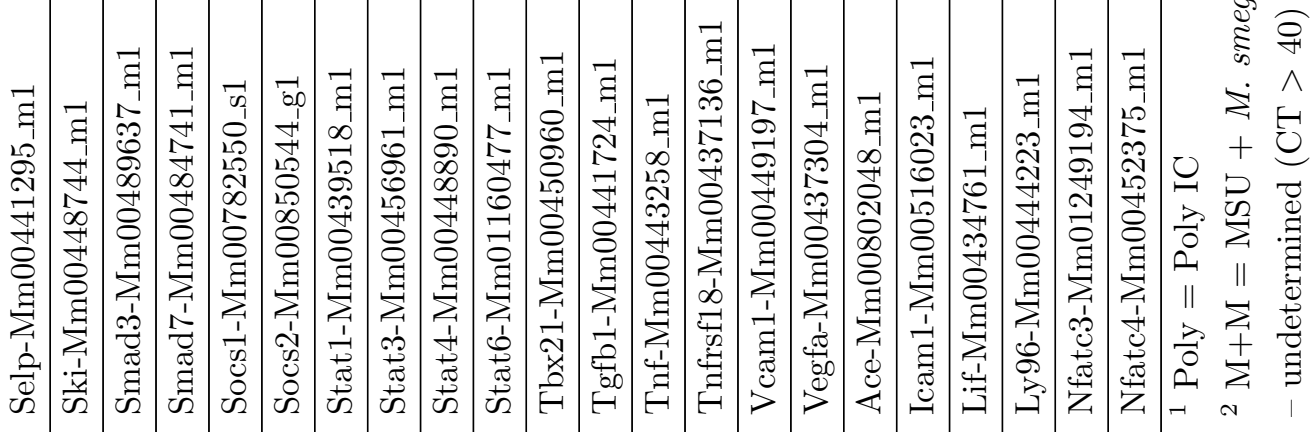




\section{Spleen monocytes frequency, numbers and suppressive func- tion}

A.

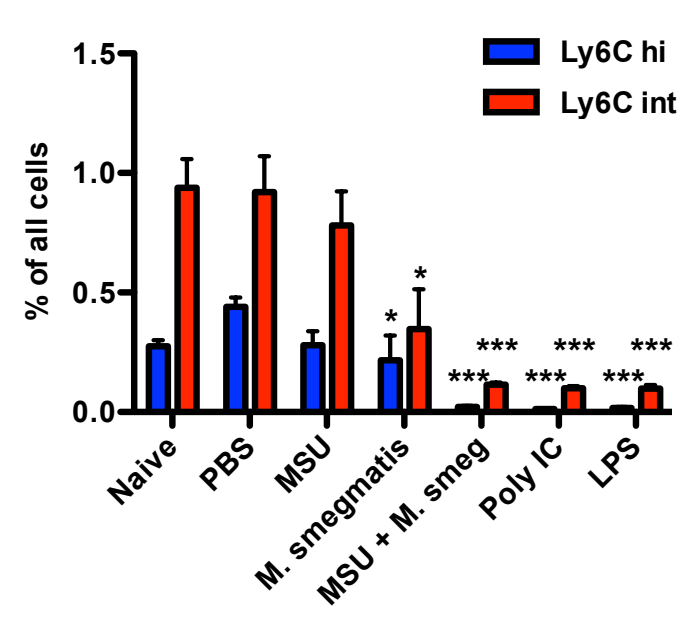

B.

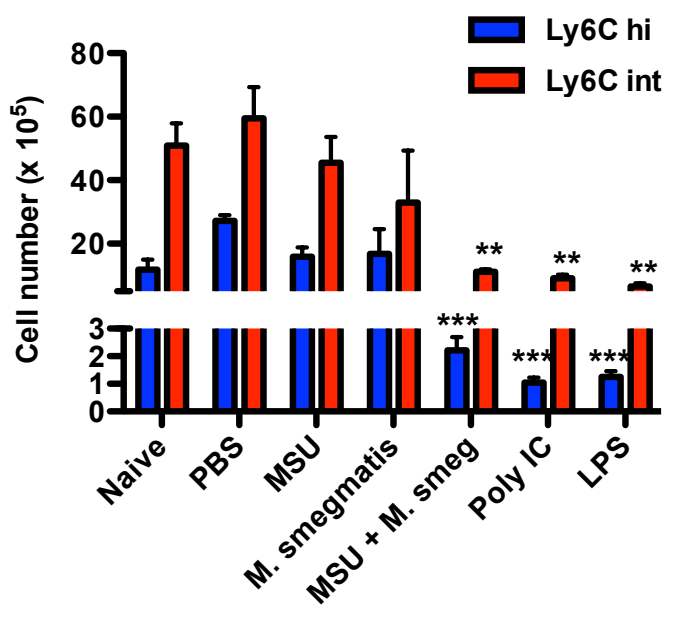

Figure D.1: Frequency and number of monocytes in spleens of adjuvant treated tumour bearing mice. C57 mice bearing established B16F1 tumours were injected around the tumour with the indicated adjuvants on day 7, 9, 11 and $14.24 \mathrm{~h}$ after the last treatment, spleens were analysed by flow cytometry. Ly6Chi monocytes were identified as live CD11b+ Ly6Chi Ly6G- cells and Ly6Cint monocytes were identified as live CD11b+ Ly6Cint Ly6G+ cells. (A) The percentages of both monocyte populations in spleens of naïve and adjuvant treated tumour bearing mice is shown. (B) The total numbers of both monocyte populations in spleens are graphed. Data are expressed as mean $+\mathrm{SE}$ and are from one experiment with 5 mice per group. The analysis of spleen monocytes has been performed by Lisa Shaw. ${ }^{*} p<0.05,{ }^{* *} p<0.01$ and ${ }^{* * *} p<0.001$ according to 1-way-ANOVA with Tukey's post test. 
Naive

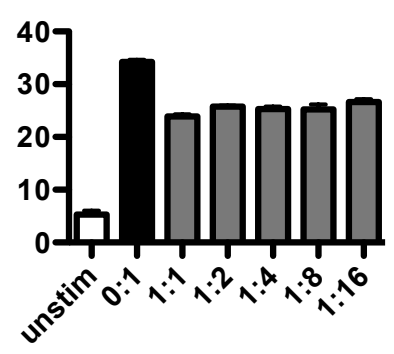

PBS

MSU
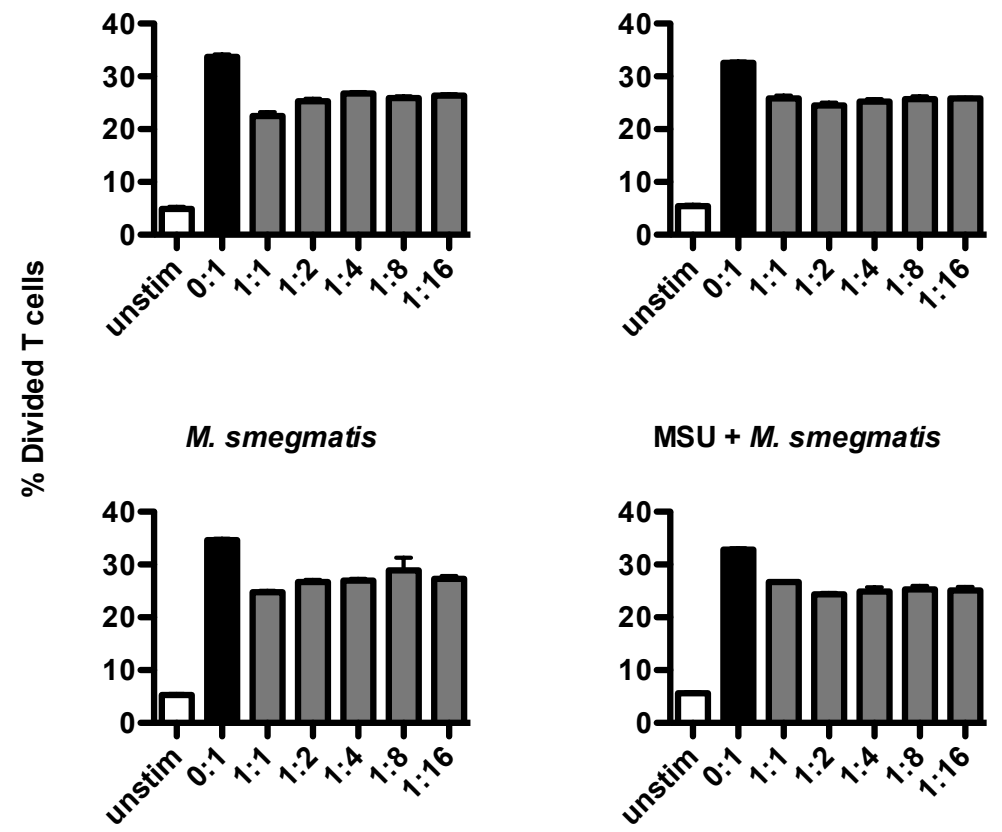

MSU + M. smegmatis

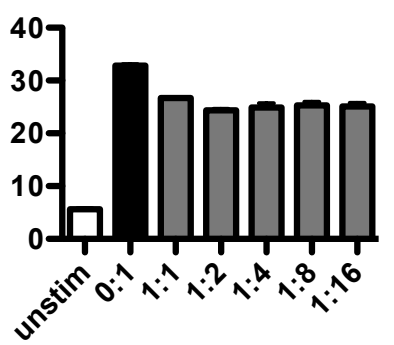

Poly IC

LPS
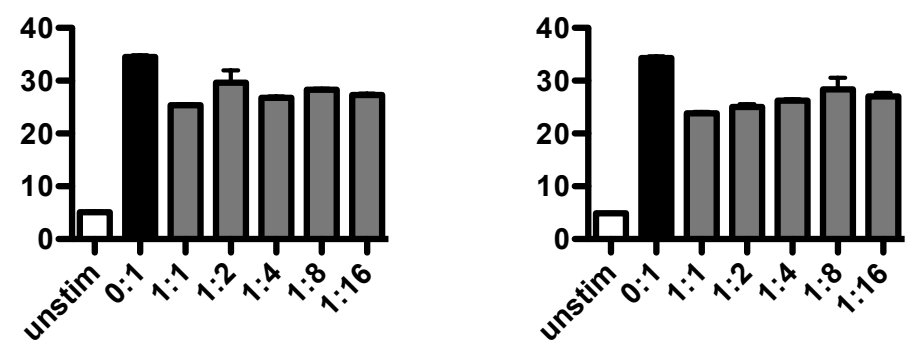

MDSC : T cell ratio

Figure D.2: Suppression of T cell proliferation by Ly6Chi monocytes. Data are from the same experiment as Figure D.1. After red blood cell lysis, spleen cells were separated through a lympholyte centrifugation. Cells from the buffy layer were then magnetically depleted of Ly6G+, CD3+ and B220+ cells, followed by positive magnetic selection for Gr$1+$ cells. Ly6Chi monocytes were enriched to 7-10 \%, with the remaining cells consisting mainly of B cells and T cells. Ly6Chi monocytes were plated at the indicated ratios with naïve CFSE labelled LN cells stimulated with anti-CD3 and anti-CD28. After 3 days T cell proliferation was assessed by CFSE dilution using flow cytometry. Data are expressed as mean $+\mathrm{SE}$ and are from one experiment with 5 mice per group. The analysis of spleen monocytes has been performed by Lisa Shaw. 
Naive

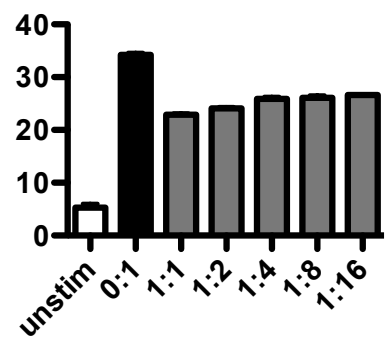

PBS

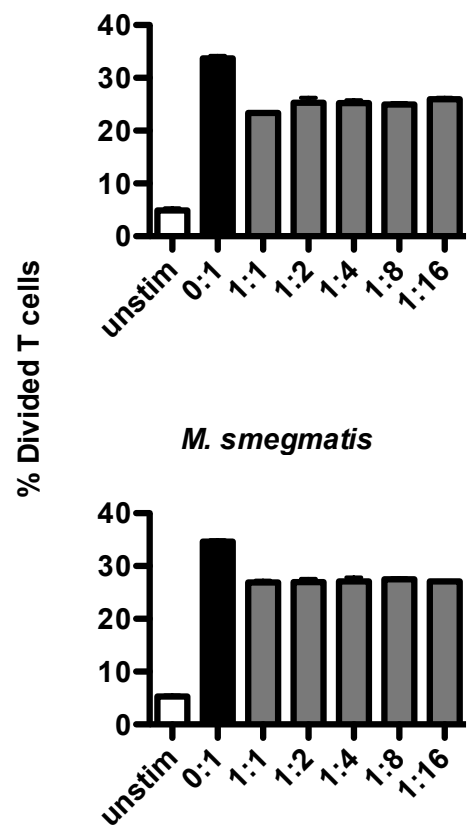

Poly IC

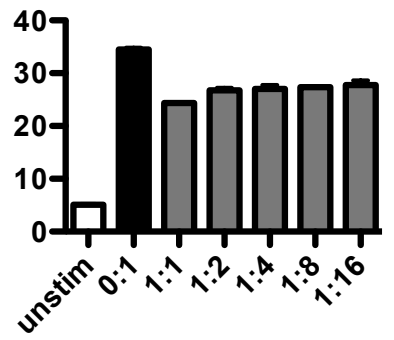

MSU

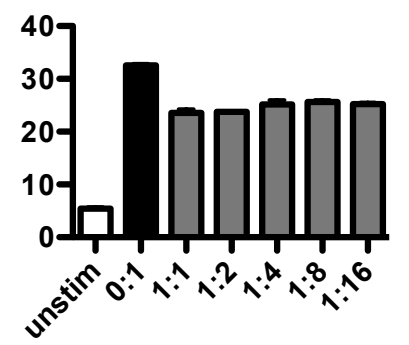

MSU + M. smegmatis

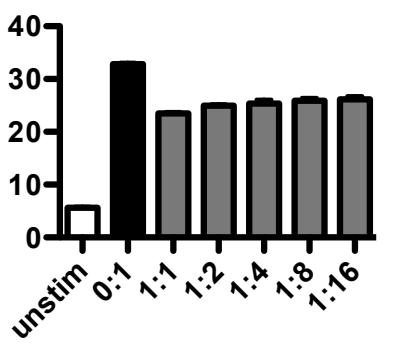

LPS

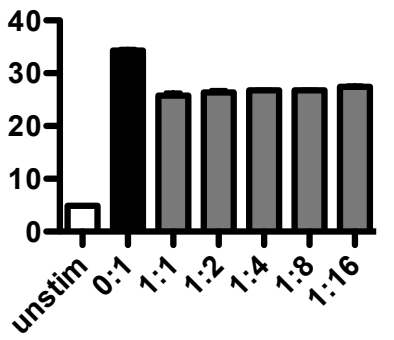

MDSC : T cell ratio

Figure D.3: Suppression of $\mathbf{T}$ cell proliferation by Ly6G+ cells. Data are from the same experiment as Figure D.1. After red blood cell lysis, spleen cells were separated through a lympholyte centrifugation. Cells from the pellet were then magnetically depleted of Ly6C+, CD3+ and B220+ cells. Ly6G+ cells were enriched to 40-50\%, with the remaining cells consisting mainly of B cells and T cells. Ly6G+ cells were plated at the indicated ratios with naïve CFSE labelled LN cells stimulated with anti-CD3 and anti-CD28. After 3 days $\mathrm{T}$ cell proliferation was assessed by CFSE dilution using flow cytometry. Data are expressed as mean $+\mathrm{SE}$ and are from one experiment with 5 mice per group. The analysis of spleen monocytes has been performed by Lisa Shaw. 


\section{E Publications}

Publications arising from the work presented in this thesis:

Fenella J Rich, Sabine Kuhn, Evelyn J Hyde, Jacquie L Harper, Franca Ronchese, Joanna R Kirman. Induction of $\mathrm{T}$ cell responses and recruitment of an inflammatory dendritic cell subset following tumor immunotherapy with Mycobacterium smegmatis. Cancer Immunol Immunother, [Epub ahead of print], 2012.

So Nai Lim, Sabine Kuhn, Evelyn Hyde, Franca Ronchese. Combined TLR stimulation with Pam3Cys and Poly I:C enhances Flt3-Ligand dendritic cell activation for tumor immunotherapy. J Immunother, 35 (9): 670-9, 2012.

Sabine Kuhn, Evelyn J Hyde, Fenella J Rich, Jacquie L Harper, Joanna R Kirman, Franca Ronchese. Peri-tumoral administration of adjuvants that induce inflammatory dendritic cells activates anti-tumor T cells and NK cells. Manuscript in preparation 